\title{
Vaccine development against carp viruses Integrating adaptive immunity
}

Carmen W. E. Embregts 


\section{Propositions}

1. The practical implementation of DNA vaccines against fish pathogens deserves immediate attention.

(this thesis)

2. The most crucial step in the validation of efficacy of experimental vaccines is the establishment of an appropriate challenge mode.

(this thesis)

3. Scientific data should be valued primarily on their biological relevance, not on their p-value.

4. When it comes to trends in food, it is safer to trust the expert's advice than one's own gut feeling.

5. As in Jurassic Park, genetic modification should be allowed when animals are contained properly.

6. The right to parental leave when adopting pets should be integrated in the collective labour agreement.

7. Given that creativity is an essential skill that benefits from life-long training, regular whole-office make-overs should be practised until retirement.

Propositions belonging to the thesis, entitled

Vaccine development against carp viruses - Integrating adaptive immunity

Carmen W.E. Embregts

Wageningen, $23^{\text {th }}$ of May 2018 


\section{Vaccine development against carp viruses Integrating adaptive immunity}

Carmen W.E. Embregts 


\section{Thesis committee}

\section{Promotor}

Prof. Dr G.F. Wiegertjes

Personal chair at Cell Biology and Immunology Wageningen University \& Research

\section{Co-promotor}

Dr M. Forlenza

Associate professor, Cell Biology and Immunology Wageningen University \& Research

\section{Other members}

Prof. Dr M.M. van Oers, Wageningen University \& Research

Dr C. Tafalla, National Institute for Agriculture and Food Research and Technology (INIA), Madrid, Spain

Prof. A. Adams, University of Stirling, UK

Dr S. Koumans, MSD Animal Health, Boxmeer

This research was conducted under the auspices of the Graduate School Wageningen Institute of Animal Sciences 


\title{
Vaccine development against carp viruses Integrating adaptive immunity
}

\author{
Carmen W.E. Embregts
}

Thesis

submitted in fulfilment of the requirements for the degree of doctor at Wageningen University

by the authority of the Rector Magnificus,

Prof. Dr. A.P.J. Mol,

in the presence of the

Thesis Committee appointed by the Academic Board

to be defended in public

on Wednesday 23 May 2018

at 1.30 p.m. in the Aula 
Carmen W.E. Embregts

Vaccine development against carp viruses - Integrating adaptive immunity 308 pages.

$\mathrm{PhD}$ thesis, Wageningen University, Wageningen, the Netherlands (2018) With references, with summaries in Dutch and English

ISBN: 978-94-6343-746-2

doi: $10.18174 / 441974$ 


Chapter 1 General introduction

Chapter 2 Oral vaccination of fish: Lessons from humans and veterinary species

Chapter 3 Intramuscular DNA vaccination of juvenile carp against Spring Viraemia of Carp Virus induces full protection and establishes a viral-specific $\mathrm{B}$ and $\mathrm{T}$ cell response

Chapter 4 Vaccination of carp against SVCV with an oral DNA

vaccine or an insect cells-based subunit vaccine

Chapter 5 Intra-muscular and oral vaccination of common carp

(Cyprinus carpio L.) using a Koi Herpesvirus ORF25

DNA vaccine

Chapter 6 Characterization of $\mathrm{T}$ cell populations of common carp 184

- a molecular and cellular approach

Chapter 7 Preliminary characterization of $\operatorname{Igm}^{+}$and $\operatorname{Igt}^{+} \mathrm{B}$ cell populations in carp and their role during systemic and mucosal infections

Chapter 8 General discussion

Summaries

Summary (English)

Samenvatting (Nederlands)

About the author

Curriculum vitae

List of publications

Overview of completed training activities

Acknowledgements 


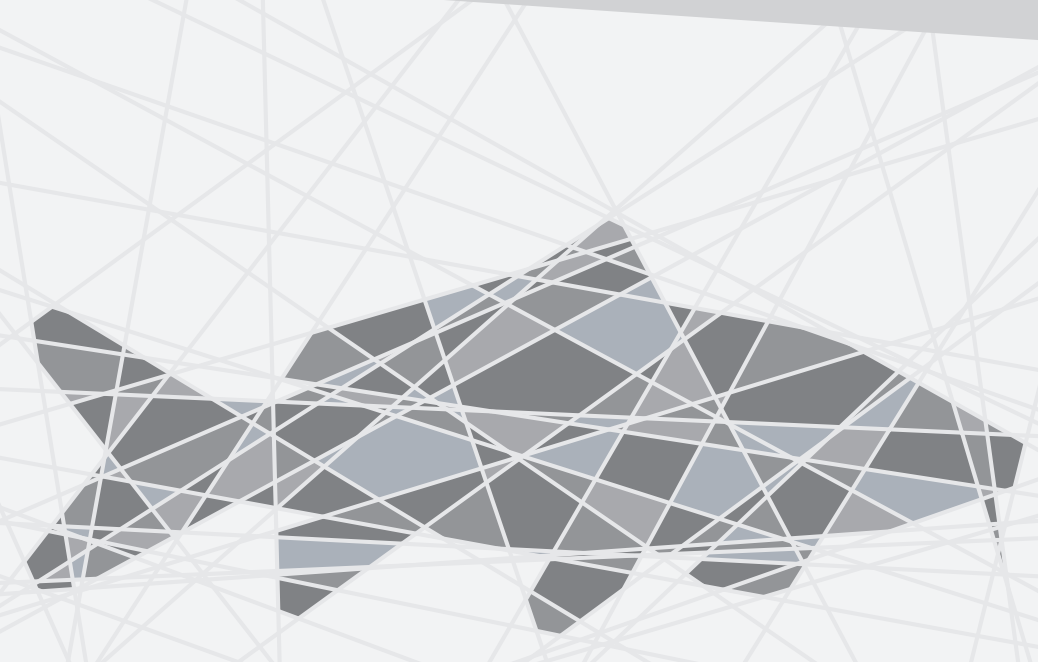




\section{General introduction}

Title page 2 


\section{Aquaculture}

\section{The pros and contras of intensification}

Over the last decades, the aquaculture and fisheries sectors underwent a complete metamorphosis. While fisheries have stabilized capture over the last 20 years, aquaculture production has made a tremendous rise. To illustrate, where aquaculture in 1974 contributed only $7 \%$ of the total human consumption of fish, this rose to $26 \%$ in 1994 and to $44 \%$ in 2014. This rise in production has also led to an increase in consumed fish products per capita worldwide; from $9.9 \mathrm{~kg}$ in the $1960 \mathrm{~s}$ to $14.4 \mathrm{~kg}$ in the 1990s and to more than $20 \mathrm{~kg}$ in 2015 [1]. To meet this growth in production output, intensification of the whole aquaculture sector took place over the last decades, resulting in a shift from (semi-)extensive to intensive aquaculture systems [2]. The obvious advantage of this development is an increase in production of fish proteins, badly needed to feed a growing world population.

The disadvantages of intensification include increased environmental impacts, as well as a strong increase in disease outbreaks, caused by multiple stress factors such as high stocking densities and poor water quality [3-7]. Alarmingly, these outbreaks are not only associated with well-known diseases but can also come from an array of emerging diseases, which can be a new form (different host or severity) of a known disease, or the outbreak of an existing disease in a new geographical area [8-10]. Besides placing the aquaculture sector at high risk, outbreaks of (emerging) diseases can also form a threat for wildlife fish species, especially in open aquaculture systems (e.g. nets, cages) [11]. In general, the increasing incidence of existing and emerging diseases calls for an urgent need to develop novel, effective vaccines for fish kept in (intensive) aquaculture systems, since effective treatments and vaccines are lacking for most diseases.

\section{Common carp - a major aquaculture species facing threats}

Cyprinids are the most cultured group of aquaculture fish species, with grass carp, silver carp and common carp comprising the top three of most produced freshwater fish species [12,13]. Common carp (Cyprinus carpio L.) are omnivorous and are either kept in high densities in monoculture in closed ponds or tanks (cold climate regions including Europe) or at low densities in closed polyculture systems [12,14]. These intensive culture systems often allow for limited water quality measures, resulting in multiple stressors such as crowding (high stocking densities in $\mathrm{kg} / \mathrm{m}^{3}$ ), 
high loading (high weight per unit flow rate in $\mathrm{kg} /(\mathrm{L} / \mathrm{min}))$ and competition for feed. The impacts of these stressors on the immune system of the fish have been known for long and have been characterized for multiple fish species. Various studies on common carp have shown that high stocking densities induced acute as well as chronic stress, characterized by increased plasma glucose, cortisol, and free fatty acid levels, decreased levels of lysozyme activity and phagocytic capacity as well as a lower number of lymphocytes. All these parameters can possibly affect levels of resistance to pathogens $[15,16]$. Indeed, carp aquaculture is threatened by a large number of different pathogens including bacteria (Mycobacterium spp., Flavobacterium branciophyla, Aeromonas salmonicida achromogenes), endo- and ectoparasites (Eimeria spp., Lernea spp., Argulus spp.) and viruses (Carp pox, Spring Viremia of Carp Virus (SVCV), Koi Herpes Virus (KHV; Cyprinid Herpesvirus-3 CyHV3)) (FAO, 2010-2017; Hoole et al., 2008). While sometimes chemical treatments can allow for elimination of certain pathogens, effective treatments and vaccines are still lacking for most of the important pathogens of carp.

\section{TargetFish: a targeted vaccine strategy for six major fish species in Europe}

Since outbreaks of infectious diseases lead to tremendous losses in the aquaculture sector, vaccines targeted against the most important diseases of fish are urgently needed. To this end, the project TargetFish (2012-2017) was funded by the European Commission $7^{\text {th }}$ Framework program. TargetFish focussed on six aquaculture species important to Europe, being Atlantic salmon (Salmo salar), rainbow trout (Oncorhynchus mykiss), European sea bass (Dicentrarchus labrax), gilthead seabream (Sparus aurata), turbot (Scophthalmus maximus) and common carp (Cyprinus carpio). The major aim of TargetFish was to bring together scientists leading on fish immunology and vaccinology and enterprises from the biotechnology and veterinary sectors, and to design vaccines for aquaculture species and bring them close to the European market. Specifically, TargetFish aimed to enhance targeted disease prophylaxis in Europe aquaculture by 1) generating knowledge about potential antigens and adjuvants for mucosal routes of vaccination while characterizing underlying immune mechanisms and 2) validating the obtained knowledge through response assays that monitor vaccine efficacy and safety. The project consortium consisted of 30 partners, representing an equal number of Research and Technology Departments (RTDs) and Small- to Medium-sized Enterprises (SMEs). They decided to cooperate closely while communicating with the larger vaccine and 
nutrition industries via an Industry Forum. Within the TargetFish project, The Cell Biology and Immunology Group of Wageningen University focused on common carp as target species and targeted Spring Viremia of Carp Virus (SVCV) and Koi Herpes Virus (KHV) as important pathogens. The results on the development of (oral) vaccines for carp against these two viruses and the corresponding immune responses, in particular the characterization of adaptive $\mathrm{B}$ and $\mathrm{T}$ cell responses and their role during infection or in response to vaccination, are subject of this thesis (Carmen Embregts).

\section{Viruses of major importance to common carp}

\section{Spring Viraemia of Carp Virus (SVCV)}

SVCV is a highly contagious Sprivivirus (family Rhabdoviridae) and causes a notifiable disease, meaning that occurrences should immediately be reported to the Office International des Epizooties (OIE). Typical outbreaks of SVCV occur during spring, when temperatures rise relatively quickly to values between $11^{\circ} \mathrm{C}$ and $17^{\circ} \mathrm{C}$ [18]. Under these conditions, mortalities can reach up to $70 \%$, mainly in juvenile fish [19-21], indicating its potentially severe impact on the carp aquaculture sector. SVCV mainly infects common carp but natural outbreaks have been reported in other freshwater species as well. The virus is thought to enter through the gills and spreads to systemic organs (liver, kidney, spleen and air bladder), where it causes haemorrhages and necrosis $[19,22]$. SVCV bullet shaped virions are $\sim 80-180 \mathrm{~nm}$ in length and 60-90 nm in diameter [19]. The linear, negative single-stranded RNA genome of 11.019 nucleotides (nt) contains 5 open reading frames (ORFs) with identical $10 \mathrm{nt}$ untranslated regions (UTRs) upstream of the genes that signal the start of translation [23,24]. The virion structure and genomic organization are depicted in Fig. 1.

The RNA-dependent RNA polymerase (L) is responsible for viral transcription and translation, which occurs upon interaction of the L protein with the nucleoprotein (N) and phosphoprotein $(\mathrm{P})[25,26]$. The $\mathrm{N}$ protein is involved in modulating transcription and forms the nucleocapsid together with the $\mathrm{P}$ protein and the viral RNA. Furthermore, part of the N protein (around 1/3) is directly associated with the viral RNA, giving rise to the helical symmetry of the nucleocapsid [27]. While the N 
protein is the most abundant viral protein, the glycoprotein $(\mathrm{G})$ is required for viral entry and is the target of neutralizing antibodies against SVCV. This appears to be a feature of Rhabdoviridae shared with the other fish viruses Infectious Haematopoietic Necrosis Virus (IHNV; Genus Novirhabdovirus) [28,29] and Viral Haemorrhagic Septicemia Virus (VHSV; Genus Novirhabdovirus) [29,30]. Together with the G protein, the matrix $(\mathrm{M})$ protein forms the bullet shape of the virion membrane by linking the nucleocapsid with the cytoplasmic domains of the $G$ protein. The $G$ protein is present at the virion surface as trimeric spikes or peplomers that are able to bind the cellular receptor that will subsequently induce endocytosis of the virus into the targeted host cell [19]. Given these traits, the G protein is the main antigen for vaccine development against SVCV and is also used in the experimental vaccines against SVCV described in this thesis.

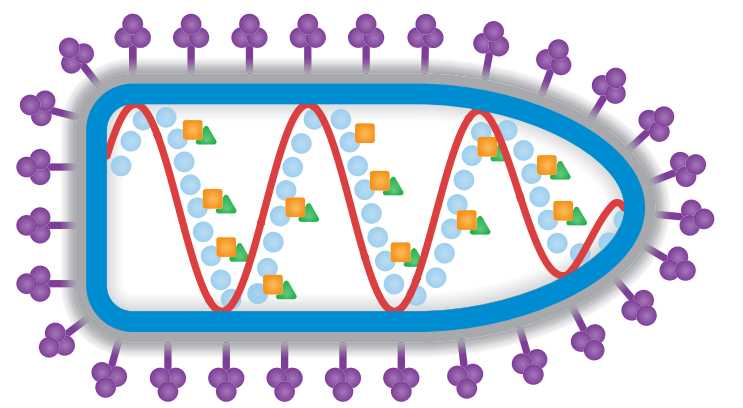

- Negative ssRNA

- N protein

$\triangle$ P protein

M protein

- $G$ protein

- L protein

Envelope

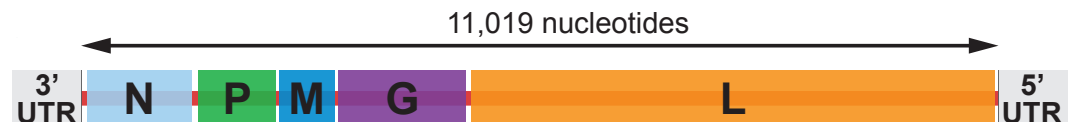

Fig. 1. Structure and genomic organization of SVCV. The upper part shows the bulletshaped virus containing the -ssRNA and the associated nucleoprotein (N), RNA-dependent RNA polymerase (L) and phosphoprotein (P). The matrix protein (M) links the cytoplasmic domains of the glycoprotein $(G)$, hereby creating the typical bullet shape. The lower part shows the genomic organization of SVCV, including the $10 \mathrm{nt} 3^{\prime}$ and 5' untranslated regions (UTRs). Except for the $10 \mathrm{nt}$ UTRs, coding region sizes for the different proteins (N, P, M, G, $\mathrm{L})$ are scaled based on their size (nt) in the SVCV genome.

\section{Koi Herpes Virus (KHV)}

KHV (also known as Cyprinid Herpesvirus-3, CyHV-3) is considered to be one of the most important factors affecting populations of common carp [31,32] and leads to a notifiable disease. KHV readily infects highly valuable ornamental koi carp (Cyprinus carpio koi) and it is hypothesized that koi shows, and especially the transport and mixing of koi at exhibition shows, has led to the global spread 
of this virus [31]. KHV enters through the skin, from where it spreads to internal tissues [33]. Symptoms of the disease include mass discoloration, proliferation of gill epithelium with necrotic lesions, skin lesions, necrosis of parenchyma cells in the liver, spleen, kidney and gastro-intestinal (GI) tract [34-36]. Outbreaks appear independent of the age of the fish and can lead to mortality rates of up to $100 \%$ [37]. $\mathrm{KHV}$ is a Herpesvirus belonging to the family of Alloherpesviridae and has a double stranded DNA genome of $295 \mathrm{kbp}$, encoding for 156 functional ORFs [38]. Of these ORFs, a number of 40-43 structural proteins have been identified in various strains $[39,40]$. The 40 structural proteins identified by Michel et al. could be further divided into 3 capsid proteins, 13 envelope proteins, 2 tegument proteins and 22 unclassified structural proteins. KHV virions (170-230 nm) consist of an icosahedral inner capsid containing the genome, surrounded by a lipid envelope bearing the viral glycoproteins. In between the capsid and envelope the tegument can be found; an amorphous layer of proteins [36]. A schematic overview of the virion with its identified proteins is depicted in Fig. 2.

The functional characterization of the different KHV proteins is still in its infancy due to the high number of structural proteins and the quick attenuation of KHV in culture. These factors make it difficult to select the best antigen, or antigens, for use in a vaccine against KHV. This scientific challenge, as well as the initial testing of a DNA vaccine encoding one of the major glycoproteins (ORF25) are described in this thesis.

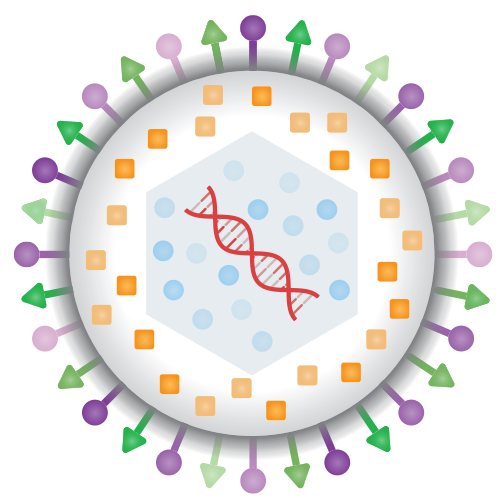

$\infty \times$ DNA

Capsid proteins

$72,78,92$

- Tegument proteins 62, 123

- Envelope proteins $32,81,136$

- Envelope glycoproteins

$25,59,65,99,108$, $115,131,132,148,149$

Fig. 2. Structure of KHV. The ds-DNA is surrounded by an icosahedral capsid and a surrounding envelope with both envelope proteins and glycoproteins. The tegument layer is in between the capsid and the surrounding envelope. The identified Open Reading Frames of structural proteins (ORFs, [39]) with their corresponding numbers are mentioned within each protein group. 


\section{Vaccine development for aquaculture fish species}

\section{Vaccine delivery routes}

Traditionally, vaccination of fish is performed by intraperitoneal (i.p.) injection or by bath/immersion treatment $[41,42]$. While injection has proven to be a very effective route of vaccination, it also has some major downsides: fish have to be caught, anesthetized and injected individually (either manually or with a vaccination machine), causing significant stress [43]. Furthermore, local tissue inflammation and tissue adhesion are often associated with injected vaccines, especially when strong adjuvants are used $[44,45]$. Ultimately, severe tissue damage can lead to downgrading of the filet (Reviewed in [46]). This emphasizes the need to develop efficient vaccines that can be administered in a relatively easy, non-invasive and stress-free way. Mucosal vaccines (i.e. immersion but also oral vaccines) allow for vaccine uptake through the skin and/or gills (immersion/bath) or intestine (oral vaccination) and can be delivered relatively stress-free, certainly when compared to injection vaccines. Bath vaccination allows for the vaccination of large quantities of fish but is only practical for small fish and requires high amounts of vaccine. Furthermore, bath vaccination is less suitable for fish kept in open systems such as cages. Oral vaccination, on the other hand, allows for vaccination of large groups of any sized fish and is independent of the system that the fish are housed in. However, a major downside of oral vaccines is the need to prevent degradation in the gastrointestinal tract, especially since the most important absorptive sites for antigens are found in the hindgut of fish [47-51]. While several efficient oral vaccines have been reported based on the use of inactivated (whole) pathogens, effective oral DNA vaccines or oral subunit vaccines are still scarce (Reviewed in [52]). Attempts to design oral vaccines (DNA, subunit) based on the G protein of SVCV are described in this thesis. Oral vaccination probably remains the most favourable approach to mucosal and stress-free vaccination of fish, as reviewed and discussed in this thesis.

\section{Vaccine types and recent advances in vaccine development}

While the first vaccines for use in aquaculture were based on inactivated or live attenuated pathogens, new vaccine types include DNA vaccines, recombinant subunit vaccines, virus-like-particles (VLPs), recombinant viral or bacterial vectors and synthetic peptides (Fig. 3). These new vaccine types aim to induce a targeted immune response against specific immunogenic proteins or peptide(s) or use a 
pathogen that has a crucial deletion(s) that leaves it infective and immunogenic but no longer pathogenic. While live attenuated vaccines generally induce strong immune responses, often correlated with high protection, their risk of residual or reversion to virulence raises safety concerns and argues in favour of new vaccine types. Inactivated vaccines, but also new approaches, such as those based on recombinant proteins, are generally regarded as safe but often require the use of adjuvants to trigger a sufficient immune response.

DNA vaccines combine many advantages; they are safe to use, relatively easy and cheap to produce and have a long shelf-life. Successful examples in fish include intramuscular (i.m.) DNA vaccination against VHSV [30], IHNV [28] and ISAV [53] in rainbow trout, i.m. and oral DNA vaccination against Infectious Pancreatic Necrosis Virus (IPNV) [54,55], and i.m. DNA vaccination against SVCV in common carp (this thesis, [21]). Besides the development of various new vaccine types over the past decades, legislation of vaccines has also advanced. Recently, the CLYNAV vaccine against Salmon Alphavirus Virus 3 (SAV3) was the first DNA vaccine for fish that was given a positive recommendation for marketing authorization by the European Union [56]. This breakthrough indicates a bright future for DNA vaccines against other fish diseases as well.

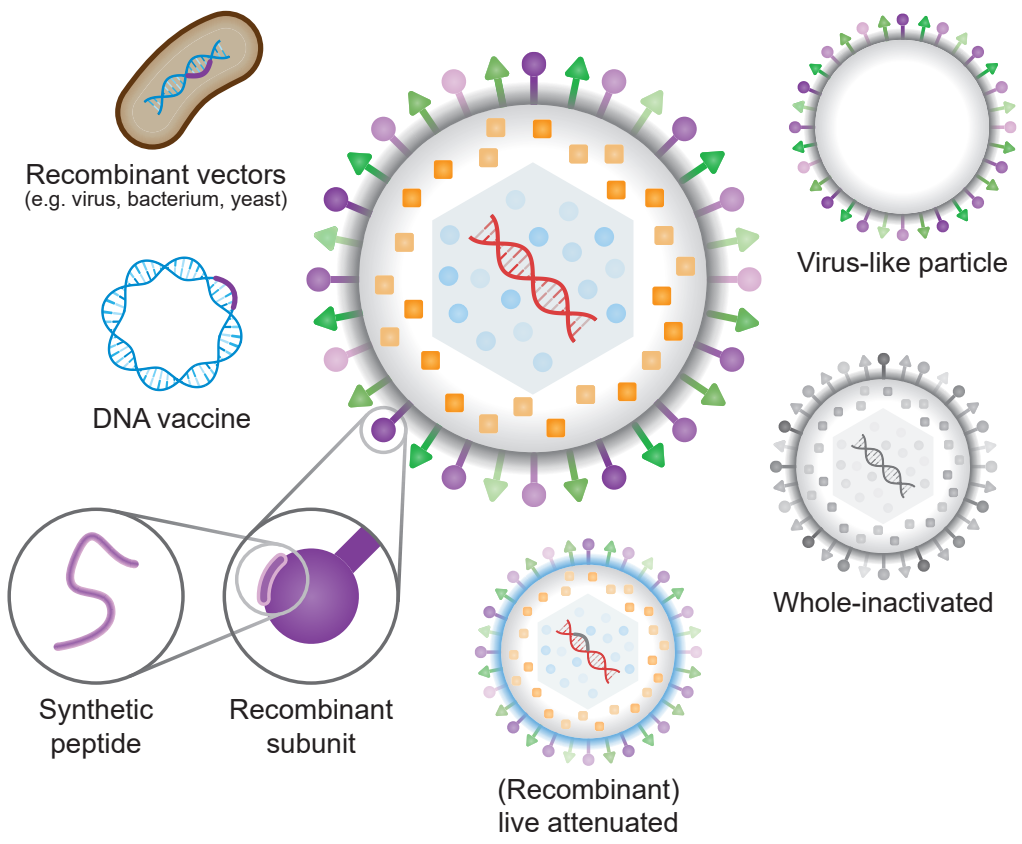


Fig. 3. Overview of different vaccine types available against viral diseases.Vaccines against viruses are taken as a reference but all approaches shown, except for the viruslike-particles (VLPs) are applicable to other pathogens as well. Included are: the "traditional" whole-inactivated vaccines; (recombinant) live attenuated vaccines with an indicated alteration in the genome; virus-like-particles composed of selected capsid proteins that retain virus size and mimic virus tropism; subunit vaccines or synthetic peptides relying on the recombinant expression or synthesis of (parts of) viral protein(s) in an heterologous system; DNA vaccines based on plasmids encoding a viral protein; and recombinant vectors that carry the information or expressed the target protein.

\section{DNA vaccination: what makes it so successful in fish?}

Based on the experience gained with the use of the above-mentioned DNA vaccines for fish, we can generally conclude that, at least when injected intramuscularly, DNA vaccines are effective without the need for adjuvants. This effectiveness is possibly caused by the presence of unmethylated $\mathrm{CpG}$ motifs in the plasmid backbone that give a "non-self" signal to pattern recognition receptors, a response also shown in fish [57,58]. Furthermore, where subunit or inactivated vaccines mainly induce antibody-driven immune responses, DNA vaccines induce both humoral and cellmediated immune responses [21,59-62].

DNA vaccines can be delivered using multiple routes, of which the intramuscular (i.m.) injection route is the most commonly used one. Upon i.m. injection, the DNA is either taken up by local cells, degraded, or redistributed through circulation (reviewed in [63]). When it enters the circulation, it has been shown to reach other organs away from the site of injection (e.g. spleen, kidney and gills in the case of fish), although part of the circulating DNA will be degraded by blood nucleases $[64,65]$. Although the mechanisms of i.m. DNA vaccination have not yet been revealed in detail, myocytes can take up the DNA by different routes (reviewed in [65]), leading to transport to the nucleus and subsequent transcription and translation. Next, viral proteins will be expressed on the host cell surface $[21,66]$ and viral peptides will be presented on Mhc-I molecules on myocytes, as well as on Mhc-II molecules on antigen-presenting cells after being processed. Together, this will directly activate humoral as well as cellular responses, including classical complement activation and $\mathrm{Cd} 8^{+} \mathrm{T}$ cells-mediated cytotoxicity. It is suggested that part of the success of i.m. DNA vaccination of fish is linked to the endogenous expression of the viral protein by host cells, possibly mimicking a natural infection and thus triggering 'proper' protective immune responses [63,67-70]. 


\section{Studying adaptive immune responses after vaccination}

In immunized animals, or animals that have survived an infection, the pool of memory B and T cells will guarantee protection upon (re-)exposure to the pathogen. The presence of memory B cells can lead to the rapid production of high amounts of antigen-specific antibodies, and the presence of memory $\mathrm{T}$ cells can lead to the rapid expansion of specific $\mathrm{Cd} 8^{+}$cytotoxic $\mathrm{T}$ cells or $\mathrm{Cd} 4^{+}$helper $\mathrm{T}$ cells (including regulatory $\mathrm{T}$ cells) which regulate immune responses through cytokine secretion. A thorough knowledge of the adaptive immune mechanisms (i.e. humoral and/or cellular responses) triggered by protective vaccines might help in the development of diagnostic tools that can predict the level of protection after vaccination, thus circumventing laborious vaccine potency tests that require many experimental animals. Furthermore, this knowledge will give insight into the lack of protection of suboptimal vaccines and will aid their further optimization. Given the importance of measuring adaptive immune responses after vaccination, in this thesis we studied $\mathrm{B}$ and $\mathrm{T}$ cell responses in naïve, vaccinated and infected carp using existing and newly developed tools. In the next two sections, we will introduce some of the assays most commonly used to study T- and B-cell responses in fish, including the ones we used to investigate adaptive responses after vaccination.

\section{Studying $T$ cell responses after vaccination}

There is good evidence that similar to mammals, fish $\mathrm{T}$ cells can be divided in $\mathrm{Cd} 4+$ helper $\mathrm{T}$ cells and $\mathrm{Cd} 8+$ cytotoxic $\mathrm{T}$ cells. Generally, helper and regulatory $\mathrm{T}$ cells recognize peptides presented by MHC class-II, and cytotoxic $\mathrm{T}$ cells recognize peptides presented by MHC class-I [71-74]. One method to study cytotoxicity T cell function and memory is the cytotoxicity assay, which does not require $\mathrm{T}$ cell specific antibodies but does require Mhc-matched clonal fish. Briefly, in this in vitro assay, effector cells (peripheral blood leukocytes, PBLs) isolated from a vaccinated fish are mixed with MHC-matched target cells infected with the same virus targeted by the vaccine. If virus-specific cytotoxicity $\mathrm{T}$ cells are present in the pool of effector cells, they will kill infected target cells. The percentage of killed cells can be quantified through lactate dehydrogenase (LDH)-release, an enzyme that is released upon cell damage $[71,75,76]$. The use of this assay has already allowed for the identification of virus-specific "memory" cytotoxic $\mathrm{T}$ cell responses after vaccination against VHSV 
[62], and IHNV [77] in trout and against Carp Hematopoietic Necrosis Virus in carp [78].

Another method to study T cell memory is the in vitro proliferation assay. Here, cells are stained with a fluorescent dye and then exposed to the vaccine antigen or to the pathogen. Any memory cells present will divide, leading to halving of the intensity of the fluorescent dye at each round of division. Double staining of the cells with a T cellspecific antibody allows for visualization of proliferating T cells. Comparison of the proliferation of cells isolated from naïve and vaccinated fish, will give an estimation of the "memory" response. Alternatively, fish can be injected intravenously with a fluorescent dye that binds specifically to proliferating cells, allowing for the analysis of proliferating "memory" cells in vivo e.g. after a booster vaccination or challenge with the selected pathogen. While flow cytometry had not been used to study T cell proliferation after vaccination, it was used to characterize proliferating "memory" $\mathrm{T}$ cells in common carp that survived an infection with the blood-borne parasite Trypanoplasma borreli [79]. In this thesis the in vitro proliferation assay was used to study T cell memory after DNA vaccination against SVCV [21].

Recent advances in molecular biology open up even more opportunities for studying antigen-specific T cells. Spectratyping approaches can determine specific changes of $\mathrm{T}$ cell receptor (tcr) profiles by describing the lengths of the corresponding complementary-determining region 3 (CDR3) [80]. To illustrate, where a highly diverse and polyclonal $\mathrm{T}$ cell repertoire was observed in naïve fish, a less diverse and more skewed distribution was observed after primary and secondary infection with VHSH, indicating the clonal expansion of VHSV-specific ("memory") cells $[59,81]$. A similar approach can also be used to study proliferation of B cells, as will be discussed in the next section.

\section{Studying B cell responses to understand protection after vaccination}

While immunoglobulins (Igs) of mammals can be divided in $\operatorname{IgG}$, IgA, IgD, IgM and IgE isotypes, teleost species have Igm, Igd and Igt. Similar to mammals, fish Igm plays a major role in systemic immunity, although Igm responses at mucosal surfaces have been described as well [82-87]. Due to the limited availability of anti-Igd antibodies, Igd populations have only been studied in catfish (Ictalurus punctatus) [88] and rainbow trout [89]. Interestingly, both $\operatorname{Igm}^{+} \operatorname{Igd}^{+}$and $\operatorname{Igm}^{-\operatorname{Igd}^{+}}$ populations were identified in both species, in contrast to mammals where $\operatorname{IgD}$ is 
mostly co-expressed with IgM on mature B cells $[90,91]$ and only minor populations of $\operatorname{IgM}-\operatorname{IgD}^{+}$cells have been described in the upper aerodigestive mucosa $[92,93]$ or in the blood as naïve self-reactive anergic B cells [94]. The function of the different $\operatorname{Igd}^{+}$populations has not yet been studied in detail, although in rainbow trout, a $\mathrm{Ccr} 7^{+} \mathrm{Igd}^{+} \mathrm{Igm}^{-}$population was found mainly in gills [95]. While their number decreased in gills after bath challenge with VHSH, at the same time their number increased in the head kidney, suggesting a functional role for $\mathrm{Ccr}^{+} \mathrm{Igd}^{+} \mathrm{Igm}^{-}$cells. Igt is proposed to be the main immunoglobulin involved in mucosal responses strongly reacting to gill- and intestinal parasites, at least in rainbow trout $[83,89]$. However, further insights in the protective role of Igt in other fish species is emerging slowly. Carp has (at least) two copies of Igt, named Igt1 and Igt2, with respectively four and two immunoglobulin domains. Of these two, based on gene expression studies, Igt2 was proposed to be more involved in mucosal responses and Igt1 more in systemic responses. While igt 2 is expressed more than igt 1 in mucosal organs and increased in gene expression during infection with the mucosal parasite Lernea, igt1 expression increased during infection with the blood parasite Trypanoplasma borelli [96].

With respect to vaccination, protection can often be correlated with the presence or absence of neutralizing antibodies. To illustrate, the induction of neutralizing antibodies after vaccination has been described for multiple aquatic viruses including IPNV [54], IHNV [97] and VHSV [29]. In this thesis, a neutralization assay was used to quantify neutralizing serum antibodies against SVCV. However, the relative contribution of different Ig subtypes cannot be determined in this assay. In contrast to this technique, antigen-specific ELISAs or ELISPOTs can be used to detect antigen-specific antibody subtypes. These assays have been used to measure antibody responses after vaccination against several aquatic pathogens including the bacteria Vibrio harveyi [98] and Vibrio vulnificus [99] and the virus IPNV [100]. While these assays can be applied to study B cell responses after any type of vaccination, they require the availability of antibodies specific for each Ig-subtype, which are still lacking in many fish species. Furthermore, since Igd and Igt titres are usually much lower than Igm titres, quantification of (antigen-specific) Igd and Igt in serum and mucus requires highly sensitive assays and is often problematic. Besides examining antibody responses, one can also study the proliferation of the $\mathrm{B}$ cell subtypes themselves using in vitro and in vivo proliferation assays, as explained in the previous paragraph. To illustrate the potential "memory" B cells specific for 
Trypanoplasma borreli [79] or Ichthyophthirius multifiliis [101] were described using the in vitro proliferation assay. Similar to what has been described for T cells, studying the use of specific VDJ combinations of the Igh chain through spectratyping allows for alternative ways to characterize antigen-specific VDJ profiles. Using this technique, VHSV-specific clonal Igm and Igt responses were identified after infection with VHSV [102]. A similar approach can be used to study antigen-specific clonal B cell responses after vaccination. 


\section{Outline of this thesis}

While Spring Viremia of Carp virus (SVCV) and Koi Herpes Virus (KHV) are probably the two most important viruses affecting carp production, no effective vaccines for world-wide use were available at the start of this project. In addition, a reliable experimental challenge that best complies with the natural route of infection, essential to test vaccine efficacy, was not available for SVCV. Traditionally, fish vaccines are administered by injection, resulting in stress and local side-effects. Since oral vaccines allow for stress-free delivery their development is desirable. Furthermore, deeper insight into (adaptive) immune responses of carp after vaccination would help to develop vaccination strategies.

For these reasons, the two main aims of this thesis were 1) to design experimental (injection and/or oral) vaccines against SVCV and KHV and to assess their efficacy following experimental challenge, and 2) to characterize protective immune responses of carp after infection and vaccination, with a focus on adaptive immune responses. The results described in this thesis will contribute to future vaccination strategies for common carp against two major viral pathogens - SVCV and KHV. Furthermore, detailed knowledge on the (adaptive) immune system will be of great value for the design of new vaccines against other important diseases in carp.

First, in chapter 2, we review the current state of oral vaccines for aquaculture fish species. We discuss all factors considered essential for the development of oral vaccines: choosing the protective antigen, the correct antigen expression system, and an effective encapsulation matrix. Furthermore, we examine whether the nature of the pathogen and the fish species are important determinants for the efficacy of oral vaccines. Finally, we discuss whether oral vaccination should be considered the best route for vaccination against all pathogens.

In chapter 3 we develop a novel DNA vaccine encoding the SVCV-G protein, and show that it confers $90-100 \%$ protection after intramuscular (i.m.) injection of juvenile carp. This protection was validated with an optimized experimental bath challenge, resembling the natural route of infection of SVCV. We investigated the early inflammatory response at the site of injection in the muscle as well as induction of long-term systemic immune responses. Our results show for the first time that both $\mathrm{B}$ and $\mathrm{T}$ cell mediated responses are involved in the protection against SVCV. With the effectiveness of i.m. injection of the SVCV-G DNA vaccine in mind, we 
considered an oral delivery route for the same vaccine. In chapter $\mathbf{4}$ we describe the inefficacy of the SVCV-G DNA vaccine when applied via an oral route. Despite the use of multiple vaccination regimes, doses and the addition of the mucosal adjuvant lymphotoxin B (LTB), no protection could be observed from the experimental challenge with SVCV. To learn from the absence of protection, we studied the local and systemic immune responses induced by oral vaccination. We also describe the development of an experimental subunit vaccine based on a recombinant baculovirus expression of the SVCV-G protein. Unfortunately, no protection was afforded when administering the subunit vaccine through multiple routes.

Building on the experience with SVCV, in chapter 5 we set out to develop an experimental prototype vaccine against KHV taking a DNA vaccination approach. The DNA vaccine encoded either a soluble or a transmembrane form of ORF25, one of the major membrane proteins of KHV considered an important target for neutralizing antibodies. We performed vaccination through the i.m. or oral route using multiple vaccine doses. Unfortunately, we could not induce a strong protection against a subsequent challenge. We discuss the influence of the (lack of) induction of local immune responses in the observed effect. Finally, we propose new strategies for the design of future vaccines against KHV.

The characterization of adaptive immune response in fish lags behind mainly because of a lack of tools to study B and T cell subsets. To this end, we have developed antibodies and assays, and used them in the following two chapters. In chapter 6 , we characterized $\mathrm{T}$ cell populations in common carp, showing that both the antiCd4-1 and anti-Cd8a1 antibody react to small lymphoid-like cells and do not react to B cells, neutrophilic granulocytes, thrombocytes or monocytes/macrophages. Furthermore, we describe the existence of multiple $\mathrm{Cd} 4$ genes and isoforms ( $c d 4-1 a$, $c d 4-1 b, c d 4-2 a$ and $c d 4-2 b)$ and characterized their expression profiles in multiple organs and sorted cells. Finally, we gain new insights into the cells recognized by the WCL38 antibody, which was previously described to recognize putative mucosal T cells.

In chapter 7 we use our newly developed antibodies to characterize Igt1 and Igt2 populations in multiple organs. Using both a mucosal (Sphaerospora molnari) and a systemic (Trypanoplasma borreli) infection we show that, in contrast to what was reported previously, Igt1 and Igt 2 play a role in both mucosal and systemic infections. Finally, in chapter $8 \mathrm{I}$ place all outcomes of the previous chapters in a larger 
framework and discuss the limitations and challenges as well as future perspectives of vaccine development for carp. Furthermore, I discuss how the advances in molecular biology can aid characterization of (adaptive) immune responses in carp, and how this knowledge can help in developing assays to predict vaccine efficacy. In conclusion, this thesis contributes to the protection of common carp against SVCV and KHV by 1) developing various vaccine types, each tested through multiple delivery routes and 2) investigating immune responses after vaccination, increasing knowledge on carp immunology in general, and on protective mechanisms of vaccination in particular. The insights into antigen selection, vaccine design and vaccine delivery are also valuable for vaccine development for fish species.

\section{References}

1. FAO. The State of World Fisheries and Aquaculture 2016. (2016). Available at: http://www. fao.org/3/a-i5555e.pdf [Accessed September 18, 2017]

2. EU. The Long-Term Economic and Ecologic Impact of Larger Sustainable Aquaculture. (2014). doi:10.1007/s13398-014-0173-7.2

3. Iwama GK, Picktering AD, Sumpter JP. Fish stress and health in aquaculture. 62nd ed. Cambridge University Press (2011).

4. Costas B, Aragão C, Mancera JM, Dinis MT, Conceição LEC, Refojos BC. High stocking density induces crowding stress and affects amino acid metabolism in Senegalese sole Solea senegalensis (Kaup 1858) juveniles. Aquac Res (2008) 39:1-9. doi:10.1111/j.13652109.2007.01845.x

5. Chebaani N, Guardiola FA, Sihem M, Nabil A, Oumouna M, Meseguer J, Esteban MA, Cuesta A. Innate humoral immune parameters in Tilapia zillii under acute stress by low temperature and crowding. Fish Physiol Biochem (2014) 40:797-804. doi:10.1007/s10695013-9886-3

6. Sadhu N, Sharma SRK, Joseph S, Dube P, Philipose KK. Chronic stress due to high stocking density in open sea cage farming induces variation in biochemical and immunological functions in Asian seabass (Lates calcarifer, Bloch). Fish Physiol Biochem (2014) 40:11051113. doi:10.1007/s10695-014-9909-8

7. Yarahmadi P, Miandare HK, Fayaz S, Caipang CMA. Increased stocking density causes changes in expression of selected stress- and immune-related genes, humoral innate immune parameters and stress responses of rainbow trout (Oncorhynchus mykiss). Fish Shellfish Immunol (2016) 48:43-53. doi:10.1016/j.fsi.2015.11.007

8. Murray AG, Peeler EJ. A framework for understanding the potential for emerging diseases in aquaculture. in Preventive Veterinary Medicine, 223-235. doi:10.1016/j. prevetmed.2004.10.012

9. Pridgeon J. Major bacterial diseases in aquaculture and their vaccine development. CAB Rev Perspect Agric Vet Sci Nutr Nat Resour (2012) 7: doi:10.1079/PAVSNNR20127048

10. Walker PJ, Winton JR. Emerging viral diseases of fish and shrimp. Vet Res (2010) 41: doi:10.1051/vetres/2010022

11. Jones SRM, Bruno DW, Madsen L, Peeler EJ. Disease management mitigates risk of pathogen transmission from maricultured salmonids. Aquac Environ Interact (2014) 6:119134. doi:10.3354/aei00121 
12. FAO. Cultured Aquatic Species Information Program. Cyprinus carpio. (2017) Available at: http://www.fao.org/fishery/culturedspecies/Cyprinus_carpio/en\#tcNA00D6 [Accessed September 18, 2017]

13. Rahman MM. Role of common carp (Cyprinus carpio) in aquaculture production systems. Front Life Sci (2015) 8:399-410. doi:10.1080/21553769.2015.1045629

14. Rahman MM, Verdegem MCJ, Nagelkerke LAJ, Wahab MA, Milstein A, Verreth JAJ. Growth, production and food preference of rohu Labeo rohita (H.) in monoculture and in polyculture with common carp Cyprinus carpio (L.) under fed and non-fed ponds. Aquaculture (2006) 257:359-372. doi:10.1016/j.aquaculture.2006.03.020

15. Yin Z, Lam T., Sin Y. The effects of crowding stress on the non-specific immune response in fancy carp (Cyprinus carpio L.). Fish Shellfish Immunol (1995) 5:519-529. doi:10.1016/ s1050-4648(95)80052-2

16. Ruane NM, Carballo EC, Komen J. Increased stocking density influences the acute physiological stress response of common carp Cyprinus carpio (L.). Aquac Res (2002) 33:777-784. doi:10.1046/j.1365-2109.2002.00717.x

17. Hoole D, Bucke D, Burgess P, Wellby I. Diseases of carp and other cyprinid fishes. Fishing News Books (2008). doi:10.1002/9780470999752

18. Ahne W. The influence of environmental temperature and infection route on the immune response of carp (Cyprinus carpio) to spring viremia of carp virus (SVCV). Vet Immunol Immunopathol (1986) 12:383-386. doi:10.1016/0165-2427(86)90144-3

19. Ahne W, Bjorklund HV, Essbauer S, Fijan N, Kurath G, Winton JR. Spring viremia of carp (SVC). Dis Aquat Organ (2002) 52:261-272. doi:10.3354/dao052261

20. Fijan N, Petrinec Z, Sulimanovic D, Zwillenberg LO. Isolation of the viral causative agent from the acute form of infectious dropsy of carp. Vet Arh (1971) 41:125-138.

21. Embregts CWE, Rigaudeau D, Veselý T, Pokorová D, Lorenzen N, Petit J, Houel A, Dauber M, Schütze H, Boudinot P, et al. Intramuscular DNA Vaccination of Juvenile Carp against Spring Viremia of Carp Virus Induces Full Protection and Establishes a Virus-Specific B and T Cell Response. Front Immunol (2017) 8:1340. doi:10.3389/fimmu.2017.01340

22. Ahne W. Uptake and multiplication of spring viraemia of carp virus in carp, Cyprinus carpio L. J Fish Dis (1978)265-268. doi:10.1111/j.1365-2761.1978.tb00029.x

23. Björklund H V., Higman KH, Kurath G. The glycoprotein genes and gene junctions of the fish rhabdoviruses spring viremia of carp virus and hirame rhabdovirus: analysis of relationships with other rhabdoviruses. Virus Res (1996) 42:65-80. doi:10.1016/01681702(96)01300-7

24. Hoffmann B, Schütze H, Mettenleiter TC. Determination of the complete genomic sequence and analysis of the gene products of the virus of Spring Viremia of Carp, a fish rhabdovirus. Virus Res (2002) 84:89-100. Available at: www.elsevier.com/locate/virusres [Accessed July 20, 2017]

25. Roy P, Clewley JP. Spring viremia of carp virus RNA and virion-associated transcriptase activity. J Virol (1978) 25:912-6. Available at: http://www.ncbi.nlm.nih.gov/pubmed/565417 [Accessed December 26, 2017]

26. Roy P. Phosphoproteins of Spring Viremia of Carp virus. Virology (1981) 112:274-281. doi:10.1016/0042-6822(81)90632-2

27. Sokol F, Koprowski H. Structure-function relationships and mode of replication of animal rhabdoviruses. Proc Natl Acad Sci U S A (1975) 72:933-6. doi:10.1073/pnas.72.3.933

28. Anderson E., Mourich DV, Fahrenkrug SC, Lapatra SE, Shepherd J, Leong JA. Genetic immunization of rainbow trout (Oncorhynchus mykiss) against infectious hematopoietic necrosis virus. Mol Mar Biol Biotechnol (1996) 5:114-122.

29. Boudinot P, Blanco M, de Kinkelin P, Benmansour A. Combined DNA immunization with the glycoprotein gene of viral hemorrhagic septicemia virus and infectious hematopoietic necrosis virus induces double-specific protective immunity and nonspecific response in rainbow trout. Virology (1998) 249:297-306. doi:10.1006/viro.1998.9322 
30. Lorenzen N, Lorenzen E, Einer-Jensen K, Heppell J, Wu T, Davis H. Protective immunity to VHS in rainbow trout (Oncorhynchus mykiss, Walbaum) following DNA vaccination. Fish Shellfish Immunol (1998) 8:261-270.

31. Haenen OLM, Way K, Bergmann SM, Ariel E. The emergence of koi herpesvirus and its significance to European aquaculture. Bull Eur Assoc Fish Pathol (2004) 24:293-307. Available at: http://library.wur.nl/WebQuery/wurpubs/fulltext/198497 [Accessed September 20, 2017]

32. Pokorova D, Vesely T, Pickova V, Reschova S, Hulova J. Current knowledge on koi herpesvirus (KHV): a review. Vet Med (Praha) (2005) 50:139-147.

33. Costes B, Raj VS, Michel B, Fournier G, Thirion M, Gillet L, Mast J, Lieffrig F, Bremont $\mathrm{M}$, Vanderplasschen A. The major portal of entry of koi herpesvirus in Cyprinus carpio is the skin. J Virol (2009) 83:2819-30. doi:10.1128/JVI.02305-08

34. Oh M-J, Jung SJ, Choi TJ, Kim HR, Rajendran KV, Kim YJ, Park MA, Chun, S.K. MJ. A viral disease occurring in cultured carp Cyprinus carpio in Korea. Fish Pathol (2001) 36:147-151. doi:10.3147/jsfp.36.147

35. Hedrick RP, Waltzek TB, McDowell TS. Susceptibility of koi carp, common carp, goldfish, and goldfish x common carp hybrids to cyprinid herpesvirus-2 and herpesvirus-3. J Aquat Anim Health (2006) 18:26-34. doi:10.1577/H05-028.1

36. Miyazaki T, Kuzuya Y, Yasumoto S, Yasuda M, Kobayashi T. Histopathological and ultrastructural features of Koi herpesvirus (KHV)-infected carp Cyprinus carpio, and the morphology and morphogenesis of KHV. Dis Aquat Organ (2008) 80:1-11. doi:10.3354/ dao01929

37. Ilouze M, Dishon A, Kotler M. Characterization of a novel virus causing a lethal disease in carp and koi. Microbiol Mol Biol Rev (2006) 70:147-156. doi:10.1128/MMBR.70.1.147156.2006

38. Aoki T, Hirono I, Kurokawa K, Fukuda H, Nahary R, Eldar A, Davison AJ, Waltzek TB, Bercovier H, Hedrick RP. Genome Sequences of Three Koi Herpesvirus Isolates Representing the Expanding Distribution of an Emerging Disease Threatening Koi and Common Carp Worldwide. J Virol (2007) 81:5058-5065. doi:10.1128/JVI.00146-07

39. Michel B, Leroy B, Raj VS, Lieffrig F, Mast J, Wattiez R, Vanderplasschen AF, Costes B. The genome of cyprinid herpesvirus 3 encodes 40 proteins incorporated in mature virions. $J$ Gen Virol (2010) 91:452-462. doi:10.1099/vir.0.015198-0

40. Yi Y, Zhang H, Lee X, Weng S, He J, Dong C. Extracellular virion proteins of two Chinese CyHV-3/KHV isolates, and identification of two novel envelope proteins. Virus Res (2014) 191:108-116. doi:10.1016/j.virusres.2014.07.034

41. Sommerset I, Krossøy B, Biering E, Frost P. Vaccines for fish in aquaculture. Expert Rev Vaccines (2005) 4:89-101. doi:10.1586/14760584.4.1.89

42. Dhar AK, Manna SK, Thomas Allnutt FC. Viral vaccines for farmed finfish. Virusdisease (2014) 25:1-17. Available at: http://www.pubmedcentral.nih.gov/articlerender. fcgi?artid=3889245\&tool=pmcentrez\&rendertype=abstract [Accessed October 15, 2015]

43. Espelid S, Løkken GB, Steiro K, Bøgwald J. Effects of cortisol and stress on the immune system in Atlantic Salmon (Salm o salar L.). Fish Shellfish Immunol (1996) 6:95-110. doi:10.1006/fsim.1996.0011

44. Midtlyng PJ, Reitan LJ, Speilberg L. Experimental studies on the efficacy and side-effects of intraperitoneal vaccination of Atlantic salmon (Salmo salar L.) against furunculosis. Fish Shellfish Immunol (1996) 6:335-350. doi:10.1006/fsim.1996.0034

45. Mutoloki S, Reite OB, Brudeseth B, Tverdal A, Evensen Ø. A comparative immunopathological study of injection site reactions in salmonids following intraperitoneal injection with oil-adjuvanted vaccines. Vaccine (2006) 24:578-588. doi:10.1016/j. vaccine.2005.08.070

46. Tafalla C, Bøgwald J, Dalmo R a. Adjuvants and immunostimulants in fish vaccines: Current knowledge and future perspectives. Fish Shellfish Immunol (2013) 35:1740-1750. 
doi:10.1016/j.fsi.2013.02.029

47. Rombout JHWM, Berg AA van den, Witte P, Egberts E. Immunological importance of the second gut segment of carp . 111. Systemic and / or mucosal immune responses after immunization with soluble or particulate antigen. (1989)179-186.

48. Vigneulle M, Laurencin FB. Uptake of Vibrio anguillarum bacterin in the posterior intestine of rainbow trout Oncorhynchus mykiss, sea bass Dicentrarchus labrax and turbot or anal intubation. Dis Aquat Organ (1991) 11:85-92.

49. Rombout JHWM, Lamers CHJ, Helfrich MH, Dekker a., Taverne-Thiele JJ. Uptake and transport of intact macromolecules in the intestinal epithelium of carp (Cyprinus carpio L.) and the possible immunological implications. Cell Tissue Res (1985) 239:519-530. doi:10.1007/BF00219230

50. Fuglem B, Jirillo E, Bjerkås I, Kiyono H, Nochi T, Yuki Y, Raida M, Fischer U, Koppang EO. Antigen-sampling cells in the salmonid intestinal epithelium. Dev Comp Immunol (2010) 34:768-774. doi:10.1016/j.dci.2010.02.007

51. Løkka G, Koppang EO. Antigen sampling in the fish intestine. Dev Comp Immunol (2015) 64: doi:10.1016/j.dci.2016.02.014

52. Embregts CWE, Forlenza M. Oral vaccination of fish: Lessons from humans and veterinary species. Dev Comp Immunol (2016) 64:118-137. doi:10.1016/j.dci.2016.03.024

53. Mikalsen AB, Sindre H, Torgersen J, Rimstad E. Protective effects of a DNA vaccine expressing the infectious salmon anemia virus hemagglutinin-esterase in Atlantic salmon. Vaccine (2005) 23:4895-4905. doi:10.1016/j.vaccine.2005.05.025

54. de las Heras AI, Rodríguez Saint-Jean S, Pérez-Prieto SI. Immunogenic and protective effects of an oral DNA vaccine against infectious pancreatic necrosis virus in fish. Fish Shellfish Immunol (2010) 28:562-570. doi:10.1016/j.fsi.2009.12.006

55. Frost P, Ness A. Vaccination of Atlantic salmon with recombinant VP2 of infectious pancreatic necrosis virus (IPNV), added to a multivalent vaccine, suppresses viral replication following IPNV challenge. Fish Shellfish Immunol (1997) 7:609-619. doi:10.1006/ fsim. 1997.0113

56. EMA. First DNA vaccine in the EU recommended for use in salmon. 2016-04-22 (2016)1. Available at: http://www.ema.europa.eu/ema/index.jsp?curl=pages/news_and_events/ news/2016/04/news_detail_002516.jsp\&mid=WC0b01ac058004d5c1 [Accessed September $18,2017]$

57. Krieg AM, Yi AK, Schorr J, Davis HL. The role of CpG dinucleotides in DNA vaccines. Trends Microbiol (1998) 6:23-27. doi:10.1016/S0966-842X(97)01145-1

58. Martinez-Alonso S, Martinez-Lopez A, Estepa A, Cuesta A, Tafalla C. The introduction of multi-copy $\mathrm{CpG}$ motifs into an antiviral DNA vaccine strongly up-regulates its immunogenicity in fish. Vaccine (2010) 29:1289-1296. doi:10.1016/j.vaccine.2010.11.073

59. Boudinot P, Bernard D, Boubekeur S, Thoulouze MI, Bremont M, Benmansour A. The glycoprotein of a fish rhabdovirus profiles the virus-specific T-cell repertoire in rainbow trout. J Gen Virol (2004) 85:3099-3108. doi:10.1099/vir.0.80135-0

60. Kanellos T, Sylvester ID, D'Mello F, Howard CR, Mackie A, Dixon PF, Chang KC, Ramstad A, Midtlyng PJ, Russell PH. DNA vaccination can protect Cyprinus Carpio against spring viraemia of carp virus. Vaccine (2006) 24:4927-4933. doi:10.1016/j.vaccine.2006.03.062

61. Lorenzen N, Lorenzen E, Einer-Jensen K, Heppell J, Davis HL. Genetic vaccination of rainbow trout against viral haemorrhagic septicaemia virus: small amounts of plasmid DNA protect against a heterologous serotype. Virus Res (1999) 63:19-25. Available at: www. elsevier.com/locate/virusres [Accessed October 30, 2017]

62. Utke K, Kock H, Schuetze H, Bergmann SM, Lorenzen N, Einer-Jensen K, Köllner B, Dalmo RA, Vesely T, Ototake M, et al. Cell-mediated immune responses in rainbow trout after DNA immunization against the viral hemorrhagic septicemia virus. Dev Comp Immunol (2008) 32:239-252. doi:10.1016/j.dci.2007.05.010

63. Tonheim TC, Bøgwald J, Dalmo RA. What happens to the DNA vaccine in fish? A review of 
current knowledge. Fish Shellfish Immunol (2008) 25:1-18. doi:10.1016/j.fsi.2008.03.007

64. Seternes T, Tonheim TC, Løvoll M, Bøgwald J, Dalmo R a. Specific endocytosis and degradation of naked DNA in the endocardial cells of cod (Gadus morhua L.). $J$ Exp Biol (2007) 210:2091-103. doi:10.1242/jeb.003186

65. Tonheim TC, Dalmo RA, Bøgwald J, Seternes T. Specific uptake of plasmid DNA without reporter gene expression in Atlantic salmon (Salmo salar L.) kidney after intramuscular administration. Fish Shellfish Immunol (2008) 24:90-101. doi:10.1016/j.fsi.2007.09.006

66. Lorenzen E, Lorenzen N, Einer-Jensen K, Brudeseth B, Evensen Ø. Time course study of in situ expression of antigens following DNA-vaccination against VHS in rainbow trout (Oncorhynchus mykiss Walbaum) fry. Fish Shellfish Immunol (2005) 19:27-41. doi:10.1016/j.fsi.2004.10.009

67. Lorenzen N, LaPatra SE. DNA vaccines for aquacultured fish. Rev Sci Tech (2005) 24:201213. doi:10.3354/dao056031

68. Shedlock DJ, Weiner DB. DNA vaccination: antigen presentation and the induction of immunity. J Leukoc Biol (2000) 68:793-806.

Available at: http:/eutils.ncbi.nlm.nih.gov/entrez/eutils/elink.

fcgi?dbfrom=pubmed\&id=11129646\&retmode $=$ ref \&cmd=prlinks\%5Cnpapers $3: / /$ publication/uuid/B97A25AA-E2EE-447E-A100-EB9846198866

69. Kutzler MA, Weiner DB. DNA vaccines: Ready for prime time? Nat Rev Genet (2008) 9:776-788. doi:10.1038/nrg2432

70. Liu MA. DNA vaccines: An historical perspective and view to the future. Immunol Rev (2011) 239:62-84. doi:10.1111/j.1600-065X.2010.00980.x

71. Nakanishi T, Toda H, Shibasaki Y, Somamoto T. Cytotoxic T cells in teleost fish. Dev Comp Immunol (2011) 35:1317-1323. doi:10.1016/j.dci.2011.03.033

72. Nakanishi T, Shibasaki Y, Matsuura Y. T Cells in Fish. Biology (Basel) (2015) 4:640-63. doi:10.3390/biology4040640

73. Mutoloki S, Jørgensen JB. "The Adaptive Immune Response in Fish," in Fish Vaccination (John Wiley \& Sons Ltd.), 105-115.

74. Fischer U, Koppang EO, Nakanishi T. Teleost T and NK cell immunity. Fish Shellfish Immunol (2013) 35:197-206. doi:10.1016/j.fsi.2013.04.018

75. Somamoto T, Koppang EO, Fischer U. Antiviral functions of CD8+ cytotoxic T cells in teleost fish. Dev Comp Immunol (2014) 43:197-204. doi:10.1016/j.dci.2013.07.014

76. Fischer U, Utke K, Ototake M, Dijkstra JM, Köllner B. Adaptive cell-mediated cytotoxicity against allogeneic targets by CD8-positive lymphocytes of rainbow trout (Oncorhynchus mykiss). Dev Comp Immunol (2003) 27:323-337. doi:10.1016/S0145-305X(02)00100-3

77. Fischer U, Utke K, Somamoto T, Köllner B, Ototake M, Nakanishi T. Cytotoxic activities of fish leucocytes. Fish Shellfish Immunol (2006) 20:209-226. doi:10.1016/j.fsi.2005.03.013

78. Sato A, Okamoto N. Induction of virus-specific cell-mediated cytotoxic responses of isogeneic ginbuna crucian carp, after oral immunization with inactivated virus. Fish Shellfish Immunol (2010) 29:414-421. doi:10.1016/j.fsi.2010.04.017

79. Piazzon MC, Savelkoul HSJ, Pietretti D, Wiegertjes GF, Forlenza M. Carp I110 Has AntiInflammatory Activities on Phagocytes, Promotes Proliferation of Memory T Cells, and Regulates B Cell Differentiation and Antibody Secretion. J Immunol (2015) 194:187-99. doi:10.4049/jimmunol.1402093

80. Six A, Mariotti-Ferrandiz ME, Chaara W, Magadan S, Pham HP, Lefranc MP, Mora T, Thomas-Vaslin V, Walczak AM, Boudinot P. The past, present, and future of immune repertoire biology - the rise of next-generation repertoire analysis. Front Immunol (2013) 4:1-16. doi:10.3389/fimmu.2013.00413

81. Castro R, Takizawa F, Chaara W, Lunazzi A, Dang TH, Koellner B, Quillet E, Six A, Fischer U, Boudinot P. Contrasted TCR $\beta$ Diversity of CD8+ and CD8- T Cells in Rainbow Trout. PLoS One (2013) 8: doi:10.1371/journal.pone.0060175

82. Bradshaw CM, Richard AS, Sigel MM. IgM Antibodies in Fish Mucus. Exp Biol Med (1971) 
136:1122-1124. doi:10.3181/00379727-136-35443

83. Salinas I, Zhang Y-A, Sunyer JO. Mucosal immunoglobulins and B cells of teleost fish. Dev Comp Immunol (2011) 35:1346-1365. doi:10.1016/j.dci.2011.11.009

84. Rombout JHWM, Taverne N, van de Kamp M, Taverne-Thiele AJ. Differences in mucus and serum immunoglobulin of carp (Cyprinus carpio L.). Dev Comp Immunol (1993) 17:309317. doi:10.1016/0145-305X(93)90003-9

85. Parra D, Korytár T, Takizawa F, Sunyer JO. B cells and their role in the teleost gut. Dev Comp Immunol (2016) 64:150-166. doi:10.1016/j.dci.2016.03.013

86. Lumsden JS, Ostland VE, Byrne PJ, Ferguson HW. Detection of a distinct gill-surface antibody response following horizontal infection and bath challenge of brook trout Salvelinus fontinalis with Flavobacterium branchiophilum, the causative agent of bacterial gill disease. Dis Aquat Organ (1993) 16:21-27. doi:10.3354/dao016021

87. Von Gersdorff Jørgensen L, Heinecke RD, Skjødt K, Rasmussen KJ, Buchmann K. Experimental evidence for direct in situ binding of IgM and IgT to early trophonts of Ichthyophthirius multifiliis (Fouquet) in the gills of rainbow trout, Oncorhynchus mykiss (Walbaum). J Fish Dis (2011) 34:749-755. doi:10.1111/j.1365-2761.2011.01291.x

88. Edholm E-S, Bengtén E, Stafford JL, Sahoo M, Taylor EB, Miller NW, Wilson M. Identification of two IgD+ B cell populations in channel catfish, Ictalurus punctatus. $J$ Immunol (2010) 185:4082-94. doi:10.4049/jimmunol.1000631

89. Zhang Y-A, Salinas I, Li J, Parra D, Bjork S, Xu Z, LaPatra SE, Bartholomew J, Sunyer JO. IgT, a primitive immunoglobulin class specialized in mucosal immunity. Nat Immunol (2010) 11:827-35. doi:10.1038/ni.1913

90. Geisberger R, Lamers M, Achatz G. The riddle of the dual expression of IgM and IgD. Immunology (2006) 118:429-437. doi:10.1111/j.1365-2567.2006.02386.x

91. Kerr WG, Henderschot LM, Burrows PD. Regulation of IgM and IgD expression in human B-lineage cells. J Immunol (1991) 146:3314-3321.

92. Chen K, Cerutti A. New insights into the enigma of immunoglobulin D. Immunol Rev (2010) 237:160-179. doi:10.1111/j.1600-065X.2010.00929.x

93. Chen K, Xu W, Wilson M, He B, Miller NW, Bengtén E, Edholm E-S, Santini PA, Rath P, Chiu A, et al. Immunoglobulin D enhances immune surveillance by activating antimicrobial, proinflammatory and B cell-stimulating programs in basophils. Nat Immunol (2009) 10:889. Available at: http://dx.doi.org/10.1038/ni.1748

94. Duty JA, Szodoray P, Zheng N-Y, Koelsch KA, Zhang Q, Swiatkowski M, Mathias M, Garman L, Helms C, Nakken B, et al. Functional anergy in a subpopulation of naive B cells from healthy humans that express autoreactive immunoglobulin receptors. $J$ Exp Med (2009) 206:139-151. doi:10.1084/jem.20080611

95. Castro R, Bromage E, Abos B, Pignatelli J, Gonzalez Granja A, Luque A, Tafalla C. CCR7 Is Mainly Expressed in Teleost Gills, Where It Defines an IgD+IgM- B Lymphocyte Subset. $J$ Immunol (2014) 192:1257-1266. doi:10.4049/jimmunol.1302471

96. Ryo S, Wijdeven RHM, Tyagi A, Hermsen T, Kono T, Karunasagar I, Rombout JHWM, Sakai M, Kemenade BMLV van, Savan R. Common carp have two subclasses of bonyfish specific antibody IgZ showing differential expression in response to infection. Dev Comp Immunol (2010) 34:1183-1190. doi:10.1016/j.dci.2010.06.012

97. Corbeil S, LaPatra SE, Anderson ED, Jones J, Vincent B, Hsu Y-L, Kurath G. Evaluation of the protective immunogenicity of the N, P, M, NV and G proteins of infectious hematopoietic necrosis virus in rainbow trout Oncorhynchus mykiss using D N A vaccines. (1999) 39:2936. Available at: http://www.int-res.com/articles/dao/39/d039p029.pdf [Accessed October 30, 2017]

98. Xu Z, Chen CF, Mao ZJ, Zhu WY. Detection of serum and mucosal antibody production and antibody secreting cells (ASCs) in large yellow croaker (Pseudosciaena crocea) following vaccination with Vibrio harveyi via different routes. Aquaculture (2009) 287:243-247. doi:10.1016/j.aquaculture.2008.10.026 
99. Park JH, Park WJ, Jeong HD. Immunological efficacy of Vibrio vulnificus bacterins given as an oral vaccine in the flounder, Paralichthys olivaceus. Aquaculture (2001) 201:187-197. doi:10.1016/S0044-8486(01)00647-0

100. Abós B, Wang T, Castro R, Granja AG, Leal E, Havixbeck J, Luque A, Barreda DR, Secombes CJ, Tafalla C. Distinct Differentiation Programs Triggered by IL-6 and LPS in Teleost IgM(+) B Cells in The Absence of Germinal Centers. Sci Rep (2016) 6:30004. doi:10.1038/srep30004

101. Findly RC, Zhao X, Noe J, Camus AC, Dickerson HW. B cell memory following infection and challenge of channel catfish with Ichthyophthirius multifiliis. Dev Comp Immunol (2013) 39:302-311. doi:10.1016/j.dci.2012.08.007

102. Castro R, Jouneau L, Pham H-P, Bouchez O, Giudicelli V, Lefranc M-P, Quillet E, Benmansour A, Cazals F, Six A, et al. Teleost fish mount complex clonal IgM and IgT responses in spleen upon systemic viral infection. PLoS Pathog (2013) 9:e1003098. doi:10.1371/journal.ppat.1003098 


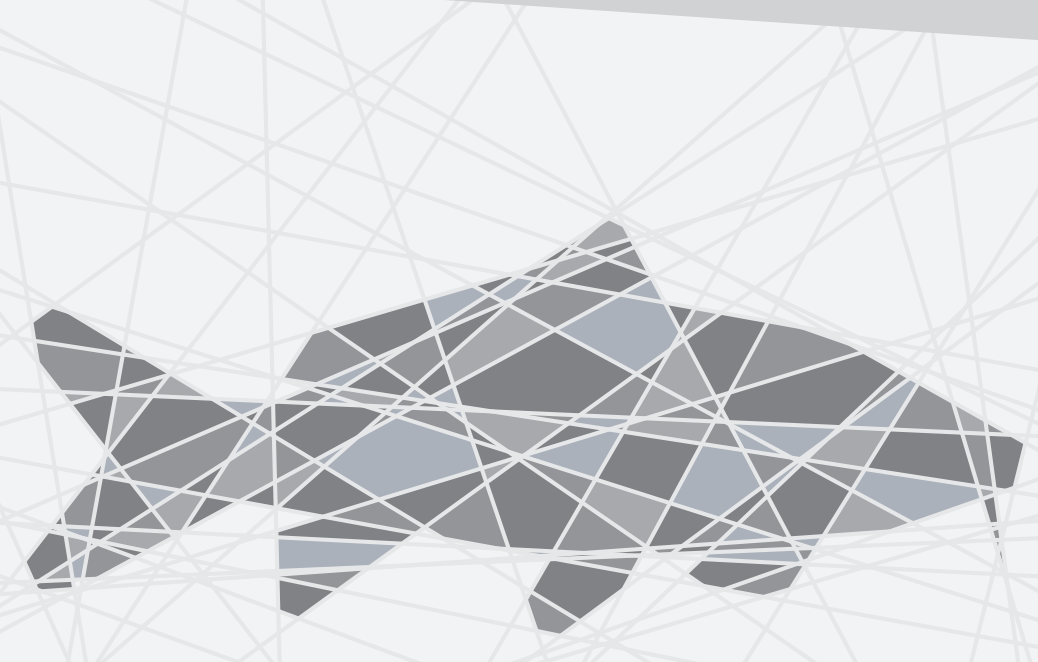




\section{Oral vaccination of fish: Lessons from humans and veterinary species}

\section{Carmen W.E. Embregts', Maria Forlenza'}

Title page 2

' Cell Biology and Immunology Group, Department of Animal Sciences, Wageningen University, Wageningen, The Netherlands.

Developmental and Comparative Immunology 64 (2016), I I 8-I 37 doi: 10.1016/j.dci.2016.03.024 


\section{Abstract}

The limited number of oral vaccines currently approved for use in humans and veterinary species clearly illustrates that development of efficacious and safe oral vaccines has been a challenge not only for fish immunologists. The insufficient efficacy of oral vaccines is partly due to antigen breakdown in the harsh gastric environment, but also to the high tolerogenic gut environment and to inadequate vaccine design. In this review we discuss current approaches used to develop oral vaccines for mass vaccination of farmed fish species. Furthermore, using various examples from the human and veterinary vaccine development, we propose additional approaches to fish vaccine design also considering recent advances in fish mucosal immunology and novel molecular tools. Finally, we discuss the pros and cons of using the zebrafish as a pre-screening animal model to potentially speed up vaccine design and testing for aquaculture fish species.

Abbreviations: TLR: Toll-like receptor, NLR: NOD-like receptor, RLR: RIG-like receptor 


\section{Introduction}

Aquaculture has been the fastest growing food-producing sector for years and the yield of aquaculture has overgrown the yield of wild capture fisheries [1]. As a downside of this sector-wide intensification, increasing stocking densities give rise to high stress levels which in turn make fish more vulnerable to infections. Prevention of disease outbreaks is therefore essential to prevent serious economic losses and thus the development or refinement of targeted vaccines for aquaculture species is imperative.

To date vaccines are available for most aquaculture fish species; most are targeting bacterial pathogens and only a few are raised against viruses. Depending on the age and size of the fish, commercial vaccines are administered either orally (by mixing with the feed), by immersion (dip or bath) or by injection through the intraperitoneal (i.p.) or intramuscular (i.m.) route. Intraperitoneal injection is conventionally used to deliver water-in-oil (w/o)-based injectable vaccines whereas intramuscular injection is most often used to deliver DNA plasmids (Reviewed in [2]. While protection is generally highest with injection-vaccination, it is also associated with intensive handling and stress for the fish. Furthermore, depending on the type of adjuvant used, w/o-based injectable vaccines have been associated with local side effects including tissue inflammation, adhesion and necrosis. Fish that are too small to be injected are usually vaccinated orally or by immersion, but these routes usually result in low efficacy and short protection. To ensure protection throughout the entire production cycle, vaccination regimes have been developed for various species in which a combination of immersion, oral and injection vaccination is used. In most cases, it is only after injection with w/o-based vaccines that strong and long lasting protection is achieved.

There is no doubt that with respect to animal welfare and handling costs, the mucosal route of vaccination, and in particular the oral route, would be the ideal method of vaccine delivery. Nevertheless, owing to the high costs of vaccine production required for immersion vaccination or the limited efficacy of the current oral formulations, mass vaccination of fish, exclusively via the mucosal routes, is not common practice. While nowadays improvement of current oral vaccination strategies is a major topic in fish vaccine development, the first report on a successful oral vaccine was already reported in 1942. The study showed protection of trout against a challenge with Bacterium salmonicida after prolonged feeding (64-70 consecutive days) with 
chloroform-inactivated bacteria [3]. In the $80 \mathrm{~s}$ and 90 s considerable attention was drawn to the development of oral vaccines for fish. During this period, morphological and functional differences within the intestine were investigated and the second segment was identified as the main place of antigen uptake [4-10]. This knowledge highlighted the necessity of protecting antigens from being broken down in the stomach and foregut of the fish. The need for antigen protection was confirmed by comparing the uptake of antigens, induced immune responses and vaccine efficacy after oral or anal administration. Higher vaccine efficacy of anal vaccination in comparison to oral vaccination using the same vaccine was reported for many studies including vaccination against Vibrio anguillarum ( $V$. anguillarum) and Yersinia ruckeri (Y. ruckeri) for salmon [11] or against $V$. anguillarum in carp [12].

The overall limited efficacy of oral vaccines in fish, as in other veterinary species or in humans, is not only due to problems linked to antigen breakdown in the harsh gastric environment, but also to the highly tolerogenic gut environment. The phenomenon of (oral) tolerance is well known in fish as well [13-16],but the mechanisms associated to its development have not been systematically addressed. The potential risk of tolerance induction upon oral vaccination, especially in immunologically immature young fish, is therefore an additional factor that fish immunologists have to take into account while developing mucosal vaccines.

From studies in mammals it is well established that mucosal tolerance is dependent on antigen dose and route of administration [17]. Mucosal tolerance is driven by the expression of high local levels of anti-inflammatory cytokines (IL-10 and TGF $\beta$ ) that sustain the generation and maintenance of tolerogenic regulatory $\mathrm{T}$ cells and dendritic cells (DCs) [18]. Therefore potent mucosal adjuvants as well as targeted delivery strategies are being used for oral vaccine development in humans and veterinary species. For example, the use of live attenuated vaccines targeting mucosal dendritic cells or microfold epithelial cells (M cells), administered along with strong adjuvants (i.e. bacterial toxins) has received significant attention and holds great promise to facilitate the induction of effective mucosal responses [19,20].

This review will summarize current knowledge on experimental oral vaccines in human and veterinary species, with a particular focus on fish, focusing on antigen type, dose, encapsulation as well as delivery methods. First, based on all summarised work we will try to extrapolate communalities among all (successful) approaches with a special focus on the nature of the pathogen, nature of the fish and vaccination 
strategy. Next, using several examples from the human and veterinary field, we will dissect the conditions that might lead to tolerance to orally delivered antigens in fish and later, the protective immune mechanisms that need to be rationally targeted to overcome tolerance and achieve successful oral vaccination. Among others, we will discuss the possibility to target local antigen presenting cells (APCs) or M-like antigen-sampling cells [21] as well as the choice of mucosal adjuvants that can either promote antigen adhesion to M-like cells, or strongly activate local innate and adaptive immune responses. Finally, we will discuss the pros and cons of using zebrafish as a pre-screening platform for novel oral vaccination approaches for cultured fish species.

\section{Is development of fish oral vaccines really lagging behind? Current status on human and veterinary oral vaccines}

In mammals it is well known that parenteral antigen administration triggers weak mucosal responses, whereas antigen administration at mucosal surfaces efficiently triggers local as well as systemic humoral and cell-mediated responses [20]. The importance of triggering specific immune responses at mucosal surfaces for protection against mucosal pathogens is well recognized. Despite this, in humans, as in many veterinary species, the development of effective oral vaccines has gone at a slow paste. In fact, after more than 100 years of research, only five mucosal vaccines have been approved for human use. These include four oral vaccines against polio virus, rotavirus, Salmonella typhi (S. thypi), and Vibrio cholera (V. cholera) and one nasal vaccine against influenza virus [20,22,23]. Perhaps not surprising, but also these vaccines do not trigger a long-lasting protection and they all require boosting after 2 years from first administration in adults, and 6 months in children aged 2-5 years. Many oral vaccines for human application are currently in various developmental phases and clinical testing, but often safety issues or adverse side effects slow their implementation. Altogether this underlines how, also in humans, optimal conditions to achieve effective oral vaccination have not been defined yet and how challenging the field of mucosal vaccine development can be.

The situation for veterinary oral vaccines (for non-fish species) is perhaps a little better, with a very successful oral vaccine against rabies virus, which helped eradicate rabies disease from wildlife reservoirs in Europe and most of the United States, and one oral vaccine for pigs against the intracellular bacterium Lawsonia intracellularis 
[24]. Several mucosal (oral) vaccines are available from various vaccine companies for pigs and cows against rotavirus and for poultry against several pathogens: turkey adenovirus; infectious bronchitis virus; Newcastle virus; infectious bursitis virus; chicken herpesvirus; turkey herpesvirus; reovirus; Bordetella avium; Pasteurella multocida [25].

Considering the total number of commercially available vaccines against fish pathogens, only a small but significant proportion is administered orally (Reviewed in $[26,27,2]$. For salmonid species, commercial oral vaccines are available for Atlantic salmon and coho salmon against piscirickettsia salmonis (P. salmonis), infectious pancreatic virus (IPNV), and infectious salmon anaemia virus (ISAV); for rainbow trout against IPNV, Y. ruckeri, P. salmonis and for rainbow trout and sea bass against $V$. anguillarum. For Great amberjack one oral vaccine has been licensed in Japan against Lactococcus garviae (L. garviae). Despite their commercialisation however, oral vaccines for fish are in use in a few countries only (mainly Chile, Norway and Scotland) [2]. Similar to the human oral vaccines, oral vaccines for fish provide only a weak or short protection and are therefore mostly used as prime and/or booster vaccination. Depending on the fish species and production cycle, farmers will optimally choose for a vaccination regime starting with dip vaccination, followed by booster vaccination (dip or oral) and finally an injection vaccination [2]. Such vaccination regime generally confers strong and long-lasting protection during the entire production cycle. However, it causes more stress to the fish and is more costly and labour intensive for the farmer. Therefore considerable effort is still dedicated to the improvement of currently available vaccines and to design new oral vaccination strategies that can provide stronger and longer lasting protection in fish.

\section{Successes and concerns of current mucosal human and veterinary vaccines}

When considering the nature of the pathogen, all oral vaccines licensed for use in humans and veterinary species are against mucosal pathogens that either infect the mucosal surface itself or use the mucosa as portal of entry to then establish a systemic infection. Furthermore, with only few exceptions, successful oral vaccines are based on live attenuated viruses or bacteria that closely mimic the route of infection of the pathogen and trigger strong local immune responses without the need for any additional adjuvant. For example, the live attenuated poliovirus and $S$. typh $i$ vaccines 
are derived from pathogens that preferentially adhere to $\mathrm{M}$ cells and exploit M-cell transport to invade organized mucosal lymphoid tissues in the intestine [28,29].

As a downside, live attenuated vaccines are less safe as it cannot be guaranteed that the pathogen would not revert to the virulent form or cause pathogenesis in few individuals. This has been already observed for the oral polio vaccine in humans for which 1 in a million doses caused a so-called 'vaccine-associated paralytic polio' [30]. This reflects the importance and risks associated to the use of live attenuated vaccines and the need for development of even safer and rationally designed vaccines. The challenges however are not only in the development of safer vaccines for humans, but also for veterinary species including poultry. It is now been ascertained that imperfect vaccination against Marek's disease virus (MDV) can drive the generation and enhance the transmission of highly virulent pathogens [31]. Spray-vaccination of chickens with live attenuated MDV gives rise to the considerable risk that not all chickens are exposed to the same dose of the vaccine, if at all. The vaccine effectively protects vaccinated individuals but fails to induce sterile immunity, as vaccinated chickens are still able to shed the vaccine virus. In this situation, in order to be able to survive in a population of largely vaccinated animals, the virus is driven towards increased virulence. This example clearly highlights how not only the design but also the administration route of a successful vaccine plays a crucial role in tilting the balance between immunity and pathogenesis.

When considering the currently licensed oral vaccines for fish we realise that all are based on heat- or formalin-killed pathogens. Most of these pathogens are however either enteric pathogens, such as Y. ruckeri and $V$. anguillarum, or enter and affect mucosal organs, such as $P$. salmonis or IPNV. Furthermore, although no specific adjuvants are mentioned to be administered along with the vaccine antigen, most vaccines are either incorporated in an Antigen Protecting Vehicle (Y. ruckeri, V. anguillarum and IPNV vaccines, MSD-Animal Health) or in patented MicroMatrix ${ }^{\mathrm{TM}}$ delivery system (P. salmonis, ISAV and IPNV, Centrovet). Such encapsulations or delivery methods are aimed at protecting the antigens from gastric degradation, but might also promote antigen uptake by enhancing adhesion to mucosal surfaces. Moreover, they might possibly act as adjuvants by providing local inflammatory signals [32]. The use of inactivated pathogens certainly poses an advantage with respect to (environmental) safety but, together with the lack of strong mucosal adjuvants, it might still be one of the causes of the weak and short protection provided 
by these vaccines.

Interestingly, prolonged protection against $P$. salmonis and ISA virus can be achieved in farmed salmonids, but only upon administration of MicroMatrix ${ }^{\mathrm{TM}}$ encapsulated oral vaccines at repeated intervals of approximately 1200 degree-days (approximately 3 month for salmon kept at 11-12 degrees) [33]. Protection was found to correlate to high serum IgM levels whereas the window of disease susceptibility was shown to coincide with the decline in IgM serum levels. Therefore, repeated oral administrations assured that serum IgM remained high throughout the entire production cycle and this confirmed once again that oral antigen administration can elicit systemic responses [16]. Furthermore, while it is clear that on the one hand fish do not develop a strong memory response to the vaccine, they on the other hand also do not develop tolerance. The possible mechanisms behind such phenomenon are not fully understood. It is possible that the vaccine dose combined with the MicroMatrix ${ }^{\mathrm{TM}}$ provides sufficient signals (inflammatory and co-stimulatory) to activate B cells directly and drive them towards few rounds of division and antibody production. Nevertheless, as it will be further discussed in section 6.2, the lack of immune memory and tolerance upon antigen re-exposure might be explained by the failure of the vaccine to activate cell-mediated $\mathrm{T}$ cell responses required for effective $\mathrm{B}$ cell activation and memory formation. Given the promising but insufficient progress on fish oral vaccine development, substantial research effort is still being dedicated to the study of mucosal immune responses in fish and the generation of novel oral vaccines.

\section{Current strategies and efficacy of experimental non-encapsulated oral fish vaccines}

Given the relative success of the currently licensed fish vaccines, as well as the convenience and safety of inactivated pathogens, a large number of studies focused on the use of non-encapsulated (inactivated) bacteria, yeast or plants expressing bacterial or viral antigens. This approach is largely based on the hypothesis that antigens expressed in whole cells might not need further encapsulation since the cell wall itself might act as natural protection barrier against the intestinal environment. A selected overview of the current experimental approaches for the development of oral vaccines against bacterial and viral pathogens using non-encapsulated antigens is presented in Table 1 and will be discussed in this section. A more extensive and 
detailed summary is presented in Supplementary Table 1. Although many reports could be found describing vaccine uptake and local responses, we selectively focused on those studies that also tested vaccine efficacy upon pathogen challenge (see references in (supplementary) Table 1).

Independently of the dose, regime, nature of the pathogen or vaccine type, it is apparent that when protection could be observed, this was either assessed after only a very short time following last vaccine administration or was found to decrease within 2-3 months after vaccination. In most cases single or consecutive administrations, or consecutive administration with varying time intervals, did not make a significant difference in survival. This suggests that the dose achieved by a single administration or by consecutive administration is sufficient to induce a response and confer protection, at least within the time tested.

Interestingly, two studies showed that the addition of common injectable adjuvants (alum and Freund's incomplete) to the oral vaccine had a positive effect on vaccine efficacy. Addition of $25 \% \mathrm{v} / \mathrm{v}$ of $10 \%$ potassium aluminium sulphate (alum) increased survival of rainbow trout against $V$. anguillarum using different types of whole cell vaccines, when compared to the same vaccines without adjuvant [34], supplementary Table 1 . The addition of incomplete Freund's adjuvant $(20 \% \mathrm{v} / \mathrm{v})$ to a formalin-killed Streptococcus agalactiae (S. agalactiae) vaccine increased survival of red tilapia upon i.p. challenge with $S$. agalactiae, when compared to the nonadjuvanted vaccine [35], Table 1. Nevertheless, long-term protection and potential side effects were not investigated.

Of interest, few approaches showed the potential of using enteric pathogens not affecting fish or commensal microbes as vaccine vehicles (Table 1). Formalininactivated Escherichia coli (E. coli) expressing S. agalactiae or grass carp reovirus (GCRV) antigens, and live attenuated Salmonella typhimurium (S. typhimurium) expressing surface immunogenic protein (Sip) of $S$. agalactiae generally conferred good protection at various vaccination doses. Similarly, live Lactococcus lactis expressing A. hydrophila antigens, Lactobacillus plantarum (L. plantarum) expressing Spring Viremia of Carp Virus (SVCV) or Cypinid Herpesvirus-3 (CyHV3) antigens, and Lactobacillus casei expressing IPNV non-structural proteins, all showed promising levels of protection. These results together suggest that enteric microbes might be suitable vaccine vehicles. Live commensals might retain their ability to colonize the gut environment and persist long enough to deliver the antigen 
at mucosal surfaces. However, as a result of the intrinsic ability to be tolerated by the host, on their own they might not be sufficient to trigger strong local responses. In contrast, inactivated enteric pathogens might still be able to diffuse through the mucus layer and adhere to enterocytes. By doing so, they might possibly provide inflammatory signals for local immune reactions owing to the presence of Pathogen Associated Molecular Patterns (PAMPs) triggering danger signals.

Production of vaccine antigens in plants including algae, potatoes, tobacco or rice has been proposed as a viable approach for the development of mucosal vaccines especially in veterinary species, owing among others, to their relatively low production cost and scalability [36]. Studies in mice demonstrated the ability of riceexpressed proteins to effectively deliver antigens to $\mathrm{M}$ cells and trigger local as well as systemic response [37]. In fish, the first report on an effective oral vaccine produced in plants was reported only recently [38]. In the latter study, lyophilised rice calli of Oriza sativa expressing the major capsid protein of Rock Bream Iridovirus (RBIV) were mixed with the fish feed and were shown to confer very good protection against a RBIV challenge.

Another expression system exploited for the generation of vaccine antigens is yeast. Antigens expressed and produced in yeast benefit from a similar protection from its host as antigens expressed in bacteria. The wall of yeast makes the antigens less vulnerable to degradation and decreases the need for further encapsulation. Furthermore, yeast can easily be mixed with fish feed and might even act as an adjuvant by its richness in $\beta$-glucans. When administered orally with the fish feed, the yeast Pichia pastoris expressing the major capsid protein of RBIV conferred significant protection against RBIV challenge. However, protection was only evaluated shortly after vaccination. The yeast expression system has also been exploited for its ability to produce large quantities of Virus-Like Particles (VLPs), which are multimeric protein complexes whose shape mimic natural occurring virions. VLPs composed of the capsid protein of the Red-spotted Grouper Nervous Necrosis Virus (RGNNV) were produced in Saccharomyces cerevisiae (S. cerevisiae) and conferred low but significant protection against viral challenge when administered orally [39].

Despite the many and promising approaches summarised in Table 1 (and Supplementary Table 1), it is difficult to draw general conclusions on dose, vaccine type, and vaccine efficacy since long-term protection ( $>3$ months) was not systematically addressed. Most studies performed challenges 10-30 days after last vaccination, and only a 
few also assessed protection at later time points [40-44]. Furthermore, in most of the summarized studies, pathogen challenge was not performed using the natural route of infection. It has already been mentioned that oral vaccination can lead to increased serum IgM levels, and that in some infections serum IgM levels correlated to diseases resistance or susceptibility $[33,45]$. Nonetheless, it cannot be excluded that bypassing the mucosa by directly injecting the pathogen into the peritoneal cavity or muscle might also bypass crucial mucosal immune mechanisms. As a consequence, the subsequent lack of activation of local as well as systemic responses might lead to an underestimation of vaccine efficacy.

Table 1. Experimental approaches for the development of oral vaccines using nonencapsulated antigens

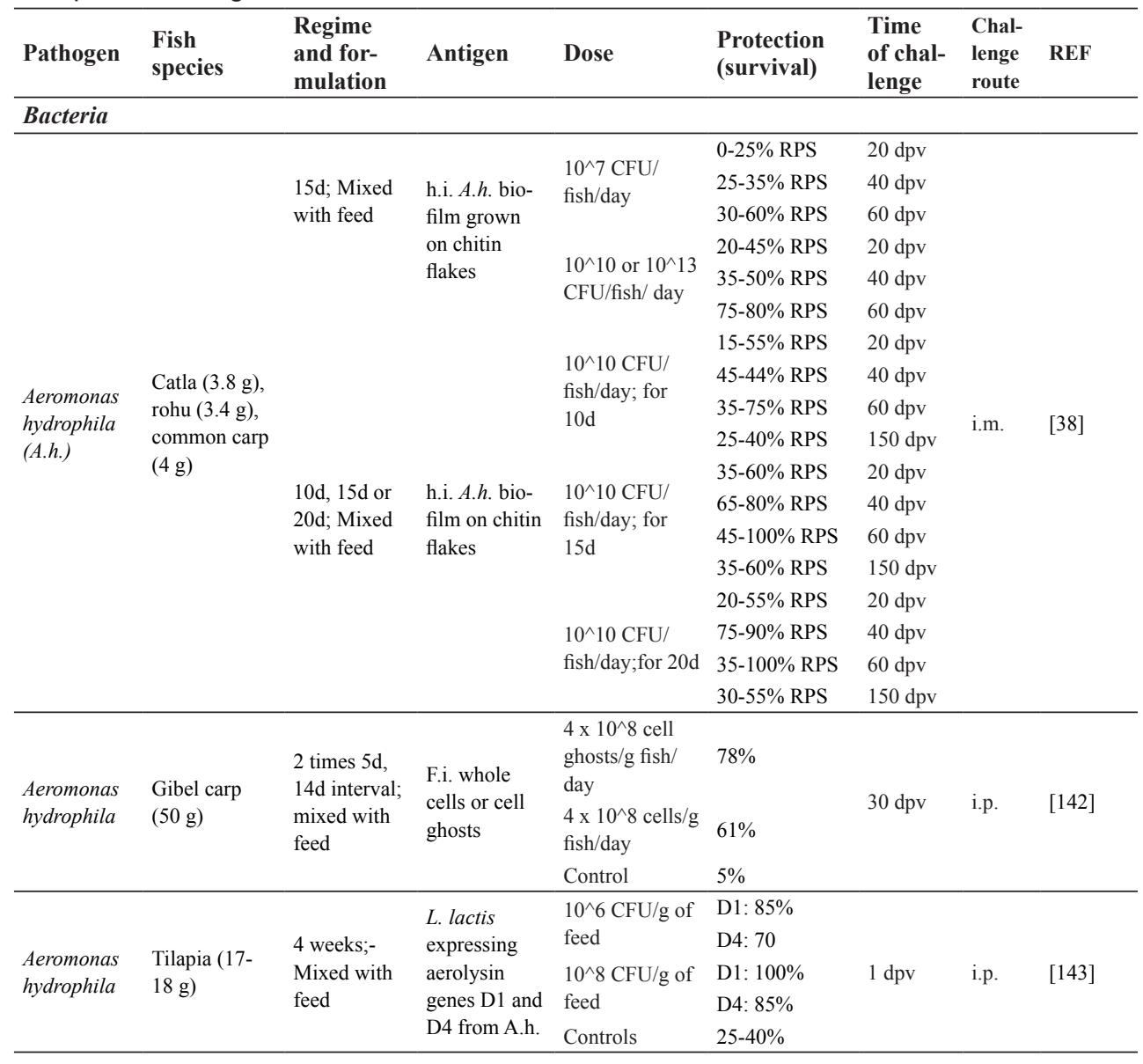




\begin{tabular}{|c|c|c|c|c|c|c|c|c|}
\hline \multirow{4}{*}{$\begin{array}{l}\text { Edwardsi- } \\
\text { ella tarda } \\
\text { (E.t.) }\end{array}$} & \multirow{4}{*}{ Olive flounder } & \multirow{4}{*}{$\begin{array}{l}\text { One time } \\
\text { only or with } \\
\text { boost at } 14 \mathrm{~d} \text {; } \\
\text { Gavage }\end{array}$} & \multirow{4}{*}{$\begin{array}{l}\text { Autotrophic } \\
\text { mutant } \\
\Delta \text { alr } \Delta \text { asd } \\
\text { E.tarda }\end{array}$} & $10^{\wedge} 8 \mathrm{CFU} /$ fish & $90-100 \%$ & \multirow{4}{*}{$3 \mathrm{wpv}$} & \multirow{4}{*}{$\begin{array}{l}\text { i.p., } \\
\text { bath }\end{array}$} & \multirow{4}{*}{ [87] } \\
\hline & & & & $10^{\wedge} 9 \mathrm{CFU} /$ fish & $100 \%$ & & & \\
\hline & & & & $\begin{array}{l}10^{\wedge} 8 \mathrm{CFU} / \text { fish, } \\
10^{\wedge} 8 \mathrm{CFU} / \text { fish } \\
\text { for boost } \\
10^{\wedge} 9 \mathrm{CFU} / \text { fish, } \\
10^{\wedge} 8 \mathrm{CFU} / \text { fish } \\
\text { for boost }\end{array}$ & $60-65 \%$ & & & \\
\hline & & & & Controls & $0-18 \%$ & & & \\
\hline \multirow{4}{*}{$\begin{array}{l}\text { Edwardsiel- } \\
\text { la tarda }\end{array}$} & \multirow{4}{*}{$\begin{array}{l}\text { Olive flounder } \\
(4-5 \mathrm{~g})\end{array}$} & \multirow{4}{*}{$\begin{array}{l}\text { One time } \\
\text { only, or with } \\
\text { boost at } 14 \mathrm{~d} \text {; } \\
\text { Gavage }\end{array}$} & \multirow{4}{*}{$\begin{array}{l}\text { Autotrophic } \\
\text { mutant } \\
\Delta \text { alr } \Delta \text { asd } \\
\text { E.tarda }\end{array}$} & $10^{\wedge} 8 \mathrm{CFU}$ fish & $90-100 \%$ & \multirow{4}{*}{$3 \mathrm{wpv}$} & \multirow{4}{*}{$\begin{array}{l}\text { i.p., } \\
\text { bath }\end{array}$} & \multirow{4}{*}[87]{} \\
\hline & & & & $10^{\wedge} 9 \mathrm{CFU} /$ fish & $100 \%$ & & & \\
\hline & & & & $\begin{array}{l}10^{\wedge} 8 \mathrm{CFU} / \text { fish, } \\
10^{\wedge} 8 \mathrm{CFU} \text { for } \\
\text { boost } \\
10^{\wedge} 9 \mathrm{CFU} / \text { fish, } \\
10^{\wedge} 8 \mathrm{CFU} \text { for } \\
\text { boost }\end{array}$ & $60-65 \%$ & & & \\
\hline & & & & Controls & $0-18 \%$ & & & \\
\hline \multirow{4}{*}{$\begin{array}{l}\text { Edwardsi- } \\
\text { ella ictaluri } \\
\text { (E.i.) }\end{array}$} & \multirow{4}{*}{$\begin{array}{l}\text { Channel } \\
\text { catfish (7-9 } \\
\mathrm{cm})\end{array}$} & \multirow{4}{*}{$\begin{array}{l}\text { One time; } \\
\text { Mixed with } \\
\text { feed }\end{array}$} & \multirow{4}{*}{$\begin{array}{l}\text { Live attenuat- } \\
\text { ed } E \text {. ictaluri }\end{array}$} & $4.6-6 \times 10^{\wedge} 6$ & $92-100 \%$ & \multirow{4}{*}{$29 \mathrm{dpv}$} & \multirow{4}{*}{$\begin{array}{l}\text { bath, } \\
\text { cohab }\end{array}$} & \multirow{4}{*}{ [144] } \\
\hline & & & & $\begin{array}{l}4.6-6 \times 10^{\wedge} 7 \\
\text { CFU/g feed }\end{array}$ & $97-100 \%$ & & & \\
\hline & & & & $\begin{array}{l}\text { Field trial; } 1.9 \\
\times 10^{\wedge} 6 \mathrm{CFU} / \mathrm{g}\end{array}$ & V: $54 \%$ & & & \\
\hline & & & & feed & C: $28 \%$ & & & \\
\hline \multirow{3}{*}{$\begin{array}{l}\text { Photobacte- } \\
\text { rium damse- } \\
\text { la (P.d.) }\end{array}$} & \multirow{3}{*}{$\begin{array}{l}\text { Sea bass } \\
(20 \mathrm{~g})\end{array}$} & \multirow{3}{*}{$\begin{array}{l}\text { One time; } \\
\text { Gavage }\end{array}$} & \multirow{3}{*}{$\begin{array}{l}\text { F.i. whole } \\
\text { P.d. and } \\
\text { extracellular } \\
\text { components }\end{array}$} & \multirow{3}{*}{$500 \mu 1$} & V: $64 \%$ & & \multirow{3}{*}{ bath } & \multirow{3}{*}[41]{} \\
\hline & & & & & $\begin{array}{l}\text { C: } 31 \% \\
\text { V: } 55 \%\end{array}$ & $12 \mathrm{wpv}$ & & \\
\hline & & & & & C: $19 \%$ & & & \\
\hline \multirow{5}{*}{$\begin{array}{l}\text { Flavobacte- } \\
\text { rium psy- } \\
\text { chrophilum } \\
\text { (F.p.) }\end{array}$} & \multirow{5}{*}{ Ayu $(0.5 \mathrm{~g})$} & \multirow{5}{*}{$\begin{array}{l}3 \mathrm{~d} \text { or } 15 \mathrm{~d} \\
\text { with } 5 \mathrm{~d} \\
\text { interval; } \\
\text { repeated } 5 \\
\text { or } 15 \text { times; } \\
\text { Mixed with } \\
\text { feed }\end{array}$} & & & $\begin{array}{l}5 \text { times: } 53- \\
94 \%\end{array}$ & & & \\
\hline & & & & & $\begin{array}{l}15 \text { times: } 92- \\
97 \%\end{array}$ & 3 wpv & & \\
\hline & & & $\begin{array}{l}\text { F.i. F.psy- } \\
\text { chrophilum }\end{array}$ & $\begin{array}{l}0.1-0.2 \mathrm{~g} \\
\text { bacteria } / \mathrm{kg}\end{array}$ & $\begin{array}{l}\text { C: } 35-69 \% \\
5 \text { times: } 76- \\
87 \%\end{array}$ & & bath & {$[145]$} \\
\hline & & & & & $\begin{array}{l}15 \text { times: } 79- \\
88 \%\end{array}$ & $7 \mathrm{wpv}$ & & \\
\hline & & & & & C: $42-76 \%$ & & & \\
\hline & & & & & $\begin{array}{l}\text { SP cells: } 66- \\
68 \%\end{array}$ & & & \\
\hline & & & Membrane & & LP cells: $94 \%$ & & & \\
\hline & & 10 times on & $\begin{array}{l}\text { vesicles (su- } \\
\text { pernatatant) }\end{array}$ & $1250 \mu 1$ super- & $\begin{array}{l}\text { SP+SN: } 47- \\
53 \%\end{array}$ & & & \\
\hline rium psy- & $\begin{array}{l}\text { Rainbow trout } \\
(1.6 \mathrm{~g})\end{array}$ & $\begin{array}{l}\text { alternating } \\
\text { days; Mixed }\end{array}$ & $\begin{array}{l}\text { and f.i. F.p. } \\
\text { of stationary }\end{array}$ & $\begin{array}{l}\text { natant }(\mathrm{SN}) \text { and } \\
0.2 \mathrm{~g} \text { cells } / \mathrm{kg}\end{array}$ & $\mathrm{LP}+\mathrm{SN}: 51 \%$ & $3 \mathrm{wpv}$ & bath & [146] \\
\hline chrophilum & & with feed & $\begin{array}{l}(\mathrm{SP}) \text { and } \\
\text { logarithmic }\end{array}$ & of fish & $\begin{array}{l}\mathrm{LP}+\mathrm{SN}+\mathrm{SP}: \\
59 \%\end{array}$ & & & \\
\hline & & & phase (LP) & & $\begin{array}{l}\text { SP+SN+SP: } \\
97-100 \%\end{array}$ & & & \\
\hline & & & & & C: $56.3-44.1 \%$ & & & \\
\hline
\end{tabular}




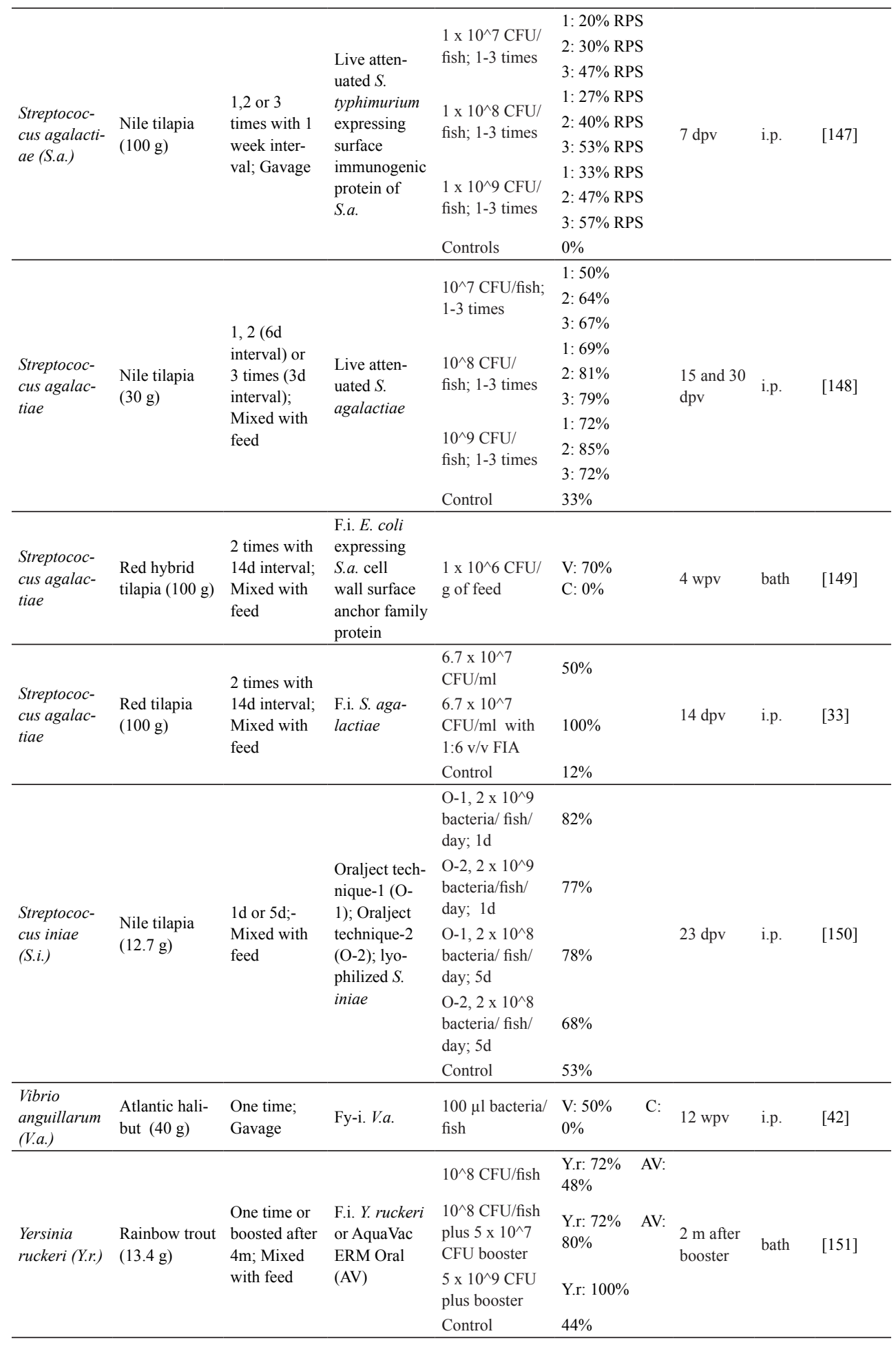




\begin{tabular}{|c|c|c|c|c|c|c|c|c|}
\hline \multicolumn{9}{|l|}{ Viruses } \\
\hline $\begin{array}{l}\text { Viral } \\
\text { heamorrhag- } \\
\text { ic septice- } \\
\text { mia virus } \\
\text { (VHSV) }\end{array}$ & $\begin{array}{l}\text { Rainbow trout } \\
(40-80 \mathrm{~g})\end{array}$ & $\begin{array}{l}\text { 3d; Mixed } \\
\text { with feed }\end{array}$ & $\begin{array}{l}\text { Attenuated } \\
\text { lyophilized } \\
\text { VHSV, PEG } \\
\text { pellets }\end{array}$ & $\begin{array}{l}4.8-5.7 \mathrm{TCID}_{50} \\
\text { /fish total }\end{array}$ & $\begin{array}{l}\text { V: } 77-91 \% \\
\text { C: } 30-41 \%\end{array}$ & $6 \mathrm{wpv}$ & bath & [152] \\
\hline $\begin{array}{l}\text { Viral } \\
\text { heamorrhag- } \\
\text { ic septice- } \\
\text { mia virus } \\
\text { (VHSV) }\end{array}$ & $\begin{array}{l}\text { Olive flounder } \\
(4-5 \mathrm{~g})\end{array}$ & $\begin{array}{l}1 \text { time or } \\
\text { boost after } \\
2 \mathrm{w} ; \text { Gavage }\end{array}$ & $\begin{array}{l}\text { Live recom- } \\
\text { binant VHSV } \\
\text { eGFP-NV } \\
(\Delta \mathrm{NV})\end{array}$ & $\begin{array}{l}10^{\wedge} 4 \mathrm{PFU} / \text { fish } \\
10^{\wedge} 5 \mathrm{PFU} / \text { fish } \\
2 \times 10^{\wedge} 5 \mathrm{PFU} / \\
\text { fish } \\
\text { Control }\end{array}$ & $\begin{array}{l}25-60 \% \\
50-75 \% \\
70-90 \% \\
0 \%\end{array}$ & 4 wpv & i.m. & [88] \\
\hline $\begin{array}{l}\text { Red-spotted } \\
\text { grouper } \\
\text { nervous ne- } \\
\text { crosis virus } \\
\text { (RGNNV) } \\
\end{array}$ & $\begin{array}{l}\text { Convict grou- } \\
\text { per }(71.5 \mathrm{~g})\end{array}$ & $\begin{array}{l}1 \text { time; } \\
\text { Gavage }\end{array}$ & $\begin{array}{l}\text { RGNNV } \\
\text { VLPs pro- } \\
\text { duced in } S \text {. } \\
\text { cerevisiae }\end{array}$ & $\begin{array}{l}50 \mu \mathrm{g} \text { of puri- } \\
\text { fies VLPs/fish }\end{array}$ & $\begin{array}{l}\text { V: } 100 \% \\
37 \%\end{array}$ & C: $\quad 56 \mathrm{dpv}$ & i.p. & [37] \\
\hline $\begin{array}{l}\text { Grass carp } \\
\text { reovirus } \\
(\mathrm{GCRV})\end{array}$ & $\begin{array}{l}\text { Grass carp } \\
(150 \mathrm{~g})\end{array}$ & $\begin{array}{l}\text { 21d; Mixed } \\
\text { with feed }\end{array}$ & $\begin{array}{l}\text { f.i. } E \text {. coli } \\
\text { expressing } \\
\text { capsid pro- } \\
\text { teins VP5- } \\
\text { VP7 }\end{array}$ & $\begin{array}{l}10^{\wedge} 8 \text { bacteria/ } \\
\text { feed pellet }\end{array}$ & $\begin{array}{l}\text { V: } 90 \% \\
\text { C: } 10 \%\end{array}$ & $7 \mathrm{dpv}$ & i.p. & [153] \\
\hline $\begin{array}{l}\text { Cyprinid } \\
\text { Herpes } \\
\text { Virus-3 } \\
\text { (CyHV3), } \\
\text { Spring } \\
\text { Viremia of } \\
\text { Carp Virus } \\
\text { (SVCV) }\end{array}$ & $\begin{array}{l}\text { Common carp } \\
(500 \mathrm{~g})\end{array}$ & $\begin{array}{l}3 \mathrm{~d} \text {; followed } \\
\text { by } 2 \text { boosts } \\
\text { for } 3 \mathrm{~d} \text { after } \\
8 \mathrm{~d} \text { and } 25 \mathrm{~d} \\
\text { from first } \\
\text { administra- } \\
\text { tion; Mixed } \\
\text { with feed }\end{array}$ & $\begin{array}{l}\text { SVCV-G } \\
\text { and Cy- } \\
\text { HV3-ORF81 } \\
\text { expressed in } \\
\text { L. plantarum } \\
\text { SVCV-G } \\
\text { and Cy- } \\
\text { HV3-ORF81 } \\
\text { expressed in } \\
\text { L. plantarum }\end{array}$ & $\begin{array}{l}10^{\wedge} 9 \mathrm{CFU} / \mathrm{g} \\
\text { feed }\end{array}$ & $\begin{array}{l}\text { V: } 71 \% \\
\text { (SVCV) } \\
\text { C: } 11 \% \\
\text { (SVCV) } \\
\text { V: } 53 \% \\
\text { (CyHV3) } \\
\text { C: } 8 \% \\
\text { (CyHV3) }\end{array}$ & $5 \mathrm{dpv}$ & oral & [154] \\
\hline $\begin{array}{l}\text { Rock bream } \\
\text { iridovirus } \\
\text { (RBIV) }\end{array}$ & $\begin{array}{l}\text { Rock bream } \\
(7-8 \mathrm{~g})\end{array}$ & $\begin{array}{l}4 \text { times, } \\
\text { weekly } \\
\text { interval; } \\
\text { Mixed with } \\
\text { feed }\end{array}$ & $\begin{array}{l}\text { Major cap- } \\
\text { sid protein } \\
\text { expressed } \\
\text { in Pichia } \\
\text { pastoris }\end{array}$ & $\begin{array}{l}1 \mathrm{x} 10^{\wedge} 6 \text { yeast } \\
\text { cells } 2.5 \mathrm{~g} \text { feed/ } \\
\text { fish }(250 \mu \mathrm{g} \\
\text { recombinant/ } \\
\text { fish })\end{array}$ & $\begin{array}{l}\mathrm{V}: 92 \% \\
\mathrm{C}: 0 \%\end{array}$ & $10 \mathrm{dpv}$ & bath & [155] \\
\hline $\begin{array}{l}\text { Rock bream } \\
\text { iridovirus }\end{array}$ & $\begin{array}{l}\text { Rock bream } \\
(10 \mathrm{~g})\end{array}$ & $\begin{array}{l}3 \text { times, 10d } \\
\text { interval; } \\
\text { Mixed with } \\
\text { feed }\end{array}$ & $\begin{array}{l}\text { Major capsid } \\
\text { protein } \mathrm{rMCP} \\
\text { in rice callus }\end{array}$ & $\begin{array}{l}10 \mu \mathrm{g} \text { lyo- } \\
\text { philized calli } \\
\text { powder/ fish } \\
30 \mu \mathrm{g} \text { lyo- } \\
\text { philized calli } \\
\text { powder/ fish } \\
\text { Control }\end{array}$ & $\begin{array}{l}90 \% \\
0 \%\end{array}$ & $10 \mathrm{dpv}$ & bath & {$[36]$} \\
\hline
\end{tabular}

Abbreviations: d: $\operatorname{day}(\mathrm{s})$; w: week(s); dpv: days post vaccination; wpv: weeks post vaccination, mpv: months post vaccination; h.i.: heat-inactivated; f.i.: Formalin-inactivated; fy-i. formaldehyde-inactivated, UV-:. UV-inactivated; cohab: cohabitation; FIA: Freund's incomplete adjuvant; BW: body-weight; i.p.: intraperitoneal; i.m: intramuscular.

\section{Current strategies and efficacy of experimental encapsulated oral fish vaccines}

In contrast to the abovementioned whole bacteria or yeast- and plant-derived vaccines, more vulnerable vaccines such as DNA plasmids, purified recombinant proteins and sub-unit vaccines certainly need a form of protection to prevent 
breakdown and ensure antigen uptake in the second gut segment of fish. In many animal species, soluble, non-adherent antigens are generally found to be taken up at low levels, if at all, and in the intestine, such antigens commonly induce immune tolerance $[20,62,63]$. On the contrary, enhanced mucosal immune responses were observed when using antigens that could be retained at mucosal surfaces by delivery in adherent polymers or were coupled to proteins that themselves are adherent to epithelial surfaces $[20,25,62]$.

It was clearly shown in mice and several other veterinary species that microencapsulated vaccines have several advantages for mucosal delivery. Microfold (M) cells are particularly accessible to microparticles and actively transport them into Peyer's patches, which consist of large clusters of lymphoid follicles localized in the mucosa of the distal small intestine. Microparticles that are both small (up to $1 \mu \mathrm{m}$ diameter) and adherent to M cells are taken up most efficiently [64-66]. Furthermore, specific glycolipids and protein-linked oligosaccharides on the apical surfaces of villus enterocytes, follicle-associated enterocytes above Peyer's patches, and M cells play a crucial role in antigen adherence $[23,65,66]$. Cells that are functionally equivalent, but phenotypically distinct, from mammalian $\mathrm{M}$ cells have been identified in the gut of Atlantic salmon [21]. The role of M-like cells in gut immunity together with gut barrier function and anatomical construction are reviewed in [67].

Given the presence of specialized antigen-sampling (M-like) cells in the fish intestine, together with the already described antigen-sampling ability of enterocytes and gutassociated macrophages $[62,68]$, we can safely state that micro-encapsulation can be exploited to further enhance uptake of the abovementioned inactivated bacteria or yeast- and plant-derived vaccines (section 4). In this way vaccines that show a suboptimal protection when delivered 'naked', possibly due to high intestinal antigen breakdown, can be improved upon encapsulation. An overview of the current experimental approaches for the development of oral vaccines against bacterial and viral pathogens using (bio)encapsulated antigens is presented in Table 2 and will be discussed in this section.

Bio-encapsulation in live vehicles such as artemia, rotifer and water flea was investigated for its suitability to vaccinate fish larvae that do not yet feed on pelleted food (Table 2). Although some reports describe the induction of protection after oral delivery of bio-encapsulated vaccines for fry and larvae, protection was generally assessed shortly after antigen administration pointing more towards 
an immunostimulatory effect of the vaccine. Furthermore, maximum dosage of vaccination is restricted by the daily feed intake and encapsulation efficiency of the vaccine since the bio-encapsulated vaccines are incorporated in the natural starter diet of the fish fry or larvae. This efficiency strongly depends on the chosen vehicle in combination with the state of the bacteria to be encapsulated and their stability after bioencapsulation [69]. Factors such as vaccine dose and time of exposure, especially in young animals, are extremely crucial for vaccine efficacy. As it will be further discussed in section 7.2, these factors can certainly make the difference between induction of immune responses or tolerance.

Micro-encapsulation of vaccines in polymers such as chitosan, Poly D,L-lactic-coglycolic acid (PLGA), alginates, liposome, and MicroMatrix ${ }^{\mathrm{TM}}$ are more practical and efficient for juvenile and older fish as they can be incorporated in the feed. The properties and formulation of the aforementioned micro-encapsulation methods have been extensively reviewed elsewhere $[32,70]$. Given this, we will concentrate on a few studies that specifically focused on vaccine efficacy upon pathogen challenge (Table 2). Most evident is the success of alginate-encapsulated live attenuated or avirulent enteric pathogens (e.g. Edwardsiella tarda, Staphylococcus iniae, Vibrio $s s p$ ) and DNA vaccines against viruses. As further discussed later in section 7.3, such success might reside in the ability of the alginates to protect the microbe and the plasmid while passing through the digestive tract, and to diffuse through the gut mucus layer, thereby reaching the enterocyte surface. Once in contact with the epithelium, alginates might be actively taken up by antigen-sampling cells and deliver the microbe or DNA plasmid. Enteric pathogens will most likely have the intrinsic ability to activate local mucosal responses, whereas DNA plasmids can then enter the nucleus and trigger antigen expression in the host cell in a manner similar to that triggered during a viral infection. The latter mechanism has been previously described as the base of the great success of i.m. injected DNA vaccines in fish [71]. The efficacy of DNA vaccines also confirms that delivering nucleic acids fulfils the requirements of closely mimicking antigen expression and presentation during an intracellular pathogen infection. This in turn will most likely trigger cellular as well as humoral immune responses appropriate to the pathogen. 
Table 2. Experimental approaches for the development of oral vaccines using (bio) encapsulated antigens

\begin{tabular}{|c|c|c|c|c|c|c|c|c|c|}
\hline Pathogen & $\begin{array}{l}\text { Encap- } \\
\text { sulation } \\
\text { method }\end{array}$ & $\begin{array}{l}\text { Fish } \\
\text { species }\end{array}$ & Regime & Antigen & Dose & $\begin{array}{l}\text { Protection } \\
(\%)\end{array}$ & $\begin{array}{l}\text { Time } \\
\text { of chal- } \\
\text { lenge }\end{array}$ & $\begin{array}{l}\text { Chal- } \\
\text { lenge } \\
\text { route }\end{array}$ & REF \\
\hline \multicolumn{10}{|l|}{ Bacteria } \\
\hline $\begin{array}{l}\text { Vibrio } \\
\text { anguillarum } \\
\text { (V.a.) }\end{array}$ & $\begin{array}{l}\text { Bioencap- } \\
\text { sulation }\end{array}$ & $\begin{array}{l}\text { Ayu } \\
(63 \mathrm{mg})\end{array}$ & $22 d$ & $\begin{array}{l}\text { F.i. } V . a \text {. in } \\
\text { water flea and } \\
\text { rotifer }\end{array}$ & $\begin{array}{l}0.64 \mu \mathrm{g} \\
\mathrm{LPS} / \text { fish } \\
\text { detected }\end{array}$ & $\begin{array}{l}\text { V: } 92.4 \% \mathrm{C}: \\
64.2 \%\end{array}$ & $1 \mathrm{dpv}$ & bath & {$[51]$} \\
\hline $\begin{array}{l}\text { Vibrio } \\
\text { harveyi }\end{array}$ & Alginates & $\begin{array}{l}\text { Rainbow } \\
\text { trout } \\
(15 \mathrm{~g})\end{array}$ & $\begin{array}{l}6 \text { times, } \\
1 \mathrm{w} \text { inter- } \\
\text { val }\end{array}$ & $\begin{array}{l}\text { Outer mem- } \\
\text { brane proteins } \\
\text { (OMPs) }\end{array}$ & $\begin{array}{l}50 \mu \mathrm{g} \\
\text { OMPs/ } \\
\text { fish/ day }\end{array}$ & $\begin{array}{l}\text { V: } 90 \% \quad \text { C: } \\
10 \%\end{array}$ & $12 \mathrm{wpv}$ & i.p. & [156] \\
\hline $\begin{array}{l}\text { Edwardsi- } \\
\text { ella tarda } \\
\text { (E.t.), Strep- } \\
\text { tococcus } \\
\text { iniae (S.i.) }\end{array}$ & Alginates & $\begin{array}{l}\text { Japanese } \\
\text { flounder } \\
(12.4 \mathrm{~g})\end{array}$ & $\begin{array}{l}3 \mathrm{~d} \text {; fol- } \\
\text { lowed by } \\
\text { oral and } \\
\text { immersion } \\
\text { boost at } \\
2 \mathrm{~m}\end{array}$ & $\begin{array}{l}\text { Attenuated } E . t \text {. } \\
\text { expressing } S . i \text {. } \\
\text { antigen Sia } 10\end{array}$ & $\begin{array}{l}10^{\wedge} 8 \mathrm{CFU} / \\
\text { fish/ day } \\
\\
10^{\wedge} 8 \mathrm{CFU} / \\
\text { fish/day, } 8 \mathrm{~h} \\
\text { immersion } \\
\text { boost } 10^{\wedge} 7 \\
\mathrm{CFU} / \mathrm{ml}\end{array}$ & $\begin{array}{l}\text { V: } 82 \% \\
\text { (E.t.) } \\
\text { C: } 26 \% \\
\text { (E.t.) } \\
\text { V: } 84 \% \\
\text { (S.i.) } \\
\text { C: } 14 \% \\
\text { (S.i.) } \\
\text { V: } 76 \% \\
\text { (E.t.) } \\
\text { C: } 22 \% \\
\text { (E.t.) } \\
\text { V: } 86 \% \text { (S.i.) } \\
\text { C: } 26 \% \\
\text { (S.i.) }\end{array}$ & $1 \mathrm{mpv}$ & $\begin{array}{l}\text { bath } \\
(E . t .) \text {; } \\
\text { i.p. } \\
(S . i)\end{array}$ & [157] \\
\hline $\begin{array}{l}\text { Edwardsiel- } \\
\text { la tarda }\end{array}$ & Alginates & $\begin{array}{l}\text { Japanese } \\
\text { flounder } \\
(10 \mathrm{~g})\end{array}$ & $5 \mathrm{~d}$ & $\begin{array}{l}\text { Avirulent } E \text {. } \\
\text { tarda } \text { strain } \\
\text { ATCC } 15947\end{array}$ & $\begin{array}{l}10^{\wedge} 9 \mathrm{CFU} / \\
\text { fish/ day }\end{array}$ & $\begin{array}{l}\text { V: } 64-71 \% \\
\text { C: } 14-18 \%\end{array}$ & $5 \mathrm{wpv}$ & $\begin{array}{l}\text { i.p. and } \\
\text { bath }\end{array}$ & [76] \\
\hline $\begin{array}{l}\text { Edwardsi- } \\
\text { ella tarda } \\
\text { (E.t.) }\end{array}$ & Alginates & $\begin{array}{l}\text { Japanese } \\
\text { flounder } \\
(9.6 \mathrm{~g})\end{array}$ & $5 \mathrm{~d}$ & $\begin{array}{l}\text { Pseudomonas } \\
\text { strain FP3 } \\
\text { expressing E.t. } \\
\text { antigen Esa1 }\end{array}$ & $\begin{array}{l}10^{\wedge} 9 \mathrm{CFU} / \\
\text { fish/day }\end{array}$ & $\begin{array}{l}\text { V: } 56 \% \quad \text { C: } \\
8 \%\end{array}$ & $1 \mathrm{mpv}$ & i.p. & [158] \\
\hline $\begin{array}{l}\text { Edwardsi- } \\
\text { ella tarda, } \\
\text { Vibrio } \\
\text { harveji (V.h.) }\end{array}$ & Alginates & $\begin{array}{l}\text { Turbot } \\
(14 \mathrm{~g})\end{array}$ & $3 d$ & $\begin{array}{l}\text { Live attenuated } \\
\text { E.t. expressing } \\
\text { V.h. DegQ sol- } \\
\text { uble antigen }\end{array}$ & $\begin{array}{l}10^{\wedge} 8 \mathrm{CFU} / \\
\text { fish/day }\end{array}$ & $\begin{array}{l}\text { V: } 91 \% \\
\text { (E.t.) } \\
\text { C: } 20 \% \\
\text { (E.t.) } \\
\text { V: } 94 \% \\
\text { (V.h.) } \\
\text { C: } 28 \% \\
\text { (V.h.) }\end{array}$ & $1 \mathrm{mpv}$ & bath & [159] \\
\hline $\begin{array}{l}\text { Flavo- } \\
\text { bacterium } \\
\text { columnare }\end{array}$ & Alginates & $\begin{array}{l}\text { Nile } \\
\text { tilapia } \\
(15.7 \mathrm{~g})\end{array}$ & $7 \mathrm{~d}$ & $\begin{array}{l}\text { F.i. F. co- } \\
\text { lumnare }\end{array}$ & $\begin{array}{l}2.65 \mathrm{x} \\
10^{\wedge} 10 \\
\mathrm{CFU} / \mathrm{fish} / \\
\text { day }\end{array}$ & $\begin{array}{l}\text { No protec- } \\
\text { tion }\end{array}$ & $21 \mathrm{dpv}$ & bath & [160] \\
\hline $\begin{array}{l}\text { Flavobacte- } \\
\text { rium psy- } \\
\text { chrophilum }\end{array}$ & Alginates & $\begin{array}{l}\text { Rainbow } \\
\text { trout } \\
(1 \mathrm{~g})\end{array}$ & $\begin{array}{l}3 \text { times } \\
\text { for } 7 \mathrm{~d} \text {, } \\
\text { followed } \\
\text { by } 11 \mathrm{~d} \\
\text { standard } \\
\text { food }\end{array}$ & $\begin{array}{l}\text { Live attenuated } \\
\text { F. psychroph- } \\
\text { ilum }\end{array}$ & $\begin{array}{l}7.7 \times 10^{\wedge} 5 \\
\mathrm{CFU} / \text { fish/ }\end{array}$ & $\begin{array}{l}\mathrm{V}: 8 \% \\
0 \%\end{array}$ & $3 \mathrm{dpv}$ & $\begin{array}{l}\text { Sub- } \\
\text { cuta- } \\
\text { neous }\end{array}$ & [161] \\
\hline $\begin{array}{l}\text { Aeromonas } \\
\text { hydrophila }\end{array}$ & $\begin{array}{l}\text { Chitosan } \\
(600 \mathrm{~nm}) ; \\
\text { alginate- } \\
\text { chitosan } \\
(1100 \mathrm{~nm})\end{array}$ & $\begin{array}{l}\text { Indian } \\
\text { major } \\
\text { carp }(100 \\
\text { g) }\end{array}$ & $7 \mathrm{~d}$ & $\begin{array}{l}\text { F.i. A. hydroph- } \\
\text { ila }\end{array}$ & $\begin{array}{l}1 \times 10^{\wedge} 10 \\
\mathrm{CFU} / \mathrm{fish} / \\
\text { day }\end{array}$ & $\begin{array}{l}\text { V: } 20 \% \quad C: \\
10 \%\end{array}$ & $7 \mathrm{wpv}$ & i.p. & [162] \\
\hline
\end{tabular}




\begin{tabular}{|c|c|c|c|c|c|c|c|c|c|}
\hline $\begin{array}{l}\text { Vibrio an- } \\
\text { guillarum }\end{array}$ & Chitosan & $\begin{array}{l}\text { Asian } \\
\text { sea bass } \\
(10 \mathrm{~g})\end{array}$ & $21 d$ & $\begin{array}{l}\text { Porin gene, } \\
\text { outer mem- } \\
\text { brane protein } \\
\text { OMP38 DNA } \\
\text { plasmid }\end{array}$ & $\begin{array}{l}50 \mu \mathrm{g} \\
\text { plasmid/ } \\
\text { fish total }\end{array}$ & $\begin{array}{l}\text { V: } 55 \% \\
\text { C: } 10-15 \%\end{array}$ & $1 \mathrm{dpv}$ & i.m. & [163] \\
\hline $\begin{array}{l}\text { Vibrio } \\
\text { parahaemo- } \\
\text { lyticus }\end{array}$ & Chitosan & $\begin{array}{l}\text { Black } \\
\text { seabream } \\
(80 \mathrm{~g})\end{array}$ & $3 d$ & $\begin{array}{l}\text { Outer mem- } \\
\text { brane protein } \\
\mathrm{K} \text { in DNA } \\
\text { plasmid }\end{array}$ & $\begin{array}{l}50 \mu \mathrm{g} \\
\text { plasmid/ } \\
\text { fish total }\end{array}$ & $\begin{array}{l}\text { V: } 80 \% \\
\text { C: } 17 \%\end{array}$ & $21 \mathrm{dpv}$ & i.m. & [164] \\
\hline $\begin{array}{l}\text { Aeromonas } \\
\text { hydrophila }\end{array}$ & $\begin{array}{l}\text { Liposome } \\
(5-10 \mu \mathrm{m})\end{array}$ & $\begin{array}{l}\text { Common } \\
\text { carp } \\
(30 \mathrm{~g})\end{array}$ & $3 d$ & $\begin{array}{l}\text { F.i. A. hydroph- } \\
\text { ila }\end{array}$ & $\begin{array}{l}10 \mu \mathrm{l} \text { of } \\
\text { liposomes/ } \\
\text { day }(33 \mu \mathrm{g} / \\
\mathrm{ml} \text { protein) }\end{array}$ & $\begin{array}{l}\mathrm{V}: 60-80 \\
\mathrm{C}: 55-100 \%\end{array}$ & $22 \mathrm{dpv}$ & $\begin{array}{l}\text { intra- } \\
\text { subcu- } \\
\text { taneous }\end{array}$ & [102] \\
\hline $\begin{array}{l}\text { Aeromonas } \\
\text { salmonicida }\end{array}$ & Liposome & $\begin{array}{l}\text { Common } \\
\text { carp } \\
(350 \mathrm{~g})\end{array}$ & $\begin{array}{l}3 \text { times, } \\
2 \mathrm{w} \text { inter- } \\
\text { val }\end{array}$ & $\begin{array}{l}\text { Ultrasound-in- } \\
\text { activated } A \text {. } \\
\text { salmonicida }\end{array}$ & $\begin{array}{l}100 \mu \mathrm{g} / \\
\text { vaccination }\end{array}$ & $\begin{array}{l}\mathrm{V}: 84 \% \\
\mathrm{C}: 63 \%\end{array}$ & $2 \mathrm{wpv}$ & bath & [165] \\
\hline $\begin{array}{l}\text { Piscirickett- } \\
\text { sia salmonis }\end{array}$ & $\begin{array}{l}\text { MicroMa- } \\
\text { trix }\end{array}$ & $\begin{array}{l}\text { Atlantic } \\
\text { salmon } \\
(30 \mathrm{~g})\end{array}$ & $\begin{array}{l}10 \text { times, } \\
2 \mathrm{~d} \text { interval }\end{array}$ & P. salmonis & $\begin{array}{l}1 \times 10^{\wedge} 10 \\
\text { cells/g feed } \\
(2 \% \mathrm{BW} \\
\text { fed every } \\
3 \mathrm{~d})\end{array}$ & $\begin{array}{l}\text { V: } 80 \% \\
\text { C: } 10-20 \%\end{array}$ & $\begin{array}{l}300 \text { or } \\
600 \\
\text { degree- } \\
\text { dpv }\end{array}$ & i.p. & [166] \\
\hline \multirow[t]{2}{*}{$\begin{array}{l}\text { Stenotro- } \\
\text { phomonas } \\
\text { maltophilia }\end{array}$} & \multirow[t]{2}{*}{$\begin{array}{l}\text { PLGA } \\
(2.2 \mu \mathrm{m})\end{array}$} & \multirow[t]{2}{*}{$\begin{array}{l}\text { Channel } \\
\text { catfish } \\
(100 \mathrm{~g})\end{array}$} & \multirow[t]{2}{*}{$\begin{array}{l}3 \text { times, } \\
14 \mathrm{~d} \\
\text { interval }\end{array}$} & \multirow[t]{2}{*}{$\begin{array}{l}\text { Ultrasone-in- } \\
\text { activated } S \text {. } \\
\text { maltophilia }\end{array}$} & $\begin{array}{l}50 \mu \mathrm{g} / \text { fish } \\
100 \mu \mathrm{g} / \\
\text { fish } \\
200 \mu \mathrm{g} / \\
\text { fish }\end{array}$ & $\begin{array}{l}43.3 \% \\
65 \% \\
75 \%\end{array}$ & \multirow[t]{2}{*}{$35 \mathrm{dpv}$} & \multirow[t]{2}{*}{ i.p. } & \multirow[t]{2}{*}{ [167] } \\
\hline & & & & & Control & $0 \%$ & & & \\
\hline $\begin{array}{l}\text { Lactococcus } \\
\text { garviae }\end{array}$ & $\begin{array}{l}\text { PLGA, } \\
\text { alginates }\end{array}$ & $\begin{array}{l}\text { Rainbow } \\
\text { trout } \\
(20 \mathrm{~g})\end{array}$ & $\begin{array}{l}7 \mathrm{~d} \text {; booster } \\
\text { at } 61 \mathrm{~d}\end{array}$ & $\begin{array}{l}\text { Formalin-in- } \\
\text { activated } L . \\
\text { garviae in } \\
\text { PLGA or } \\
\text { alginate (Alg) } \\
\text { microspheres }\end{array}$ & $\begin{array}{l}1 \times 10^{\wedge} 10 \\
\text { bacteria/ } \\
\text { fish/ day } \\
\text { in PLGA } \\
\text { or alginate } \\
\text { micro- } \\
\text { spheres } \\
\text { (alg); boost } \\
\text { at 61d }\end{array}$ & $\begin{array}{l}\text { PLGA: } 68 \% \\
\text { Alg: } 60 \% \\
\text { C: } 14 \% \\
\text { PLGA: } 50 \% \\
\text { Alg: } 44 \% \\
\text { C: } 10 \% \\
\text { PLGA: } 76 \% \\
\text { Alg: } 72 \% \\
\text { C: } 14 \% \\
\text { PLGA: } 70 \% \\
\text { Alg: } 68 \% \\
\text { C: } 14 \%\end{array}$ & $\begin{array}{l}60 \mathrm{dpv} \\
90 \mathrm{dpv} \\
\text { (with } \\
\text { boost) } \\
120 \mathrm{dpv} \\
\text { (with } \\
\text { boost) }\end{array}$ & i.p. & [168] \\
\hline \multicolumn{10}{|l|}{ Viruses } \\
\hline $\begin{array}{l}\text { Nervous ne- } \\
\text { crosis virus } \\
(\mathrm{NNV})\end{array}$ & $\begin{array}{l}\text { Bioencap- } \\
\text { sulation }\end{array}$ & $\begin{array}{l}\text { Orange } \\
\text { spotted } \\
\text { grouper } \\
(18 \mathrm{dph})\end{array}$ & $2 d$ & $\begin{array}{l}\text { Fy.-i. E. coli } \\
\text { expressing } \\
\text { NNV capsid } \\
\text { protein in } \\
\text { Artemia }\end{array}$ & $\begin{array}{l}10^{\wedge} 5 \\
\text { bacteria/ } \\
\text { Artemia }\end{array}$ & $\begin{array}{l}\text { V: } 80-86 \% \\
\text { C: } 44-54 \%\end{array}$ & $35 \mathrm{dph}$ & i.p. & [79] \\
\hline \multirow[t]{2}{*}{$\begin{array}{l}\text { Nervous ne- } \\
\text { crosis virus } \\
(\mathrm{NNV})\end{array}$} & \multirow[t]{2}{*}{$\begin{array}{l}\text { Bioencap- } \\
\text { sulation }\end{array}$} & \multirow[t]{2}{*}{$\begin{array}{l}\text { Orange } \\
\text { spotted } \\
\text { grouper } \\
(30 \mathrm{dph})\end{array}$} & \multirow[t]{2}{*}{$2 d$} & \multirow[t]{2}{*}{$\begin{array}{l}\text { h.i. } V . a \text {. or } E \text {. } \\
\text { coli }(E . c) \text { ex- } \\
\text { pressing NNV } \\
\text { coat protein in } \\
\text { Artemia }\end{array}$} & $\begin{array}{l}8.3 \times 10^{\wedge} 4 \\
\text { CFU } E . c / \\
\text { Artemia } \\
(200 / \text { fish/ } \\
\text { day) } \\
4.1 \times 10^{\wedge} 6 \\
\text { CFU } V . a / \\
\text { Artemia } \\
(200 / \text { fish/ } \\
\text { day) }\end{array}$ & $52.5-87.5 \%$ & \multirow[t]{2}{*}{$7 \mathrm{dpv}$} & \multirow[t]{2}{*}{ i.p. } & \multirow[t]{2}{*}[78]{} \\
\hline & & & & & Control & $2.5-42.5 \%$ & & & \\
\hline
\end{tabular}




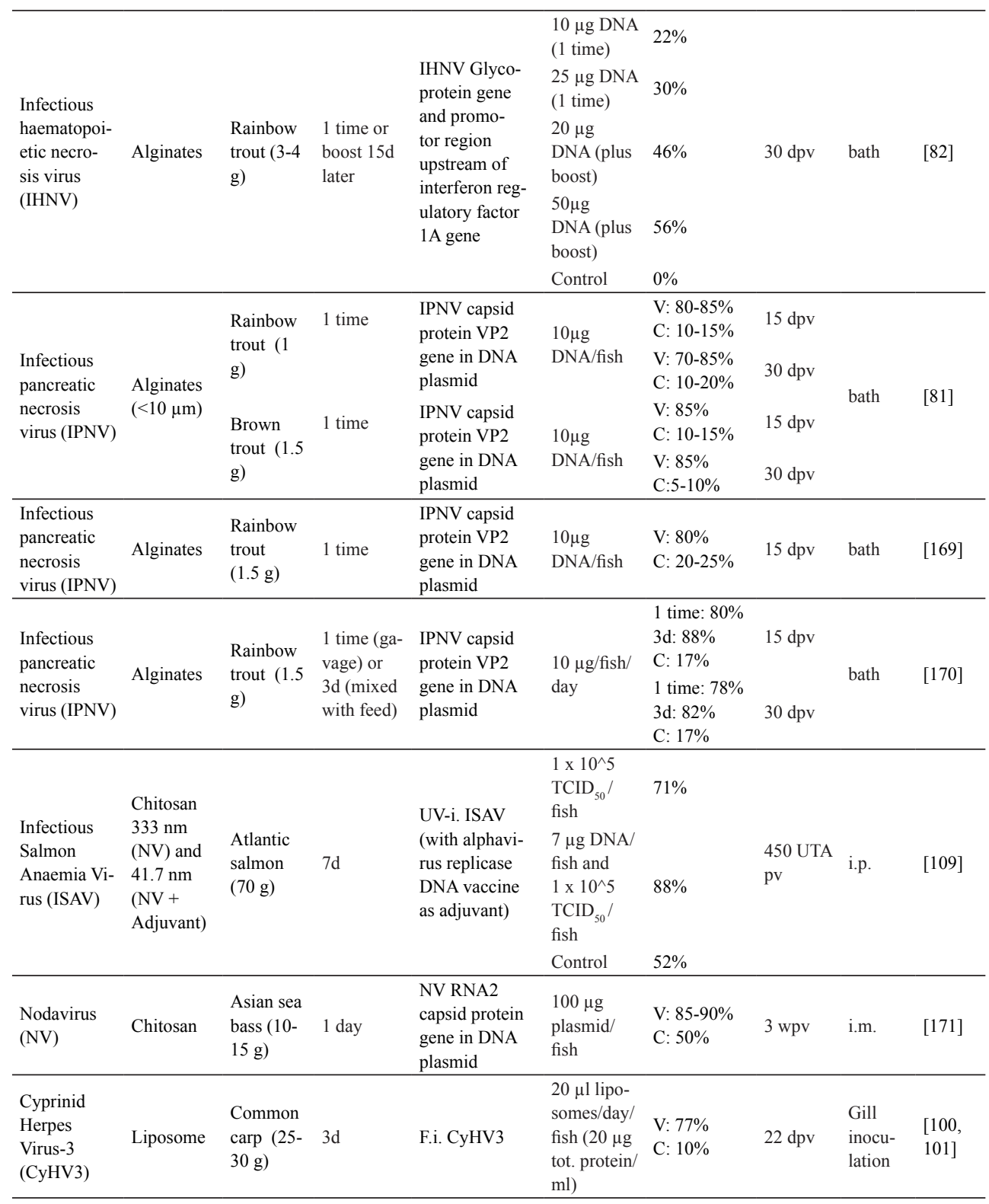




\begin{tabular}{|c|c|c|c|c|c|c|c|c|c|}
\hline \multirow{6}{*}{$\begin{array}{l}\text { Infecious } \\
\text { heamato- } \\
\text { poietic ne- } \\
\text { crosis virus } \\
\text { (IHNV) }\end{array}$} & \multirow{6}{*}{$\begin{array}{l}\text { PLGA } \\
(500 \mathrm{~nm} \\
\text { average, } \\
200-1000 \\
\mathrm{~nm})\end{array}$} & \multirow{6}{*}{$\begin{array}{l}\text { Rainbow } \\
\text { trout } \\
(5 \mathrm{~g})\end{array}$} & \multirow{6}{*}{$4 d$ or $8 d$} & \multirow{6}{*}{$\begin{array}{l}\text { IHNV Gly- } \\
\text { coprotein } \\
\text { gene in DNA } \\
\text { plasmid }\end{array}$} & $\begin{array}{l}22 \mu \mathrm{g} \\
\text { plasmid } \\
\text { DNA }\end{array}$ & $17 \%$ & \multirow{4}{*}{$6 \mathrm{wpv}$} & \multirow{6}{*}{ i.p. } & \multirow{6}{*}{ [126] } \\
\hline & & & & & $\begin{array}{l}43 \mu \mathrm{g} \\
\text { plasmid } \\
\text { DNA }\end{array}$ & $27 \%$ & & & \\
\hline & & & & & Control & $7 \%$ & & & \\
\hline & & & & & $\begin{array}{l}22 \mu \mathrm{g} \\
\text { plasmid } \\
\text { DNA }\end{array}$ & $17 \%$ & & & \\
\hline & & & & & $\begin{array}{l}43 \mu \mathrm{g} \\
\text { plasmid } \\
\text { DNA }\end{array}$ & $33 \%$ & \multirow[t]{2}{*}{$10 \mathrm{wpv}$} & & \\
\hline & & & & & Control & $17 \%$ & & & \\
\hline
\end{tabular}

Abbreviations: d: day(s); w: week(s); dpv: days post vaccination; dph: days post hatch; wpv: weeks post vaccination, mpv: months post vaccination; h.i.: heat-inactivated; f.i.: Formalin-inactivated; fy-i. formaldehyde-inactivated, UV-.. UV-inactivated; cohab: cohabitation; FIA: Freund's incomplete adjuvant; BW: body-weight; i.p.: intraperitoneal; i.m: intramuscular.

\section{Rational immunological approach to oral vaccine design for fish}

There is no doubt that, independently of the administration routes, vaccines that best mimic the natural pathogen infection and trigger appropriate immune pathways against the pathogen, are most effective; but how is this appropriate immune response achieved?

\subsection{Triggering humoral responses}

For pathogens against which an antibody-dependent systemic humoral response is appropriate to confer protection, killed or inactivated microbes, protein-adjuvant vaccines, virus-like particles (VLPs), or other subunit vaccines are sufficient to trigger adequate systemic immune responses. When inactivating the pathogen or producing the subunits in heterologous expression systems, care should be taken to assure that the antigenic epitope against which the antibody response is directed remains intact. Parenteral administration of such vaccines in the presence of adjuvants will in fact elicit antigen-specific neutralizing antibodies that will protect the host upon natural infection. Briefly, B cells can directly recognize protein antigens and can be stimulated to proliferate and secrete antibodies. In parallel, the adjuvant present in the vaccine formulation will trigger the activation of local innate immune cells, including Antigen Presenting Cells (APCs), through recognition of viral PAMPs via Pattern Recognition Receptors (e.g. TLRs, NLRs, RLRs). APCs will then upregulate the expression of pro-inflammatory cytokines (i.e. IL-12, IFN $\alpha / \beta$ and TNF $\alpha$ ) and co- 
stimulatory molecules (i.e. CD80/86). These molecules are crucial for the activation of cell-mediated immunity through antigen presentation to CD4+ helper T cells via the MHC-II pathway. In turn, CD4+ helper T cells, will differentiate to the Th1 subset under the influence of the pro-inflammatory cytokines. Subsequent production of IFN $\gamma$ by Th1 T cells will sustain antibody production, affinity maturation and memory formation of antigen-specific B cells. In most mammals and in birds these antibodies, mostly of the IgG isotype, will be able to fix complement and will be present in the circulation and peripheral immune organs.

Vaccines that exclusively elicit systemic humoral immunity, however, might not be efficacious against mucosal (enteric) pathogens, as parenteral immunization does not effectively elicit mucosal responses [20]. Pathogens that undergo rapid antigenic variation will also not be stopped by vaccines exclusively inducing humoral immunity, as cross-protection will not be achieved. Most importantly, pathogens against which not only humoral but also cell-mediated cytotoxic $\mathrm{T}$ cell responses are required (e.g intracellular microbes) will also not be affected by vaccines that mainly elicit humoral responses. This last category, which also includes most mucosal pathogens, comprises the largest group of microbes against which effective vaccines are lacking in most animal species. Perhaps it is not surprising that most target pathogens are viruses and intracellular bacteria, with the exception of few protozoan subspecies (e.g. plasmodium, trypanosomes) [98].

\subsection{Triggering cell-mediated $\mathrm{T}$ cell responses}

To address the need to elicit systemic cell-mediated $\mathrm{T}$ cell responses in humans and several veterinary species, live attenuated pathogens (bacteria or viruses) and non-replicating DNA plasmids have been developed that are administered via the parenteral route in the presence of strong adjuvants. One of the advantages of for example live attenuated viruses is their ability to replicate in the host but at much lower rate than the wild type virulent pathogen. Such feature allows for the use of relatively low vaccine doses. Next, and most importantly, vaccine replication in the host and the presence of the adjuvant will trigger the activation of local innate immune cells and APCs. This latter aspect is extremely crucial for the activation of cell-mediated immunity through antigen presentation to CD8+ T cells via the MHC-I pathway, as well as to CD4+ helper T cells via the MHC-II pathway through crosspresentation. The presence of viral particles as well as the expression of viral antigens 
on the APC surface will also activate antigen-specific B cells. IFN $\gamma$-secreting CD4+ Th1 cells will further sustain B cells activation, antibody production and isotype switch in a manner similar to the one described above (section 6.1). Altogether, such response contributes to a full activation of effector humoral as well as cellmediated responses against viruses or other intracellular pathogens. Interestingly, DNA plasmids, although not able to replicate in the host cells, have been shown to effectively trigger both arms of the immune system. Especially in fish, they have been proven very successful and can be used at doses 100 times lower than in larger mammals $[24,71]$. Unfortunately, mainly due to legislative concerns, only the DNA vaccine for vaccination of salmon again IHNV in Canada is commercially available. In fish, most of the aforementioned immune mechanisms are believed to be generally conserved, although they have not been investigated in details in all farmed fish species, and are reviewed elsewhere [99]. Humoral as well as cell-mediated immune responses have been associated with protection upon systemic as well as mucosal vaccination in fish $[100,101]$. Serum IgM levels have been associated with protection in several vaccination strategies, especially against extracellular pathogens. The relatively recent development of monoclonal antibodies in ginbuna crucian carp and trout against the T cell markers CD8 $\alpha$ and CD4 [102,103] has allowed for a better characterization of $\mathrm{T}$ cell responses to infection and vaccination [104]. Most of the signature cytokines associated to Th1, Th2 and Th17 responses have been identified in fish, although their full functional characterization is still underway in most relevant fish species. Finally, the discovery of a novel immunoglobulin type in 2005, named IgT (for Teleost [105]), and the generation of monoclonal antibodies against trout IgT, has provided fish vaccinologists with a new tool to better characterize systemic and mucosal responses $[106,107]$.A detailed description of innate as well as adaptive immune responses identified and characterized thus far in fish has been recently reviewed [101,108]. A recent review focusing especially on the immunological mechanisms following mucosal vaccination of finfish is also available [109].

\section{Triggering protective humoral and cell-mediated responses upon oral vaccination}

\subsection{Targeting $M-($ like) cells and antigen presenting cells in the fish gut}

When antigens or vaccines are administered via the oral route they are faced with the same host defence mechanisms as do commensals and enteric microbes. Oral 
vaccines will have to survive the gastric environment, attacks by proteases or nucleases, and stick to and penetrate the thick mucus to finally reach the epithelial barrier. For these reasons relatively large amounts of antigens need to be delivered orally, as it is impossible to exactly determine the amount of antigen that is delivered to the epithelial layer. When and if the vaccine will be in contact with the apical epithelium, it will have to be actively taken up and delivered to the basal side. At this side, the lamina propria, innate immune cells and specifically APCs would process it. In mammals specialized antigen sampling cells, M cells, are present in the follicle associated epithelium surmounting Payer's patches but also on the apical part of the villus epithelium $[66,110]$. Owing to their location, antigen sampling ability, and structure, $\mathrm{M}$ cells play a key role in exposing leukocytes in the lamina propria to the variety of antigens and microbes present in the lumen of the gut. Furthermore, M cells efficiently sample luminal content (bacteria, viruses, soluble microbial products or particulate antigens) and deliver it to leukocytes on their basolateral membrane by using vesicles that are transported through the cytosol [64]. M cells form a pocket within which leukocytes can aggregate. This pocket reduces the distance between the apical and basal membrane thereby increasing the efficiency at which antigens are delivered to underlining leukocytes [111]. Besides M cells, specialized dendritic cells (DCs) extend protrusion through the epithelial layer and directly sample antigens in the lumen $[112,113]$. As a result of their efficient sampling ability, strategic positions and specialized roles in activating local immune responses, $\mathrm{M}$ cells and intraepithelial DCs have become specific targets for vaccine delivery [114]. For example, a monoclonal antibody carrying a vaccine antigen has been used to specifically deliver antigens to murine $\mathrm{M}$ cells [37], and several immunostimulatory molecules are exploited to target DCs [19]. In this way local mucosal responses can be rationally triggered.

In salmon, a distinct population of M-like cells has been identified. These cells were found to be intermingled within the epithelial layer, extend cytoplasmic protrusion to the luminal side and be able to sample gold-BSA microparticles [21]. Although M-like cells specific markers have not yet been identified in fish, salmon M-like cells show a staining similar to mammalian M cells as were found positive for Ulex europaeus agglutinin (UEA-1 from gorse) and negative for wheat germ agglutinin (WGA). UEA-1 single-positive cells were only found deep in the mucosal folds of the villi and not in the apical region. This suggests that they might have a strategic 
position that allows them to interact with microbes that gain access to the deeper area of the intestinal folds preventing uptake and possible reaction to luminal (commensal) microbes. Although leukocytes were not present in the basal pocket underneath the antigen-sampling cells, macrophage-like cells have been seen associated to the basal side of M-like cells. In fish, both enterocytes and intraepithelial macrophages have been shown to play a role in antigen uptake in the second gut segment. Intraepithelial macrophages were shown to efficiently take up and transport antigens especially when antigens where coupled to enteric adhesion molecules [62]. Besides this, the presence of motile non-resident macrophages that can potentially transport antigens at peripheral sites has also been observed [8]. Markers for fish macrophages are available only in a few fish species [115-117]. Nevertheless, they could be used as a proof of concept to specifically target this putative APC in a model fish species to demonstrate the suitability of cell-specific antigen delivery approaches.

\subsection{Induction of anergy or tolerance and antigen dose}

Perhaps expected, but in mammals the majority of antigens or microorganisms sampled from the lumen through $\mathrm{M}$ cells or DCs do not trigger a local immune response. The lack of responsiveness to ingested or orally administered antigens is referred to as oral tolerance, which is speculated to be the prevention of harmful responses to otherwise beneficial food components and commensal bacteria. This intrinsic homeostatic mechanism is the major obstacle to overcome when designing oral vaccines. The mechanisms leading to oral tolerance are still not fully understood, not even in mammals. Nevertheless there is a general consensus that $\mathrm{T}$ regulatory cells (Tregs) and tolerogenic DCs (tol-DCs) play an important role in gut immunity and induction of oral tolerance [18]. In mammals, Tregs express high levels of the IL-2 receptors and may deprive other cell populations of this growth factor by adsorbing IL-2. Tregs also express high levels of the inhibitory receptor CTLA4 and through this compete with normal T cells for co-stimulatory molecules on APCs. Finally, both Tregs and tol-DC secrete high amounts of the anti-inflammatory cytokines IL-10 and TGF $\beta$ that greatly increase the threshold required for leukocyte activation [18]. In fact, under these conditions, an orally administered antigen that is taken up and presented by DC to antigen-specific T cells will never trigger an appropriate stimulatory signal, unless the antigen itself has the ability to trigger strong pro-inflammatory responses. The latter signals should then 
translate into the upregulation of MHC-II molecules and co-stimulatory molecules (CD80/86) on the APC surface as well as secretion of pro-inflammatory cytokines. In the absence of pro-inflammatory signals and in the presence of IL-10 and TGF $\beta$, antigen presentation by non-activated APCs will trigger the development of Tregs and render antigen-specific T cells anergic or drive them to apoptosis (deletion) [18]. This in turn will contribute to depletion or unresponsiveness of the pool of antigenspecific T cells leading to tolerance upon re-exposure to the same antigens.

From a vaccination point of view it is important to note that induction of Tregs and anergy has been associated to antigen dose. In mammals, high doses of orally administered antigens will induce anergy and deletion of antigen-specific $\mathrm{T}$ cell whereas low antigen dose will rather trigger Tregs development. In fish, although the mechanisms of tolerance induction have not been systematically addressed, antigen dose and route of administration have also been associated to tolerance [13,14]. Given the difficulty to precisely quantify the amount of orally administered antigen that is taken up at mucosal surfaces, it might prove extremely difficult to standardize the conditions at which the vaccine will not induce tolerance. From an oral vaccine development perspective, the vaccine antigen should therefore not only be actively taken up and delivered to APCs in the lamina propria, but should also trigger strong stimulatory signals of a magnitude sufficient to break intestinal tolerance. In this respect, the use of strong mucosal adjuvants or replicating vaccines will prove instrumental.

\subsection{Mimicking pathogen entry and activation of mucosal responses}

\subsubsection{Live attenuated enteric pathogens as vaccine vectors}

Live attenuated enteric pathogens, even in the absence of adjuvants, will have the ability to enter and replicate at the mucosal surface and trigger appropriate mucosal responses, owing to the presence of PAMPs that can trigger stimulatory signals. One of the major hurdles is in the development of safe attenuated vaccines that are least likely to revert to virulence. Attenuation by repeated passages of viruses or bacteria in vitro does not always allow controlling the number and sites of the mutations, making it more difficult to predict how likely the vaccine is to revert to the virulent form. Furthermore, based on the nature of the pathogen, live attenuated vaccines might not confer sterile immunity implying that the vaccinated host will be carrier and shedder of the vaccine pathogen. As discussed above, lack of sterile immunity might come at great costs for the entire vaccinated and non-vaccinated population. 
The development of safe vaccines is of utmost importance when considering animals, including fish, that are reared at high stocking densities, under relatively stressful conditions and are transported all over the world. In this situation vaccines should preferably induce sterile immunity and not be harmful to any kind of animal, the environment as well as the end consumer.

Due to the safety concerns, to date, one live bacterial vaccine has been licensed for sale in aquaculture in Canada and Chile (Renogen ${ }^{\circledR}$ only) and three in the US (Shoemaker et al., 2009). These vaccines include Renogen ${ }^{\circledR}$ against bacterial kidney disease (BKD), AQUAVAC-ESC ${ }^{\circledR}$ against enteric septicemia of catfish, and AQUAVACCOL ${ }^{\circledR}$ against columnaris disease of catfish. A live viral hemorrhagic septicemia virus (VHSV) vaccine is available in Germany (Gomez-Casado et al., 2011). Of interest the Renogen ${ }^{\circledR}$ vaccine is based on cross-reactive immunity generated upon vaccination with an environmental avirulent bacterium Arthrobacter (Ar.) davidanieli, phylogenetically and antigenically related to Renibacterium salmoninarum, which is the causative agent of BKD (Griffiths et al. 1998). Unfortunately all aforementioned live attenuated vaccines are delivered by immersion in catfish fry one week posthatch and are not sufficiently protective when administered orally at this stage $[118,119]$. A similar approach was used to orally vaccinate Japanese flounder against Edwardsiella tarda (E. tarda). Very good protection upon i.p. and bath challenge was found after oral administration of avirulent strains encapsulated in alginate microparticles [74]. Moreover, alginate encapsulated avirulent strains of E. tarda were used to generate a cross-protective divalent oral vaccine against E. tarda and $V$. harveji or E. tarda and Streptococcus iniae and were found to induce very good protection (Table 2).

Of note is the fact that E. tarda is an enteric pathogen with a very wide host and temperature range. For what discussed above, it might retain its intrinsic ability to adhere to mucosal surfaces and trigger strong mucosal responses even in the absence of adjuvants. Using avirulent E. tarda as vehicle to express heterologous antigens might prove a viable option against microbes for which attenuated strains still show safety concerns or for which environmental avirulent strains are not available. The safety level of avirulent or attenuated E. tarda strains, together with serotype variability, certainly needs to be systematically evaluated before it can find its commercial application. Regardless of the aforementioned challenges when developing live enteric pathogens as vaccine vehicles, the large body of experimental 
work performed so far and the availability of a few commercial vaccines that can be administered by immersion, shows their great potential $[2,120]$. Similar approaches have been pursued using other enteric pathogens such as E. coli and $V$. anguillarum that were engineered to express the nervous necrosis virus (NVV) capsid protein and were delivered as inactivated vehicles through artemia (Table 2), [87,88]. Although the length of the vaccine administration as well as the duration of protection need further optimization, the principle of using enteric pathogens as oral vehicle seems very promising in fish as well.

\subsubsection{Encapsulated DNA plasmid and activation of effective mucosal responses}

As summarized in Table 2, it appears that alginate-encapsulated DNA plasmid might also fulfil most requirements for effective antigen administration at mucosal surfaces followed by activation of local immune responses. Considering their biochemical properties, and possibly those of fish gut mucus, alginates might assure plasmid protection, migration through the mucus layer, and efficient uptake by the epithelial layer. Once the plasmid enters the cytoplasm, $\mathrm{CpG}$ motives present in the plasmid backbone might deliver stimulatory signals acting as adjuvants. Once expressed by host cells, the protein antigen encoded by the plasmid can be recognized as a PAMP, activating local innate immune cells but also antigen-specific B cells or NK cells through antibody-dependent cell-mediated cytotoxicity (ADCC).

Of interest, alginate microspheres were used to successfully vaccinate brown trout and rainbow trout against IPNV using VP2-encoding DNA plasmid or against IHNV using glycoprotein $(\mathrm{G})$-encoding plasmid [89,90,121,122]. Microspheres were generated using a $\mathrm{CaCl}_{2}$ method that generally leads to relatively large particles (10$100 \mu \mathrm{m}$ or larger). This size is much bigger than the one considered to be most effectively taken up by $M$ cells in mammals [65]. This suggests that cells other than antigen-sampling M-like cells might be involved in the uptake of alginate microparticles in fish. In our laboratory, an heterogeneous suspension of alginate microparticles was generated using an alternative approach. Microspheres ranging in size between 1.6 and $9 \mu \mathrm{m}$ containing a total of $20 \mu \mathrm{g}$ of DNA plasmid encoding for the SVCV-G protein (unpublished data) were delivered by oral gavage to carp of 20 g. Immuno-histochemical analysis revealed strong $\mathrm{G}$ protein expression throughout the epithelial layer of the second gut segment at 14 days post-vaccine administration. 
Furthermore, discrete macrophage-like cells and other leukocytes in the lamina propria were also found to be strongly positive for $\mathrm{G}$ protein expression. Our results, combined with the results in trout, suggest that alginates, independently of the size, might be particularly suitable to deliver antigens at mucosal surfaces in fish. They seem to be effectively taken up or perhaps can directly fuse with the gut epithelial membrane. Such efficient antigen uptake by enterocytes was observed previously [8] but can however also pose a limitation to the efficacy of oral vaccines: on the one hand, persistence of the antigen in the enterocytes might guarantee sufficient antigen exposure and activation of local immune responses, on the other hand however it might create conditions leading to tolerance due to high local antigen dose if the antigen persists for too long. Additionally, in fish kept at $20^{\circ} \mathrm{C}$, the intestinal epithelium in the second gut segment is completely renewed within 10-15 days $[123,124]$. This time is of course temperature and fish species dependent. In zebrafish kept at $26^{\circ} \mathrm{C}$, renewal was faster (7-10 days) in the second gut segment and took 5-7 days in the first gut segment [125]. Although the renewal period is significantly longer than observed in mammals (3-4 days), the antigen might be lost unless it is efficiently transferred to leukocytes at the basal side of the epithelium, or taken up by intraepithelial macrophages. This might partly help explain why relatively high plasmid doses need to be used upon oral administration when compared to i.m. injection. Antigens might then have to be delivered repeatedly, increasing the costs of oral DNA vaccination. Furthermore, considering the importance of the antigen dose in preventing the tilting of the balance towards induction of oral tolerance, optimizing the time and dose of DNA plasmid delivery will be of utmost importance for successful oral DNA vaccination.

\subsubsection{Recombinant attenuated live vaccine vectors}

Rather than relying on live attenuated pathogens, avirulent strains, or DNA plasmids, a safer and rational attenuation of candidate vaccine pathogens would be preferred. This could circumvent risks linked to the potential reversion to virulence or legislative concerns linked to the use of DNA vaccinated animals. Attenuation can be achieved by engineering the pathogen by for example selectively deleting metabolic pathway(s) or virulence gene(s). Such approach also allows the generation of DIVA vaccines facilitating the Discrimination of Infected from Vaccinated Animals, by the addition of tags or other markers. DIVA vaccines are particularly relevant in the veterinary 
vaccine industry to allow transport and trading of vaccinated animals. As a trade-off, a great deal of knowledge needs to be gathered about the pathogen infectious cycle and genes function in order to be able to rationally modify the pathogen. For large, complex viruses such as herpesviruses, or bacteria, this can greatly delay and increase the costs of vaccine development. For pathogens containing a segmented genome, such as influenza virus, engineering of genes in one or few particular segments might not prevent resorting of genomic segments among influenza serotypes within the populations, thereby quickly diluting out and losing the vaccine strain.

Rational pathogen attenuation has been attempted in fish as well (Table 1). For example, live attenuated E. tarda mutants were generated in which the alanine racemase gene and aspartase semialdehyde dehydrogenase gene were knocked out, rendering the mutants dependent on exogenous alanine and aspartate [48]. The mutants conferred good protection upon challenge with virulent strains when administered to Olive flounder by oral gavage. Live attenuated recombinant VHSV was generated by removing the non-structural protein $\mathrm{NV}(\Delta \mathrm{NV})$ and inserting eGFP [58]. Such attenuated strain was able to confer good protection, in a dose-dependent manner, when administered orally. Despite the promising results, only few studies investigated the efficacy of recombinant attenuated vaccines for oral vaccine delivery. Still, long-term protection needs to be validated for the abovementioned studies as challenge was performed relatively shortly after last vaccination. Nonetheless, the use of enteric pathogens, like E. tarda, proves again to be a viable strategy for oral vaccine delivery.

\section{Rational vaccine design and novel approaches for the vaccines of the future}

\subsection{Live viral vectors as vaccine vehicles}

Is it therefore at all possible to generate safe live vaccines? In the last 30 years a great effort has been directed towards the identification of live viral or bacterial vectors that are not necessarily derived from the pathogen against which the vaccine is developed. For this purpose baculoviruses, lentiviruses, retroviruses, alphaviruses and adenoviruses have received great attention. All these vectors have several features in common: i) they can be modified or are naturally non-pathogenic to the species in which they will be used in; ii) they are able to deliver nucleic acids in eukaryotic cells, allowing for vaccine antigen expression by the host cell itself; iii) 
through viral pseudotyping, they can be designed to specifically target the cells to be infected; iv) they have very straightforward cloning and modification strategy.

Due to their ability to insert their genome in the host chromosome, baculoviruses, lentiviruses and retroviruses have been removed from the list of suitable vaccine vectors as they are considered unsafe. Incorporation of viral genes in a random location in the host genome can cause the onset of cancer (oncogenic insertion), or in the case of species used for human or animal consumption, the animals might be considered a Genetically Modified Organism (GMO). The latter is a problem especially linked to consumers' perception of safety; in fact, even though the foreign gene might not necessarily incorporate in the gonads of the vaccinated animals and therefore cannot be transmitted to the progeny, consumers and legislative bodies are not willing to take the risk. A very good case about how extremely successful vaccines are currently not widely used on the market, is the one about DNA vaccines in fish. As extensively discussed above, i.m. injection of DNA plasmid or oral administration of alginate-encapsulated DNA can be very effective in protecting fish against a variety of viral pathogens. Unfortunately, with the only exception of the DNA vaccine against IHN virus licensed in Canada for vaccination of salmon, no other DNA vaccine is available on the marked for edible species.

\subsubsection{Alphaviruses}

Alphaviruses are positive-sense, single-stranded RNA viruses with a genome of approximately $11.5 \mathrm{~kb}$ in length that do not insert their genome in the host chromosome. For this and for the relative simplicity and ease of manipulation of their RNA genome, they also represent interesting targets for vaccine vector development. Two types of Alphavirus-based vectors have been designed [126] but none of them have so far been approved by any regulatory agency for use in animals or humans. The first vector is based on the generation of a self-replicating RNA (replicon) originating from a DNA plasmid also containing the sequence of the gene of interest. The second vector is based on Alphavirus replicon particles (RPs) that are single-cycle, propagation-defective particles that carry RNA encoding for the gene of interest but are not able to spread beyond the initial infected cells. As discussed above, the second generation of Alphavirus RPs carrying RNA certainly have an increased safety profile with respect to DNA-based replicon RNA as it does not require delivery of DNA sequences to the host. Nonetheless, DNA-based 
salmonid alphavirus (SAV)-based replicon vaccines encoding the infectious salmon anemia virus (ISAV) hemagglutinin-esterase (HE) have been shown to be extremely effective when delivered i.m, whereas i.p. administration did not provide the same protection [127]. Intramuscular delivery triggered strong local as well as systemic innate, humoral and possibly cell-mediated responses, further supporting the suitability of alphavirus-based vectors to trigger strong immune responses. Another study also showed the broad temperature range $\left(4^{\circ} \mathrm{C}-37^{\circ} \mathrm{C}\right)$ and tropism of the SAVbased replicon as it was functional in fish, mammalian and insect cells in vitro as well as in shrimps in vivo [128]. The level of heterologous protein expression by the SAV-based replicon is however lower than observed in mammalian replicon-based systems. To date, such vectors have not been tested for oral delivery. For this reason it will be interesting to raise their safety profile by developing Alphavirus replicon particles that could deliver RNA rather than DNA. Furthermore, based on their proven ability to trigger the immune system of fish, immunity to the vaccine vector will also have to be evaluated if Alphaviruses are to be used as vaccine vehicles.

\subsubsection{Adenoviruses}

Taking into account the abovementioned safety considerations, adenoviruses are currently at the vanguard of live viral vectors for use in humans and veterinary species [129]. Adenoviruses are medium-sized, non-enveloped double-stranded DNA viruses. Adenoviruses have been isolated from all vertebrate species, including fish [122], and are known to cause mild to severe respiratory disease in warm-blooded animals and enteric/renal diseases in aquatic animals. The best characterized adenoviruses are those of mammalian and avian origin [130]. In fish, only one representative has been isolated from white sturgeon and has been temporarily assigned to a separate new genus [131]. Advantages of adenoviral vectors include their large packaging capacity $(>8 \mathrm{~kb})$, high titres and high levels of transgene expression when placed under a strong promotor. Moreover, they are able to target a broad range of dividing and non-dividing cell types with almost $100 \%$ efficiency. Unlike lentiviruses or retroviruses, adenoviruses do not integrate into the host genome which greatly increases their safety profile.

Good examples of the great success of adenoviruses as vaccine vectors are the recently developed experimental vaccines against Ebola virus [132], malaria [133,134], Respiratory Syncytia Virus (RSV) [135] Foot-and-Mouth Disease 
[136]. They are generally based on a combined vaccination regime starting with prime vaccination with a modified non-human adenovirus, followed by booster vaccination with a modified poxvirus (mostly Modified Vaccinia Ankara (MVA)). The adenovirus vector, very effectively triggers strong humoral and cell-mediated responses to the vaccine antigen but also to the adenovirus vector itself. Therefore booster vaccination with an heterologous vector is required to prevent elimination of the adenovirus-based vaccine in primed vaccinated individuals.

The discovery and pioneering work performed on mammalian and avian adenoviruses, combined with the presence of cold-blooded adenoviruses, represents a novel, unexploited strategy that could be undertaken by fish vaccinologists for future vaccine development. For this, the further characterization of the currently identified adenovirus genome, the search for additional strains infecting fish species other than white sturgeon, and the generation of tools for their genetic manipulation, will be essential to ascertain the potential of adenoviruses as effective vaccine vehicles also in fish.

\section{Improvement of currently suboptimal oral vaccines: mucosal adjuvants}

Non-replicating antigens such as soluble proteins, plasmid DNA or killed pathogens are poorly immunogenic when delivered orally. Moreover, they generally induce tolerance because of their inability to trigger appropriate inflammatory stimuli and costimulatory signals. Therefore, the use of potent mucosal adjuvants can greatly enhance the efficacy of such suboptimal vaccines. The use of novel adjuvant formulations for improvement of fish vaccine efficacy has been extensively reviewed elsewhere [70], therefore in this section we will focus on mucosal adjuvants that can specifically enhance oral vaccine efficacy.

The relative success of the few commercial oral vaccines for fish can perhaps be partly attributed to their protective encapsulation method: antigen protective vehicle (APV) by MSD or MicroMatrix ${ }^{\mathrm{TM}}$ by Centrovet [2]. Even though these vehicles might provide stimulatory signals, they are obviously not sufficient to trigger a strong memory response [33] as the oral vaccines alone are not sufficient to provide longlasting protection. These vaccines however, can be used as a base to start testing novel combinations of mucosal adjuvant-vaccine antigens. Suboptimal vaccines could be administered in combination with conventional adjuvants or molecules 
that have been shown to exert strong immunostimulatory activities also in fish and are therefore able to trigger danger signals. Examples of the latter type include enterotoxins or PAMPs such as: the non-toxic part of the Escherichia coli heat-labile enterotoxin (LTB), cholera toxin $\beta$-subunit (CTB), polyI:C, beta-glucans, bacterial flagellin or $\mathrm{CpG}$ motifs.

\subsection{Conventional adjuvants}

Some of the adjuvants generally used for injection vaccination in humans or veterinary species (i.e. alum and Freund's incomplete) have also been tested in fish in combination with oral vaccines (section 4 and Table 1). Although the reports showed the applicability of using standard injection vaccine adjuvants in an oral vaccination strategy, possible side effects need to be assessed.

Other promising adjuvants components considered in experimental human and veterinary vaccines are liposomes, saponins (e.g Quil-A, QS-21) or highly immunogenic immune stimulating complex (ISCOMS). At this moment, Pharmaq is studying the introduction of the latter adjuvants in commercialised fish vaccines [70]. Two studies already investigated the efficacy or liposomes for oral vaccination of fish (Table 2). The first study showed successful oral vaccination of carp against CyHV-3 using liposome-encapsulated formalin-inactivated (f.i) CyHV-3 $[95,96]$. The second study, showed more variable degrees of protection against $A$. hydrophila using liposome-encapsulated f.i. A. hydrophila [82]. In both cases, long term protection and the underlining protective mechanisms have not been investigated in details. The saponing Quil-A was found to increase systemic antibody levels against the model antigen Human Gamma Globulin (HGG) after oral delivery to Mozambique tilapia but protection after challenge was not assessed [137].

Additional adjuvants that are included in licensed human and veterinary vaccines and can be used for both, parenteral or mucosal administration include: MF59 ${ }^{\circledR}$ (oil-in-water emulsion), a squalene-based adjuvant system $03\left(\mathrm{ASO}^{\circledR}\right)$ and $\mathrm{AS}^{\circledR} 4^{\circledR}$ (monophosphoryl lipid A (MPL-A) + alum) [138]. They all have been shown to effectively induce humoral as well as cell-mediated responses to various degrees, at least when administered via the parenteral route. Squalene is a cholesterol precursor that is added to adjuvant emulsions (i.e. MF59 and AS03) and was shown to enhanced antigen uptake by DCs. Local reactions and its persistence in oil residues however, drive the demand for alternatives. Non-toxic MPL-A, derived from LPS, is a TLR4- 
targeting adjuvant that was shown to greatly enhance APCs activity as well as T and B cell memory responses. Muramyl dipeptide (MDP) present on bacterial cell walls is currently included in various experimental formulation and, similarly to MPL-A, is a strong activator of APCs and of cellular responses. To date, none of the abovementioned licensed adjuvants or their components have been tested for oral vaccine delivery in fish. Considering their full characterization, safety profile, and most of all, their ability to induce humoral and cell-mediated responses, the aforementioned adjuvants or their components, represent promising novel tools to further improve currently insufficient oral vaccines.

\subsection{Enterotoxins as strong mucosal adjuvants}

To improve delivery and uptake of antigens to the hindgut, antigens can be fused to enteric carrier molecules that also retain the ability to induce strong inflammatory signals. For example, fusion of Green Fluorescent Protein (GFP) to LTB (LTBGFP) led to increased GFP uptake in the carp mucosa and enhanced GFP-specific serum antibody levels. While almost no GFP was detected after non-LTB-fused GFP administration, high levels of GFP were detected in enterocytes and macrophagelike cells after delivery of LTB-GFP [62]. In earlier studies, the enteric protein CTB was found to increase systemic antibody levels against the model antigen Human Gamma Globulin (HGG) after oral delivery to Mozambique tilapia [139]. Enterotoxins hold great promise as mucosal adjuvants as very little amounts have been shown to trigger strong mucosal responses in humans and in veterinary species, including fish. Modified subunits have been developed that retain their immunostimulatory capacity while limiting the exacerbated inflammatory response $[140,141]$. These toxins are effective as mucosal adjuvants, presumably because they retain the ability to penetrate the mucus layer, are taken up by $\mathrm{M}$ cells or mucosal DCs, and ultimately activate key innate signalling pathways. Activated DCs in turn orchestrate adaptive immune responses that are appropriate for defence against live pathogens. Given the already ascertained ability of these molecules to enhance uptake by intraepithelial macrophages, it will be interesting to evaluate their possible uptake by the recently described fish M-like antigen-sampling cells and follow their faith within the vaccinated animal. This information might certainly contribute to a better understanding of the protective mechanisms that need to be triggered to elicit protective mucosal responses. 
Perhaps acting in a similar manner as enterotoxins, a new microparticle-based oral adjuvant containing LPS was described. In this study, LPS from meningococcus bacteria was found to significantly induce IgM production and protection against $A$. hydrophila in African catfish after oral administration [142]. LPS molecules, similarly to enterotoxins, might in fact retain the ability to trigger strong local responses and enhance the efficacy of suboptimal inactivated vaccines.

As discussed previously, not only enterotoxins but also enteric pathogens themselves (e.g. E. tarda, V. anguillarum, E. coli, Y. ruckeri), either as live vehicles or as inactivated pathogens, can provide adequate stimulatory signals and act as adjuvants for currently suboptimal oral vaccine formulations.

\subsection{Molecular adjuvants}

Although studies show the potential of using molecular adjuvants such as DNA plasmid-encoded molecules or RNA-based vectors, their use in oral vaccination for fish is very limited. For example, Alphavirus replicon vaccines have been widely used in mammalian vaccines and are known to activate the innate and adaptive immune system at various levels. This approach was also used in an oral DNA vaccine for Atlantic salmon against Infectious Salmon Anaemia Virus (ISAV). It was observed that addition of the alphavirus replicon DNA sequence significantly increased protection upon ISAV challenge when compared to the oral DNA vaccine without the molecular adjuvant [93]. The large number of dsRNA sequences accumulated upon delivery of the Alphavirus replicon might contribute to the observed adjuvant effect.

A chitosan-encapsulated DNA vector encoding heat shock protein 70 (hsp70) of the protozoan Cryptocaryon irritans (C. irritans) was found to significantly increase survival of orange spotted grouper when administered orally along with a DNA plasmid encoding the immobilization antigen (iAg) of C. irritans. This study not only showed that oral DNA vaccination is also effective against extracellular parasites, but also that heat shock proteins can act as molecular adjuvants when administered orally [143].

Several cytokines and chemokines have been tested as molecular adjuvants in injected DNA vaccines [70] For example, strong adjuvant effects have been described for ifna, ifnb and ifnc in Atlantic salmon that were vaccinated with a suboptimal DNA vaccine against ISAV. Besides increased protection, significantly higher antibody levels and 
expression of several B- and T-cell markers was found in the muscle, indicating an interferon-induced influx of leukocytes to the site of injection [144]. Other cytokines such as II 8 and Il1 $\beta$ (peptides) have also been used but never in combination with oral vaccine delivery. Given the necessity to trigger strong inflammatory signals that are able to overcome the high tolerogenic threshold of the gut environment, cytokines such as I112, Ifn $\gamma$ or Tnfo could also be used alone or in combination. Most of these cytokine induce activation of APCs by triggering the upregulation of pro-inflammatory cytokines, MHC-II and CD80/86 co-stimulatory molecules. For this reason, they could be used in combination with inactivated/killed pathogens that alone trigger little activation of APCs.

\subsection{PAMPs as mucosal adjuvants}

Great advances have been made in fish research in trying to identify and characterize several Pattern Recognition Receptors (PRRs), scavenger receptors, C-type lectins etc, because these in fact, represent the targets for rational adjuvant design. In fish, PAMPs such as bacterial flagellin (targeting TLR5) or CpG motives (potentially targeting TLR9), have been shown to exert strong immunostimulatory activities and to have adjuvant activities when administered with suboptimal parenteral vaccines, as reviewed in [70]. Flagellin, especially from enteric pathogens (i.e. Vibrio ssp, Salmonella ssp), holds great promise. In fact, similar to enterotoxins, it has the ability to withstand the gastric environment and the potential to target receptors on leukocytes at mucosal surfaces, hereby triggering the necessary costimulatory signals on APCs. CpG motives, either incorporated in the plasmid backbone of DNA vaccines or administered along with antigens, have been shown to have significant immunostimulatory activities. Their effects are, however, sequence- and speciesdependent [145] suggesting that the choice of CpG motives has to be tailored to the fish species. This implicates that, when administered to different fish, the same vaccine antigen might have to be combined to different $\mathrm{CpGs}$ in order to exert its adjuvant effect. Despite their extensive characterization in vitro, and in vivo upon parenteral administration of antigens, the use of both flagellin and CpGs as mucosal adjuvants awaits further characterization.

Among the most promising immunostimulants that could be used as mucosal adjuvant in oral vaccine formulations, beta-glucans are perhaps the best known and characterized [146-151] (REF). Beta-glucans are easily incorporated into the 
fish standard feed, making them suitable candidate adjuvants for oral vaccination. Adjuvant activities of beta-glucans have been demonstrated in fish most often in combination with i.p. vaccination against bacterial diseases [152,153]. Their potential in oral vaccination, however, has not yet been investigated.

In addition to the use as an immunostimulant, beta-glucans can also function as a vehicle to encapsulate and protect antigens. Although this has not yet been exploited for fish vaccines, sub-cutaneous injection of mice with OVA-loaded beta-glucan particles (GPs) induced an increased $\mathrm{CD}^{+}{ }^{+} \mathrm{T}$-cell proliferation when compared to mice injected with OVA absorbed to the adjuvant alum [154]. The potential use of GPs in oral vaccination was investigated using human intestinal cell lines (Caco-2 and HT-29) and mice [155]. It was found that in vitro GP-OVA complexes were internalized by Caco-2 and HT-29 cells and that the complexes did not affect cell viability. Internalization induced an increased expression of $i l 23 p 19$, ils, and a downregulation of $\operatorname{tg} f \beta$. The above mentioned results show the adjuvant potential of GPs as vaccine vehicle but future research is necessary to test their applicability for fish vaccines.

Another well-known and widely used adjuvant is polyinosinic:polycytidylic acid (poly I:C), which has been used in multiple fish vaccination studies by injection vaccination but not yet as an mucosal adjuvant. In mammals, poly I:C is most often delivered in nano- or microspheres because of its vulnerability to serum nucleases and because high doses of systemic poly I:C are toxic and can induce autoimmunity [156]. In fish, co-delivery of chitosan-encapsulated poly I:C and inactivated whole VHSV by i.p. injection was found to induce significant protection in zebrafish against a challenge with VHSV. However, there was no significant difference in survival between groups vaccinated with or without poly I:C as adjuvant [157]. Similar protective effects were found when zebrafish were co-vaccinated with the VHSV-G protein and poly I;C (Kavaliauskis, manuscript in preparation). Poly I:C can also be combined with other adjuvants to further enhance its immunostimulating properties. Combinations of poly I:C and $\mathrm{CpG}$ were found to increase protection in Atlantic salmon against SAV when combined with i.p. injection of an inactivated SAV vaccine $[158,159]$. Altogether, the above studies show the potency of poly I:C as an adjuvant for fish vaccines. However, its use as an adjuvant for oral vaccination is yet to be evaluated. 


\subsection{Plant-based proteins as immunostimulants to break mucosal tolerance}

Plant-based proteins, including soy bean meal and concentrates thereof, are becoming increasingly important as protein source to reduce fishmeal content. However, it is known that the substitution of fishmeal with soy bean meal may have adverse effects such as induction of intestinal lesions and subacute enteritis [160,161]. Enteritis leads to a widening of the lamina propria and a subsequent influx of immune cells. The influx of immune cells interferes with the barrier function of the intestine and increases the ease of pathogens or antigens to pass the intestinal barrier [162]. While intestinal lesions and severe enteritis needs to be prevented, soy bean meal might be used as a feed additive to increase vaccine antigen uptake by increasing permeability of the intestinal wall. This possibility was evaluated in rainbow trout orally vaccinated with PLGA nanospheres containing a DNA vaccine against IHNV. Fish were fed diets either containing $35 \%$ soy bean meal or $35 \%$ soy bean concentrate but no significant differences were found in nanoparticle uptake or survival when being challenged ten weeks after vaccination [97]. Since severe enteritis induced by high soy bean meal concentration leads to endocytosis block rather than an increase in uptake in a species-specific manner, care should be taken to find an optimal low dose for every fish species and size [163].

Altogether, a rational selection of enteric (live) vectors or encapsulation methods might aid the development of new effective, and the improvement of current suboptimal, oral vaccines for fish. Moreover, it will certainly provide a matrix of tools when combined with a more targeted selection of mucosal adjuvants based on their immunostimulatory ability and affinity for the gut environment.

\section{Consideration on the nature of the fish and its environment}

Oral vaccine development in fish might present an additional degree of complexity when considering the vast number of cultured fish species and the diversity within. Therefore, different fish species may require a different strategy for the development of oral vaccines, even against the same pathogen. Major differences in gut morphology as well as intestinal environment can be found between stomachless fish and fish with a stomach, and between carnivorous, herbivorous and omnivorous fish species. Oral vaccination of fish that do not have a stomach may require less protection of the antigen since they are not exposed to the harsh environment and low $\mathrm{pH}$ of the 
stomach. However, factors others than stomach $\mathrm{pH}$ are involved and even within the group of fish that possess a stomach, large differences exist. For example, fish with a thin stomach wall, like rainbow trout, have high stomach acidity because they are more dependent on $\mathrm{pH}$ than on muscle strength for food kneading and breakdown. This is in contrast to fish with a thick muscular stomach wall, like African catfish, that are better able to knead food and are therefore less dependent on stomach $\mathrm{pH}$ for food breakdown [164].

Carnivorous fish species have higher protease activity compared to herbivorous and omnivorous species. Non-carnivorous fish might have high $\alpha$-amylase activity, since plant materials are difficult to digest. It is important to gain knowledge on the fish intestinal tract and its environment to make an estimate of the level of stress that the vaccine will encounter. Depending on the estimated degree and source of breakdown, an antigen protection or encapsulation technique can be chosen or designed. Besides feeding preferences, differences in digestion enzymes and intestinal environment are caused by several other factors including age of the fish, temperature and season $[165,166]$.

In humans, success or failure of mucosal vaccines in Latin American, European, American individuals versus African patients has been largely ascribed to differences in microbial compositions [167]. In mice it has been shown that gut microbiota largely influences the development of an healthy mucosal immune system and most importantly that oral tolerance could not be induced in the absence of signals derived from the gut flora [168-172]. Studies have demonstrated that also in zebrafish the composition the gut microbiota plays a crucial role of in the onset of enterocolitis [173] and recent reviews have summarised how also in teleost fish a delicate arm race between the host, commensals and pathogens is taking place at mucosal surfaces [174]. Therefore, considering the immense heterogeneity of fish species, their environment and eating habits, a careful consideration of the enterotypes (gut microbiota community profiles) of aquaculture species should also be taken into account when developing oral vaccines.

While antigen coating or encapsulation can be the key to ensure sufficient antigen delivery to the hindgut, the biochemical properties of the encapsulation vehicle can strongly influence the degree of uptake and antigen release. Consequently, choosing a suboptimal antigen coating or encapsulation system can have detrimental effects on the efficacy of the delivered vaccine. As an example, oral vaccination of 
rainbow trout against IHNV using PLGA-microencapsulated DNA vaccine resulted in low protection, while uptake of the microspheres was significant. This apparent discrepancy was found to be caused by the property of PLGA to not dissolve efficiently at 14 degrees, the water temperature at which vaccination was performed [97]. This study shows the importance of choosing an encapsulation method whose characteristics match with the environment of the fish.

Last but not least, in experimental vaccination and challenge experiments of fish, temperature might be a key element determining the level of success. It is commonly known that the fish immune system is rather slow compared to mammals, especially that of fish living in cold environments. Besides temperature, changes in water type and culturing conditions will also play a role as for example salmon having to move from fresh to salt water conditions and from tanks to sea cages. Only few studies systematically addressed the effect of temperature on vaccine efficacy. For example, vaccination of coho salmon against $V$. anguillarum at temperatures ranging from 3.9 and 20.6 degrees did not lead to significant differences in vaccine efficacy as all groups showed very good protection [175]. On the contrary, a temperature-dependent effect was observed on the protective innate and adaptive mechanisms induced upon i.m. DNA vaccination of rainbow trout against VHSV. While the vaccine protected the fish well at temperatures of 5,10 and 15 degrees, no neutralizing antibodies and a delayed $m \times 3$ expression were observed only in fish vaccinated at 5 degrees [176]. Altogether, given the vast diversity of fish species, heterogeneity of their environment and culturing conditions, testing the vaccine in the target species and under field conditions will prove to be crucial to finally validate vaccine efficacy.

\section{The zebrafish as animal model for aquaculture animals}

Vaccine validation should certainly be performed in the species of interest and under conditions that best resemble the natural rearing conditions and environment. Still, the use of fish models might help speed up part of the process linked to the characterization of the vaccine and of the immune response of the host. In the past 20 years, the small zebrafish has managed to climb the pyramid of animal models commonly used for biomedical research in humans. Despite its established reputation in the biomedical field, zebrafish has been largely underestimated and poorly used as a model in the aquaculture field. The availability of numerous transgenic fish lines, including those specifically marking $\operatorname{IgM}+\mathrm{B}$ cells, $\mathrm{T}$ cells, macrophages, or 
neutrophils, creates an unique opportunity to investigate the real-time kinetics of cell recruitment, proliferation and migration in response to specific vaccine antigens. Double transgenic and reporter fish lines for specific cytokines or chemokines are also available. These lines might help elucidate which cell types express which molecules, when and where, in response to antigens or adjuvants. This type of analysis is in general rarely possible in most fish species due to the scarcity of antibodies.

The use of transgenic fish lines in combination with labelled antigens might help predict the faith of for example orally delivered antigens and the relative contribution of specific cell types in the uptake, presentation and activation of the immune response. A detailed review of zebrafish gut physiology and its potential use as a model to study intestinal responses is reviewed elsewhere in this issue (Brugman, 2016). Fluorescently labelled inactivated or live vectors could be easily traceable in zebrafish. Delivery and uptake of (encapsulated) antigens could be monitored in all gut segments and suitability of various encapsulation methods, based on size or stability, could be performed in real-time. Chemical ablation of specific cell types is also possible in zebrafish [177], allowing the determination of the role of specific leukocytes in the response to the vaccine. For example, the ultimate proof that M-like sampling cells transport and deliver antigens to leukocytes in the lamina propria could come from the generation of novel transgenic zebrafish lines, as soon as M-like cell markers are identified in fish. Besides the availability of transgenic lines, several mutants are also available. For example, I11 $0^{-/-}$knockout zebrafish are available and could be used to investigate the role of 1110 in the onset or maintenance of gut tolerance. Furthermore, live imaging is not only limited to the transparent larval stages, but with the combined use of for example two-photon microscopy and casper mutant fish that lack pigmentation, it can be extended to the juvenile and adult fish as well.

Despite the many advantages of the zebrafish model, including the ones mentioned above, the use of zebrafish as a model for aquaculture species poses some limitations. Zebrafish is a cyprinid fish living in fresh waters at an optimal temperature of 27 degrees and is a stomachless, omnivorous fish. Due to these characteristics, the zebrafish is certainly a suitable pre-screening model for cyprinids and some fresh water fish. In contrary, it might be less suitable for other commercially relevant species, including salmon, trout, turbot, seabream, or sea bass. As discussed above, the microbiota plays an extremely crucial role in influencing gut development, 
homeostasis as well as induction of tolerance. As a consequence the microbiota and the gut environment will be extremely different between omnivores and carnivores. Nevertheless, zebrafish can provide the proof of principle of the validity of novel approaches and may help accelerate the selection of antigens, adjuvant, vaccine vehicles or encapsulation methods, which without doubt, will ultimately have to be validated in the species of interest.

\section{Concluding remarks}

Based on the status of oral vaccines in humans and veterinary species, there is no doubt that the generation of safe and efficacious oral vaccines is among one of the most difficult tasks of immunologists. This is illustrated by the very limited number or oral vaccines approved for use in humans and the slightly larger number approved for use in poultry, pigs and cattle. In this respect fish are not lagging much behind, with 5 oral vaccines available on the market. These vaccines however, are against only a very limited number of pathogens and are available for an even smaller number of fish species. When considering the vast diversity of cultured fish species and their pathogens, the current oral vaccines are by far insufficient to fulfil the market requirements. Such species diversity is in fact larger than the diversity in species and pathogens faced by for example the poultry or cattle vaccine industry.

Nonetheless, fish oral vaccine development can greatly profit from the progress made on human and veterinary oral vaccines. For example, the use of live vectors, e.g adenoviruses, or a more rational attenuation of enteric pathogens, e.g E. tarda or $V$. anguillarum, as well as the combination of weak oral antigens with strong mucosal adjuvants, e.g enterotoxins, leaves a vast number of combinations that have not been fully exploited in fish vaccine development. Fish mucosal immunology, despite the large body of work performed in the last 30 years, is still in its infancy, mostly due to the great heterogeneity in teleost species. Nevertheless, the discovery of new players in fish mucosal immunity within the last 5-10 years, including IgT or M-like sampling cells, keeps the field of mucosal immunology and vaccinology a dynamic and developing area. The possibility to specifically target M-like cells or putative APCs in the fish gut is becoming a viable option in fish vaccine delivery as well. This is being realized through the great advances in gene discovery and the several genome sequencing initiatives for several fish species and their pathogens. The bottleneck will of course be the functional characterization of most of the novel 
genes and the translation of this fundamental knowledge into practical applications linked to vaccine development. Molecular traceable and genetically modifiable models such as transgenic or mutant zebrafish can support and accelerate fish vaccine development as much as other animal models have helped the human and veterinary field. Information on host mucosal responses, together with insights in how fish gut microbiota might influence the response to oral vaccination is increasing at a rapid paste. This information will be essential to design strategies aimed at breaking mucosal tolerance while preventing inflammation for a greater variety of fish species. Finally, collaborations between academia, industrial partners and farmers will be instrumental to produce safe and efficacious vaccines for most commercially relevant fish species.

\section{Acknowledgements}

This research was partly funded by the European Commission under the $7^{\text {th }}$ Framework Programme for Research and Technological Development (FP7) of the European Union (Grant Agreement 311993 TARGETFISH) and by the Netherlands Organisation for Scientific Research (NWO) under Veni project number 11200.

\section{References}

1. FAO. The state of world fisheries and aquaculture. Rome (2014). doi:92-5-105177-1

2. Brudeseth BE, Wiulsrød R, Fredriksen BN, Lindmo K, Løkling K-EE, Bordevik M, Steine N, Klevan A, Gravningen K. Status and future perspectives of vaccines for industrialised finfish farming. Fish Shellfish Immunol (2013) 35:1759-1768. doi:10.1016/j.fsi.2013.05.029

3. Duff DCB. The oralimmunization of trout against Bacterium salmonicida. J Immunol (1942) 44:87-94. Available at: http://www.jimmunol.org/content/44/1/87

4. Fujino Y, Ono S-I, Nagai A. Studies on uptake of rabbit's immunoglobulin into the columnar epithelial cells in the gut of rainbow trout Salmo gairdneri. Nippon Suisan Gakkaishi (1987) 53:367-370.

5. Georgopoulou U, Sire MF, Vernier JM. Immunological demonstration of intestinal absorption and digestion of protein macromolecules in the trout (Salmo gairdneri). Cell Tissue Res (1986) 245:387-395.

6. Iida H, Yamamoto T. Intracellular transport of horseradish peroxidase in the absorptive cells of goldfish hindgut in vitro, with special reference to the cytoplasmic tubules. Cell Tissue Res (1985)553-560.

7. Noaillac-Depeyre J, Gas N. Absorption of protein macromolecules by the enterocytes of the carp (Cyprinus carpio L.). Ultrastructural and cytochemical study. Z Zellforsch Mikrosk Anat (1973) 146:525-541. doi:10.1007/BF02347181

8. Rombout JHWM, van den Berg AA. Immunological importance of the second gut segment of carp. I. Uptake and processing of antigens by epithelial cells and macrophages. J Fish Biol 
(1989) 35:13-22. doi:10.1111/j.1095-8649.1989.tb03388.x

9. Rombout JHWM, Bot HE, Taverne-Thiele JJ. Immunological importance of the second gut segment of carp.. II. Characterization of mucosal leucocytes. J Fish Biol (1989) 35:167-178. doi:10.1111/j.1095-8649.1989.tb02966.x

10. Stroband HWJ, Kroon A. The development of the stomach in Clarias lazera and the intestinal absorption of protein macromolecules. Cell Tissue Res (1981) 215: doi:10.1007/BF00239123

11. Johnson KA, Amend DF. Efficacy of Vibrio anguillarum and Yersinia ruckeri bacterins applied by oral and anal intubation of salmonids. J fish Dis Oxford (1983) 6:473-476. doi:10.1111/j.1365-2761.1983.tb00101.x

12. Rombout JWHM, Blok LJ, Lamers CHJ, Egberts E, Immunology C. Immunization of carp (Cyprinus carpio) with a Vibrio anguillarum bacterin: Indications for a common mucosal immune system. Dev Comp Immunol (1986) 10:341-351. doi:10.1016/0145$305 \times(86) 90024-8$

13. Joosten PHMH, Engelsma MYY, van der Zee MDD, Rombout JHWMH. Induction of oral tolerance in carp (Cyprinus carpio L.) after feeding protein antigens. Vet Immunol Immunopathol (1997) 60:187-196. Available at: http://www.sciencedirect.com/science/ article/pii/S0165242797001244 [Accessed December 4, 2015]

14. Maurice S, Nussinovitch A, Jaffe N, Shoseyov O, Gertler A. Oral immunization of Carassius auratus with modified recombinant A-layer proteins entrapped in alginate beads. Vaccine (2004) 23:450-459. doi:10.1016/j.vaccine.2004.06.022

15. Rombout JHWM, Kiron V. "Mucosal Vaccination of Fish," in Fish Vaccination (John Wiley \& Sons Ltd.), 56-67.

16. Rombout JHWM, Berg AA van den, Witte P, Egberts E. Immunological importance of the second gut segment of carp . 111. Systemic and / or mucosal immune responses after immunization with soluble or particulate antigen. (1989)179-186.

17. Faria AMC, Weiner HL. Oral tolerance. Immunol Rev (2005) 206:232-259. doi:10.1111/ j.0105-2896.2005.00280.x

18. Weiner HL, Wu HY. Oral tolerance. Immunol Res (2011) 241:241-259. doi:10.1385/ IR:28:3:265

19. Fujkuyama Y, Tokuhara D, Kataoka K, Gilbert RS, McGhee JR, Yuki Y, Kiyono H, Fujihashi $\mathrm{K}$. Novel vaccine development strategies for inducing mucosal immunity. Expert Rev Vaccines (2012) 11:367-79. doi:10.1586/erv.11.196

20. Neutra MR, Kozlowski PA. Mucosal vaccines: the promise and the challenge. Nat Rev Immunol (2006) 6:148-158. doi:10.1038/nri1777

21. Fuglem B, Jirillo E, Bjerkås I, Kiyono H, Nochi T, Yuki Y, Raida M, Fischer U, Koppang EO. Antigen-sampling cells in the salmonid intestinal epithelium. Dev Comp Immunol (2010) 34:768-774. doi:10.1016/j.dci.2010.02.007

22. Böhles N, Busch K, Hensel M. Vaccines against human diarrheal pathogens: current status and perspectives. Hum Vaccin Immunother (2014) 10:1522-35. doi:10.4161/hv.29241

23. Lamichhane A, Azegamia T, Kiyonoa H, Azegami T, Kiyono H. The mucosal immune system for vaccine development. Vaccine (2014) 32:6711-23. doi:10.1016/j. vaccine.2014.08.089

24. Meeusen ENT, Walker J, Peters A, Pastoret P-P, Jungersen G. Current status of veterinary vaccines. Clin Microbiol Rev (2007) 20:489-510, table of contents. doi:10.1128/ CMR.00005-07

25. Gerdts V, Mutwiri GK, Tikoo SK, Babiuk LA. Mucosal delivery of vaccines in domestic animals. Vet Res 37:487-510. doi:10.1051/vetres:2006012

26. Dhar AK, Manna SK, Thomas Allnutt FC. Viral vaccines for farmed finfish. Virusdisease (2014) 25:1-17. Available at: http://www.pubmedcentral.nih.gov/articlerender. fcgi?artid=3889245\& tool=pmcentrez\&rendertype $=$ abstract $[$ Accessed October 15, 2015]

27. Sommerset I, Krossøy B, Biering E, Frost P. Vaccines for fish in aquaculture. Expert Rev Vaccines (2005) 4:89-101. doi:10.1586/14760584.4.1.89 
28. Jones BBD, Ghori N, Falkow S. Salmonella typhlrnurium Initiates Murine Infection by nPenetrating and Destroying the Specialized EpitheliallnM Cells of the Peyer's Patches. $J$ Exp Med (1994) 180:

29. Sicinski P, Rowinski J, Warchol JB, Jarzabek Z, Gut W, Szczygiel B, Bielecki K, Koch G. Poliovirus type 1 enters the human host through intestinal M cells. Gastroenterology (1990) 98:56-58. Available at: http://www.ncbi.nlm.nih.gov/pubmed/2152776

30. WHO. Adverse events following Oral Poliovirus Vaccine. Inf sheet Obs rate vaccine React Polio vaccines (2014)1-5. Available at: http://www.who.int/vaccine_safety/initiative/tools/ polio_vaccine_rates_information_sheet.pdf

31. Read AF, Baigent SJ, Powers C, Kgosana LB, Blackwell L, Smith LP, Kennedy DA, Walkden-Brown SW, Nair VK. Imperfect Vaccination Can Enhance the Transmission of Highly Virulent Pathogens. PLoS Biol (2015) 13:e1002198. doi:10.1371/journal. pbio. 1002198

32. Plant KP, LaPatra SE. Advances in fish vaccine delivery. Dev Comp Immunol (2011) 35:1256-1262. doi:10.1016/j.dci.2011.03.007

33. Tobar I, Arancibia S, Torres C, Vera V, Soto P, Carrasco C, Alvarado M, Neira E, Arcos S, Tobar J a. Successive Oral Immunizations Against Piscirickettsia Salmonis and Infectious Salmon Anemia Virus are Required to Maintain a Long-Term Protection in Farmed Salmonids. Front Immunol (2015) 6:1-7. doi:10.3389/fimmu.2015.00244

34. Agius C, Horne MT, Ward, PD.Immunization of rainbow trout, Salmo gairdneri Richardson, against vibriosis: comparison of an extract antigen with whole cell bacterins by oral and intraperitoneal routes. J Fish Dis (1983) 6:129-134. doi:10.1111/j.1365-2761.1983. tb00060.x

35. Firdaus-Nawi M, Yusoff SM, Yusof H, Abdullah SZ, Zamri-Saad M. Efficacy of feed-based adjuvant vaccine against Streptococcus agalactiae in Oreochromis spp. in Malaysia. Aquac Res (2013) 45:87-96. doi:10.1111/j.1365-2109.2012.03207.x

36. Liew PS, Hair-Bejo M. Farming of Plant-Based Veterinary Vaccines and Their Applications for Disease Prevention in Animals. Adv Virol (2015) 2015:1-12. doi:10.1155/2015/936940

37. Nochi T, Takagi H, Yuki Y, Yang L, Masumura T, Mejima M, Nakanishi U, Matsumura A, Uozumi A, Hiroi T, et al. Rice-based mucosal vaccine as a global strategy for cold-chain- and needle-free vaccination. Proc Natl Acad Sci U S A (2007) 104:10986-10991. doi:10.1073/ pnas.0703766104

38. Shin YJ, Kwon TH, Seo JY, Kim TJ. Oral immunization of fish against iridovirus infection using recombinant antigen produced from rice callus. Vaccine (2013) 31:5210-5215. doi:10.1016/j.vaccine.2013.08.085

39. Wi GR, Hwang JY, Kwon M-G, Kim HJ, Kang HA, Kim H-J. Protective immunity against nervous necrosis virus in convict grouper Epinephelus septemfasciatus following vaccination with virus-like particles produced in yeast Saccharomyces cerevisiae. Vet Microbiol (2015) 177:214-218. doi:10.1016/j.vetmic.2015.02.021

40. Azad I., Shankar K., Mohan C., Kalita B. Biofilm vaccine of Aeromonas hydrophilastandardization of dose and duration for oral vaccination of carps. Fish Shellfish Immunol (1999) 9:519-528. doi:10.1006/fsim.1998.0206

41. Bakopoulos V, Volpatti D, Gusmani L, Galeotti M, Adams A, Dimitriadis GJ. Vaccination trials of sea bass, Dicentrarchus labrax (L.), against Photobacterium damsela subsp. piscicida, using novel vaccine mixtures. J Fish Dis (2003) 26:77-90. doi:10.1046/j.13652761.2003.00438.x

42. Bowden TJ, Menoyo-Luque D, Bricknell IR, Wergeland H. Efficacy of different administration routes for vaccination against Vibrio anguillarum in Atlantic halibut (Hippoglossus hippoglossus L.). Fish Shellfish Immunol (2002) 12:283-285. doi:10.1006/ fsim.2001.0386

43. Nayak DK, Asha a., Shankar KM, Mohan C V. Evaluation of biofilm of Aeromonas hydrophila for oral vaccination of Clarias batrachus - A carnivore model. Fish Shellfish 
Immunol (2004) 16:613-619. doi:10.1016/j.fsi.2003.09.012

44. Siriyappagouder P, Shankar KM, Naveen Kumar BT, Patil R, Byadgi O V. Evaluation of biofilm of Aeromonas hydrophila for oral vaccination of Channa striatus. Fish Shellfish Immunol (2014) 41:581-585. doi:10.1016/j.fsi.2014.09.021

45. Rombout JHWM, Berg a a V a NDEN, Witte P, Egberts E. Immunological importance of the second gut segment of carp . 111. Systemic and / or mucosal immune responses after immunization with soluble or particulate antigen. (1989)179-186.

46. Tu FP, Chu WH, Zhuang XY, Lu CP. Effect of oral immunization with Aeromonas hydrophila ghosts on protection against experimental fish infection. Lett Appl Microbiol (2010) 50:13-17. doi:10.1111/j.1472-765X.2009.02746.x

47. Anuradha K, Foo HL, Mariana NS, Loh TC, Yusoff K, Hassan MD, Sasan H, Raha a. R. Live recombinant Lactococcus lactis vaccine expressing aerolysin genes D1 and D4 for protection against Aeromonas hydrophila in tilapia (Oreochromis niloticus). J Appl Microbiol (2010) 109:1632-1642. doi:10.1111/j.1365-2672.2010.04789.x

48. Choi SH, Kim MS, Kim KH. Protection of olive flounder (Paralichthys olivaceus) against Edwardsiella tarda infection by oral administration of auxotrophic mutant E. $\operatorname{tarda}(\Delta$ alr $\Delta$ asd E. tarda). Aquaculture (2011) 317:48-52. doi:10.1016/j.aquaculture.2011.04.021

49. Wise DJ, Greenway TE, Byars TS, Griffin MJ, Khoo LH. Oral vaccination of Channel catfish against Enteric Septicemia of Catfish using a live attenuated Edwardsiella ictaluri isolate. $J$ Aquat Anim Health (2015) 27:135-143. doi:10.1080/08997659.2015.1032440

50. Kondo M, Kawai K, Okabe M, Nakano N, Oshima SI. Efficacy of oral vaccine against bacterial coldwater disease in ayu Plecoglossus altivelis. Dis Aquat Organ (2003) 55:261264. doi:10.3354/dao055261

51. Aoki M, Kondo M, Nakatsuka Y, Kawai K, Oshima SI. Stationary phase culture supernatant containing membrane vesicles induced immunity to rainbow trout Oncorhynchus mykiss fry syndrome. Vaccine (2007) 25:561-569. doi:10.1016/j.vaccine.2006.07.047

52. Huang LY, Wang KY, Xiao D, Chen DF, Geng Y, Wang J, He Y, Wang EL, Huang JL, Xiao GY. Safety and immunogenicity of an oral DNA vaccine encoding Sip of Streptococcus agalactiae from Nile tilapia Oreochromis niloticus delivered by live attenuated Salmonella typhimurium. Fish Shellfish Immunol (2014) 38:34-41. doi:10.1016/j.fsi.2014.02.017

53. Li LP, Wang R, Liang WW, Huang T, Huang Y, Luo FG, Lei AY, Chen M, Gan X. Development of live attenuated Streptococcus agalactiae vaccine for tilapia via continuous passage in vitro. Fish Shellfish Immunol (2015) 45:955-963. doi:10.1016/j.fsi.2015.06.014

54. Nur-Nazifah M, Sabri MY, Siti-Zahrah a. Development and efficacy of feed-based recombinant vaccine encoding the cell wall surface anchor family protein of Streptococcus agalactiae against streptococcosis in Oreochromis sp. Fish Shellfish Immunol (2014) 37:193200. doi:10.1016/j.fsi.2014.01.011

55. Shoemaker C a., Vandenberg GW, Désormeaux A, Klesius PH, Evans JJ. Efficacy of a Streptococcus iniae modified bacterin delivered using Oralject ${ }^{\mathrm{TM}}$ technology in Nile tilapia (Oreochromis niloticus). Aquaculture (2006) 255:151-156. doi:10.1016/j. aquaculture.2005.12.016

56. Villumsen KR, Neumann L, Ohtani M, Strøm HK, Raida MK. Oral and anal vaccination confers full protection against Enteric Redmouth Disease (ERM) in rainbow trout. PLoS One (2014) 9:e93845. doi:10.1371/journal.pone.0093845

57. Adelmann M, Köllner B, Bergmann SM, Fischer U, Lange B, Weitschies W, Enzmann PJ, Fichtner D. Development of an oral vaccine for immunisation of rainbow trout (Oncorhynchus mykiss) against viral haemorrhagic septicaemia. Vaccine (2008) 26:837-844. doi:10.1016/j.vaccine.2007.11.065

58. Kim MS, Kim DS, Kim KH. Oral immunization of olive flounder (Paralichthys olivaceus) with recombinant live viral hemorrhagic septicemia virus (VHSV) induces protection against VHSV infection. Fish Shellfish Immunol (2011) 31:212-216. doi:10.1016/j.fsi.2011.05.003

59. Lu L, Xu H, He Y, Li J. Protection of grass carp, Ctenopharyngon idellus (Valenciennes), 
through oral administration of a subunit vaccine against reovirus. J Fish Dis (2011) 34:939942. doi:10.1111/j.1365-2761.2011.01310.x

60. Cui L-C, Guan X-TX-T, Liu Z-MZ-M, Tian C-YC-Y, Xu YGY-G. Recombinant lactobacillus expressing $\mathrm{G}$ protein of spring viremia of carp virus (SVCV) combined with ORF81 protein of koi herpesvirus (KHV): A promising way to induce protective immunity against SVCV and KHV infection in cyprinid fish via oral vaccination. Vaccine (2015) 33: doi:10.1016/j. vaccine.2015.05.002

61. Seo JY, Chung HJ, Kim TJ. Codon-optimized expression of fish iridovirus capsid protein in yeast and its application as an oral vaccine candidate. J Fish Dis (2013) 36:763-768. doi:10.1111/jfd.12037

62. Companjen AR, Florack DEA, Slootweg T, Borst JW, Rombout JHW. Improved uptake of plant-derived LTB-linked proteins in carp gut and induction of specific humoral immune responses upon infeed delivery. Fish Shellfish Immunol (2006) 21:251-260. doi:10.1016/j. fsi.2005.12.001

63. Mayer L, Shao L. Therapeutic potential of oral tolerance. Nat Rev Immunol (2004) 4:407419. doi: $10.1038 /$ nri1370

64. Neutra MR, Mantis NJ, Kraehenbuhl JP. Collaboration of epithelial cells with organized mucosal lymphoid tissues. Nat Immunol (2001) 2:1004-9. doi:10.1038/ni1101-1004

65. Frey A, Giannasca KT, Weltzin R, Giannasca PJ, Reggio H, Lencer WI, Neutra MR. Role of the glycocalyx in regulating access of microparticles to apical plasma membranes of intestinal epithelial cells: implications for microbial attachment and oral vaccine targeting. $J$ Exp Med (1996) 184:1045-59. Available at: http://www.pubmedcentral.nih.gov/articlerender. fcgi?artid=2192803\&tool=pmcentrez\&rendertype=abstract [Accessed November 7, 2015]

66. Mantis NJ, Frey A, Neutra MR. Accessibility of glycolipid and oligosaccharide epitopes on rabbit villus and follicle-associated epithelium. Am J Physiol Gastrointest Liver Physiol (2000) 278:G915-23. Available at: http://www.ncbi.nlm.nih.gov/pubmed/10859221 [Accessed November 7, 2015]

67. Løkka G, Koppang EO. Antigen sampling in the fish intestine. Dev Comp Immunol (2015) 64: doi:10.1016/j.dci.2016.02.014

68. Rombout Jan JHWM, Abelli L, Picchietti S, Scapigliati G, Kiron V. Teleost intestinal immunology. Fish Shellfish Immunol (2011) 31:616-626. doi:10.1016/j.fsi.2010.09.001

69. Kawai K, Yamamoto S, Kusuda R. Plankton-mediated oral delivery of Vibrio anguillarum vaccine to juvenile ayu. Nippon Suisan Gakkaishi (1989) 55:35-40.

70. Tafalla C, Bøgwald J, Dalmo R a. Adjuvants and immunostimulants in fish vaccines: Current knowledge and future perspectives. Fish Shellfish Immunol (2013) 35:1740-1750. doi:10.1016/j.fsi.2013.02.029

71. Lorenzen N, LaPatra SE. DNA vaccines for aquacultured fish. Rev Sci Tech (2005) 24:201213. doi:10.3354/dao056031

72. Arijo S, Brunt J, Chabrillón M, Díaz-Rosales P, Austin B. Subcellular components of Vibrio harveyi and probiotics induce immune responses in rainbow trout, Oncorhynchus mykiss (Walbaum), against V. harveyi. J Fish Dis (2008) 31:579-90. doi:10.1111/j.13652761.2008.00932.x

73. Sun Y, Hu YH, Liu CS, Sun L. A Streptococcus iniae DNA vaccine delivered by a live attenuated edwardsiella tarda via natural infection induces cross-genus protection. Lett Appl Microbiol (2012) 55:420-426. doi:10.1111/j.1472-765X.2012.03307.x

74. Cheng S, Hu YH, Zhang M, Sun L. Analysis of the vaccine potential of a natural avirulent Edwardsiella tarda isolate. Vaccine (2010) 28:2716-2721. doi:10.1016/j.vaccine.2010.01.023

75. Sun Y, Liu CS, Sun L. Identification of an Edwardsiella tarda surface antigen and analysis of its immunoprotective potential as a purified recombinant subunit vaccine and a surfaceanchored subunit vaccine expressed by a fish commensal strain. Vaccine (2010) 28:66036608. doi:10.1016/j.vaccine.2010.07.050

76. Hu YH, Cheng S, Zhang M, Sun L. Construction and evaluation of a live vaccine against 
Edwardsiella tarda and Vibrio harveyi: Laboratory vs. mock field trial. Vaccine (2011) 29:4081-4085. doi:10.1016/j.vaccine.2011.04.025

77. Leal C a G, Carvalho-Castro G a., Sacchetin PSC, Lopes CO, Moraes a. M, Figueiredo HCP. Oral and parenteral vaccines against Flavobacterium columnare: Evaluation of humoral immune response by ELISA and in vivo efficiency in Nile tilapia (Oreochromis niloticus). Aquac Int (2010) 18:657-666. doi:10.1007/s10499-009-9287-x

78. Ghosh B, Bridle AR, Nowak BF, Cain KD. Assessment of immune response and protection against bacterial coldwater disease induced by a live-attenuated vaccine delivered orally or intraperitoneally to rainbow trout, Oncorhynchus mykiss (Walbaum). Aquaculture (2015) 446:242-249. doi:10.1016/j.aquaculture.2015.04.035

79. Behera T, Swain P. Antigen encapsulated alginate-coated chitosan microspheres stimulate both innate and adaptive immune responses in fish through oral immunization. Aquac Int (2014) 22:673-688. doi:10.1007/s10499-013-9696-8

80. Rajesh Kumar S, Ishaq Ahmed VP, Parameswaran V, Sudhakaran R, Sarath Babu V, Sahul Hameed a. S. Potential use of chitosan nanoparticles for oral delivery of DNA vaccine in Asian sea bass (Lates calcarifer) to protect from Vibrio (Listonella) anguillarum. Fish Shellfish Immunol (2008) 25:47-56. doi:10.1016/j.fsi.2007.12.004

81. Li L, Lin S-L, Deng L, Liu Z-G. Potential use of chitosan nanoparticles for oral delivery of DNA vaccine in black seabream Acanthopagrus schlegelii Bleeker to protect from Vibrio parahaemolyticus. J Fish Dis (2013) 36:987-95. doi:10.1111/jfd.12032

82. Yasumoto S, Yoshimura T, Miyazaki T. Oral immunization of common carp with a liposome vaccine containing Aeromonas hydrophila antigen. Fish Pathol (2006) 41:45-49. doi: $10.3147 /$ jsfp. 41.45

83. Irie T, Watarai S, Iwasaki T, Kodama H. Protection against experimental Aeromonas salmonicida infection in carp by oral immunisation with bacterial antigen entrapped liposomes. Fish Shellfish Immunol (2005) 18:235-242. doi:10.1016/j.fsi.2004.07.006

84. Tobar J a., Jerez S, Caruffo M, Bravo C, Contreras F, Bucarey S a., Harel M. Oral vaccination of Atlantic salmon (Salmo salar) against salmonid rickettsial septicaemia. Vaccine (2011) 29:2336-2340. doi:10.1016/j.vaccine.2010.12.107

85. Wang K, Deng L, Huang J, Fu X, Chen D, Geng Y. Study on the immunogencity of poly (D , L-lactide-co- glycolide) (PLGA) microspheres-encapsulated vaccine preparation against Stenotrophomonas maltophilia infection in channel catfish (Ictalurus punctatus). African $J$ Biotechnol (2011) 10:2751-2761. doi:10.5897/AJB10.175

86. Altun S, Kubilay A, Ekici S, Didinen BI, Diler O. Oral vaccination against lactococcosis in rainbow trout (Oncorhynchus mykiss) using sodium alginate and poly (lactide-co-glycolide) carrier. Kafkas Univ Vet Fak Derg (2009) 16:211-217. doi:10.9775/kvfd.2009.1327

87. Lin C-C, Lin JH-Y, Chen M-S, Yang H-L. An oral nervous necrosis virus vaccine that induces protective immunity in larvae of grouper (Epinephelus coioides). Aquaculture (2007) 268:265-273. doi:10.1016/j.aquaculture.2007.04.066

88. Chen YM, Shih CH, Liu HC, Wu CL, Lin CC, Wang HC, Chen TY, Yang HL, Lin JHY. An oral nervous necrosis virus vaccine using Vibrio anguillarum as an expression host provides early protection. Aquaculture (2011) 321:26-33. doi:10.1016/j.aquaculture.2011.08.035

89. Ballesteros NA, Alonso M, Rodríguez Saint-Jean S, Perez-Prieto SI. An oral DNA vaccine against infectious haematopoietic necrosis virus (IHNV) encapsulated in alginate microspheres induces dose-dependent immune responses and significant protection in rainbow trout (Oncorrhynchus mykiss). Fish Shellfish Immunol (2015) 45:877-888. doi:10.1016/j.fsi.2015.05.045

90. de las Heras AI, Rodríguez Saint-Jean S, Pérez-Prieto SI. Immunogenic and protective effects of an oral DNA vaccine against infectious pancreatic necrosis virus in fish. Fish Shellfish Immunol (2010) 28:562-570. doi:10.1016/j.fsi.2009.12.006

91. Ballesteros N a., Rodriguez Saint-Jean S, Perez-Prieto SI. Immune responses to oral pcDNAVP2 vaccine in relation to infectious pancreatic necrosis virus carrier state in rainbow trout 
Oncorhynchus mykiss. Vet Immunol Immunopathol (2015) 165:127-137. doi:10.1016/j. vetimm.2015.04.001

92. Ballesteros NA, Rodriguez Saint-Jean S, Perez-Prieto S. Food pellets as an effective delivery method for a DNA vaccine against infectious pancreatic necrosis virus in rainbow trout (Oncorhynchus mykiss, Walbaum). Fish Shellfish Immunol (2014) 37:220-8. doi:10.1016/j. fsi.2014.02.003

93. Rivas-Aravena A, Fuentes Y, Cartagena J, Brito T, Poggio V, La Torre J, Mendoza H, Gonzalez-Nilo F, Sandino AM, Spencer E. Development of a nanoparticle-based oral vaccine for Atlantic salmon against ISAV using an alphavirus replicon as adjuvant. Fish Shellfish Immunol (2015) 45:157-166. doi:10.1016/j.fsi.2015.03.033

94. Vimal S, Abdul Majeed S, Nambi KSN, Madan N, Farook M a., Venkatesan C, Taju G, Venu S, Subburaj R, Thirunavukkarasu a. R, et al. Delivery of DNA vaccine using chitosantripolyphosphate (CS/TPP) nanoparticles in Asian sea bass, Lates calcarifer (Bloch, 1790) for protection against nodavirus infection. Aquaculture (2014) 420-421:240-246. doi:10.1016/j. aquaculture.2013.11.017

95. Miyazaki T, Yasumoto S, Kuzuya Y, Yoshimura T. "A primary study on oral vaccination with liposomes entrapping Koi Herpesvirus ( KHV) antigens against KHV infection in carp," in Diseases in Asian Aquaculture, ed. R. P. Bondad-Reantaso, M.G., Mohan, C.V., Crumlish, M. and Subasinghe (Manila: Asian Fisheries Society), 99-184.

96. Yasumoto S, Kuzuya Y, Yasuda M, Yoshimura T, Miyazaki T. Oral immunization of common carp with a liposome vaccine fusing Koi Herpesvirus antigen. Fish Pathol (2006) 41:141145. doi:10.3147/jsfp.41.141

97. Adomako M, St-Hilaire S, Zheng Y, Eley J, Marcum RD, Sealey W, Donahower BC, LaPatra $\mathrm{S}$, Sheridan PP. Oral DNA vaccination of rainbow trout, Oncorhynchus mykiss (Walbaum), against infectious haematopoietic necrosis virus using PLGA [Poly(D,L-Lactic-Co-Glycolic Acid)] nanoparticles. J Fish Dis (2012) 35:203-214. doi:10.1111/j.1365-2761.2011.01338.x

98. Koff WC, Burton DR, Johnson PR, Walker BD, King CR, Nabel GJ, Ahmed R, Bhan MK, Plotkin SA. Accelerating next-generation vaccine development for global disease prevention. Science (2013) 340:1232910. doi:10.1126/science.1232910

99. Mutoloki S, Jørgensen JB, Evensen Ø. Fish vaccination., eds. R. Gudding, A. Lillehaug, Ø. Evensen Chichester, UK: Wiley-Blackwell (2014). doi:10.1002/9781118806913.ch9

100. Salinas I. The Mucosal Immune System of Teleost Fish. Biology (Basel) (2015) 4:525-39. doi:10.3390/biology4030525

101. Mutoloki S, Jørgensen JB. The Adaptive Immune Response in Fish. Fish Vaccin (2014)105115.

102. Toda H, Shibasaki Y, Koike T, Ohtani M, Takizawa F, Ototake M, Moritomo T, Nakanishi T. Alloantigen-specific killing is mediated by CD8-positive T cells in fish. Dev Comp Immunol (2009) 33:646-652. Available at: http://www.sciencedirect.com/science/article/B6T5X4V4MJ6H-1/2/298d5db7b081269bflada5cdf9d97c34

103. Toda H, Saito Y, Koike T, Takizawa F, Araki K, Yabu T, Somamoto T, Suetake H, Suzuki Y, Ototake M, et al. Conservation of characteristics and functions of CD4 positive lymphocytes in a teleost fish. Dev Comp Immunol (2011) 35:650-60. doi:10.1016/j.dci.2011.01.013

104. Fischer U, Koppang EO, Nakanishi T. Teleost T and NK cell immunity. Fish Shellfish Immunol (2013) 35:197-206. doi:10.1016/j.fsi.2013.04.018

105. Hansen JD, Landis ED, Phillips RB. Discovery of a unique Ig heavy-chain isotype (IgT) in rainbow trout: Implications for a distinctive B cell developmental pathway in teleost fish. Proc Natl Acad Sci U S A (2005) 102:6919-24. doi:10.1073/pnas.0500027102

106. Xu Z, Parra D, Gómez D, Salinas I, Zhang Y-A, von Gersdorff Jørgensen L, Heinecke RD, Buchmann K, LaPatra S, Sunyer JO. Teleost skin, an ancient mucosal surface that elicits gut-like immune responses. Proc Natl Acad Sci U S A (2013) 110:13097-102. doi:10.1073/ pnas. 1304319110

107. Salinas I, Zhang Y-A, Sunyer JO. Mucosal immunoglobulins and B cells of teleost fish. Dev 
Comp Immunol (2011) 35:1346-1365. doi:10.1016/j.dci.2011.11.009

108. Jørgensen JB. The Innate Immune Response in Fish. Fish Vaccin (2014)85-103.

109. Munang'andu HM, Mutoloki S, Evensen Ø. A Review of the Immunological Mechanisms Following Mucosal Vaccination of Finfish. Front Immunol (2015) 6:427. Available at: http:// journal.frontiersin.org/article/10.3389/fimmu.2015.00427/abstract [Accessed October 15, 2015]

110. Wang M, Gao Z, Zhang Z, Pan L, Zhang Y. Roles of M cells in infection and mucosal vaccines. Hum Vaccin Immunother (2014) 10:3544-51. doi:10.4161/hv.36174

111. Jang MH, Kweon M-N, Iwatani K, Yamamoto M, Terahara K, Sasakawa C, Suzuki T, Nochi T, Yokota Y, Rennert PD, et al. Intestinal villous M cells: an antigen entry site in the mucosal epithelium. Proc Natl Acad Sci U S A (2004) 101:6110-5. doi:10.1073/pnas.0400969101

112. Scott CL, Aumeunier AM, Mowat AM. Intestinal CD103+ dendritic cells: master regulators of tolerance? Trends Immunol (2011) 32:412-9. Available at: http://www.sciencedirect.com/ science/article/pii/S1471490611001001 [Accessed September 27, 2015]

113. Owen JL, Sahay B, Mohamadzadeh M. New generation of oral mucosal vaccines targeting dendritic cells. Curr Opin Chem Biol (2013) 17:918-24. Available at: http://www. sciencedirect.com/science/article/pii/S1367593113001154 [Accessed October 14, 2015]

114. Kim S-H, Jang Y-S. Antigen targeting to M cells for enhancing the efficacy of mucosal vaccines. Exp Mol Med (2014) 46:e85. doi:10.1038/emm.2013.165

115. Mulero I, Pilar Sepulcre M, Roca FJ, Meseguer J, García-Ayala A, Mulero V. Characterization of macrophages from the bony fish gilthead seabream using an antibody against the macrophage colony-stimulating factor receptor. Dev Comp Immunol (2008) 32:1151-9. Available at: http://www.ncbi.nlm.nih.gov/pubmed/18420271 [Accessed December 11, 2015]

116. Ellett F, Pase L, Hayman JW, Andrianopoulos A, Lieschke GJ. Mpeg1 Promoter Transgenes Direct Macrophage-Lineage Expression in Zebrafish. Blood (2010) 117:e49-e56. doi:10.1182/blood-2010-10-314120

117. Weyts FAA, Rombout JHWM, Flik G, Verburg-van Kemenade BML. A common carp (Cyprinus carpioL.) leucocyte cell line shares morphological and functional characteristics with macrophages. Fish Shellfish Immunol (1997) 7:123-133. Available at: http://www. sciencedirect.com/science/article/pii/S1050464896900698 [Accessed December 22, 2015]

118. Shoemaker CA, Klesius PH. "Replicating Vaccines," in Fish vaccination, 99-117. doi:10.1007/978-3-0346-0277-8

119. Lillehaug A. Vaccination Strategies and Procedures. Fish Vaccin (2014)140-152.

120. Park S Bin, Aoki T, Jung TS. Pathogenesis of and strategies for preventing Edwardsiella tarda infection in fish. Vet Res (2012) 43:1. doi:10.1186/1297-9716-43-67

121. Ballesteros N a., Saint-Jean SSR, Encinas P a., Perez-Prieto SI, Coll JM. Oral immunization of rainbow trout to infectious pancreatic necrosis virus (Ipnv) induces different immune gene expression profiles in head kidney and pyloric ceca. Fish Shellfish Immunol (2012) 33:174185. doi:10.1016/j.fsi.2012.03.016

122. Davison AJ, Benko M, Harrach B. Genetic content and evolution of adenoviruses. $J$ Gen Virol (2003) 84:2895-2908. doi:10.1099/vir.0.19497-0

123. Stroband HW, Debets FM. The ultrastructure and renewal of the intestinal epithelium of the juvenile grasscarp, Ctenopharyngodon idella (Val.). Cell Tissue Res (1978) 187:181-200. Available at: http://www.ncbi.nlm.nih.gov/pubmed/630592 [Accessed December 5, 2015]

124. Rombout JHWM, Lamers CHJ, Helfrich MH, Dekker a., Taverne-Thiele JJ. Uptake and transport of intact macromolecules in the intestinal epithelium of carp (Cyprinus carpio L.) and the possible immunological implications. Cell Tissue Res (1985) 239:519-530. doi:10.1007/BF00219230

125. Wallace KN, Akhter S, Smith EM, Lorent K, Pack M. Intestinal growth and differentiation in zebrafish. Mech Dev (2005) 122:157-73. doi:10.1016/j.mod.2004.10.009

126. Vander Veen RL, Harris DLH, Kamrud KI. Alphavirus replicon vaccines. Anim Health Res 
Rev (2012) 13:1-9. doi:10.1017/S1466252312000011

127. Wolf A, Hodneland K, Frost P, Hoeijmakers M, Rimstad E. Salmonid alphavirus-based replicon vaccine against infectious salmon anemia (ISA): impact of immunization route and interactions of the replicon vector. Fish Shellfish Immunol (2014) 36:383-92. doi:10.1016/j. fsi.2013.12.018

128. Olsen CM, Pemula AK, Braaen S, Sankaran K, Rimstad E. Salmonid alphavirus replicon is functional in fish, mammalian and insect cells and in vivo in shrimps (Litopenaeus vannamei). Vaccine (2013) 31:5672-9. doi:10.1016/j.vaccine.2013.09.058

129. Appaiahgari MB, Vrati S. Adenoviruses as gene/vaccine delivery vectors: promises and pitfalls. Expert Opin Biol Ther (2015) 15:337-51. doi:10.1517/14712598.2015.993374

130. Bangari DS, Mittal SK. Development of nonhuman adenoviruses as vaccine vectors. Vaccine (2006) 24:849-62. doi:10.1016/j.vaccine.2005.08.101

131. Kovács GM, LaPatra SE, D’Halluin JC, Benko M. Phylogenetic analysis of the hexon and protease genes of a fish adenovirus isolated from white sturgeon (Acipenser transmontanus) supports the proposal for a new adenovirus genus. Virus Res (2003) 98:27-34.

132. Gilbert SC. Adenovirus-vectored Ebola vaccines. Expert Rev Vaccines (2015) 14:1347-1357. doi:10.1586/14760584.2015.1077122

133. Schuldt NJ, Amalfitano A. Malaria vaccines: focus on adenovirus based vectors. Vaccine (2012) 30:5191-8. doi:10.1016/j.vaccine.2012.05.048

134. Ogwang C, Kimani D, Edwards NJ, Roberts R, Mwacharo J, Bowyer G, Bliss C, Hodgson $\mathrm{SH}$, Njuguna P, Viebig NK, et al. Prime-boost vaccination with chimpanzee adenovirus and modified vaccinia Ankara encoding TRAP provides partial protection against Plasmodium falciparum infection in Kenyan adults. Sci Transl Med (2015) 7:286re5. doi:10.1126/ scitranslmed.aaa2373

135. Green CA, Scarselli E, Sande CJ, Thompson AJ, Lara CM De, Taylor KS, Haworth K, Sorbo M Del, Angus B, Siani L, et al. Chimpanzee adenovirus - and MVA-vectored respiratory syncytial virus vaccine is safe and immunogenic in adults. Sci Transl Med (2015) 7:1-17. doi:10.1126/scitranslmed.aac5745

136. Porta C, Kotecha A, Burman A, Jackson T, Ren J, Loureiro S, Jones IM, Fry EE, Stuart DI, Charleston B. Rational engineering of recombinant picornavirus capsids to produce safe, protective vaccine antigen. PLoS Pathog (2013) 9:e1003255. doi:10.1371/journal. ppat. 1003255

137. Jenkins P, Harris J, Pulsford A. Enhanced enteric uptake of human gamma globulin by Quil-A saponin in Oreochromis mossambicus. Fish Shellfish Immunol (1991) 1:279-295. doi:10.1016/S1050-4648(05)80066-X

138. Savelkoul HFJ, Ferro VA, Strioga MM, Schijns VEJC. Choice and Design of Adjuvants for Parenteral and Mucosal Vaccines. Vaccines (2015) 3:148-71. doi:10.3390/vaccines3010148

139. Jenkins PG, Wrathmell AB, Harris JE, Pulsford AL. The effects of different adjuvants on intestinal absorption and subsequent immune responses of the tilapian Oreochromis mossambicus. Fish Shellfish Immunol (1994)167-177.

140. Lycke N. From toxin to adjuvant: Basic mechanisms for the control of mucosal IgA immunity and tolerance. Immunol Lett (2005) 97:193-198. doi:10.1016/j.imlet.2004.12.008

141. Elson CO, Dertzbaugh MT. "Mucosal adjuvants," in Mucosal immunology, ed. Ogra R. et al. (New York: Academic Press), 967-986.

142. Pérez O, Romeu B, Cabrera O, González E, Batista-Duharte A, Labrada A, Pérez R, Reyes LM, Ramírez W, Sifontes S, et al. Adjuvants are key factors for the development of future vaccines: Lessons from the Finlay adjuvant platform. Front Immunol (2013) 4:1-12. doi:10.3389/fimmu.2013.00407

143. Josepriya T a., Chien K-H, Lin H-Y, Huang H-N, Wu C-J, Song Y-L. Immobilization antigen vaccine adjuvanted by parasitic heat shock protein 70C confers high protection in fish against cryptocaryonosis. Fish Shellfish Immunol (2015) 45:517-527. doi:10.1016/j.fsi.2015.04.036

144. Chang C-J, Sun B, Robertsen B. Adjuvant activity of fish type I interferon shown in a virus 
DNA vaccination model. Vaccine (2015) 33:2442-2448. doi:10.1016/j.vaccine.2015.03.093

145. Pietretti D, Wiegertjes GF. Ligand specificities of Toll-like receptors in fish: Indications from infection studies. Dev Comp Immunol (2014) 43:205-222. doi:10.1016/j.dci.2013.08.010

146. Bonaldo a, Bonaldo a, Thompson KD, Thompson KD, Manfrin a, Manfrin a, Adams a, Adams a, Murano E, Murano E, et al. The influence of dietary beta-glucans on the adaptive and innate immune responses of European sea bass (Dicentrarchus labrax) vaccinated against vibriosis. Ital J Anim Sci (2007) 6:151-164.

147. Ogier de Baulny M, Quentel C, Fournier V, Lamour F, Le Gouvello R. Effect of long-term oral administration of beta-glucan as an immunostimulant or an adjuvant on some nonspecific parameters of the immune response of turbot Scophthalmus maximus. Dis Aquat Organ (1996) 26:139-147. doi:10.3354/dao026139

148. Selvaraj V, Sampath K, Sekar V. Adjuvant and immunostimulatory effects of $\beta$-glucan administration in combination with lipopolysaccharide enhances survival and some immune parameters in carp challenged with Aeromonas hydrophila. Vet Immunol Immunopathol (2006) 114:15-24. doi:10.1016/j.vetimm.2006.06.011

149. Selvaraj V, Sampath K, Sekar V. Administration of yeast glucan enhances survival and some non-specific and specific immune parameters in carp (Cyprinus carpio) infected with Aeromonas hydrophila. Fish Shellfish Immunol (2005) 19:293-306. doi:10.1016/j. fsi.2005.01.001

150. Skov J, Kania PW, Holten-Andersen L, Fouz B, Buchmann K. Immunomodulatory effects of dietary $\beta$-1,3-glucan from Euglena gracilis in rainbow trout (Oncorhynchus mykiss) immersion vaccinated against Yersinia ruckeri. Fish Shellfish Immunol (2012) 33:111-120. doi:10.1016/j.fsi.2012.04.009

151. De Smet R, Demoor T, Verschuere S, Dullaers M, Ostroff GR, Leclercq G, Allais L, Pilette C, Dierendonck M, De Geest BG, et al. Beta-Glucan microparticles are good candidates for mucosal antigen delivery in oral vaccination. J Control Release (2013) 172:671-678. doi:10.1016/j.jconrel.2013.09.007

152. Suanyuk N, Itsaro A. Efficacy of inactivated Streptococcus iniae vaccine and protective effect of ??-(1,3/1,6)-glucan on the effectiveness of vaccine in red tilapia Oreochromis niloticus x O. mossambicus. Songklanakarin J Sci Technol (2011) 33:143-149.

153. Fredriksen BN, Sævareid K, McAuley L, Lane ME, Bøgwald J, Dalmo R a. Early immune responses in Atlantic salmon (Salmo salar L.) after immunization with PLGA nanoparticles loaded with a model antigen and $\beta$-glucan. Vaccine (2011) 29:8338-8349. doi:10.1016/j. vaccine.2011.08.087

154. Huang H, Ostroff GR, Lee CK. Robust Stimulation of Humoral and Cellular Immune Responses following Vaccination with Antigen-Loaded $\beta$-Glucan Particles Robust Stimulation of Humoral and Cellular Immune Responses following Vaccination with Antigen-Loaded $\beta$-Glucan Particles. MBio (2010)1-7. doi:10.1128/mBio.00164-10.Editor

155. De Smet R, Demoor T, Verschuere S, Dullaers M, Ostroff GR, Leclercq G, Allais L, Pilette C, Dierendonck M, De Geest BG, et al. B-Glucan microparticles are good candidates for mucosal antigen delivery in oral vaccination. J Control Release (2013) 172:671-678. doi:10.1016/j.jconrel.2013.09.007

156. De Clercq E. Degradation of Poly(inosinic acid). poly(cytidylic acid) [(I)n . (Cn)] by Human Plasma. Eur J Biochem (1979) 93:165-172. doi:10.1111/j.1432-1033.1979.tb12807.x

157. Kavaliauskis A, Arnemo M, Kim S-H, Ulanova L, Speth M, Novoa B, Dios S, Evensen Ø, Griffiths GW, Gjøen T. Use of Poly(I:C) Stabilized with Chitosan As a Vaccine-Adjuvant Against Viral Hemorrhagic Septicemia Virus Infection in Zebrafish. Zebrafish (2015) 12:111. doi:10.1089/zeb.2015.1126

158. Thim HL, Iliev DB, Christie KE, Villoing S, McLoughlin MF, Strandskog G, Jørgensen JB. Immunoprotective activity of a Salmonid Alphavirus Vaccine: Comparison of the immune responses induced by inactivated whole virus antigen formulations based on $\mathrm{CpG}$ class B oligonucleotides and poly I:C alone or combined with an oil adjuvant. Vaccine (2012) 
30:4828-4834. doi:https://doi.org/10.1016/j.vaccine.2012.05.010

159. Thim H, Villoing S, McLoughlin M, Christie K, Grove S, Frost P, Jørgensen J. Vaccine Adjuvants in Fish Vaccines Make a Difference: Comparing Three Adjuvants (Montanide ISA763A Oil, CpG/Poly I:C Combo and VHSV Glycoprotein) Alone or in Combination Formulated with an Inactivated Whole Salmonid Alphavirus Antigen. Vaccines (2014) 2:228-251. doi:10.3390/vaccines2020228

160. Chikwati EM, Sahlmann C, Holm H, Penn MH, Krogdahl Å, Bakke AM. Alterations in digestive enzyme activities during the development of diet-induced enteritis in Atlantic salmon, Salmo salar L. Aquaculture (2013) 402-403:28-37. doi:10.1016/j. aquaculture.2013.03.023

161. Couto A, Kortner TM, Penn M, Bakke AM, Krogdahl Å, Oliva-Teles A. Effects of dietary phytosterols and soy saponins on growth, feed utilization efficiency and intestinal integrity of gilthead sea bream (Sparus aurata) juveniles. Aquaculture (2014) 432:295-303. doi:10.1016/j.aquaculture.2014.05.009

162. Knudsen D, Jutfelt F, Sundh H, Sundell K, Koppe W, Frøkiaer H. Dietary soya saponins increase gut permeability and play a key role in the onset of soyabean-induced enteritis in Atlantic salmon ( Salmo salar L.). Br J Nutr (2008) 100:120-129. doi:10.1017/ S0007114507886338

163. Urán PA., Aydin R, Schrama JW, Verreth J a J, Rombout JHWM. Soybean meal-induced uptake block in Atlantic salmon Salmo salar distal enterocytes. J Fish Biol (2008) 73:25712579. doi:10.1111/j.1095-8649.2008.02091.x

164. Weber ES. A veterinary guide to the fish gastrointestinal tract. Vet Clin North Am Exot Anim Pract (2014) 17:123-43. doi:10.1016/j.cvex.2014.01.001

165. Chakrabarti I, Gani MA, Chaki KK, Sur R, Misra KK. Digestive enzymes in 11 freshwater teleost fish species in relation to food habit and niche segregation. Comp Biochem Physiol Part A Physiol (1995) 112:167-177. doi:10.1016/0300-9629(95)00072-F

166. Kolkovski S. Digestive enzymes in fish larvae and juveniles - implications and applications to formulated diets. Aquaculture (2001) 200:181-201. doi:10.1016/S0044-8486(01)00700-1

167. Valdez Y, Brown EM, Finlay BB. Influence of the microbiota on vaccine effectiveness. Trends Immunol (2014) 35:526-537. doi:10.1016/j.it.2014.07.003

168. Hill DA, Artis D. Intestinal Bacteria and the Regulation of Immune Cell Homeostasis. Annu Rev Immunol (2010) 28:623-667. doi:10.1146/annurev-immunol-030409-101330

169. Hapfelmeier S, Macpherson AJ. In remembrance of commensal intestinal microbes. Commun Integr Biol (2010) 3:569-71. doi:10.4161/cib.3.6.13011

170. Bauer H, Horowitz R, Levenson S, Popper H. The response of the lymphatic tissue to the microbial flora. Am J Pathol (1963) 42:471-483.

171. Sudo N, Sawamura S, Tanaka K, Aiba Y, Kubo C, Koga Y. The requirement of intestinal bacterial flora for the development of an IgE production system fully susceptible to oral tolerance induction. J Immunol (1997) 159:1739-1745.

172. Kiyono H, McGhee JR, Wannemuehler MJ, Michalek SM. Lack of oral tolerance in C3H/ HeJ mice. J Exp Med (1982) 155:605-610.

173. Brugman S, Liu KY, Lindenbergh-Kortleve D, Sansom JN, Furuta GT, Renshaw SA, Willemsen R, Nieuwenhuis EES. Oxazolone-induced enterocolitis in zebrafish depends on the composition of the intestinal microbiota. Gastroenterology (2009) 137:1757-1767. doi:10.1053/j.gastro.2009.07.069

174. Gomez D, Sunyer JO, Salinas I. The mucosal immune system of fish: The evolution of tolerating commensals while fighting pathogens. Fish Shellfish Immunol (2013) 35:17291739. doi:10.1016/j.fsi.2013.09.032

175. Rohovec J. S. PLG, Fryer JL. Immunization of fish for the control of vibriosis.20-23.

176. Lorenzen E, Einer-Jensen K, Rasmussen JS, Kjær TE, Collet B, Secombes CJ, Lorenzen $\mathrm{N}$. The protective mechanisms induced by a fish rhabdovirus DNA vaccine depend on temperature. Vaccine (2009) 27:3870-3880. doi:10.1016/j.vaccine.2009.04.012 
177. Gray C, Loynes CA, Whyte MKB, Crossman DC, Renshaw SA, Chico TJA. Simultaneous intravital imaging of macrophage and neutrophil behaviour during inflammation using a novel transgenic zebrafish. Thromb Haemost (2011) 105:811-819. doi:10.1160/TH10-080525

178. Midtlyng PJ, Reitan LJ, Lillehaug A, Ramstad A. Protection, immune responses and side effects in Atlantic salmon (Salmo salar. L.) vaccinated against furunculosis by different procedures. Fish Shellfish Immunol (1996) 6:599-613. doi:10.1006/fsim.1996.0055

179. Piganelli JD, Wiens GD, Zhang JA, Christensen JM, Kaattari SL. Evaluation of a whole cell p57- vaccine against Renibacterium salmoninarum. Dis Aquat Organ (1999) 36:37-44. doi: $10.3354 /$ dao036037

180. Salati F, Ono K, Kusuda R. Oral vaccination of glass eel of Anguilla japonica against Edwardsiella tarda infection. Fish Shellfish Immunol (1991) 1:309-310.

181. Plumb J a., Vinitnantharat S, Abe V, Phelps RP. Density-dependent effect on oral vaccination of channel catfish against Edwardsiella ictaluri. Aquaculture (1994) 122:91-96. doi:10.1016/0044-8486(94)90501-0

182. Dec C, Angelidis P, Laurencin FB. Effects of oral vaccination against vibriosis in turbot, Scophthalmus maximus (L.), and sea bass, Dicentrarchus labrax (L.). J Fish Dis (1990) 13:369-376. doi:10.1111/j.1365-2761.1990.tb00795.x

183. Horne MT, Tatner M, McDerment S, AGIUS C, WARD P. Vaccination of rainbow trout, salmo gairdneri Richardson, at low temperatures and the long persistence of protection. $J$ Fish Dis (1982) 5:343-345. doi:10.1111/j.1365-2761.1982.tb00490.x

184. Fryer JL, Rohovec JS GR. Immunization of fish for the control of vibriosis. Mar Fish Rev (1978) 40:20-23. Available at: http://spo.nmfs.noaa.gov/mfr403/mfr4035.pdf

185. Lillehaug A. Oral immunization of rainbow trout, Salmo gairdneri Richardson, against vibriosis with vaccines protected against digestive degradation. J Fish Dis (1989) 12:579584. doi:10.1111/j.1365-2761.1989.tb00567.x

186. Esteve-Gassent MD, Fouz B, Amaro C. Efficacy of a bivalent vaccine against eel diseases caused by Vibrio vulnificus after its administration by four different routes. Fish Shellfish Immunol (2004) 16:93-105. doi:10.1016/S1050-4648(03)00036-6

187. Xu Z, Chen CF, Mao ZJ, Zhu WY. Detection of serum and mucosal antibody production and antibody secreting cells (ASCs) in large yellow croaker (Pseudosciaena crocea) following vaccination with Vibrio harveyi via different routes. Aquaculture (2009) 287:243-247. doi:10.1016/j.aquaculture.2008.10.026

188. Ji R, Zou W, Hu S, Yan Q. Vaccination in three different ways against vibriosis of Seriola dumerili caused by Vibrio hollisae. Chinese J Oceanol Limnol (2008) 26:233-237. doi:10.1007/s00343-008-0233-y

189. Gravningen K, Kestin S, Thorarinsson R, Syvertsen C. Oral vaccination against enteric red mouth disease in rainbow trout (Oncorhynchus mykiss Walbaum). The effect of vaccine dose rate on protection against the disease. J Appl Ichthyol (1998) 14:163-166. Available at: http://www.scopus.com/inward/record.url?eid=2-s2.0-0032460010\&partnerID=40\&md5=6f 7f6506cf6ba1535bb31ec1c69fd93e

190. Gomez-Casado, E, Estepa, A, Coll, JM. A comparative review on European- farmed finfish RNA viruses and their vaccines. Vaccine (2011) 29. http://dx.doi.org/ 10.1016/j. vaccine.2011.01.097, 2657e2571.

191. Griffiths, SG, Melville, KJ, Salonius, K. Reduction of Renibacterium salmo- ninarumculture activity in Atlantic salmon following vaccination with avirulent strains. Fish Shellfish Immunol. (1998) 8, 607e619. http://dx.doi.org/10.1006/ fsim.1998.0169 
Supplementary Table 1. Experimental approaches for the development of oral vaccines using non-encapsulated antigens

\begin{tabular}{|c|c|c|c|c|c|c|c|c|}
\hline Pathogen & $\begin{array}{l}\text { Fish } \\
\text { species }\end{array}$ & $\begin{array}{l}\text { Regime and } \\
\text { formulation }\end{array}$ & Antigen & Dose & $\begin{array}{l}\text { Protection } \\
\text { (survival) }\end{array}$ & $\begin{array}{l}\text { Time of } \\
\text { chal- } \\
\text { lenge }\end{array}$ & $\begin{array}{l}\text { Challenge } \\
\text { route }\end{array}$ & REF \\
\hline \multicolumn{9}{|l|}{ Bacteria } \\
\hline $\begin{array}{l}\text { Aeromonas } \\
\text { hydrophila } \\
\text { (A.h.) } \\
\end{array}$ & $\begin{array}{l}\text { Murrel } \\
(15 \mathrm{~g})\end{array}$ & $\begin{array}{l}\text { 20d; } \\
\text { Mixed with } \\
\text { feed }\end{array}$ & $\begin{array}{l}\text { h.i. } A . h \text { free- } \\
\text { cells or biofilm } \\
\text { on chitin flakes }\end{array}$ & $\begin{array}{l}10^{\wedge} 10 \text { cells/fish/ } \\
\text { day; Free-cell or } \\
\text { biofilm }\end{array}$ & $\begin{array}{l}\text { Free-cell: } 49 \% \\
\text { Biofilm: } 92 \% \\
\text { C: } 28 \%\end{array}$ & $60 \mathrm{dpv}$ & i.m. & [39] \\
\hline $\begin{array}{l}\text { Aeromonas } \\
\text { hydrophila }\end{array}$ & $\begin{array}{l}\text { Walking } \\
\text { catfish } \\
(140-260 \\
\text { g) }\end{array}$ & $\begin{array}{l}20 \mathrm{~d} ; \\
\text { Mixed with } \\
\text { feed }\end{array}$ & $\begin{array}{l}\text { h.i. } A . h \text { free- } \\
\text { cells or biofilm } \\
\text { on chitin flakes }\end{array}$ & $\begin{array}{l}4.29 \times 10^{\wedge} 10 \\
\text { (biofilm); } 6.79 \\
\times 10^{\wedge} 10 \text { (free- } \\
\text { cell) cells/g fish/ } \\
\text { day }\end{array}$ & $\begin{array}{l}\text { Free-cell: } 46-55 \% \\
\text { Biofilm: } 93-100 \% \\
\text { C: } 0 \%\end{array}$ & $60 \mathrm{dpv}$ & i.m. & {$[40]$} \\
\hline $\begin{array}{l}\text { Aeromonas } \\
\text { salmonicida }\end{array}$ & $\begin{array}{l}\text { Atlantic } \\
\text { salmon } \\
(35 \mathrm{~g})\end{array}$ & $\begin{array}{l}9 \mathrm{~d} ; \\
\text { Mixed with } \\
\text { feed }\end{array}$ & $\begin{array}{l}\text { Oravacc F Vet } \\
\text { commercial }\end{array}$ & $\begin{array}{l}0.9 \% \mathrm{w} / \mathrm{v} \text { of } \\
\text { pelleted feed }\end{array}$ & No protection & $\begin{array}{l}8 \text { and } 16 \\
\text { wpv }\end{array}$ & i.p. & {$[172]$} \\
\hline $\begin{array}{l}\text { Renibacterium } \\
\text { salmoninarum } \\
\text { (R.s.) }\end{array}$ & $\begin{array}{l}\text { Coho } \\
\text { salmon } \\
(45 \mathrm{~g})\end{array}$ & $\begin{array}{l}15 \text { times on } \\
\text { alternating } \\
\text { days followed } \\
\text { by i.p. boost; } \\
\text { Mixed with } \\
\text { feed }\end{array}$ & $\begin{array}{l}\text { f.i. } R . s . \mathrm{p} 57- \\
\text { attenuated } \\
\text { on } 40-50 \mathrm{~nm} \\
\text { dextrose beads, } \\
\text { coated with } \\
\text { Eugradit L-30D }\end{array}$ & $\begin{array}{l}100 \mu \mathrm{g} / \text { fish/day } \\
\text { with boost with } \\
500 \mu \mathrm{g} \text { in FIA }\end{array}$ & No protection & $20 \mathrm{dpv}$ & bath & [173] \\
\hline \multirow{7}{*}{$\begin{array}{l}\text { Edwardsiella } \\
\text { tarda }\end{array}$} & \multirow{7}{*}{$\begin{array}{l}\text { Eel } \\
(0.1 \mathrm{~g})\end{array}$} & \multirow{7}{*}{$\begin{array}{l}\text { 10d; Mixed } \\
\text { with feed }\end{array}$} & \multirow{7}{*}{$\begin{array}{l}\text { F.i. or sonicated } \\
\text { whole cell } E \text {. } \\
\text { tarda }\end{array}$} & \multirow{7}{*}{$\begin{array}{l}1 \mathrm{mg} / \mathrm{cells} / \mathrm{fish} / \\
\text { day }\end{array}$} & formalin: $30 \%$ & \multirow{7}{*}{$10 \mathrm{dpv}$} & \multirow{7}{*}{ bath } & \multirow{7}{*}[174]{} \\
\hline & & & & & sonicated: $75 \%$ & & & \\
\hline & & & & & C: $15 \%$ & & & \\
\hline & & & & & $\begin{array}{l}\text { SP+SN: } 47-53 \% \\
\mathrm{LP}+\mathrm{SN}: 51 \%\end{array}$ & & & \\
\hline & & & & & LP+SN+SP: $59 \%$ & & & \\
\hline & & & & & $\begin{array}{l}\text { SP+SN+SP: } 97- \\
100 \%\end{array}$ & & & \\
\hline & & & & & C: $56.3-44.1 \%$ & & & \\
\hline $\begin{array}{l}\text { Edwardsiella } \\
\text { ictaluri }\end{array}$ & $\begin{array}{l}\text { Channel } \\
\text { Catfish } \\
(0.044 \mathrm{~g})\end{array}$ & $\begin{array}{l}\text { Two periods } \\
\text { of } 5 \mathrm{~d}, 10 \mathrm{~d} \\
\text { interval; } \\
\text { Mixed with } \\
\text { feed }\end{array}$ & F.i. E. ictaluri & $\begin{array}{l}1 \% \mathrm{w} / \mathrm{v} \text { of } \\
\text { pelleted feed }\end{array}$ & No protection & 4 wpv & bath & [175] \\
\hline $\begin{array}{l}\text { Streptococcus } \\
\text { iniae }\end{array}$ & $\begin{array}{l}\text { Red } \\
\text { tilapia } \\
(7.7 \mathrm{~g}) \\
\end{array}$ & $\begin{array}{l}7 \mathrm{~d} ; \\
\text { Mixed with } \\
\text { feed }\end{array}$ & $\begin{array}{l}\text { Freeze-dried } S . \\
\text { iniae }\end{array}$ & $\begin{array}{l}10 \mathrm{~g} \text { cells } / \mathrm{kg} \\
\text { feed }\end{array}$ & No protection & $\begin{array}{l}1 \text { and } 3 \\
\text { wpv }\end{array}$ & i.p. & [119] \\
\hline \multirow{5}{*}{$\begin{array}{l}\text { Vibrio } \\
\text { anguillarum } \\
\text { (V.a.) }\end{array}$} & \multirow{3}{*}{$\begin{array}{l}\text { Turbot } \\
(5 \mathrm{~g})\end{array}$} & \multirow{3}{*}{$\begin{array}{l}5 \mathrm{~d} \text {; Mixed } \\
\text { with feed }\end{array}$} & \multirow{3}{*}{$\begin{array}{l}\text { F.i. } V . \\
\text { anguillarum }\end{array}$} & \multirow{3}{*}{$\begin{array}{l}2 \times 10^{\wedge} 11 \text { cells/ } \\
\mathrm{ml}\end{array}$} & $\begin{array}{l}\text { V: } 81 \% \\
\text { C: } 35 \%\end{array}$ & $33 \mathrm{dpv}$ & \multirow{5}{*}{ i.p. } & \multirow{5}{*}{ [176] } \\
\hline & & & & & $\begin{array}{l}\text { V: } 98 \% \\
\text { C: } 80 \%\end{array}$ & $81 \mathrm{dpv}$ & & \\
\hline & & & & & $\begin{array}{l}\text { V: } 61 \% \\
\text { C: } 52 \% \\
\text { (survivors of } 81 \\
\text { dpv challenge) }\end{array}$ & $91 \mathrm{dpv}$ & & \\
\hline & \multirow{2}{*}{$\begin{array}{l}\text { Sea bass } \\
(10-50 \mathrm{~g})\end{array}$} & \multirow{2}{*}{$\begin{array}{l}5 \mathrm{~d} ; \text { Mixed } \\
\text { with feed }\end{array}$} & \multirow{2}{*}{$\begin{array}{l}\text { F.i. } V \text {. } \\
\text { anguillarum }\end{array}$} & \multirow{2}{*}{$\begin{array}{l}2 \times 10^{\wedge} 11 \text { cells/ } \\
\mathrm{ml}\end{array}$} & $\begin{array}{l}\mathrm{V}: 89 \% \\
\mathrm{C}: 59 \%\end{array}$ & $33 \mathrm{dpv}$ & & \\
\hline & & & & & $\begin{array}{l}\text { V: } 29 \% \\
\text { C: } 7.2 \%\end{array}$ & $79 \mathrm{dpv}$ & & \\
\hline $\begin{array}{l}\text { Vibrio } \\
\text { anguillarum }\end{array}$ & $\begin{array}{l}\text { Rainbow } \\
\text { trout } \\
(100-120 \\
\text { g) }\end{array}$ & $\begin{array}{l}16 \text { times on } \\
\text { alternating } \\
\text { days; Mixed } \\
\text { with feed }\end{array}$ & $\begin{array}{l}\text { F.i. } V \text {. } \\
\text { anguillarum } \\
\text { with } 2.5 \mathrm{w} / \mathrm{v} \\
\text { alum }\end{array}$ & $\begin{array}{l}3.5 \mathrm{mg} \text { bacteria/ } \\
\text { fish }\end{array}$ & $\begin{array}{l}\text { V: } 6-22 \% \\
C: 0 \%\end{array}$ & $85 \mathrm{dpv}$ & i.p. & {$[177]$} \\
\hline
\end{tabular}




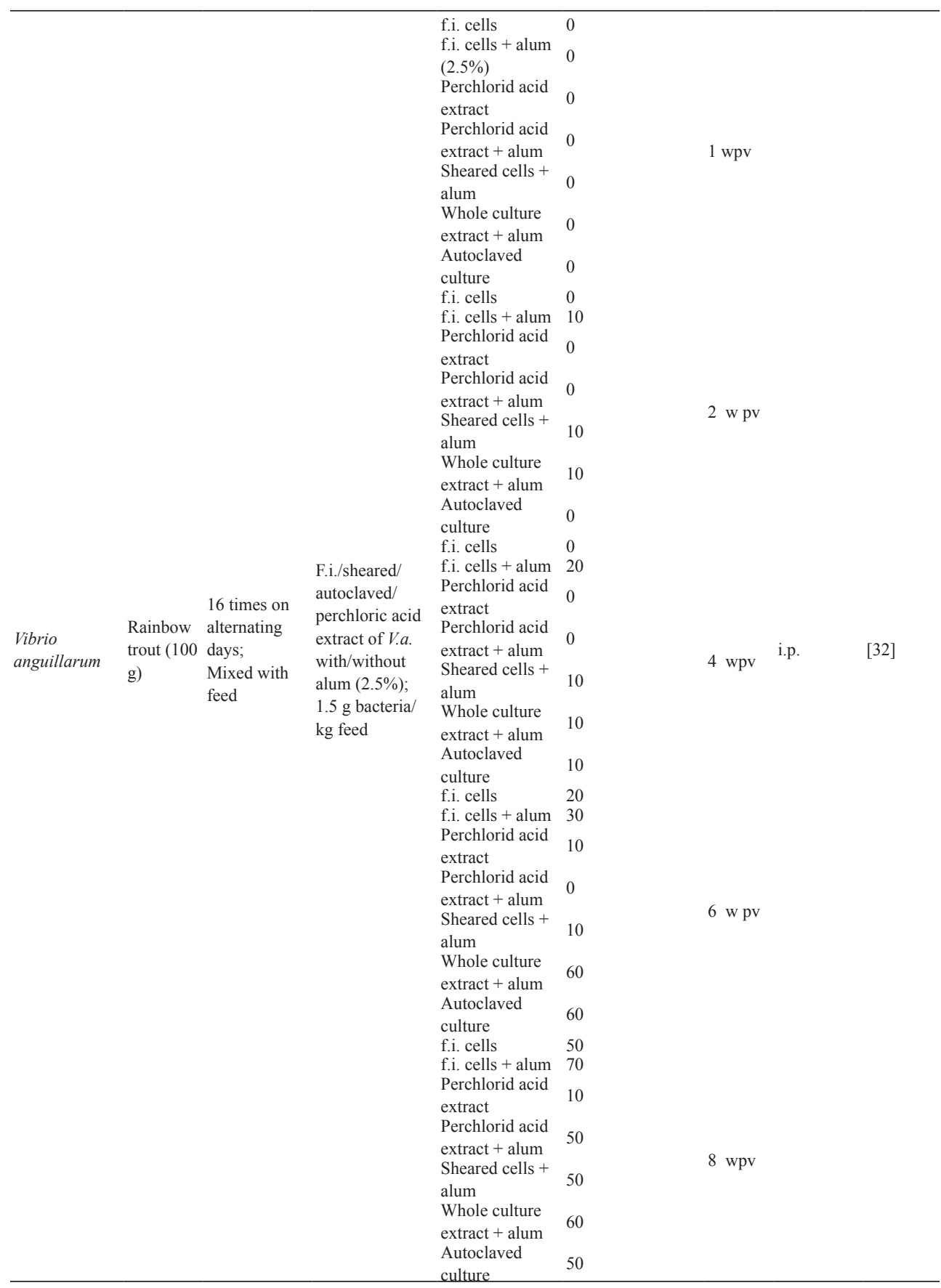




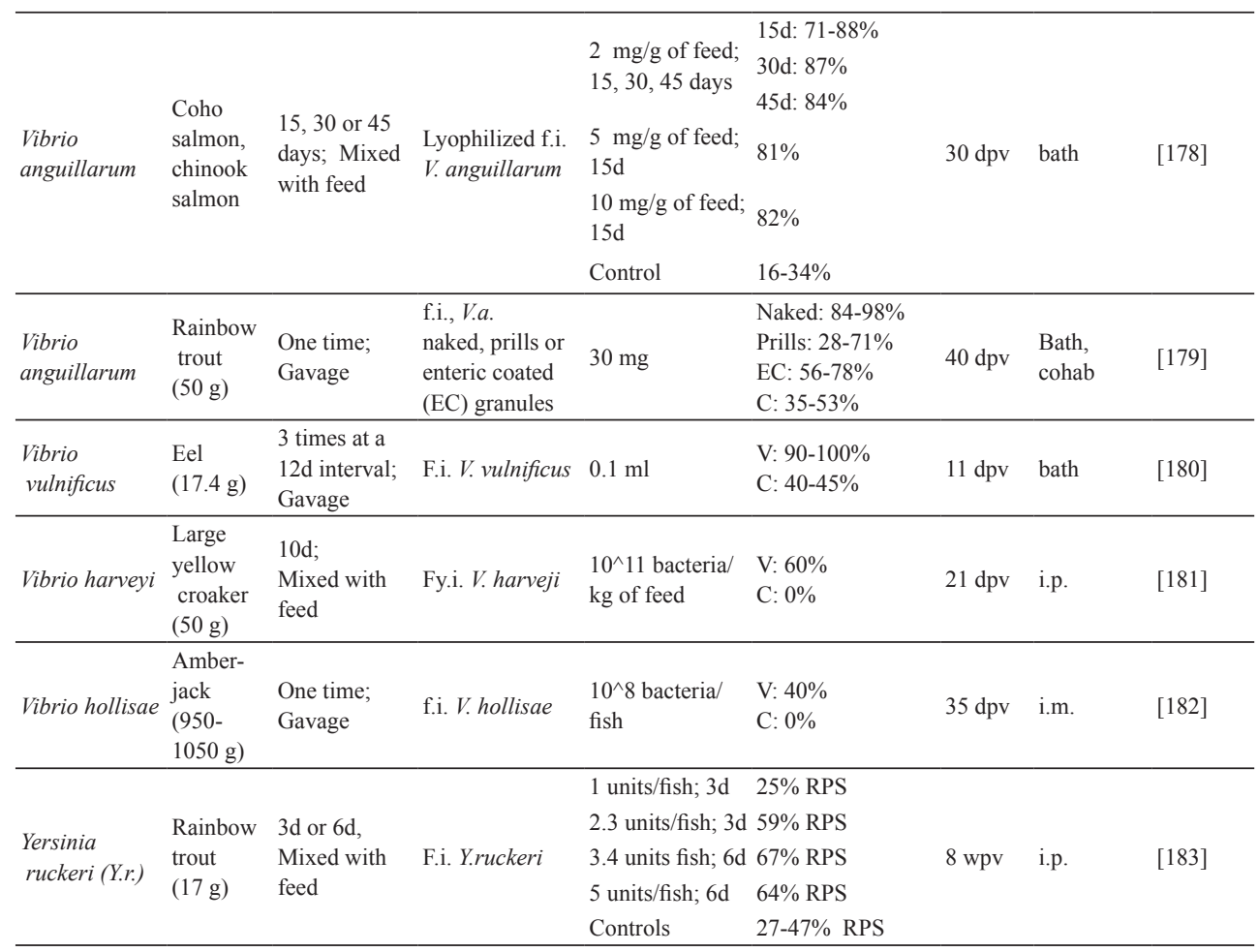

Abbreviations: d: day(s); w: week(s); dpv: days post vaccination; wpv: weeks post vaccination, mpv: months post vaccination; h.i.: heat-inactivated; f.i.: Formalin-inactivated; fy-i. formaldehyde-inactivated, UV-:. UV-inactivated; cohab: cohabitation; FIA: Freund's incomplete adjuvant; BW: body-weight; i.p.: intraperitoneal; i.m: intramuscular. 


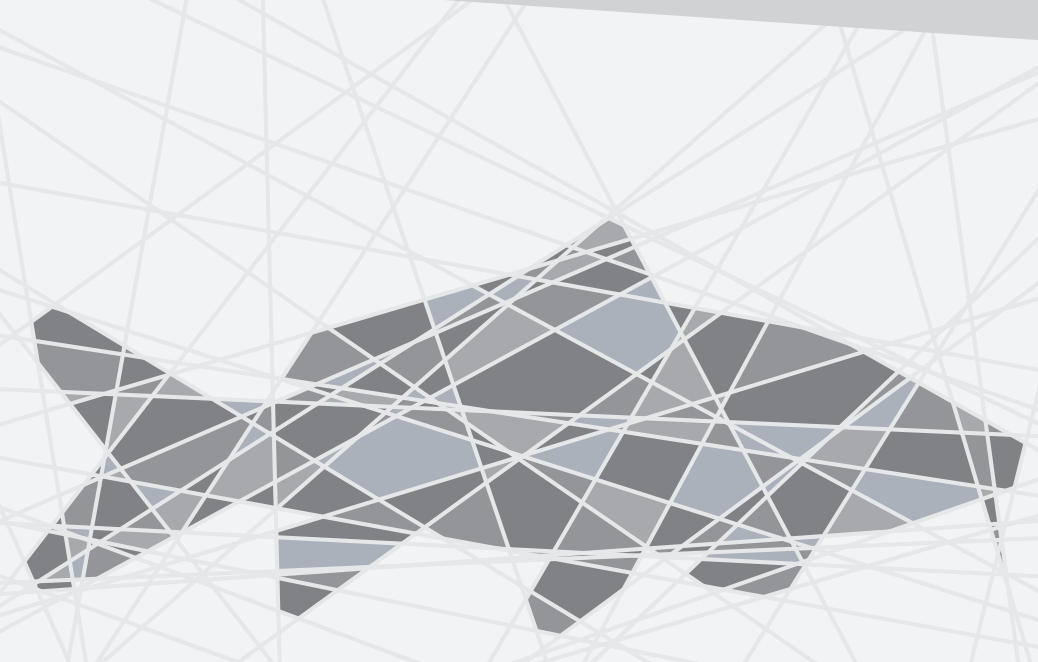




\section{Intramuscular DNA vaccination of juvenile carp against Spring Viraemia of Carp Virus induces full protection and establishes a viral-specific B and $T$ cell response}

\section{Carmen W.E. Embregts', Dimitri Rigaudeau², Tomáš Veselý3, Dagmar Pokorová3, Niels Lorenzen", Jules Petit ${ }^{1}$, Armel Houel${ }^{5}$, Malte Dauber, Heike Schütze ${ }^{6}$, Pierre Boudinot ${ }^{5}$, Geert F.Wiegertjes', Maria Forlenza'}

' Cell Biology and Immunology Group, Department of Animal Sciences, Wageningen University, Wageningen, The Netherlands.

${ }^{2}$ INRA, Infectiologie Expérimentale Rongeurs Poissons, Université Paris-Saclay, Jouy-en-Josas, France ${ }^{3}$ Veterinary Research Institute, Brno, Czech Republic

${ }^{4}$ National Veterinary Institute, DTU, Lyngby, Denmark ${ }^{5}$ INRA, Virologie et Immunologie Moléculaires, Université Paris-Saclay, Jouy-en-Josas, France

${ }^{6}$ Friedrich-Loeffler-Institut, Federal Research Institute for Animal Health, Institute for Infectiology, Insel Riems, Germany

Frontiers of Immunology 8(20I 7), article 1340 doi: 10.3389/fimmu.2017.01340 


\section{Abstract}

Although Spring Viraemia of Carp Virus (SVCV) can cause high mortalities in common carp, a commercial vaccine is not available for worldwide use. Here, we report a DNA vaccine based on the expression of the SVCV glycoprotein $(\mathrm{G})$ which, when injected in the muscle even at a single low dose of $0.1 \mu \mathrm{g} \mathrm{DNA} / \mathrm{g}$ of fish, confers up to $100 \%$ protection against a subsequent bath challenge with SVCV. Importantly, to best validate vaccine efficacy, we also optimized a reliable bath challenge model closely mimicking a natural infection, based on a prolonged exposure of carp to SVCV at $15^{\circ} \mathrm{C}$. Using this optimized bath challenge we showed a strong agedependent susceptibility of carp to SVCV, with high susceptibility at young age (3 months) and a full resistance at 9 months. We visualized local expression of the $\mathrm{G}$ protein and associated early inflammatory response by immunohistochemistry, and describe changes in the gene expression of pro-inflammatory cytokines, chemokines and anti-viral genes in the muscle of vaccinated fish. Adaptive immune responses were investigated by analyzing neutralizing titers against SVCV in the serum of vaccinated fish and the in vitro proliferation capacity of peripheral SVCV-specific $\mathrm{T}$ cells. We show significantly higher serum neutralizing titers and the presence of SVCV-specific T cells in the blood of vaccinated fish, which proliferated upon stimulation with SVCV. Altogether, this is the first study reporting on a protective DNA vaccine against SVCV in carp and the first to provide a detailed characterization of local innate as well as systemic adaptive immune responses elicited upon DNA vaccination that suggest a role not only of $\mathrm{B}$ cells but also of $\mathrm{T}$ cells in the protection conferred by the SVCV-G DNA vaccine. 


\section{Introduction}

The strong increase in the consumption or use of fish products over the last decades has been the result of the ongoing intensification of the whole aquaculture sector. This intensification, however, has led to the increasing incidence of infectious diseases for which no effective vaccines are yet available. In response to this, an increasing emphasis has been placed on the development of experimental vaccines for fish and the investigation of fish immune responses after vaccination [1-4]. Besides the various vaccination strategies currently employed in the aquaculture sector, including intraperitoneal injection or immersion vaccination, experimental DNA vaccination has been reported for a broad range of fish viruses and was shown to be especially effective against fish rhabdoviruses when administered by intra-muscular injection. Furthermore, a major step forwards towards the commercialization of DNA vaccines for fish was made in April 2016 when the European Medicine Agency gave, for the first time, a positive advice towards their use in Europe by granting marketing authorization for the CLYNAV DNA vaccine against Salmon Pancreatic Disease [5]. Effective DNA vaccines against fish rhabdoviruses are reported against Viral Hemorrhagic Septicemia Virus (VHSV) in rainbow trout (Oncorhynchus mykiss) [6], Japanese flounder (Paralichthys olivaceus) [7], turbot (Scophthalmus maximus) [8] and Pacific herring (Clupea pallasii) [9]; against Infectious Hematopoietic Necrosis Virus (IHNV) in Chinook salmon (Oncorhynchus tshawytscha), sockeye salmon (Oncorhynchus nerka) [10] and rainbow trout [11]; and against Hirame Rhabdovirus (HIRRV) in Japanese flounder [12]. Furthermore, combined DNA vaccination against VHSV and IHNV was shown to induce protection against both viruses in rainbow trout [13]. In all these successful vaccines, the DNA plasmid coded for the rhabdovirus glycoprotein $(\mathrm{G})$.

Carp is the most cultured fish species worldwide and the ornamental variant, koi carp, are very high value fish [14]. Their production however is threatened by several bacterial and viral diseases. Among those, Spring Viraemia of Carp (SVC) is caused by SVC Virus (SVCV), a cytopathic virus belonging to the genus Sprivivirus of the Rhabdoviridae family causing an acute systemic infection in several cyprinid species [15] SVC is widespread throughout Europe and has been diagnosed in other parts of the world including the US [16,17] and China [18]. SVCV virions contain one linear negative-sense single-stranded RNA molecule that codes for five structural proteins. 
The G protein of SVCV, which is the only viral protein present on the virion surface and forms trimeric peplomers, binds to cellular receptors to induce viral endocytosis and is the target of protective neutralizing antibodies $[15,19,20]$. Outbreaks of SVCV cause severe losses in carp production, especially during spring, and mainly affect juvenile carp for which mortality rates can be as high as 90\% [15].

To date, DNA vaccines against SVCV have been shown to induce only limited protection [21,22], much lower than reported for G protein-based DNA vaccines against other fish Rhabdoviruses including IHNV and VHSV [23]. Recently, a recombinant Lactococcus plantarum expressing both the SVCV G protein and the Koi Herpes Virus (KHV, Cyprinid Herpesvirus 3) Open Reading Frame 25 (ORF25) was reported to induce moderate protection against both viruses after oral administration [24]. The latter study indicates that oral vaccination against SVCV is also possible, which would allow for relatively stress-free vaccination of large groups of fish simultaneously. However, despite its benefits, only few successful oral vaccines for fish have been reported so far (Reviewed in [25]). While the aforementioned studies show the potential of G protein-based vaccines against SVCV, strong protection against SVCV viral challenge has not been reported thus far for any experimental vaccine. Furthermore, the lack of an optimized challenge model, closely resembling the natural route of SVCV infection, hampered vaccine evaluation as most studies report the use of intraperitoneal injections as the preferred challenge route.

Characterization of local and systemic immune responses after DNA vaccination against rhabdoviruses has been performed for various fish species. A rapid induction of type-I interferons and Interferon Stimulated Genes (ISGs) like $m x$, isg15 and viperin was reported in multiple studies [13,26-28] and can therefore be considered one of the hallmarks of the rapid, non-specific, antiviral response induced by DNA vaccination. The rapid local upregulation of $m x$ was suggested to be the main determinant for the observed cross-protection against IHNV after DNA vaccination using plasmids encoding the G protein of either Snakehead Rhabdovirus (SHRV) or SVCV [29]. The expression of two micro-RNAs (miRNAs), which are strongly induced in rainbow trout either infected with VHSV or DNA vaccinated against VHSV, is apparently correlated with upregulation of type-I ifns, if $n \gamma$ and $m x$ genes and may play a role in the modulation of the response [30].

DNA vaccination of fish also induces an adaptive immune response. In trout, it was found that protection against VHSV was essentially based on the presence of 
neutralizing antibodies, which are detectable at 2-3 weeks after vaccination [13]. $\mathrm{T}$ cell responses have also been studied and it was shown that VHSV-G DNA vaccination induced a specific $\mathrm{T}$ cells response that comprised the same public response as induced by the virus itself [31]. Furthermore, PBLs isolated from VHSV-G DNA vaccinated trout specifically killed VHSV-infected but not IHNVinfected target cells [32]. To date, detailed characterization of the immune response of carp after DNA vaccination against SVCV has not been performed.

In this study, we report the establishment of a reliable SVCV bath challenge for common carp. Using this challenge model we first examined the age-related susceptibility of carp to SVCV, to estimate the best timing of vaccination and the window of high disease susceptibility. We found that carp were most susceptible at the age of 3 months and were fully resistant at 9 months. Having assessed the importance of protecting carp at a young age, we vaccinated 3 -month-old carp at $20^{\circ} \mathrm{C}$, with two doses of an intra-muscularly (i.m.) injected SVCV-G protein-based DNA vaccine, and showed the ability of the vaccine to induce $95-100 \%$ protection against SVCV, even when administered at a low dose $(0.1 \mu \mathrm{g} / \mathrm{g}$ fish $)$. To investigate the rapid local immune response induced after DNA vaccination, we raised an antibody against the SVCV G protein and used it together with leukocyte-specific antibodies to examine the expression of the $\mathrm{G}$ protein in the muscle and the recruitment of leukocytes to the site of injection. By real-time quantitative PCR (RT-qPCR) we characterized the expression of a panel of immune genes related to the innate and adaptive response. Through analysis of virus-specific humoral and cellular responses we investigated the neutralizing activity in serum of vaccinated fish and the presence of antigenspecific T-cells by performing an in vitro proliferation assay.

Altogether this is the first study reporting 1) a reliable SVCV bath challenge model, 2) age-related susceptibility of carp to SVCV 3) the optimization of a G proteinbased DNA vaccine conferring full protection against SVCV and 4) the detailed characterization of local as well as systemic humoral and cellular immune responses triggered upon DNA vaccination in carp. Overall, this report contributes to the understanding of the protective mechanisms triggered by DNA vaccination in carp and will play an essential role in the design of future SVCV-G-based vaccination strategies in carp, the species representing the biggest fish production in the global aquaculture. 


\section{Materials and Methods}

\section{Animals}

European common carp (Cyprinus carpio carpio) R3xR8 were used that originated from a cross between the Hungarian R8 strain and the Polish R3 strain [33]. In this study we will refer to carp as the European common carp subspecies, unless stated otherwise. Carp were bred in the Aquatic Research Facility (ARC) of University's animal facility, Carus at Wageningen University, the Netherlands and were either kept at the local facility, transported to the Veterinary Research Institute (VRI, Brno, Czech Republic), or to the Institut National de la Recherche Agronomique (INRA, Paris, France) for viral challenge experiments. Carp were raised at $20-23^{\circ} \mathrm{C}$ in recirculating UV-treated water and fed pelleted carp food (Skretting, Nutreco) twice daily.

\section{Virus}

The reference SVCV strain VR-1390, isolate stock of the INRA laboratory [34,35], was propagated in Epithelioma Papulosum Cyprinid (EPC) cells grown in Glasgow's modified Eagle's medium (GMEM)-25mM HEPES (Eurobio) supplemented with $10 \%$ foetal calf serum (FCS; Eurobio), 1\% tryptose phosphate broth (Eurobio), $2 \mathrm{mM}$ L-glutamine (PAA), $100 \mu \mathrm{g} / \mathrm{ml}$ penicillin (Biovalley) and $100 \mu \mathrm{g} / \mathrm{ml}$ streptomycin (Biovalley) in the absence of $\mathrm{CO}_{2}$. The SVCV CAPM V 539 strain [36] was propagated in Common Carp Brain (CCB) cells at $27^{\circ} \mathrm{C}$ or in EPC cells at $20^{\circ} \mathrm{C}$. EPCs were grown in MEM medium (Gibco) supplemented with $10 \%$ FCS, 2 $\mathrm{mM}$ L-glutamine, $100 \mu \mathrm{g} / \mathrm{ml}$ penicillin and $100 \mu \mathrm{g} / \mathrm{ml}$ streptomycin in the presence of $\mathrm{CO}_{2}$. For CCBs the same medium was supplemented with $3.5 \mathrm{~g} / \mathrm{L} \mathrm{D}$-glucose and $1 \%$ non-essential amino acids (Gibco). Virus titers were determined by the method of Reed and Muench [37] and were given as plaque-forming units (pfu).

\section{Characterization and validation of a monoclonal antibody against the SVCV G protein}

SVCV of the Fijan strain $[34,38]$ was used to raise antibodies against the SVCV G protein in mice. Before immunization, female Balb/c mice were given a tolerizing treatment in order to reduce reactions to cell proteins, as described before [39]. After such treatment, mice were immunized with $1.4 \times 10^{8}$ pfu of concentrated and purified SVCV in complete Freund's adjuvant. The same viral dose was given 4 weeks later in 
incomplete Freund's adjuvant, followed by two more doses in the following 85 days. Three days after the last immunization mouse spleen cells were isolated and fused with Sp2/0 myeloma cells. Obtained hybridoma supernatants were screened through Western blot and immunofluorescence of SVCV infected and pcDNA3-SVCV-G transfected cells. For Western blot analysis, purified SVCV and lysates from noninfected EPC cells were resolved on 15\% SDS-PAGE gels. Proteins were transferred to nitrocellulose membranes and incubated with hybridoma supernatants $(1: 10)$ or with anti-SVCV rabbit polyclonal serum (1:2000) as positive control. Western blot development was performed as described before [38,39]. For immunofluorescence analysis, EPCs were infected with an MOI of 1 for $24 \mathrm{~h}$ at $20^{\circ} \mathrm{C}$. In parallel, EPCs were seeded in 6-well plates, transfected with $2 \mu \mathrm{g}$ of pcDNA3-SVCV-G or pcDNA3 using $7 \mu$ l of FuGENE HD (Promega) following the manufacturer's guidelines. EPC were imaged $48 \mathrm{~h}$ after transfection. Infected or transfected cells were fixed with $4 \%$ PFA for 15 min at $4^{\circ} \mathrm{C}$ and incubated with hybridoma supernatant from selected clone $13 \mathrm{C} 10 \mathrm{c}(1: 150)$ for $1 \mathrm{~h}$ and with goat-anti-mouse-RPE (BioLegend, 1:500) for $30 \mathrm{~min}$ at room temperature. A counterstaining with DAPI (Thermo Scientific) was included to stain the cells nuclei. Images were acquired with a EVOS fl LED fluorescence microscope (Advanced Microscopy Group (AMG)).

\section{Optimization of SVCV challenge}

For all viral challenges the water temperature was gradually lowered from $20^{\circ} \mathrm{C}$ to $15^{\circ} \mathrm{C}$ at a rate of $1-2^{\circ} \mathrm{C}$ per day. Optimization of the SVCV challenge was performed at INRA, using the VR-1390 strain. Three-month-old carp ( $n=20$ per group, 2-4 g) were challenged by immersion, i.m. or intra-peritoneal (i.p.) injection. For immersion challenge, carp were exposed to a dilution of SVCV-containing EPC supernatants (8 $\mathrm{x} 10^{6} \mathrm{pfu} / \mathrm{ml}$ ) for either $3 \mathrm{~h}$ or $48 \mathrm{~h}$ in a volume of $4 \mathrm{~L}$ (biomass $15 \mathrm{~g} / \mathrm{L}$ ). A control group $(n=20)$ was exposed to uninfected EPC cell culture supernatant by immersion and was treated similarly. Fish were also challenged by injection; they were anesthetized using $0.3 \mathrm{~g} / 1$ Tricaine Methane Sulfonate (TMS, Crescent Research Chemicals) before i.m. injection of $1000 \mathrm{pfu} /$ fish or i.p injection of 5000 or $10000 \mathrm{pfu} / \mathrm{fish}$. Water quality monitoring included $\mathrm{pH}(8)$ and oxygen $(>7 \mathrm{mg} / \mathrm{L})$. Fish were observed daily and moribund fish were removed from the tanks.

Age-related sensitivity to SVCV infection was investigated at VRI, using the SVCV CAMP V 539 strain and juvenile carp between 3 and 9 months (20 g). R3xR8 carp, all 
from the same hatching batch, were raised under the same condition (water flow (15 $\mathrm{L} / \mathrm{h}) \mathrm{pH}(7.5-8)$, oxygen $(10-12 \mathrm{mg} / \mathrm{L})$ and $\mathrm{N}-\mathrm{NH} 4(<0.2 \mathrm{mg} / \mathrm{L}))$ up until 3 months of age. At that time, a subgroup of fish ( $n=20$ per group) was transferred to $100 \mathrm{~L}$ tanks having the same water conditions, were acclimatized to the temperature of $15^{\circ} \mathrm{C}$ and challenged by immersion for $30 \mathrm{~h}$ with $8 \times 10^{6} \mathrm{pfu} / \mathrm{ml}$. The remaining fish were kept under controlled water quality condition up until 7 and 9 months, and then challenged following the same protocol. Fish were observed daily and moribund fish were removed from the tanks.

\section{DNA vaccination}

The pcDNA3-SVCV-G DNA vaccine was prepared as described previously [32] using the G protein sequence of the CAPM V 539 strain Jaroslavicky 97 (Accession number: KU934300). All vaccinations were performed at $20^{\circ} \mathrm{C}$. Carp of 3 months ( $n=10$ per group, $1.5-2 \mathrm{~g}$ ) were assigned to either the pcDNA3 empty plasmid group (negative control) or the pcDNA3-SVCV-G group (vaccine). Carp were anesthetized in $0.3 \mathrm{~g} / 1 \mathrm{TMS}$ and vaccinated i.m. in the epaxial muscle, below the dorsal fin with $1 \mu \mathrm{g}$ (first experiment) or $0.1 \mu \mathrm{g}$ (second experiment) of DNA plasmid/g of fish in $10 \mu 1$ PBS.

For assessment of vaccine efficacy, carp were challenged 2.5-3 months after vaccination, at $15^{\circ} \mathrm{C}$, using the optimized challenge method, and survival was monitored over a period of 3-5 weeks. In parallel, mid kidneys were isolated from a subgroup of carp $(n=6)$ to confirm SVCV infection through analysis of $s v c v n$ gene expression. The mid kidney is one of the organs in which virus replication occurs and can be used for virus re-isolation of monitoring of viral infection.

\section{RNA isolation and CDNA synthesis}

For gene expression analysis of the local response at the injection site, carp injected with the low plasmid dose $(0.1 \mu \mathrm{g} / \mathrm{g}$ fish $)$ were sacrificed at 3 and 5 days post-injection (dpi). Carp were euthanized in $0.6 \mathrm{~g} / \mathrm{l} \mathrm{TMS}$ and bled through the caudal vein. Muscle at the injection site was isolated, immediately snap frozen in liquid nitrogen, and stored at -80 until further processing. For the analysis of $s v c v-n$ gene expression, mid kidney was isolated at various time-points post infection, since this is the most suitable organ for detection of SVCV at early time points post-infection [40].

Total RNA was isolated from muscle and mid kidney tissue using the RNeasy Mini Kit (Qiagen) according to the manufacturer's instructions including on-column 
DNase treatment using the RNase-free DNase set (Qiagen). For RNA isolation from muscle tissue an additional Proteinase-K (Qiagen) treatment was included. RNA concentrations were measured using a Nanodrop-1000, the integrity was verified on a $1 \%$ agarose gel and RNA was stored at $-80^{\circ} \mathrm{C}$ until further use. Prior to cDNA synthesis of $1 \mu \mathrm{g}$ total RNA, a second DNase treatment was performed using DNAse I, Amplification Grade (Invitrogen). Reverse transcription of the RNA was performed using random primers (300 ng) and Superscript ${ }^{\mathrm{TM}}$ III (200U) First Strand Synthesis Systems for RT-PCR (Invitrogen). cDNA samples were further diluted 25 times in nuclease-free water and stored at $-20^{\circ} \mathrm{C}$.

\section{Gene expression analysis}

Real-time quantitative PCR (RT-qPCR) was performed using a Rotor-Gene ${ }^{\text {TM }} 6000$ (Qiagen). Fluorescence data were analyzed using Rotor-Gene Q series software version 2.3.1. Briefly, $5 \mu 1$ of 25 times diluted cDNA was mixed with $2 \mu 1$ of forward and reverse primers $(2.1 \mu \mathrm{M}$ of each primer) and $7 \mu 1$ of $2 \mathrm{x}$ ABsolute qPCR SYBR Green Mix (Thermo Scientific) as detection chemistry. The list of primers can be found in Table 1. The take-off value for each sample and the average reaction efficiencies $(E)$ for each primer set were obtained upon comparative quantitation analysis from the Rotor-Gene software [41]. The relative expression ratio (R) of each sample was calculated according to the Pfaffl method [42] based on the take-off deviation of sample versus each of the unhandled controls at time point $0 \mathrm{~h}$ and normalized relative to the $s 11$ protein of the $40 s$ subunit as reference gene. For analysis of the $s v c v-n$ gene during infection with SVCV the housekeeping gene $\beta$-actin was used, since it was found to be the most stable under these circumstances.

\section{Immunohistochemistry}

In order to visualize the expression of the SVCV G protein at the site of injection, carp (3-4 g) were i.m. injected with $20 \mu \mathrm{g}$ of pcDNA3 (empty plasmid) or pcDNA3SVCV-G plasmid in $20 \mu \mathrm{PBS}$ containing $0.01 \%$ green tattoo dye (Eickemeyer). Carp were sacrificed 7 and 14 days dpi and bled through the caudal vein before collecting muscle tissue at the site of injection.

Cryosections $(5 \mu \mathrm{m})$ from muscle sections were stained with specific antibodies as described before [43]. For the detection of the SVCV-G protein, slides were stained with anti-SVCV-G clone $13 \mathrm{C} 10 \mathrm{c}$ diluted $1: 150$ and alkaline phosphatase (AP) conjugated goat-anti-mouse (Dako) (1:200). Development was performed using AP 
Table 1. Primers used in RT-qPCR

\begin{tabular}{|c|c|c|c|}
\hline Primer & FW primer 5'-3', & RV primer 3'- 5' & Acc. No. \\
\hline \multicolumn{4}{|c|}{ Housekeeping genes } \\
\hline $40 s$ & CCGTGGGTGACATCGTTACA & $\begin{array}{l}\text { TCAGGACATTGAACCTCA- } \\
\text { CTGTCT }\end{array}$ & AB012087 \\
\hline$\beta$-actin & $\begin{array}{l}\text { CAACAGGGAAAAGATGAC- } \\
\text { ACAGATC }\end{array}$ & GGGACAGCACAGCCTGGAT & ССАСТВА \\
\hline \multicolumn{4}{|c|}{ SVCV detection } \\
\hline$s v c v-n$ & TGAGGTGAGTGCTGAGGATG & $\begin{array}{l}\text { CCATCAGCAAAGTCCCGGT- } \\
\text { AT }\end{array}$ & NC_002803 \\
\hline \multicolumn{4}{|l|}{ Cytokines } \\
\hline cxca & CTGGGATTCCTGACCATTGGT & $\begin{array}{l}\text { GTTGGCTCTCTGTTTCAATG- } \\
\text { CA }\end{array}$ & AJ421443 \\
\hline$c x c b 1$ & GGGCAGGTGTTTTTGTGTTGA & AAGAGCGACTTGCGGGTATG & AB082985 \\
\hline$c x c b 2$ & $\begin{array}{l}\text { AGGCAGGTGCTTCTGTGCTG- } \\
\text { ACA }\end{array}$ & $\begin{array}{l}\text { TTCATGCATTTCCGCTCTGCG- } \\
\text { CT }\end{array}$ & JN104598 \\
\hline$i l 1 b$ & AAGGAGGCCAGTGGCTCTGT & $\begin{array}{l}\text { CCTGAAGAAGAGGAGGAGG- } \\
\text { CTGTCA }\end{array}$ & AJ245635 \\
\hline il6a & CAGATAGCGGACGGAGGGGC & GCGGGTCTCTTCGTGTCTT & KC858890 \\
\hline$i l 6 b$ & GGCGTATGAAGGAGTGAGGG & TGCTCCTCTCTCGGTCAGAT & KC858889 \\
\hline tnfa & GCTGTCTGCTTCACGCTCAA & $\begin{array}{l}\text { CCTTGGAAGTGACATTTGCT- } \\
\text { TTT }\end{array}$ & $\begin{array}{l}\text { AJ311800 } \\
\& \text { AJ311801 }\end{array}$ \\
\hline \multicolumn{4}{|c|}{ Transcription factors } \\
\hline stat1 & GAGACGGAGGAATCACC & GGATGTCTGGGTAAAGGTAG & KJ782028 \\
\hline \multicolumn{4}{|c|}{ Interferons } \\
\hline ifn $\gamma 2 a / 2 b$ & $\begin{array}{l}\text { CGATCAAGGAAGATGACCCA- } \\
\text { GTC }\end{array}$ & $\begin{array}{l}\text { GTTGCTTCTCTGTAGACACG- } \\
\text { CTTC }\end{array}$ & AM168523 \\
\hline ifn $\varphi 1$ & GCACGTATACAAAGATGAACC & TGATCCAAGGTCAAGACAAG & GQ168341 \\
\hline ifn $\varphi 2$ & TTGGTGTAAAAAAGGCAACC & GCTGCTTTCTCGTCATAATAC & JN741616 \\
\hline \multicolumn{4}{|c|}{ Interferon stimulated genes } \\
\hline$m x 1$ & ACAATTTGCGGTCTTTGAGA & CCCTGCCATTTCTCTTCG & cypCar_00015892 \\
\hline$m \times 2$ & GCTTACGGTCTCTGGGG & $\begin{array}{l}\text { TGGTTTCATCTTTAGTTCTTA- } \\
\text { TCATC }\end{array}$ & cypCar_00029512 \\
\hline vip2 & CTGTCGGACACATCAGC & TCAATGGGCAAGACGAAA & cypCar_00024055 \\
\hline pkr3 & CACGGTGTTTGAAAAGAGC & GACTGGGTCTCAGCATTC & cypCar_00039221 \\
\hline isg15.2 & AGTGTTCGTCAAGAATGAGG & CCTCGCAGACGGAAAAC & cypCar_00039111 \\
\hline \multicolumn{4}{|c|}{ Adaptive immune genes } \\
\hline igm & CACAAGGCGGGAAATGAAGA & $\begin{array}{l}\text { GGAGGCACTATATCAACA- } \\
\text { GCA }\end{array}$ & AB004105 \\
\hline igt1 & AAAGTGAAGGATGAAAGTGT & TGGTAACAGTGGGCTTATT & AB598367 \\
\hline igt2 & GATTCTACTGGGT8CTTCAC & GACATCACTCAACTC8TTCT & AB598368 \\
\hline zap70 & GGAACAAGCCATCATTAGCC & GTCGTCTCTCACCCTCCTG & Scaf $2523 \& 63374$ \\
\hline
\end{tabular}


substrate $(4.5 \mu \mathrm{l} / \mathrm{ml}$ nitro-blue-tetrazolium (Roche Applied Science) and $3.5 \mu \mathrm{l} /$ ml 5'-bromo-4'-chloro-3'indolyl phosphatase (BCIP; Roche Applied Science) in AP buffer $\left(0.1 \mathrm{M}\right.$ Tris- $\left.\mathrm{Cl}, 0.1 \mathrm{M} \mathrm{NaCl}, 0.05 \mathrm{M} \mathrm{MgCl}_{2}\right)$ until sufficient staining was observed. For the detection of neutrophilic granulocytes, slides were stained with the TCLBE8 antibody $(1: 50,[43,44])$ and GAM-AP (1:200, Dako). Development was performed as described above. Tissue morphology was examined using a Hemacolor ${ }^{\circledR}$ eosin-azur staining (Merck Millipore). Pictures were made using a Leica DM6 microscope and analyzed using the Leica LAS X program.

\section{Neutralization assay}

Sera from fish injected with $1 \mu \mathrm{g} / \mathrm{g}$ of either pcDNA3 or pcDNA3-SVCV-G plasmid were collected 2.5-3 months after vaccination and were used to quantify neutralizing titers. Blood was drained from the caudal vein and was let to clot at $4{ }^{\circ} \mathrm{C}$ overnight. Serum extraction was performed by centrifugation at $2000 \mathrm{~g}$ for $10 \mathrm{~min}$ and the obtained supernatant was centrifuged at $10.000 \mathrm{~g}$ for $20 \mathrm{~min}$. Serum was heat-treated at $56^{\circ} \mathrm{C}$ for $30 \mathrm{~min}$, aliquoted and frozen at $-20^{\circ} \mathrm{C}$ prior to use in titration assays. To determine SVCV neutralization titers, carp serum was mixed with an equal volume of GMEM 2\% FCS, DEAE 1 X (Sigma), containing carp complement (standardized serum pool from naïve carp, diluted $1: 80$ ), and incubated $4 \mathrm{~h}$ at $20^{\circ} \mathrm{C}$ with $2.1 \times 10^{2}$ pfu SVCV (strain VR-1390). One hundred $\mu 1$ of the mixture was then transferred onto confluent EPC monolayers in 24 wells and incubated for one hour at $14^{\circ} \mathrm{C}$. After this step, melted methylcellulose was added to the wells and plates were kept at $24^{\circ} \mathrm{C}$ for three days. After three days, plates were fixed with $4 \%$ formaldehyde and stained with crystal violet to reveal viral plaques. Controls included non-infected wells and wells infected with SVCV without pre-incubation with carp serum. Plaques were counted manually and an upper threshold on the number of counted plaques, indicating a fully infected well, was set at 100 plaques.

\section{In vitro antigen-specific $B$ and $T$ cell proliferation assays}

Carp were vaccinated i.m. with $1 \mu \mathrm{g}$ DNA/g of fish of pcDNA3-SVCV-G DNA vaccine. The same amount of pcDNA3 plasmid was used as injection control. PBLs were isolated 3 months after vaccination, separated on Ficoll Paque (GE Healthcare) as described previously $[45,46]$, and stained with Carboxyfluorescein succinimidyl ester (CFSE) [47]. Part of the CFSE-labelled cells $\left(2 \times 10^{7}\right.$ cells $\left./ \mathrm{ml}\right)$ were transferred to round-bottom 96-well culture plates (Corning) and stimulated with SVCV (MOI 
of 25) or, as a control, with equivalent volumes of CCB-conditioned culture medium, for 2 hours at $27^{\circ} \mathrm{C}$. Cells were then seeded in 48-well plates (Corning) at a density of $2 \times 10^{6} /$ well in Advanced DMEM/F-12 (Life Technologies) supplemented with 2 $\mathrm{mM} \mathrm{L}$-glutamine, $100 \mathrm{U} / \mathrm{ml}$ penicillin $\mathrm{G}, 50 \mathrm{mg} / \mathrm{ml}$ streptomycin sulphate, $1 \%$ FCS and $10^{-5} \mathrm{M}$ 2-Mercaptoethanol (Sigma). In parallel, a fraction of both SVCV-treated or mock-treated cells was incubated in the presence of recombinant carp interferon gamma (Ifn $\gamma 2,100 \mathrm{ng} / \mathrm{ml}$ ) or interleukin-10b (rIl10b, $0.25 \mathrm{U} / \mathrm{ml}$ ) since we reported before that these cytokines are able to enhance carp leukocytes pro-inflammatory activities [46] and (antigen-specific) proliferation of memory cells [45,47-49]. Cells were incubated for 6 days at $27^{\circ} \mathrm{C}$ in the presence of $5 \% \mathrm{CO}_{2}$.

Proliferation of Zap70 ${ }^{+} \mathrm{T}$ cells and $\mathrm{Igm}^{+} \mathrm{B}$ cells was analyzed by flow cytometry using a cross-reactive antibody for the pan T cell marker Zap70 [47] and the mouse monoclonal antibody (WCI12) against carp Igm [52]. Cells were collected after 6 days, washed once with PBS and incubated for 30 min at RT with Zombie Red ${ }^{\mathrm{TM}}$ fixable viability dye (1:1000 in PBS, BioLegend). For the subsequent analysis of T cell proliferation, cells were washed once with FACS buffer (0.5\% BSA (Roche), $0.01 \% \mathrm{NaN}_{3}$ in PBS) and were fixed and permeabilized using the Cytofix/Cytoperm Kit (BD Biosciences) according to the manufacturer's protocol. Cells were washed once in FACS buffer and incubated for $30 \mathrm{~min}$ on ice in $35 \mu \mathrm{l}$ of anti-Zap70 rabbit mAb (99F2; Cell Signaling, 1:100). After two washes with FACS buffer, cells were incubated in $35 \mu 1$ of PE-conjugated goat-anti-rabbit IgG (Santa Cruz, 1:100). For B cell proliferation, cells were washed and incubated as described above with WCI12 $\mathrm{mAb}(1: 100)$ and goat anti-mouse IgG-PE (Santa Cruz, 1:100). After subsequent washes, cells were analyzed on a FACS CantoA (BD Biosciences) and data were analyzed using FlowJo V10 (BD Biosciences). Proliferation of B or T cells was analyzed by first gating on the live cells (Zombie $\operatorname{Red}^{\mathrm{TM}}$, negative) subsequently for the specific cell staining (either WCI12 for Igm ${ }^{+} \mathrm{B}$ cells or Zap70 for total T cells, PE channel), and finally for the CFSE staining (visible in the FITC channel) of the identified population. The intensity of the CFSE staining at day zero was used to set the thresholds for proliferation. At day six, a decrease in CFSE fluorescence intensity was indicative of cell proliferation. Obtained percentages of proliferation in each treated group were corrected with their respective conditioned medium-only control by subtraction. 


\section{Statistical analysis}

Statistical analysis was performed for gene expression, neutralization assay and proliferation assay data. All data was analyzed using SPSS Software 22 (IBM). For gene expression data, relative expression ratios $R$ were transformed (LN(R)) and significant differences $(\mathrm{p}<0.05)$ between pcDNA3 and pcDNA3-SVCV-G at the indicated time point were determined by a one-way ANOVA followed by the Tukey post-hoc test. Plaque count of the neutralization assay and the percentages of proliferating Zap $70^{+} \mathrm{T}$ cells from the proliferation assay were analyzed using a oneway ANOVA.

\section{Results}

\section{Prolonged exposure of juvenile carp to SVCV at $15^{\circ} \mathrm{C}$, results in a reliable bath challenge model of infection}

To evaluate vaccine efficacy, the establishment of a reliable and reproducible infection model is of utmost importance. To our knowledge, the first optimization of a SVCV bath challenge was described in 1978 by Ahne [53] exposing 25-30 g carp fish to $2 \mathrm{x}$ $10^{3} \mathrm{pfu}$ for $2 \mathrm{~h}$ at $13^{\circ} \mathrm{C}$. Subsequent reports describe similar procedures. In our hands, however, such protocol resulted in high variability between replicate challenges and generally low mortality rates (data not shown). Therefore we first established a reliable and robust (bath) infection model closely mimicking the natural route of virus infection. We initially investigated the effect of virus dose, time of exposure and infection route on infection efficacy.

Carp of 3 months were exposed to SVCV (VR-1390 strain) by i.m. or i.p. injection as well as by bath at a temperature of $15^{\circ} \mathrm{C}$. Challenge by i.m. or i.p. injection resulted in mortality rates of $35 \%$ and $15-25 \%$ respectively, independent of the viral dose used (Fig. 1A). Similar mortality rates were observed after bath challenge for $3 \mathrm{~h}$ at $8 \times 10^{6} \mathrm{pfu} / \mathrm{ml}$. Bath challenge using the same viral load but with an exposure time of $48 \mathrm{~h}$ resulted in high mortality (up to 90\%) within 15 days. Altogether the data indicate that prolonged exposure of juvenile carp to SVCV, in a bath challenge at $15^{\circ} \mathrm{C}$, results in high mortality rates.

Using the optimized bath challenge method we next investigated the effect of age on SVCV susceptibility in order to look for the best time window to later on investigate vaccine efficacy. To test the robustness of the bath challenge we used the closely 
related SVCV CAMP V 539 strain in an inter-laboratory experimental setup. For this, 3, 7 or 9-month-old carp were exposed to $8 \times 10^{6} \mathrm{pfu} / \mathrm{ml}$ of the CAPM V 539 SVCV strain by bath for $30 \mathrm{~h}$ hours at $15^{\circ} \mathrm{C}$. A strong age-dependent effect on disease susceptibility was observed (Fig. 1B). While again up to $90 \%$ mortality could be observed in 3-month-old carp, mortality rates rapidly decreases to $50 \%$ in 7-monthold fish and only $0-20 \%$ mortality was observed in 9-month-old carp. Altogether we developed a reliable bath challenge with optimized temperature, viral load and exposure time. Using this optimized bath challenge we show that susceptibility of carp to SVCV is age-dependent and that the optimized challenge is reliable independent on the viral strain used.

\section{Intra-muscular vaccination using a G protein-based DNA vaccine induces 95- $100 \%$ protection against SVCV, even when administered at a low dose}

Optimization of the bath challenge model showed that carp are most susceptible to SVCV at young age (between 3 and 6 months). Therefore, to test the efficiency of DNA vaccination, carp were vaccinated at an age of 3 months and subsequently challenged at an age of 6 months (2.5-3 months after vaccination). This assured that we were still within the age-window of high susceptibility to SVCV ( $<6$ months), but also that the strong non-specific, type-I IFN-dependent protection induced by DNA vaccination in fish would have faded [23].

Carp were vaccinated at $20^{\circ} \mathrm{C}$ with $1 \mu \mathrm{g}$ of pcDNA3-SVCV-G/g fish and challenged 2.5 months later at $15^{\circ} \mathrm{C}$ using the aforementioned bath challenge. While survival in the pcDNA3-injected group was around $40 \%$, the pcDNA3-SVCV-G-vaccinated group had $90 \%$ survival (Fig.1C), which is equivalent to a relative percent survival (RPS) of 83.3. To investigate whether a lower dose of vaccine would be sufficient to protect against SVCV, in a subsequent experiment carp were vaccinated with $0.1 \mu \mathrm{g}$ of the vaccine/g of fish and challenged as described. In this experiment, full protection (100 RPS) in the vaccinated group was observed 2.5 months after vaccination (Fig. 1C). In the group vaccinated with the high vaccine dose, the development of a SVCV infection was verified by analysis of the SVCV N gene expression in mid kidneys of vaccinated and non-vaccinated fish after SVCV challenge. Svcv $n$ gene expression was detected from 2 days post-infection onwards in non-vaccinated fish (pcDNA3) but not in vaccinated fish (pcDNA3-SVCV-G) (Fig.1D), confirming that vaccination with the pcDNA3-SVCV-G vaccine strongly suppressed SVCV infection. No expression of the $\mathrm{N}$ gene was observed in non-challenged fish (data not shown). 
Altogether, the results indicate that the pcDNA3-SVCV-G vaccine, even at a low dose of $0.1 \mu \mathrm{g} / \mathrm{g}$ fish, is able to confer protection against SVCV for a period long enough to cover the age-window during which carp are most susceptible to SVCV.

A

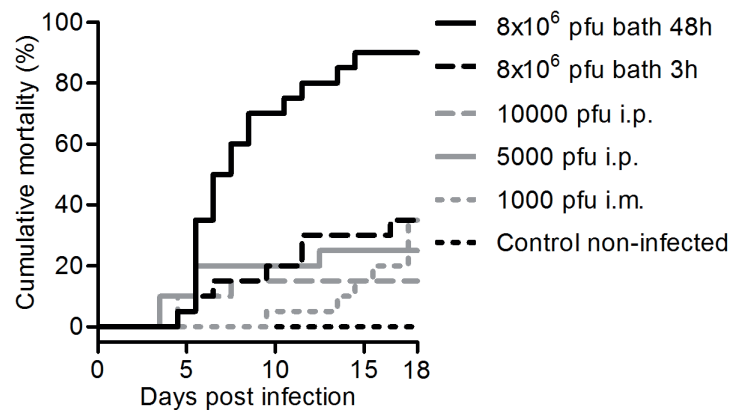

C

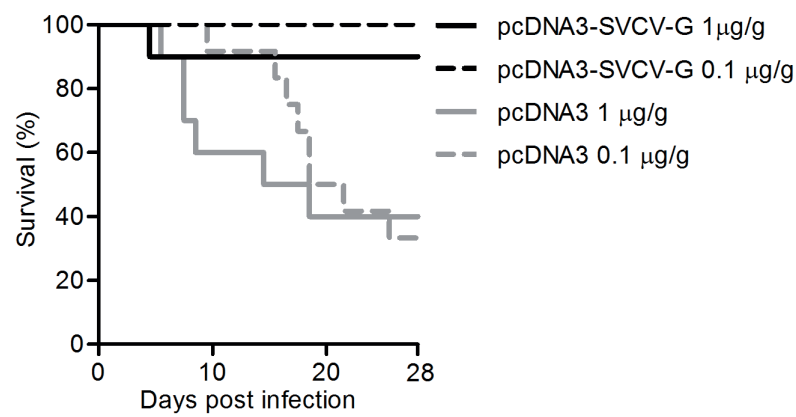

B

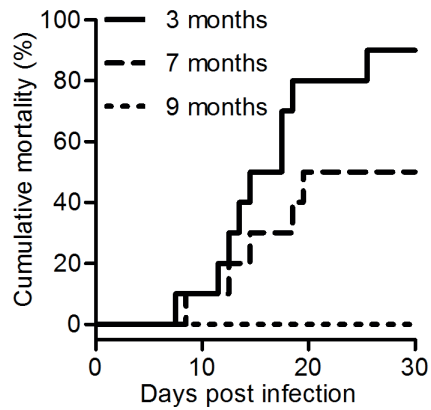

D

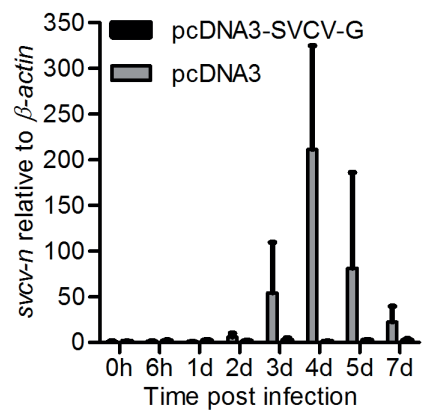

Fig. 1. Establishment of a bath challenge for SVCV and validation of a protective DNA vaccine. (A) Carp ( $n=20 /$ group) of 3 months were acclimatized to a temperature of $15^{\circ} \mathrm{C}$, exposed to the indicated doses of SVCV (VR-1390 strain), for the indicated time, and mortality was recorded. (B) Carp ( $n=10)$ were challenged at 3, 7 or 9 months of age by bath for 30 hours at $15^{\circ} \mathrm{C}$ using $8 \times 10^{6} \mathrm{pfu} / \mathrm{ml}$ of the CAPM V 539 SVCV strain and mortality was recorded. (C) Three-month-old carp ( $n=10 / \mathrm{group}$ ) were i.m. injected with $1 \mu \mathrm{g} / \mathrm{g}$ of fish of either pcDNA3 or pcDNA3-SVCV-G and challenged 2.5 months post-vaccination, for 48 hours at $15^{\circ} \mathrm{C}$ using $8 \times 10^{6} \mathrm{pfu} / \mathrm{ml}$ SVCV (VR-1390). In a subsequent experiment, fish were vaccinated with 0.1 $\mu \mathrm{g} / \mathrm{g}$ of fish of the same plasmids and challenged as described. Survival was monitored over a period of 4 weeks. (D) Carp were treated as in C) using $1 \mu \mathrm{g} / \mathrm{g}$ of fish of DNA plasmid and upon challenge mid kidneys were isolated at the indicated time points. svcv-n gene expression was analyzed by RT-qPCR. Gene expression was normalized relative to $\beta$-actin as a housekeeping gene and expressed relative to the unhandled controls collected at time point Oh. Data are shown as average + SD of $n=6$ fish. Abbreviations: h: hour, d: day.

\section{SVCV-G protein is expressed in the muscle after DNA vaccination and triggers a strong local immune response}

In order to visualize the expression of the SVCV G protein after i.m. administration, and to investigate the tissue damage as well as the local immune response, carp were injected with $20 \mu \mathrm{g}$ of either pcDNA3 or pcDNA3-SVCV-G plasmid. Muscle tissue from the site of injection was excised at 7 and 14 dpi from both groups and the 
anti-SVCV-G antibody was used to visualize G protein expression. To visualize the influx of leukocytes after vaccination a Hemacolor ${ }^{\circledR}$ eosin-azur staining was used, as well as specific antibody staining for neutrophilic granulocytes and macrophages. A strong influx of leukocytes was observed at $7 \mathrm{dpi}$ in muscle tissue of both pcDNA3 injected (Fig. 2A.2) and pcDNA3-SVCV-G vaccinated group (Fig. 2A.3), indicating that the influx is strongly damage- and inflammation-driven, mainly caused by the injection itself. Myocytes expressing the viral $G$ protein on their cell membrane were detected at 7 dpi as well as 14 dpi (Fig. 2B.2-3 and 2C). At 7 dpi, myocytes expressing the $\mathrm{G}$ protein were found to be surrounded by a large number of leukocytes and were disconnected from neighboring cells. Furthermore, the leukocytes appeared to infiltrate myocytes positive for G-protein expression (Fig. 2B.3, black arrows).

In order to verify the presence of specific leukocytes subtypes in the area surrounding the $\mathrm{G}$ protein expressing cells, an antibody specific to neutrophilic granulocytes was used (Fig. 2B.4-6). An influx of neutrophils was observed at 7 dpi in muscle injected with the pcDNA3plasmid (Fig. 2B.4) and to a larger extent in the pcDNA3-SVCV-G injected tissue (Fig. 2B.5-6). Moreover, the neutrophil-specific staining revealed that a large proportion of leukocytes surrounding $\mathrm{G}$ protein-expressing myocytes are neutrophils. Macrophages were recruited to the site of injection at this time point as well, but in lower numbers than neutrophils (data not shown).

A prominent change in muscle morphology at the injection site was observed at 14 dpi, with a clear deterioration of the muscle tissue. At this time-point the $G$ proteinexpressing myocytes were condensed, as indicated by the concentrated $G$ protein staining, detached from the surrounding myocytes and completely surrounded by leukocytes (Fig. 2C).

In conclusion, we found an injection-related inflammation in the muscle. $G$ proteinexpressing myocytes were clearly surrounded by large numbers of leukocytes, especially neutrophils. At 14 dpi a complete isolation of the $G$ protein-expressing myocytes from the surrounding muscle tissue was observed, along with cell condensation. Altogether this suggests that $G$ protein expression in the tissue leads to a robust response against $\mathrm{G}$ protein-expressing cells, which in turn might favor activation of protective mechanisms. 
A
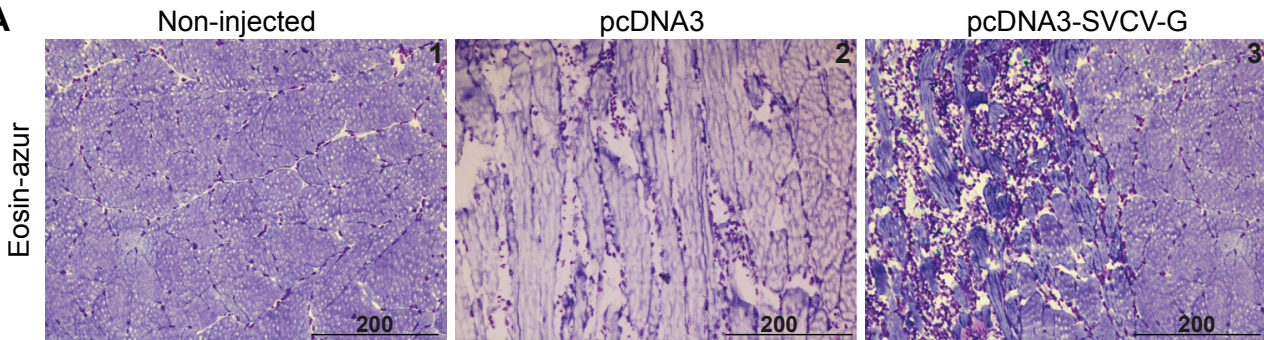

B
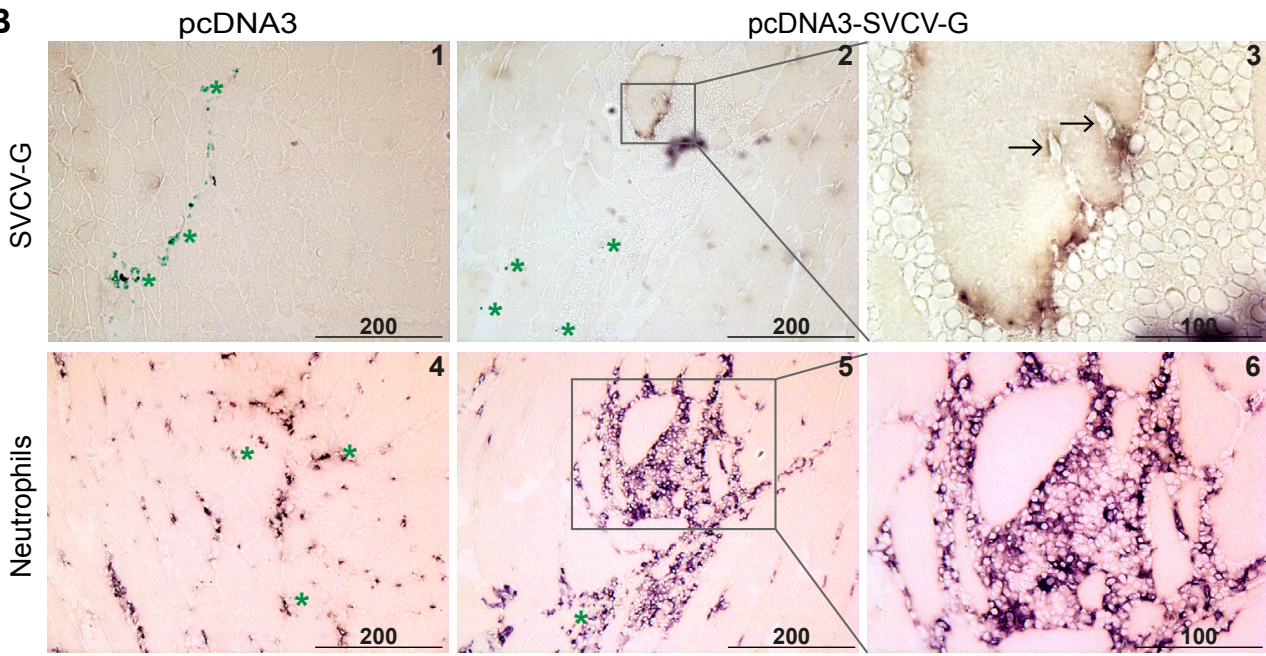

C
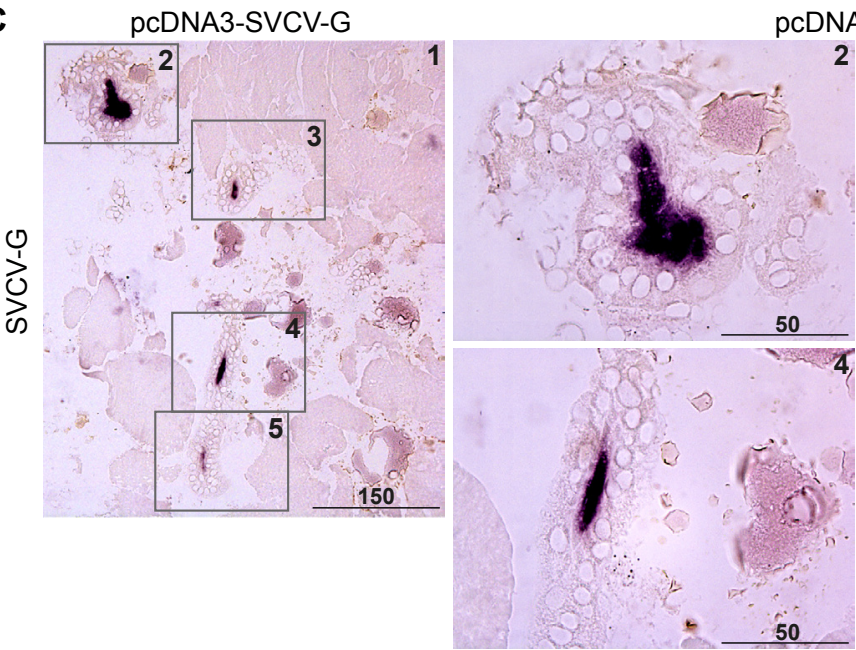

Fig. 2. Immunohistochemical analysis of $\mathrm{G}$ protein expression and leukocyte recruitment after DNA vaccination. Carp were injected with $20 \mu \mathrm{g}$ of pcDNA3 or pcDNA3SVCV-G. (A) Muscle was isolated at the site of injection at $7 \mathrm{dpi}$ and cryosections $(5 \mu \mathrm{m})$ were stained with Hemacolor (eosin-azur) to visualize leukocyte recruitment in non-injected (A.1), pcDNA3 injected (A.2), or pcDNA3-SVCV-G injected (A.3) tissue. (B) Muscle at the site of injection was isolated $7 \mathrm{dpi}$ from carp injected with pcDNA3 (B.1) or pcDNA3-SVCV-G (B.23 ) and stained with anti-SVCV-G antibody (clone 13C10c). Green asterisks in B.1 indicate ink particles along the needle path. Brown color in B.2 indicates G protein expression. Note 
that the $\mathrm{G}$ protein expressing cell is detached from the rest of the tissue and surrounded by leukocytes. A magnification of B.2 shows the G protein reactivity (B.3, purple/brown color) on the myocyte surface and the presence of leukocytes around and infiltrating (black arrows) the myocyte. A consecutive slide of the tissue at the same time point was stained using an antibody specific for carp neutrophilic granulocytes (B.4-6); the inset in B.5 identifies the same G protein-expressing cell as in B2 and B3. (C) Muscle at the site of injection isolated $14 \mathrm{dpi}$ was stained as described in B.1-3; C.1: overview of the muscle area in which four G proteinexpressing myocytes could be detected; C2-5: higher magnification of the areas indicated by the insets in C.1; note the condensation of the G protein staining (purple color) and the presence of leukocytes surrounding the $\mathrm{G}$ protein-expressing myocytes. Scale bars indicate distance in $\mu \mathrm{m}$.

\section{Intra-muscular DNA vaccination induces a rapid upregulation of immune- related genes at the site of injection}

Given the high protection conferred by the DNA vaccine plasmid (Fig. 1C), and the strong inflammatory response observed locally at the site of injection at 7 and 14 dpi (Fig. 2), we next investigated the early local gene expression profile induced by vaccination. A panel of pro-inflammatory cytokines and chemokines, antiviral genes as well as adaptive immune markers were selected to reveal which pathways were activated prior to the observed leukocyte recruitment (Fig. 2). Carp were injected with $0.1 \mu \mathrm{g} / \mathrm{g}$ plasmid and muscle tissue at the site of injection was isolated at 3 and 5 dpi for subsequent gene expression analysis (Fig. 3).

At 3 dpi the chemokine $c x c b 1$ [54,55] and the cytokines inf $2 a b$ and ifn $\varphi 2$, were specifically upregulated by the injection of pcDNA3-SVCV-G plasmid (vaccine group), but not by the empty plasmid. At $5 \mathrm{dpi}$, ifn $\varphi 1$ was also significantly upregulated in the vaccine group. In contrast, in both the pcDNA3 and in the pcDNA3-SVCV-G injected groups, the pro-inflammatory molecules $c x c b 2$, thf $\alpha$, il $6 b$ and $i l 1 \beta$ and the antiviral genes $m x 1, m x 2$, vip2, pkr3, stat1, and isg15.2 were all elevated at 3 dpi. The overall upregulation of pro-inflammatory genes confirmed the previous observation (Fig. 3) that a local inflammatory response marked by a strong recruitment of leukocytes, is triggered in part by the damage caused by the injection and by the plasmid backbone, and in part is specific for the vaccine plasmid. Interestingly, when looking at markers of adaptive immune cells, a vaccine-specific upregulation was observed at 3 dpi for igt 1 and zap70, whereas igm transcripts were elevated, but not significantly compared to the control group, suggesting that pcDNA3-SVCV-G promoted an early recruitment of $\mathrm{B}$ and $\mathrm{T}$ cells at the site of injection. At $5 \mathrm{dpi}$, the increased expression of these markers was no longer specific for the vaccine as transcription was elevated in both groups.

Altogether, our results indicate that injection of plasmid DNA in the muscle of carp 
induces a strong local inflammatory response, that is in part specific for the vaccine plasmid. Considering the efficacy of the DNA vaccine (Fig. 1C), the combined inflammatory response induced by the damage, the plasmid backbone and in part by the SVCV-G protein, might all contribute to provide the optimal conditions for the onset of a specific adaptive response to the SVCV-G protein.
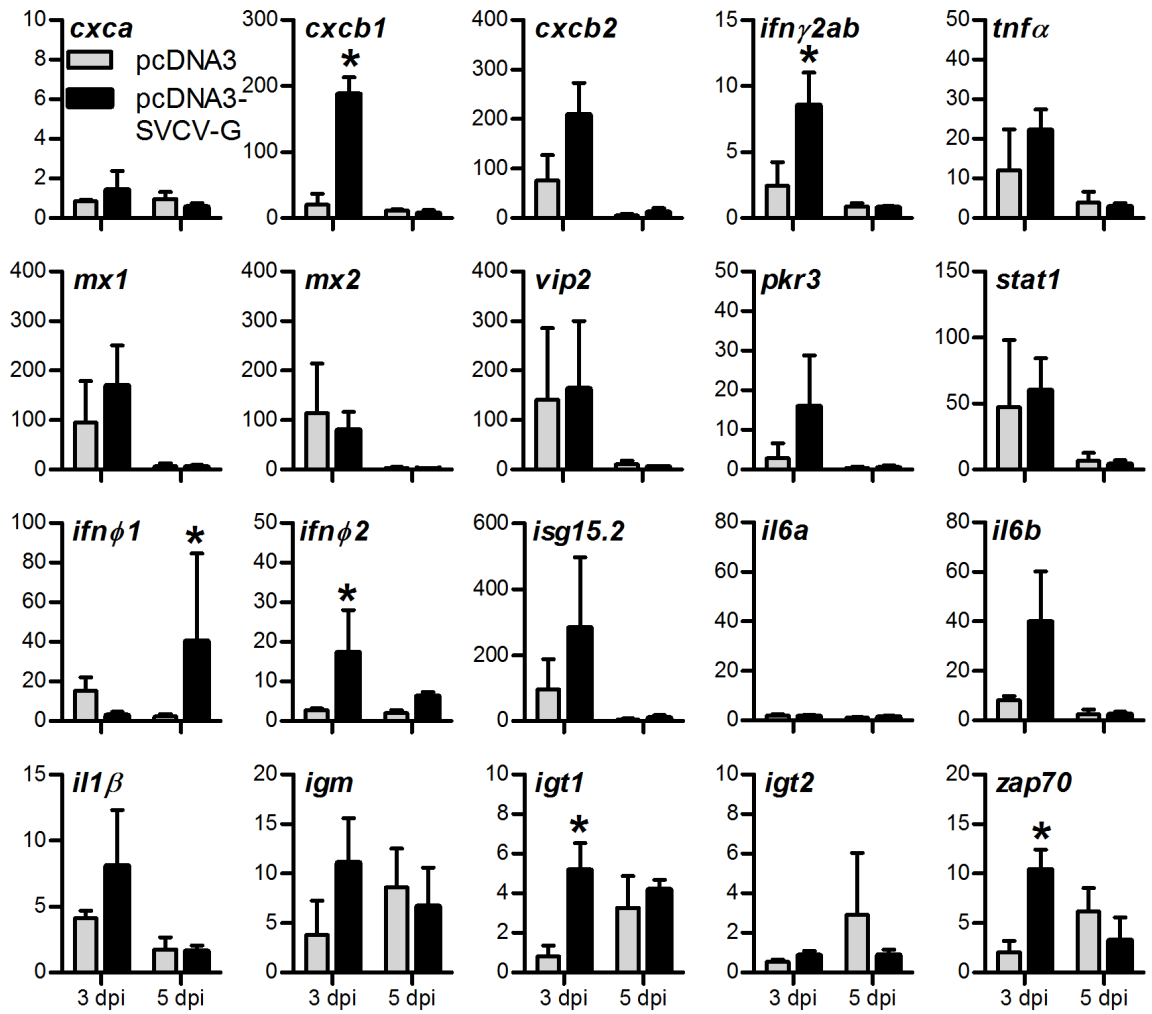

Fig. 3. Gene expression analysis of the local immune response after i.m. DNA vaccination against SVCV. Carp were injected with $0.1 \mu \mathrm{g} / \mathrm{g}$ of either pcDNA3 or pcDNA3SVCV-G. Muscle tissue at the site of injection was excised at 3 and $5 \mathrm{dpi}$. Expression of the indicated immune-relevant genes was normalized against the housekeeping gene s11 of the ribosomal subunit $40 S$ and expressed relative to the unhandled control at time point Oh. Asterisks $\left(^{*}\right)$ indicate significant differences $(p<0.05)$ between the pcDNA3 and pcDNA3SVCV-G group at the respective time point as assessed by One-way ANOVA, followed by a Tukey post-hoc test. Bars indicate average and SD of $n=3$ fish per time point.

DNA vaccination against SVCV leads to detectable virus neutralizing titers in the serum of vaccinated fish.

To investigate the role of humoral responses induced by i.m. DNA vaccination, we analyzed the neutralizing capacity of serum from vaccinated carp 3 months after vaccination. This is of importance since protection against rhabdoviruses has been shown to strongly rely on the presence of neutralizing antibodies, although they are 
not always present at detectable titers [23]. While no clear inhibition of viral growth was observed when using the serum of pcDNA3 injected carp (non-vaccinated), a significant neutralizing capacity was observed in the serum of pcDNA3-SVCV-G injected carp (vaccinated) (Fig. 4A). Although only at a serum dilution of 1:10 the number of plaques was significantly different from the controls, a clear decrease in viral plaques was observed also at the 1:100 dilution. This result shows that a single i.m. injection of DNA vaccine encoding for the SVCV G protein, is sufficient to induce virus neutralizing activity in the serum of vaccinated fish, which is most likely mediated by virus neutralizing antibodies.

ANOVA analysis showed that the overall proliferative response of Zap $70^{+} \mathrm{T}$ cells significantly different between vaccinated and non-vaccinated fish.

A

A

pcDNA3

pcDNA3-

B

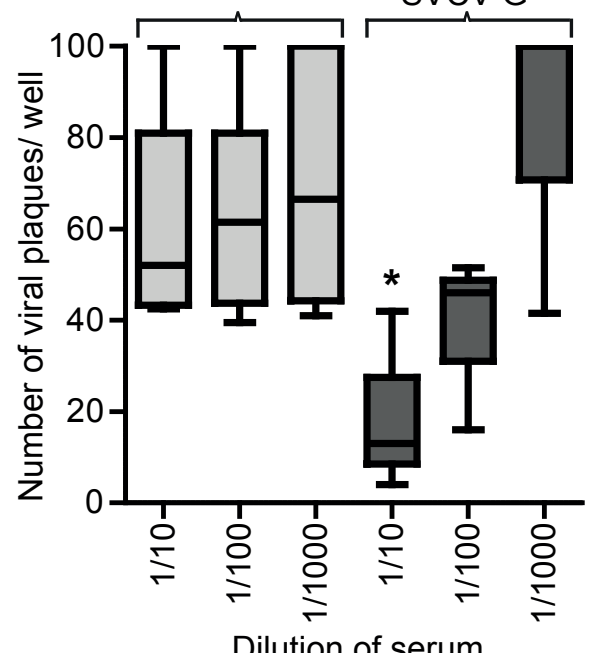

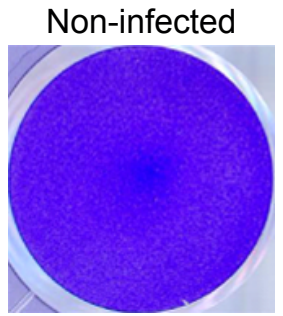

pcDNA3

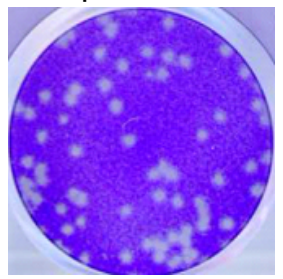

SVCV control

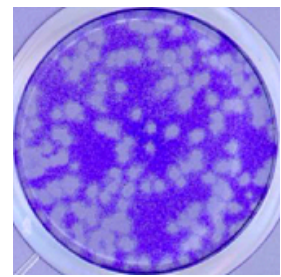

pcDNA3-SVCV-G

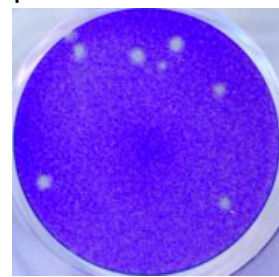

Fig. 4. Neutralizing capacity of serum from i.m. DNA vaccinated carp. Serum $(n=5 /$ group) was collected from vaccinated (pcDNA3-SVCV-G) and non-vaccinated (pcDNA3) carp 3 months after vaccination and used in a neutralization assay using the SVCV VR1390 strain. EPC cells were fixed and stained with crystal violet after three days of infection. (A) Quantification of the number of virus plaques per well. Box plots indicate the minimum, maximum and average plaques count. The upper threshold on the number of counted plaques, indicating a fully infected well, was set at 100 plaques. Asterisk $\left(^{*}\right)(p<0.05)$ indicate a significant difference between the pcDNA3 and the pcDNA3-SVCV-G group at the given dilution as assessed by an One-way ANOVA. (B) Representative pictures of wells containing non-infected EPCs monolayers (non-infected), EPC infected with SVCV only in the absence of carp serum (SVCV control), SVCV incubated with serum from pcDNA3 injected control fish (pcDNA3) or with serum from pcDNA3-SVCV-G vaccinated 


\section{DNA vaccination against SVCV induces formation of SVCV-specific Zap70+ $T$ cells}

We next investigated the presence of SVCV-specific T cells in the blood of vaccinated fish. To this end, we analyzed the proliferative capacity of antigen-specific T cells in vitro. PBLs were isolated from carp 3 months after vaccination, stimulated in vitro with SVCV alone, or in combination with recombinant Ifn $\gamma 2$ or Il10b. These two cytokines were selected for their known capacity to promote $\mathrm{T}$ cell proliferation in carp. As was reported before, carp I110b promoted proliferation of "memory" $\mathrm{T}$ cells in an in vitro study using PBLs and HKLs from carp that survived an infection with the blood-borne parasite Trypanoplasma borelli [47]. IFN $\gamma$ enhances antigen-specific $\mathrm{T}$ cell proliferation, and in ginbuna crucian carp (Carassius auratus langsdorfii) recombinant Ifnyrel was found to enhance numbers of $\mathrm{CD} 4^{+}$and $\mathrm{CD} 8 \alpha^{+}$ $\mathrm{T}$ cells during allograft rejection [51]. Furthermore, a concomitant upregulation of tbet, if $n \gamma 1$ and ifn 2 was observed upon stimulation of leukocytes from multiple organs with phytohemagglutinin (PHA) [56]. However, the effect of recombinant Il10b and Ifn $\gamma 2$ on virus-specific T cells generated upon DNA vaccination is still unknown. Proliferation of Zap $70^{+}$cells from vaccinated fish was quantified 6 days post-stimulation. Proliferation of cells stimulated with recombinant Ifn $\gamma 2$ or rll10b alone did not differ between the non-vaccinated (pcDNA3) and vaccinated (pcDNA3SVCV-G) groups (Fig. 5A) however, I110b, but not Ifn $\gamma 2$, induced proliferation in both groups. In contrast, stimulation with SVCV induced a proliferative response in the vaccinated group only, and the proliferation was further enhanced by costimulation with Ifn $\gamma 2$ (Fig. 5A and 5B) and, to a lesser extent, by rIl10b. One-way ANOVA analysis showed that the overall proliferative response of Zap $70^{+} \mathrm{T}$ cells significantly different between vaccinated and non-vaccinated fish.

A similar approach was used to measure the proliferation of $\operatorname{Igm}^{+}$B cells in PBLs from vaccinated and non-vaccinated fish. This revealed a high proliferative response upon SVCV stimulation in both groups and therefore, no significant differences were observed (data not shown). Altogether, these data indicate that i.m. DNA vaccination against SVCV induces the formation of $\mathrm{T}$ cells that recirculate in the blood of vaccinated fish 3 months after vaccination and proliferate when re-stimulated in vitro with SVCV . This proliferation capacity can be further enhanced by Ifn $\gamma 2$ and to a lesser extent by rIl10b. Whether these $\mathrm{T}$ cells are $\mathrm{Cd} 4^{+}$and/or $\mathrm{Cd} 8^{+}$will require further investigation. 
A

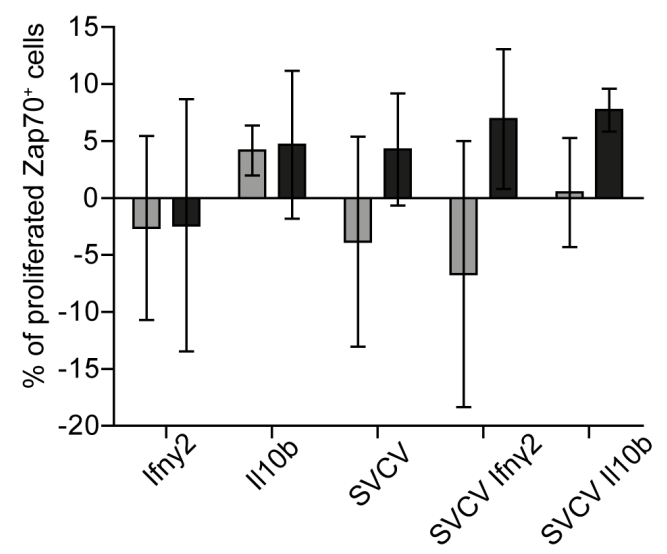

$\left.\begin{array}{l}\text { pcDNA3 } \\ \text { pcDNA3-SVCV-G }\end{array}\right\}$ *

B

pcDNA3

PcDNA3-SVCV-G
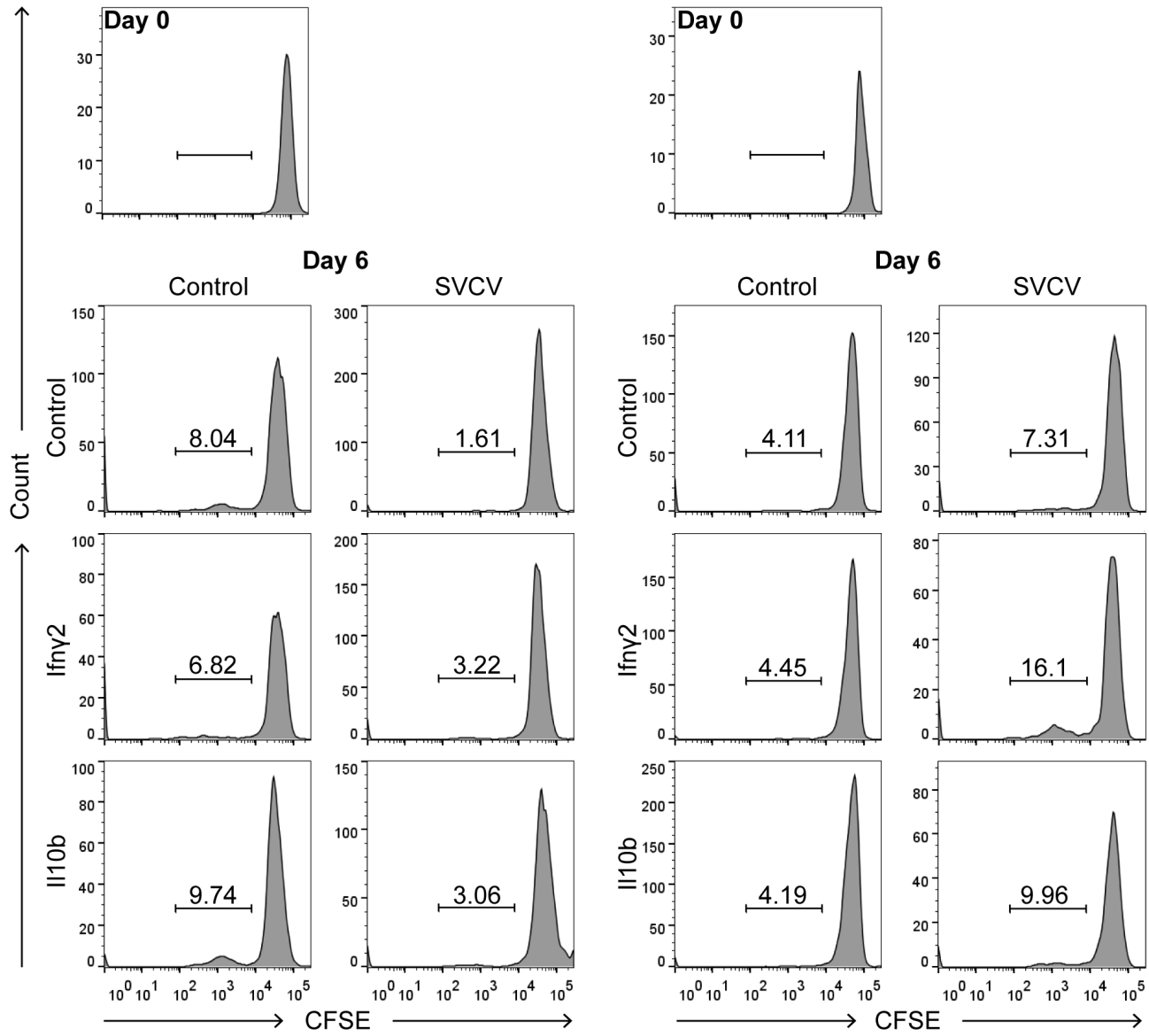
Fig. 5. DNA vaccination against SVCV induces the formation of virus-specific $T$ cells. PBLs were isolated 3 months after vaccination from non-vaccinated (pcDNA3) and vaccinated (pcDNA3-SVCV-G) carp. CFSE-labeled PBLs were stimulated for $6 \mathrm{~d}$ at $27^{\circ} \mathrm{C}$ with recombinant Ifny2 $(100 \mathrm{ng} / \mathrm{ml})$ or Il10b $(0.25 \mathrm{U} / \mathrm{ml})$ alone, or in combination with SVCV (MOI of 25). As a control, conditioned medium was used. T-cells were identified using a cross-reactive anti-Zap70 antibody, and proliferation was analyzed by flow cytometry. (A) Percentages of proliferating Zap70 $+\mathrm{T}$ cells are expressed relative to the respective conditioned medium control after subtraction of the percentage of proliferating cells in the medium control. For each group, bars represent average and SD of $n=7$ fish/stimulus, except for the $1110 \mathrm{~b}$ stimulated samples for which $n=3$ fish were used. Asterisk $\left(^{*}\right)$ indicates a significant differences between the pcDNA3 and pcDNA3-SVCV-G group as assessed by One-Way ANOVA. (B) Representative histogram plots of CFSE-labelled Zap70+ T cells from non-vaccinated (pcDNA3, left panel) and vaccinated (pcDNA3-SVCV-G, right panel) carp of the experiment in A.

\section{Discussion}

The efficacy of DNA vaccination to protect carp against SVCV has been controversial, mainly because it is notoriously difficult to set up challenge models with this virus. Here, we used an efficient bath challenge method, which likely mimics the natural infection, to demonstrate that i.m. injection of a plasmid encoding the SVCV G protein affords a high level of protection against SVCV. We did not only investigate the challenge method but also the plasmid dose, the temperature of vaccination and the age of the fish at the time of vaccination, taking into account that carp were found to be most susceptible to SVCV within the first 6 months of age. When investigating the local response induced by i.m. vaccination, our findings reveal a substantial recruitment of neutrophils and macrophages during the first two weeks following vaccination. Cells expressing the SVCV-G protein were surrounded by leukocytes, progressively disconnected from the neighboring cells and likely targeted by an immune response. Furthermore, our data show that DNA vaccination leads to the presence of virus neutralizing activity in the serum of vaccinated fish, which is most likely mediated by neutralizing antibodies, and to the presence of SVCV- specific $\mathrm{T}$ cells in the blood of vaccinated fish, which proliferate in vitro upon SVCV restimulation. Altogether, these responses are likely responsible for the long-term protection of carp observed 3 months after vaccination upon challenge with SVCV via the natural route of infection.

Challenge models that comply with the natural route of infection are of utmost importance for the proper validation of protective effects of experimental vaccines. While intra-peritoneal injections are often used because of their ease of standardization, cohabitation or bath challenges, although typically more difficult 
to standardize, better resemble the natural route of infection. Here, we report a standardized and reproducible bath challenge procedure for common carp based on prolonged ( $>30 \mathrm{~h}$ ) exposure to SVCV $\left(8 \times 10^{6} \mathrm{pfu} / \mathrm{ml}\right)$, typically leading to $>90 \%$ mortality rates in juvenile carp. Previously reported bath challenge procedures for SVCV showed mortality rates of $67-75 \%$, possibly because of the relatively short exposure time $(60 \mathrm{~min})$ to the virus and a lower viral load used $\left(5 \times 10^{3} \mathrm{pfu} / \mathrm{ml}\right)$ [22]. In our hands, such short exposure to the virus led to much lower mortality, typically lower than $30 \%$. While shorter bath challenges of a few hours may be preferred because of practical reasons, longer exposure times generally enhance antigen uptake and possibly vaccine effectiveness [57]. Indeed, longer exposure time ( $>30 \mathrm{~h}$ ) clearly improved the reproducibility of our bath challenge with SVCV. To test the efficacy of our DNA vaccine against SVCV, we used different temperatures for vaccination $\left(23^{\circ} \mathrm{C}\right)$ and for viral challenge $\left(15^{\circ} \mathrm{C}\right)$. Higher temperature at vaccination is crucial for a rapid onset of specific immune responses, especially for $\mathrm{T}$ cell help and antibody formation, and $23^{\circ} \mathrm{C}$ falls within the temperature range optimal for carp [58-61]. Possibly, vaccination at higher temperature might have contributed to the formation of virus neutralizing antibodies, most likely responsible for the neutralizing activity observed in the serum of vaccinated fish. In agreement, carp kept at $10^{\circ} \mathrm{C}$ and $15^{\circ} \mathrm{C}$ showed a delay in SVCV clearance from the blood and a delayed development of neutralizing antibodies, when compared to fish kept at $20^{\circ} \mathrm{C}$ [62]. Conversely, a lower temperature is crucial for viral replication and $15^{\circ} \mathrm{C}$ falls within the temperature range optimal for SVCV, with typical field outbreaks in Spring and associated mortalities occurring at water temperatures between 11$17^{\circ} \mathrm{C}$. In fact, carp challenged at $20-22^{\circ} \mathrm{C}$ typically show no mortalities whereas carp challenged at $10-12^{\circ} \mathrm{C}$ showed $90 \%$ mortality $[62,63]$. In our hands, carp older than 9 months of age, when kept at $10^{\circ} \mathrm{C}$, showed up to $30 \%$ mortality when challenged using our optimized challenge method (data not shown), while no mortality was observed when carp of the same age were challenged at $15^{\circ} \mathrm{C}$ (Fig. 1B). Altogether these data suggest that it is likely advantageous to vaccinate carp at high temperature, to allow for optimal development of protective response. Also in rainbow trout, temperature-dependent differences in the kinetics and immune compartment involved in the response have been described after DNA vaccination against VHSV [64]. For example, neutralizing activity was observed in the plasma of fish DNA vaccinated at $15^{\circ} \mathrm{C}$ whereas negligible or no neutralizing activity was detected in fish 
vaccinated at $10^{\circ} \mathrm{C}$ and $5^{\circ} \mathrm{C}$. In agreement, also the specificity of the protection was shown to be temperature-dependent; in fact, only trout kept at $15^{\circ} \mathrm{C}$ and vaccinated with a DNA plasmid encoding the VHSV-G protein, and not those vaccinated with a plasmid encoding the IHNV-G protein, showed a specific protection against VHSV upon challenged 40 days later. Conversely, trout kept and vaccinated at $10^{\circ} \mathrm{C}$ or $5^{\circ} \mathrm{C}$ showed a protection against a VHSV challenge independently of whether they were vaccinated with either of the VHSV-G or the IHNV-G DNA plasmids, suggesting a role for non-specific innate immune mechanisms.

We also studied how the 'natural' resistance of carp to bath exposure to SVCV increased with age, leading to almost full resistance from 9 months onwards, which might be related to the gradual increase of cross-reactive (natural) antibodies developing over time [65]. Age-dependent susceptibility to rhabdoviruses has also been reported for rainbow trout and pike to VHSV [66] and for rainbow trout to IHNV [67]. In fact, it is a general observation for many other pathogens in fish and other vertebrates [68]. This underlines the necessity of vaccinating carp at a young but immune-competent age of 3 months [69] to overcome the age period when they are most sensitive to SVCV (3-6 months). Finally, our data also make clear the need to verify vaccine efficacy within the age period of susceptibility, using the natural route of infection. Our vaccination protocol based on a low dose (0.1-1 $\mu \mathrm{g}$ DNA/g of fish) of pcDNA3-SVCV-G, protected carp against a lethal bath challenge with SVCV 2.5 months after vaccination. Lower doses might be investigated since in rainbow trout, a dose of $0.01 \mu \mathrm{g}$ DNA/g of fish could protect against IHNV [70]. Previous DNA vaccination studies in common carp required higher doses (10-25 $\mu \mathrm{g}$ DNA/g) and/or up to two booster injections [21,22] but were still less successful in achieving protection against a subsequent $\mathrm{SVCV}$ challenge, possibly caused by vaccination at a slightly lower temperature $\left(20^{\circ} \mathrm{C}\right)$, or due to difference in the challenge protocol (challenge route, viral strain).

In our study, challenge at $>2.5$ months after vaccination indicates that the protection is most likely due to the virus specific immune response and based on immune memory. Indeed, the non-specific interferon-induced response that typically arises quickly after DNA vaccination, is generally short-lived [29,71,72]. The importance of specific immunity in the protection is further supported by the induction of neutralizing antibodies and the presence of virus-specific $\mathrm{T}$ cells in the blood of vaccinated fish. 'Long term' (> 2.5 months) protective effects of DNA vaccination 
against SVCV remain to be investigated in carp and would be interesting from an immunological point of view. However, they seem to be of lower practical relevance because of the relatively short window of susceptibility that needs to be covered between the time of immune maturity ( $>3$ months) and development of natural resistance against $\mathrm{SVCV}$ ( $>6-9$ months).

Histological analysis of the muscle tissue after injection revealed a strong and rapid influx of leukocytes at the site of injection. This was largely damage- and/ or inflammation-driven rather than antigen-specific because it was also seen after injection of the empty plasmid. Yet, the influx of leukocytes into myocytes expressing the SVCV-G protein as well as the time-dependent condensation and degradation of SVCV-G-expressing cells that were completely surrounded by leukocytes, was specific for the SVCV-G injected group. This supports the notion that SVCV-Gexpressing cells can be seen by the host as non-self and can trigger both innate as well as SVCV-specific (adaptive) immune responses, at least in part similar to the one triggered by a natural virus infection [23]. A similar elimination of myocytes expressing the vaccine antigen was observed in rainbow trout; lymphocytes and macrophages were found in close proximity and infiltrating the $\mathrm{G}$ protein positive myocytes [73]. In carp, the influx of leukocytes and the following inflammatory reaction at the site of injection is consistent with the general upregulation of proinflammatory genes observed in the groups injected with both, the control or vaccine plasmid. Few genes (cxcb1, ifn $\gamma 2$, il6b, ifn $\varphi 1$ and ifn $\varphi 2)$ among those investigated within our panel were upregulated specifically in the $G$ protein-vaccinated group. Recombinant carp Cxcb was previously shown to stimulate chemotaxis of carp macrophages and granulocytes in vitro [74]. Also recombinant Il6 has been shown to have synergistic effects on antigen-specific Igm responses of trout, in vivo, when co-injected with inactivated infectious pancreatic necrosis virus (IPNV) [75].

The SVCV-G protein induced upregulation of type-I interferons (ifn $\varphi 1$ and ifn $\varphi 2$ ) appears an intrinsic property of the G protein since it was also noted for IHNV in rainbow trout [26,29] and for VHSV in Atlantic salmon [27] and rainbow trout $[13,76,77]$. In salmonids, the antiviral interferon response appears to be $G$ proteinspecific because $m x$ was found upregulated only after i.m vaccination with VHSV-G, but not with VHSV-N [28]. In conclusion, although a limited number of genes were specifically upregulated by injection of the pcDNA3-SVCV-G vaccine plasmid, it cannot be excluded that the inflammation caused by the injection-related damage, by 
the plasmid backbone, and finally by the SVCV-G protein all contribute to the onset of a subsequent specific response towards the $G$ protein.

Of interest, a rapid (7 days) influx of $\mathrm{Igm}^{+}$and $\mathrm{Igt}^{+} \mathrm{B}$ lymphocytes in the muscle of trout DNA vaccinated with VHSV-G [78], suggests a role not only for innate immune cells, but also for B lymphocytes in the early response to vaccination. In carp, SVCV-G-specific upregulation of zap70 and igtl gene expression was noted already at 3 days post-vaccination, hinting at a role also in carp of adaptive immune cells in the initial response to DNA vaccination against SVCV. Alternatively, this early wave of B cells may indirectly contribute to fight the virus via cytokine production. The importance of B cells in protection against SVCV could be confirmed by the detection of neutralizing activity, most likely mediated by neutralizing antibodies, in the serum of vaccinated, but not control carp. Indeed, neutralizing antibodies have also been reported in early studies following vaccination with inactivated SVCV $[53,62]$, although this could not be confirmed in a later study with inactivated SVCV [79].

We also examined whether DNA vaccination can induce a $\mathrm{T}$ cell response, which would lead to long lasting virus-specific clones. SVCV-G-specific upregulation of the pan T cell marker zap70 was noted at 3 days after vaccination. Previous studies already suggested a role for cell-mediated immune responses in the protection against SVCV, based on the upregulation of various T-cell markers after SVCV challenge in carp [80] and on a strong lymphocyte proliferation also in the absence of SVCV-specific antibodies in goldfish [21]. To gain further insights in the T cell response upon DNA vaccination, we analyzed the proliferative capacity of $\left(Z a p 70^{+}\right)$ $\mathrm{T}$ cells by stimulating PBLs from vaccinated carp with SVCV in vitro. We also examined the potential of two (recombinant) cytokines, I110b and Ifn $\gamma 2$, to modulate such proliferative response. Interleukin-10 can have multiple effects on B and $\mathrm{T}$ lymphocytes, including regulation of proliferation and differentiation (as reviewed in Piazzon et al., 2016), and carp Il10b was shown to promote survival and enhance proliferation of antigen-specific B and T cells [47]. Ifn $\gamma 2$ has multiple effects and in carp was found to enhance antigen-specific responses during in vitro stimulation of carp leukocytes and phagocytes [48]. We observed an SVCV-specific T cell proliferation in PBLs from vaccinated carp stimulated in vitro with SVCV, which could be enhanced by Il10b or Ifn $\gamma 2$. Despite the large variation in the individual response of PBLs, in vitro re-stimulation with the virus led to an overall significantly 
higher proliferation of T cells in PBLs isolated from vaccinated fish when compared to the overall $\mathrm{T}$ cells proliferation in PBLs from non-vaccinated fish. This suggests that the frequency of peripheral SVCV-specific T cells is higher in vaccinated than in non-vaccinated fish. Although the development of a cell-mediated cytotoxicity response after DNA vaccination against VHSV has been described in rainbow trout [32], in this report we show for the first time virus-specific proliferation of carp $\mathrm{T}$ cells in vitro after a single low dose injection of DNA vaccine against SVCV. The presence of virus-specific $\mathrm{T}$ cells three months after vaccination raises the issue of the importance of a $\mathrm{T}$ cell-dependent response after the recall: while it is often considered that viral particles provide a perfect matrix of repetitive antigens to induce $\mathrm{T}$ cell-independent $\mathrm{B}$ cell responses, it is possible that $\mathrm{T}$ cell help plays an important role in the immune response of vaccinated fish. The virus-specific $\mathrm{T}$ cells present in vaccinated fish may also comprise cytotoxic $\mathrm{T}$ cells, of which the contribution to protection would have to be assessed in vivo. The development of antibodies against specific subsets of $\mathrm{T}$ cells will allow for further investigation of the role of $\mathrm{T}$ cells in the establishment of protection against SVCV.

From our data it appears that already a single low dose of the SVCV-G DNA vaccine is sufficient to trigger both arms of the adaptive immune system. Our data show that DNA vaccination against SVCV induces neutralizing antibodies, and suggest that SVCV-specific T cells might contribute to the protection.

Altogether, we for the first time report on a fully protective G protein-based DNA vaccine in carp against SVCV. We also describe age-related susceptibility of carp to SVCV, an optimized bath challenge method, along with the characterization of local as well as systemic protective immune responses after i.m. DNA vaccination against SVCV. Our data provide new insights into the respective implication of B and $\mathrm{T}$ cells in the response to the vaccine: an early role for the adaptive immune response and a possible early recruitment of $\mathrm{B}$ and $\mathrm{T}$ cells to the site of injection. In a later phase of the response we showed the induction of neutralizing antibodies, and the presence of antigen-specific 'memory' $\mathrm{T}$ cells. This latter finding raises the issue of the relative importance of $\mathrm{T}$ cells in the response. Most likely the combination of humoral as well as cell-mediated responses are key to the success of the current DNA vaccine. Given the recent developments in legislation of DNA vaccines for aquaculture species, marked by the approval on the use of the CLYNAV vaccine against Pancreatic Disease in Atlantic salmon, our data might contribute 
to the increasing need on the study of DNA vaccines for fish and their underlying mechanisms of protection [5]. Furthermore, new developments on mucosal delivery of vaccines, and especially oral vaccines (Reviewed in Embregts and Forlenza, 2016) would bypass some major disadvantages of i.m. injection vaccines and might allow for easier commercialization and wider application of DNA vaccines.

\section{Author Contributions}

CE, MF, JP, DR, TV, GW and PB contributed to the design of the experiments, acquisition of samples and analysis of data. PB, GW and MF acquired funding. NL, $\mathrm{DP}, \mathrm{MD}, \mathrm{HS}, \mathrm{AH}$ contributed with reagents, materials and analysis tools. $\mathrm{CE}, \mathrm{MF}$, GW, NL, and PB wrote the manuscript.

\section{Acknowledgements}

This work was supported by the European Commission under the $7^{\text {th }}$ Framework Programme for Research and Technological Development (FP7) of the European Union (Grant Agreement 311993 TARGETFISH). PB received institutional support from INRA and was partly funded by the EU INFRAIA project VetBioNet (EU H2020 project 731014); TV and DP were also partly supported by the Ministry of Agriculture of the Czech Republic (MZE-RO0517).

\section{References}

1. Tonheim TC, Bøgwald J, Dalmo RA. What happens to the DNA vaccine in fish? A review of current knowledge. Fish Shellfish Immunol (2008) 25:1-18. doi:10.1016/j.fsi.2008.03.007

2. Mutoloki S, Munang'andu HM, Evensen O, Munang'andu HM, Evensen Ø. Oral vaccination of fish- antigen preparations, uptake and immune induction. Front Immunol (2015) 6:519. doi:10.3389/fimmu.2015.00519

3. Parra D, Reyes-Lopez FE, Tort L. Mucosal Immunity and B Cells in Teleosts: Effect of Vaccination and Stress. Front Immunol (2015) 6:1-12. doi:10.3389/fimmu.2015.00354

4. Munang'andu HM, Mutoloki S, Evensen Oø. An overview of challenges limiting the design of protective mucosal vaccines for finfish. Front Immunol (2015) 6: doi:10.3389/ fimmu.2015.00542

5. EMA. First DNA vaccine in the EU recommended for use in salmon. 2016-04-22 (2016)1. Available at: http://www.ema.europa.eu/ema/index.jsp?curl=pages/news_and_events/ news/2016/04/news_detail_002516.jsp\&mid=WC0b01ac058004d5c1 [Accessed September 
6. Lorenzen N, Lorenzen E, Einer-Jensen K, Heppell J, Wu T, Davis H. Protective immunity to VHS in rainbow trout (Oncorhynchus mykiss, Walbaum) following DNA vaccination. Fish Shellfish Immunol (1998) 8:261-270.

7. Byon JY, Ohira T, Hirono I, Aoki T. Comparative immune responses in Japanese flounder, Paralichthys olivaceus after vaccination with viral hemorrhagic septicemia virus (VHSV) recombinant glycoprotein and DNA vaccine using a microarray analysis. Vaccine (2006) 24:921-30. doi:10.1016/j.vaccine.2005.08.087

8. Pereiro P, Martinez-Lopez A, Falco A, Dios S, Figueras A, Coll JM, Novoa B, Estepa A. Protection and antibody response induced by intramuscular DNA vaccine encoding for viral haemorrhagic septicaemia virus (VHSV) G glycoprotein in turbot (Scophthalmus maximus). Fish Shellfish Immunol (2012) 32:1088-1094. doi:10.1016/j.fsi.2012.03.004

9. Hart LM, Lorenzen N, Lapatra SE, Grady CA, Roon SE, O’Reilly J, Gregg JL, Hershberger PK. Efficacy of a glycoprotein DNA vaccine against viral haemorrhagic septicaemia (VHS) in pacific herring, clupea pallasii valenciennes. J Fish Dis (2012) 35:775-779. doi:10.1111/ j.1365-2761.2012.01364.x

10. Garver KA, LaPatra SE, Kurath G. Efficacy of an infectious hematopoietic necrosis (IHN) virus DNA vaccine in Chinook Oncorhynchus tshawytscha and sockeye O. nerka salmon. Dis Aquat Organ (2005) 64:13-22. doi:10.3354/dao064013

11. Kurath G, Garver KA, Corbeil S, Elliott DG, Anderson ED, LaPatra SE. Protective immunity and lack of histopathological damage two years after DNA vaccination against infectious hematopoietic necrosis virus in trout. Vaccine (2006) 24:345-354. doi:10.1016/j. vaccine. 2005.07.068

12. Takano T, Iwahori A, Hirono I, Aoki T. Development of a DNA vaccine against hirame rhabdovirus and analysis of the expression of immune-related genes after vaccination. Fish Shellfish Immunol (2004) 17:367-374. doi:10.1016/j.fsi.2004.04.012

13. Boudinot P, Blanco M, de Kinkelin P, Benmansour A. Combined DNA immunization with the glycoprotein gene of viral hemorrhagic septicemia virus and infectious hematopoietic necrosis virus induces double-specific protective immunity and nonspecific response in rainbow trout. Virology (1998) 249:297-306. doi:10.1006/viro.1998.9322

14. FAO. Cultured Aquatic Species Information Program. Cyprinus carpio. (2017) Available at: http://www.fao.org/fishery/culturedspecies/Cyprinus_carpio/en\#tcNA00D6 [Accessed September 18, 2017]

15. Ahne W, Bjorklund HV, Essbauer S, Fijan N, Kurath G, Winton JR. Spring viremia of carp (SVC). Dis Aquat Organ (2002) 52:261-272. doi:10.3354/dao052261

16. Garver KA, Dwilow AG, Richard J, Booth TF, Beniac DR, Souter BW. First detection and confirmation of spring viraemia of carp virus in common carp, Cyprinus carpio L., from Hamilton Harbour, Lake Ontario, Canada. J Fish Dis (2007) 30:665-671. doi:10.1111/ j.1365-2761.2007.00851.x

17. Dikkeboom AL, Radi C, Toohey-Kurth K, Marcquenski S, Engel M, Goodwin AE, Way K, Stone DM, Longshaw C. First report of Spring Viremia of Carp Virus (SVCV) in wild common carp in North America. J Aquat Anim Health (2004) 16:169-178. doi:10.1577/H03064.1

18. Liu H, Gao L, Shi X, Gu T, Jiang Y, Chen H. Isolation of spring viraemia of carp virus (SVCV) from cultured koi (Cyprinus carpio koi) and common carp (C. carpio carpio) in P.R. China. Bull Eur Assoc Fish Pathol (2004) 24:194-202.

19. Dauber M, Schütze H, Fichtner D. Determination of the complete genomic sequence and analysis of the gene products of the virus of Spring Viremia of Carp, a fish rhabdovirus. Bull Eur Assoc Fish Pathol (2001) 21:170-177. doi:10.1016/S0168-1702(01)00441-5

20. Teng Y, Liu H, Lv JQ, Fan WH, Zhang QY, Qin QW. Characterization of complete genome sequence of the spring viremia of carp virus isolated from common carp (Cyprinus carpio) in China. Arch Virol (2007) 152:1457-1465. doi:10.1007/s00705-007-0971-8 
21. Kanellos T, Sylvester ID, D'Mello F, Howard CR, Mackie A, Dixon PF, Chang KC, Ramstad A, Midtlyng PJ, Russell PH. DNA vaccination can protect Cyprinus Carpio against spring viraemia of carp virus. Vaccine (2006) 24:4927-4933. doi:10.1016/j.vaccine.2006.03.062

22. Emmenegger EJ, Kurath G. DNA vaccine protects ornamental koi (Cyprinus carpio koi) against North American spring viremia of carp virus. Vaccine (2008) 26:6415-6421. doi:10.1016/j.vaccine.2008.08.071

23. Lorenzen N, LaPatra SE. DNA vaccines for aquacultured fish. Rev Sci Tech (2005) 24:201213. doi:10.3354/dao056031

24. Cui L-C, Guan X-TX-T, Liu Z-MZ-M, Tian C-YC-Y, Xu YGY-G. Recombinant lactobacillus expressing $\mathrm{G}$ protein of spring viremia of carp virus (SVCV) combined with ORF81 protein of koi herpesvirus (KHV): A promising way to induce protective immunity against SVCV and KHV infection in cyprinid fish via oral vaccination. Vaccine (2015) 33: doi:10.1016/j. vaccine.2015.05.002

25. Purcell MK, Kurath G, Garver KA, Herwig RP, Winton JR. Quantitative expression profiling of immune response genes in rainbow trout following infectious haematopoietic necrosis virus (IHNV) infection or DNA vaccination. Fish Shellfish Immunol (2004) 17:447-462. doi:10.1016/j.fsi.2004.04.017

26. Acosta F, Petrie A, Lockhart K, Lorenzen N, Ellis AE. Kinetics of Mx expression in rainbow trout (Oncorhynchus mykiss) and Atlantic salmon (Salmo salar L.) parr in response to VHSDNA vaccination. Fish Shellfish Immunol (2005) 18:81-89. doi:10.1016/j.fsi.2004.06.005

27. Yasuike M, Kondo H, Hirono I, Aoki T. Difference in Japanese flounder, Paralichthys olivaceus gene expression profile following hirame rhabdovirus (HIRRV) G and N protein DNA vaccination. Fish Shellfish Immunol (2007) 23:531-541. doi:10.1016/j.fsi.2006.12.006

28. Kim CH, Johnson MC, Drennan JD, Simon BE, Thomann E, Leong JA. DNA vaccines encoding viral glycoproteins induce nonspecific immunity and Mx protein synthesis in fish. $J$ Virol (2000) 74:7048-7054. doi:10.1128/JVI.74.15.7048-7054.2000

29. Bela-ong DB, Schyth BD, Zou J, Secombes CJ, Lorenzen N. Involvement of two microRNAs in the early immune response to DNA vaccination against a fish rhabdovirus. Vaccine (2015) 33:3215-3222. doi:10.1016/j.vaccine.2015.04.092

30. Boudinot P, Bernard D, Boubekeur S, Thoulouze MI, Bremont M, Benmansour A. The glycoprotein of a fish rhabdovirus profiles the virus-specific T-cell repertoire in rainbow trout. J Gen Virol (2004) 85:3099-3108. doi:10.1099/vir.0.80135-0

31. Utke K, Kock H, Schuetze H, Bergmann SM, Lorenzen N, Einer-Jensen K, Köllner B, Dalmo RA, Vesely T, Ototake M, et al. Cell-mediated immune responses in rainbow trout after DNA immunization against the viral hemorrhagic septicemia virus. Dev Comp Immunol (2008) 32:239-252. doi:10.1016/j.dci.2007.05.010

32. Irnazarow I. Genetic variability of Polish and Hungarian carp lines. Aquaculture (1995) 129:215. doi:10.1016/0044-8486(95)91961-T

33. Fijan N, Petrinec Z, Sulimanovic D, Zwillenberg LO. Isolation of the viral causative agent from the acute form of infectious dropsy of carp. Vet Arh (1971) 41:125-138.

34. Betts AM, Stone DM, Way K, Torhy C, Chilmonczyk S, Benmansour A, De Kinkelin P. Emerging vesiculo-type virus infections of freshwater fishes in Europe. Dis Aquat Organ (2003) 57:201-212. doi:10.3354/dao057201

35. Koutná M, Veselý T, Pšikal I, Hůlová J. Identification of spring viraemia of carp virus (SVCV) by combined RT-PCR and nested PCR. Dis Aquat Organ (2003) 55:229-235. doi:10.3354/dao055229

36. Reed LJ, Muench H. A simple method of estimating fifty per cent endpoints. Am J Epidemiol (1938) 27:493-497. Available at: http://www.scopus.com/inward/record.url?eid=2-s2.033745158157\&partnerID=tZOtx3y1

37. Hoffmann B, Schütze H, Mettenleiter TC. Determination of the complete genomic sequence and analysis of the gene products of the virus of Spring Viremia of Carp, a fish rhabdovirus. Virus Res (2002) 84:89-100. Available at: www.elsevier.com/locate/virusres [Accessed July 
38. Dauber M, Schütze H, Fichtner D. Development and characterization of monoclonal antibodies raised against a viral haemorrhagic septicaemia virus (VHSV) isolate which failed to be identified by a commercial kit. Bull Eur Assoc Fish Pathol (2001) 21:170-177.

39. Forlenza M, Kaiser T, Savelkoul HFJ, Wiegertjes GF. The use of real-time quantitative PCR for the analysis of cytokine mRNA levels. Methods Mol Biol (2012) 820:7-23. doi:10.1007/978-1-61779-439-1

40. Pfaffl MW. A new mathematical model for relative quantification in real-time RT-PCR. Nucleic Acids Res (2001) 29:e45-e45. doi:10.1093/nar/29.9.e45

41. Forlenza M, Scharsack JP, Kachamakova NM, Taverne-Thiele AJ, Rombout JHWM, Wiegertjes GF. Differential contribution of neutrophilic granulocytes and macrophages to nitrosative stress in a host-parasite animal model. Mol Immunol (2008) 45:3178-3189. doi:10.1016/j.molimm.2008.02.025

42. Nakayasu C, Omori M, Hasegawa S, Kurata O, Okamoto N. Production of a monoclonal antibody for carp (Cyprinus carpio L.) phagocytic cells and separation of the cells. Fish Shellfish Immunol (1998) 8:91-100. Available at: http://ac.els-cdn.com/ S105046489790125X/1-s2.0-S105046489790125X-main.pdf?_tid=f2ecacd2-4167-11e7b339-00000aab0f01\&acdnat=1495730217_68caa61b6a51021c584816f36ce0e663 [Accessed May 25, 2017]

43. Koumans-van Diepen JE, van de Lisdonk MHM, Taverne-Thiele AJ, Verburg-van Kemenade BML, Rombout JHWM. Characterisation of immunoglobulin-binding leucocytes in carp (Cyprinus carpio L.). Dev Comp Immunol (1994) 18:45-56. doi:10.1016/0145305X(94)90251-8

44. Forlenza M, Walker PD, de Vries BJ, Wendelaar Bonga SE, Wiegertjes GF. Transcriptional analysis of the common carp (Cyprinus carpio L.) immune response to the fish louse Argulus japonicus Thiele (Crustacea: Branchiura). Fish Shellfish Immunol (2008) 25:76-83. doi:10.1016/j.fsi.2007.12.013

45. Piazzon MC, Savelkoul HSJ, Pietretti D, Wiegertjes GF, Forlenza M. Carp I110 Has AntiInflammatory Activities on Phagocytes, Promotes Proliferation of Memory T Cells, and Regulates B Cell Differentiation and Antibody Secretion. J Immunol (2015) 194:187-99. doi:10.4049/jimmunol.1402093

46. Arts JAJ, Tijhaar EJ, Chadzinska M, Savelkoul HFJ, Verburg-van Kemenade BML. Functional analysis of carp interferon- $\gamma$ : Evolutionary conservation of classical phagocyte activation. Fish Shellfish Immunol (2010) 29:793-802. doi:10.1016/j.fsi.2010.07.010

47. Piazzon MC, Wentzel AS, Tijhaar EJ, Rakus KŁ, Vanderplasschen A, Wiegertjes GF, Forlenza M. Cyprinid Herpesvirus 3 Il10 Inhibits Inflammatory Activities of Carp Macrophages and Promotes Proliferation of Igm ${ }^{+} \mathrm{B}$ Cells and Memory T Cells in a Manner Similar to Carp I110. J Immunol (2015) 195:3694-3704. doi:10.4049/jimmunol.1500926

48. Piazzon MC, Wentzel AS, Wiegertjes GF, Forlenza M. Carp I110a and Il10b exert identical biological activities in vitro, but are differentially regulated in vivo. Dev Comp Immunol (2017) 67:350-360. doi:10.1016/j.dci.2016.08.016

49. Shibasaki Y, Hatanaka C, Matsuura Y, Miyazawa R, Yabu T, Moritomo T, Nakanishi T. Effects of IFN $\gamma$ administration on allograft rejection in ginbuna crucian carp. Dev Comp Immunol (2016) 62:108-115. doi:10.1016/j.dci.2016.04.021

50. Secombes CJ, van Groningen JJ, Egberts E. Separation of lymphocyte subpopulations in carp Cyprinus carpio L. by monoclonal antibodies: immunohistochemical studies. Immunology (1983) 48:165-175.

51. Ahne W. Uptake and multiplication of spring viraemia of carp virus in carp, Cyprinus carpio L. J Fish Dis (1978)265-268. doi:10.1111/j.1365-2761.1978.tb00029.x

52. Huising MO, Stet RJM, Kruiswijk CP, Savelkoul HFJ, Lidy Verburg-van Kemenade BM. Molecular evolution of CXC chemokines: extant CXC chemokines originate from the CNS. Trends Immunol (2003) 24:306-312. doi:10.1016/S1471-4906(03)00120-0 
53. Huising MO, Stolte E, Flik G, Savelkoul HFJ, Verburg-van Kemenade BML. CXC chemokines and leukocyte chemotaxis in common carp (Cyprinus carpio L.). Dev Comp Immunol (2003) 27:875-888. doi:10.1016/S0145-305X(03)00082-X

54. Stolte EH, Savelkoul HFJ, Wiegertjes G, Flik G, Lidy Verburg-van Kemenade BM. Differential expression of two interferon- $\gamma$ genes in common carp (Cyprinus carpio L.). Dev Comp Immunol (2008) 32:1467-1481. doi:10.1016/j.dci.2008.06.012

55. Moore JD. Particulate antigen uptake during immersion immunisation of fish: The effectiveness of prolonged exposure and the roles of skin and gill. Fish Shellfish Immunol (1998) 8:393-407. Available at: http://ac.els-cdn.com/S1050464898901437/1-s2.0S1050464898901437-main.pdf?_tid=f9091fe8-451c-11e7-9ac7-00000aab0f01\&acdnat=1496 137820_6ba01bb2ca9977975cdf392af11a49df [Accessed May 30, 2017]

56. Avtalion RR. Temperature effect on antibody production and immunological memory, in carp (Cyprinus carpio) immunized against bovine serum albumin (BSA). Immunology (1969) 17:927-931.

57. Avtalion RR, Wojdani A, Malik Z, Shahrabani R, Duczyminer M. "Influence of Environmental Temperature on the Immune Response in Fish," in Current Topics in Microbiology and Immunology / Ergebnisse der Mikrobiologie und Immunitätsforschung (Berlin, Heidelberg: Springer Berlin Heidelberg), 1-35. doi:10.1007/978-3-642-65531-9_1

58. Rijkers GT, Frederix-Wolters EM, van Muiswinkel WB. The immune system of cyprinid fish. Kinetics and temperature dependence of antibody-producing cells in carp (Cyprinus carpio). Immunology (1980) 41:91-97.

59. Le Morvan C, Troutaud D, Deschaux P. Differential effects of temperature on specific and nonspecific immune defences in fish. J Exp Biol (1998) 201:165-168.

60. Ahne W. The influence of environmental temperature and infection route on the immune response of carp (Cyprinus carpio) to spring viremia of carp virus (SVCV). Vet Immunol Immunopathol (1986) 12:383-386. doi:10.1016/0165-2427(86)90144-3

61. Ahne W. Rhabdovirus carpio-Infektion beim karpfen (Cyprinus carpio): Untersuchungen über reaktionen des wirtsorganismus. Fortschritte der Veterinärmedizin (1980) 30:180-183.

62. Lorenzen E, Einer-Jensen K, Rasmussen JS, Kjær TE, Collet B, Secombes CJ, Lorenzen $\mathrm{N}$. The protective mechanisms induced by a fish rhabdovirus DNA vaccine depend on temperature. Vaccine (2009) 27:3870-3880. doi:10.1016/j.vaccine.2009.04.012

63. Pokorova D, Reschova S, Vesely T. Kinetics of anti-svev antibodies in the serum of the common carp (Cyprinus carpio 1.) post experimental infection. in Fish and Shellfish Immunology (Fish \& Shellfish Immunology), 1730. doi:10.1016/J.FSI.2013.03.287

64. Meier W, Schmitt M, Wahli T. Viral hemorrhagic septicemia (VHS) of nonsalmonids. Annu Rev Fish Dis (1994) 4:359-373. doi:10.1016/0959-8030(94)90035-3

65. Bergmann SM, Fichtner D, Skall HF, Schlotfeldt HJ, Olesen NJ. Age- and weight-dependent susceptibility of rainbow trout Oncorhynchus mykiss to isolates of infectious haematopoietic necrosis virus (IHNV) of varying virulence. Dis Aquat Organ (2003) 55:205-210. doi:10.3354/dao055205

66. Kollmann TR, Levy O, Montgomery RR, Goriely S. Innate Immune Function by Toll-like Receptors: Distinct Responses in Newborns and the Elderly. Immunity (2012) 37:771-783. doi:10.1016/j.immuni.2012.10.014

67. Huttenhuis HBT, Taverne-Thiele AJ, Grou CPO, Bergsma J, Saeij JPJ, Nakayasu C, Rombout JHWM. Ontogeny of the common carp (Cyprinus carpio L.) innate immune system. Dev Comp Immunol (2006) 30:557-574. doi:10.1016/j.dci.2005.08.001

68. Corbeil S, LaPatra S. E, Anderson E. D, Kurath G. Nanogram quantities of a DNA vaccine protect rainbow trout fry against heterologous strains of infectious hematopoietic necrosis virus. Vaccine (2000) 18:2817-2824. doi:10.1016/S0264-410X(00)00078-5

69. LaPatra SE, Corbeil S, Jones GR, Shewmaker WD, Lorenzen N, Anderson ED, Kurath G. Protection of rainbow trout against infectious hematopoietic necrosis virus four days after specific or semi-specific DNA vaccination. Vaccine (2001) 19:4011-4019. doi:10.1016/ 
S0264-410X(01)00113-X

70. Lorenzen N, Lorenzen E, Einer-Jensen K, LaPatra SE. Immunity induced shortly after DNA vaccination of rainbow trout against rhabdoviruses protects against heterologous virus but not against bacterial pathogens. Dev Comp Immunol (2002) 26:173-179. doi:10.1016/S0145305X(01)00059-3

71. van der Aa LM, Chadzinska M, Golbach LA, Ribeiro CMS, Lidy Verburg-van Kemenade BM. Pro-inflammatory functions of carp CXCL8-like and CXCb chemokines. Dev Comp Immunol (2012) 36:741-750. doi:10.1016/j.dci.2011.11.011

72. Abós B, Wang T, Castro R, Granja AG, Leal E, Havixbeck J, Luque A, Barreda DR, Secombes CJ, Tafalla C. Distinct Differentiation Programs Triggered by IL-6 and LPS in Teleost IgM(+) B Cells in The Absence of Germinal Centers. Sci Rep (2016) 6:30004. doi:10.1038/srep30004

73. McLauchlan PE, Collet B, Ingerslev E, Secombes CJ, Lorenzen N, Ellis AE. DNA vaccination against viral haemorrhagic septicaemia (VHS) in rainbow trout: Size, dose, route of injection and duration of protection - Early protection correlates with Mx expression. Fish Shellfish Immunol (2003) 15:39-50. doi:10.1016/S1050-4648(02)00137-7

74. Cuesta A, Tafalla C. Transcription of immune genes upon challenge with viral hemorrhagic septicemia virus (VHSV) in DNA vaccinated rainbow trout (Oncorhynchus mykiss). Vaccine (2009) 27:280-9. doi:10.1016/j.vaccine.2008.10.029

75. Castro R, Martínez-Alonso S, Fischer U, Haro NÁ De, Soto-Lampe V, Wang T, Secombes CJ, Lorenzen N, Lorenzen E, Tafalla C. DNA vaccination against a fish rhabdovirus promotes an early chemokine-related recruitment of B cells to the muscle. Vaccine (2014) 32:1160-1168. doi:10.1016/j.vaccine.2013.11.062

76. Dixon P. Immunization with viral antigens: viral diseases of carp and catfish. Dev Biol Stand (1997) 90:221-232.

77. Forlenza M, de Carvalho Dias JDA, Veselý T, Pokorová D, Savelkoul HFJ, Wiegertjes GF. Transcription of signal-3 cytokines, IL-12 and IFN alpha beta, coincides with the timing of CD8 alpha beta up-regulation during viral infection of common carp (Cyprinus carpio L). Mol Immunol (2008) 45:1531-47. doi:10.1016/j.molimm.2007.10.010

78. Piazzon MC, Lutfalla G, Forlenza M. IL10, A Tale of an Evolutionarily Conserved Cytokine across Vertebrates. Crit Rev Immunol (2016) 36:99-129. doi:10.1615/ CritRevImmunol.2016017480 


\section{Supplementary data 1}

\section{A monoclonal raised against the SVCV G protein is able to specifically recognize the native $G$ protein on infected and transfected EPC cells}

Antibodies were raised in mice by immunization with purified SVCV virus and the obtained supernatants were screened and validated through Western blot and immunofluorescence analyses of both infected and transfected EPC cells. Western blot analysis showed that clone $13 \mathrm{C} 10 \mathrm{c}$ recognizes a single protein at around 90 $\mathrm{kDa}$ (Supplementary fig. 1, A.2), which is higher than the calculated $57 \mathrm{kDa}$ protein size of the SVCV G protein but is in accordance with the size detected using the previously validated anti-SVCV polyclonal rabbit antibodies [1]. This difference in size can be most likely ascribed to the presence of carbohydrate moieties on the glycoprotein. Next, infected and transfected EPC cells were used to show the ability of the antibody to also recognize the native SVCV G protein. EPCs were infected with SVCV at an MOI of 1 for $24 \mathrm{~h}$ at $20^{\circ} \mathrm{C}$ and subsequently stained with the selected a-SVCV-G monoclonal antibody (13C10c). In parallel, EPC cells were transfected with pcDNA3-SVCV-G or pcDNA3 and were treated similarly. The antibody showed a strong reactivity to virus plaques in infected wells, but does not react with non-infected cells (Supplementary fig. 1 B). In addition, a specific staining was observed in EPCs transfected with the pcDNA3-SVCV-G plasmid, but not in EPCs transfected with the pcDNA3 vector control (Supplementary fig. 1C). Together this indicates that clone $13 \mathrm{C} 10 \mathrm{c}$ is able to specifically recognize the native SVCV $\mathrm{G}$ protein and it is suitable for immunoblotting and immunohistochemical analysis.

\section{Reference}

1. Hoffmann B, Schütze H, Mettenleiter TC. Determination of the complete genomic sequence and analysis of the gene products of the virus of Spring Viremia of Carp, a fish rhabdovirus. Virus Res (2002) 84:89-100. Available at: www.elsevier.com/locate/virusres [Accessed July 20, 2017] 
A

$$
\begin{aligned}
& \begin{array}{lllll}
1 & 2 & 3 & 4 & \mathrm{kDa}
\end{array}
\end{aligned}
$$

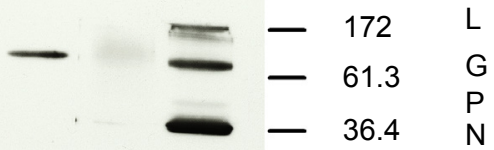

$$
\begin{aligned}
& \begin{array}{r}
\text { - } 24.7 \mathrm{M} \\
-\quad 19.2 \mathrm{M}
\end{array} \\
& -13.2 \\
& -9.3
\end{aligned}
$$

B

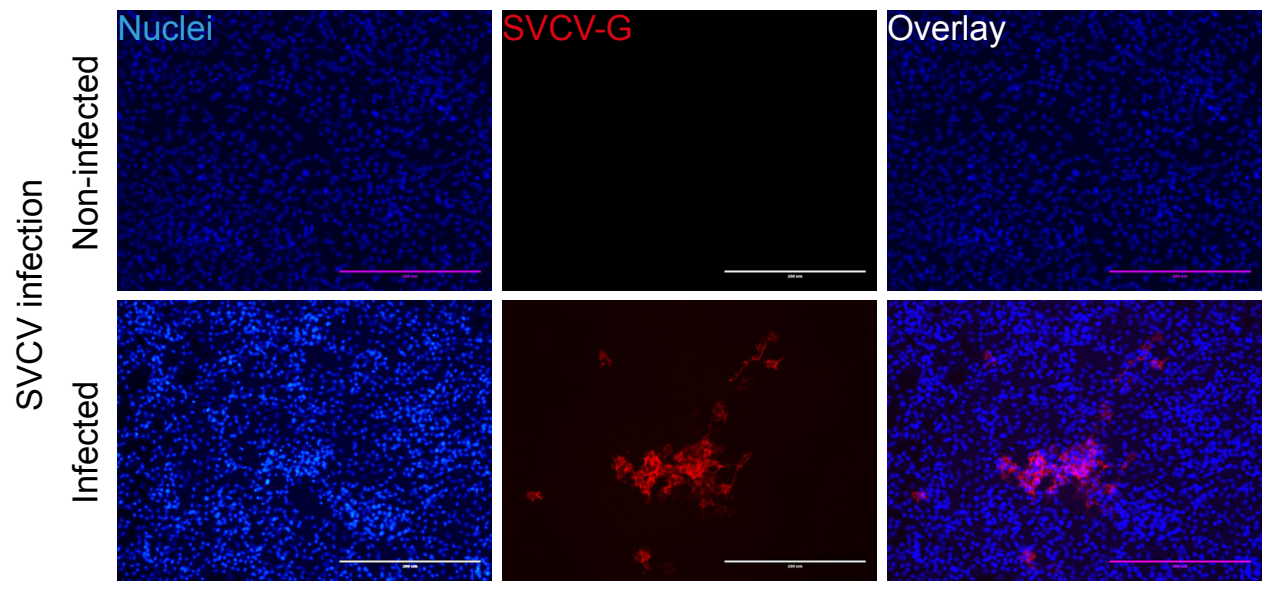

C

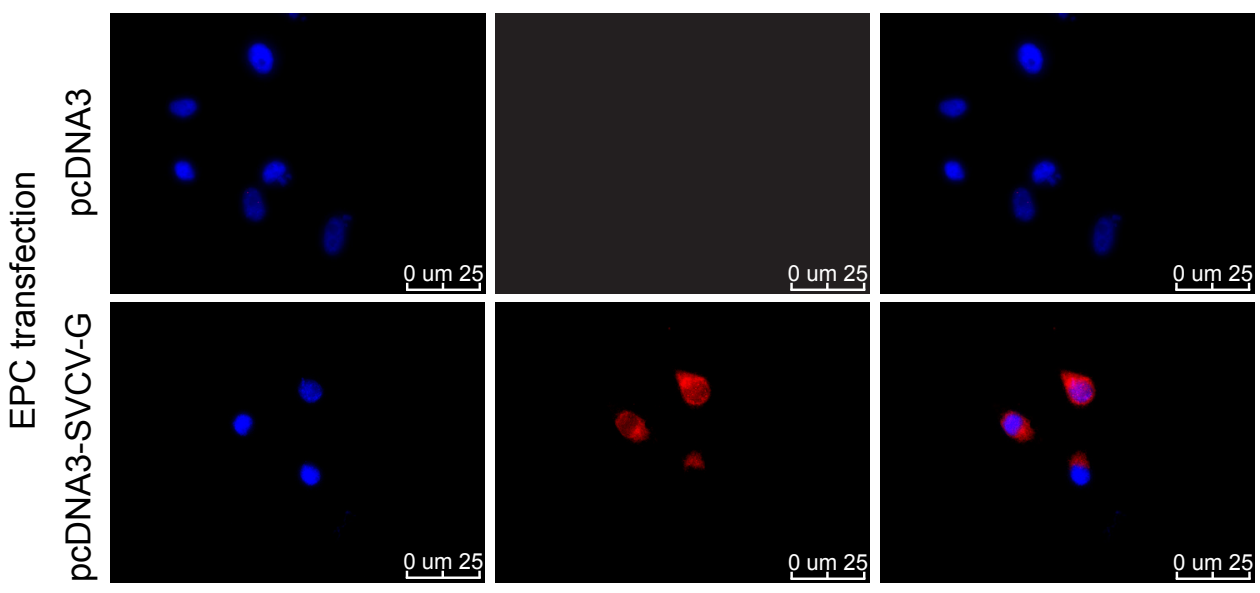


Supplementary fig. 1. Validation of a monoclonal antibody against the SVCV G protein. (A) Proteins from non-infected EPCs (lanes 1 and 3) and purified virions (lanes 2 and 4) were resolved on a 15\% SDS-PAGE gel. Proteins were visualized using hybridoma $13 \mathrm{C} 10 \mathrm{c}$ supernatant (1:10; lanes 1-2) and anti-SVCV polyclonal rabbit serum (1:2000; lanes 3-4). (B) EPC cells were infected with SVCV $(\mathrm{MOI}=5)$ and imaged $24 \mathrm{~h}$ later. (C) EPCs were transfected with $2 \mu \mathrm{g}$ or pcDNA3-SVCV-G or pcDNA3 and imaged 48h later. Infected and transfected EPC were fixed, permeabilized and incubated with hybridoma $13 \mathrm{C} 10 \mathrm{c}$ supernatant $(1: 150)$ followed by incubation with goat-anti-mouse RPE (1:500). DAPI (blue) counterstaining was used to visualize nuclei. Fluorescent images were acquired using a EVOS fI LED fluorescence microscope. 


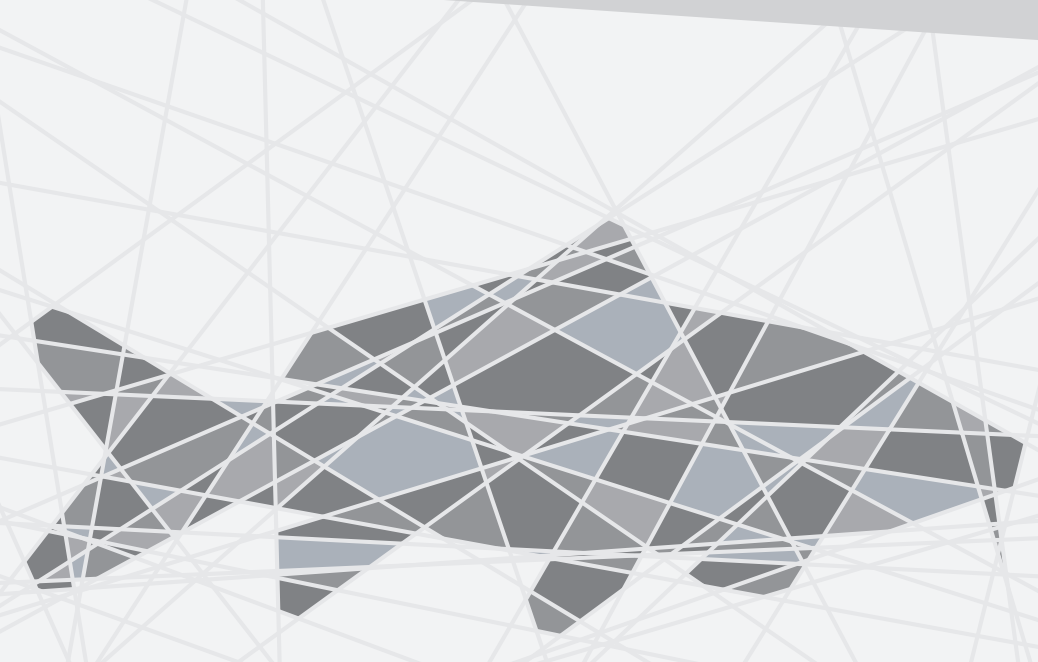




\section{Vaccination of carp against SVCV with an oral DNA vaccine or an insect cells-based subunit vaccine}

\section{Carmen W.E. Embregts', Dimitri Rigaudeau², Luca Tacchi', Gorben P. Pijlman³, Linde Kampers',3, Tomáš Veselý, ${ }^{4}$ Dagmar Pokorová ${ }^{4}$ Pierre Boudinot ${ }^{5}$, Geert F. Wiegertjes', Maria Forlenza'}

I Cell Biology and Immunology Group, Department of Animal Sciences, Wageningen University, Wageningen, The Netherlands

2 INRA, Infectiologie Expérimentale Rongeurs Poissons, Université Paris-Saclay, Jouy-en-Josas, France

${ }^{3}$ Laboratory of Virology, Wageningen University, the Netherlands ${ }^{4}$ Veterinary Research Institute, Brno, Czech Republic ${ }^{5}$ INRA, Virologie et Immunologie Moléculaires, Université Paris-Saclay, Jouy-en-Josas, France

Fish \& Shellfish Immunology (accepted for publication) doi: 10.1016/j.fsi.2018.03.028 


\section{Abstract}

We recently reported on a successful vaccine for carp against SVCV based on the intramuscular injection of a DNA plasmid encoding the SVCV glycoprotein (SVCV-G). This shows that the intramuscular (i.m.) route of vaccination is suitable to trigger protective responses against SVCV, and that the SVCV G-protein is a suitable vaccine antigen. Yet, despite the general success of DNA vaccines, especially against fish rhabdoviruses, their practical implementation still faces legislative as well as consumer's acceptance concerns. Furthermore, the i.m. route of plasmid administration is not easily combined with most of the current vaccination regimes largely based on intraperitoneal or immersion vaccination. For this reason, in the current study we evaluated possible alternatives to a DNA-based i.m. injectable vaccine using the SVCV-G protein as the vaccine antigen. To this end, we tested two parallel approaches: the first based on the optimization of an alginate encapsulation method for oral delivery of DNA and protein antigens; the second based on the baculovirus recombinant expression of transmembrane SVCV-G protein in insect cells, administered as whole-cell subunit vaccine through the oral and injection route. In addition, in the case of the oral DNA vaccine, we also investigated the potential benefits of the mucosal adjuvants Escherichia coli lymphotoxin subunit B (LTB). Despite the use of various vaccine types, doses, regimes, and administration routes, no protection was observed, contrary to the full protection obtained with our reference i.m. DNA vaccine. The limited protection observed under the various conditions used in this study, the nature of the host, of the pathogen, the type of vaccine and encapsulation method, will therefore be discussed in details to provide an outlook for future vaccination strategies against SVCV. 


\section{Introduction}

Spring Viremia of Carp Virus (SVCV) is a cytopathic virus belonging to the genus Sprivivirus of the family Rhabdoviridae and is one of the main viruses affecting carp production. Outbreaks occur mainly during Spring, causing an acute systemic infection in several cyprinid species leading to mortality rates of up to $90 \%$, mainly in juvenile fish $[1,2]$. SVCV virions contain a single, linear, negative-sense, singlestranded RNA molecule, coding for five structural proteins. The glycoprotein $(G)$ is the only one present on the virion surface forming trimeric peplomers that bind to cellular receptors to induce viral endocytosis. For these reasons, the SVCV-G protein is the likely target of protective neutralizing antibodies [2-4].

Recently we reported on an experimental DNA vaccine for European common carp (Cyprinus carpio carpio) against SVCV able to confer up to 100\% protection upon bath challenge with SVCV 2.5 months after vaccination [5]. This protection was achieved by a single i.m. injection of $0.1 \mu \mathrm{g}$ DNA/g of fish of a pcDNA3 vector encoding the SVCV-G protein. We also showed that besides the local inflammation triggered at the site of injection, the DNA vaccine triggered the production of serum neutralizing antibodies and that SVCV-specific T cells were detectable in the blood of vaccinated fish for at least 3 months after vaccination. Thus, similar to the glycoprotein of other fish rhabdoviruses (i.e. viral haemorrhagic septicaemia virus (VHSV) and infectious haematopoietic necrosis virus (IHNV)) (Reviewed in [6]), the glycoprotein of SVCV is an excellent vaccine antigen, at least when administered through i.m. DNA vaccination.

Although to date i.m or intraperitoneal (i.p.), injection vaccination is most effective, oral vaccination would be the ideal delivery method from an animal welfare and handling costs' point of view, (Reviewed in [7]). However, owing to the limited efficacy of the current experimental oral formulations, mass vaccination of fish via the oral route is not common practice. The difficulties in the development of effective oral vaccines are linked to the need to use relatively high vaccine doses, the necessity to protect the antigen against intestinal degradation, as well as the challenges in finding optimal conditions to overcome oral tolerance (Reviewed in [7-10]). To date, a strong interest in the use of DNA-based or subunit vaccines also for oral delivery is currently increasing. For example, oral delivery of alginate encapsulated DNA vaccines against infectious haemorrhagic necrosis virus (IHNV) or against infectious 
pancreatic necrosis virus (IPNV) was shown to confer various degrees of protection in brown trout (Salmo trutta L.) and rainbow trout (Oncorhynchus mykiss) [11,12], showing the potential of oral DNA vaccination of fish against viruses. Interestingly, high protection against IPNV was achieved not only after oral administration of the vaccine by oral gavage, but also after mixing the alginate-encapsulated DNA vaccine [13], or the chitosan-triphosphate (CS-TPP) nanoparticles containing the DNA vaccine, in feed pellets [14]. Most recently, CLYNAV was the first DNA vaccine to receive a positive recommendation for marketing authorization in the European Union for vaccination of salmon against Salmon Alphavirus 3 (SAV3) [15]. Although this is a major breakthrough in the European legislation, the implementation of DNA vaccines in the daily practice is far from being complete. Alternatives to DNA-based vaccines remain of interest and include inactivated pathogens or subunit vaccines.

While successful oral vaccines against SVCV have not been reported thus far, one study showed the potential of oral vaccination of common carp and koi carp (Cyprinus carpio koi) against SVCV and Koi Herpes Virus (KHV), using recombinant Lactococcus plantarum (L. plantarum) expressing both the SVCV-G protein and the KHV-ORF81 protein [16].

Based on the assessed potential of oral vaccination against SVCV, and on the efficacy of the SVCV-G-based i.m. DNA vaccine [5], in the current study we used two parallel approaches to vaccinate carp against SVCV: one based on the oral administration of the SVCV-G DNA vaccine, and the other based on the use of the SVCV-G protein as subunit vaccine for i.m., i.p. or oral delivery. In the first approach we examined the efficacy of alginate microspheres in assuring intact delivery of protein antigens or DNA plasmid to the carp intestine. Next, we compared various vaccination regimes and antigen doses, either or not in combination with the potent mucosal adjuvant Escherichia coli lymphotoxin-beta (LTB) [17]. Furthermore, we analysed local as well as systemic immune responses, based on the expression analysis of immunerelevant genes and the distribution of $\operatorname{~Igm}^{+} \mathrm{B}$ cells, mucosal $\mathrm{T}$ cells, neutrophilic granulocytes and macrophages in the spleen and intestine of carp vaccinated orally with the SVCV-G DNA plasmid. For the second approach, we generated two recombinant Autographa californica multicapsid nucleopolyhedrovirus (AcMNPV) baculoviruses for transmembrane SVCV-G expression in insect cells, based on the proven efficiency of this system to produce membrane-bound and soluble glycoproteins from other rhabdoviruses [18,19]. Indeed, analysis revealed high 
expression of SVCV-G protein on the membrane of recombinant baculovirusinfected insect cells, which allowed us to use whole-cell preparations as SVCV-G subunit vaccine, using various delivery routes.

Despite the various approaches adopted in this study with regard to vaccine design, dose, vaccination regime, the vaccines did not lead to sufficient protection. These factors, as well as the nature of the pathogen, of the host and of the encapsulation method will be discussed in details with the aim to provide an outlook for future vaccination strategies.

\section{Materials and methods}

\section{Animals}

European common carp (Cyprinus carpio carpio) R3xR8, originated from crossbreeding of the Hungarian R8 strain and the Polish R3 strain [20], were used in all experiments. In this study we will refer to carp as the European common carp subspecies, unless stated otherwise. Carp were bred in the Aquatic Research Facility Carus of the animal facility at Wageningen University, the Netherlands. Carp eggs were either kept and raised at the local facility or transported to the Institut National de la Recherche Agronomique (INRA, Paris, France) for viral challenge experiments. Carp were raised at $20-23^{\circ} \mathrm{C}$ in recirculating UV-treated water and fed pelleted carp food (Skretting, Nutreco) twice daily. All animals were handled in accordance with good animal practice as defined by the European Union guidelines for the handling of laboratory animals (http://ec.europa.eu/environment/chemicals/lab_animals/ home_en.htm). All vaccination and challenge studies were performed at INRA. All animal work at INRA was approved by the Direction of the Veterinary Services of Versailles and COMETHEA (authorization number 78-28, project authorization \#2707-2016011318282761), as well as fish facilities (authorization number B78720). Animal work in Wageningen University was approved by the local animal committee (DEC number 2015098).

\section{SVCV}

The reference SVCV strain VR-1390 (isolate stock of the INRA laboratory [21,22]), was propagated in Epithelioma Papulosum Cyprinid (EPC) cells grown in Glasgow's 
modified Eagle's medium (GMEM)-25mMHEPES (Eurobio), supplemented with $10 \%$ foetal calf serum (FCS; Eurobio), 1\% tryptose phosphate broth (Eurobio), $2 \mathrm{mM}$ L-glutamine (PAA), $100 \mu \mathrm{g} / \mathrm{mL}$ penicillin (Biovalley) and $100 \mu \mathrm{g} / \mathrm{mL}$ streptomycin (Biovalley). Virus titers were determined by the method of Reed and Muench [23] and were given as plaque-forming units (pfu).

\section{Insect cells}

Spodoptera frugiperda 21 (Sf21) cells were used for the construction of the recombinant baculoviruses and initial validation of the constructs; S. frugiperda 9 (Sf9) cells were used for the preparation of the SVCV-G subunit vaccine for in vivo vaccination experiments.

Sf21 cells were cultured in Grace's insect medium (Gibco) supplemented with 10\% foetal calf serum (FCS) (Gibco) and $10 \mu \mathrm{g} / \mathrm{mL}$ Gentamycin at $27^{\circ} \mathrm{C}$. Sf9 cells were cultured in Sf-900 II SFM (Thermo Fisher) supplemented with 5\% FCS and 10 $\mu \mathrm{g} / \mathrm{mL}$ Gentamycin. For infection of both cell lines, medium without addition of Gentamycin was used.

\section{Construction of recombinant AcMnPV baculoviruses expressing SVCV-G}

Three recombinant AcMnPV baculoviruses were constructed: one encoding the SVCV-G protein under the control of the polyhedrin $(\mathrm{PH})$ promotor and the reporter gene green fluorescent protein (GFP) under the control of the p10 promotor (bAcGFP-SVCV-G); the second encoding the SVCV-G protein alone under the $\mathrm{PH}$ promotor (bAc-SVCV-G); and the third encoding the GFP protein alone under the p10 promotor (Fig 1). The SVCV-G coding sequence was obtained by PstI-BamHI (NEB) digestion of the pcDNA3-SVCV-G vector [5,24], followed by ligation in the PstI-BamHI restriction sites of the pFastBac Dual-GFP/Polyhedrin vector (Invitrogen), thereby replacing the polyhedrin gene. The $\mathrm{pFastBac}$ Dual vectors were then used to transform competent DH10Bac cells (Thermo Fisher) for subsequent bacmid isolation.

For construction of bAc-SVCV-G, the SVCV G gene was amplified using primers containing a PstI restriction site and Gateway AttB1 or AttB2 sites (AttB1-SVCV-G_ FW GGGGACAAGTTTGTACAAAAAAGCAGGCTTAGAATTCACCATGTCTA TCATCAGCTACATC and AttB2-SVCV-G_RV GGGGACCATTTGTACAAGAA AGCTGGGTACTGCAGTCAAACTAAAGACCGCATTT). The amplicon was 
inserted into a pDONR207 (Thermo Fisher) and a pDEST8 vector (Thermo Fisher) subsequently, using Gateway cloning. Finally, the product was used to transform competent DH10Bac cells for subsequent bacmid isolation.

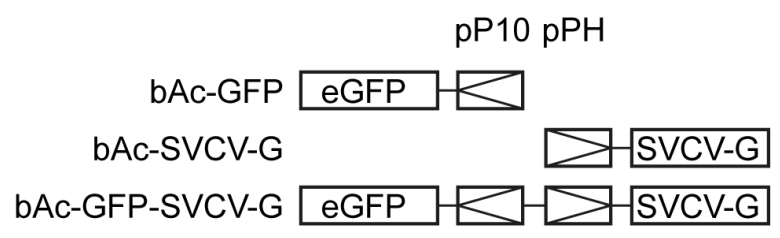

Fig. 1. Schematic representation of the construct design of the three recombinant bacmids encoding GFP under the p10 promotor (pP10), SVCV-G under the polyhedrin promotor $(\mathrm{PH})$ or both.

\section{Insect cell transfection, infection and SVCV-G protein expression}

For generation of baculovirus stocks, Sf21 cells were transfected with the recombinant bacmids using FectoFly (Polyplus Westburg) in non-supplemented Grace's insect medium. Virus was collected at 4-5 days post-transfection and was used to infect a T75 flask of Sf21 to generate the virus master stock for further studies. Virus titres were determined using an end-point dilution assay and calculated according to the formula of Reed and Muench [23].

For subsequent infection and recombinant protein expression, Sf21 or Sf9 cells were infected with the obtained viruses with an multiplicity of infection (MOI) of 4. Cells were harvested for subsequent immunohistochemical analysis or for use as vaccine antigens in vaccination trials. Expression of the SVCV G protein on the cell membrane of infected Sf21 cells was verified by immunofluorescence analysis. Cell were harvested by centrifugation and fixed in $4 \%$ PFA for $15 \mathrm{~min}$ at $4^{\circ} \mathrm{C}$, followed by staining with the mouse monoclonal antibody anti-SVCV-G (clone 13C10c) [5] diluted 1:150 and with a goat-anti-mouse RPE (1:200, BioLegend). Pictures were made using a Zeiss Observer Z1m inverted microscope (Zeiss).

\section{Encapsulation of DNA plasmid and proteins in alginate microspheres}

The pcDNA3-SVCV-G vaccine was prepared as described previously $[5,24]$ using the G protein sequence of the CAPM V 539 strain Jaroslavicky 97 (Accession number: KU934300). The pcDNA3 empty plasmid and PBS were used as negative controls. Alginate encapsulation was performed according to the method used for oral DNA vaccination of brown trout and rainbow trout against IPNV [11] with slight modifications. Briefly, 3\% alginate (alginic acid sodium salt from brown algae, Sigma) in distilled water was mixed in a 1:1 (v/v) ratio with the DNA vaccine (at various concentrations), with or without $5 \mu \mathrm{g}$ LTB/vaccine dose, in PBS. LTB 
kindly provided by Dr. John D. Clemens [17]. The mixture was added dropwise to an Erlenmeyer with paraffin oil or cod liver oil containing 1\% v/v Span-80 surfactant (Sigma). The obtained emulsion was mixed for $10 \mathrm{~min}$ at $1000 \mathrm{rpm}$. Paraffin oil was used in the first challenge experiment including the histological and gene expression analysis, cod liver oil was used in all follow-up in vivo experiments. Microspheres were generated by addition of $0.15 \mathrm{M} \mathrm{CaCl}_{2}$ (equal volume to $3 \%$ alginate solution) and stirring for $2 \mathrm{~h}$ at $1000 \mathrm{rpm}$. With this method, alginate particles between 1.6 and $9 \mu \mathrm{m}$ were obtained. Microspheres were then pelleted by centrifugation for 10 minutes at $1000 \mathrm{x} g$ and the oil supernatant was removed. Alginate preparations were immediately used for oral vaccination or stored at $4{ }^{\circ} \mathrm{C}$.

For analysis of the efficacy of delivery of alginate-encapsulated protein antigens to the intestine of carp, Sf21 cells were infected with bAc-GFP using an MOI of 4 and harvested $72 \mathrm{~h}$ post-infection. Cells were pelleted, washed once with PBS and disrupted by passing 10 times through a 22 -gauge needle on ice, after which the lysate was centrifuged at $21.000 \mathrm{x} g$ to pellet the nuclei and collect the cytosolic fraction containing the recombinant GFP. The obtained lysate was encapsulated in alginates as described above, to obtain an alginate-antigen solution equivalent to 2 x $10^{7}$ cell $/ \mathrm{mL}$ alginate suspension. Non-encapsulated lysates were used as control. Alginate preparations were immediately used for oral administration and subsequent immunofluorescence analysis.

\section{Immunofluorescence}

Carp of six months were anesthetized using $0.3 \mathrm{~g} / \mathrm{L}$ Tricaine Methane Sulfonate (TMS, Crescent Research Chemicals) and received $100 \mu \mathrm{L}$ of encapsulated insect cells lysate containing recombinant GFP by oral gavage with a $200 \mu \mathrm{L}$ pipet. Carp were euthanized $24 \mathrm{~h}$ later using $0.6 \mathrm{~g} / \mathrm{L}$ TMS and bled through the caudal vein. The first, second and third part of the intestine were isolated and cleaned, mounted in CryoCompound mounting medium (Klinipath) and snap-frozen in liquid nitrogen. Five $\mu \mathrm{m}$ cryosections were air-dried, fixed in 4\% paraformaldehyde in PBS for 30 minutes at room temperature, rinsed twice with PBS and embedded in Vectashield with DAPI nuclear staining (Vectorlabs). GFP uptake was visualized using a M205 FA fluorescence stereomicroscope (Leica). 


\section{Immunohistochemistry}

The efficacy of encapsulation and oral delivery of the DNA vaccine was examined by immunohistochemical analysis of $G$ protein expression in the intestine of orally vaccinated carp. For this purpose, carp (3-month-old) received 2 x $10 \mu \mathrm{g}$ of alginateencapsulated pcDNA3 or pcDNA3-SVCV-G by oral gavage, with a $48 \mathrm{~h}$ interval. After 14 days, carp were sacrificed using an overdose of TMS $(0.6 \mathrm{~g} / \mathrm{L})$ and bled through the caudal vein before collection of the second segment of the intestine. Cryosections $(5 \mu \mathrm{m})$ from the intestine were stained with an antibody against the $\mathrm{G}$ protein as described before [5]. Briefly, slides were stained with anti-SVCV-G clone $13 \mathrm{C} 10 \mathrm{c}$ diluted 1:150 followed by incubation with alkaline phosphatase (AP) conjugated goat-anti-mouse (Dako, 1:200). Development was performed using AP substrate $(4.5 \mu \mathrm{l} / \mathrm{mL}$ nitro-blue-tetrazolium (Roche Applied Science) and $3.5 \mu \mathrm{L} /$ mL 5'-bromo-4'-chloro-3'indolyl phosphatase (BCIP; Roche Applied Science)) in AP buffer $\left(0.1 \mathrm{M}\right.$ Tris-Cl, $\left.0.1 \mathrm{M} \mathrm{NaCl}, 0.05 \mathrm{M} \mathrm{MgCl}_{2}\right)$ until sufficient staining was observed.

\section{Vaccination and challenge trials}

Carp of 3 months (2-4 g, $n=20$ per group) were vaccinated at $20^{\circ} \mathrm{C}$ with the alginateencapsulated DNA vaccine ( $10 \mu \mathrm{L} /$ fish $)$ by oral gavage using a $10 \mu \mathrm{L}$ pipet. Controls included i.m. injections of the pcDNA3 empty plasmid or the pcDNA3-SVCV-G vaccine $(0.1 \mu \mathrm{g} \mathrm{DNA} / \mathrm{g}$ of fish, $10 \mu \mathrm{L} / \mathrm{fish})$ and alginate-encapsulated PBS. All orally delivered vaccines were encapsulated in alginate microspheres as described in paragraph 2.3. In the first experiment, carp were orally vaccinated either once with $3 \mu \mathrm{g}$ DNA/g of fish, or three times with $1 \mu \mathrm{g}$ DNA/g fish with a 72h interval. In the second experiment, carp received $20 \mu \mathrm{g}$ or $100 \mu \mathrm{g}$ encapsulated DNA, with or without the addition of $5 \mu \mathrm{g} E$. coli lymphotoxin-beta (LTB) ([17], kindly provided by Dr. John D. Clemens).

In the third vaccination trial, the SVCV-G subunit vaccine was used for injection and oral vaccination. Sf9 cells were infected with bAc-SVCV-G, or with bAc-GFP as control, at an MOI of 4 . After 3 days, cells were collected and the number of $\mathrm{SVCV}_{-\mathrm{G}^{+}}$or $\mathrm{GFP}^{+}$cells was estimated by counting the number of syncytia or the number of $\mathrm{GFP}^{+}$cells. Cells were harvested by centrifugation at $300 \mathrm{x} g$, washed in PBS, fixed in 4\% PFA for 10 minutes at $4^{\circ}$ and washed twice with PBS again. Carp (3-month-old, 2-4 g, $n=20$ per group) were vaccinated with PFA-fixed whole-Sf9 
cells expressing the recombinant SVCV-G. For injection vaccination, the equivalent of $100,1.000$ or 10.000 non-encapsulated $\mathrm{SVCV}_{-\mathrm{G}^{+}}$cells were resuspended in 10 $\mu \mathrm{L}$ PBS and administered through i.m. or i.p. injection. For oral vaccination, the equivalent of $1.000,10.000$ or $100.000 \mathrm{SVCV}^{-\mathrm{G}^{+}}$cells were encapsulated in a final volume of $10 \mu \mathrm{L}$ alginate microspheres and administered through oral gavage. As controls, GFP-expressing cells were used: 10.000 non-encapsulated $\mathrm{GFP}^{+}$cells for i.m. and i.p. injection, or 100.000 encapsulated $\mathrm{GFP}^{+}$cells for oral gavage. As a positive control, i.m. injection of the pcDNA3-SVCV-G vaccine $(0.1 \mu \mathrm{g}$ DNA/g of fish) was used. Prior to vaccination, carp were anesthetized using $0.3 \mathrm{~g} / \mathrm{L}$ TMS. All vaccinations were performed at $20^{\circ} \mathrm{C}$ and before bath challenge with SVCV, 2.5 months after vaccination, the water temperature was gradually lowered to $15^{\circ} \mathrm{C}$ at a rate of $1-2^{\circ} \mathrm{C}$ per day. Challenge was performed by bath, using a viral exposure time of 48 hours and a viral load of $8 \times 10^{6} \mathrm{pfu} / \mathrm{mL}$, as previously optimized [5]. Mortality was recorded over a period of 3-4 weeks.

\section{RNA isolation and CDNA synthesis}

Carp (3-month-old, $n=3 /$ treatment) received two times $10 \mu \mathrm{g}$ of alginate-encapsulated pcDNA3 or pcDNA3-SVCV-G with an 48h interval and were sacrificed 5 days later to collect the intestine (all three segments separately), spleen and gills. Spleen and gills were stored in RNA later, the intestinal segments were snap-frozen immediately. All organs were stored at $-80^{\circ} \mathrm{C}$ until further processing. Total RNA was isolated using the RNeasy Mini Kit (Qiagen) according to the manufacturer's instructions including on-column DNase treatment using the RNase-free DNase set (Qiagen). RNA concentrations were measured using a Nanodrop-1000, the integrity was verified on a $1 \%$ agarose gel and RNA was stored at $-80^{\circ} \mathrm{C}$ until further use. Prior to cDNA synthesis of $1 \mu \mathrm{g}$ total RNA, a second DNase treatment was performed using DNAse I, Amplification Grade (Invitrogen). Reverse transcription of the RNA was performed using random primers (300 ng) and Superscript ${ }^{\mathrm{TM}}$ III (200U) First Strand Synthesis Systems for RT-PCR (Invitrogen). cDNA samples were further diluted 25 times in nuclease-free water and stored at $-20^{\circ} \mathrm{C}$.

\section{Gene expression analysis}

Real-time quantitative PCR (RT-qPCR) was performed using a Rotor-Gene ${ }^{\mathrm{TM}} 6000$ (Qiagen). Fluorescence data were analysed using Rotor-Gene Q series software 
version 2.3.1. Briefly, $5 \mu \mathrm{L}$ of 25 times diluted cDNA was mixed with $2 \mu 1$ of forward and reverse primers $(2.1 \mu \mathrm{M}$ of each primer) and $7 \mu \mathrm{L}$ of $2 x$ ABsolute $\mathrm{qPCR}$ SYBR Green Mix (Thermo Scientific) as detection chemistry. The list of primers can be found in Table 1. The take-off value for each sample and the average reaction efficiencies $(E)$ for each primer set were obtained upon comparative quantitation analysis from the Rotor-Gene software [25]. The relative expression ratio (R) of each sample was calculated based on the take-off deviation of sample versus each of the unhandled control and normalized relative to the $s 11$ protein of the $40 s$ subunit as reference gene.

Table 1. Primers used for Real-Time quantitative PCR analysis

\begin{tabular}{|c|c|c|c|}
\hline Primer & FW primer 5'-3' & RV primer 3'- 5' & Acc. No. \\
\hline \multicolumn{4}{|c|}{ Housekeeping genes } \\
\hline $40 s$ & CCGTGGGTGACATCGTTACA & $\begin{array}{l}\text { TCAGGACATTGAACCTCACT- } \\
\text { GTCT }\end{array}$ & AB012087 \\
\hline \multicolumn{4}{|l|}{ Cytokines } \\
\hline$c x c b 1$ & $\begin{array}{l}\text { GGGCAGGTGTTTTTGTGTTG- } \\
\mathrm{A}\end{array}$ & AAGAGCGACTTGCGGGTATG & AB082985 \\
\hline$i l 1 \beta$ & $\underset{\mathrm{T}}{\mathrm{AAGGAGGCCAGTGGCTCTG-}}$ & $\begin{array}{l}\text { CCTGAAGAAGAGGAGGAG- } \\
\text { GCTGTCA }\end{array}$ & AJ245635 \\
\hline if $n \gamma 1$ & TGCACTTGTCAGTCTCTGCT & TGTACTTGTCCCTCAGTATTT & AM261214 \\
\hline ifn $\gamma 2 a / 2 b$ & $\begin{array}{l}\text { CGATCAAGGAAGATGACCC- } \\
\text { AGTC }\end{array}$ & $\begin{array}{l}\text { GTTGCTTCTCTGTAGACACG- } \\
\text { CTTC }\end{array}$ & AM168523 \\
\hline \multicolumn{4}{|c|}{ Interferon stimulated genes } \\
\hline$m x 1$ & ACAATTTGCGGTCTTTGAGA & CCCTGCCATTTCTCTTCG & cypCar_00015892 \\
\hline vip2 & CTGTCGGACACATCAGC & TCAATGGGCAAGACGAAA & cypCar_00024055 \\
\hline pkr3 & CACGGTGTTTGAAAAGAGC & GACTGGGTCTCAGCATTC & cypCar_00039221 \\
\hline isg15.2 & AGTGTTCGTCAAGAATGAGG & CCTCGCAGACGGAAAAC & cypCar_00039111 \\
\hline \multicolumn{4}{|c|}{ Adaptive immune genes } \\
\hline$i g m$ & $\begin{array}{l}\text { CACAAGGCGGGAAATGAAG- } \\
\text { A }\end{array}$ & $\begin{array}{l}\text { GGAGGCACTATATCAACAGC- } \\
\text { A }\end{array}$ & AB004105 \\
\hline igt1 & AAAGTGAAGGATGAAAGTGT & TGGTAACAGTGGGCTTATT & AB598367 \\
\hline igt2 & GATTCTACTGGGT8CTTCAC & GACATCACTCAACTC8TTCT & AB598368 \\
\hline zap70 & GGAACAAGCCATCATTAGCC & GTCGTCTCTCACCCTCCTG & $\begin{array}{l}\text { Scaf } 2523 \\
\& 63374\end{array}$ \\
\hline
\end{tabular}

\section{Flow cytometry}

Carp received $10 \mu \mathrm{g}$ of encapsulated pcDNA3 or pcDNA3-SVCV-G by oral gavage, or were left unhandled. After 3, 5, 7 and 14 days the intestine (all segments together) and spleen ( $n=3 /$ treatment/time-point)were isolated for flow cytometric analysis of 
cell populations as described before [26,27]. Briefly, single-cell suspensions were loaded onto a 1.02-1.083 Percoll (GE Healthcare) density gradient and centrifuged for $15 \mathrm{~min}$ at $800 \mathrm{x} g$. The obtained total leukocyte populations were stained with specific antibodies against carp Igm (WCI12, 1:100; [28]), putative mucosal T cells (WCL38, 1:100, [29]), macrophages (WCL15, 1:50; [30]) or neutrophilic granulocytes (TCLBE8, 1:50; [31-32]) and goat-anti-mouse PE (1:200, BioLegend). Cells were analysed on a FACS CantoA (BD Biosciences) and data were analysed using FlowJo V10 (BD Biosciences).

\section{Statistics}

For gene expression data, statistical analysis was performed using SPSS Software 22 (IBM). Relative expression ratios $\mathrm{R}$ were transformed $(\mathrm{LN}(\mathrm{R}))$ and significant differences $(\mathrm{p}<0.05)$ were determined by Student's T tests.

\section{Results}

\section{Delivery of alginate-encapsulated pcDNA3-SVCV-G results in G protein expression in the intestine}

To investigate whether alginate encapsulation of our pcDNA3-SVCV-G vaccine would allow for delivery of intact DNA to the second intestinal segment, we analysed the local expression of the SVCV-G protein after oral DNA delivery.

Carp received 2 x $10 \mu \mathrm{g}$ of alginate-encapsulated pcDNA3-SVCV-G by oral gavage. The second intestinal segment was isolated 14 days later and was processed for immunohistochemical detection of the SVCV-G protein. While no G protein staining was observed in the intestinal epithelium of carp receiving the empty pcDNA3 plasmid (Fig. 2A), a clear signal of the G protein could be observed in the pcDNA3SVCV-G group (Fig. 2B). The G protein was clearly expressed along the epithelium, as indicated by the strong purple staining. Furthermore, it was found in concentrated areas throughout the lamina propria, possibly indicating SVCV-G-expressing leukocytes (Fig. 2B). Overall, we show that encapsulation of the pcDNA3-SVCV-G vaccine in alginate microspheres effectively protects the DNA against intestinal degradation thereby ensuring delivery of intact DNA and subsequent protein expression in the second intestinal segment. 
A

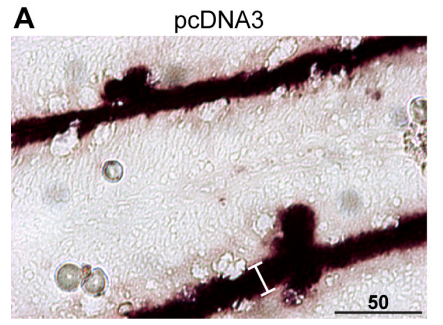

B

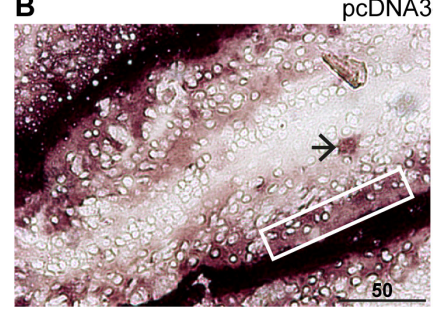

pcDNA3-SVCV-G

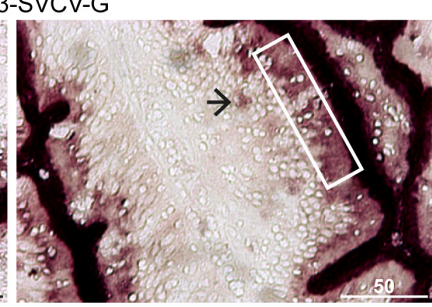

Fig 2. Immunohistochemical analysis of SVCV-G protein expression after oral administration of alginate-encapsulated pcDNA3-SVCV-G vaccine. Carp (3-month-old) received $2 \times 10 \mu \mathrm{g}$ of DNA plasmid (pcDNA3 or pcDNA3-SVCV-G) with a $48 \mathrm{~h}$ interval. The intestine was isolated 14 days later and stained with an antibody specific for the SVCV-G protein. A) Intestinal villi of the second gut segment from fish that received pcDNA3 control plasmid. White brackets indicate the regions of a-specific background staining due to endogenous phosphatase activity. B) Intestinal villi of two different regions of the second gut segment from fish that received the pcDNA3-SVCV-G vaccine plasmid. White boxes indicate strong specific staining of the SVCV-G protein in the epithelial layer; arrows point to putative $\mathrm{G}$ protein-positive cells present throughout the lamina propria, most likely indicating $\mathrm{G}$ proteinexpressing leukocytes. Scale bars indicate $\mu \mathrm{m}$.

\section{Oral administration of low doses of pcDNA3-SVCV-G does not confer protection} against SVCV, nor does it induce a strong local or systemic immune response

After confirming the delivery of the intact DNA plasmid to the intestine of carp, characterized by a strong local expression of the SVCV-G protein, we next examined the protection induced by oral delivery of relatively low doses of the pcDNA3SVCV-G DNA vaccine. Carp were vaccinated orally with 1 dose of $3 \mu \mathrm{g}$ DNA/g of fish or with $1 \mu \mathrm{g}$ DNA/g of fish 3 times with a $72 \mathrm{~h}$ interval. All orally delivered vaccines were encapsulated in alginate microspheres. The naked DNA vaccine injected intramuscularly was used as positive control and all vaccinations where performed at $20^{\circ} \mathrm{C}$. At 2.5 months post-vaccination (mpv) carp were challenged at $15^{\circ} \mathrm{C}$ by bath using $8 \times 10^{6} \mathrm{pfu} / \mathrm{mL}$ for $48 \mathrm{~h}$ and mortality was recorded over a period of 3 weeks. As expected, i.m. vaccination with pcDNA3-SVCV-G induced a 95\% survival (RPS of 92) (Fig. 3). In contrast, no protection was induced by oral delivery of the same DNA vaccine at any of the given regimes. The group receiving a single dose of pcDNA3-SVCV-G had a survival of $20 \%$ and had similar kinetics as all control groups that received either a low or a high dose of pcDNA3 plasmid, with the highest incidence around 8-11 days. Surprisingly, the group receiving three times a low dose of pcDNA3-SVCV-G resulted in only 5\% survival after 21 days, with most of the mortalities occurring already within the first 3 days after challenge. 


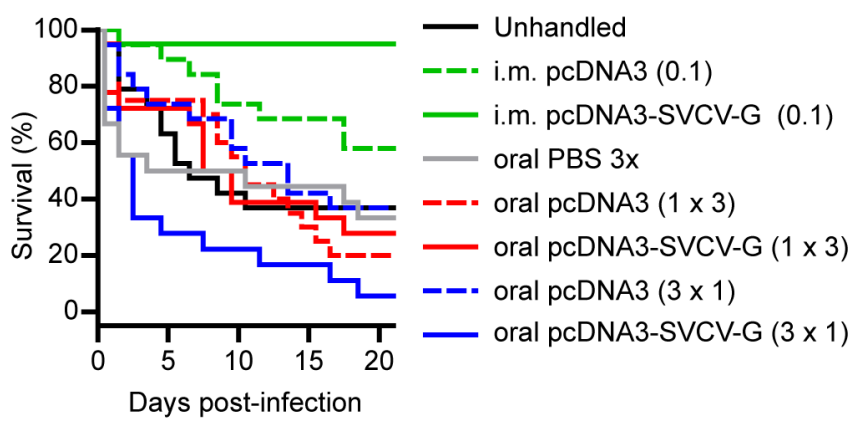

Fig 3. Efficacy of orally administered alginate-encapsulated pcDNA3-SVCV-G vaccine. Carp (3-month-old, $2-4 \mathrm{~g}, n=20 / \mathrm{group})$ were kept at $20^{\circ} \mathrm{C}$ and were orally vaccinated with $1 \times 3 \mu \mathrm{g} \mathrm{DNA} / \mathrm{g}$ of fish or $3 \times 1 \mu \mathrm{g}$ alginate-encapsulated DNA/g of fish with a $72 \mathrm{~h}$ interval. I.m. injection of $0.1 \mu \mathrm{g} \mathrm{DNA} / \mathrm{g}$ of fish was used as a positive control and oral gavage of alginate-encapsulated PBS or pcDNA3 plasmid was included as negative controls. Carp were challenged at $15^{\circ} \mathrm{C}$ by bath for $48 \mathrm{~h}$, using $8 \times 10^{6} \mathrm{pfu} / \mathrm{mL}$ at $2.5 \mathrm{mpv}$. Mortality was recorded for 3 weeks.

After observing the lack of protection induced by oral delivery of the pcDNA3SVCV-G, we next set out to investigate the local response to higher doses of the orally delivered DNA vaccine through gene expression analysis. The panel of genes was selected based on previous analysis of the local response triggered by the same plasmid upon i.m. DNA vaccination, which was previously shown to induce full protection against SVCV [5]. In parallel, we also investigated whether oral vaccination of carp would lead to changes in the relative distribution of leukocytes in the intestine and spleen.

For analysis of local gene expression, carp received $2 \times 10 \mu \mathrm{g}$ of alginate-encapsulated plasmid (pcDNA3 or pcDNA3-SVCV-G) with a $48 \mathrm{~h}$ interval. Intestine (all three segments separate), spleen and gills were isolated 5 days later and used for subsequent gene expression analysis. In the intestine, although a significant upregulation was observed only for $c x c b 1$, in the first segment, in the group receiving the vaccine plasmid (Fig. 4A), a similar but lower response was observed in the second and third segment. Ifn $\gamma 1$, ifn $2 a b, m x 1, p k r 3$ and isg15.2 were all elevated in the intestine but their upregulation was not significantly different from the one observed in the group receiving the empty plasmid. Similarly, the panel of B cell- and T cell-related genes (igm, igt1, igt2, zap70) did not show a vaccine-specific response. In parallel, we analysed the expression of selected genes also in spleen (Fig. 4B) and gills (Fig. 4C) of control and vaccinated carp. While most of the genes showed some degree of response, this was often observed in both, the control and vaccinated group. 
A Intestinal segments
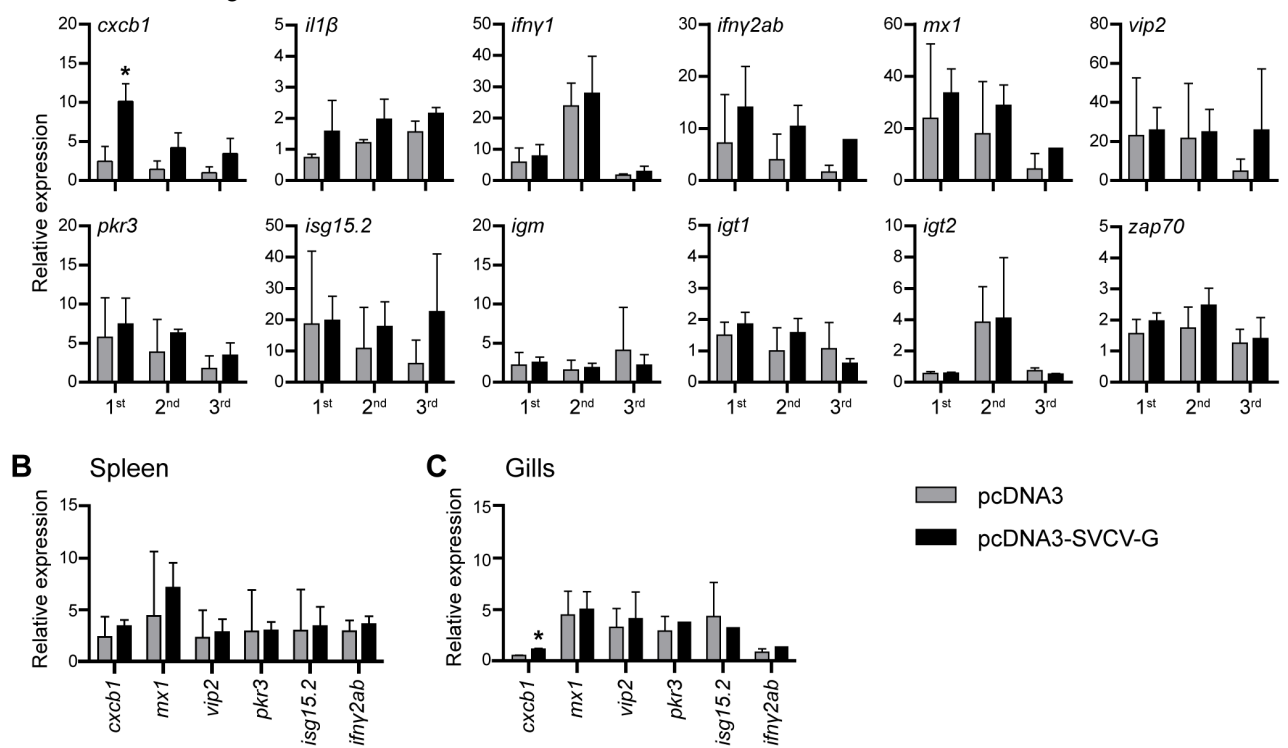

Fig 4. Gene expression analysis of immune-related genes after oral administration of pcDNA3-SVCV-G. Carp (3-months-old) received $2 \times 10 \mu \mathrm{g}$ of plasmid (either pcDNA3 or pcDNA3-SVCV-G) with an interval of $48 \mathrm{~h}$ and organs were isolated 5 days later. Gene expression analysis in the three intestinal segments (A), spleen (B), and gills (C) was normalized against the housekeeping gene s11 of the ribosomal subunit $40 \mathrm{~S}$ and expressed relative to the unhandled control. Significant differences $(p<0.05$, indicated with an asterisk *) were analysed by a Student's T-test. Bars indicate average and SD of $n=3$ fish per time point.

For the flow cytometric analysis of leukocyte distribution in the intestine and spleen of vaccinated carp, organs were isolated at various time points after oral administration of $10 \mu \mathrm{g}$ of either pcDNA3 (control) or pcDNA3-SVCV-G (vaccine) and leukocytes were stained with specific antibodies against $\operatorname{Igm}^{+} \mathrm{B}$ cells, putative mucosal $\mathrm{T}$ cells, macrophages and neutrophilic granulocytes. In the intestine, no apparent changes in the relative percentage of $\operatorname{Igm}^{+} \mathrm{B}$ cells, mucosal $\mathrm{T}$ cells and neutrophilic granulocytes could be observed in the control or vaccine group when compared to the unhandled group (Fig 5A). The relative percentage of macrophages however, was elevated in both the control and vaccinated group, but such increase was not significantly different between the two groups, suggesting that macrophage recruitment is most likely due to the administration of alginate microspheres rather than of the plasmid. In the spleen, moderate changes in the percentage of $\operatorname{Igm}^{+} \mathrm{B}$ cells, mucosal $\mathrm{T}$ cells and macrophages was observed in both groups (Fig. 5B), again suggesting that leukocyte recruitment might be triggered by the alginate administration and not specifically by the DNA plasmid. 
In conclusion, analysis of the local response to the orally delivered DNA vaccine revealed no clear specific response to the oral vaccine and most changes could be ascribed to the administration of alginate microspheres. This moderate response might in part explain the lack of protection observed.

A Intestine
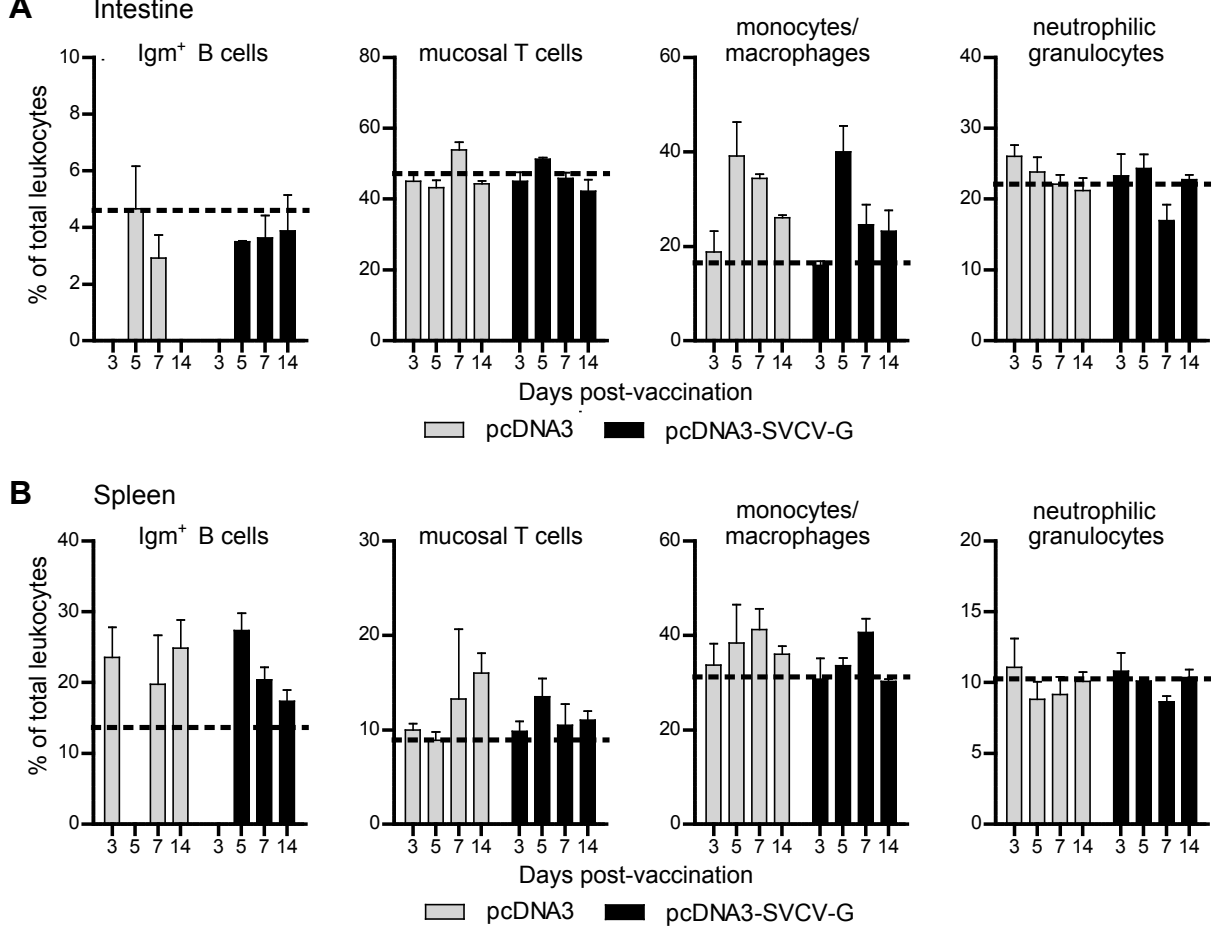

Fig 5. Leukocytes distribution in intestine and spleen after oral delivery of alginate encapsulated pcDNA3-SVCV-G. Carp (3-month-old) received $10 \mu \mathrm{g}$ of plasmid (either pcDNA3 or pcDNA3-SVCV-G), or were left unhandled; 3, 5, 7 and 14 days later the intestine (A) and the spleen (B) were isolated for flow cytometric analysis. Cells were stained with monoclonal antibodies against Igm $^{+} \mathrm{B}$ cells, mucosal T cells, monocytes/macrophages and neutrophilic granulocytes. The dotted line at each graph indicates the average $\%$ of the indicated cell type in unhandled fish. Bars indicate average and SD of $n=3$ fish per time point.

High doses of orally delivered pcDNA3-SVCV-G, with or without the mucosal adjuvant LTB, do not confer protection against SVCV

After observing a higher and faster mortality in the group receiving repeated administration of a low dose of the DNA vaccine (Fig. 2), in the follow-up experiment we used single high doses of encapsulated DNA vaccine, in the presence or absence of a mucosal adjuvant. Carp received $20 \mu \mathrm{g}$ or $100 \mu \mathrm{g}$ of DNA (pcDNA3 or pcDNA3-SVCV-G), with or without the addition of $5 \mu \mathrm{g}$ LTB. Carp were challenged as described above and mortality was recorded over a period of 4 weeks. The i.m. pcDNA3-SVCV-G vaccinated group showed full protection against 
the bath challenge (Fig. 6). Strikingly, in none of the orally vaccinated groups, independently of the presence of the LTB adjuvant, protection could be observed. As already observed in Fig. 3, the group orally vaccinated with a high plasmid dose, this time also in combination with LTB adjuvant, showed a faster kinetic of mortality. In conclusion, we found that under the tested vaccination regimes, oral administration of alginate-encapsulated pcDNA3-SVCV-G vaccine, even in the presence of a potent mucosal adjuvant as LTB, does not confer protection against a bath challenge with SVCV.
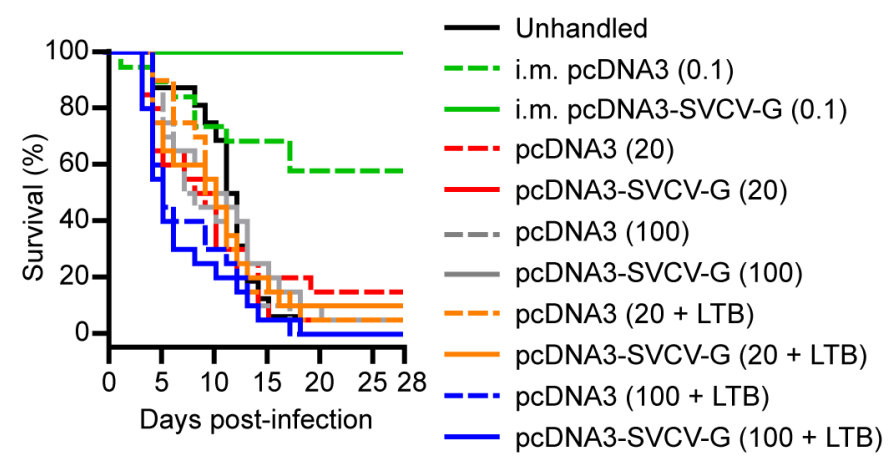

Fig 6. Efficacy of orally administered alginate-encapsulated pcDNA3-SVCV-G vaccine. Carp (3-month-old, $2-4 \mathrm{~g}, n=20 / \mathrm{group}$ ) were kept at $20^{\circ} \mathrm{C}$ and orally vaccinated with $20 \mu \mathrm{g}$ or $100 \mu \mathrm{g}$ alginate-encapsulated DNA plasmid/fish, with or without the addition of $5 \mu \mathrm{g}$ LTB. I.m. injection of $0.1 \mu \mathrm{g} \mathrm{DNA} / \mathrm{g}$ of fish was used as a control. Carp were challenged at $15^{\circ} \mathrm{C}$ by bath for $48 \mathrm{~h}$, using $8 \times 10^{6} \mathrm{pfu} / \mathrm{mL}$ at $2.5 \mathrm{mpv}$. Mortality was recorded for 4 weeks.

\section{Validation of recombinant baculovirus for the production of a SVCV-G subunit vaccine}

In an attempt to generate a vaccine that would allow for a DNA-independent administration of the vaccine antigen, we designed an insect cell-based subunit vaccine. To this end, recombinant AcMNPV baculoviruses encoding the transmembrane form of the SVCV-G protein together with GFP (bAc-GFP-SVCV-G), the SVCV-G protein alone (bAc-SVCV-G) or the GFP protein alone (bAc-GFP) were constructed and were used to infect $\mathrm{Sf} 21$ or Sf9 cells. The expression of the SVCV-G protein on the cell surface was analysed by examining cell morphology and by using a specific monoclonal antibody against the SVCV-G protein [5]. All cells infected with the recombinant baculoviruses encoding for the SVCV-G protein showed clear syncytia formation 3 days after infection, whereas no syncytia could be observed in cells infected with bAc-GFP (Fig. 7A upper panel). Upon extracellular staining with the anti-SVCV-G antibody, expression of the SVCV-G protein on the Sf21 cell membrane could be confirmed (Fig. 7A, middle panel), also coinciding with GFP 
expression in the bAc-GFP-SVCV-G-infected group (Fig. 7A, lower panel) and with the syncytia in the bAc-GFP-SVCV-G and bAc-SVCV-G group (Fig. 7A, middle and lower panel). A similar picture was observed in infected $\mathrm{Sf} 9$ cells (data not shown). Together, the formation of syncytia and the use of the specific antibody against the SVCV-G protein allowed us to confirm that the SVCV-G protein is expressed on the membrane of insect cells infected with the recombinant baculoviruses.

After having assessed that alginate encapsulation allows for delivery of intact DNA plasmid to the intestine, we used the same encapsulation method to assess whether encapsulation was required for efficient delivery of protein antigens to the intestine of carp. To this end, the cytosolic fraction of Sf21 cells infected with bAc-GFP was encapsulated in alginate microspheres and administered to carp by oral gavage; unhandled fish or fish that received non-encapsulated antigens were used as control (Fig. 7B upper panel). After 24h, the intestine was isolated and examined for the presence of GFP. A strong GFP signal was detected in all intestinal segments of carp that received encapsulated antigens (Fig. 7B lower panel), whereas GFP was detected at lower level, only in the last intestinal segment, in carp that received non-encapsulated antigens (Fig. 7B, middle panel). Altogether, this confirms that encapsulation of proteins greatly enhances protein delivery to all intestinal segments of carp and might thus be suitable for the formulation of subunit vaccines for oral delivery.

\section{Insect cells-based SVCV-G subunit vaccine does not confer protection against SvcV}

After verifying the expression of the SVCV-G protein on bAc-SVCV-G-infected insect cells, whole-cells were used as source of the subunit vaccine and administered to carp using different administration routes. Carp received PFA-fixed whole Sf9 cells expressing the recombinant SVCV-G protein by oral gavage of alginateencapsulated cells $\left(1.000,10.000\right.$ or $100.000 \mathrm{SVCV}-\mathrm{G}^{+}$cells/fish) or by i.m. or i.p.

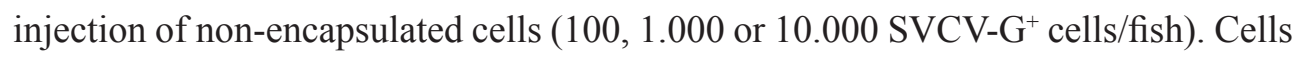
expressing recombinant GFP were used as control using the maximum dose used of the SVCV-G-expressing cells and the administration route of the corresponding vaccinated group. In addition, a group vaccinated by i.m. injection with the pcDNA3SVCV-G vaccine $(0.1 \mu \mathrm{g}$ DNA/g of fish) was used as a positive control.

Carp were challenge 2.5 months later. In the orally vaccinated groups, $25 \%$ of carp survived in the control group, whereas only $10 \%$ or $5 \%$ survived in the group 
vaccinated with 1.000 or 10.000 cells, respectively, and none of the fish survived in the group receiving the highest dose (Fig. 8A). In the i.p. injected groups, all vaccinated fish showed a survival ranging between $5 \%$ and $30 \%$, which was lower than the corresponding injected control (Fig. 8B). In the i.m. injected groups, the highest survival (25\%) and a delayed onset of mortality was observed in the group

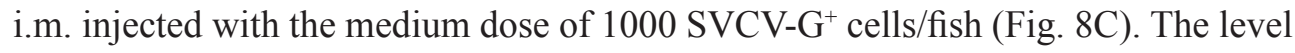
of protection however, was much lower than the one observed in the corresponding positive control group i.m. injected with the pcDNA3-SVCV-G plasmid in which $90 \%$ survival was observed. In general, compared to the groups vaccinated through the i.m. or i.p. route, the group vaccinated orally showed the fastest kinetics of mortality, with the highest incidence at 5 days after challenge, and the highest mortality in the group receiving the highest vaccine dose. In conclusion, the use of an insect cell-based subunit vaccine against SVCV, under the conditions, doses and vaccination regimes used in our study, was not able to induce significant protection against a bath challenge with SVCV.

Despite the lack of protection observed under the various conditions used, the fact that we used different vaccine types, administration routes, and vaccine doses, in the presence or not of an adjuvant, provides us with valuable information to discuss in details the design of future vaccination strategies.

\section{Discussion}

In this study we tested two approaches for vaccination of carp against SVCV. The first approach focused on the oral delivery of alginate-encapsulated SVCV-G plasmid DNA; the second approach consisted of a baculovirus-based recombinant expression of the transmembrane SVCV-G protein in insect cells, which were subsequently used as a whole-cell subunit vaccine for oral or injection delivery. We did not only test different vaccine types but also various doses, regimes, administration routes and, in the case of the DNA-based oral vaccine, the use of a strong mucosal adjuvant (E. coli lymphotoxin-beta, LTB). Protection under all tested conditions proved insufficient, certainly when compared with the full protection obtained with the reference pcDNA3-SVCV-G vaccine when injected i.m. We discuss how our results may have been influenced by the nature of the pathogen, host species and encapsulation 
A
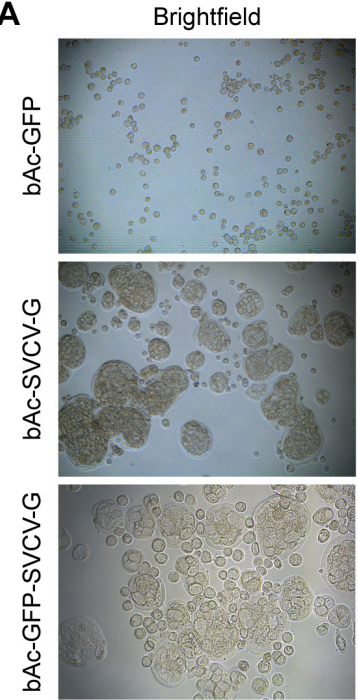

B
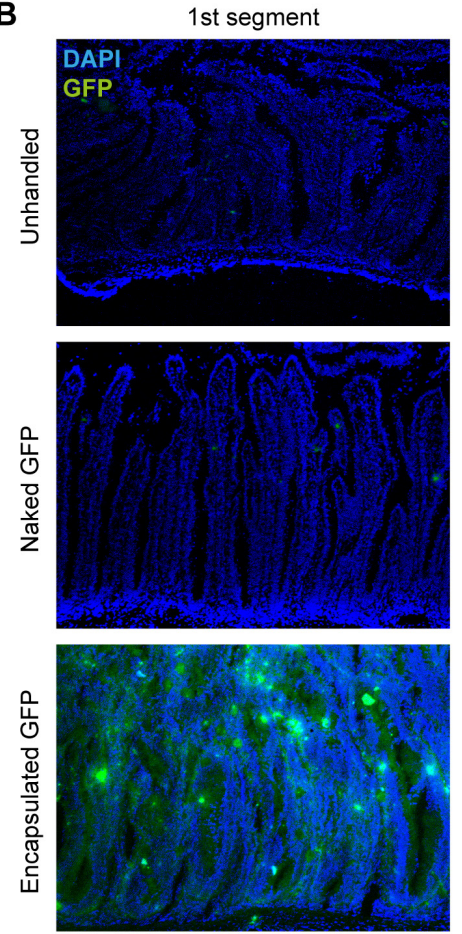

GFP
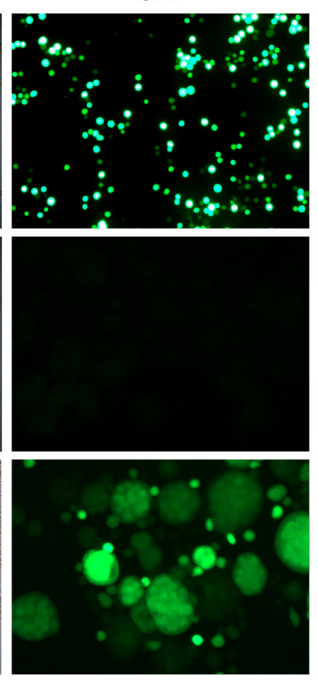

SVCV-G
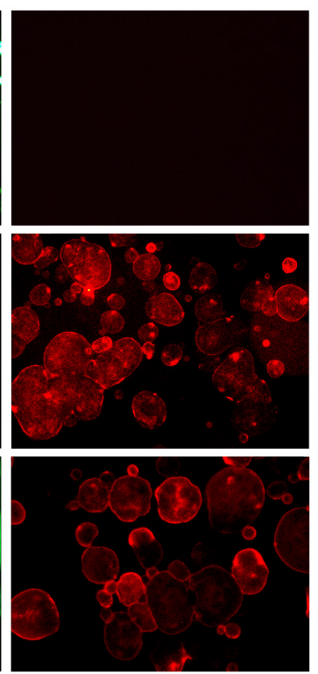

2nd segment
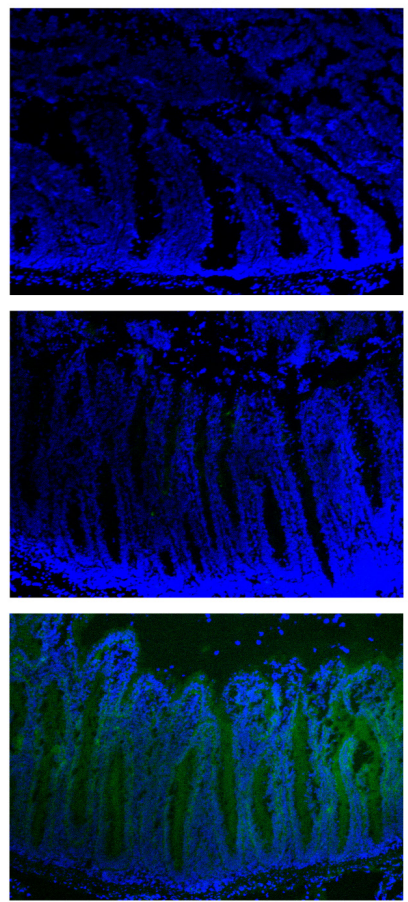

Overlay
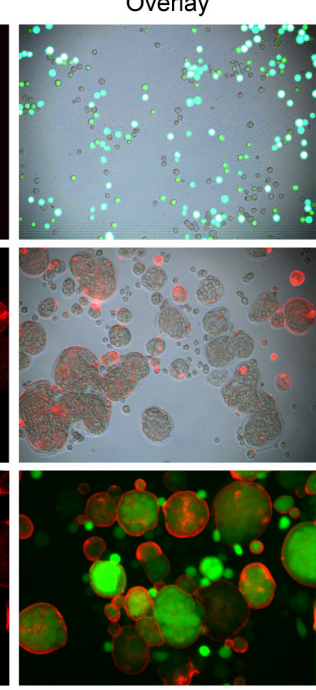

3rd segment
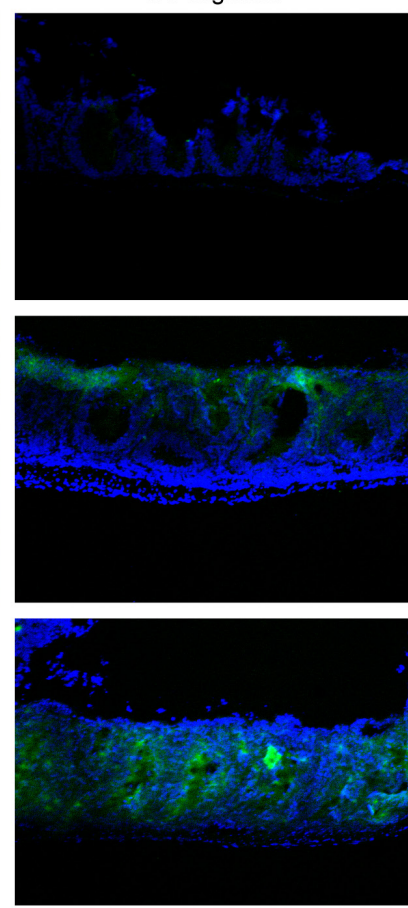

Fig 7. Validation of an insect-cell based SVCV-G expression system and encapsulation efficacy of protein antigens. A) Sf21 cells were infected with the indicated recombinant baculoviruses (bAc) at an MOI of 4 and after 48 hours GFP and SVCV-G protein expression was visualized. Cells were fixed with PFA and stained with a monoclonal antibody against the SVCV-G protein (1:150) and goat-anti-mouse RPE (1:200). Images were acquired with a Zeiss Observer Z1m inverted microscope and all images were obtained using a 20 times magnification. Note the formation of syncytia only in the cells infected with the baculovirus 
encoding the SVCV-G protein. For clarity, the brightfield channel is not shown in the overlay picture in the bottom tight panel. B) Sf21 cells were infected with the bAc-GFP baculovirus at an $\mathrm{MOI}$ of 4 for $72 \mathrm{~h}$. Cells were harvested and the cytosolic fraction containing the GFP was encapsulated in alginate microspheres. Carp (6-month-old) received $100 \mu \mathrm{L}$ of encapsulated cell lysate (equivalent to $150.000 \mathrm{GFP}^{+}$cells). After $24 \mathrm{~h}$, the intestine was isolated and the presence of GFP signal was examined in cryosections. A counterstaining with DAPI was included to visualize the cell nuclei. Images were acquired using a M205 FA fluorescence stereomicroscope (Leica).

A

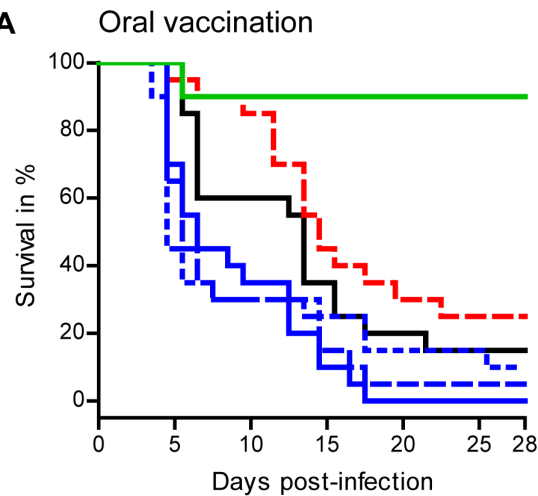

C

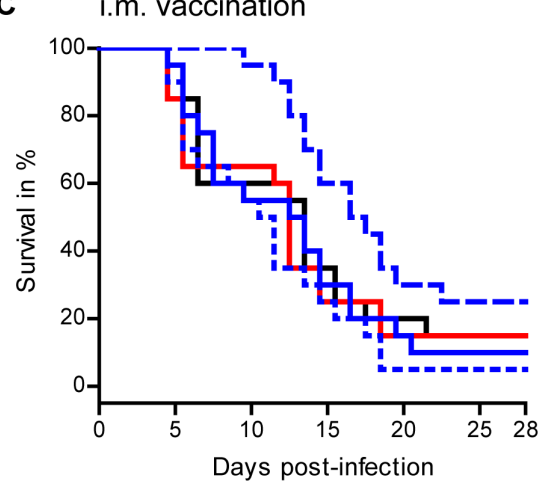

B i.p. vaccination

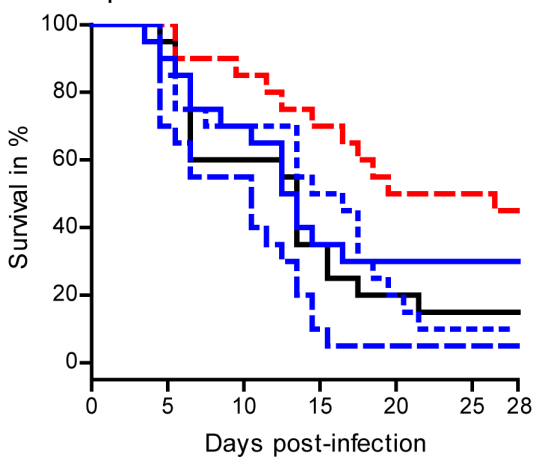

- Unhandled

pcDNA3-SVCV-G $0.1 \mu \mathrm{g} / \mathrm{g}$

- bAc-GFP 100.000

-- bAc-SVCV-G 1.000

- bAc-SVCV-G 10.000

bAc-SVCV-G 100.000

Fig. 8. Efficacy of injection and oral vaccination with a recombinant SVCV-Gbased subunit vaccine. Carp (3-month-old, $n=20 /$ group) were kept at $20^{\circ} \mathrm{C}$ and were vaccinated with SVCV-G+ PFA-fixed whole Sf9 cells using various dose and administration routes. For clarity, the vaccinated groups and respective controls are shown in separate graphs, whereas the unhandled group is shown in each graph as a reference. (A) Carp received $1.000,10.000$ or 100.000 alginate-encapsulated SVCV-G+ cells, or 100.000 $\mathrm{GFP}^{+}$cells through oral gavage. (B) Carp received $100,1.000$ or 10.000 SVCV-G ${ }^{+}$ cells, or $10.000 \mathrm{GFP}^{+} \mathrm{Sf9}$ cells through i.p. injection or (C) through i.m. injection. The group vaccinated i.m. with pcDNA3-SVCV-G $(0.1 \mu \mathrm{g} / \mathrm{g}$ of fish) served as positive control. 
method used in our study.

In our first approach, we used an encapsulation method based on alginate microspheres. Immunohistochemical analysis revealed high levels of SVCV-G protein expression in the intestinal epithelium after oral administration of encapsulated pcDNA3SVCV-G plasmid, confirming that the plasmid was delivered intact, was taken up by epithelial cells, finally resulting in SVCV-G protein translation.

In a first vaccination trial we tested relatively low DNA vaccine doses, administered orally either once ( $3 \mu \mathrm{g}$ DNA/g of fish) or three times ( $1 \mu \mathrm{g}$ DNA/g of fish with a $72 \mathrm{~h}$ interval). Under these conditions, no protection was obtained and a steeper and quicker mortality was observed in the group vaccinated three times with the lowest dose of the vaccine. As further discussed later, this might be indicative of tolerance induction. Despite the ascertained SVCV-G protein expression, oral delivery of the encapsulated DNA vaccine did not induce strong local and systemic immune responses (antiviral genes expression, recruitment of neutrophilic granulocytes, macrophages, $\operatorname{Igm}^{+} \mathrm{B}$ cells, and putative mucosal T cells) as those typically observed upon i.m. injection of the same DNA vaccine [5].

In our second trial, we used a single but higher vaccine dose and orally administered either $20 \mu \mathrm{g}$ or $100 \mu \mathrm{g}$ of alginate-encapsulated plasmid per fish, with or without further addition of the mucosal adjuvant LTB. Despite the use of a higher dose and addition of an adjuvant, no protection was observed. Again, a slightly steeper mortality curve in the group vaccinated with the highest dose of plasmid in the presence of LTB was observed. Bacterial toxins and their derivatives, although among the strongest mucosal adjuvants, are often most effective when administered nasally (not orally) and coupled to antigens [33-35], which may partly explain our observation. In addition, in our study, the absence of a clear effect of LTB on vaccine efficacy, could be explained by mistiming: possibly immune stimulation by LTB could have occurred prior to sensing of the SVCV-G protein by immune cells since SVCV-G protein expression first required uptake of the plasmid, transcription, and finally translation. Future studies focusing on oral delivery of DNA plasmid could deliver LTB as a plasmid thereby synchronizing its expression with the one of the vaccine antigens.

With regard to the host species, it is of interest to compare our study to previous studies in trout in which oral DNA vaccination against IPNV and IHNV was reported with various degrees of success. The encapsulation method, plasmid dose, regime 
and age of vaccination used in our first trial were comparable to those previously reported for oral DNA vaccination of trout against IHNV and IPNV. However, while a single oral administration of $10 \mu \mathrm{g}$ of alginate-encapsulated plasmid induced full protection against IPNV in 3-month-old brown trout and rainbow trout of 1-1.5 g [11], the same plasmid dose and vaccination regime was not successful in protecting rainbow trout against IHNV [12]. In the latter study, a significant degree of protection (RPS=56) was observed only in trout receiving a very high dose $(100$ $\mu \mathrm{g}$ ) of encapsulated DNA plasmid. Although significant, protection was lower than the one achieved after i.m. injection of $5 \mu \mathrm{g}$ of the same plasmid (RPS=76) [12]. The clear difference with respect to the plasmid dose required to achieve protection, suggests that the nature of the pathogen (IPNV versus IHNV) might play a crucial role in achieving protection after oral DNA vaccination. While IPNV is a mucosal pathogen, mainly targeting intestine and pancreas, SVCV is a systemic pathogen similarly to IHNV. SVCV mainly targets kidney and spleen and a strong systemic response might therefore be more important than a strong local mucosal immune response. Although oral vaccination is known to trigger both a local and a systemic response [9,36,37], the systemic response induced by our oral DNA vaccine may not have been strong enough to induce protection. This could maybe also explain the difference in success between oral DNA vaccination against IPNV and against IHNV, since IHNV is a rhabdovirus requiring a stronger systemic response than the mucosal pathogen IPNV [12]. In addition, the time of challenge post-vaccination, 30 days (450 degree days approximately) in the trout studies versus 2.5 months (1500 degree days approximately) in our study, is a well-known determining factor when evaluating protection. It could be informative to further investigate the duration of protection induced by the oral DNA vaccines against IPNV and IHNV.

In our second approach, we generated an SVCV-G-based subunit vaccine and tested its efficacy after both, injection (i.m and i.p.) and oral vaccination. To this end, formalin-fixed whole Sf9 cells expressing the recombinant transmembrane SVCV-G protein where used to vaccinate carp through i.p., i.m., and oral delivery. Only the cells used for oral delivery were encapsulated in alginate microspheres, whereas the cells used for injection vaccination were whole-cell suspensions. Despite the use of various doses and routes of administration, none of the tested regimes induced significant protection in carp when challenged with SVCV 2.5 months after vaccination. We hypothesised that the insect cells would act both as vaccine 
vehicle by displaying the recombinant SVCV-G protein on the cell surface in its native conformation, and as adjuvant by expressing non-self-proteins. To illustrate the potency of our approach, i.m. injection of only few thousands cells expressing the rabies virus $G$ protein was shown to be sufficient to achieve $100 \%$ protection in mice [38]. Furthermore, a strong protection was achieved also in raccoons vaccinated orally with lysate of insect cells infected with a baculovirus encoding the rabies G protein [39]. Yet, we did not observe a similar protection in our carp study. In the mouse study, animals received two i.m. injections with 1-week interval and protection was assessed 14 days post-immunization. Only mice receiving $2 \mathrm{x}$ 1000 cells, but not lower number of cells, showed full protection. Comparison of the weight of these mice (approximately $20 \mathrm{~g}$ at 6 weeks) with the weight of the fish used in our study (2-4 $g$ at 3 months), indicates that the relative antigen dose (i.e. absolute number of SVCV-G ${ }^{+}$insect cells) was higher in our study, and thus dose may not explain the difference in protection observed in carp injected with whole Sf9 insect cells expressing the recombinant transmembrane SVCV-G protein.

While injection by i.m. route of the pcDNA3-SVCV-G vaccine, already at a low dose of $0.1 \mu \mathrm{g} / \mathrm{g}$ of fish, was shown to confer full protection against challenge with SVCV [5], i.m injection of the SVCV-G $\mathrm{G}^{+}$insect cell-based recombinant vaccine induced $20 \%$ protection at best. This suggests that crucial protective mechanisms that are triggered by the injection of DNA plasmid, are not (sufficiently) triggered by the injection of the whole-cell-based subunit vaccine. These could include inflammatory responses seen in the muscle upon i.m. injection of DNA plasmid and ascribed to vaccine-induced local damage and recognition of molecular patterns on the plasmid backbone (i.e. CpG motifs) [5,40,41]. Possibly, also local antigen expression by host cells, normally induced by the DNA vaccine, assuring peptide presentation on MHC-I molecules and likely activation of the cytotoxic T cell compartment, may not have been triggered by the subunit vaccine. Yet, the co-presence of insect cells as non-self-antigens, and the damage inflicted by the injection should have mimicked at least some of the conditions normally induced by i.m. injection of DNA plasmid. For example, the presence of the native $G$ protein on insect cells should have activated specific humoral responses. One possible explanation could be that the insect cells are (too) rapidly eliminated by the host: it is not possible to accurately estimate the total amount and persistence of G protein locally in the muscle after injection of DNA plasmids since several copies of plasmid DNA can be incorporated in 
each cell and transcribed at various rates. However, what we and others observed, was that $\mathrm{G}^{+}$myocytes could be detected up to 14-21 days after plasmid injection although myocytes were in the process of being eliminated by host cells $[5,42]$. If indeed insect cells are (too) rapidly eliminated, this would reduce the duration of antigen persistence in vivo and negatively affect the required antigen presentation and subsequent induction of a protective immune response. Given the importance of the three-dimensional conformation of the SVCV-G protein and the possible impact of detergents used to lyse cells, we decided to administer the SVCV-G-expressing insect cells as (encapsulated) whole cells instead of (non-encapsulated) lysates as in the raccoon study [39]. Possibly, the presence of an encapsulation matrix and/or the lower availability of damage-associated molecular patterns (DAMPs) might partly explain the observed differences in vaccine efficacy between the two studies.

Altogether, when considering the host species and the oral vaccination approach we took, although alginates were very successful for oral vaccination of brown trout and rainbow trout against IPNV and IHNV [11,13], alginate might not be a universal encapsulation matrix for all fish species. Salmonids are carnivorous species and plantbased alginates are not part of their standard diet. In contrast, carp are omnivorous species and might tolerate plant-based alginate much better, minimizing the costimulatory effect seen in salmonids. Therefore, encapsulation matrixes that are not plant-based, like the copolymer poly(lactic-co-glycolic acid) (PLGA) or chitosan, might prove more effective in carp. Finally, the ability of alginates to protect or release their cargo in the intestinal lumen can be highly affected by the $\mathrm{pH}$ of the intestinal environment (Joosten et al. 1997), which is different between fish species. Thus, the maximum release should be assessed and optimized for each species.

Furthermore, with respect to the adjuvant used, while linking of GFP to LTB was previously found to enhance GFP uptake and specific humoral responses in carp [43], LTB did not increase protection in our case when administered as protein antigen together with the DNA vaccine mix. Possibly, other adjuvants or immunostimulants may be more suitable for oral vaccination of carp, and may include saponins, flagellin, $\beta$-glucans, and molecular adjuvants including cytokines $[8,44]$. Some of these could be administered as plasmids along with DNA vaccines, or directly as proteins or polysaccharides along with subunit vaccines, in both cases assuring similar kinetics of uptake and expression of the adjuvant and vaccine antigen.

With respect to vaccine efficacy, in all our trials a faster kinetic of mortality was 
generally observed in the groups orally vaccinated using alginate-encapsulated DNA or proteins. Although the induction and mechanisms of tolerance in fish are still elusive, some reports hint at the possible induction of tolerance in carp when low doses of antigens are delivered over a longer period of time (Joosten et al., 1997; Rombout et al., 1989). In our study, we administered antigens orally once, two or three times with a short time interval. Whether the lack of protection was due to insufficient antigen dose or to the induction of oral tolerance, possibly due to the regimes used, is not known and was not the focus of the current study. Perhaps, using our SVCV-G DNA vaccine as model, it will be possible in the future to more systematically compare which protective mechanisms are triggered upon injection vaccination, and which are triggered (or are absent) upon (unsuccessful) oral vaccination using the same vaccine. The paucity of tools to thoroughly investigate mucosal responses, in particular B and $\mathrm{T}$ cell responses at mucosal surfaces, greatly hampers such in-depth type of analysis. There is no doubt however, that more insights into the mechanisms behind induction of oral tolerance in fish will greatly advance future oral vaccine design.

To conclude, while injection vaccination regimes can provide a situation where the exact vaccine dose and exact location of the antigen uptake are known, oral vaccination regimes appear more difficult to optimize and standardize. This is mostly caused by the large surface area of the intestine, the possibility of antigen breakdown in the gastro-intestinal tract and/or the highly tolerogenic environment. Furthermore, an improved understanding of the mechanisms important for optimal functioning of mucosal adjuvants would help to steer the subtle balance between tolerance and activation of protective responses. It may be clear that in order to formulate an effective vaccine, parameters such as vaccine dose, vaccination regime, antigen formulation, encapsulation, and choice of adjuvants, all need to be tailored to the fish species and targeted to the specific pathogen of interest.

\section{Competing interests}

The authors declare to have no conflict of interests. 


\section{Acknowledgements}

This work was supported by the European Commission under the $7^{\text {th }}$ Framework Programme for Research and Technological Development (FP7) of the European Union (Grant Agreement 311993 TARGETFISH). PB received institutional support from INRA. TV and DP were also partly supported by the Ministry of Agriculture of the Czech Republic (MZE-RO0517). The authors wish to thank Dr. John D. Clemens from the Department of Microbiology and Immunology, Tulane University School of Medicine, New Orleans US for providing the LTB adjuvant and the support staff of the Aquatic Research Facility Carus, of the animal facility at Wageningen University for fish breading and rearing.

\section{References}

1. Ahne W. The influence of environmental temperature and infection route on the immune response of carp (Cyprinus carpio) to spring viremia of carp virus (SVCV). Vet Immunol Immunopathol (1986) 12:383-386. doi:10.1016/0165-2427(86)90144-3

2. Ahne W, Bjorklund HV, Essbauer S, Fijan N, Kurath G, Winton JR. Spring viremia of carp (SVC). Dis Aquat Organ (2002) 52:261-272. doi:10.3354/dao052261

3. Dauber M, Schütze H, Fichtner D. Determination of the complete genomic sequence and analysis of the gene products of the virus of Spring Viremia of Carp, a fish rhabdovirus. Bull Eur Assoc Fish Pathol (2001) 21:170-177. doi:10.1016/S0168-1702(01)00441-5

4. Teng Y, Liu H, Lv JQ, Fan WH, Zhang QY, Qin QW. Characterization of complete genome sequence of the spring viremia of carp virus isolated from common carp (Cyprinus carpio) in China. Arch Virol (2007) 152:1457-1465. doi:10.1007/s00705-007-0971-8

5. Embregts CWE, Rigaudeau D, Veselý T, Pokorová D, Lorenzen N, Petit J, Houel A, Dauber M, Schütze H, Boudinot P, et al. Intramuscular DNA Vaccination of Juvenile Carp against Spring Viremia of Carp Virus Induces Full Protection and Establishes a Virus-Specific B and T Cell Response. Front Immunol (2017) 8:1340. doi:10.3389/fimmu.2017.01340

6. Lorenzen N, LaPatra SE. DNA vaccines for aquacultured fish. Rev Sci Tech (2005) 24:201213. doi:10.3354/dao056031

7. Embregts CWE, Forlenza M. Oral vaccination of fish: Lessons from humans and veterinary species. Dev Comp Immunol (2016) 64:118-137. doi:10.1016/j.dci.2016.03.024

8. Tafalla C, Bøgwald J, Dalmo R a. Adjuvants and immunostimulants in fish vaccines: Current knowledge and future perspectives. Fish Shellfish Immunol (2013) 35:1740-1750. doi:10.1016/j.fsi.2013.02.029

9. Mutoloki S, Munang'andu HM, Evensen O, Munang'andu HM, Evensen Ø. Oral vaccination of fish- antigen preparations, uptake and immune induction. Front Immunol (2015) 6:519. doi:10.3389/fimmu.2015.00519

10. Munang'andu HM, Mutoloki S, Evensen Oø. An overview of challenges limiting the design of protective mucosal vaccines for finfish. Front Immunol (2015) 6: doi:10.3389/ fimmu.2015.00542

11. de las Heras AI, Rodríguez Saint-Jean S, Pérez-Prieto SI. Immunogenic and protective effects of an oral DNA vaccine against infectious pancreatic necrosis virus in fish. Fish 
Shellfish Immunol (2010) 28:562-570. doi:10.1016/j.fsi.2009.12.006

12. Ballesteros NA, Alonso M, Rodríguez Saint-Jean S, Perez-Prieto SI. An oral DNA vaccine against infectious haematopoietic necrosis virus (IHNV) encapsulated in alginate microspheres induces dose-dependent immune responses and significant protection in rainbow trout (Oncorrhynchus mykiss). Fish Shellfish Immunol (2015) 45:877-888. doi:10.1016/j.fsi.2015.05.045

13. Ballesteros NA, Rodriguez Saint-Jean S, Perez-Prieto S. Food pellets as an effective delivery method for a DNA vaccine against infectious pancreatic necrosis virus in rainbow trout (Oncorhynchus mykiss, Walbaum). Fish Shellfish Immunol (2014) 37:220-8. doi:10.1016/j. fsi.2014.02.003

14. Ahmadivand S, Soltani M, Behdani M, Evensen Ø, Alirahimi E, Hassanzadeh R, Soltani E. Oral DNA vaccines based on CS-TPP nanoparticles and alginate microparticles confer high protection against infectious pancreatic necrosis virus (IPNV) infection in trout. Dev Comp Immunol (2017) 74:178-189. doi:10.1016/j.dci.2017.05.004

15. EMA. First DNA vaccine in the EU recommended for use in salmon. 2016-04-22 (2016)1. Available at: http://www.ema.europa.eu/ema/index.jsp?curl=pages/news_and_events/ news/2016/04/news_detail_002516.jsp\&mid=WC0b01ac058004d5c1 [Accessed September $18,2017]$

16. Cui L-C, Guan X-T, Liu Z-M, Tian C-Y, Xu YG. Recombinant lactobacillus expressing $\mathrm{G}$ protein of spring viremia of carp virus (SVCV) combined with ORF81 protein of koi herpesvirus (KHV): A promising way to induce protective immunity against SVCV and KHV infection in cyprinid fish via oral vaccination. Vaccine (2015) 33: doi:10.1016/j. vaccine.2015.05.002

17. Norton EB, Lawson LB, Freytag LC, Clements JD. Characterization of a mutant Escherichia coli heat-labile toxin, LT(R192G/L211A), as a safe and effective oral adjuvant. Clin Vaccine Immunol (2011) 18:546-51. doi:10.1128/CVI.00538-10

18. Huang F, Li Q, Cui X, Cao S, Xiong C, Wang M, Lu Y, Wang W, Ye J, Liu X. Characterization of Spring Viremia of Carp Virus Glycoprotein Expressed by Recombinant Baculovirus. Pak Vet J (2012) 32:334-338. Available at: http://pvj.com.pk/pdffiles/32_3/334-338.pdf [Accessed August 8, 2017]

19. Encinas P, Gomez-Sebastian S, Nunez M, Gomez-Casado E, Escribano JM, Estepa A, Coll J. Antibody recognition of the glycoprotein $g$ of viral haemorrhagic septicemia virus (VHSV) purified in large amounts from insect larvae. BMC Res Notes (2011) 4:210. doi:10.1186/1756-0500-4-210

20. Irnazarow I. Genetic variability of Polish and Hungarian carp lines. Aquaculture (1995) 129:215. doi:10.1016/0044-8486(95)91961-T

21. Fijan N, Petrinec Z, Sulimanovic D, Zwillenberg LO. Isolation of the viral causative agent from the acute form of infectious dropsy of carp. Vet Arh (1971) 41:125-138.

22. Betts AM, Stone DM, Way K, Torhy C, Chilmonczyk S, Benmansour A, De Kinkelin P. Emerging vesiculo-type virus infections of freshwater fishes in Europe. Dis Aquat Organ (2003) 57:201-212. doi:10.3354/dao057201

23. Reed LJ, Muench H. A simple method of estimating fifty per cent endpoints. Am J Epidemiol (1938) 27:493-497. Available at: http://www.scopus.com/inward/record.url?eid=2-s2.033745158157\&partnerID=tZOtx3y1

24. Utke K, Kock H, Schuetze H, Bergmann SM, Lorenzen N, Einer-Jensen K, Köllner B, Dalmo RA, Vesely T, Ototake M, et al. Cell-mediated immune responses in rainbow trout after DNA immunization against the viral hemorrhagic septicemia virus. Dev Comp Immunol (2008) 32:239-252. doi:10.1016/j.dci.2007.05.010

25. Forlenza M, Kaiser T, Savelkoul HFJ, Wiegertjes GF. The use of real-time quantitative PCR for the analysis of cytokine mRNA levels. Methods Mol Biol (2012) 820:7-23. doi:10.1007/978-1-61779-439-1

26. Forlenza M, Walker PD, de Vries BJ, Wendelaar Bonga SE, Wiegertjes GF. Transcriptional 
analysis of the common carp (Cyprinus carpio L.) immune response to the fish louse Argulus japonicus Thiele (Crustacea: Branchiura). Fish Shellfish Immunol (2008) 25:76-83. doi:10.1016/j.fsi.2007.12.013

27. Koumans-van Diepen JE, van de Lisdonk MHM, Taverne-Thiele AJ, Verburg-van Kemenade BML, Rombout JHWM. Characterisation of immunoglobulin-binding leucocytes in carp (Cyprinus carpio L.). Dev Comp Immunol (1994) 18:45-56. doi:10.1016/0145305X(94)90251-8

28. Secombes CJ, van Groningen JJ, Egberts E. Separation of lymphocyte subpopulations in carp Cyprinus carpio L. by monoclonal antibodies: immunohistochemical studies. Immunology (1983) 48:165-175.

29. Rombout JHWM, Joosten PHM, Engelsma MY, Vos AP, Taverne N, Taverne-Thiele JJ. Indications for a distinct putative T cell population in mucosal tissue of carp (Cyprinus carpio L.). Dev Comp Immunol (1998) 22:63-77. doi:10.1016/S0145-305X(97)00048-7

30. Weyts FAA, Rombout JHWM, Flik G, L Verburg-van Kemenade BM, Weyts FAA, Rombout JHWM, Flik G, Verburg-van Kemenade BML. A common carp (Cyprinus carpio L.) leucocyte cell line shares morphological and functional characteristics with macrophages. Fish Shellfish Immunol (1997) 7:123-133. doi:10.1006/fsim.1996.0069

31. Nakayasu C, Omori M, Hasegawa S, Kurata O, Okamoto N. Production of a monoclonal antibody for carp (Cyprinus carpio L.) phagocytic cells and separation of the cells. Fish Shellfish Immunol (1998) 8:91-100. Available at: http:/ac.els-cdn.com/ S105046489790125X/1-s2.0-S105046489790125X-main.pdf?_tid=f2ecacd2-4167-11e7b339-00000aab0f01\&acdnat=1495730217_68caa61b6a51021c584816f36ce0e663 [Accessed May 25, 2017]

32. Forlenza M, Scharsack JP, Kachamakova NM, Taverne-Thiele AJ, Rombout JHWM, Wiegertjes GF. Differential contribution of neutrophilic granulocytes and macrophages to nitrosative stress in a host-parasite animal model. Mol Immunol (2008) 45:3178-3189. doi:10.1016/j.molimm.2008.02.025

33. Rhee JH, Lee SE, Kim SY. Mucosal vaccine adjuvants update. Clin Exp Vaccine Res (2012) 1:50-63. doi:10.7774/cevr.2012.1.1.50

34. Srivastava A, Gowda DV, Madhunapantula S V., Shinde CG, Iyer M. Mucosal vaccines: a paradigm shift in the development of mucosal adjuvants and delivery vehicles. Apmis (2015) 123:275-288. doi:10.1111/apm.12351

35. George-Chandy A, Eriksson K, Lebens M, Nordström I, Schön E, Holmgren J. Cholera toxin B subunit as a carrier molecule promotes antigen presentation and increases CD40 and CD86 expression on antigen-presenting cells. Infect Immun (2001) 69:5716-25. doi:10.1128/ IAI.69.9.5716-5725.2001

36. Rombout JHWM, Berg AA van den, Witte P, Egberts E. Immunological importance of the second gut segment of carp . 111 . Systemic and / or mucosal immune responses after immunization with soluble or particulate antigen. (1989)179-186.

37. Munang'andu HM, Mutoloki S, Evensen Ø. A Review of the Immunological Mechanisms Following Mucosal Vaccination of Finfish. Front Immunol (2015) 6:427. Available at: http:// journal.frontiersin.org/article/10.3389/fimmu.2015.00427/abstract [Accessed October 15, 2015]

38. Prehaud C, Takehara K, Flamand A, Bishop DHL. Immunogenic and Protective Properties of Rabies Virus Glycoprotein Expressed by Baculovirus Vectors. (1989) Available at: http:// ac.els-cdn.com/0042682289905515/1-s2.0-0042682289905515-main.pdf?_tid=a41dc28c8337-11e7-a55d-00000aacb35e\&acdnat=1502966246_f09d6613c0869b58590e31b36afffd95 [Accessed August 17, 2017]

39. Fu ZF, Rupprecht CE, Dietzschold B, Saikumar P, Niu HS, Babka I, Wunner WH, Koprowski H. Oral vaccination of racoons (Procyon Iotor) with baculovirus-expressed rabies virus glycoprotein. Vaccine (1993) 11:925-928. Available at: https://s3.amazonaws. com/academia.edu.documents/46388708/0264-410x_2893_2990379-c20160610-24433- 
1 dbfoyi.pdf?AWSAccessKeyId=AKIAIWOWYYGZ2Y53UL3A\&Expires=15029690 04\&Signature $=$ SVbPWIU1 mi\%252FgwlXxkXyyvDXi9Kw\%253D\&response-contentdisposition=inline $\% 253 \mathrm{~B}$ file [Accessed August 17, 2017]

40. Martinez-Alonso S, Martinez-Lopez A, Estepa A, Cuesta A, Tafalla C. The introduction of multi-copy CpG motifs into an antiviral DNA vaccine strongly up-regulates its immunogenicity in fish. Vaccine (2010) 29:1289-1296. doi:10.1016/j.vaccine.2010.11.073

41. Krieg AM, Yi AK, Schorr J, Davis HL. The role of CpG dinucleotides in DNA vaccines. Trends Microbiol (1998) 6:23-27. doi:10.1016/S0966-842X(97)01145-1

42. Lorenzen E, Lorenzen N, Einer-Jensen K, Brudeseth B, Evensen Ø. Time course study of in situ expression of antigens following DNA-vaccination against VHS in rainbow trout (Oncorhynchus mykiss Walbaum) fry. Fish Shellfish Immunol (2005) 19:27-41. doi:10.1016/j.fsi.2004.10.009

43. Companjen AR, Florack DEA, Slootweg T, Borst JW, Rombout JHW. Improved uptake of plant-derived LTB-linked proteins in carp gut and induction of specific humoral immune responses upon infeed delivery. Fish Shellfish Immunol (2006) 21:251-260. doi:10.1016/j. fsi.2005.12.001

44. Petit J, Wiegertjes GF. Long-lived effects of administering $\beta$-glucans: Indications for trained immunity in fish. Dev Comp Immunol (2016) 64:93-102. doi:10.1016/j.dci.2016.03.003 


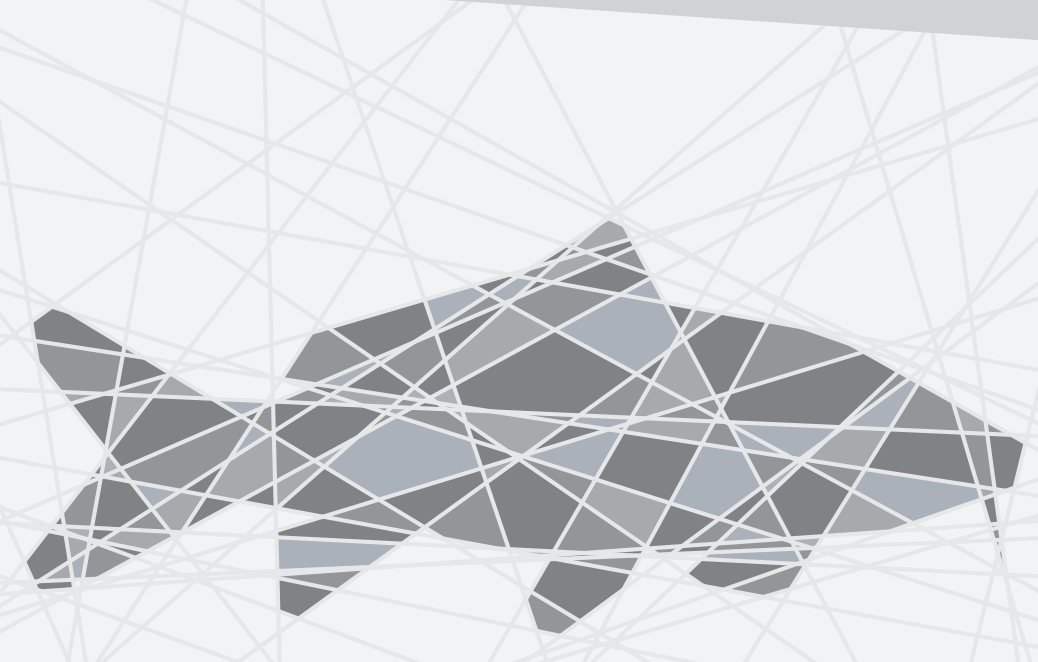




\section{Intra-muscular and oral vaccination using a Koi Herpesvirus ORF25 DNA vaccine does not confer protection in common carp (Cyprinus carpio L.)}

\section{Carmen W.E. Embregts', Roni Tadmor-Levi², Tomáš Veselý', Dagmar Pokorová3, Lior David ${ }^{2}$, Geert F.Wiegertjes', Maria Forlenza'}

' Cell Biology and Immunology Group, Department of Animal Sciences, Wageningen University, Wageningen, The Netherlands

${ }^{2}$ Department of Animal Sciences, RH Smith Faculty of Agriculture Food and Environment, The Hebrew University of Jerusalem, Rehovot, Israel ${ }^{3}$ Veterinary Research Institute, Brno, Czech Republic 


\section{Abstract}

Koi Herpes Virus (KHV or Cyprinid Herpesvirus 3, CyHV-3) is among the most threatening pathogens affecting common carp production as well as the highly valuable ornamental koi carp. To date, no effective commercial vaccine is available for worldwide use. A previous study reported that three intramuscular injections with an ORF25-based DNA vaccine, led to the generation of neutralizing antibodies and conferred significant protection against an intraperitoneal challenge with KHV. In the present study, we set out to optimize an ORF25-based DNA vaccination protocol that required fewer injections, and would confer protection upon a challenge that better resembled the natural route of infection. To this end, ORF25 was cloned in pcDNA3 either as a soluble protein or as a full-length transmembrane GFP-fusion protein. We tested our ORF25-based DNA vaccines in multiple vaccination trials using different doses, vaccination routes (i.m. injection and oral gavage) and challenge methods (bath and cohabitation). Furthermore, we analysed local and systemic responses to the i.m. injected DNA vaccine through histological and RT-qPCR analysis. We observed a strong protection when fish received three injections of either of the two DNA vaccines. However, this protection was observed only after bath challenge and not after cohabitation challenge. Furthermore, protection was insufficient when fish received one injection only, or received the plasmid orally. The importance of choosing a challenge model that best reflects the natural route of infection and the possibility to include additional antigens in future DNA vaccination strategies against KHV will be discussed. 


\section{Introduction}

Common carp (Cyprinus carpio carpio L.) is among the five most cultured species worldwide but its sharp increase in production, along with the global intensification of aquaculture, have led to the increasing incidence of infectious diseases outbreaks [1,2]. Koi Herpes Virus (KHV), also known as Cyprinid Herpesvirus 3 (CyHV3 ), is one of the pathogens causing high losses in common carp aquaculture [3,4]. Furthermore, KHV affects ornamental koi carp (Cyprinus carpio koi) and wild carp populations, resulting not only in high economical losses but also in environmental damage $[3,5,6]$. KHV is part of the Alloherpesviridae family and has a linear double stranded DNA genome of $\sim 295 \mathrm{kB}$, encoding 156 functional Open Reading Frames (ORFs) [7,8]. While effective inactivated, live attenuated or live recombinant experimental vaccines against KHV have been reported [9-17], no commercial vaccine is yet available for worldwide use due to legislative restrictions concerning associated risks of reactivation [18] or reversion to virulence. The only available live attenuated immersion vaccine is commercialized and used exclusively in Israel (KV3, KoVax Ltd, Israel).

In contrast to live virus vaccines, subunit or DNA vaccines would provide suitable alternatives owing to their safety profile. The large size of the KHV genome and the complexity of the virion require identification and characterization of ORFs before they can be selected as vaccine candidates. While 40-43 functional proteins has been identified in various KHV isolates [7,8,19], the immunogenicity of only a few has been characterized [15,20,22-24]. Recently, two studies showed the potential of using the membrane proteins ORF25 and ORF81 as candidates for intra-muscular (i.m.) DNA vaccination of carp against KHV $[25,26]$. In these studies, three consecutive i.m. injections of 1,10 or $50 \mu \mathrm{g}$ of pcDNA3 encoding the soluble form of the surface glycoproteins ORF25 or ORF81, triggered the formation of neutralizing antibodies, and induced up to $87.5 \%$ survival when carp were challenged with KHV by intraperitoneal (i.p.) injection. While this is promising, fewer injections or vaccination routes other than injection would be preferred from a practical point of view. Furthermore, a challenge method better resembling the natural route of infection might be more reliable to assess vaccine efficacy. The potential of oral vaccination against KHV was assessed using liposomes containing formalin-inactivated KHV $[16,27]$ and using recombinant Lactococcus plantarum (L. plantarum) encoding the KHV ORF81 [28]. In both studies, protection around 70-75\% was obtained, 
indicating that oral vaccination against KHV is possible but needs further refinement. More recently, during the course of this study, a report described the generation of an ORF25-deleted KHV virus and its efficacy as vaccine strain. Despite its proven safety and level of attenuation in vivo, its efficacy as a vaccine was suboptimal, suggesting that the presence of ORF25 is required to trigger KHV-specific protective responses [14].

Given the promising results from previous studies, we set out to investigate the potential of a DNA vaccine encoding the ORF25 for oral and injection vaccination. We hypothesized that since previous studies on DNA vaccination against KHV used constructs expressing soluble proteins, a transmembrane ORF25 would trigger a more appropriate immune response already after a single injection. In the current study, a construct encoding the extracellular soluble domain of the ORF25 (pcDNA3solORF25), and one encoding a transmembrane ORF25-GFP fusion protein (pcDNA3-tmORF25-GFP) were constructed. We here describe their application as injection as well as oral DNA vaccines using various doses and vaccination regimes. We did not only assess vaccine efficacy upon KHV challenge using a bath and a cohabitation method, closely mimicking the natural route of infection, but we also characterized the local immune response upon injection vaccination. Since moderate degree of protection was observed only after repeated administrations of the DNA plasmid encoding the transmembrane ORF25 protein, further optimization is required to achieve full protection against KHV. Accordingly, the use of additional $\mathrm{KHV}$ proteins as vaccine antigens for future (DNA) vaccination strategies will be discussed.

\section{Materials and methods}

\section{Animals}

European common carp (Cyprinus carpio carpio) R3xR8 that originated from a cross between the Hungarian R8 strain and the Polish R3 strain [29] were used, unless stated otherwise. Specific pathogen free (SPF) carp were bred in the Aquatic Research Facility (ARC) of the Carus animal facility at Wageningen University, the Netherlands. Fish were either raised at the local facility or eggs were transported to the Veterinary Research Institute (VRI, Brno, Czech Republic) for viral challenge 
experiments. Carp were raised at $20-23{ }^{\circ} \mathrm{C}$ in recirculating UV-treated water and fed pelleted carp food (Skretting, Nutreco) twice daily. As a control, susceptible naïve Koi carp were purchased at the Czech koi breeding center ALCEDOR s.r.o.

For the experiments in Israel, common carp of the Yugoslavian (YxY) strain ( $7 \mathrm{~g}$ in weight) were used. As a control, susceptible koi carp of mixed varieties were used. Näive koi were obtained at $\sim 10 \mathrm{~g}$ weight from Kibbutz Gan Shmuel and Kibbutz Ma'agan Michael fish breeding centers in Israel. Common carp were bred and reared in recirculating water as described previously [30,31], at the fish facility of the Robert H. Smith Faculty of Agriculture, Food and Environment of the Hebrew University of Jerusalem in Rehovot, Israel.

All animals were handled in accordance with good animal practice as defined by the European Union guidelines for the handling of laboratory animals (http://ec.europa. eu/environment/chemicals/lab_animals/home_en.htm). All the work performed in Israel was approved by the Animal research ethics committee of the Hebrew University of Jerusalem under permit \# AG-13059-5. Animal work in Wageningen University was approved by the local experimental animal committee (DEC number 2014098). Animal work at VRI was approved by the Branch Commission for Animal Welfare of the Ministry of Agriculture of the Czech Republic (permission No MZe 1717).

\section{Cells}

Common carp brain (CCB) cells were cultured in Eagle's minimal essential medium (MEM) supplemented with 1\% penicillin/streptomycin, 1\% L-glutamine, 1\% nonessential amino acids, $10 \% \mathrm{FCS}$ and $3.5 \mathrm{~g} / \mathrm{L} \mathrm{D}$-glucose at $27^{\circ} \mathrm{C}$, in the presence of $5 \% \mathrm{CO}_{2}$.

\section{Cloning of KHV ORF25}

The full-length KHV ORF25 was amplified from genomic DNA isolated from KHV isolate C250 (kindly provided by Dr. Keith Way, CEFAS, Waymouth, UK) by PCR using proofreading Taq polymerase (Roche). Primers were designed based on alignments of the genome sequences of the following KHV strains; KHV-U (Acc. Nr, DQ657948), KHV-I (Acc. Nr. DQ177346) and KHV-J (AP008984). All primers used for cloning can be found in Table 1, and a schematic representation of the constructs can be found in Fig. 1. The obtained products were verified on a $1 \%$ agarose gel and were purified using S400 Sephacryl columns (GE Healthcare). For 
the transmembrane ORF25 fused to GFP (tmORF25-GFP) construct, the purified PCR product amplifying the full-length ORF25 sequence was ligated using T4 ligase (Promega) in the pcDNA3-GFP vector [32] between the KpnI and XhoI sites generating the pcDNA3-tmORF25-GFP. Cloning in this vector allowed for insertion of the tmORF25 upstream of the GFP sequence separated by a short linker sequence encoding the amino acids GGSGG, as described previously [32]. For the soluble ORF25 (solORF25) construct, the purified PCR product encoding for amino acid 1-444 followed by a C-terminal HA tag, with the first amino acid being the first methionine, was ligated in the pcDNA3 vector between the KpnI and XhoI sites generating the HA-tagged pcDNA3-solORF25-. Constructs were verified by sequencing and plasmids were purified using the endotoxin-free plasmid isolation midi kit (Invitrogen) prior to in vitro transfection or in vivo i.m. injection.

\section{Transfection of CCB cells and staining of tmORF25-GFP}

CCB cells were seeded on glass cover slips in 12 well-plates at a density of $250.000 /$ well. Cells were transfected with $1 \mu \mathrm{g}$ pcDNA3-tmORF25-GFP or pcDNA3-GFP and $4 \mu \mathrm{L}$ of FuGENE HD transfection reagent (Promega) using the manufacturer's guidelines. After $48 \mathrm{~h}$ cells were rinsed twice with Tris-buffered saline (TBS, 10mm TRIS, $135 \mathrm{~mm} \mathrm{NaCl}, \mathrm{pH} 7.5$ ), fixed with 4\% paraformaldehyde (PFA) in TBS for 10 min at RT and blocked with $1 \%$ bovine serum albumin (BSA) in TBS. Serum from carp that were vaccinated once with $0.5 \mu \mathrm{g}$ pcDNA3-tmORF25-GFP plasmid/g fish and subsequently exposed to KHV, as further described in paragraph 2.6, was used to visualize tmORF25 surface expression. Serum was collected from survivor fish 30 days after the cohabitation challenge. Serum was diluted 1:50 in PBS and was incubated on the slides for 45 minutes. Subsequently, slides were stained with an antibody against carp Igm (WCI12; [33], diluted 1:100 in TBS containing $1 \%$ BSA) and PE-conjugated goat-anti-mouse (Invitrogen, 1:200). Cells were visualized using an EVOS fl LED fluorescence microscope (Advanced Microscopy Group (AMG)).

\section{Virus}

Wild type KHV virus was used for the challenges performed in Israel and was kindly provided by KoVax, Ltd. (Israel). KHV (Isolate Hedrick KHV 261) was used for the challenges performed in the Czech Republic. KHV isolate Hedrick KHV 261 was propagated in CCB cells. Virus titres were determined using the method of Reed and Muench [34] and are displayed as plaque-forming units (pfu). 
Table 1. Primers used in this study.

\begin{tabular}{|c|c|c|c|}
\hline & FW primer 5'-3' & RV primer 3'- 5' & Acc. Nr. \\
\hline \multicolumn{4}{|c|}{ Primers used for amplification and cloning of KHV ORF25 } \\
\hline tmORF25 & $\begin{array}{l}\text { ATATACGGTACCATGGC- } \\
\text { GGGTTGTGGGGTT }\end{array}$ & $\begin{array}{l}\text { ATATATCTCGAGGGGCC- } \\
\text { TCCGGGAAACCTGGGC }\end{array}$ & \\
\hline solORF25 & $\begin{array}{l}\text { ATATACGGTACCATGGC- } \\
\text { GGGTTGTGGGGTT }\end{array}$ & $\begin{array}{l}\text { ATATATCTCGAGTTAAG- } \\
\text { CGTAGTCTGGGACGTCG- } \\
\text { TATGGGTAGGTGGCGTT- } \\
\text { GAGGTCCT }\end{array}$ & \\
\hline \multicolumn{4}{|c|}{ Primers used for RT-qPCR } \\
\hline \multicolumn{4}{|c|}{ Housekeeping genes } \\
\hline $40 s$ & $\begin{array}{l}\text { CCGTGGGTGACATCGTTA- } \\
\text { CA }\end{array}$ & $\begin{array}{l}\text { TCAGGACATTGAACCTC- } \\
\text { ACTGTCT }\end{array}$ & AB012087 \\
\hline \multicolumn{4}{|l|}{ Cytokines } \\
\hline$c x c a$ & $\begin{array}{l}\text { CTGGGATTCCTGACCATT- } \\
\text { GGT }\end{array}$ & $\begin{array}{l}\text { GTTGGCTCTCTGTTTCA- } \\
\text { ATGCA }\end{array}$ & AJ421443 \\
\hline $\operatorname{cxcbl}$ & $\begin{array}{l}\text { GGGCAGGTGTTTTTGTG- } \\
\text { TTGA }\end{array}$ & $\begin{array}{l}\text { AAGAGCGACTTGCGGG- } \\
\text { TATG }\end{array}$ & AB082985 \\
\hline$i l 1 \beta$ & $\begin{array}{l}\text { AAGGAGGCCAGTGGCTC- } \\
\text { TGT }\end{array}$ & $\begin{array}{l}\text { CCTGAAGAAGAGGAGG- } \\
\text { AGGCTGTCA }\end{array}$ & AJ 245635 \\
\hline if $n \gamma 2 a / 2 b$ & $\begin{array}{l}\text { CGATCAAGGAAGATGAC- } \\
\text { CCAGTC }\end{array}$ & $\begin{array}{l}\text { GTTGCTTCTCTGTAGAC- } \\
\text { ACGCTTC }\end{array}$ & AM168523 \\
\hline \multicolumn{4}{|c|}{ Interferon stimulated genes } \\
\hline$m x 1$ & $\begin{array}{l}\text { ACAATTTGCGGTCTTTG- } \\
\text { AGA }\end{array}$ & CCCTGCCATTTCTCTTCG & cypCar_00015892 \\
\hline vip2 & CTGTCGGACACATCAGC & $\begin{array}{l}\text { TCAATGGGCAAGACGA- } \\
\text { AA }\end{array}$ & cypCar_00024055 \\
\hline pkr3 & $\begin{array}{l}\text { CACGGTGTTTGAAAAGA- } \\
\text { GC }\end{array}$ & $\begin{array}{l}\text { GACTGGGTCTCAGC- } \\
\text { ATTC }\end{array}$ & cypCar_00039221 \\
\hline isg15.2 & $\begin{array}{l}\text { AGTGTTCGTCAAGAATG- } \\
\text { AGG }\end{array}$ & CCTCGCAGACGGAAAAC & cypCar_00039111 \\
\hline \multicolumn{4}{|c|}{ Adaptive immune genes } \\
\hline igm & $\begin{array}{l}\text { CACAAGGCGGGAAATGA- } \\
\text { AGA }\end{array}$ & $\begin{array}{l}\text { GGAGGCACTATATCAAC- } \\
\text { AGCA }\end{array}$ & AB004105 \\
\hline igt1 & $\begin{array}{l}\text { AAAGTGAAGGATGAAAG- } \\
\text { TGT }\end{array}$ & $\begin{array}{l}\text { TGGTAACAGTGGGCTTA- } \\
\text { TT }\end{array}$ & AB598367 \\
\hline igt2 & $\begin{array}{l}\text { GATTCTACTGGGT8CTTC- } \\
\text { AC }\end{array}$ & $\begin{array}{l}\text { GACATCACTCAACTC8T- } \\
\text { TCT }\end{array}$ & AB598368 \\
\hline
\end{tabular}

Restriction sites ( $\mathrm{Kpnl}$ in the $\mathrm{FW}$ and $\mathrm{Xhol}$ in the RV primer) are in bold; the Kozak sequence is underlined in the common FW primers. The HA tag is underlined in the RV primer used for the soluble ORF25 construct.

\section{Vaccination and challenge experiments}

In the first experiment, carp (R3xR8 strain) of 24-30 g ( $n=20 /$ group) received $0.5 \mu \mathrm{g}$ DNA/g of fish by i.m. injection, either once or three times with a three-weeks interval. Carp were anesthetized in $0.3 \mathrm{~g} / \mathrm{L}$ of Tricaine Methane Sulfonate (TMS; Crescent Research Chemicals) before vaccination. Vaccination groups included the pcDNA3 empty vector, pcDNA3-solORF25 and pcDNA-tmORF25-GFP. Susceptible koi carp 


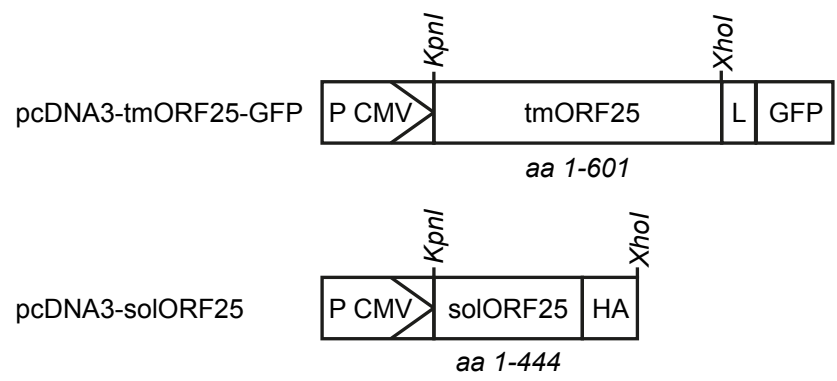

Fig. 1. Schematic representation of the two pcDNA3 constructs encoding ORF25. tmORF25: transmembrane ORF25; solORF25: soluble ORF25; P CMV: cytomegalovirus promotor; restriction sites are indicated in italics; numbers indicate the first and last nucleotide relative to the full-length sequence. C-terminal GFP and HA tags are indicated, as well as the GGSGG linker sequence (L) between the tmORF25 and the GFP sequence.

were included as unhandled (non-vaccinated) controls. Carp were challenged 3 months after the first vaccination by bath ( $2 \mathrm{~h}$ exposure to $0.7 \times 10^{4} \mathrm{pfu} / \mathrm{mL}$ ) or by cohabitation (1:5 ratio of shedders to vaccinated carp). Shedders were susceptible koi carp infected on forehand by intraperitoneal (i.p.) injection of $150 \mu \mathrm{L}$ of undiluted virus suspension (approximately 5000 pfu) of wild-type KHV (strain Hedrick KHV 261) and mortalities were recorded daily for 30 days.

In the second experiment, carp (YxY strain) of $\sim 7 \mathrm{~g}$ were vaccinated by i.m. injection of $1 \mu \mathrm{g}$ of DNA/g of fish (pcDNA3-GFP or pcDNA3-tmORF25-GFP, $n=15$ / group) or by i.m. injection of $20 \mu \mathrm{l}\left(>0.7 \times 10^{5} \mathrm{pfu} / \mathrm{ml}\right)$ of the KV3 live attenuated KHV vaccine (KoVax, Ltd). Immersion vaccination with the KV3 vaccine for $1 \mathrm{~h}$ in $2 \mathrm{~L}$ water containing $>0.7 \times 10^{5} \mathrm{pfu} / \mathrm{ml}$ of $\mathrm{KV} 3$ vaccine was used as a positive vaccine control ( $n=12 /$ group) and unhandled (non-vaccinated) fish as a negative control. Fish were challenged by cohabitation two months later as described above.

In a third experiment, carp (YxY strain) of $2 \mathrm{~g}$ ( $n=30$ / group, divided over duplicate tanks) received $1.5 \mu \mathrm{g}$ DNA/g of fish by i.m. injection (pcDNA3-GFP or pcDNA3tmORF25-GFP) or $2.5 \mu \mathrm{g}$ of DNA/g of fish by oral gavage, either once or three times with a $72 \mathrm{~h}$ interval. DNA plasmids (pcDNA3-GFP or pcDNA3-tmORF25GFP) were encapsulated in alginate microspheres as described previously (Embregts et al., 2017, this issue) and administered in $10 \mu \mathrm{L}$ by oral gavage using a $10 \mu \mathrm{L}$ pipette. Carp were challenged by cohabitation 3 months later.

\section{Histological analysis}

Carp were i.m. injected with $20 \mu \mathrm{g}$ of DNA (pcDNA3 or pcDNA3-tmORF25-GFP, 
$n=3$ per plasmid) to examine the expression of the GFP-fused tmORF25 protein. Fish were euthanized in $0.6 \mathrm{~g} / \mathrm{L}$ (Crescent Research Chemicals) and bled through the caudal vein 14 days later. The site of injection was isolated, snap-frozen in liquid nitrogen and stored at $-80{ }^{\circ} \mathrm{C}$ until further processing. Cryosections $(5 \mu \mathrm{m})$ from muscle sections were mounted on poly-lysine slides, fixed for 15 min at $4{ }^{\circ} \mathrm{C}$ with $4 \%$ PFA in PBS, stained with DAPI (4’,6-Diamidino-2-Phenylindole, Dilactate) to visualize cell nuclei (Thermo Scientific), and mounted with Vectashield (Vectorlabs). Sections were imaged using a EVOS fl LED fluorescence microscope (Advanced Microscopy Group (AMG)).

\section{RNA isolation and cDNA synthesis}

Carp were vaccinated by i.m. injection with $1 \mu \mathrm{g}$ DNA/g fish and were sacrificed 3 and 5 days later to analyze the local response to the vaccine. Carp were euthanized in $0.6 \mathrm{~g} / \mathrm{L}$ tricaine methane sulfonate (TMS, Crescent Research Chemicals) and bled through the caudal vein. Muscle at the injection site was isolated, snap frozen in liquid nitrogen, and stored at $-80^{\circ} \mathrm{C}$ until further processing.

Total RNA was isolated from muscle tissue using the RNeasy Mini Kit (Qiagen) according to the manufacturer's instructions including on-column DNase treatment using the RNase-free DNase set (Qiagen) and an additional Proteinase-K (Qiagen) treatment. RNA concentrations were measured using a Nanodrop-1000 (Thermo Scientific), the integrity was verified on a $1 \%$ agarose gel and RNA was stored at $-80{ }^{\circ} \mathrm{C}$ until further use. Prior to cDNA synthesis, $1 \mu \mathrm{g}$ total RNA was subjected to a second DNase treatment using DNAse I, Amplification Grade (Invitrogen). Reverse transcription of the RNA was performed using random primers (300 ng) and Superscript ${ }^{\mathrm{TM}}$ III (200U) First Strand Synthesis Systems for RT-PCR (Invitrogen). cDNA samples were diluted 25 times in nuclease-free water and stored at $-20^{\circ} \mathrm{C}$.

\section{Gene expression analysis}

Real-time quantitative PCR (RT-qPCR) was performed using a Rotor-Gene ${ }^{\text {TM }} 6000$ (Qiagen). Fluorescence data were analyzed using Rotor-Gene Q series software version 2.3.1 as described previously [35,36]. Briefly, $5 \mu \mathrm{L}$ of 25 times diluted cDNA was mixed with $2 \mu \mathrm{L}$ of forward and reverse gene-specific primers $(2.1 \mu \mathrm{M}$ of each primer) and $7 \mu \mathrm{L}$ of $2 x$ ABsolute qPCR SYBR Green Mix (Thermo Scientific) as detection chemistry. Thirty-five cycles were used for the detection of all selected genes. The list of primers can be found in Table 1. The take-off $(\mathrm{Ct})$ value for each 
sample and the average reaction efficiencies $(E)$ for each primer set were obtained upon comparative quantitation analysis from the Rotor-Gene software [37]. The relative expression ratio $(\mathrm{R})$ of each sample was calculated according to the Pfaffl method [38] based on the take-off deviation of sample versus each of the unhandled controls at time point $0 \mathrm{~h}$, and normalized relative to the $s 11$ protein of the $40 \mathrm{~s}$ subunit (referred to as $40 s$ ) as reference gene.

\section{Results}

\section{Expression of KHV tmORF25-GFP in vitro and in vivo}

For the in vitro validation of the tmORF25-GFP, CCB cells were transfected with the pcDNA3-tmORF25-GFP construct or with pcDNA3-GFP. A strong GFP signal was detected 48 hours after transfection with either of the two constructs. Surface expression of tmORF25 was confirmed upon labelling with serum from fish that were vaccinated with pcDNA3-tmORF25-GFP and that survived a subsequent bath challenge with KHV (Fig. 2A, upper panel). No reactivity was observed in CCB cells transfected with the pcDNA3-GFP construct (Fig. 2A, lower panel), confirming the specific of the reaction. Surface labelling using control serum from pcDNA3injected carp showed a similar pattern to Fig. 2A, lower panel (not shown).

For the in vivo validation of the same construct, muscle tissue at the site of injection and spleen of fish that were injected i.m. with $20 \mu \mathrm{g}$ of plasmid were examined. Tissues were processed for cryosectioning and expression of ORF25-GFP was clearly observed locally in the muscle as well as in the spleen 14 days after injection of the pcDNA3-tmORF25-GFP plasmid (Fig. 2B-C). Together this data shows that ORF25-GFP was expressed and detected in vivo both locally and systemically.

Fig 2. Detection of tmORF25-GFP in vitro and in vivo. (A) CCB cells were transfected with pcDNA3-tmORF25-GFP or pcDNA3-GFP; 48 hours later cells were fixed with PFA and analysed for surface expression of tmORF25-GFP. Upon labelling with serum from pcDNA3-tmORF25-GFP vaccinated fish that survived a subsequent KHV bath challenge, surface expression of tmORF25 was detected only in pcDNA3-tmORF25-GFP transfected cells (upper panel) and not in pcDNA3-GFP transfected cells (lower panel). (B-C) For in vivo validation of the tmORF25-GFP construct, carp were injected with $20 \mu \mathrm{g}$ of either pcDNA3 or pcDNA3-tmORF25-GFP and muscle (B) and spleen (C) were collected 14 days after injection. Direct fluorescence detection of the tmORF25-GFP in $5 \mu \mathrm{m}$ PFA-fixed cryosections revealed clear tmORF25-GFP expression (upper panels). A counterstaining with DAPI was included to visualize the nuclei. Images were acquired using an EVOS fl LED fluorescence microscope (Advanced Microscopy Group (AMG)). Scale bars indicate $\mu \mathrm{m}$. 
A CCB cells
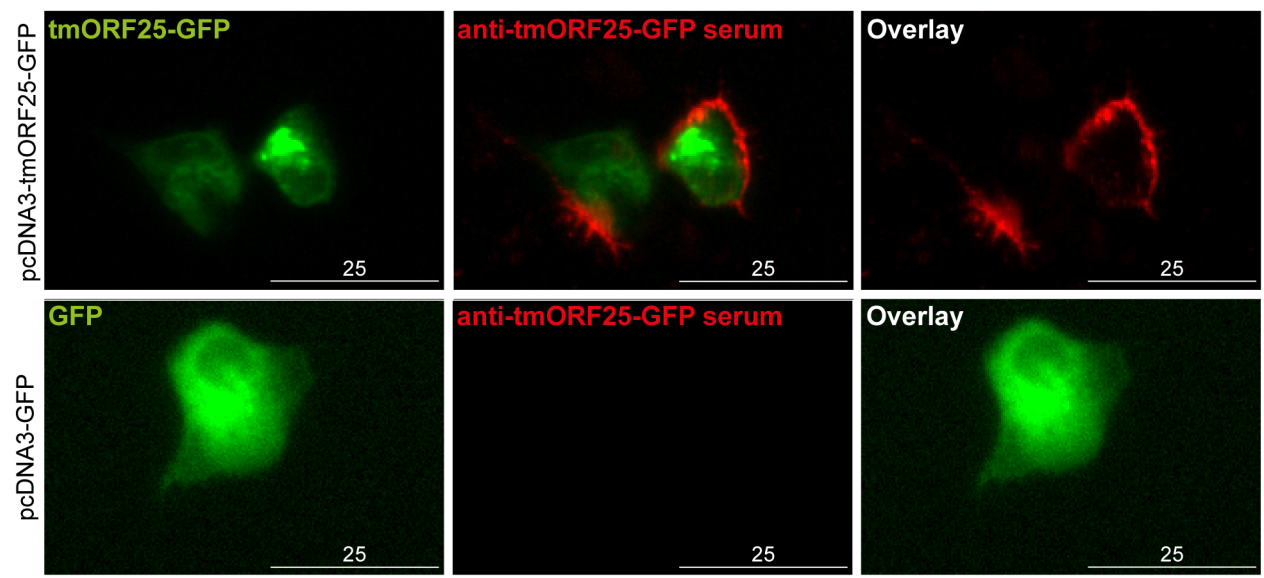

B Muscle
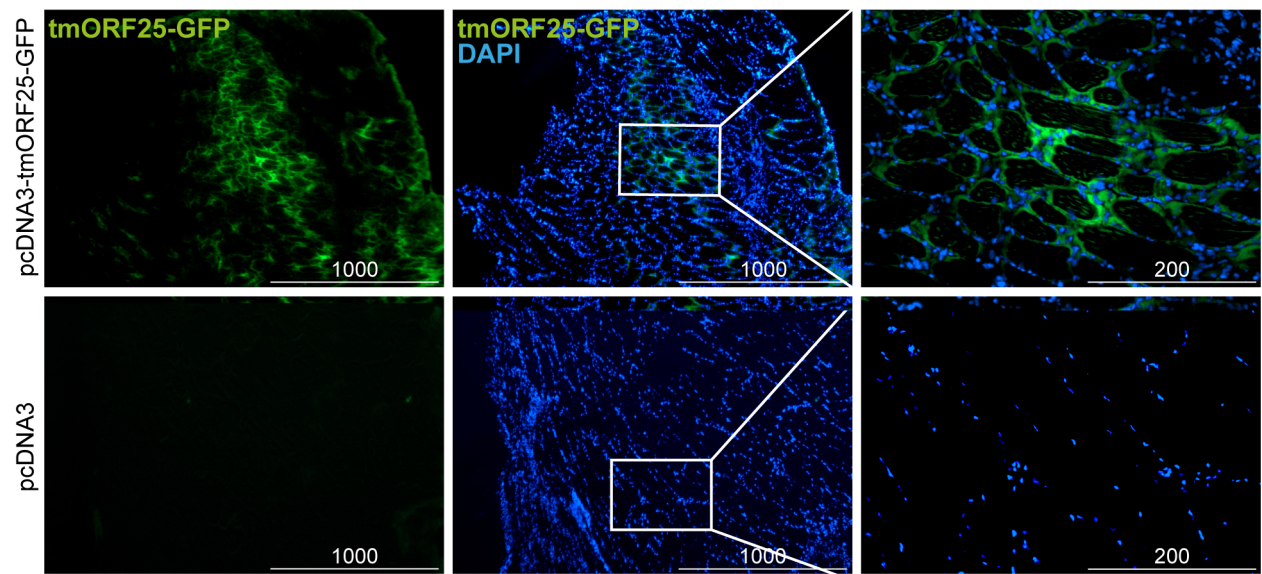

\section{Spleen}
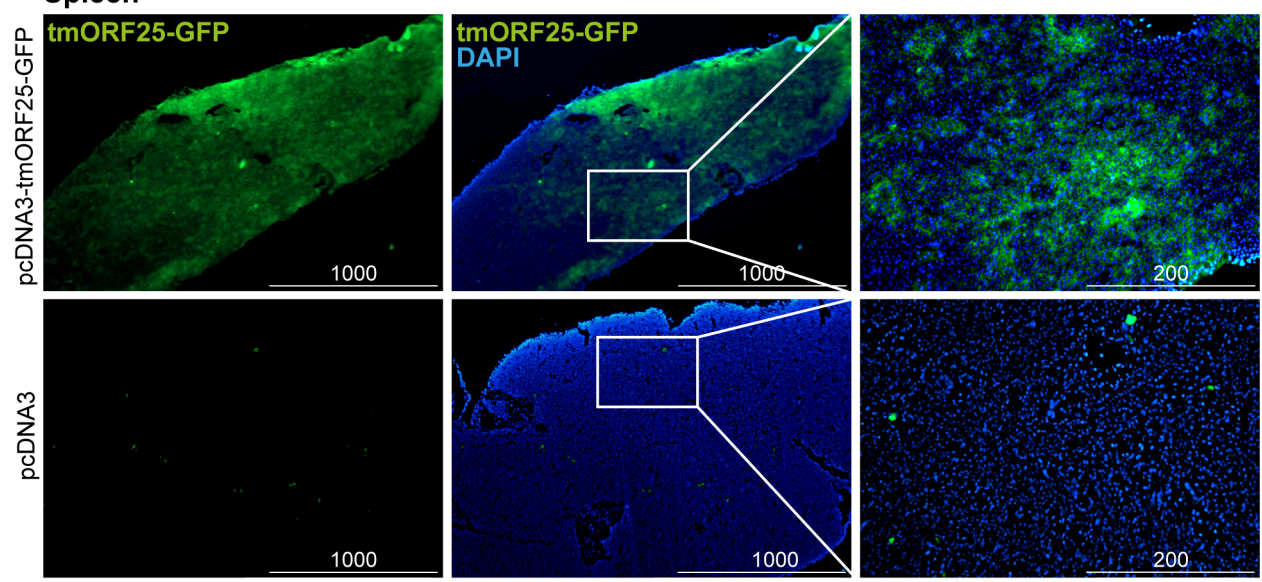

Figure legend on previous page 


\section{Protection against KHV after i.m. and oral DNA vaccination using ORF25}

To investigate whether a single i.m. DNA injection is sufficient to protect carp against $\mathrm{KHV}$, and to compare the efficacy of the constructs encoding either the soluble ORF25 or the native transmembrane ORF25, we tested the pcDNA3-solORF25 as well as the pcDNA3-tmORF25-GFP plasmids. To this end carp received $0.5 \mu \mathrm{g}$ DNA/g of fish, either once or three times with 3-weeks intervals and were challenged three months after the first injection (six weeks after the $3^{\text {rd }}$ injection). Fish injected with the same dose of the empty pcDNA3 plasmid served as control. For the KHV challenge, we chose to infect by either bath or cohabitation since these best resemble natural routes of infection. Strikingly, large differences in survival rate were observed between the cohabitation and bath challenge (Fig. 3A). After cohabitation challenge, mortalities were generally high. The highest survival (relative percent of survival (RPS) of 26) was observed in the groups vaccinated with either 1 or 3 injections of the tmORF25 construct. In the groups injected with the solORF25 construct, survival was less than $20 \%$. After bath challenge, highest survival was observed in the group receiving three injections of the tmORF25 construct (RPS of 89), followed by the solORF25injected group (RPS of 84). In the groups injected once, survival was generally lower; the highest survival was observed again in the tmORF25-injected group (RPS of 45). Based on these results, we selected the pcDNA3-tmORF25-GFP construct for follow up experiments and decided to optimize the dose of the single injection regime, since this is preferred over multiple injections. Despite the generally higher mortality observed upon cohabitation challenge, we decided to continue with this challenge method in all follow-up experiments, since this better reflects a natural mode of infection.

In our second experiment, fish were i.m. injected once with $1 \mu \mathrm{g} \mathrm{DNA} / \mathrm{g}$ of fish. This time, the KV3 (KoVax, Ltd, Israel) vaccine was included as positive control and fish were either i.m. injected with $20 \mu \mathrm{L}$ (>1400 pfu/fish) of the KV3 solution or immersed according to the recommended protocol. All groups were challenged two months later by cohabitation. The groups vaccinated with the KV3 vaccine, either by i.m. injection or by immersion, showed very good protection (RPS of 88) (Fig. 3B). In contrast, no survival was observed in the group vaccinated with the pcDNA3-tmORF25-GFP plasmid. These results indicate that vaccination through the i.m. can protect carp against KHV, at least when using the live attenuated KV3 vaccine, whereas i.m. DNA vaccination needs further optimization. 
In a third experiment, we performed a preliminary assessment of the efficacy of the pcDNA3-tmORF25-GFP vaccine when delivered orally. Fish received one or three administrations (with a $72 \mathrm{~h}$ interval) of $2.5 \mu \mathrm{g}$ of alginate-encapsulated DNA/g of fish or one i.m injection of $1.5 \mu \mathrm{g}$ DNA/g of fish. Upon cohabitation challenge three months after vaccination, no protection was observed in all tested groups (Fig. 3C). Overall, we conclude that i.m. and oral DNA vaccination against KHV using a constructs encoding tmORF25, using the chosen vaccine doses and regimes, does not confer strong protection against a cohabitation challenge with KHV.

\section{Effects of pcDNA3-tmORF25-GFP injection on expression of immune-related genes}

Given that a single injection of DNA vaccine induced only limited protection, we set out to examine the local response to the DNA vaccine. We analysed the expression of a selected panel of immune-related genes in muscle of carp injected with $1 \mu \mathrm{g} / \mathrm{g}$ of fish of the pcDNA3-tmORF25-GFP vaccine. Samples from the site of injection were taken after 3 and 5 days. Our data show that although the expression of proinflammatory molecules and interferon-stimulated genes (ISGs) $m x 1$, vip 2 and isg 15.2 was increased up to 100 folds at 3 or 5 days post-injection (dpi), the levels were not significantly different from those observed in the control group not expressing the ORF25 (pcDNA3-GFP) (Fig. 4). A similar pattern was observed for genes linked to adaptive immune responses; despite a 40-fold upregulation of $I g m$ at 5 dpi in carp receiving the pcDNA3-tmORF25-GFP plasmid, this upregulation was also observed in the pcDNA3-GFP-injected group. Overall, we conclude that given the absence of a significant ORF25-specific response, the observed early local response is likely due to both the damage-related inflammation caused by the injection as well as the plasmid backbone.

\section{Discussion}

While effective inactivated, live recombinant or attenuated experimental vaccines have been described for KHV [9-13,16,17], owing to safety concerns, the only commercially available live attenuated vaccine is restricted for use in Israel. To circumvent some of the concerns associated with the use of live recombinant or attenuated viral vaccines, there is a strong drive for the development of alternative 
A Cohabitation challenge

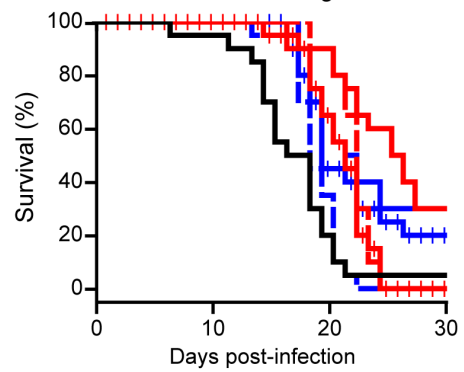

Bath challenge

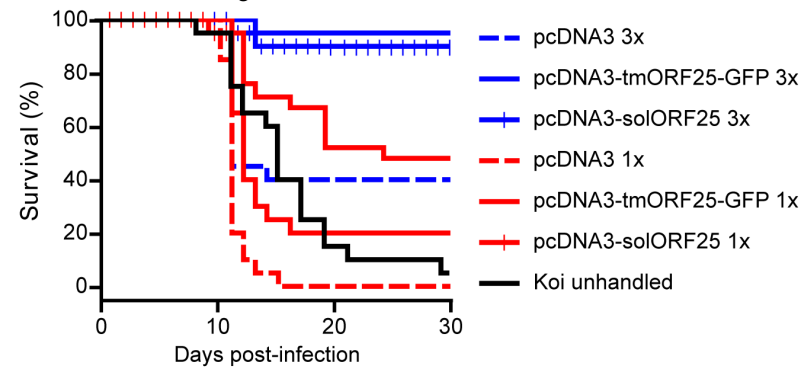

B

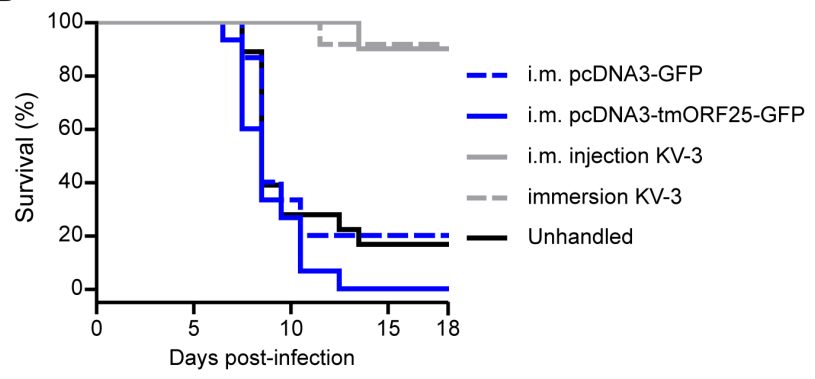

C

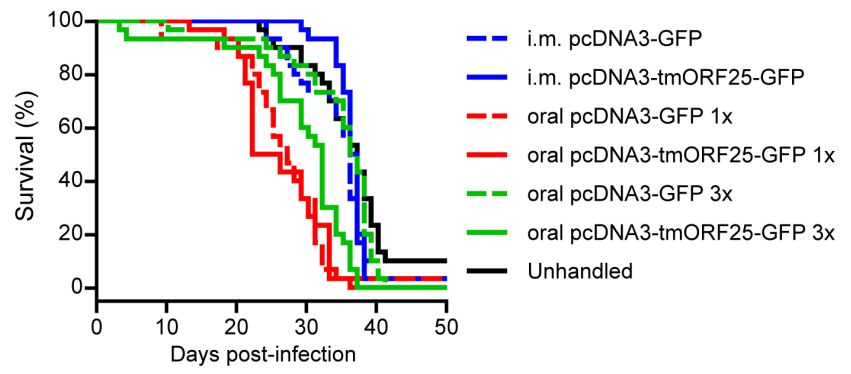

Fig 3. Survival of carp i.m. and orally vaccinated using ORF25 DNA vaccines. (A) Carp of 24-30 g ( $n=20 /$ group) were injected with $0.5 \mu \mathrm{g} D N A / g$ of fish of the indicated plasmids, either once $(1 \mathrm{x})$ or three times $(3 \mathrm{x})$, with a 3 -week interval. Carp were challenged 3 months after the first injection (six weeks after the $3^{\text {rd }}$ injection) by cohabitation or by bath. (B) Carp of $7 \mathrm{~g}$ ( $n=15 /$ group) were injected with $1 \mu \mathrm{g}$ DNA/g of fish of the indicated plasmids. The control groups ( $n=12$ / group) were vaccination with the KV3 vaccine by i.m. injection ( $20 \mu \mathrm{L}, 1400 \mathrm{pfu}$ ) or by immersion $(200 \mu \mathrm{L} / 2 \mathrm{~L}, 7000 \mathrm{pfu} / \mathrm{L})$. Carp were challenged 2 months after vaccination by cohabitation. (C) Carp of 2 grams ( $n=30 /$ group) were injected i.m. with $1.5 \mu \mathrm{g} \mathrm{DNA} / \mathrm{g}$ of fish of the indicated plasmids or received $2.5 \mu \mathrm{g}$ of alginate-encapsulated DNA/g of fish of the same plasmids through oral gavage, either once (1x) or three times (3x), with a $72 \mathrm{~h}$ interval. Carp were challenged 3 months after vaccination by cohabitation. Mortality was recorded for the indicated time. 


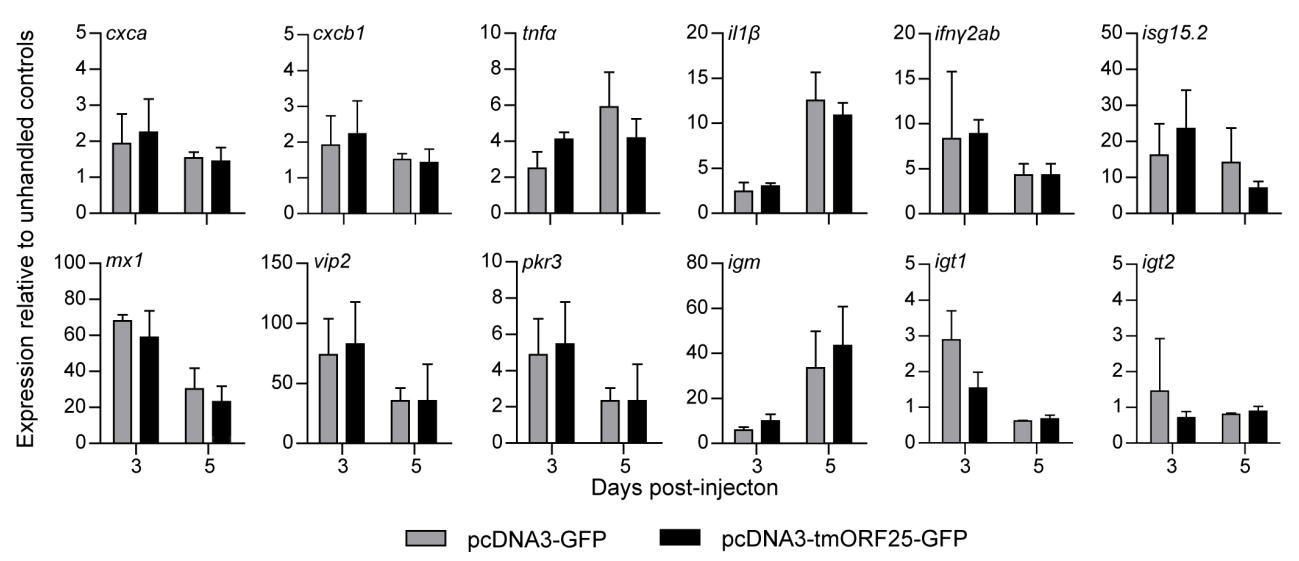

Fig. 4. Gene expression analysis of immune-related genes in the muscle after i.m. injection of pcDNA3-tmORF25-GFP. Carp were injected with $1 \mu \mathrm{g}$ DNA/g of fish with either pcDNA3-GFP or pcDNA3-tmORF25-GFP. The site of injection was isolated 3 and 5 days postinjection and processed for gene expression analysis. Expression was normalized against the housekeeping gene s11 of the ribosomal subunit 40s (referred to as 40s) and expressed relative to the unhandled controls at $t=0$. Bars indicate average and SD of $n=3$ (pcDNA3-GFP) or $n=4$ (pcDNA3-tmORF25-GFP) fish per time point.

types of vaccines such as subunit vaccines or DNA vaccines. Recently, the CLYNAV DNA vaccine against Pancreatic Disease in Atlantic salmon (Salmo salar) has been granted marketing authorization in Europe [39], becoming the first commercially available DNA vaccine on the European market. This development opens up a whole new era, as well as a renewed interest in the development and application of targeted DNA vaccines for aquaculture species.

In the current study, we describe two experimental DNA vaccines encoding the KHV ORF25 protein, either as a soluble (pcDNA3-solORF25) or as a transmembrane GFP-fused protein (pcDNA3-tmORF25-GFP). The potential for ORF25 to serve as antigen for DNA vaccination of carp against KHV was previously shown in a study by Zhou et al. [25]. In the latter study, koi carp received three i.m injections of either 1, 10 or $50 \mu \mathrm{g}$ of DNA encoding for a soluble form of ORF25. Upon i.p. challenge with KHV, survival rates of $80 \%$ were obtained already with the lowest concentration of plasmid, and reached $87.5 \%$ with the highest concentration of the ORF25 DNA vaccine.

Based on the results by Zhou et al., in the current study we set out to optimize a vaccination protocol that required fewer injections and that would confer protection upon a challenge that better resembled the natural route of infection. To this end, we investigated the protection induced by pcDNA3-solORF25 and pcDNA3-tmORF25- 
GFP, using two routes of vaccination (i.m. injection and oral gavage), different vaccines doses and two challenge routes (bath and cohabitation). In agreement with the study by Zhou et al., in our first vaccination trial, we observed the strongest protection after three i.m. injections of either of the constructs when carp were challenged by bath, but not when challenged by co-habitation. Nevertheless, considering that a single injection with the tmORF25 construct conferred higher protection upon bath and cohabitation challenge than a single injection with the solORF25 construct, for our subsequent trials we decided to continue with the tmORF25 plasmid. Furthermore, since our aim was also to optimize a vaccination protocol that would confer protection upon a challenge closely resembling the natural route of infection, we decided to continue with the cohabitation challenge for the further optimization of our ORF25 DNA vaccine.

In a subsequent trials, the protection conferred by i.m. injection was validated by the KV3 vaccine that conferred the same level of protection when delivered through i.m. injection, as it did when administered through the recommended immersion route (90 $\%$ survival). However, the tmORF25-GFP DNA vaccine, when i.m. injected once, did not confer sufficient protection in any of the follow up experiments. Similar results were observed when the plasmid was delivered orally once or three times (up to $13 \mu \mathrm{g} /$ fish).

Histological analysis 14 days after i.m. injection of the pcDNA3-GFP-tmORF25 revealed a strong expression of the tmORF25-GFP protein in myocytes at the site of injection and in the spleen of vaccinated carp. This is in agreement with studies in fish showing that part of the injected DNA can be detected in multiple systemic organs within few hours, up until 1 year after i.m. injection [40,41]. Analysis of the local expression of a panel of immune-related genes revealed that injection of pcDNA3tmORF25-GFP did not induce significant vaccine-specific changes at 3 and 5 days after injection. In fact, changes in expression of various inflammatory and antiviral genes were observed, but these were not different between groups injected with the empty plasmid or with the tmORF25 construct. Recently, we reported the induction of a strong vaccine-specific local response after i.m. injection of the same dose of pcDNA3 plasmid encoding the Spring Viremia of Carp Virus (SVCV) glycoprotein (G) protein, characterized by significant increases in the cytokines $c x c b 1$, if $n \gamma 2 a b$, ifn $\varphi 1$, ifn $\varphi 2$ and even the adaptive immunity-related genes igt1 and zap70 [42]. Since this vaccine was also found to confer full protection against SVCV in juvenile 
carp, we can speculate that the expression of ORF25 alone might not be sufficient to trigger a strong local as well as systemic response required to achieve protection against KHV.

In the aforementioned ORF25 DNA vaccination study [25] koi carp received three i.m injections (with 3-weeks interval) of a DNA plasmid encoding the soluble ORF25, and were subsequently challenged by i.p. injection. The latter study used a construct encoding a soluble ORF25 protein composed of the core amino acids of the extracellular portion of the ORF25 molecule (amino acid 165-444). In our study we found that the tmORF25 construct conferred a protection similar to the one obtained by the solORF25 construct after three injections, whereas it induced a higher protection than the solORF25 construct after a single injection. The differences between the two studies in the effectiveness of the constructs encoding the soluble ORF25 protein can possibly be ascribed to the fact that our soluble construct encoded amino acid 1-444, thus including also the most N-terminal portion of the protein. Whether and how this (additional) portion of the protein would affect the correct folding of the soluble peptide would require further investigation, but it is unlikely that the additional N-terminal amino acids would have adverse effects on folding or immunogenicity. Furthermore, the approach using the full-length tmORF25 protein should exclude the possibility that important neutralizing-epitopes would be missing. Finally, it cannot be excluded that the route of challenge, i.p. in the former study and cohabitation (or bath) in our study, as well as the time of challenge after vaccination, 2 weeks after the last booster in the former study and 2.5 months in our study, altogether play a crucial role to reveal the effectiveness of the vaccine.

While 40 or more structural proteins have been identified in various KHV strains $[8,19]$, half of them are still uncharacterized, increasing the difficulty to select potential candidates for vaccine design. In a study characterizing the immunogenicity of KHV structural proteins [23], Igm reactivity in sera of carp that survived a KHV infection strongly points towards the fact that not one, but multiple proteins are the target of the KHV-specific antibody response of carp and koi. In particular, the major capsid protein ORF92 and proteins belonging to the ORF25-family (ORF25, ORF65 and ORF148, ORF149) were found to be major targets for the antibody response of carp against KHV [23]. Furthermore, as previously mentioned, neutralizing antibodies against ORF25 [25] and ORF81 [26] were generated when these antigens where used for DNA vaccination. In agreement, it was recently shown that neutralizing 
antibodies against ORF25 are (at least partly) required for the induction of a protective immune response against KHV [14]. Finally, upon KHV immunization of mice a neutralizing monoclonal antibody was generated against the capsid protein ORF72 [24].

In conclusion, while there is evidence that i.m. DNA vaccination using an ORF25 DNA vaccine holds promise for the protection against KHV, we were not able to optimize a DNA vaccination protocol that would be effective upon oral administration or upon single injection of the ORF25-based DNA plasmid. Our findings on a challenge route-dependent survival indicate that to optimally evaluate vaccine efficacy, challenge models that comply with the natural route of infection should preferably be used. Considering the complexity of the KHV proteome, we argue that a vaccine approach combining multiple KHV antigenic proteins might be more potent in triggering a protective immune response against a cohabitation challenge with KHV. More specifically, the type-I membrane proteins from the ORF25 family (ORF25, ORF148, ORF149), the glycoprotein ORF81 as well as the capsid proteins ORF72 and ORF92 are strong vaccine candidates that require further investigation.

\section{Competing interests}

The authors declare to have no conflict of interests.

\section{Acknowledgements}

This work was supported by the European Commission under the $7^{\text {th }}$ Framework Programme for Research and Technological Development (FP7) of the European Union (Grant Agreement 311993 TARGETFISH). TV and DP were also partly supported by the Ministry of Agriculture of the Czech Republic (MZE-RO0517). The authors wish to thank the support staff of the Aquatic Research Facility Carus, of the animal facility at Wageningen University for fish breading and rearing. 


\section{References}

1. FAO. The State of World Fisheries and Aquaculture 2016. (2016). Available at: http://www. fao.org/3/a-i5555e.pdf [Accessed September 18, 2017]

2. FAO. Cultured Aquatic Species Information Program. Cyprinus carpio. (2017) Available at: http://www.fao.org/fishery/culturedspecies/Cyprinus_carpio/en\#tcNA00D6 [Accessed September 18, 2017]

3. Haenen OLM, Way K, Bergmann SM, Ariel E. The emergence of koi herpesvirus and its significance to European aquaculture. Bull Eur Assoc Fish Pathol (2004) 24:293-307. Available at: http://library.wur.nl/WebQuery/wurpubs/fulltext/198497 [Accessed September 20, 2017]

4. Pokorova D, Vesely T, Pickova V, Reschova S, Hulova J. Current knowledge on koi herpesvirus (KHV): a review. Vet Med (Praha) (2005) 50:139-147.

5. Matsui K, Honjo M, Kohmatsu Y, Uchii K, Yonekura R, Kawabata Z. Detection and significance of koi herpesvirus (KHV) in freshwater environments. Freshw Biol (2008) 53:1262-1272. doi:10.1111/j.1365-2427.2007.01874.x

6. Garver KA, Al-Hussinee L, Hawley LM, Schroeder T, Edes S, LePage V, Contador E, Russell S, Lord S, Stevenson RMW, et al. Mass mortality associated with koi herpesvirus in wild common carp in Canada. J Wildl Dis (2010) 46:1242-1251. doi:10.7589/0090-355846.4.1242

7. Aoki T, Hirono I, Kurokawa K, Fukuda H, Nahary R, Eldar A, Davison AJ, Waltzek TB, Bercovier H, Hedrick RP. Genome sequences of three koi herpesvirus isolates representing the expanding distribution of an emerging disease threatening koi and common carp worldwide. J Virol (2007) 81:5058-65. doi:10.1128/JVI.00146-07

8. Michel B, Leroy B, Raj VS, Lieffrig F, Mast J, Wattiez R, Vanderplasschen AF, Costes B. The genome of cyprinid herpesvirus 3 encodes 40 proteins incorporated in mature virions. $J$ Gen Virol (2010) 91:452-462. doi:10.1099/vir.0.015198-0

9. Schmid T, Gaede L, Böttcher K, Bräuer G, Fichtner D, Beckmann R, Speck S, Becker F, Truyen U. Efficacy assessment of three inactivated koi herpes virus antigen preparations against experimental challenge virus infection in common carp. J Fish Dis (2016) 39:10071013. doi:10.1111/jfd. 12428

10. Boutier M, Ronsmans M, Ouyang P, Fournier G, Reschner A, Rakus K, Wilkie GS, Farnir FF, Bayrou C, Lieffrig FF, et al. Rational Development of an Attenuated Recombinant Cyprinid Herpesvirus 3 Vaccine Using Prokaryotic Mutagenesis and In Vivo Bioluminescent Imaging. PLoS Pathog (2015) 11: doi:10.1371/journal.ppat.1004690

11. Ronen A, Perelberg A, Abramowitz J, Hutoran M, Tinman S, Bejerano I, Steinitz M, Kotler M. Efficient vaccine against the virus causing a lethal disease in cultured Cyprinus carpio. Vaccine (2003) 21:4677-4684. doi:10.1016/S0264-410X(03)00523-1

12. Fuchs W, Fichtner D, Bergmann SM, Mettenleiter TC. Generation and characterization of koi herpesvirus recombinants lacking viral enzymes of nucleotide metabolism. Arch Virol (2011) 156:1059-1063. doi:10.1007/s00705-011-0953-8

13. Perelberg A, Ronen A, Hutoran M, Smith Y, Kotler M. Protection of cultured Cyprinus carpio against a lethal viral disease by an attenuated virus vaccine. Vaccine (2005) 23:33963403. doi:10.1016/j.vaccine.2005.01.096

14. Vancsok C, Peñaranda MMD, Raj VS, Leroy B, Jazowiecka-Rakus J, Boutier M, Gao Y, Wilkie GS, Suárez NM, Wattiez R, et al. Proteomic and Functional Analyses of the Virion Transmembrane Proteome of Cyprinid Herpesvirus 3. J Virol (2017) 91:JVI.01209-17. doi:10.1128/JVI.01209-17

15. Boutier M, Gao Y, Vancsok C, Suárez NM, Davison AJ, Vanderplasschen A. Identification of an essential virulence gene of cyprinid herpesvirus 3. Antiviral Res (2017) 145:60-69. doi:10.1016/j.antiviral.2017.07.002

16. Yasumoto S, Kuzuya Y, Yasuda M, Yoshimura T, Miyazaki T. Oral immunization of common 
carp with a liposome vaccine fusing Koi Herpesvirus antigen. Fish Pathol (2006) 41:141145. doi:10.3147/jsfp. 41.141

17. Costes B, Fournier G, Michel B, Delforge C, Raj VS, Dewals B, Gillet L, Drion P, Body A, Schynts F, et al. Cloning of the koi herpesvirus genome as an infectious bacterial artificial chromosome demonstrates that disruption of the thymidine kinase locus induces partial attenuation in Cyprinus carpio koi. J Virol (2008) 82:4955-64. doi:10.1128/JVI.00211-08

18. St-Hilaire S, Beevers N, Way K, Le Deuff R, Martin P, Joiner C. Reactivation of koi herpesvirus infections in common carp Cyprinus carpio. Dis Aquat Organ (2005) 67:15-23. doi:10.3354/dao067015

19. Yi Y, Zhang H, Lee X, Weng S, He J, Dong C. Extracellular virion proteins of two Chinese CyHV-3/KHV isolates, and identification of two novel envelope proteins. Virus Res (2014) 191:108-116. doi:10.1016/j.virusres.2014.07.034

20. Monaghan SJ, Thompson KD, Bron JE, Bergmann SM, Jung TS, Aoki T, Muir KF, Dauber M, Reiche S, Chee D, et al. Expression of immunogenic structural proteins of cyprinid herpesvirus 3 in vitro assessed using immunofluorescence. Vet Res (2016) 47: doi:10.1186/ s13567-015-0297-6

21. Kattlun J, Menanteau-Ledouble S, Gotesman M, Abd-elfattah A, Way K, Soliman H, Bergmann S, El-Matbouli M. Immunogenic potential of a membrane protein encoded by the viral gene located at ORF 81 of Immunogenic potential of a membrane protein encoded by the viral gene located at ORF 81 of Cyprinid Herpes-. Wien Tierarztl Monatsschr (2016) 103:58-67.

22. Rosenkranz D, Klupp BG, Teifke JP, Granzow H, Fichtner D, Mettenleiter TC, Fuchs W. Identification of envelope protein pORF81 of koi herpesvirus. J Gen Virol (2008) 89:896900. doi:10.1099/vir.0.83565-0

23. Fuchs W, Granzow H, Dauber M, Fichtner D, Mettenleiter TC. Identification of structural proteins of koi herpesvirus. Arch Virol (2014) 159:3257-3268. doi:10.1007/s00705-0142190-4

24. Tu C, Lu YP, Hsieh CY, Huang SM, Chang SK, Chen MM. Production of monoclonal antibody against ORF72 of koi herpesvirus isolated in Taiwan. Folia Microbiol (Praha) (2014) 59:159-165. doi:10.1007/s12223-013-0261-7

25. Zhou J-X, Wang H, Li X-W, Zhu X, Lu W-L, Zhang D-M. Construction of KHV-CJ ORF25 DNA vaccine and immune challenge test. J Fish Dis (2014) 37:319-325. doi:10.1111/ jfd.12105

26. Zhou J-X, Xue J, Wang Q, Zhu X, Li X, Lv. W, Zhang D, Lu. W, D. Z. Vaccination of plasmid DNA encoding ORF81 gene of CJ strains of KHV provides protection to immunized carp. Vitr Cell Dev Biol - Anim (2014) 50:489-495. doi:10.1007/s11626-014-9737-2

27. Miyazaki T, Yasumoto S, Kuzuya Y, Yoshimura T. "A primary study on oral vaccination with liposomes entrapping Koi Herpesvirus ( KHV) antigens against KHV infection in carp," in Diseases in Asian Aquaculture, ed. R. P. Bondad-Reantaso, M.G., Mohan, C.V., Crumlish, M. and Subasinghe (Manila: Asian Fisheries Society), 99-184.

28. Cui L-C, Guan X-T, Liu Z-M, Tian C-Y, Xu YG. Recombinant lactobacillus expressing $\mathrm{G}$ protein of spring viremia of carp virus (SVCV) combined with ORF81 protein of koi herpesvirus (KHV): A promising way to induce protective immunity against SVCV and KHV infection in cyprinid fish via oral vaccination. Vaccine (2015) 33: doi:10.1016/j. vaccine.2015.05.002

29. Irnazarow I. Genetic variability of Polish and Hungarian carp lines. Aquaculture (1995) 129:215. doi:10.1016/0044-8486(95)91961-T

30. Bar I, Kaddar E, Velan A, David L. Melanocortin receptor 1 and black pigmentation in the Japanese ornamental carp (Cyprinus carpio var. Koi). Front Genet (2013) 4:6. doi:10.3389/ fgene.2013.00006

31. Tadmor-Levi R, Asoulin E, Hulata G, David L. Studying the genetics of resistance to CyHV3 disease using introgression from feral to cultured common carp strains. Front Genet (2017) 
8:1-13. doi:10.3389/fgene.2017.00024

32. Fink IR, Pietretti D, Voogdt CGP, Westphal AH, Savelkoul HFJ, Forlenza M, Wiegertjes GF. Molecular and functional characterization of Toll-like receptor (Tlr) 1 and Tlr2 in common carp (Cyprinus carpio). Fish Shellfish Immunol (2016) 56:70-83. doi:10.1016/j. fsi.2016.06.049

33. Secombes CJ, van Groningen JJ, Egberts E. Separation of lymphocyte subpopulations in carp Cyprinus carpio L. by monoclonal antibodies: immunohistochemical studies. Immunology (1983) 48:165-175.

34. Reed LJ, Muench H. A simple method of estimating fifty per cent endpoints. Am J Epidemiol (1938) 27:493-497. Available at: http://www.scopus.com/inward/record.url?eid=2-s2.033745158157\&partnerID=tZOtx3y1

35. Forlenza M, Kaiser T, Savelkoul HFJ, Wiegertjes GF. The use of real-time quantitative PCR for the analysis of cytokine mRNA levels. Methods Mol Biol (2012) 820:7-23. doi:10.1007/978-1-61779-439-1

36. Forlenza M, Walker PD, de Vries BJ, Wendelaar Bonga SE, Wiegertjes GF. Transcriptional analysis of the common carp (Cyprinus carpio L.) immune response to the fish louse Argulus japonicus Thiele (Crustacea: Branchiura). Fish Shellfish Immunol (2008) 25:76-83. doi:10.1016/j.fsi.2007.12.013

37. Forlenza M, Kaiser T, Savelkoul HFJ, Wiegertjes GF. The use of real-time quantitative PCR for the analysis of cytokine mRNA levels. Methods Mol Biol (2012) 820:7-23. doi:10.1007/978-1-61779-439-1_2

38. Pfaffl MW. A new mathematical model for relative quantification in real-time RT-PCR. Nucleic Acids Res (2001) 29:e45.

39. EMA. First DNA vaccine in the EU recommended for use in salmon. 2016-04-22 (2016)1. Available at: http://www.ema.europa.eu/ema/index.jsp?curl=pages/news_and_events/ news/2016/04/news_detail_002516.jsp\&mid=WC0b01ac058004d5c1 [Accessed September $18,2017]$

40. Tonheim TC, Dalmo RA, Bøgwald J, Seternes T. Specific uptake of plasmid DNA without reporter gene expression in Atlantic salmon (Salmo salar L.) kidney after intramuscular administration. Fish Shellfish Immunol (2008) 24:90-101. doi:10.1016/j.fsi.2007.09.006

41. Tonheim TC, Leirvik J, Løvoll M, Myhr AI, Bøgwald J, Dalmo RA. Detection of supercoiled plasmid DNA and luciferase expression in Atlantic salmon (Salmo salar L.) 535 days after injection. (2007) doi:10.1016/j.fsi.2007.03.015

42. Embregts CWE, Rigaudeau D, Veselý T, Pokorová D, Lorenzen N, Petit J, Houel A, Dauber M, Schütze H, Boudinot P, et al. Intramuscular DNA Vaccination of Juvenile Carp against Spring Viremia of Carp Virus Induces Full Protection and Establishes a Virus-Specific B and T Cell Response. Front Immunol (2017) 8:1340. doi:10.3389/fimmu.2017.01340 


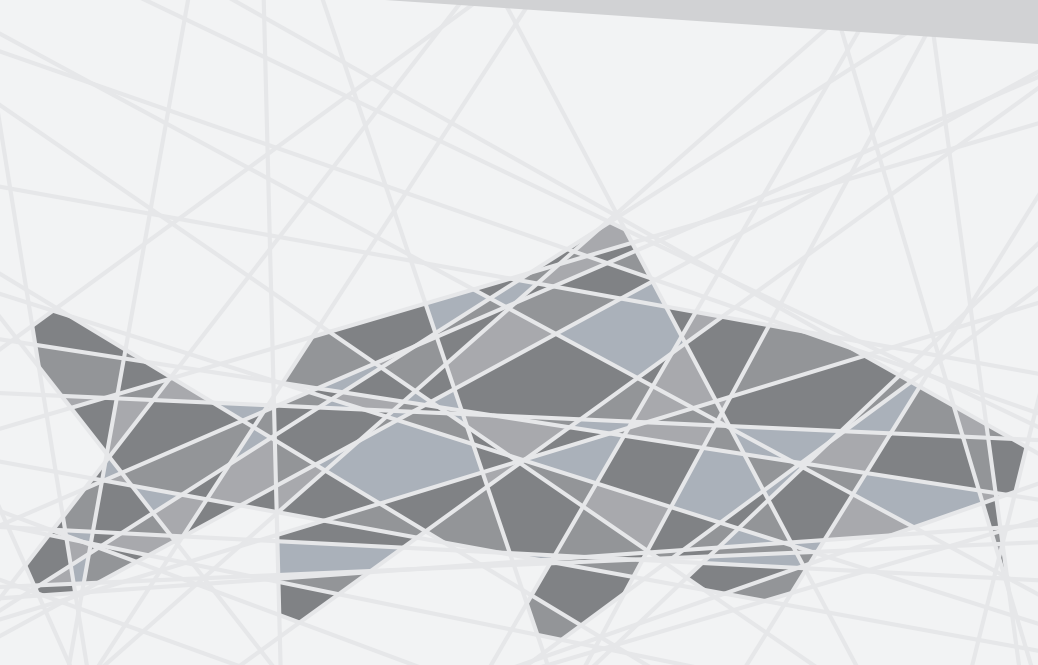




\section{Characterization of $\mathrm{T}$ cell populations of common carp - a molecular and cellular approach}

\section{Carmen W.E. Embregts', Jules Petit', Cas van den Bomen', T. Nakanishi', Ben Meijer', Geert F.Wiegertjes', Maria Forlenza'}

' Cell Biology and Immunology Group, Department of Animal Sciences, Wageningen University, Wageningen, The Netherlands 2 Department of Veterinary Medicine, Nihon University, Japan 


\section{Abstract}

In the current study, we report the molecular characterization of two $c d 4$ genes in common carp, each present in two isoforms ( $c d 4-1 a, c d 4-1 b, c d 4-2 a, c d 4-2 b)$. Using cross-reactive antibodies to carp $\mathrm{Cd} 4-1$ and $\mathrm{Cd} 8 \alpha 1$ in combination with a panel of leukocyte-specific antibodies available in our lab, we were able to characterize $\mathrm{T}$ cell populations in common carp. We show a strong double labelling with Zap $70^{+}$cells, and the highest percentage of $\mathrm{T}$ cells was found in thymus, intestine and gills. No reactivity of the anti-Cd4-1 and anti-Cd8 $\alpha 1$ antibodies was found against B-cells, thrombocytes, neutrophilic granulocytes and monocytes/macrophages. In agreement with the flow cytometry data, transcriptome data of a common carp organ library, and RT-qPCR gene expression analysis of MACS sorted cells, confirmed that all $c d 4$ genes and isoforms were highest expressed in thymus and gills, and that no expression of any of the $c d 4$ genes was found in other leukocyte types. RNA sequencing of FACS sorted Cd $4-1^{+}$and $\mathrm{Cd} 8 \alpha 1^{+}$cells led to the identification of several differentially expressed genes that can be used to develop new cell-specific markers for cellular and molecular analysis. Using an antibody previously described to recognize putative mucosal T cells (WCL38), we observed distinct WCL38 ${ }^{+} \mathrm{Cd} 8 \alpha^{+}, \mathrm{WCL} 38^{+} \mathrm{Cd} 8 \alpha^{-}$and WCL38 $\mathrm{Cd} 8 \alpha^{+}$populations in the intestine, whereas no $\mathrm{WCL} 38^{+} \mathrm{Cd} 4-1^{+}$population was observed. FACS sorting and RNA sequencing revealed an interesting pattern of genes that were differentially expressed between the WCL38 ${ }^{+}$and the Cd8 $8+$ and $\mathrm{Cd} 4-1+$ population. Based on these gene signatures, we preliminary concluded that besides conventional $\alpha \beta T$ cells, an additional population of $\mathrm{NK}(\mathrm{T})$ cells might be recognized by WCL38, potentially including ${ }_{\gamma \delta} \mathrm{T}$ cells. Finally, we discuss how we plan to complete the characterization of the population identified by the WCL38 antibody, and how we can use these information to further characterize mucosal and systemic $\mathrm{T}$ cells in common carp. 


\section{Introduction}

In mammals, $\mathrm{T}$ cells can be divided into $\mathrm{CD} 4^{+}$and $\mathrm{CD} 8^{+} \mathrm{T}$ cells, also known as helper T cells (Th) and cytotoxic T cells (CTL), respectively. CD4 and CD8 are T cell co-receptors that stabilize T cell receptor (TCR)/antigen-MHC complexes and bind to MHC class II or MHC class I, respectively . The co-receptor CD8 is expressed on $\mathrm{T}$ cells as a homodimeric $(\mathrm{CD} 8 \alpha \alpha)$ or heterodimeric $(\mathrm{CD} 8 \alpha \beta)$ transmembrane glycoprotein, whereas CD4 is a single-chain transmembrane glycoprotein.

In European common carp (Cyrpinus carpio)the genes encoding for $c d 8 \alpha 1, c d 8 \alpha 2$, $c d 8 \beta 1, c d 8 \beta 2$ and $c d 4-l i k e$ ( $c d 4-1)$ have been identified and characterized [1,2] Besides the $c d 4-1$ gene, a second $c d 4$-related gene ( $c d 4-2$ or $c d 4-r e l)$ has been described in many teleost species including Atlantic salmon (Salmo salar) [3], Japanese flounder (Paralichthys olivaceus) [4], catfish (Ictalurus punctatus) [5], Atlantic halibut (Hippoglossus hippoglossus) [6], rainbow trout (Oncorhynchus mykiss) [7], pufferfish (Takifugu rubripes) [8] and zebrafish (Danio rerio) [9]. Sequence analysis showed that all described $c d 4-2$ sequences encode two to three Ig-like domains, a TM region and a cytoplasmic tail containing the $\mathrm{p} 56^{\text {lck }}$ domain. In contrast to the description of multiple genes for $c d 8 \alpha$ and $c d 8 \beta$ in common carp, the presence of multiple genes and isoforms of cd4 has not yet been reported. However, since common carp is a tetraploid species, multiple gene copies are to be expected $[10,11]$.

Besides the division of $\mathrm{T}$ cells based on expression of either the $\mathrm{Cd} 4$ or $\mathrm{Cd} 8$ coreceptor, $\mathrm{T}$ cells can be divided based on their $\mathrm{T}$ cell receptor (TCR), resulting in a division between ${ }_{\alpha \beta} \mathrm{T}$ cells and ${ }_{\gamma \delta} \mathrm{T}$ cells [12]. In human and mouse, ${ }_{\gamma \delta} \mathrm{T}$ cells are found mainly at mucosal surfaces including skin, lungs, reproductive tract and intestine $[13,14]{ }_{\gamma \delta} \mathrm{T}$ cells recognize non-peptide antigens without interaction with APCs and express mostly the Cd8 $8 \alpha$ homodimer [15]. Teleost $t c r \gamma$ and $t c r \delta$ genes have been identified and cloned in multiple species but due to a lack of specific antibodies, the exact function of ${ }_{\gamma \delta} \mathrm{T}$ cells in fish is still elusive. Recently, antibodies raised against zebrafish Tcr $\gamma$ and Tcr $\delta$ revealed a phenotype of $\gamma^{+} \delta^{+} \mathrm{Cd} 4 \mathrm{Cd} 8 \alpha^{+}$cells that was able to phagocytose, induce $\mathrm{Cd} 4^{+} \mathrm{T}$ cell activation, B cell proliferation and Igm and Igz (Igt) production and is potentially involved in antigen presentation [16].

Besides the conventional $\mathrm{Cd} 4^{+}$or $\mathrm{Cd} 8^{+}$single positive $\mathrm{T}$ cells, peripheral $\mathrm{Cd} 4^{+} \mathrm{Cd} 8^{+}$ double-positive T-cells have been described in multiple mammalian species including 
human, mice and rats. In some species this type represents a large proportion of the total $\mathrm{T}$ cell pool, like in chickens (5-40\%), monkeys (5-20\%) or swine (60\%) (Reviewed in [17]).

Furthermore, CD4 can be expressed on monocyte subsets of human and rat [18], but not in mice and birds (Reviewed in [18,19]) whereas dendritic cells (DCs) of human, mouse and rat were found to express both $\mathrm{Cd} 4$ and $\mathrm{Cd} 8$ (Reviewed in [20]). A first description of a non-lymphoid $\mathrm{Cd} 4^{+}$cell population in teleost fish was reported only recently [21]. In this study, antibodies specific for rainbow trout Cd4-1 and Cd4-2 were used and a population of highly phagocytic Cd4-1 single-positive cells was found with a myeloid morphology, besides Cd4-2 single positive and Cd4-1/ Cd4-2 double positive lymphoid populations [21]. In agreement with this, Japanese flounder Cd4-1, but not Cd4-2, seems to be expressed on larger myeloid cells, as was characterized by in situ hybridization [4]. Furthermore, in zebrafish, lck-Cd4-1 ${ }^{+}$ mononuclear phagocytes (MNPs) were described in the thymus and skin of zebrafish [22]. In addition to these new views on $\mathrm{Cd} 4$ in fish, a $\mathrm{Cd} 8 \alpha^{+} \mathrm{MHC} \mathrm{II}^{+}$dendritic cell population was found in skin of rainbow trout [23]. Taken together, only $\mathrm{Cd} 4$ or $\mathrm{Cd} 8$ are not sufficient for the identification and characterization of T-cell subsets.

Despites the importance of T-cells in the teleost immune response, their functional characterization is still in its infancy, mainly due to a lack of specific tools and antibodies to characterize different $\mathrm{T}$ cell subsets. Furthermore, due to the low level of conservation of the $\mathrm{Cd} 4$ and $\mathrm{Cd} 8$ proteins, antibodies are most often not cross-reactive between species. While few antibodies were raised successfully against $\mathrm{T}$ cells, using whole-cell lysates for the immunization of mice, in sea bass (Dicentratchus labrax) (DTL15; [24]), catfish (CfT1; [25]) and carp (WCL9 and WCL38; [26,27]), the first antibodies against specific $\mathrm{T}$ cell subsets were only raised recently. Upon now, antibodies against $\mathrm{Cd} 4$ have been produced and validated only in zebrafish [9], Atlantic salmon [28], ginbuna crucian carp (Carassius auratus langsdorfii) [29], fugu [30] and rainbow trout [21], and for $\mathrm{Cd} 8 \alpha$ in ginbuna crucian carp [29] and rainbow trout [31]. This low number of antibodies, as well as the absence of antibodies against $\mathrm{Cd} 8 \beta$, illustrates the difficulty in producing specific antibodies. However, it was recently described that the antibodies raised against ginbuna crucian carp Cd4-1 and Cd8 $\alpha 1$ cross-reacted with zebrafish lymphocytes and also with those of other cyprinids, including common carp [32].

Combining these cross-reactive antibodies with a panel of antibodies already 
available in our laboratory, we for the first time characterized T-cell subsets in common carp by flow cytometry. Furthermore, using homology cloning and in silico analysis of available carp genome and transcriptome datasets, we were able to identify and characterize two $c d 4$ genes, each present in two isoforms. Using this renewed panel of available antibodies against carp T cell markers, we FACS sorted $\mathrm{Cd} 4-1^{+}$and $\mathrm{Cd} 8 \alpha 1^{+}$populations and performed RNA sequencing and transcriptome analysis on the obtained samples to 1 ) confirm the specificity of the antibodies to $\mathrm{T}$ cells of common carp and 2) to gain insights into T cell subset-specific transcriptome profiles, hereby greatly enhancing the knowledge on common carp T cell subsets. Using the same approach, we used the antibody WCL38 which was previously reported to recognize putative mucosal $\mathrm{T}$ cells [26], to investigate specific gene signatures of this mucosal cell population. When combining the transcriptome data set of WCL38-sorted cells with that of $\mathrm{Cd} 4-1^{+}$and $\mathrm{Cd} 8 \mathrm{a} 1^{+}$sorted cells we reveal that besides conventional ${ }_{\alpha \beta} \mathrm{T}$ cells, the WCL38 antibody might potentially recognized ${ }_{\gamma \delta}$ T-cells and a subset of NK(T) cells. Altogether, we describe the presence of two $c d 4$ genes in carp ( $c d 4-1$ and $c d 4-2$ ) each present in two isoforms and by combining cellular and molecular approaches we were able to shed an unique light on $\mathrm{T}$ cell subsets in common carp.

\section{Materials and methods}

\section{Animals}

European common carp (Cyrpinus carpio carpio) from the R3xR8 strain were used that originated from a cross between the Hungarian R8 strain and the Polish R3 strain (Irnazarow, 1995). In this study we will refer to carp as the European common carp subspecies, unless stated otherwise. Carp were bred in the Aquatic Research Facility (ARC) of Wageningen University and research animal facility, the Netherlands. Carp were raised at $20-23^{\circ} \mathrm{C}$ in recirculating UV-treated water and fed pelleted dry food (Skretting, Nutreco) twice daily. All studies were performed with approval of the local animal welfare committee (DEC) of Wageningen University.

\section{Cells}

Vero cells, originated from African green monkey kidney, were cultured in Minimum Essential Medium (DMEM, Gibco) supplemented with 1\% v/v streptomycin/ 
penicillin, 1\% L-glutamine, 10\% foetal calf serum (FCS), and $4.5 \mathrm{~g} / 1 \mathrm{D}$-glucose. Cells were kept at $37^{\circ} \mathrm{C}$ in the presence of $5 \% \mathrm{CO}_{2}$.

\section{RNA isolation and CDNA synthesis}

Total RNA was isolated from healthy carp spleen or from cells sorted (described below). Briefly, cells were resuspended in RLT lysis buffer and homogenized through a $22 \mathrm{G}$ needle; alternatively the tissue was disrupted and homogenized in the same buffer using a TissueLyserII (Qiagen). RNA was extracted using the RNeasy Mini Kit (Qiagen) for RNA isolation from tissue, or the Micro RNeasy Kit (Qiagen) for RNA isolation from sorted cells, following manufacturer's guidelines, including an on-column DNAse treatment using the RNase-free DNase kit (Qiagen). RNA concentrations were measured using a Nanodrop-1000, the integrity was verified on a $1 \%$ agarose gel and RNA was stored at $-80^{\circ} \mathrm{C}$ until further use.

Prior to cDNA synthesis from $1 \mu \mathrm{g}$ total RNA, a second DNase treatment was performed using DNAse I, Amplification Grade (Invitrogen). Reverse transcription of the RNA was performed using random primers (300 ng) and Superscript ${ }^{\mathrm{TM}}$ III (200U) First Strand Synthesis Systems for RT-PCR (Invitrogen) according to the manufacturer's instructions. cDNA samples were further diluted 25 times in nuclease-free water and stored at $-20^{\circ} \mathrm{C}$.

\section{Identification and cloning of European common carp Cd4-1 and Cd4-2 isoforms}

To obtain the European common carp Cd4-1 sequences, the Japanese common carp (Cyprinus carpio carpio) cd4-1 (Acc. Nr. DQ400124) was used to blast against the European common carp transcriptome [33]. This retrieved two sequences which in turn were used to blast against the carp genome to confirm the presence of two $c d 4$ 1 sequences, designated $c d 4-1 a$ (based on its highest similarity with the previously published sequence) and $c d 4-1 b$.

To obtain the European common carp Cd4-2 sequence, the zebrafish (Danio rerio) $c d 4-2$ (currently annotated as $c d 4-2.2-201$, Acc. Nr. ENSDART00000103971.3) was used to blast against the carp transcriptome and genome. These blasts retrieved three partial sequences, initially named $c d 4-2 a 1, c d 4-2 a 2$ and $c d 4-2 b$, of which $c d 4-2 a 1$ and $c d 4-2 a 2$ were almost identical. In order to first confirm the existence and subsequently the sequences of all $c d 4$ genes and isoforms, reverse transcriptase (RT)-PCR was performed (SuperScript One-Step RT-PCR System, Invitrogen) using Platinum Taq DNA polymerase and primers designed within the predicted 
coding region of the obtained sequences. To confirm the presence of $c d 4-1 b$, internal primers CycaCd4-1b-FW2 and CycaCd4-1b-RV2 were used to amplify a region of 900 bp using $1 \mu \mathrm{g}$ total RNA from spleen tissue as template. To confirm the presence of all three isoforms of cd4-2, a two-step approach was used. Based on the in silico sequence, common primers for all sequences were designed spanning the start and the stop codon region, as well as common internal primer (Fig. 1). The RT-PCR products from the cd4-2all_ATG_FW in combination with cd4-2all_Rv1 or cd42all_FW1 in combination with cd4-2all_STOP_RV, were used as templates for a second nested-PCR, for which isoform specific primers were used, amplifying the two $c d 4-2 a$ together and specifically the $c d 4-2 b$ sequence (Fig. 1, step 2). For each PCR reaction, $1 \mu \mathrm{g}$ of RNA was used with $200 \mathrm{nM}$ of both forward and reverse primer (Table 1) in a total volume of $50 \mu \mathrm{L}$. The PCR program consisted of a 2 min denaturation step at $94^{\circ} \mathrm{C}$ followed by 35 cycles $\left(94^{\circ} \mathrm{C} 30 \mathrm{sec}, 54-58^{\circ} \mathrm{C} 30 \mathrm{sec}\right.$, $72^{\circ} \mathrm{C} 45 \mathrm{sec}$ and a final elongation step of $7 \mathrm{~min}$ at $72^{\circ} \mathrm{C}$. Obtained products were purified using the DNA Clean \& Concentrator-5 kit (Zymo Research), ligated into the pGEM-T Easy vector (Promega) and transformed into JM109 competent cells (Promgea). Bacterial cultures were grown onto LB/ampicillin/IPTG/X-gal plates and selected colonies were sequenced (BaseClear).

Despite several attempts, sequence analysis never retrieved a sequence corresponding to the $c d 4-2 a 2$ isoforms, whereas $c d 4-2 a 1$ and $c d 4-2 b$ could be confirmed. For this reason, we concluded that, at least in the carp strains we used, only two $c d 4-2$ genes are expressed, and will thereafter be referred to as $c d 4-2 a$ and $c d 4-2 b$.

\section{Nucleotide and protein sequence analysis}

After verification, the sequences were translated into proteins and multiple tools were used to identify protein structural domains; signal peptide (SignalP 4.0; http:// www.cbs.dtu.dk/services/SignalP/), transmembrane domain and helix (TMHMM Server 2.0; http://www.cbs.dtu.dk/services/TMHMM), immunoglobulin domains (InterPro protein analysis and classification; https:/www.ebi.ac.uk/interpro/) N glycosylation sites (NetNGlyc 4.0; http://www.cbs.dtu.dk/services/NetOGlyc/) and O glycosylation sites (NetOGlyc 1.0; http:/www.cbs.dtu.dk/services/NetNGlyc/). Multiple sequence alignments were made using Clustal Omega (https:// www.ebi. ac.uk/Tools/msa/clustalo/). 


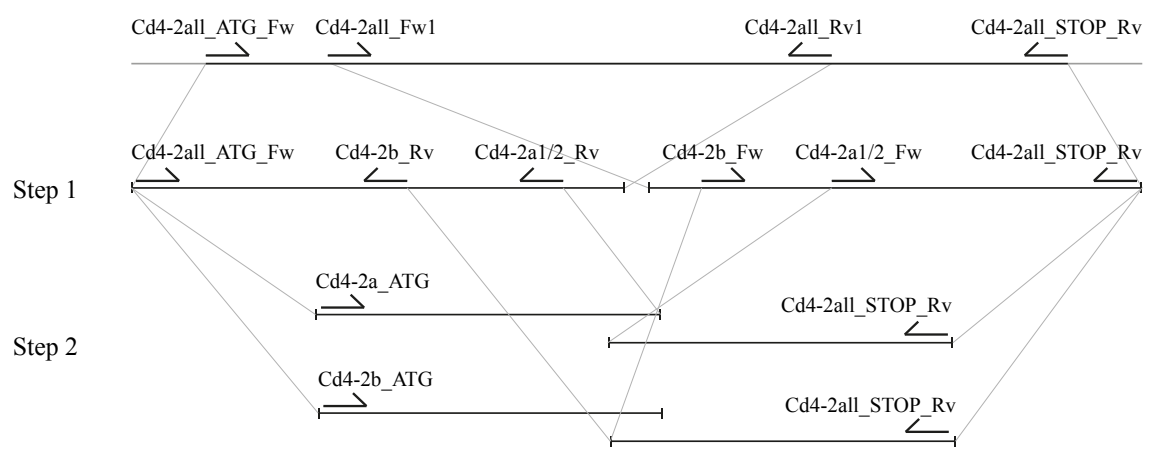

Fig. 1. Strategy used to identify and clone the European common carp cd4-2a and cd4$\mathbf{2 b}$ sequences. Primer names are indicated above the sequence and can be found in Table 1. Arrows indicate the direction of amplification, grey lines indicate downstream use of the amplicons in Step 1 and Step 2.

\section{Real Time-quantitative PCR and in silico analysis of transcriptome data}

The expression of $c d 4-1$ and $c d 4-2$ in sorted immune cells and in various organs was examined by Real Time-quantitative PCR (RT-qPCR) and in silico analysis.

For the organ distribution, manual datamining was performed, employing the previously obtained sequences for $c d 4-1 a, c d 4-1 b, c d 4-2 a$ and $c d 4-2 b$ as BLAST query against the carp organ library transcriptome [10]. Similarly to Kolder et al., reads per million per kilobase (RPKM)-expression values were used to allow between organ comparison of the genes of interest.

For the expression in various cell types, available cDNA from Magnetically-Activated Cell Sorting MACS sorted cells [34,35] was used as template for RT-qPCR using a Rotor-Gene ${ }^{\mathrm{TM}} 6000$ (Qiagen). Briefly, $5 \mu \mathrm{L}$ of 25 times diluted cDNA was mixed with $2 \mu \mathrm{L}$ of forward and reverse primers $(2.1 \mu \mathrm{M}$ of each primer) and $7 \mu \mathrm{L}$ of 2x ABsolute qPCR SYBR Green Mix (Thermo Scientific) as detection chemistry. The list of primers used for RT-qPCR can be found in Table 1. Fluorescence data were analyzed using Rotor-Gene Q series software version 2.3.1. The take-off value for each sample and the average reaction efficiencies $(E)$ for each primer set were obtained upon comparative quantitation analysis from the Rotor-Gene software [36] and were used to calculate the basal gene expression level relative to the $s 11$ protein of the $40 s$ subunit reference gene based on the Pfaffl method [37].

\section{Eukaryotic expression of European common carp Cd4-1a and Cd8a1}

In order to confirm that antibodies against ginbuna crucian carp Cd4-1 and Cd8 81 would cross-react to T cells of European common carp [32], the sequences coding 
Table 1. Primer used in this study. Asterisks $\left(^{*}\right)$ indicates that the sequences are to be submitted to the NCBI database.

\begin{tabular}{ll}
\hline Cloning of $\boldsymbol{C d} 4-1$ and $\boldsymbol{C d} 4-\mathbf{2}$ & \\
\hline Primer & Sequence 5'-> 3' \\
\hline CycaCD4-1b-Fw2 & GGAGGGAATGTTATCCTACCTAGAGA \\
CycaCD4-1b-Rv2 & AAACATTCAGAACCTCCACCTG \\
Cd4-2all_ATG_FW & AAAAAAGAATAGAATGGCAA \\
Cd4-2all_STOP_RV & CGCTCTGCTCCTCTCA \\
Cd4-2all_Fw1 & TACAGGAGACCCAAATACT \\
Cd4-2all_Rv1 & CTCCACCATCAAACTCCACTGTAC \\
Cd4-2b_Rv & GTTACACTGAGCGTCAAAGCA \\
Cd4-2a1/2_Rv & AGTTGGTGCTGAGTTGCC \\
Cd4-2b_Fw & CCTAATGGTCAAAAACATAATGAAAAGAGT \\
Cd4-2a1/2_Fw & GTGGGTGGGAGATGGAAA \\
Cd4-2a_ATG & AAGGGACAGAATGGCAAT \\
Cd4-2b_ATG & AAGAATAGAATGGCAA+CTTG \\
\hline
\end{tabular}

\section{RT-qPCR}

\begin{tabular}{|c|c|c|c|}
\hline Primer & Fw primer 5'-3' & Rv primer 3'-5' & Acc.Nr. \\
\hline $40 s$ & CCGTGGGTGACATCGTTACA & TCAGGACATTGAACCTCACTGTCT & $\mathrm{AB} 012087$ \\
\hline$c d 4-1 a$ & TGATGGAAGAGAAACAGAAGCA & CAAGACCGACCAGGGAATGT & DQ400124 \\
\hline$c d 4-1 b$ & GAAAACTCAGATTGTCATCAAAGCA & AGAAGCGGAGAGGTCTATTACAG & $*$ \\
\hline$c d 4-2 a$ & GTGGGTGGGAGATGGAAA & AGTTGGTGCTGAGTTGCC & $*$ \\
\hline$c d 4-2 b$ & $\begin{array}{l}\text { CCTA AT GG TC A A A A A C ATA AT- } \\
\text { GAAAAGAGT }\end{array}$ & GTTACACTGAGCGTCAAAGCA & * \\
\hline$c d 8 \alpha 1$ & TGCGCAGCCATGAACAACAATA & GCCATTGTTGTTGTCGATGGTA & EU025118 \\
\hline$c d 8 \alpha 2$ & TGCGCAGCCATGAACAACAATA & CGTAATCACTGGTGTTGCAGTT & EU025119 \\
\hline$c d 8 \beta 1$ & AATCAA+CGGCT+CGGAAACTATC & CGCTCTCTGCCAGCGCTGTTACA & EU025120 \\
\hline$c d 8 \beta 2$ & AAT+CAATGGCT+CGGAAGTTCTG & $\begin{array}{l}\text { C C A G C A + C T G T T GA + C ATA - } \\
\text { CACTAA }\end{array}$ & EU025121 \\
\hline
\end{tabular}

\section{Expression vectors}

\begin{tabular}{ll}
\hline Primer & Sequence 5'-3' \\
\hline pDis_SfiI_Cd4-1a_FW & GTTACGGGCCCAGCCGGCCTATGTGAACTGGTACCGTGG \\
\hline pDis_SalI_Cd4-1a_RV & CGGAAGTCGACGCTGATGGCAACACAAAGCCA \\
\hline PDis_SfiI_Cd8 $\alpha 1$ _FW & GTTACGGGCCCAGCCGGCCAATACCATTTACCAAGAAGGA- \\
& CAGG \\
\hline pDis_SalI_Cd8 1 1_RV & CGCAAGTCGACTGATTTTGTCGTAGCCATTGTTGTTG \\
\hline
\end{tabular}

for the extracellular portion of the European common carp Cd4-1a and CD8 $\alpha 1$ were cloned into the expression vector pDisplay (Promega) downstream of the sequence coding for the immunoglobulin light chain leader peptide and HA-tag, and upstream of the PDGFR transmembrane region. Briefly, the nucleotide sequence coding for amino acids 26-409 of Cd4-1a and 20-141 of Cd8 $\alpha 1$, were amplified from pGEM-T easy vectors harbouring the full-length sequences, as described above for $c d 4-1 a$ and previously for $c d 8 \alpha 1$ [34], using primers containing SfiI and SalI restriction sites (Table 1). PCR products were purified using the QIAquick gel extraction kit (Qiagen), digested with SfiI (Promega) and SalI (Promega) and ligated using T4 
ligase (Promega). Obtained plasmids were sequenced to verify the insert.

\section{Cell transfection and immunofluorescence}

Vero cells were seeded in 24 well-plates on glass cover slips at a density of 150.000/ well. Cells were transfected with $0.5 \mu \mathrm{g}$ of plasmid (pDisplay_Cd4-1a-HA or pDisplay_Cd8 $\alpha 1$-HA) and $1.75 \mu \mathrm{L}$ of FuGENE HD transfection reagent (Promega) using the manufacturer's guidelines. After $48 \mathrm{~h}$ cells were rinsed twice with phosphate buffer (PBS), fixed with 4\% paraformaldehyde (PFA) in PBS (Lonza) for $15 \mathrm{~min}$ at $4^{\circ} \mathrm{C}$, washed again and stained with an antibody against the HA-Tag (1:1000, ProteinTag) and monoclonal antibodies against ginbuna crucian carp Cd41 (clone 6D1) or Cd8 $\alpha$ (clone 2C3) [29,32] (hybridoma supernatant 1:5 diluted, kindly provided by Prof. T. Nakanishi, Nihon University, Japan). After subsequent washes, slides were incubated with FITC-conjugated goat-anti-mouse IgG (1:200, Santa Cruz) and PE-conjugated goat-anti-rat IgG (1:200, Santa Cruz). A staining with DAPI (Thermo Scientific) was included to visualize the cell nuclei. Cells were visualized using an EVOS fl LED fluorescence microscope (Advanced Microscopy Group (AMG)).

\section{Flow cytometric analysis of European common carp Cd4-1 and Cd8a1 populations}

To confirm the cross-reactivity of the monoclonal antibodies against ginbuna crucian carp Cd4-1 and Cd8 1 1 to European common carp T cells, double labelling with a cross-reactive antibody against the pan-(NK)T cell marker Zap70 was performed, as described before [38,39]. To further characterize the specificity of the antibodies, double labelling was performed with monoclonal antibodies against common carp $\mathrm{Igm}^{+} \mathrm{B}$ cells, neutrophilic granulocytes, monocytes/macrophages and putative mucosal T cells. Carp were euthanized with $0.6 \mathrm{~g} / \mathrm{L}$ Tricaine Methane Sulfonate (TMS, Crescent Research Chemicals) buffered with $1.2 \mathrm{~g} / \mathrm{L} \mathrm{NaHCO}_{3}$ and bled through the caudal vein. Total leukocytes were isolated from blood, thymus, spleen, head kidney, intestine and gills as described previously [35,40]. For the double labelling with anti-Zap70, 1 × $10^{6}$ cells were first incubated with rat anti-Cd4-1 (clone $6 \mathrm{D} 1,1: 5$ ) or rat anti-Cd8 $\alpha 1$ (clone 2C3, 1:5) monoclonal antibodies in FACS buffer $\left(0.5 \% \mathrm{BSA}, 0.01 \% \mathrm{NaN}_{3}\right.$ in PBS) for $45 \mathrm{~min}$ on ice, washed, and incubated with PE-conjugated goat-anti-rat IgG (1:200, Santa Cruz) for 30 min on ice. After subsequent washes, cells were fixed and permeabilized using the Cytofix/Cytoperm 
Kit (BD Biosciences) according to the manufacturer's protocol and incubated with anti-Zap70 rabbit mAb (99F2; Cell Signalling, 1:100). After two washes with FACS buffer, cells were incubated with FITC-conjugated goat-anti-rabbit IgG (Santa Cruz, 1:100). For all other labelling, cells were simultaneously incubated with rat anti-Cd4-1 $(1: 5)$ or rat anti-Cd8 $81(1: 5)$ and a mouse monoclonal antibody against $\operatorname{Igm}^{+} \mathrm{B}$ cells (WCI12, 1:100; [41], neutrophilic granulocytes (TCLBE8, 1:50, [42], monocytes/ macrophages (WCL15, 1:50; [43] or putative mucosal T cells (WCL38, 1:50; [26] as described above. After washes, cells were incubated with PE-conjugated goat-antimouse IgG (1:200; Santa Cruz) and FITC-conjugated goat-anti-rat IgG (1:200; Santa Cruz) in FACS buffer. Cells were analyzed on a FACS CantoA (BD Biosciences) and data were analyzed using FlowJo V10 (BD Biosciences).

\section{Cell sorting of CD4-1+, Cd8a+ or WCL38+ populations}

Total leukocytes were isolated from the intestine and fluorescently labelled as mentioned above, using rat anti-Cd4-1a (1:5), rat anti-Cd8 $\alpha 1$ (1:5) or anti-putative mucosal T cells (WCL38, mouse IgM monoclonal, 1:50) as primary antibodies and PE-conjugated goat-anti-rat IgG or PE-conjugated goat-anti-mouse (for WCL38, 1:200, Santa Cruz) as secondary antibodies. Single positive cells for each population were sorted from the lymphoid gate using an Influx Cell Sorter (BD Biosciences). Only sorted samples with $>98 \%$ purity were pelleted and immediately lysed in 300 $\mu \mathrm{L}$ RLT lysis buffer (Qiagen). The lysate was snap-frozen on dry ice and stored at $-80^{\circ} \mathrm{C}$ until subsequent RNA isolation.

\section{Illumina sequencing and data analysis}

$250 \mathrm{ng}$ of total RNA obtained from sorted cells $\left(n=3\right.$ for $\mathrm{Cd} 4-1^{+}, \mathrm{Cd} 8 \alpha^{+}, \mathrm{WCL} 38^{+}$ and $n=2$ for WCL38 $^{-}$) were used for Illumina sequencing. After confirming correct RNA size, quality and quantity by an Agilent Bioanalyzer, RNA libraries were made using the TruSeq Stranded total RNA library prep kit (Illumina Inc.) following the manufacturer's guidelines. Paired-end libraries were sequenced with a read length of 1 x 100 nucleotides using an Illumina HiSeq 2500 and Illumina software (HCS) for basecalling. TopHat version 2.0.5 [44] was used to align the obtained reads to the reference genome (Bioproject PRJNA; [10] and secondary alignments were filtered out using SAMtools version 0.1.18 [45]. Read counts were obtained from the alignment for each predicted gene using HTSeq-count version 0.5.3.p9 [46] using the intersection-strict settings in order to exclude reads that do not align with annotated 
exons. The quality of the data was assessed using the statistical package $\mathrm{R}$ (The $\mathrm{R}$ Foundation), Raw RNA-seq counts were normalized to correct for the sequencing depth and transcripts length by dividing the count number by the sequencing depth (the total number of obtained reads) or by empirical estimates of the sequencing depth (available in the edgeR package, v.3.12.0, [47].

To investigate differentially expressed genes between the different sorted cell populations, raw RNA-seq counts were analysed using the DESeq2 package (v1.6.3) from Bioconductor (v3.3) [48] in R statistical software (3.1.2) (Team, 2015). DESeq2 analysis was performed using a paired design, allowing the cell populations to be compared between individuals. The resulting $\mathrm{p}$-values were adjusted using Benjamini $\&$ Hochberg corrections for controlling the false discovery rate, as recommended [48]. Results were considered statistically significant when $\operatorname{padj}<0.05$. Subsequently, manual datamining was performed to analyse the differentially expressed genes.

In order to investigate the expression of different $\mathrm{T}$ cell receptors, corresponding cypCar genes were searched through manual BLAST using the following sequences: genes + accession (Table 2). Using the cypcar coding the corresponding RPKM values were recovered from the dataset and investigated.

Table 2. Genes used for the manual BLAST search of tcr genes of common carp in transcriptome datasets

\begin{tabular}{|c|c|c|}
\hline Gene & Accession Nr. & Cypcar \\
\hline tcr_alphal & AB120613.1 & cypCar_00002413 \\
\hline tcr_alpha2b & AB120620.1 & cypCar_00002413 \\
\hline tcr_alpha2a & AB120621.1 & cypCar_00002413 \\
\hline tcrb_partial & AB430330.2 & cypCar_00042411 \\
\hline tcrb_partial & & cypCar_00042526 \\
\hline tcrg_full & DQ367842.1 & cypCar_00042658 \\
\hline tcrg_full & & cypCar_00001016 \\
\hline tcrd_partial & AB541473.2 & cypCar_00002412 \\
\hline tcrd_partial & & cypCar_00005250 \\
\hline
\end{tabular}




\section{Results}

\section{European common carp has two Cd4-1 isoforms}

Using a combined approach of homology cloning and in silico data from available European common carp genome and transcriptomes [33], we were able to retrieve the full-length mRNA sequences of two isoforms of $c d 4-1$ which were designated as $c d 4-1 a$ and $c d 4-1 b$. Cd4- $1 a$ was designated as such based on its highest similarity to a previously reported $c d 4-1$ sequence from Japanese common carp (Fig. 2A). The obtained cDNA sequences of 1440 and $1416 \mathrm{bp}$ translated into proteins of 478 and 471 amino acids (aa) for Cd4-1a and Cd4-1b, respectively (Fig. 2A). Based on protein sequence alignment between the European Cd4-1 and other known Cd4-1 sequences, the predicted Japanese Cd4-1 protein sequence appeared to be incomplete, lacking the signal peptide (SP), most likely due to a mutation $(\mathrm{G}>\mathrm{T})$ at the level of the ATG codon (data not shown). Protein structure analysis revealed that both Cd4-1 isoforms have a SP followed by four extracellular immunoglobulin (Ig) domains, a transmembrane (TM) domain containing a transmembrane helix, and a cytoplasmic tail containing the conserved p5 $7^{\text {lck }} \mathrm{CXC}$ motif. Furthermore, four and seven possible $\mathrm{N}$-glycosylation sites were identified for Cd4-1a and Cd41b, respectively, as well as one and two possible O-glycosylation sites (Fig. 2A). Pairwise comparison revealed protein similarity of $73.2 \%$ between the two European common carp sequences (Fig. 2B). As expected, high protein similarity (97.6\%) was found between our Cd4-1a and the previously reported Japanese common carp Cd41a sequence, whereas the latter sequence showed $72.0 \%$ similarity to our Cd4-1b sequence. Common carp Cd4-1a showed a similarity of $68-69 \%$ to both ginbuna crucian carp Cd4-1 isoforms and the Cd4-1b only showed a slightly higher similarity to ginbuna crucian carp Cd4-1a (75.8\%) than to $\mathrm{Cd} 4-2 \mathrm{~b}$ (76.8\%). Interestingly, both common carp Cd4-1 isoforms show lower similarity to zebrafish Cd4-1 (56.9\% and $61.5 \%$ for). Both carp isoforms were equally different from rainbow trout $\mathrm{Cd} 4-1$ (Oncorhynchus mykiss; 37-38\%). 
A

\begin{tabular}{|c|}
\hline $\begin{array}{l}\text { JaCyca_Cd4-1a } \\
\text { EuCyca_Cd4-1a } \\
\text { EuCyca_Cd4-1b }\end{array}$ \\
\hline $\begin{array}{l}\text { JaCyca_Cd4-1a } \\
\text { EuCyca_Cd4-1a } \\
\text { EuCyca_Cd4-1b }\end{array}$ \\
\hline $\begin{array}{l}\text { JaCyca_Cd4-1a } \\
\text { EuCyca_Cd4-1a } \\
\text { EuCyca_Cd4-1b }\end{array}$ \\
\hline $\begin{array}{l}\text { JaCyca_Cd4-1a } \\
\text { EuCyca_Cd4-1a } \\
\text { EuCyca_Cd4-1b }\end{array}$ \\
\hline $\begin{array}{l}\text { JaCyca_Cd4-1a } \\
\text { EuCyca_Cd4-1a } \\
\text { EuCyca_Cd4-1b }\end{array}$ \\
\hline $\begin{array}{l}\text { JaCyca_Cd4-1a } \\
\text { EuCyca_Cd4-1a } \\
\text { EuCyca_Cd4-1b }\end{array}$ \\
\hline $\begin{array}{l}\text { JaCyca_Cd4-1a } \\
\text { EuCyca_Cd4-1a } \\
\text { EuCyca_Cd4-1b }\end{array}$ \\
\hline $\begin{array}{l}\text { JaCyca_Cd4-1a } \\
\text { EuCyca_Cd4-1a } \\
\text { EuCyca_Cd4-1b }\end{array}$ \\
\hline $\begin{array}{l}\text { JaCyca_Cd4-1a } \\
\text { EuCyca_Cd4-1a } \\
\text { EuCyca_Cd4-1b }\end{array}$ \\
\hline
\end{tabular}

B

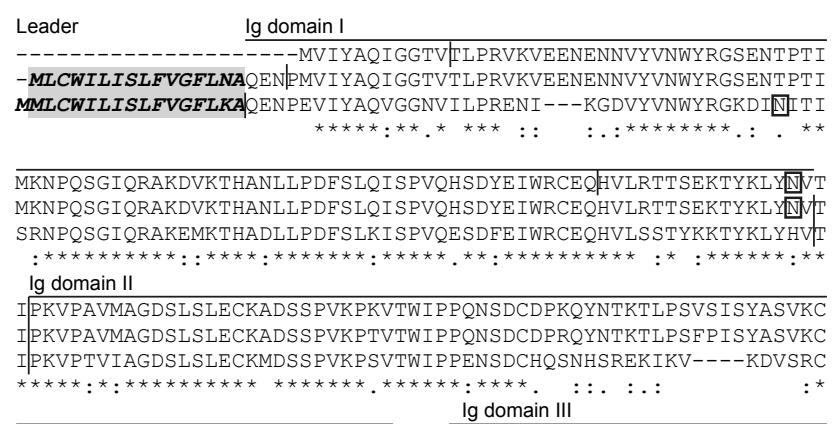

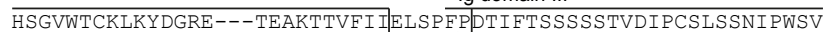
HSGVWTCKLEYDGRE---TEAKTTVFI IELSPE|PDTIFTSSSSSTVDIPCSLSSNIPWSV HSGVWTCMLEYGSKKKYYT』ATTTVSVI|DLSASPDTIYTSS--SKVNIPCSLSSKIPWSI

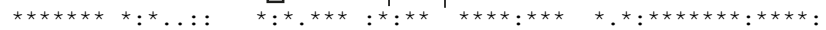

LKESGLRGGNWSFTPLSYP[NSTQSLLELSMDPVVSWS I PQGADNKVKAEKRELKDQDLSI LKEFGLRGGNWSTPLSYPNSTQSLLELSMDPVVSWS I PQGADNKVKAEKRELKDQDLSI

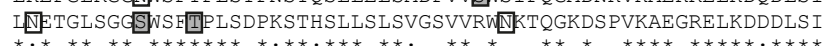
Ig domain IV

RNLPVSENVRGVYTCDLIFNTKKLSRKVTVEVIKVSSSGGSRVYEGQSVNLTCTLGHQHS RNLPVSENVRGVYTCDLIFNTKKLSRKVTVEVI KISSSGGSRVYDGQSVNLTCTLGHQHS -NLPVSEKIRGVYTCSLTFSTKTLSRKVQVEVINVSSSGGSRVYGGSVILTCSLGHQLT

SDLEVKWSCSSCSFISSLKTPHPSSLS I PEVKLKDSEKLTCELWKNGKKLTSAVFSLRIV SDLEVKWSCSSCSFISSLKPPHPSSLS I PEVKLKDSGKLTCELWKNGKKLTSAVLSLRIV SDLEVKWNTSSCS IFFGLN-LHQSTLS I PEVKVEHSGKLTCELWKKGEKLT SAVLSLKIE

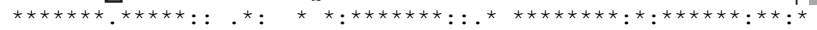
Transmembrane Cytoplasmic

KAPVDIWLCVAISGGVVGFILLLVIVIICIRRHRQMMMYRRRKTKFCCCNNPQONOKGFY KAPVDIWLCVAISGGVVGFILLLVIVI ICIRRHRQMMMYRRRKTKFCCCNNPQQNQKGEY KAPVDIWLCVAISSGVVVFILLVVVTI ICIRRHROMMMYRRRKIKYCCCKNPQONOKGFY

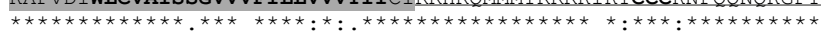

$\frac{K T}{\star \star}$

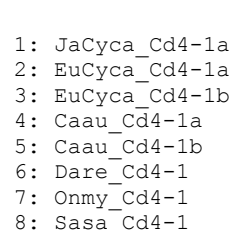

\begin{tabular}{rrrrrrrr}
1 & \multicolumn{1}{c}{2} & \multicolumn{1}{c}{3} & 4 & \multicolumn{1}{c}{5} & \multicolumn{1}{c}{6} & \multicolumn{1}{c}{${ }^{1}$} \\
100.00 & 97.60 & 72.04 & 67.04 & 67.87 & 56.95 & 37.47 & 38.50 \\
& 100.00 & 73.23 & 68.25 & 68.83 & 56.87 & 36.89 & 37.87 \\
& & 100.00 & 75.81 & 76.84 & 61.44 & 38.18 & 39.39 \\
& & & 100.00 & 95.68 & 55.58 & 37.86 & 37.99 \\
& & & & 100.00 & 56.02 & 38.07 & 37.99 \\
& & & & 100.00 & 36.88 & 37.23 \\
& & & & & 100.00 & 89.57 \\
& & & & & & 1000
\end{tabular}

Fig 2. Protein alignment and similarity matrix of European common carp Cd4-1 isoforms. A) Signal peptides are highlighted in light grey and bolded, the transmembrane (TM) region is highlighted in dark grey with the TM helix in bold. Predicted N-glycosylation sites are shown in boxes with white background, O-glycosylation sites in boxes with grey background. The conserved p57 ck CXC-domain in the cytoplasmic region (underlined) is in bold. B) Protein identity matrix showing amino acid similarities. Accession numbers used are: Japanese common carp (Cyprinus carpio ABD58988.1), Ginbuna crucian carp (Carassius autatus langsdorfii BAF94326.1 (Cd4-1a) and BAF94327.2 (Cd4-1b)), zebrafish (Danio rerio ABU95651.1), and rainbow trout (Oncorhynchus mykiss AAY42068.1). Accession numbers for the European common carp will be submitted upon confirmation of the entire sequence by sequencing. 


\section{European common carp has two Cd4-2 isoforms}

A similar approach, combining homology cloning and in silico sequence analysis from transcriptome data, was used to identify three isoforms of Cd4-2. As described in the material and method section, although BLAST analysis retrieved three $c d 4-2$ transcripts, only two of them could be confirmed to be expressed and are referred to as $c d 4-2 a$ and $c d 42 b$. Full mRNA sequences were retrieved for $c d 4-2 a$ (1277 bp) and $c d 4-2 b$ (1292 bp), which translated in proteins of 417 and 393 aa, respectively (Fig. 3A). Protein structure analysis showed that both isoforms had a signal peptide, followed by three Ig domains, a TM and a cytoplasmic region containing the conserved p57 ${ }^{\text {lck }} \mathrm{CXC}$ motif; three and two N-glycosylation sites were predicted for Cd4-2a and Cd4-2b, respectively, as well as four O-glycosylation sites for both isoforms (Fig. 3A). Pairwise protein sequence comparisons showed that $\mathrm{Cd} 4-2 \mathrm{a}$ and Cd4-2b share $68.9 \%$ similarity with each other, 55-57\% with zebrafish Cd4-2, 32$33 \%$ with rainbow trout Cd4-2 and 35-37\% with catfish (Fig. 3B).

\section{Transcriptional analysis of $c d 4$ and $c d 8$ isoforms}

After identification of the $c d 4$ genes and isoforms in common carp, we set out to characterize their relative expression in immune organs and in sorted immune cells. First, using available transcriptome datasets of various organ of common carp we characterized the expression profile of the $c d 4$ genes and isoforms (Fig. 4). The expression of European and Japanese common carp $c d 8$ genes was previously reported using Real Time-quantitative PCR analysis and Reverse Transcriptase PCR, respectively [2,34]. As expected, for all $c d 4$ genes and isoforms the highest counts per million reads were observed in thymus (Fig 4A). Besides thymus, high expression of $c d 4$ genes was observed in gills, head kidney, skin, spleen, intestine and fins. Recently it was reported that antibodies against ginbuna crucian carp Cd4-1 and Cd8 $\alpha 1$ [29,31] were cross-reactive to T cells of other cyprinid species, including Japanese common carp [32]. Given the presence of multiple isoforms for each of these proteins and the slight differences often observed between the European and Japanese sequences, we tested the suitability of the antibodies to detect T cells also in European common carp. To this end, we used the anti-Cd4-1 and anti-Cd8 $\alpha-1$ antibodies on carp thymocytes in combination with a cross-reactive antibody against the pan NK/T cell marker Zap70 [38]. In thymus, besides observing Zap70-/Cd4-1+ and $\mathrm{Zap} 70^{-} / \mathrm{Cd} 8 \alpha 1^{+}$populations, owing to the different developmental stages present 
A

Eucyca Cd4-2a

EuCyca_Cd4-2b

EuCyca Cd4-2a

EuCyca_Cd4-2b

EuCyca Cd4-2a

Eucyca-Cd4-2b

EuCyca Cd4-2a

EuCyca_Cd4-2b

EuCyca_Cd4-2a

EuCyca_Cd4-2b

EuCyca_Cd4-2a

Eucyca_Cd4-2b

EuCyca_Cd4-2a

EuCyca_Cd4-2b

EuCyca Cd4-2a

EuCyca_Cd4-2b

Leader $\frac{\lg \text { domain I }}{\text { MAICKILFFL-LALCVWCGKCEVFYRVGDEVSMKCGVDSNSNIDWKFNGESIFSITGKS }}$
MATCKILFFLFLALYISCGKCDVFYKRVGDEVSMNCGAPSNSDTEWKFDNVLIFNVKGKT

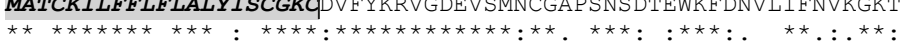

GTRRKASSHIAEKASTPGDILKVPRLETRDSGNYFCKQSGK--HHTVRVVSAFV $\longdiv { \text { KPGPVL } }$ GAKLKGPSHIVLKASTNGENLKVSRLETRDSGNYICSYSGSVKQHNIYVVSVHAKPGPVL

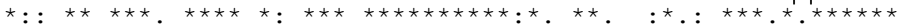
Ig domain II

LQSSNAELHCDITGDPNTEVOWORPPNGEEYKEKKOVIHLKSVTSEEAGOWTCLVEKKLK VQSSDAELHCDITGNSNTQVQWLRPPNGQKHNEKSQVIKLKSVTSKDAGQWTCQVKDALT

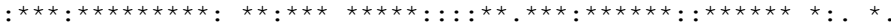
Ig domain III

LIVTLTVV-GLQ TTAVNASKGDDTELPCSLPQSVSQRVVGGRWKADHLPKVSFANLTNTA LSVTLTVVADLQTTAVNVSEGDDTKLPCSLPQSVSQRVVGGKWKADHLSDVSF ITLKNTE

$\overline{\text { GEGLHWHGNDLSKYNFTTGQLSTNFDVTLKKVQSSDDGTFVCTVEFDGGVSLSVETTLRV }}$ NKGLHWNGKDLSKVNFTTEQLSTKFDVTLKNVQHRDAGKFVCTVEFEGGASRSVEMTLTV

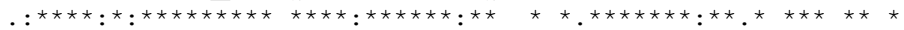
VDKPSGVKGSNNRKGKPPAVMESLTKEVYGLKLWIWIAVGASSVALIVLIVVIGVV $Q R R N$ FGKNSGGQGFNKGKGKTPS I KE I LTKNVYGIELWVWIAVGASSVVLIGLI IVTVLVRQRN

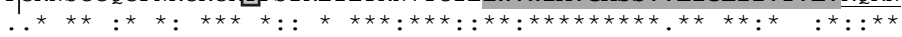
Cytoplasmic

KRMKKRVRTLRSMRQPLTDKDYCQCDRAEKEVEFGEQARPLPVPRQHRNPRTRTAGPNHT KRMKERVRKLRSMRQPLTAKDYCRCKRMNLRMK

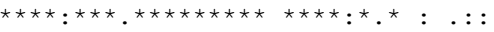

$\underline{\mathrm{N}}$

$-$

B

\begin{tabular}{|c|c|c|c|c|c|c|c|}
\hline & 1 & 2 & 3 & 4 & 5 & 6 & 7 \\
\hline 1: EuCyca Cd4-2a & 100.00 & 69.15 & 75.97 & 78.05 & 55.30 & 46.44 & 35.28 \\
\hline 2: EuCyca_Cd4-2b & & 100.00 & 65.89 & 65.95 & 56.88 & 48.29 & 36.81 \\
\hline 3: Caau C $\bar{d} 4-2.1$ & & & 100.00 & 90.05 & 55.64 & 49.60 & 35.62 \\
\hline 4: Caau_Cd4-2.1 & & & & 100.00 & 55.53 & 47.57 & 35.88 \\
\hline 5: Dare_Cd4-2.1 & & & & & 100.00 & 71.64 & 32.49 \\
\hline 6: Dare_Cd4-2.2 & & & & & & 100.00 & 24.68 \\
\hline Icpu_Cd4-rel2 & & & & & & & 100.00 \\
\hline
\end{tabular}

Fig. 3. Protein alignment and similarity matrix of European common carp Cd4-2 isoforms. A) Signal peptides are highlighted in light grey and are in bold, the transmembrane (TM) region is highlighted in dark grey with the TM helix in bold. Predicted N-glycosylation sites are shown in a box with white background, O-glycosylation sites in a box with grey background. The conserved p57 lck $\mathrm{CXC}$-domain in the cytoplasmic region (underlined) is in bold. B) Protein identity matrix showing amino acid similarities. Accession numbers used for the alignment and the identity matrix are ginbuna crucian carp (Carassius auratus langsdorfii BAO71686.1 (Cd4-2.1) and BAO71687.1 (Cd4-2.2) zebrafish (Danrio rerio Cd4-2.1 CCL97787.2 and Cd42.2 CCL97786.2), rainbow trout (Oncorhynchus mykiss AAY42069.1) and catfish (Ictalurus punctatus ABD93355.1). Accession numbers for the European common carp will be submitted upon confirmation of the entire sequence by sequencing.

in the thymus, we found clear double-positive T cell populations (Fig. 4B).

This suggested that the antibodies against ginbuna $\mathrm{Cd} 4-1$ and $\mathrm{Cd} 8 \alpha 1$ are crossreactive to carp $\mathrm{T}$ cells. Using these antibodies to sort cells from the intestine, we were able to show that $\mathrm{Cd} 4-1^{+}$sorted populations showed a strong enrichment in the expression of all $c d 4$ transcripts (Fig. 4C), strongly suggesting that Cd4-1 and 
Cd4-2 are expressed on the same T cell type. Accordingly, $c d 4$ transcripts were not detected in $\mathrm{Cd} 8 \alpha 1^{+}$sorted fractions and vice versa, confirming the specificity of the antibodies. A low expression of $c d 4-1 a$ and $c d 4-2 a$ could still be detected in the Cd4-1 - fraction; given the high basal expression level of these two genes in the Cd4$1^{+}$population, it is possible that the signal detected in the $\mathrm{Cd} 4-1^{-}$fraction is due to a low number of contaminating cells in the negative fraction. However, it cannot be excluded that, similarly to what reported in rainbow trout [21] a Cd4-2 singlepositive fraction may exist. $\mathrm{Cd} 8 \alpha 1^{+}$sorted leukocytes showed a strong enrichment in the expression of all $c d 8$ transcripts, except $c d 8 \beta 1$. Interestingly, $c d 8 \beta 1$ was equally expressed in the $\mathrm{Cd} 8 \alpha 1^{+}$andCd8 $\alpha 1^{-}$fractions (Fig. 4D), suggesting the presence of a $\mathrm{Cd} 8 \alpha 1^{-} / \mathrm{Cd} 8 \beta^{+}$population. Due to the lack of available antibodies against carp Cd4-2 and Cd8 $\beta 1$ molecules the present of additional $\mathrm{T}$ cell populations could not be further investigated.

Since it was previously reported that Cd4-1 can be expressed on cell types other than $\mathrm{T}$ cells, we preliminary assessed the presence of $c d 4$ and $c d 8$ transcripts in sorted Igm $^{+}$B cells, thrombocytes, neutrophils and 6-days cultured macrophage. Using these samples, we did not detect expression of any of the $c d 4$ or $c d 8 \mathrm{~T}$ cell markers, indicating that in carp, $\mathrm{Cd} 4$ and $\mathrm{Cd} 8$ might not be expressed on B cells, neutrophils, macrophages or thrombocytes.

\section{T cells distribution in European common carp}

Having observed a clear double-labelling of the cross-reactive anti-Cd4-1 and anti-Cd8 81 antibodies with Zap70 in the thymus, as well as a specific expression profile of $c d 4$ and $c d 8$ isoforms in $\mathrm{Cd} 4-1$ and $\mathrm{Cd} 8 \alpha 1$ sorted cell populations (Fig. 4), we set out to further validate the specificity of the antibodies and characterize the distribution of $\mathrm{T}$ cells in European common carp. First, by expressing HA-tagged common carp Cd4-1a and Cd8 $\mathrm{C} 1$ on Vero cells and by performing a double labelling with the anti-Cd4-1 or anti-Cd8 $\alpha 1$ antibodies and an anti-HA antibody, we observed a specific double-labelling (Fig. 5A). Labelling of untransfected cells, as well as a labelling of Cd4-1-expressing cells when using the anti-Cd8 $\alpha 1$ antibody, and vice versa, was not observed (data not shown). Next, using total leukocytes isolated from thymus, blood, head kidney, spleen, intestine and gills we show that both the antiCd4-1 and the anti-Cd8 $\alpha$ antibodies only react to small lymphoid cells that are low in granularity (Fig. 5B). Finally, we used the anti-Cd4-1 or anti-Cd8 $\alpha 1$ antibodies 
again in combination with the anti-Zap70 antibody for flow cytometric analysis of T cells distribution in various immune organs. In PBLs, spleen, intestine and gills we show that all leukocytes stained with either the $\mathrm{Cd} 4-1$ or $\mathrm{Cd} 8 \alpha 1$ antibody were also positive for Zap70, indicating their specificity for (NK)T cells in carp (Fig. 5B-C). In agreement with the findings on $c d 4$ and $c d 8$ genes expression in Fig. 3A, we find the highest percentage of T cells $\left(\mathrm{Cd} 4-1^{+}\right.$and $\left.\mathrm{Cd} 8 \alpha 1^{+}\right)$in thymus, intestine and gills (Fig. 5D). In spleen and head kidney $\mathrm{Cd} 4-1^{+}$cells are slightly more abundant than $\mathrm{Cd} 8 \alpha 1^{+}$cells, but the total number of T cells is generally low. $\mathrm{Cd} 8 \alpha 1^{+}$cells are more abundant than $\mathrm{Cd} 4-1^{+}$cells in intestine and in thymus.

A

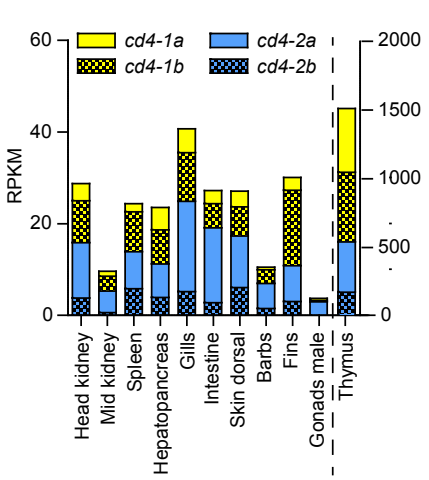

B
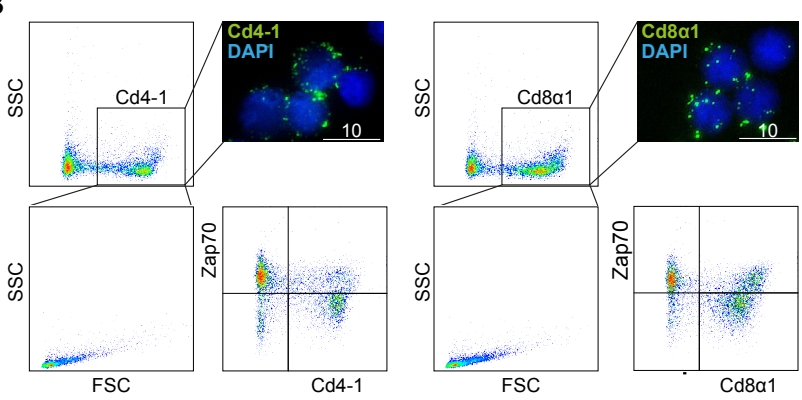

C

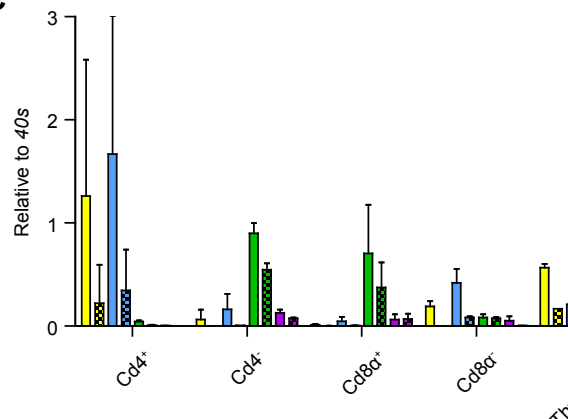

D

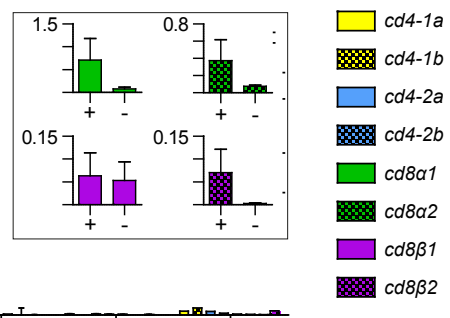

Fig. 4. Expression of $c d 4$ and $c d 8$ genes and isoforms in several organs and sorted cell types. A) Transcriptome data from a European common carp organ library showing the reads per million kilobase (RPKM) of the different $c d 4$ genes and isoforms. B) Flow cytometry labelling of thymus leukocytes with a cross-reactive antibody against Zap70 and Cd4-1 (6D1) or Cd8a1 (2C3). Cells were analysed on a FACS CantoA (BD Biosciences). Fluorescent pictures were made after a cytospin from thymocytes labelled with anti-Cd4-1 or anti-Cd8a1. C) Relative expression of $c d 4$ and $c d 8$ transcripts in sorted Cd4-1+ $(n=3)$ or Cd4-1- $(n=4)$, Cd8a1+ $(n=3)$ or Cd8a1- $(n=3)$ cells, neutrophils $(n=2)$, thrombocytes $(n=1)$, total thymocytes $(n=2)$ or 6-days cultured macrophages $(n=4)$ Data represents mean + standard deviation (SD). D) Relative expression only of the $c d 8$ genes in Cd8a1 and Cd8a1+ fractions showing that $c d 8 \beta 1$ expression is still present in Cd8a $1^{-}$fractions, whereas expression of all other $c d 8$ isoforms is completely depleted. 


\section{Carp Cd4-1 and Cd8a1 are exclusively expressed on T cells}

To investigate whether Cd4-1 or Cd8 $\alpha 1$ antigens would be expressed on leukocytes other than $\mathrm{T}$ cells, we used a panel of existing antibodies against different carp leukocytes, including antibodies against carp thrombocytes (clone WCL6), neutrophilic granulocytes (neutrophils, clone TCLBE8), Igm+ B cells and monocytes/ macrophages (clone WCL15). Using total leukocytes isolated from, blood, head kidney, and spleen we show that both the anti-Cd4-1 and the anti-Cd8 $\alpha 1$ antibodies do not react to carp thrombocytes, neutrophils, $\operatorname{Igm}^{+} \mathrm{B}$ cells or monocytes/macrophages (Fig 6). These data are also in accordance with those obtained upon gene expression analysis of $c d 4$ and $c d 8$ genes in the corresponding sorted populations (Fig. 5E).

Altogether, the data confirms that the antibodies raised against ginbuna crucian carp Cd4-1 and Cd8 $\alpha 1$ cross-react with a population of Zap70 ${ }^{+}$cells and strongly suggesting that Cd4-1 and Cd8 81 in carp are exclusively present on (NK)T cells.

\section{Carp Cd4-1+ and Cd8a1+ cells have distinct transcription profiles}

After confirming the specificity of the antibodies, we next characterized the transcriptomic profile of the $\mathrm{Cd} 4-1^{+}$and $\mathrm{Cd} 8 \alpha 1^{+}$populations in common carp. Through FACS sorting and subsequent RNA Sequencing, we were able show that while both the $\mathrm{Cd} 4-1^{+}$and $\mathrm{Cd} 8 \alpha 1^{+}$populations have high transcript counts for the pan-(NK)T cell markers zap 70 and $l c k, c d 4$ genes are expressed in the $\mathrm{Cd} 4-1^{+}$sorted fraction but not in the $\mathrm{Cd} 8 \alpha 1^{+}$sorted fraction, and vice versa (Fig. 7A). In accordance with the finding in Fig. 4E, both isoforms of $c d 4-2$ were found in the $\mathrm{Cd} 4-1^{+}$sorted fraction, further confirming that in common carp, $c d 4-2$ is expressed on $\mathrm{Cd} 4-1^{+} \mathrm{T}$ cells. As expected, high expression of tcr $\alpha$ and $t c r \beta$ transcript was observed in both sorted fraction, and moderate counts for $t c r \delta$ were also detected. Using the same dataset, we again confirmed that the antibodies do not react with B cells, neutrophils and macrophages, since no significant counts were retrieved for genes related to immunoglobulin (Ig) $\mathrm{m}$ heavy chains of Igm, Igd, Igt1 and Igt2 (igm, igd, igt1 and igt2), myeloperoxidase (mpx) and macrophage expressed gene 1 (mpeg-1) (Fig. 7B). The observation of moderate count numbers for mpeg 1.1 observed especially in the $\mathrm{Cd} 4-1^{+}$sorted fractions needs closer investigation, since we didn't observe expression of $\mathrm{c} d 4$ transcripts in cultured macrophages, nor did we observe a double labelling with our antibody against monocyte/macrophages.

When looking at genes that are differentially expressed between the Cd4- $1^{+}$and 
$\mathrm{Cd} 8 \alpha 1^{+}$populations, several transcripts can be identified and we selected the most differentially expressed genes in each population (normalized counts in positive fractions $n>500$, counts in negative fractions $n<100$; Fig. 7C). Among these, the most expressed genes are the chemokine CCL20-like in Cd4-1 $1^{+}$cells and trypsinlike/mast cell protease 1A-like in $\mathrm{Cd} 8 \alpha 1^{+}$cells. Due to the incomplete annotation of the carp genome many transcripts are referred to as predicted or uncharacterized genes and await further investigation.

A
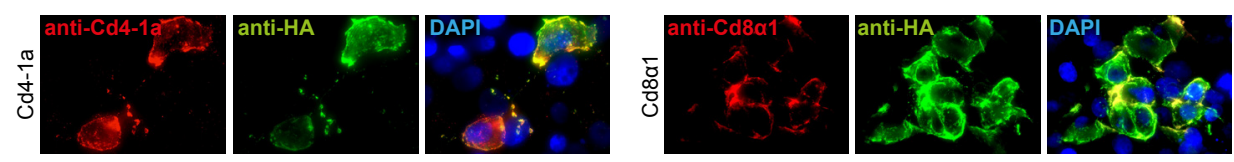

B

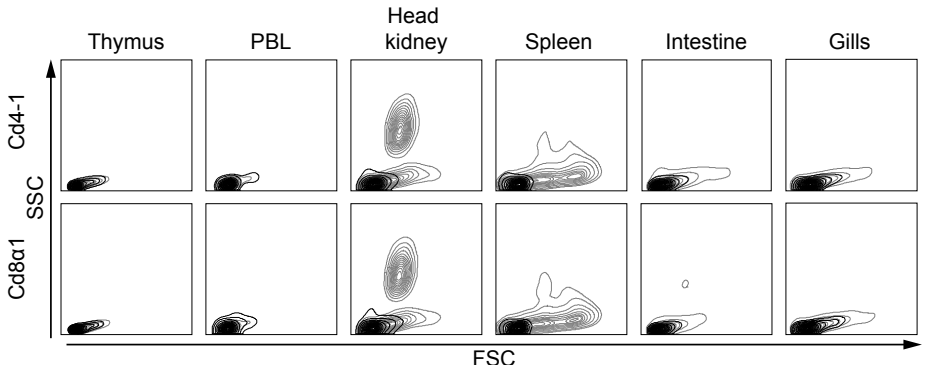

C
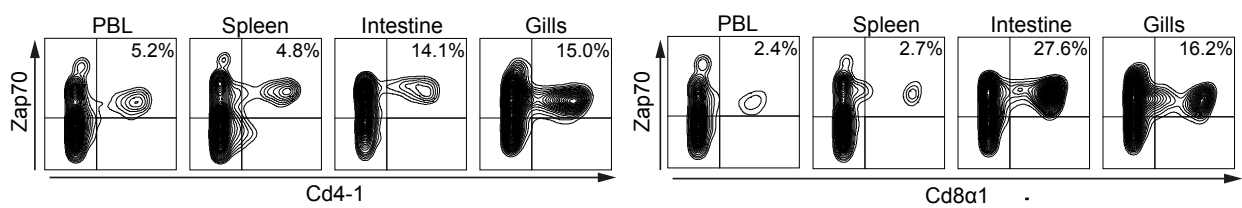

D
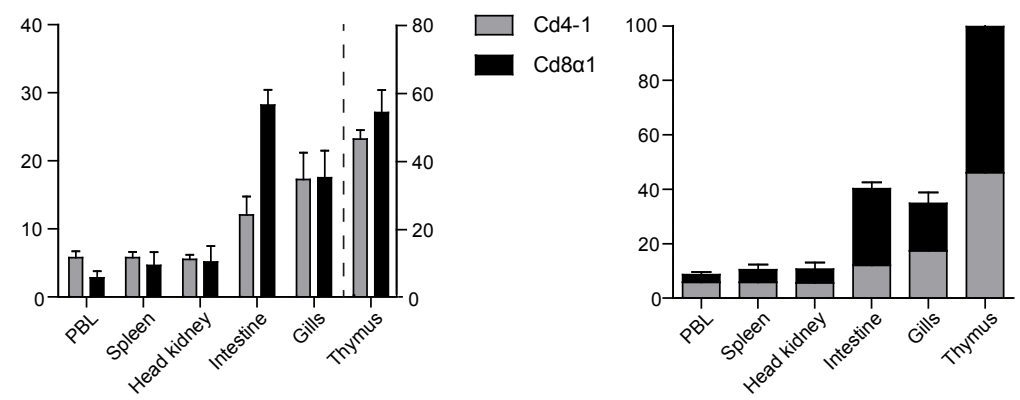

Fig 5. Antibody specificity and Cd4-1+ and Cd8a1 $1^{+}$distribution in common carp. A) Vero cells were transfected with pcDNA3-Cd4-1a or pcDNA3-Cd8a1 and were labelled 48 hours later with anti-Cd4-1 or anti-Cd8a1 in combination with anti-HA-tag. DAPI was included to visualize the nuclei and images were made using an EVOS fl LED fluorescence microscope (Advanced Microscopy Group (AMG)). B) Total leukocytes isolated from thymus, blood, head kidney, spleen, intestine and spleen were labelled with anti-Cd4-1 or Cd8a and analysed based on their forward scatter (FSC) - sideward scatter (SSC) pattern. Black lines indicate the position of the $\mathrm{Cd} 4-1^{+}$or $\mathrm{Cd} 8 \mathrm{a} 1^{+}$cells within the total leukocyte gate; grey lines indicate the remaining leukocytes. C) total leukocytes were isolated from the indicated organs and 
labelled with both anti-Cd4-1 or anti-Cd8a in combination with an anti-Zap70 antibody. Cells were analysed on a FACS CantoA (BD Biosciences) and data were analysed using FlowJo V10 (BD Biosciences. Numbers indicate average percentages of double positive cells as also indicated in D. (D) Percentages of $\mathrm{Cd} 4-1^{+}$or $\mathrm{Cd} 8 \mathrm{a} 1^{+}$cells in the total leukocyte gate of the indicated organs; bars represent mean of $n=4$ measurements + standard deviation (SD).
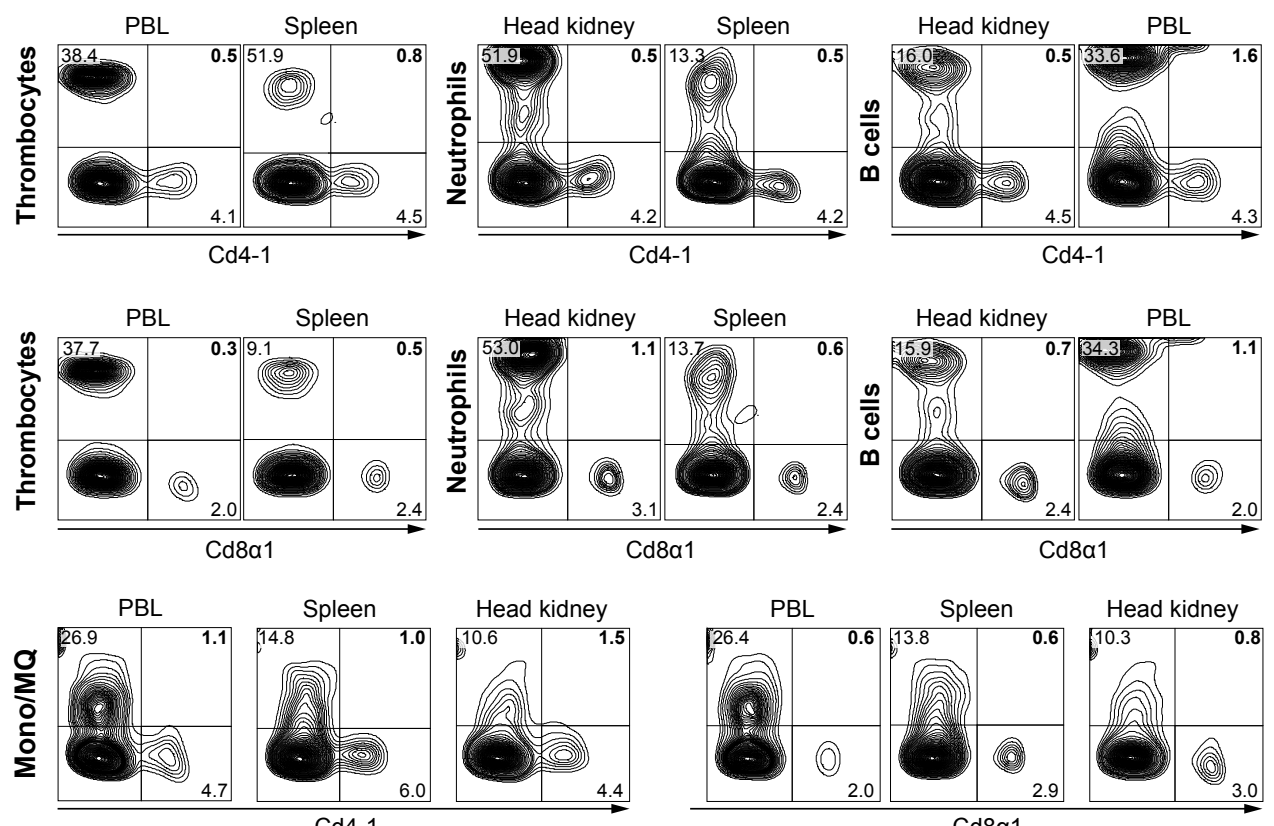

Fig 6. Cd4-1 and Cd8a1 are not expressed on carp thrombocytes, neutrophils, Igm+ B cell or monocyte/macrophages. Total leukocytes isolated from the indicated organs were labelled with anti-Cd4-1 or anti-Cd8a1 in combination with antibodies against carp thrombocytes, neutrophils, Igm ${ }^{+} B$ cells or monocytes/macrophages (Mono/MQ). Cells were analysed on a FACS CantoA (BD Biosciences) and data were analysed using FlowJo V10 (BD Biosciences).

\section{The WCL38 antibody recognizes more cell types than only T cells}

Previous reports from our group described a mouse monoclonal antibody (clone WCL38) recognizing a putative $T$ cells population which was found to be abundant in mucosal organs such as intestine, gills and skin, and in far lower numbers in peripheral organs including thymus, head kidney, spleen and blood [26]. To further characterize the population recognized by the WCL38 antibody, we performed double labelling with WCL38 and anti-Cd4-1 or anti-Cd8 $\alpha 1$ antibodies. Flow cytometric analysis of leukocyte populations isolated from the intestine revealed that while most Cd4- $1^{+}$cells are WCL38, WCL38 and Cd8 $\alpha 1$ single-positive as well as $\mathrm{Cd} 8 \alpha^{+} \mathrm{WCL} 38^{+}$double-positive populations exist, where the $\mathrm{Cd} 8 \alpha 1$ single-positive 
population is the least abundant in this organ (Fig. 8A).

To further characterize the cell populations recognized by the WCL38 antibody, cells were sorted from the intestine and analysed using RNA Sequencing. By comparing the WCL38 ${ }^{+}$and WCL38 sorted fraction we confirmed that although zap70 transcripts are enriched in the WCL $38^{+}$fraction, a high number of zap70 transcripts can still be found in the WCL38- fraction, confirming that only a subset of (NK)T cells is recognized by the WCL38 antibody (Fig. 8B). Interestingly, with the exception of $c d 4-2 a$, none of the other $c d 4-1$ and $c d 4-2$ isoforms was enriched in the WCL $38^{+}$fraction, confirming the flow cytometry data showing that most WCL $38^{+}$cells are Cd4-1- (Fig. 8B), but leaving open the possibility of a separate WCL $38^{+} \mathrm{Cd} 4-2 \mathrm{a}^{+}$positive population. Furthermore, when looking at $c d 8$ transcripts, only $c d 8 \alpha 1$ but none of the $c d 8 \beta$ isoforms was enriched in WCL $38^{+}$sorted cells, suggesting the possibility that the subset of $\mathrm{WCL}_{3} 8^{+}$cells that is positive for $\mathrm{Cd} 8 \alpha 1$, might be negative for $\mathrm{Cd} 8 \beta$. Currently, due to the lack of anti-Cd8 8 antibodies such hypothesis could not be confirmed. Since in the WCL $38^{+}$fractions only low counts for B cell, neutrophil and macrophage markers (ighvm, mpx, mpeg, respectively) could be detected (Fig. 8C), we conclude that WCL38 does not react with any of these cell types.

Based on the flow cytometry data shown in Fig. 8A, we know that approximately $50 \%$ of the total $\mathrm{WCL}_{3} 8^{+}$cells in the intestine is also positive for $\mathrm{Cd} 8 \alpha 1$ and that only a negligible amount is double positive with $\mathrm{Cd} 4-1$. Considering that the percentage of the WCL $38^{+} \mathrm{Cd} 4-1^{+}$and WCL $38^{+} \mathrm{Cd} 8 \alpha 1$ together $(\sim 30 \%)$ is lower than the total percentage of the WCL $38^{+}$population (40-55\%), we hypothesized that an additional population might be recognized by the WCL38 antibody. Although at this point of the study we were not yet able to sort the $\mathrm{WCL} 38^{+} / \mathrm{Cd} 4-1 \% \mathrm{Cd} 8 \alpha 1^{-}$cells, we could take advantage of the RNAseq dataset available for each sorted population in order to investigate genes that are specifically enriched in the WCL $38^{+}$fraction. First, when looking at the top 20 most expressed genes that are enriched in the WCL38 ${ }^{+}$ fraction when compared to the WCL38- fraction, we see a number of T cell markers and related genes including T-cell surface antigen $c d 2$, T-cell surface glycoprotein $c d 5$-like, T-cell differentiation marker $c d 6$-like, as well as the cytotoxicity-related genes granzyme-a and granzyme-b, and perforin-1-like (Fig. 9). When looking at genes that are depleted in the WCL $38^{+}$fraction we see two chains of the H-2 class II histocompatibility antigen and tumor necrosis factor-like. However, care should 
be taken with the interpretation of this dataset since most of the output is based on predicted genes only.

Secondly, we investigated highly expressed genes ( $>1000$ counts) in the WCL38 ${ }^{+}$ fraction that were significantly higher expressed than in the WCL38-, Cd4-1 ${ }^{+}$and $\mathrm{Cd} 8 \alpha 1^{+}$fractions. Since we performed single labelling for FACS sorting we should be aware that sorting for WCL38 will result in the absence of Cd4-1+ cells (Fig. 10A, gate 1) ad the presence of both $\mathrm{WCL}_{3} 8^{+} \mathrm{CD} 8 \alpha 1^{+}$and $\mathrm{WCL} 38^{+} \mathrm{Cd} 8 \alpha 1^{-}$cells (Fig. 10B gate 1). The other way around, the WCL38- fraction will contain all intestinal Cd4-1 $1^{+}$cells (Fig. 10A gate 2) and also WCL38-Cd8 $\alpha 1^{+}$cells (Fig. 10B gate 2). Therefore, if we look at genes that are enriched in the WCL38 $8^{+}$fraction (Fig. 10A,B gate 1) when compared to the WCL38-(Fig. 10A,B gate 2), Cd4-1+ (Fig. 10A gate 3) $\mathrm{Cd} 8 \alpha 1^{+}$(Fig.10B gate 4) fractions, we conclude that the genes we retrieve reflect the WCL $38^{+} \mathrm{Cd} 8 \alpha 1^{-}$population (Fig. 10B, gate 5).

The analysis only retrieved two genes (Fig. 10C, granzyme-b and nk-lysin, top two rows). The expression of these particular genes suggests that WCL38 might recognize a subpopulation of (NK)T cells. Next, we looked at genes that are $>1000$ counts in the WCL38 ${ }^{+}$fraction but $<1000$ counts in both the $\mathrm{Cd} 4-1^{+}$and $\mathrm{Cd} 8 \alpha 1^{+}$fractions. Indeed, although not completely depleted in the WCL38- fraction, the TCR-delta subunit $(t c r \delta)$ is highly expressed in WCL $38^{+}$sorted fraction but not in the $\mathrm{Cd} 4-1^{+}$or $\mathrm{Cd} 8 \alpha 1^{+}$sorted fraction (Fig. 10c lower part).

In conclusion, in this study we describe the presence of two $c d 4$ genes in European common carp, $c d 4-1$ and $c d 4-2$, each present with two isoforms, $c d 4-1 a, c d 4-1 b$ and $c d 4-2 a, c d 4-2 b$ and we show their expression profile using available transcriptome datasets. Using cross-reactive antibodies from ginbuna crucian carp we were able, for the first time in common carp, to characterize the distribution and transcription profile of distinct $\mathrm{Cd} 4-1$ and $\mathrm{Cd} 8 \alpha 1$ populations. When used in combination with antibodies specific for several carp leukocytes, we confirmed that the crossreactive anti-Cd4-1 and anti-Cd8 $\alpha 1$ antibodies specifically target $\mathrm{T}$ cells and are not expressed on Igm+ B cells, thrombocytes, neutrophils or macrophages. Finally, we performed a preliminary characterization of the population targeted by the WCL38 antibody, confirming that at least in the intestine it certainly recognizes a population of $\mathrm{Zap} 70^{+} / \mathrm{Cd} 8 \alpha 1^{+}$cells, most likely $\mathrm{T}$ cells, but also a subpopulation of $\mathrm{WCL}^{+} 8^{+}$ $\mathrm{Cd} 4-1 \% \mathrm{Cd} 8 \alpha 1^{-}$cells, suggesting the presence of an additional population which awaits further characterization. 
A

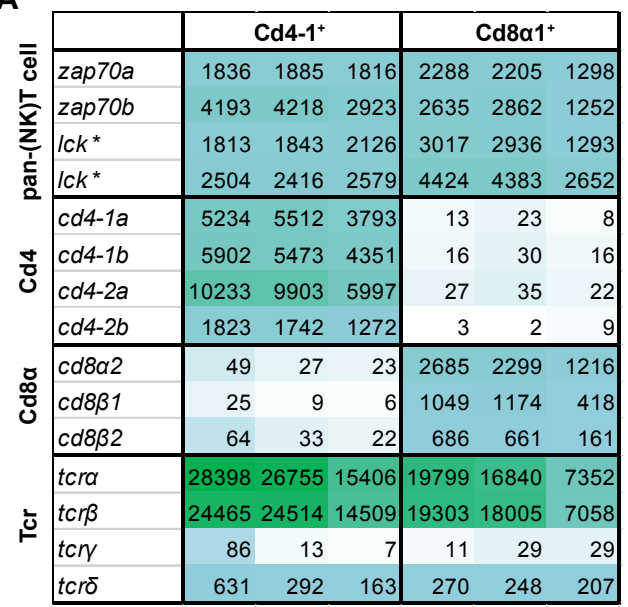

B

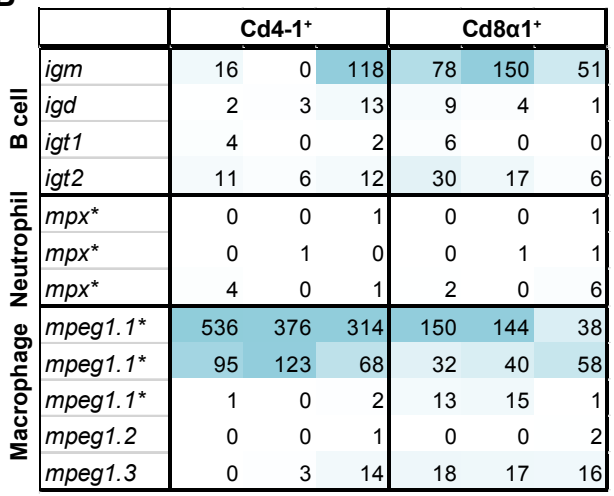

C

\begin{tabular}{|c|c|c|c|c|c|c|}
\hline \multicolumn{3}{|c|}{$\mathrm{Cd} 4-1^{+}$} & \multicolumn{3}{|c|}{$\mathrm{Cd} 8 \alpha 1^{+}$} & \multirow{2}{*}{\begin{tabular}{|l} 
Predicted gene \\
C. carpio C-C motif chemokine 20-like (LOC109112572)
\end{tabular}} \\
\hline 8453 & 5534 & 4792 & 188 & 104 & 137 & \\
\hline 2209 & 2097 & 1309 & 39 & 34 & 29 & S. rhinocerous uncharacterized LOC107719745 (LOC107719745) \\
\hline 2200 & 2067 & 1260 & 51 & 61 & 39 & C. carpio tyrosine-protein kinase-like otk (LOC109048692) \\
\hline 1796 & 2146 & 866 & 201 & 186 & 75 & C. carpio SH2 domain-containing protein 1A-like (LOC109056864) \\
\hline 1694 & 1548 & 1070 & 127 & 111 & 8 & C. carpio uncharacterized LOC109062762 (LOC109062762) \\
\hline 1829 & 1357 & 796 & 38 & 24 & 7 & S. rhinocerous transmembrane and immunoglobulin domain containing 2 (tmigd2) \\
\hline 678 & 451 & 1084 & 57 & 107 & 32 & C. carpio uncharacterized (LOC109103935) \\
\hline 656 & 391 & 815 & 21 & 21 & 18 & S. anshuiensis zinc finger and BTB domain-containing protein 7B-like (LOC107698188) \\
\hline 786 & 456 & 495 & 40 & 96 & 5 & C. carpio uncharacterized (LOC109073911) \\
\hline 158 & 117 & 71 & 1649 & 2187 & 4176 & C. carpio regulator of G-protein signaling 16-like (LOC109061921) \\
\hline 58 & 71 & 82 & 2689 & 2421 & 1473 & C. carpio trypsin-like (LOC109047095)/ mast cell protease 1A-like (LOC109047289) \\
\hline 170 & 111 & 79 & 390 & 595 & 2613 & C. carpio regulator of G-protein signaling 16-like (LOC109060642) \\
\hline 133 & 108 & 137 & 1469 & 1288 & 807 & C. carpio histone deacetylase 4-like (LOC109080789) \\
\hline 51 & 53 & 77 & 624 & 515 & 1319 & C. carpio protein lifeguard 1-like (LOC109063534) \\
\hline 56 & 34 & 88 & 826 & 969 & 658 & S. anshuiensis histone deacetylase 4-like (LOC107673471) \\
\hline 58 & 44 & 56 & 731 & 739 & 519 & C. carpio cytohesin-4-like (LOC109092102) \\
\hline 10 & 7 & 124 & 10 & 17 & 1901 & S. grahami SKI-like proto-oncogene (skil) \\
\hline 113 & 81 & 87 & 617 & 576 & 456 & C. carpio lysosome-associated membrane glycoprotein 3-like (LOC109057357) \\
\hline 31 & 34 & 61 & 203 & 179 & 1006 & C. carpio synaptotagmin-like protein 3 (LOC109087485) \\
\hline 50 & 31 & 67 & 411 & 456 & 317 & C. carpio tyrosine-protein phosphatase non-receptor type 22-like (LOC109053992) \\
\hline
\end{tabular}

Fig. 7. RNA Sequencing gene profiles of $\mathrm{Cd} 4-1^{+}$and $\mathrm{Cd} 8 \mathrm{a} 1^{+} \mathrm{FACS}$ sorted cells. Intestinal leukocytes were stained with the antibodies against Cd4-1 or Cd8 1 and were FACS-sorted. Obtained cells of $n=3$ carp were used for RNA Sequencing (Illumina sequencing, $100 \mathrm{bp}$ single reads). Values indicate reads per million kilobase (RPKM) and were obtained using HTSeq-count version 0.5.3.p9. DESeq2 (v3.3 Bioconductor) analysis was performed using a paired design. The resulting $p$-values were adjusted using Benjamini \& Hochberg corrections and results were considered statistically significant when padj<0.05.Asterisks $\left(^{*}\right)$ indicate the presence of multiple isoforms for the indicated genes, and are under further investigation. 
A

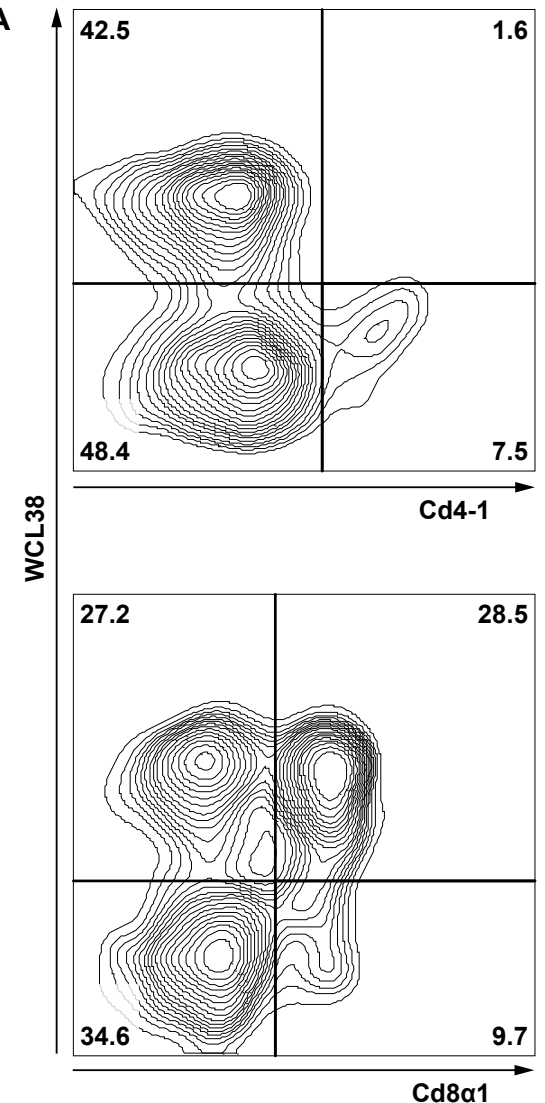

B

\begin{tabular}{|c|c|c|c|c|c|}
\hline & \multicolumn{3}{|c|}{ WCL38 ${ }^{+}$} & \multicolumn{2}{|c|}{ WCL38- } \\
\hline zap70a & 1699 & 1818 & 1722 & 1116 & 827 \\
\hline zap70b & 1995 & 1975 & 1831 & 1189 & 1074 \\
\hline Ick* & 2448 & 2606 & 2547 & 910 & 985 \\
\hline Ick* & 3334 & 3625 & 3745 & 1389 & 1340 \\
\hline$c d 4-1 a$ & 170 & 165 & 276 & 920 & 666 \\
\hline$c d 4-1 b$ & 221 & 168 & 269 & 1133 & 788 \\
\hline$c d 4-2 a$ & 608 & 417 & 665 & 2076 & 1529 \\
\hline$c d 4-2 b$ & 95 & 71 & 92 & 350 & 280 \\
\hline$c d 8 \alpha 2$ & 1003 & 1034 & 1354 & 198 & 211 \\
\hline$c d 8 \beta 1$ & 361 & 317 & 467 & 78 & 96 \\
\hline$c d 8 \beta 2$ & 205 & 144 & 284 & 750 & 622 \\
\hline $\operatorname{tcr} \alpha$ & 11371 & 11150 & 13760 & 8739 & 7554 \\
\hline $\operatorname{tcr} B$ & 13501 & 13799 & 14762 & 8511 & 7426 \\
\hline $\operatorname{tcr} \gamma$ & 349 & 204 & 177 & 77 & 172 \\
\hline $\operatorname{tcr} \delta$ & 3797 & 3176 & 2956 & 2384 & 2061 \\
\hline
\end{tabular}

C

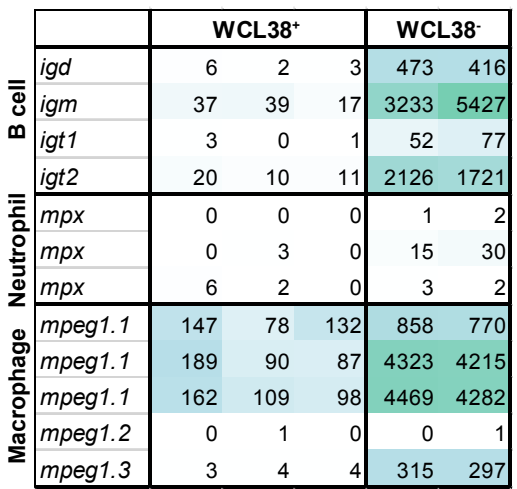

Fig. 8. Characterization of cells recognized by the WCL38 antibody using flow cytometry and RNA Sequencing. A) Total leukocytes from the intestine were labelled with WCL38 and anti-Cd4-1 or anti-Cd8a1 antibodies. Cells were analysed on a FACS CantoA (BD Biosciences) and data were analysed using FlowJo V10 (BD Biosciences. B-C) RNA sequencing (Illumina sequencing, $100 \mathrm{bp}$ single reads) was performed on WCL38 ${ }^{+}$and WCL38FACS sorted populations from the intestine and was used to $(B)$ characterize the transcription level of typical T cell markers and (C) of other cell-specific markers. Values indicate reads per million kilobase (RPKM) and obtained using HTSeq-count version 0.5.3.p9. DESeq2 (v3.3 Bioconductor) analysis was performed using a paired design. The resulting p-values were adjusted using Benjamini \& Hochberg corrections and results were considered statistically significant when padj $<0.05$ 


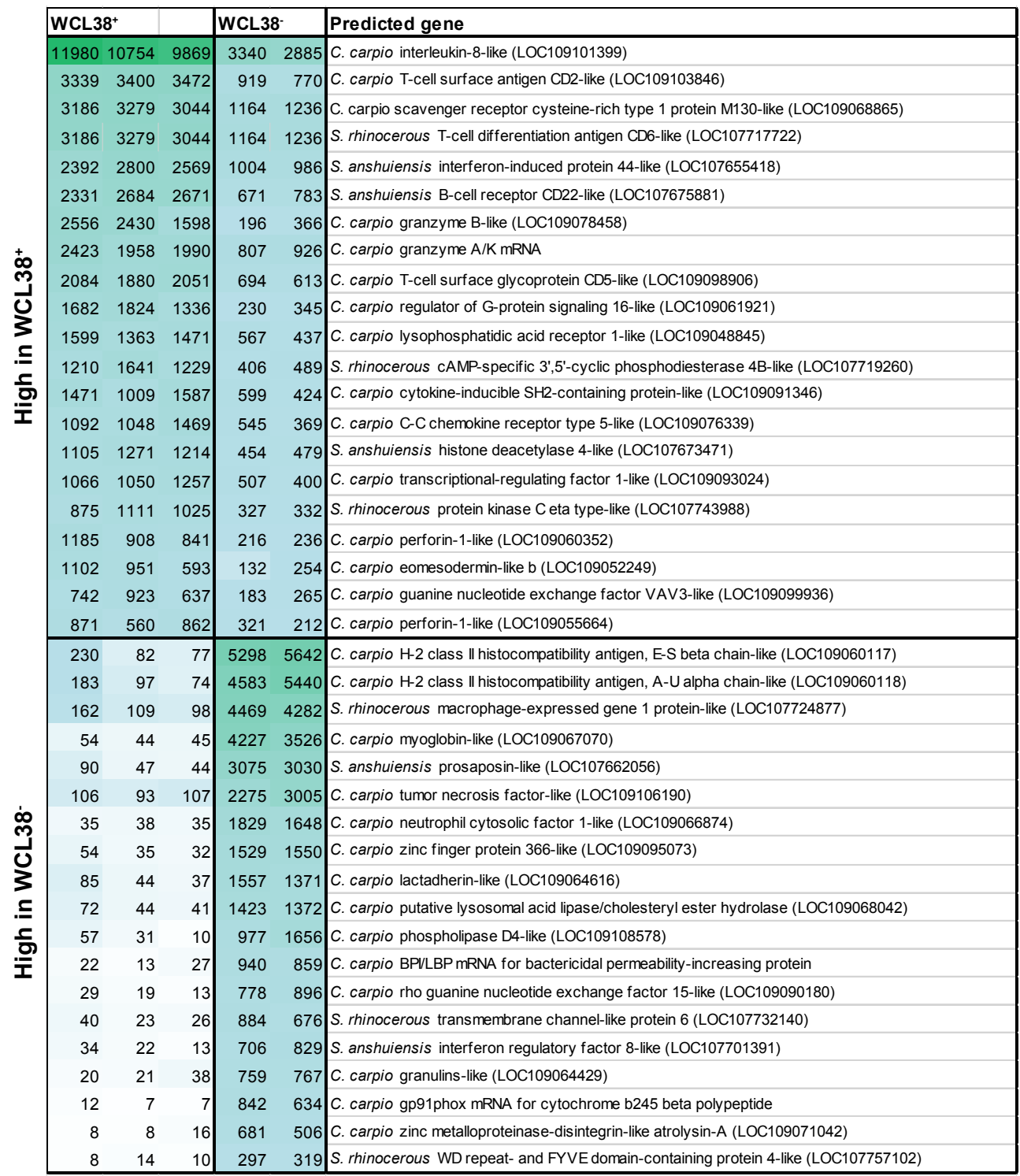

Fig. 9. Top 20 differentially expressed genes in $\mathrm{WCL}^{+} 8^{+}$and WCL38 sorted fractions. RNA sequencing (Illumina sequencing, $100 \mathrm{bp}$ single reads) was performed on WCL38+ and WCL38- FACS sorted populations from the intestine ( $n=3$ per group). Values indicate reads per million kilobase (RPKM) and were obtained using HTSeq-count version 0.5.3.p9. DESeq2 (v3.3 Bioconductor) analysis was performed using a paired design. The resulting p-values were adjusted using Benjamini \& Hochberg corrections and results were considered statistically significant when padj<0.05. The white bar at the fourth row is due the a BLAST search resulting in two predicted genes with similar predictive values. 
A

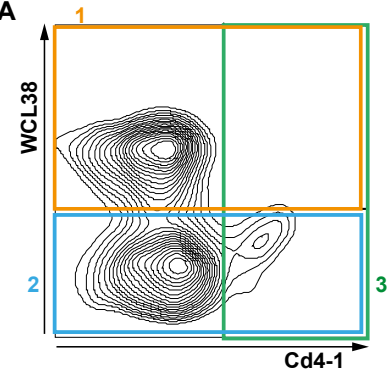

B

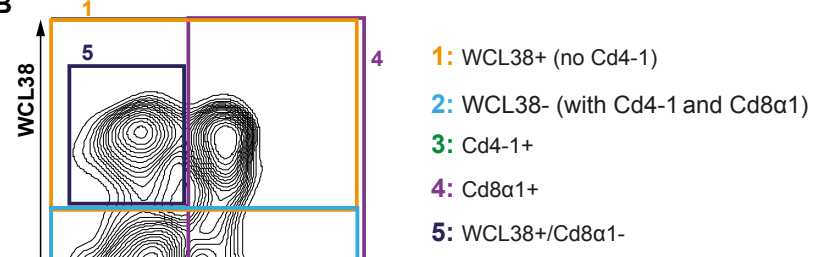

C

\begin{tabular}{|c|c|c|c|c|c|c|c|c|c|c|c|}
\hline \multicolumn{2}{|c|}{ WCL38+ } & \multirow[b]{2}{*}{1598} & \multicolumn{2}{|c|}{ WCL38- } & \multirow{2}{*}{\begin{tabular}{|l|} 
Predicted gene \\
C. carpio granzyme B-like (LOC109078458)
\end{tabular}} & \multicolumn{2}{|l|}{ Cd4-1+ } & \multirow[b]{2}{*}{104} & \multicolumn{2}{|c|}{ Cd8 $81+$} & \multirow[b]{2}{*}{142} \\
\hline 2556 & 2430 & & 196 & 366 & & 86 & 65 & & 865 & 222 & \\
\hline 1067 & 1304 & 680 & 181 & 270 & C. carpio antimicrobial peptide NK-lysin-like (LOC109107757) & 21 & 21 & 22 & 149 & 51 & 64 \\
\hline 2041 & 1712 & 1384 & 4785 & 3995 & S. rhinocerous semaphorin-4A-like (LOC107705066) & 707 & 610 & 882 & 782 & 901 & 434 \\
\hline 1662 & 1610 & 1440 & 650 & 610 & C. carpio tyrosine-protein kinase Lyn-like (LOC109069150) & 27 & 18 & 43 & 793 & 831 & 389 \\
\hline 1658 & 931 & 725 & 3872 & 2702 & S. anshuiensis myocyte-specific enhancer factor 2B-like (LOC107687723) & 31 & 36 & 53 & 55 & 54 & 136 \\
\hline 1089 & 1066 & 954 & 2142 & 1842 & C. carpio alpha-actinin-4-like (LOC109103447) & 294 & 284 & 684 & 375 & 490 & 356 \\
\hline 1102 & 951 & 593 & 132 & 254 & C. carpio eomesodermin-like b (LOC109052249) & 94 & 134 & 165 & 156 & 93 & 148 \\
\hline
\end{tabular}

Fig. 10. Comparison of RNA Sequencing profiles of WCL38+, Cd4-1+ and Cd8a1+ sorted fractions. Flow cytometry plots showing double labelling with the WCL38 antibody and ratanti-Cd4-1 (clone 6D1) (A) or rat-anti-Cd8a1 (clone 2C3) (B). Legend shows the number of the gate and the presence or absence of $\mathrm{Cd} 4-1^{+}$or $\mathrm{Cd} 8 \mathrm{a}^{+}{ }^{+}$cells within the indicated gate. C) RNA sequencing (Illumina sequencing, 100bp single reads) was performed on Cd4-1+, Cd8a1+, WCL38+ and WCL38- FACS sorted populations from the intestine and was used to investigate genes that were highly expressed (>1000 counts) in the WCL38+ dataset but not in the WCL38, Cd4-1+ or Cd8a1+ dataset. Values indicate reads per million kilobase (RPKM) for each predicted gene. DESeq2 (v3.3 Bioconductor) analysis was performed using a paired design. The resulting P-values were adjusted using Benjamini \& Hochberg corrections and results were considered significant when $P$ adj $<0.05$.

\section{Discussion}

Using cross-reactive antibodies to carp Cd4-1 and $\mathrm{Cd} 8 \alpha 1$ in combination with a panel of cell type-specific antibodies available in our lab, in this study we characterized $\mathrm{T}$ cell subsets in European common carp. We first show that European common carp has two $c d 4$ genes each present in two different isoforms. While for both Cd4-1 isoforms four Ig domains were predicted, three Ig domains were predicted for Cd4-2. This is similar to what was reported for catfish (Ictalurus punctatus) Cd4-2 [5] but not for all other species where $c d 4-2$ genes have been identified so far $[3,4,6,8,9]$, for which two Ig domains where identified. Since multiple prediction programs all show the presence of three Ig domains for both Cd4-2 proteins, their presence need to be validated in another way. 
Using RNA sequencing data from an existing organ library dataset, we confirmed that all $c d 4$ genes and isoforms are the highest expressed in thymus followed by gills, head kidney, intestine and skin and fins. Using the cross-reactive antibodies against Cd4-1 and Cd8 1 1 we confirmed that the labelled population strongly overlapped with Zap $70^{+}$cells in thymus and in peripheral organs, and that all $c d 4$ and $c d 8$ transcripts were highly enriched in the respective $\mathrm{Cd} 4-1$ and $\mathrm{Cd} 8 \alpha 1$ sorted fractions. Furthermore, $c d 4$ and $c d 8$ transcripts were absent in cells sorted with an antibody against B cells [41], neutrophilic granulocytes [42], monocytes/macrophages [43] or thrombocytes [49]. In agreement to this, no double labelling with any of these antibodies was observed in flow cytometry.

In contrast to these observations, $\mathrm{Cd} 4-1^{+} \mathrm{Cd} 4-2^{-}$macrophages were identified in rainbow trout [21], and large myeloid-like $\mathrm{Cd} 4-1^{+} \mathrm{Cd} 4-2-$ cells were identified in flounder by in situ hybridization [4]. In zebrafish, a transgenic line expressing a reported protein under the control of the zebrafish $c d 4$ promotor, marked not only a $\mathrm{Lck}^{+} / \mathrm{Cd} 4^{+}$lymphoid population, but also an $\mathrm{Lck}^{-} / \mathrm{Cd}^{+}$population with myeloid morphology [22]. Although flow cytometric analysis did not reveal the presence of large $\mathrm{Cd} 4^{+}$cells, nor of a double positive population when using our monocyte/ macrophage-specific monoclonal antibody, our RNA Sequencing datasets of Cd4$1^{+}$cells, revealed the presence of moderate counts of a predicted isoform of the macrophage-expressed gene-1 (mpeg1.1) which requires further investigation.

While gene expression analysis showed that all $c d 4$ genes were highly enriched in Cd4 $-1^{+}$sorted cells, indicating that also in common carp Cd4- $1^{+} \mathrm{Cd} 4-2^{+} \mathrm{T}$ cells exist, a moderate expression of $c d 4-1 a$ and $c d 4-2 a$ was detected in the Cd4-1- fraction. Whether this is caused by a contamination or by the presence of an additional population needs further investigation. Besides this residual expression of $c d 4$ genes in the Cd4-1' fraction, we observed the presence of $\mathrm{c} d 8 \beta 1$ transcription in the $\mathrm{Cd} 8 \alpha 1^{-}$ fraction. Although the levels were low, we clearly show that while $c d 8 \alpha 1, c d 8 \alpha 2$ and $c d 8 \beta 2$ are almost completely depleted in the $\mathrm{Cd} 8 \alpha^{-}$fraction, $c d 8 \beta 1$ is almost equally expressed in the $\mathrm{Cd} 8 \alpha 1^{+}$and $\mathrm{Cd} 8 \alpha 1^{-}$fraction. While in mice only $\mathrm{CD} 8 \alpha \beta$ heterodimers and CD8 $\alpha \alpha$ homodimers occur, in humans $\operatorname{CD} \beta$ can be expressed on the surface without the need for $\mathrm{CD} 8 \alpha$ co-expression [50] but the $\mathrm{CD} 8 \beta \beta$ is unable to bind MHC-I molecules. Based on the differential expression of $c d 8 \alpha$ and $c d 8 \beta$ genes in carp, together with the presence of a potential signalling motif in the cytoplasmic domain of $\mathrm{Cd} 8 \beta 1$ and $\mathrm{Cd} 8 \beta 2$ [1], $\mathrm{Cd} 8 \beta \beta$ homodimers might occur. The presence of 
different dimerization forms of $\mathrm{Cd} 8$ has not been characterized, hampered by the lack of specific antibodies against any teleostCd $8 \beta$.

The RNA sequencing datasets revealed that $\mathrm{Cd} 4-1^{+}$cells from the intestine express high levels of the chemokine $c c l-20$, which in mammals is known as a prototypical Th17 chemokine and was found to be involved by the formation and functioning of mucosal immune structures by its chemoattractant activities [51,52]. Although the function of $\mathrm{Ccl} 20$ has not yet been fully studied in fish, our data suggests that it is produced by $\mathrm{Cd} 4-1^{+}$but not by $\mathrm{Cd} 8 \alpha 1^{+} \mathrm{T}$ cells. The most expressed gene in the $\mathrm{Cd} 8 \mathrm{al}^{+}$sorted fraction was predicted to be the regulator of G-protein signalling 16-like, indicating that this type of signalling molecule might be specific for $\mathrm{Cd} 8 \alpha 1$ cells, since many different regulators of $\mathrm{G}$ protein signalling have been identified [53]. While this dataset shows that we have many differentially expressed genes between $\mathrm{Cd} 4-1^{+}$and $\mathrm{Cd} 8 \alpha 1^{+}$cells, the outcomes are often based on predictions only, caused by the incompletes of the annotation of the common carp genome [10]. Therefore, care should be taken when interpreting the data.

While only few $\mathrm{T}$ cell subset-specific antibodies have been successfully produced for teleost species so far, two $\mathrm{T}$ cell antibodies were developed in our lab; one against early thymocytes (WCL9; [27]) and one against putative mucosal T cells (WCL38; [26]). It was suggested that WCL38 does not react with NK cells since no reactivity was observed against non-specific cytotoxic (NCC) cells when using a cross-reactive antibody (5C6; [54]) raised against channel catfish NK cells [26,54]. Since the WCL38 and 5C6 antibodies were raised by immunizing mice with whole-cell extract, their exact epitopes are yet undiscovered. Interestingly, double labelling with WCL38 and the anti-Cd4-1 or anti-Cd8 $\alpha 1$ antibodies revealed that while almost all $\mathrm{Cd} 4^{+}$cells in the intestine were WCL38-, WCL38 ${ }^{+} \mathrm{Cd} 8 \alpha 1^{-}$, WCL38$\mathrm{Cd} 8 \alpha 1^{+}$and $\mathrm{WCL} 38^{+} \mathrm{CD} 8 \alpha 1^{+}$populations exist and in total, the $\mathrm{WCL} 38^{+}$fraction accounts for almost $60 \%$ of the total intestinal leukocytes. In order to gain insights into the population recognized by the WCL38 antibody, we sorted cells from the intestine and performed RNA sequencing on both the WCL38 ${ }^{+}$and WCL38- sorted populations. In agreement to the observation that WCL38 does not recognize all $\mathrm{T}$ cells in the intestine, $c d 8 \alpha, c d 8 \beta 1$ and $c d 4-2 a$, but not $c d 8 \beta 2$ and the other $c d 4$ isoforms, are enriched in the WCL $38^{+}$sorted fraction. Interestingly, while the Cd4-1 ${ }^{+}$ and $\mathrm{Cd} 8 \alpha 1^{+}$sorted cells showed high expression in tcr $\alpha$ and $\operatorname{tcr} \beta$ only, the WCL38 ${ }^{+}$ dataset shows high expression of $t c r \delta$ and moderate counts for $t c r \gamma$ as well. $t c r \delta$ 
transcripts are not fully depleted in the WCL38- fraction indicating that not all Tcr $\gamma^{+}$ cells are recognized by the antibody. This, together with absence of transcripts for B cell- neutrophil- and monocyte/macrophage-related genes, indicates that the WCL38 antibody might potentially recognize a subset of $(\mathrm{NK}) \mathrm{T}$ cells, possibly including ${ }_{\gamma \delta}^{\mathrm{T}}$ cells. It is proposed that ${ }_{\gamma \delta}^{\mathrm{T}}$ cells can also develop in the absence of a functional thymus [55] and indeed, also in carp, WCL38 ${ }^{+}$cells were detected earlier in intestine and gills than in thymus [56]. These cells were detected together with the expression of recombination activating gene 1 ( $r a g l)$, strengthening the hypothesis that WCL38 might recognize (a subset of) ${ }_{\gamma \delta} \mathrm{T}$ cells [56].

However, when looking at the relative abundance of $\operatorname{tcr} \alpha$ and $\operatorname{tcr} \beta$ transcrips, it is possible that the majority of cells recognized by WCL38 are ${ }_{\alpha \beta} \mathrm{T}$ cells. Conversely, only low counts of tcr $\gamma$ and $t c r \delta$ were detected in the $\mathrm{Cd} 8 \alpha^{+}$fraction, indicating that the ${ }_{\gamma \delta}^{\mathrm{T}}$ cells detected in the WCL38 $38^{+}$fraction might be $\mathrm{Cd} 8 \alpha$. While this phenotype has not been described in teleost fish, it is the major ${ }_{\gamma \delta} \mathrm{T}$ cell subset in mice $[55,57,58]$. Since mammalian $\gamma \delta-T$ cells have cytolytic activities that function via multiple routes including granzyme, perforin and TRAIL [59], the findings on the granzyme and $n k$-lysin genes exclusively found in the WCL38 $8^{+}$fraction would agree with the hypothesis that WCL38 recognizes a subset of $\mathrm{NK}(\mathrm{T})$ and ${ }_{\gamma \delta} \mathrm{T}$ cells. While in zebrafish it was recently shown that ${ }_{\gamma \delta} \mathrm{T}$ cells express $m h c I I$ [16], our data shows that $m h c I I$ is almost complete absent in the WCL38 $8^{+}$fraction. Since $m h c I I$ is preferentially expressed on professional antigen presenting cells and our data is still largely based on gene predictions, more studies are needed to verify whether $m h c I I$ is expressed on common carp ${ }_{\gamma \delta} \mathrm{T}$ cells or whether $m h c I I$ is differentially expressed between common carp and zebrafish ${ }_{\gamma \delta} \mathrm{T}$ cells.

The only two genes that were found exclusively expressed in the WCL $38^{+}$fraction and not in the WCL38- $\mathrm{Cd} 4-1^{+}$and $\mathrm{Cd} 8 \alpha 1^{+}$fractions, were granzyme- $b$ and $n k$-lysin, two typical markers for cytotoxic cells. The observation that these genes are not expressed in the $\mathrm{Cd} 8 \alpha^{+}$sorted fraction suggests that this expression comes from the $\mathrm{Cd} 8 \alpha{ }^{-W C L} 38^{+}$population. While this argues for NK(T)/ T-cells, it is striking that the WCL38 antibody recognizes only few cells in organs other than intestine and gills [26]. In fact, NK(T) cells are expected also in other organs as shown by the high basal levels of NK-enhancing factor $n k e f a$ and $n k e f b$ in PBLs, gills and kidney in common carp [60]. Still, the recognition of NK cells-only is less likely since NK cells do not bear tcr (Reviewed in [61]) and previously WCL38 was found not to 
react to NCC cells [26]. While NKT cells in teleost species have not yet been fully characterized, mammalian NKT cells express tcr $\alpha \beta$ [62], which is strongly enriched in our WCL38 $8^{+}$sorted fraction. Conversely, while we see only low counts of $c d 4$ genes in our WCL38 $8^{+}$dataset, CD4-CD8 $\alpha^{-}, \mathrm{CD}^{+} \mathrm{CD} 8 \alpha^{-}$, and CD4-CD8 $\alpha^{+} \mathrm{NKT}$ cells exist in mammals [62,63]. Unfortunately, when trying to retrieve typical NK(T)associated genes including cd56/ncam, nccrp-1, nkef, kir, nk1.1, cd122, nkg2d, or dap homologues, we found that none of these genes was present in the database, either because the sequence has not yet been identified, or the annotation was incomplete. Interestingly, the T-box transcription factor eomes- $b$ was found to be upregulated in the WCL38+ sorted fraction and was previously identified as one of the markers that was differentially expressed between systemic and mucosal T cells; where eomes- $a$ was mostly expressed in $\mathrm{Cd} 8 \alpha$ sorted cells from systemic organs, eomes- $b$ was highly expressed in $\mathrm{Cd} 8 \alpha$ sorted cells from gills [64]. Similarly, in our study, using lymphocytes from the intestine, eomes- $a$ was lowly expressed (data not shown) whereas eomes- $b$ was highly expressed in WCL $38^{+}$sorted fractions.

While antibodies against Tcry and Tcro are available only for zebrafish [9], gene expression analysis and in situ hybridization showed that in the posterior part of the intestine of sea bass (Dicentrarchus labrax) a $\operatorname{Tcr} \beta^{-}$population is present, and it was suggested that these cells are ${ }_{\gamma \delta} \mathrm{T}$ cells. This suggests that differences between $\mathrm{T}$ cell subsets might not only exist between organs, but also within the intestine. While in rainbow trout it was shown that intestinal intra-epithelial $\mathrm{T}$ cells and systemic $\mathrm{T}$ cells have the same $t c r \alpha \beta$ repertoire and respond to pathogens in a similar manner [65], other differences might exist between mucosal and systemic T cells, as was for example shown by differences in eomes- $a$ and eomes- $b$ expression in rainbow trout [64]. Since our WCL $38^{+}$dataset now includes two populations, Cd8 $\alpha 1^{-}{ }^{-W C L} 38^{+}$ and $\mathrm{Cd} 8 \alpha 1^{+} \mathrm{WCL} 38^{+}$, it is difficult to draw definitive conclusions on the cell type(s) recognized by the WCL38 antibody or the targeted protein or epitope. Future studies where all populations are sorted and processed for RNA sequencing, will provide further insights in the cells targeted by this antibody. Approaches including immunoprecipitation, mass spectrometry and protein sequencing will be valuable for the identification of the epitope recognized by WCL38, which was previously described to recognize a dimeric membrane protein existing of two subunits of 38 kDa [26].

In conclusion, in the present study we used cross-reactive antibodies against Cd4-1 and 
$\mathrm{Cd} 8 \alpha 1$ to characterize $\mathrm{T}$ cell subsets in naïve European common carp. Furthermore, we used multiple bioinformatics approaches to 1) show the differential expression of the newly identified $c d 4-1$ and $c d 4-2$ genes and isoforms ( $c d 4-1 a, c d 4-1 b, c d 4-2 a$ and $c d 4-2 b$ ) and to 2) characterize T cell populations by RNA Sequencing. Using the latter approach we will eventually be able to identify additional markers specific for Cd4-1 $1^{+}$and $\mathrm{Cd} 8 \alpha 1^{+}$cells but currently, a better annotation of the common carp genome is required to yield the best result from this data. Furthermore, the RNA sequencing results on the WCL38 sorted cells revealed that multiple cell populations, including $\mathrm{NK}(\mathrm{T})$ cell or ${ }_{\gamma \delta} \mathrm{T}$ cells, might be recognized. However, the exact nature of these populations requires further investigation.

\section{Acknowledgements}

We wish to thank Pierre Boudinot (INRA, Jouy-en-Josas) for his fruitful discussions and valuable input on the data mining for the $c d 4$ genes and isoforms. This work was supported by the European Commission under the Seventh Framework Programme for Research and Technological Development (FP7) of the European Union (Grant Agreement 311993 TARGETFISH).

\section{References}

1. Forlenza M, Walker PD, de Vries BJ, Wendelaar Bonga SE, Wiegertjes GF. Transcriptional analysis of the common carp (Cyprinus carpio L.) immune response to the fish louse Argulus japonicus Thiele (Crustacea: Branchiura). Fish Shellfish Immunol (2008) 25:76-83. doi:10.1016/j.fsi.2007.12.013

2. Sun X-F, Shang N, Hu W, Wang Y-P, Guo Q-L. Molecular cloning and characterization of carp (Cyprinus carpio L.) CD8 $\beta$ and CD4-like genes. Fish Shellfish Immunol (2007) 23:1242-1255. doi:10.1016/j.fsi.2007.06.004

3. Moore LJ, Dijkstra JM, Koppang EO, Hordvik I. CD4 homologues in Atlantic salmon. Fish Shellfish Immunol (2009) 26:10-18. doi:10.1016/j.fsi.2008.09.019

4. Kato G, Goto K, Akune I, Aoka S, Kondo H, Hirono I. CD4 and CD8 homologues in Japanese flounder, Paralichthys olivaceus: Differences in the expressions and localizations of CD4-1, CD4-2, CD8 $\alpha$ and CD8ß. Dev Comp Immunol (2013) 39:293-301. doi:10.1016/j. dci.2012.09.004

5. Edholm E-S, Stafford JL, Quiniou SM, Waldbieser G, Miller NW, Bengtén E, Wilson M. Channel catfish, Ictalurus punctatus, CD4-like molecules. Dev Comp Immunol (2007) 31:172-187. doi:10.1016/j.dci.2006.05.012

6. Øvergård A-C, Nerland AH, Patel S. Cloning, characterization, and expression pattern of Atlantic halibut (Hippoglossus hippoglossus L.) CD4-2, Lck, and ZAP-70. Fish Shellfish 
Immunol (2010) 29:987-997. doi:10.1016/j.fsi.2010.08.011

7. Laing KJ, Zou JJ, Purcell MK, Phillips R, Secombes CJ, Hansen JD. Evolution of the CD4 Family: Teleost Fish Possess Two Divergent Forms of CD4 in Addition to Lymphocyte Activation Gene-3. J Immunol (2006) 177:3939-3951. doi:10.4049/jimmunol.177.6.3939

8. Dijkstra JM, Somamoto T, Moore L, Hordvik I, Ototake M, Fischer U. Identification and characterization of a second CD4-like gene in teleost fish. Mol Immunol (2006) 43:410-419. doi:10.1016/j.molimm.2005.03.005

9. Yoon S, Mitra S, Wyse C, Alnabulsi A, Zou J, Weerdenburg EM, Van Der Sar AM, Wang D, Secombes CJ, Bird S. First demonstration of antigen induced cytokine expression by CD4-1 ${ }^{+}$ lymphocytes in a poikilotherm: Studies in zebrafish (Danio rerio). PLoS One (2015) 10:1-26. doi:10.1371/journal.pone.0126378

10. Kolder ICRM, van der Plas-Duivesteijn SJ, Tan G, Wiegertjes GF, Forlenza M, Guler AT, Travin DY, Nakao M, Moritomo T, Irnazarow I, et al. A full-body transcriptome and proteome resource for the European common carp. BMC Genomics (2016) 17:701. doi:10.1186/ s12864-016-3038-y

11. Petit J, David L, Dirks R, Wiegertjes GF. Genomic and transcriptomic approaches to study immunology in cyprinids: What is next? Dev Comp Immunol (2017) 75:48-62. doi:10.1016/j.dci.2017.02.022

12. Castro R, Bernard D, Lefranc MP, Six A, Benmansour A, Boudinot P. T cell diversity and TcR repertoires in teleost fish. Fish Shellfish Immunol (2011) 31:644-654. doi:10.1016/j. fsi.2010.08.016

13. Goodman T, Lefrancois L. Intraepithelial lymphocytes. Anatomical site, not T cell receptor form, dictates phenotype and function. J Exp Med (1989) 170:1569-81. doi:10.1084/ jem.170.5.1569

14. Hayday AC. $\gamma \delta$ Cells: A Right Time and a Right Place for a Conserved Third Way of Protection. Annu Rev Immunol (2000) 18:975-1026. doi:10.1146/annurev.immunol.18.1.975

15. Holtmeier W, Kabelitz D. "Gamma delta T Cells Link Innate and Adaptive Immune Responses," in Mechanisms of Epithelial Defense (Basel: KARGER), 151-183. doi:10.1159/000086659

16. Wan F, Hu C Bin, Ma JX, Gao K, Xiang LX, Shao JZ. Characterization of $\gamma \delta$ T cells from zebrafish provides insights into their important role in adaptive humoral immunity. Front Immunol (2017) 7: doi:10.3389/fimmu.2016.00675

17. Overgaard NH, Jung J-W, Steptoe RJ, Wells JW. CD4+/CD8+ double-positive T cells: more than just a developmental stage? J Leukoc Biol (2015) 97:31-38. doi:10.1189/jlb. 1RU0814-382

18. Gordon S, Taylor PR. Monocyte and macrophage heterogeneity. Nat Rev Immunol (2005) 5:953-964. doi:10.1038/nri1733

19. Chan MM, Chen CL, Ager LL, Cooper MD, Chan MM, Chen CH, Ager LL, Cooper MAXD. Identification of the avian homologues of mammalian CD4 and CD8 antigens. J Immunol (1988) 140:2133-2138.

20. Gibbings D, Befus AD. CD4 and CD8: an inside-out coreceptor model for innate immune cells. J Leukoc Biol (2009) 86:251-259. doi:10.1189/jlb.0109040

21. Takizawa F, Magadan S, Parra D, Xu Z, Korytáŕ T, Boudinot P, Sunyer JO. Novel Teleost CD4-Bearing Cell Populations Provide Insights into the Evolutionary Origins and Primordial Roles of CD4 ${ }^{+}$Lymphocytes and CD4 ${ }^{+}$Macrophages. J Immunol (2016) 196:4522-4535. doi:10.4049/jimmunol.1600222

22. Dee CT, Nagaraju RT, Athanasiadis EI, Gray C, Fernandez del Ama L, Johnston SA, Secombes CJ, Cvejic A, Hurlstone AFL. CD4-Transgenic Zebrafish Reveal Tissue-Resident Th2- and Regulatory T Cell-like Populations and Diverse Mononuclear Phagocytes. J Immunol (2016) 197:3520-3530. doi:10.4049/jimmunol.1600959

23. Granja AG, Leal E, Pignatelli J, Castro R, Abós B, Kato G, Fischer U, Tafalla C. Identification of Teleost Skin CD8 $\alpha+$ Dendritic-like Cells, Representing a Potential Common 
Ancestor for Mammalian Cross-Presenting Dendritic Cells. J Immunol (2015) 195:1825-37. doi:10.4049/jimmunol.1500322

24. Scapigliati G, Mazzini M, Mastrolia L, Romano N, Abelli L. Production and characterisation of a monoclonal antibody against the thymocytes of the sea bass Dicentrarchus labrax(L.) (Teleostea, Percicthydae). Fish Shellfish Immunol (1995) 5:393-405. doi:10.1006/ fsim.1995.0039

25. Passer BJ, Chen CLH, Miller NW, Cooper MD. Identification of a T lineage antigen in the catfish. Dev Comp Immunol (1996) 20:441-450. doi:10.1016/S0145-305X(96)00033-X

26. Rombout JHWM, Joosten PHM, Engelsma MY, Vos AP, Taverne N, Taverne-Thiele JJ. Indications for a distinct putative $\mathrm{T}$ cell population in mucosal tissue of carp (Cyprinus carpio L.). Dev Comp Immunol (1998) 22:63-77. doi:10.1016/S0145-305X(97)00048-7

27. Rombout J, vandeWal JW, Companjen A, Taverne N, TaverneThiele JJ. Characterization of a T cell lineage marker in carp (Cyprinus carpio L). Dev Comp Immunol (1997) 21:35-46.

28. Maisey K, Montero R, Corripio-Miyar Y, Toro-Ascuy D, Valenzuela B, Reyes-Cerpa S, Sandino AM, Zou J, Wang T, Secombes CJ, et al. Isolation and Characterization of Salmonid CD4 + T Cells. J Immunol (2016) 196:4150-4163. doi:10.4049/jimmunol.1500439

29. Toda H, Saito Y, Koike T, Takizawa F, Araki K, Yabu T, Somamoto T, Suetake H, Suzuki Y, Ototake M, et al. Conservation of characteristics and functions of CD4 positive lymphocytes in a teleost fish. Dev Comp Immunol (2011) 35:650-660. doi:10.1016/j.dci.2011.01.013

30. Kono T, Korenaga H. Cytokine Gene Expression in CD4 Positive Cells of the Japanese Pufferfish, Takifugu rubripes. PLoS One (2013) 8: doi:10.1371/journal.pone.0066364

31. Takizawa F, Dijkstra JM, Kotterba P, Korytáŕ T, Kock H, Köllner B, Jaureguiberry B, Nakanishi T, Fischer U. The expression of CD8 $\alpha$ discriminates distinct $\mathrm{T}$ cell subsets in teleost fish. Dev Comp Immunol (2011) 35:752-763. doi:10.1016/j.dci.2011.02.008

32. Miyazawa R, Matsuura Y, Shibasaki Y, Imamura S, Nakanishi T. Cross-reactivity of monoclonal antibodies against CD4-1 and CD8a of ginbuna crucian carp with lymphocytes of zebrafish and other cyprinid species. Dev Comp Immunol (2016) 80:15-23. doi:10.1016/j. dci.2016.12.002

33. Henkel C V., Dirks RP, Jansen HJ, Forlenza M, Wiegertjes GF, Howe K, van den Thillart GEEJM, Spaink HP. Comparison of the Exomes of Common Carp (Cyprinus carpio) and Zebrafish (Danio rerio). Zebrafish (2012) 9:59-67. doi:10.1089/zeb.2012.0773

34. Forlenza M, de Carvalho Dias JDA, Veselý T, Pokorová D, Savelkoul HFJ, Wiegertjes GF. Transcription of signal-3 cytokines, IL-12 and IFN alpha beta, coincides with the timing of CD8 alpha beta up-regulation during viral infection of common carp (Cyprinus carpio L). Mol Immunol (2008) 45:1531-47. doi:10.1016/j.molimm.2007.10.010

35. Forlenza M, Scharsack JP, Kachamakova NM, Taverne-Thiele AJ, Rombout JHWM, Wiegertjes GF. Differential contribution of neutrophilic granulocytes and macrophages to nitrosative stress in a host-parasite animal model. Mol Immunol (2008) 45:3178-3189. doi:10.1016/j.molimm.2008.02.025

36. Forlenza M, Kaiser T, Savelkoul HFJ, Wiegertjes GF. The use of real-time quantitative PCR for the analysis of cytokine mRNA levels. Methods Mol Biol (2012) 820:7-23. doi:10.1007/978-1-61779-439-1_2

37. Pfaffl MW. A new mathematical model for relative quantification in real-time RT-PCR. Nucleic Acids Res (2001) 29:e45-e45. doi:10.1093/nar/29.9.e45

38. Piazzon MC, Savelkoul HSJ, Pietretti D, Wiegertjes GF, Forlenza M. Carp I110 Has Anti-Inflammatory Activities on Phagocytes, Promotes Proliferation of Memory T Cells, and Regulates B Cell Differentiation and Antibody Secretion. J Immunol (2015) 194:187-99. doi:10.4049/jimmunol.1402093

39. Embregts CWE, Rigaudeau D, Veselý T, Pokorová D, Lorenzen N, Petit J, Houel A, Dauber M, Schütze H, Boudinot P, et al. Intramuscular DNA Vaccination of Juvenile Carp against Spring Viremia of Carp Virus Induces Full Protection and Establishes a Virus-Specific B and T Cell Response. Front Immunol (2017) 8:1340. doi:10.3389/fimmu.2017.01340 
40. Koumans-van Diepen JE, van de Lisdonk MHM, Taverne-Thiele AJ, Verburg-van Kemenade BML, Rombout JHWM. Characterisation of immunoglobulin-binding leucocytes in carp (Cyprinus carpio L.). Dev Comp Immunol (1994) 18:45-56. doi:10.1016/0145305X(94)90251-8

41. Secombes CJ, van Groningen JJ, Egberts E. Separation of lymphocyte subpopulations in carp Cyprinus carpio L. by monoclonal antibodies: immunohistochemical studies. Immunology (1983) 48:165-175.

42. Nakayasu C, Omori M, Hasegawa S, Kurata O, Okamoto N. Production of a monoclonal antibody for carp (Cyprinus carpio L.) phagocytic cells and separation of the cells. Fish Shellfish Immunol (1998) 8:91-100. Available at: http://ac.els-cdn. com/S105046489790125X/1-s2.0-S105046489790125X-main.pdf?_tid=f2ecacd2-4167-11e7-b339-00000aab0f01\&acdnat=1495730217_68caa61b6a51021c584816f36ce0e663 [Accessed May 25, 2017]

43. Weyts FAA, Rombout JHWM, Flik G, L Verburg-van Kemenade BM, Weyts FAA, Rombout JHWM, Flik G, Verburg-van Kemenade BML. A common carp (Cyprinus carpio L.) leucocyte cell line shares morphological and functional characteristics with macrophages. Fish Shellfish Immunol (1997) 7:123-133. doi:10.1006/fsim.1996.0069

44. Kim D, Pertea G, Trapnell C, Pimentel H, Kelley R, Salzberg SL. TopHat2 : accurate alignment of transcriptomes in the presence of insertions, deletions and gene fusions. Genome Biol (2013) 14:0-9. doi:10.1101/000851

45. Li H, Handsaker B, Wysoker A, Fennell T, Ruan J, Homer N, Marth G, Abecasis G, Durbin R. The Sequence Alignment/Map format and SAMtools. Bioinformatics (2009) 25:20782079. doi:10.1093/bioinformatics/btp352

46. Anders S, Pyl PT, Huber W. HTSeq-A Python framework to work with high-throughput sequencing data. Bioinformatics (2015) 31:166-169. doi:10.1093/bioinformatics/btu638

47. Robinson MD, McCarthy DJ, Smyth GK. edgeR: A Bioconductor package for differential expression analysis of digital gene expression data. Bioinformatics (2009) 26:139-140. doi:10.1093/bioinformatics/btp616

48. Love MI, Huber W, Anders S. Moderated estimation of fold change and dispersion for RNAseq data with DESeq2. Genome Biol (2014) 15:1-21. doi:10.1186/s13059-014-0550-8

49. Rombout JHWM, Koumans-Van Diepen JCE, Emmer PM, Taverne-Thiele JJ, Taverne N. Characterization of carp thrombocytes with specific monoclonal antibodies. J Fish Biol (1996) 49:521-531. doi:10.1006/jfbi.1996.0178

50. Devine L, Kieffer LJ, Aitken V, Kavathas PB. Human CD8 beta, but not mouse CD8 beta, can be expressed in the absence of CD8 alpha as a beta beta homodimer. J Immunol (2000) 164:833-838. doi:10.4049/jimmunol.164.2.833

51. Baba M, Imai T, Nishimura M, Kakizaki M, Takagi S, Hieshima K, Nomiyama H, Yoshie O. Identification of CCR6, the specific receptor for a novel lymphocyte-directed CC chemokine LARC. J Biol Chem (1997) 272:14893-8. doi:10.1074/jbc.272.23.14893

52. Castro G, Liu X, Ngo K, De Leon-Tabaldo A, Zhao S, Luna-Roman R, Yu J, Cao T, Kuhn R, Wilkinson P, et al. ROR $\gamma \mathrm{t}$ and ROR $\alpha$ signature genes in human Th17 cells. PLoS One (2017) 12:1-22. doi:10.1371/journal.pone.0181868

53. Vries L De, Zheng B, Fischer T, Elenko E, Farquhar MG. The regulator of G protein signaling family. (2000)

54. Evans DL, Jaso-Friedmann L, Smith Jr. EE, St John A, Koren HS, Harris DT. Identification of a putative antigen receptor on fish nonspecific cytotoxic cells with monoclonal antibodies. J Immunol (1988) 141:324-332. Available at: http://www.ncbi.nlm.nih.gov/entrez/query. fcgi?cmd=Retrieve \&db=PubMed\&dopt=Citation\&list_uids $=2454262$

55. Itohara S, Nakanishi N, Kanagawa O, Kubo R, Tonegawa S. Monoclonal antibodies specific to native murine T-cell receptor gamma delta: analysis of gamma delta $\mathrm{T}$ cells during thymic ontogeny and in peripheral lymphoid organs. Proc Natl Acad Sci U S A (1989) 86:5094-8. doi:10.1073/pnas.86.13.5094 
56. Huttenhuis HBT, Romano N, Van Oosterhoud CN, Taverne-Thiele AJ, Mastrolia L, Van Muiswinkel WB, Rombout JHWM. The ontogeny of mucosal immune cells in common carp (Cyprinus carpio L.). Anat Embryol (Berl) (2006) 211:19-29. doi:10.1007/s00429-005-00620

57. Bluestone JA, Cron RQ, Cotterman M, Houlden BA, Matis LA. Structure and specificity of $\mathrm{T}$ cell receptor g/d on major histocompatibilty complex antigen-specific CD3+, CD4-, CD8T lymphocytes. JExpMed (1988) 168:1899-1916.

58. Sheridan BS, Romagnoli PA, Pham QM, Fu HH, Alonzo F, Schubert WD, Freitag NE, Lefrançois L. $\gamma \delta$ T Cells Exhibit Multifunctional and Protective Memory in Intestinal Tissues. Immunity (2013) 39:184-195. doi:10.1016/j.immuni.2013.06.015

59. Koizumi H, Liu CC, Zheng LM, Joag S V, Bayne NK, Holoshitz J, Young JD. Expression of perforin and serine esterases by human gamma/delta T cells. J Exp Med (1991) 173:499-502. Available at: http://eutils.ncbi.nlm.nih.gov/entrez/eutils/elink.fcgi?dbfrom=pubmed\&amp;id=1703210\&amp;retmode=ref\&amp;cmd=prlinks

60. Huang R, Gao LY, Wang YP, Hu W, Guo QL. Structure, organization and expression of common carp (Cyprinus carpio L.) NKEF-B gene. Fish Shellfish Immunol (2009) 26:220-229. doi:10.1016/j.fsi.2008.10.013

61. Fischer U, Koppang EO, Nakanishi T. Teleost T and NK cell immunity. Fish Shellfish Immunol (2013) 35:197-206. doi:10.1016/j.fsi.2013.04.018

62. Godfrey DI, MacDonald HR, Kronenberg M, Smyth MJ, Van Kaer L. NKT cells: what's in a name? Nat Rev Immunol (2004) 4:231-237. doi:10.1038/nri1309

63. Godfrey DI, Hammond KJL, Poulton LD, Smyth MJ, Baxter AG. NKT cells: Facts, functions and fallacies. Immunol Today (2000) 21:573-583. doi:10.1016/S0167-5699(00)01735-7

64. Takizawa F, Araki K, Ohtani M, Toda H, Saito Y, Lampe VS, Dijkstra JM, Ototake M, Moritomo T, Nakanishi T, et al. Transcription analysis of two Eomesodermin genes in lymphocyte subsets of two teleost species. Fish Shellfish Immunol (2014) 36:215-222. doi:10.1016/j.fsi.2013.11.004

65. Bernard D, Six A, Rigottier-Gois L, Messiaen S, Chilmonczyk S, Quillet E, Boudinot P, Benmansour A. Phenotypic and functional similarity of gut intraepithelial and systemic T cells in a teleost fish. J Immunol (2006) 176:3942-3949. doi:10.4049/jimmunol.176.7.3942 


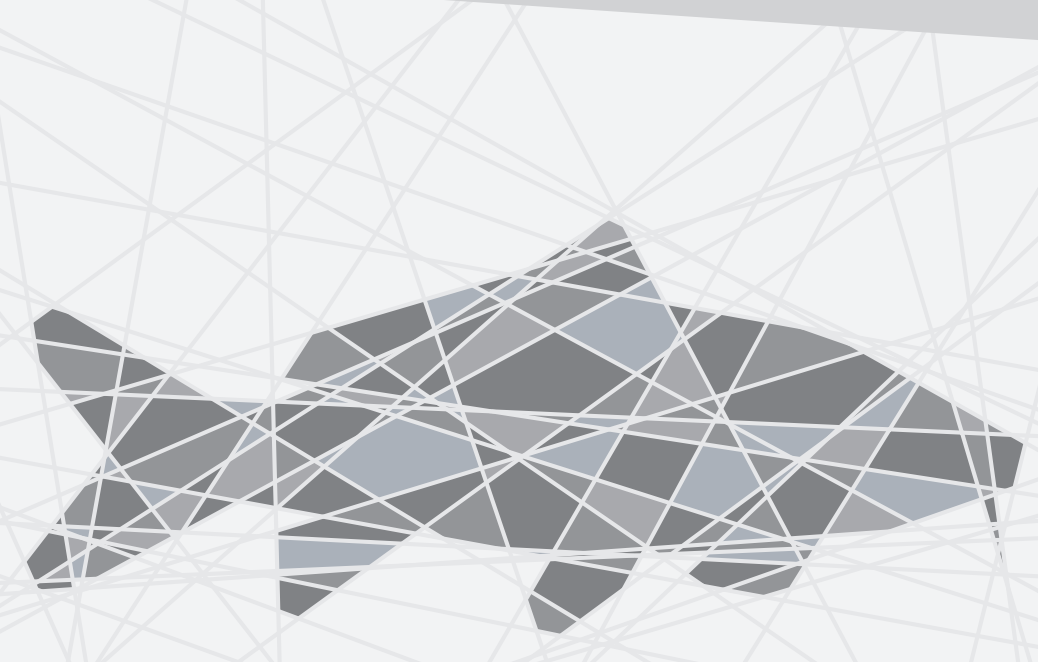




\section{Preliminary characterization of lgm $^{+}$ and $\mathrm{lgt}^{+} \mathrm{B}$ cell populations in carp and their role during systemic and mucosal infections}

\section{Carmen W.E. Embregts', Danilo Pietretti', Ingrid Govers', Lucky Marufa', Erik v.d. Brink', David Parra' ${ }^{2}$, Astrid S. Holzer ${ }^{3}$, Marleen Scheer', Geert F.Wiegertjes', Maria Forlenza'}

I Cell Biology and Immunology Group, Department of Animal Sciences, Wageningen University, Wageningen, The Netherlands

${ }^{2}$ Department of Cell Biology, Physiology and Immunology, Universitat Autònoma de Barcelona, Spain ${ }^{3}$ Institute of Parasitology, Czech Academy of Sciences, Czech Republic 


\section{Abstract}

Common carp igt1 (igzl) and igt2 (igz2) were recently characterized at the molecular level. Their functional characterization however has been hampered by the lack of specific antibodies. Here, we report on the development of polyclonal antibodies against Igt 1 and Igt 2 raised in both rabbit and chicken. While the antibodies against Igt1 were found to be specific and recognized the native molecule under various conditions, antibodies against Igt2 showed reactivity also to carp Igm (rabbit-antiIgt2) or to a yet unknown high molecular weight protein (chicken-anti-Igt2). By flow cytometric analysis we show the highest percentage of Igt1 $1^{+} \mathrm{B}$ cells in blood and spleen, and similar to $\operatorname{Igm}^{+} \mathrm{B}$ cells, Igt $1^{+} \mathrm{B}$ cells are able to phagocytose fluorescent beads of different sizes and form phagolysosomes. Using Western blot we show that while Igm and Igt2 are most abundant in mucus and on bacteria of skin and gills, Igt1 was found to be the predominant Ig coating intestinal bacteria. Interestingly, we found Igt $1^{+}$cells present at the epithelial layer in the intestine while in contrast, $\operatorname{Igm}^{+} /$ Igt $2^{+}$cells were found only in the lamina propria. Carp infected with the gill parasite Sphaerospora molnari had elevated numbers of Igt $1^{+}$cells in the gill filaments and concomitant upregulation of igt1 and igt 2 expression in their gills. Noteworthy, Igt1 and Igt 2 also responded to an infection with the extracellular blood parasite Trypanoplasma borreli. The response was characterized by an elevated parasitebinding capacity in the serum, and an increase in Igt $1^{+}$and $\mathrm{Igm}^{+} / \operatorname{Igt}^{+}$cells in the spleen. Gene expression analysis showed differences in the kinetics of igm, igt 1 and igt2, suggesting that they all might play a role during infection. In conclusion, we for the first time characterized $\mathrm{Igm}^{+}$and $\mathrm{Igt}^{+} \mathrm{B}$ cell populations in common carp and most importantly, show that Igt1 and Igt2, similarly to Igm, respond to both mucosal and systemic infections. 


\section{Introduction}

Together with cartilaginous fish, teleost fish are the earliest organisms that have an adaptive immune system composed of $\mathrm{B}$ and $\mathrm{T}$ cells. The constant $(\mathrm{C})$ domain of the immunoglobulin (Ig) defines its isotype (class) and in teleost fish igm, igd and igt (alias: igz) have been identified. While the presence of igm in teleost species was already known for long [1], igd and igt have been identified decades later [2-4].

Although identified in fish in 1997 [2], the first antibody against Igd was successfully produced in 2012, and was used to describe a soluble form in rainbow trout (Oncorhynchus mykiss) [5]. More recently double-positive $\operatorname{Igm}^{+} \operatorname{Igd}^{+}$as well as Igm $\mathrm{Igd}^{+} \mathrm{B}$ cells in rainbow trout and catfish, of which a $\mathrm{Ccr} 7^{+} \mathrm{Igd}^{+} \mathrm{Igm}^{-} \mathrm{B}$ cell population was exclusively found in gills of trout [6,7]. Although the presence of the ighd gene has been reported in all teleost species studied so far, only in catfish (Ictalurus punctatus) [7] and rainbow trout a soluble Igd has been described, [8,9]. However, although the gene has been identified in many teleost species, the exact role of $\operatorname{Igd}^{+}$ $B$ cells remains to be identified.

With respect to $\operatorname{Igt}^{+} \mathrm{B}$ cells, single-positive Igt $^{+} \operatorname{Igm}^{-}$Igd $^{-} \mathrm{B}$ cells have been described so far [10]. The exclusive expression of igt on B cells is based on the gene arrangement on the igh locus; in the zebrafish (Danio rerio), grass carp (Ctenopharyngodon idella), and fugu (Takifugu rubripes) for example, the D $\tau$-J $\tau$ $\mathrm{C} \tau$ cluster(s) encoding igt-specific genes are generally located between the region containing the $\mathrm{Vh}$ segments and the $\mathrm{D} \mu / \delta-\mathrm{J} \mu / \delta-\mathrm{C} \mu-\mathrm{C} \delta$ locus $[3,4,11]$. Since most Vh genes are located upstream of both $\mathrm{D} \tau$ and $\mathrm{D} \mu / \delta$, they can probably be used by Igt, Igm, and Igd. The arrangement and orientation of gene segments within the igh loci imposes the exclusive production of either Igt or Igm/d, since the recombination of $\mathrm{Vh}$ to $\mathrm{D} \mu / \delta$ deletes the $\mathrm{D} \tau-\mathrm{J} \tau-\mathrm{C} \tau$ region(s). After its first identification in 2005 in zebrafish [4] and rainbow trout [3], igt has been identified in all teleost species studied so far, except for medaka (Oryzias latipes) and catfish [11]. Even more, the presence of a second igt gene has been described in multiple teleost species including stickleback (Gasterosteus aculeatus) [12,13], zebrafish [14] and common carp (Cyprinus carpio) [15]. In the latter species the second Igt subtype, composed of two constant $\mathrm{Ch} \tau$ domains, was previously described as an Igm-Igt chimera [16] due to the high similarity of the $\mathrm{Ch} 1 \tau 2$ and $\mathrm{Ch} 2 \tau 2$ domain to $\mathrm{Ch} 1 \mu$ and $\mathrm{Ch} 4 \tau$ domain, respectively. Even more, a third Igt subtype has been described in Atlantic salmon 
(Salmo salar) [17] and rainbow trout [18]. When looking at the complex diversity of Ig loci the presence of multiple Igt subtypes is to be expected for other species as well, especially for diploid or tetrapoloid species [11].

While igt genes have been described in many teleost species, the characterization of the role of $\mathrm{Igt}^{+} \mathrm{B}$ cells remains poorly studied. Until now, only one monoclonal antibody is available, recognizing rainbow trout Igt1 (referred to as Igt). Using this antibody it was found that Igt plays a major role in mucosal immunity in the intestine [10], skin [19], gills [20] and in the nasopharynx-associated lymphoid tissue (NALT) [21]. These studies together show that, at least in rainbow trout, Igt is the predominant immunoglobulin in mucus and on mucosal bacteria, and that Igt, but not Igm, reacts specifically to gill- and intestinal parasites. Despite the general lack of antibodies to study B cell subtypes in teleost fish, gene expression data pointed out a similar mucosal role for igt in multiple species including sea bass (Dicentrarchus labrax) [22-24], Atlantic salmon [25], flounder (Paralichthys olivaceus) [26] and common carp [15]. While these studies emphasize the strong mucosal role for Igt, the role of Igm in mucosal immunity should not be ignored. To illustrate, both Igm and Igt were found to bind to the parasite Ichthyophthirius multifiliis in gills of rainbow trout [27], and in ayu (Plecoglossus altivelis) both igm and igt responded to Vibrio anguillarum vaccination by intra-peritoneal (i.p.) injection or immersion [28].

Furthermore, besides its role in mucosal immunity, Igt was found to have a role in systemic immunity. This was exemplified by the recruitment Igt $^{+} \mathrm{B}$ cells to the site of injection after intra-muscular (i.m.) DNA vaccination of rainbow trout [29], and by the igt response to pathogens measured in systemic organs as reported for rohu (Labeo rohita) [30], common carp [15] and mandarin fish (Siniperca chuatsi) [31]. Moreover, a clonal igt response, as assessed by repertoire analysis, was reported in spleen of rainbow trout infected with Viral Hemorrhagic Septicemia Virus (VHSV), suggesting that $\operatorname{Igt}^{+} \mathrm{B}$ cells might play a role also in systemic responses to viral infections [32]. The presence of a higher basal number of $\operatorname{~gt~}^{+} \mathrm{B}$ cells in naïve rainbow trout naturally resistant to Flavobacterium psychrophilum, as well as the strong increase in Igt $^{+} \mathrm{B}$ cells and igt expression during infection, suggests a role for Igt in the response to this bacterium [33]. In rainbow trout, were three igt subtypes were found, only igt 1 was detected in mucosal tissues, igt 2 mostly in systemic organs and thymus, and Igt3 was only detected as a protein in serum [18].

Also in common carp, in which two subtypes of igt (igz) were described [16], gene 
expression analysis showed that igt1 (igzl) was slightly higher expressed in systemic organs than igt2 (igz2), and that igt2 (igz2) was higher expressed in mucosal organs [15]. Furthermore, the observation that igt1 (igz1) but not igt2 (igz2) transcription level increased significantly during infection with the extracellular blood parasite Trypanoplasma borreli ( $T$. borreli), and that igt2 (igz2) but not igt1 (igz1) was increased during infection with the skin parasite Lernea, suggests a differential role for the two Igt subclasses [15]. While all above points at differential and specialized roles for the various Igt subclasses within and between teleost species, specific antibodies are required to characterize the distribution and the response of $\operatorname{Igt}^{+} \mathrm{B}$ cells and their secreted Igs.

To this end, in the present study, we report the development and validation of antibodies against common carp Igt1 and Igt2. Using these newly developed antibodies, combined with an available antibody against common carp Igm, we aimed to characterize $\operatorname{Igm}^{+}$and $\operatorname{Igt}^{+} \mathrm{B}$ cell subsets in naïve carp, as well as during systemic and mucosal infections. We observed spatial segregation of the different B cell subtypes in the intestine, as well as differential expression and binding of soluble Ig protein in mucus and on mucosal bacteria. Our data point towards a more pronounced role for Igt1 in the intestine, and for Igm and Igt2 in skin and gills. Furthermore, we observed that Igm, Igt1 and Igt 2 all responded to both a systemic and a mucosal infection. This indicates that, at least in common carp, Igt cannot be categorized as a strict mucosal Ig.

\section{Materials and Methods}

\section{Animals}

European common carp (Cyprinus carpio carpio) from the R3xR8 strain were used that originated from a cross between the Hungarian R8 strain and the Polish R3 strain (Irnazarow, 1995). In this study, we will refer to carp as the European common carp subspecies, unless stated otherwise. Carp were bred in the Aquatic Research Facility (ARC) of the Wageningen University animal facility, the Netherlands. Carp were raised at $20-23^{\circ} \mathrm{C}$ in recirculating UV-treated water and fed pelleted carp food (Skretting, Nutreco) twice daily. All studies were performed with approval of the local animal welfare committee (DEC) of Wageningen University. Infections with 
Sphaerospora molnari (S. molnari) were performed at the Institute of Parasitology, Czech Academy of Sciences, (Czech Republic). Here, carp of a Czech strain were obtained from the Faculty of Fisheries and Protection of Waters (University of South Bohemia, Czech Republic), were kept at $20-22^{\circ} \mathrm{C}$ in recirculating UV-treated water and were fed Sinking Coarse Carp Feed Pellets (Skretting) twice daily. Infections were performed in accordance with Czech legislation (section 29 of Act No.246/1992 Coll., on Protection of animals against cruelty, as amended by Act No. 77/2004 Coll.).

\section{Cloning, bacterial expression and purification of Igt1 and lgt2}

The sequences corresponding to the published Japanese common carp igzl and igz2, from now on referred to as igt 1 and igt2, were blasted against the European common carp genome [34]. The retrieved sequences were used to design synthetic sequences spanning the $\mathrm{CH} 2 \tau 1-\mathrm{CH} 3 \tau 1$ of igt 1 and the $\mathrm{CH} 1 \tau 2-\mathrm{CH} 2 \tau 2$ of igt2. Sequences were codon optimized for bacterial expression and synthetized (BaseClear). Obtained sequences were subcloned in the expression plasmid pQE-30UA (QIAgen) between the BamHI and HindIII cloning sites, downstream of the sequence coding for six $\mathrm{N}$-terminal histidines (6xHis-tag). Ligation products were cloned in M15 competent Escherichia coli, plated onto lysogeny broth (LB) agar plates supplemented with ampicillin $(100 \mu \mathrm{g} / \mathrm{ml})$, kanamycin $(25 \mu \mathrm{g} / \mathrm{mL})$, and incubated overnight at $37^{\circ} \mathrm{C}$. Positive clones were identified by colony PCR using vector-specific primers and the products were sequenced to verify correct orientation and frame. Selected positive clones were used for protein production as previously described [35]. Briefly, bacteria were plated on LB-ampicillin-kanamycin plates, and incubated overnight at $37^{\circ} \mathrm{C}$. Bacterial suspension $(40 \mathrm{~mL})$ from an overnight culture was transferred to $1 \mathrm{~L} \mathrm{~TB}$ medium (Bacto-tryptone $24 \mathrm{~g} / \mathrm{L}$, Bacto-yeast extract $12 \mathrm{~g} / \mathrm{L}, \mathrm{NaCl} 5 \mathrm{~g} / \mathrm{L}$ and Gycerol $4 \mathrm{~g} / \mathrm{L}$ ) and incubated at $37^{\circ}$ until $\mathrm{OD}_{600} 0.6-0.8$. Protein expression was induced by addition of $1 \mathrm{mM}$ isopropyl $\beta$-d-thiogalactoside (IPTG) for $4 \mathrm{~h}$ at $37^{\circ} \mathrm{C}$. Protein was purified from solubilized inclusion bodies under denaturing conditions using a $\mathrm{Ni}^{2+}$ NTA agarose beads (Qiagen) by gravity flow. Briefly, the column was washed with 5-column volumes of cold $\left(4^{\circ} \mathrm{C}\right) 20 \mathrm{mM}$ Tris- $\mathrm{HCl}, 500 \mathrm{mM} \mathrm{NaCl}, 6 \mathrm{M}$ Guanadine$\mathrm{HCl}, 25 \mathrm{mM}$ imidazole, $1 \%(\mathrm{v} / \mathrm{v})$ Triton X-100; 10-column volumes of cold 150 $\mathrm{mM} \mathrm{NaCl}, 4 \mathrm{M}$ Guanadine- $\mathrm{HCl}, 50 \mathrm{mM}$ imidazole; Elution was performed with cold $150 \mathrm{mM} \mathrm{NaCl}, 4 \mathrm{M}$ Guanadine- $\mathrm{HCl}, 250 \mathrm{mM}$ imidazole. Purified proteins were immediately used for coupling to Aminolink Coupling Resin (Thermofisher) for 
subsequent affinity purification of antibodies. Alternatively, proteins were refolded by rapid 20x dilution in refolding buffer (1 mM EDTA, 2 mM DTT, 50 mM Trisbase, $500 \mathrm{mM} \mathrm{NaCl}, 5 \mathrm{mM} \mathrm{GSSG}, 1.25 \mathrm{M}$ guanidine; $\mathrm{pH}$ 10.5), incubated overnight at $4^{\circ} \mathrm{C}$ and then dialysed against $50 \mathrm{mM}$ Tris-base, $500 \mathrm{mM} \mathrm{NaCl}(\mathrm{pH} \mathrm{10.5).} \mathrm{The}$ protein solution was centrifuged to remove any precipitate and concentrated again by binding on $\mathrm{Ni}^{2+}$-NTA agarose beads. Protein was eluted in $50 \mathrm{mM}$ Tris-base, 250 $\mathrm{mM}$ Imidazole, $\mathrm{pH}$ 10.5. Eluted, refolded proteins were used for immunization of rabbit and chicken as described below.

\section{Antibody production and purification}

Polyclonal rabbit serum anti-Igt1 and anti-Igt 2 was produced by immunization of rabbits with purified refolded bacterial recombinant proteins according to a 3-month standard protocol (Naxo). Polyclonal chicken IgY anti-Igt1 and anti-Igt2 were produced in-house by immunization of chicken with purified recombinant proteins ( 2 x $100 \mu \mathrm{g}$ of protein using Specol as adjuvant, with four weeks interval). Eggs containing highest titers of anti-Igt1 and anti-Igt2 IgY were selected by ELISA, using the recombinant proteins as antigens, and pooled. Antibodies were purified from the egg yolk by the "water dilution method" followed by ammonium sulphate precipitation according to the procedure described by Hansen et al., 1998, pp. 1-7 [36]. Total rabbit IgG was purified using the Melon Gel IgG purification kit (Thermo Scientific) with gravity columns (Supelco), following the manufacturer's protocol. Protein concentrations of the purified IgY and IgG fractions were measured at 280 nm using a Nanodrop-1000 (Thermo Scientific) and were stored either at $-20{ }^{\circ} \mathrm{C}$ or at $4{ }^{\circ} \mathrm{C}$ after addition of $0.01 \%$ sodium azide. Antigen-specific rabbit IgG were affinity purified from rabbit serum using an AminoLink coupling resin (Thermo Scientific) containing $2 \mathrm{mg}$ of purified recombinant Igt 1 or Igt 2 according to the manufacturer's protocol with slight modification. Briefly, protein was coupled to the resin using 150 $\mathrm{mM} \mathrm{NaCl}, 4 \mathrm{M}$ Guanadine-HCl, $250 \mathrm{mM}$ imidazole as coupling buffer. Column was used immediately or stored at $4^{\circ} \mathrm{C}$ in PBS containing $0.01 \%$ sodium azide.

\section{Western blot analysis}

The reactivity of the antibodies against common carp Igt1 or Igt2 (total IgY from egg yolk or total IgG from rabbit sera) was tested by Western blot analysis using $100 \mathrm{ng}$ of recombinant protein. For detection of Igs (Igm, Igt1, Igt2), mucus (5 $\mu \mathrm{g} /$ lane) or bacterial pellet (pellet obtained from $\sim 100 \mu \mathrm{L}$ mucus) was mixed with 
loading buffer containing $\beta$-mercaptoethanol. Samples were resolved on 4-20\% SDS-PAGE gels and proteins were transfered to nitrocellulose membranes (Protean, Thermo Scientific). The membrane was blocked for $1 \mathrm{~h}$ with $5 \%(\mathrm{w} / \mathrm{v})$ non-fat dry milk (NFDM, Elk) in Tris-buffered saline (TBS) containing $0.05 \%(\mathrm{v} / \mathrm{v})$ Tween (TBST) and subsequently incubated overnight at $4^{\circ} \mathrm{C}$ with the primary antibody (10 $\mu \mathrm{g} / \mathrm{mL}$ of affinity purified rabbit-IgG or total IgY, either anti-Igt1 or anti-Igt2) in the same buffer. After subsequent washes, membranes were incubated with HRPconjugated rabbit-anti-chicken (1:4000 ImmunoResearch Laboratories) or HRPconjugated goat-anti-rabbit (1:2000, Dako) antibodies in 10\% NFDM in TBST. Development was performed using Pierce ECL Western blotting Substrate (Thermo Scientific) and chemiluminescent signals were visualized using a ChemiDoc XRS and corresponding software (BioRad).

For testing their reactivity to naïve Igs, serum of naïve ( $8 \mu \mathrm{g} / \mathrm{lane})$ or parasite infected carp $(1 \mu \mathrm{g} / \mathrm{lane})$ was resolved on 4-20\% SDS-PAGE gels, and after transfer and blocking as described above, was incubated with affinity purified rabbit anti-Igt1 or anti-Igt2 $(10 \mu \mathrm{g} / \mathrm{ml})$, total chicken IgY anti-Igt1 or anti-Igt2 $(10 \mu \mathrm{g} / \mathrm{ml})$, or mouse monoclonal WCI12 anti-Igm (1:100 [37]). Donkey anti-mouse-IRDye-680, donkey anti-rabbit-IRDye-800, or donkey anti-chicken-IRDye-800 (all from LI-CORE) were used at a 1:5000 dilution, after which the fluorescent signal was analysed using an Odyssey scanner (LI-COR Biosciences).

\section{Indirect and sandwich ELISA}

For indirect ELISA, high-binding plates (Greiner Bio-One) were coated overnight at $4{ }^{\circ} \mathrm{C}$ with serial dilutions of recombinant Igt1 or Igt2 in carbonate/bicarbonate buffer ( $\mathrm{pH}$ 9.6). Plates were blocked for $1 \mathrm{~h}$ at room temperature (RT) with $3 \%(\mathrm{w} / \mathrm{v})$ bovine serum albumin (BSA) in phosphate-buffered saline (PBS) with $0.05 \%$ Tween (PBST). Plates were then incubated with serial dilution of the primary antibody in 3\% BSA-PBST and subsequently with HRP-conjugated rabbit-anti-chicken (1:4000; ImmunoResearch Laboratories) or HRP-conjugated goat-anti-rabbit (1:2000, Dako). The reaction was developed with 2,2'-azino-bis(3-ethylbenzothiazoline-6-sulphonic acid) (ABTS; Roche) and $\mathrm{OD}_{405 \mathrm{~nm}}$ was measured using a Filtermax F5 multi-mode microplate reader (Molecular Devices).

For sandwich ELISA, high-binding ELISA plates were coated overnight at $4^{\circ} \mathrm{C}$ with capture antibody (rabbit anti-Igt1 or Igt2 $10 \mu \mathrm{g} / \mathrm{mL}$, optimized based on reactivity to 
the recombinant proteins) in PBS. Plates were subsequently incubated for $1 \mathrm{~h}$ at RT with PBST containing 5\% NFDM (Elk), followed by incubation with recombinant protein or carp serum diluted in 2\% NFDM in PBST. Next, plates were incubated with detection antibody (anti-Igt1 IgY $9 \mu \mathrm{g} / \mathrm{mL}$, anti-Igt2 $4 \operatorname{IgY} 5 \mu \mathrm{g} / \mathrm{mL}$ ) diluted in $2 \%$ NFDM in PBST for $1 \mathrm{~h}$ at RT followed by development with the above mentioned HRP-conjugated antibodies diluted in 2\% NFDM in PBST. Development and measurements were performed as described above. To test the cross-reactivity between the rabbit anti-Igt2 antibody and carp Igm, a sandwich ELISA was set-up using the purified WCI12 anti-Igm antibody $(0.5 \mu \mathrm{g} / \mathrm{mL}$ [37]) as capture antibody, serum from naïve carp diluted in PBS was used as antigen, and the rabbit IgG $(40 \mu \mathrm{g} /$ $\mathrm{mL})$ or chicken $\operatorname{IgY}(9 \mu \mathrm{g} / \mathrm{mL}$ for Igt1 and $45 \mu \mathrm{g} / \mathrm{mL}$ for Igt 2$)$ as detection antibodies.

\section{Flow cytometry}

Carp were euthanized with $0.6 \mathrm{~g} / \mathrm{L}$ Tricaine Methane Sulfonate (TMS, Crescent Research Chemicals) buffered with $1.2 \mathrm{~g} / \mathrm{L} \mathrm{NaHCO}_{3}$ and were bled through the caudal vein. Total leukocytes were isolated from blood, spleen, head kidney, intestine and gills as described previously [38,39]. Cells ( 1 x 10\% sample) were fixed with 4\% paraformaldehyde (PFA) for 15 min on ice, washed with FACS buffer $(0.5 \%$ BSA, $0.01 \% \mathrm{NaN}_{3}$ in PBS) and incubated with chicken-anti-Igt(1/2) $(5 \mu \mathrm{g} / \mathrm{mL})$ and/or rabbit-anti-Igt(1/2) $(5 \mu \mathrm{g} / \mathrm{mL})$ for $45 \mathrm{~min}$ on ice. In order to test the reactivity of the antibodies to other cell types, double labelling was performed with an antibody against Igm $^{+}$B cells (WCI12, 1:100 [37]), or neutrophilic granulocytes (TCLBE8, 1:50 [40]), or monocytes/macrophages (WCL15, 1:50; [41]), or Cd4-1 ${ }^{+}$(clone 2C3) or $\mathrm{Cd} 8 \alpha 1^{+} \mathrm{T}$ cells (clone 6C10; both 1:5, kindly provided by Prof. T. Nakanishi, Nihon University, Japan [42]) or putative mucosal T cells (WCL38, 1:50 [43]). After washing, cells were incubated with the corresponding secondary antibodies: Alexa488-conjugated goat-anti-rabbit or rabbit-anti-chicken (1:2000, Abcam) and PE-conjugated goat-anti-mouse (1:200, Santa Cruz) or PE-conjugated goat anti-rat (1:200, Santa Cruz). Cells were analyzed on a FACS CantoA (BD Biosciences) and data were analyzed using FlowJo V10 (BD Biosciences).

\section{Immunohistochemistry}

Spleen, head kidney, intestine or gills of carp were aseptically removed and either snap-frozen in liquid nitrogen and stored at $-80^{\circ} \mathrm{C}$ for subsequent cryosectioning or fixed overnight in 4\% PFA and subsequently transferred to $70 \%$ ethanol until 
further processing for paraffin embedding. Cryosections $(5 \mu \mathrm{m})$ were stained as described before [38]. For chromogenic double staining slides were first labelled with rabbit-anti Igt1 or rabbit-anti-Igt2 $(5 \mu \mathrm{g} / \mathrm{mL})$ followed by incubation with alkaline phosphatase (AP)-conjugated goat-anti-rabbit (1:200, Dako) and development using AP substrate $(4.5 \mu \mathrm{L} / \mathrm{mL}$ nitro-blue-tetrazolium (Roche Applied Science) and 3.5 $\mu \mathrm{L} / \mathrm{mL} 5$ '-bromo-4'-chloro-3'indolyl phosphatase (BCIP; Roche Applied Science) in AP buffer ( $0.1 \mathrm{M}$ Tris-Cl, $\left.0.1 \mathrm{M} \mathrm{NaCl}, 0.05 \mathrm{M} \mathrm{MgCl}_{2}\right)$. Next, slides were incubated with the anti-Igm antibody (WCI12, 1:100), followed by incubation with HRPconjugated goat-anti-mouse (1:200, Dako) and development using AEC substrate (46 $\mu \mathrm{L}$ AEC and $1 \mu \mathrm{L} 30 \% \mathrm{H} 2 \mathrm{O} 2$ in $2 \mathrm{~mL}$ sodium-acetate buffer (pH 5) until sufficient coloration. Slides were rinsed in demi water, air-dried, embedded in Aquatex (Merck) and stored at $4^{\circ} \mathrm{C}$. Slides were visualized using a Nikon Microphot-FXA microscope with an Olympus DP50 camera and analysis-D software (Olympus).

Samples fixed in PFA and stored in 70\% ethanol were dehydrated as follows: $5 \mathrm{~min}$ $70 \%$ ethanol, $30 \mathrm{~min} 80 \%$ ethanol, $30 \mathrm{~min} 90 \%$ ethanol, $30 \mathrm{~min} 96 \%$ ethanol, $40-20$ $20 \mathrm{~min} 100 \%$ ethanol and $2 \times 30 \mathrm{~min}$ in xylene. Tissues were pre-incubated in paraffin for $1 \mathrm{~h}$ and were afterwards embedded in paraffin. Sections $(5 \mu \mathrm{m})$ were cut using a Microtome (Leica), incubated at $58{ }^{\circ} \mathrm{C}$ for $30 \mathrm{~min}$ and deparaffinised as follows; 2 x 5 min xylene, 2 x 5 min 100\% ethanol, 2 × 5 min 96\% ethanol, 2 x 5 min $70 \%$ ethanol, $2 \times 5$ min distilled water. Epitope retrieval was performed by incubation in citrate buffer $\left(10 \mathrm{mM}, \mathrm{pH}\right.$ ) for $15 \mathrm{~min}$ at $121^{\circ} \mathrm{C}$, followed by three washing steps with PBS after cooling down and peroxidase inactivation in $0.05 \%$ phenylhyrazin in PBS for $40 \mathrm{~min}$ at $37^{\circ} \mathrm{C}$. Blocking, antibody staining and development with AP- or AEC-substrate was performed as mentioned above.

\section{Mucus and bacteria isolation}

Carp were euthanized and skin mucus was gently scraped from the skin surface using a cell scraper. After collecting the skin mucus, heart perfusion was performed with PBS containing 50U heparin, after which the gills were removed. Mucus present on the outside of the gills was collected. For the collection of intestinal mucus, the gut was first cleaned by removing outside fat and vessels, after which it was gently squeezed to remove any remaining faeces. The intestine was opened longitudinally, scraped over a Petri dish and the obtained mucus was diluted in PBS containing a protease inhibitor cocktail (Roche). All obtained mucus (skin, gills, intestine) was collected on ice and immediately processed as follows: samples were vigorously 
vortexed and only the mucus was subsequently passed through a syringe using a $21 \mathrm{G}$ needle. A first centrifugation step (400 xg, $10 \mathrm{~min}$ ) was performed to pellets the cells and debris, followed by a second high-speed step (13.000xg, $10 \mathrm{~min})$ to pellet the bacteria and recover the mucus in the supernatant. Mucus was filtered using a 0.45 $\mu \mathrm{m}$ filter, aliquoted and frozen at $-80^{\circ} \mathrm{C}$.

\section{Phagocytosis assay}

The phagocytic ability of common carp B cells was examined as described previously [44]. Briefly, freshly isolated peripheral blood leukocytes (PBLs) were incubated in a 96-well flat-bottom culture plate (200.000 cells/well) containing either pHrodo particles (40 $\mu \mathrm{g} /$ well; Invitrogen) or PE-labelled beads of various sizes (1:10 cell:bead to ratio, Magsphere) in RPMI (Gibco) containing $2 \mathrm{mM} \mathrm{L-glutamine,} 100 \mu \mathrm{g} / \mathrm{mL}$ penicillin and $100 \mu \mathrm{g} / \mathrm{mL}$ streptomycin for $2 \mathrm{~h}$ at $27^{\circ} \mathrm{C}$. Cells and beads/particles were loaded onto a 3\% BSA/4.5\% glucose gradient and centrifuged for $10 \mathrm{~min}$ at $100 \mathrm{xg}$ to separate cell from non-phagocytosed beads, after which the supernatant was carefully removed and the pellet containing the cells was washed once with RPMI. The subsequent steps followed the Igm/Igt FACS staining protocol described above. Cells incubated with PE beads were analysed using a FACS CantoA (BD Biosciences) and data were analyzed using FlowJo V10 (BD Biosciences). Cells incubated with pHrodo particles were centrifuged on polysine glass slides and stained with DAPI to visualize the nuclei. Cells were imaged using a Leica DM6 microscope and analyzed using the Leica LAS X program.

\section{Infection with Sphaerospora molnari and sample processing}

Carp (7 months old) were infected by i.p. injection of 150.000 freshly isolated $S$. molnari parasites $[45,46]$ in PBS. 42 days later, when parasite levels were $7.6 \mathrm{x}$ $10^{3}$ parasites/ $\mu \mathrm{L}$ blood, fish were euthanized. Gills were isolated and were either stored in RNALater until further processing for RNA isolation and gene expression analysis, or were incubated in neutral buffered formalin for $20 \mathrm{~h}$ and transferred to $70 \%$ ethanol for subsequent processing for paraffin embedding and sectioning.

\section{RNA isolation and cDNA synthesis}

Total RNA from gills from healthy carp or carp infected with S. molnari was isolated using the RNeasy Mini Kit (Qiagen) according to the manufacturer's instructions including on-column DNase treatment using the RNase-free DNase kit (Qiagen). 
Tissue disruption and homogenization was performed using a TissueLyserII (Qiagen). RNA concentrations were measured using a Nanodrop-1000, the integrity was verified on a $1 \%$ agarose gel and RNA was stored at $-80^{\circ} \mathrm{C}$ until further use. Prior to cDNA synthesis of $1 \mu \mathrm{g}$ total RNA, a second DNase treatment was performed using DNAse I, Amplification Grade (Invitrogen). Reverse transcription of the RNA was performed using random primers (300 ng) and Superscript ${ }^{\mathrm{TM}}$ III (200U) First Strand Synthesis Systems for RT-PCR (Invitrogen). cDNA samples were further diluted 25 times in nuclease-free water and stored at $-20^{\circ} \mathrm{C}$.

\section{Gene expression analysis}

Real-time quantitative PCR (RT-qPCR) was performed on a Rotor-Gene ${ }^{\mathrm{TM}} 6000$ (Qiagen) using ABsolute qPCR SYBR Green Mix (Thermo Scientific) as detection chemistry as described before [47]. Obtained fluorescence data were analyzed using Rotor-Gene Q series software version 2.3.1. The list of primers can be found in Table 1. The take-off value for each sample and the average reaction efficiencies $(E)$ for each primer set were obtained upon comparative quantitation analysis from the Rotor-Gene software [48]. The relative expression ratio (R) of each sample was calculated according to the Pfaffl method [49] based on the take-off deviation of sample versus each of the unhandled time point controls and normalized relative to the $s 11$ protein of the ribosomal 40s subunit as reference gene.

Table 1. Primers used for Real-Time quantitative PCR analysis

\begin{tabular}{|c|c|c|c|}
\hline Primer & FW primer 5'-3' & RV primer 3'- 5' & Acc. No. \\
\hline \multicolumn{4}{|c|}{ Housekeeping genes } \\
\hline $40 s$ & CCGTGGGTGACATCGTTACA & TCAGGACATTGAACCTCACTGTCT & AB012087 \\
\hline \multicolumn{4}{|c|}{ Adaptive immune genes } \\
\hline igm & CACAAGGCGGGAAATGAAGA & GGAGGCACTATATCAACAGCA & AB004105 \\
\hline igt1 & AAAGTGAAGGATGAAAGTGT & TGGTAACAGTGGGCTTATT & AB598367 \\
\hline igt2 & GATTCTACTGGGT8CTTCAC & GACATCACTCAACTC8TTCT & AB598368 \\
\hline
\end{tabular}

\section{Infection with Trypanoplasma borreli and sample collection}

Carp (9-months-old) were intra-peritoneal (i.p.) infected with 1 x $10^{4} \mathrm{~T}$. borreli parasites [50], as described before [38]. At 10 days and 3, 4 and 6 weeks after infection, carp were euthanized and the blood was isolated from the caudal vein for 
subsequent serum isolation; in parallel spleen from $n=3$ control (non-infected) and $\mathrm{n}=5$ infected carp were isolated and either immediately snap-frozen in liquid nitrogen until processing for immunohistochemistry or stored in RNALater for subsequent RNA isolation and gene expression analysis. T. borreli parasites were isolated from the blood as described before [38].

\section{Trypanoplasma borreli binding assay}

Isolated parasites (10.000/sample) were incubated for $2 \mathrm{~h}$ at $4^{\circ} \mathrm{C}$ in $\mathrm{PBS}$ containing $0.5 \%$ BSA. After washing, parasites were incubated for $2 \mathrm{~h}$ at $4^{\circ} \mathrm{C}$ with $\mathrm{PBS}$ containing serum from either naïve carp or serum isolated at the peak of parasitaemia (3 weeks post-infection). Parasites were washed twice with cold PBS and the pellet was resuspended in loading buffer containing $\beta$-mercaptoethanol. Samples were processed for SDS-PAGE and Western blot analysis as described above, using rabbit anti-Igt1 and anti-Igt 2 antibodies. In parallel, parasites were centrifuged on polysine slides after the last wash with PBS and were labelled with anti-Igm (WCI12; 1:50) and rabbit anti-Igt1 $(5 \mu \mathrm{g} / \mathrm{mL})$ in PBS containing 0.5\% BSA. After three washes with PBS, slides were incubated with Alexa-488-conjugated goat-anti-rabbit (1:2000, Abcam) and PE-conjugated goat-anti-mouse (1:200, Santa Cruz). A staining with DAPI was included to visualise the cell nuclei. Slides were imaged using a EVOS fl LED fluorescence microscope (Advanced Microscopy Group (AMG)).

\section{Results}

\section{European common carp has two igt genes}

Previous studies in Japanese common carp reported the presence of two igt subclasses and were designated igzl and igz2 $[15,16]$. For clarity, in the current manuscript we will refer to them as igt 1 and igt 2 complying with the original nomenclature referring to a novel immunoglobulin type in teleost (Igt) [3]. Given the slight difference often observed between European and Japanese common carp, we took advantage of the availability of the European common carp genome and transcriptome [34] to further verify the protein sequences prior to recombinant protein production. Using the Japanese common carp igt sequence as reference for a BLAS search, two genes for European common carp igt were retrieved and were designated igt 1 and igt 2 based on their highest similarity to the corresponding Japanese common carp igt sequence. 
For igt 1 we retrieved a sequence of 1247 bp that translated into a protein of 414 amino acids (aa) composed of four constant Ig domains (Supplementary Fig. 1A). For the igt2, a shorter sequence of $665 \mathrm{bp}$ was retrieved that translated into a protein of 220 aa (Supplementary Fig. 1B) composed of two constant Ig domains.

\section{Preliminary validation of the specificity of antibodies against recombinant Igt1 and Igt2}

Polyclonal antibodies were raised against Igt1 and Igt 2 by immunization of chicken and rabbits with recombinant proteins corresponding to the internal $\mathrm{CH} 2 \tau_{1}$ and $\mathrm{CH} 3 \tau_{1}$ domains of Igt1, or the $\mathrm{CH} 1 \tau_{2}$ and $\mathrm{CH} 2 \tau_{2}$ domains of Igt2. The choice for the Igt1 domains was based on the low similarity to those of Igm or Igt2. For Igt2, both constant domains were chosen being aware that the $\mathrm{CH} 1 \tau_{2}$ domain shows high similarity with the $\mathrm{CH} 1 \mu$ of Igm and the $\mathrm{CH} 2 \tau_{2}$ domain shows similarity to the $\mathrm{CH} 4 \tau_{1}$ of Igt1. Total IgG (from rabbits) and total IgY (from chicken), were preliminary tested for their ability to specifically recognize the corresponding (autologous) recombinant proteins. In parallel the monoclonal antibody against carp Igm was also tested for its cross-reactivity to recombinant Igt1 and Igt2 (Fig. 1A). As expected, anti-Igt1 antibodies specifically recognized the recombinant Igt1 domains $(25 \mathrm{kDa})$, and anti-Igt2 antibodies reacted to the recombinant Igt2 domains $(25 \mathrm{kDa})$. The antiIgm monoclonal antibody did not react to any of the recombinant proteins. After assessing the specificity of the antibodies by Western blot analysis, we determined the sensitivity and specificity of the antibodies by ELISA. All antibodies could reliably recognize the autologous recombinant protein at concentrations lower than $30 \mathrm{ng} / \mathrm{ml}$ (Fig. 1B), with the exception of the IgY anti-Igt2 which showed a lower sensitivity. Both total IgG and total IgY against Igt1 did not react against recombinant Igt2, and vice versa (Fig. 1B, dotted lines). Together, we show that the antibodies raised against European common carp Igt 1 and Igt 2 show a specific reactivity to the recombinant proteins in Western blot and ELISA analysis. 

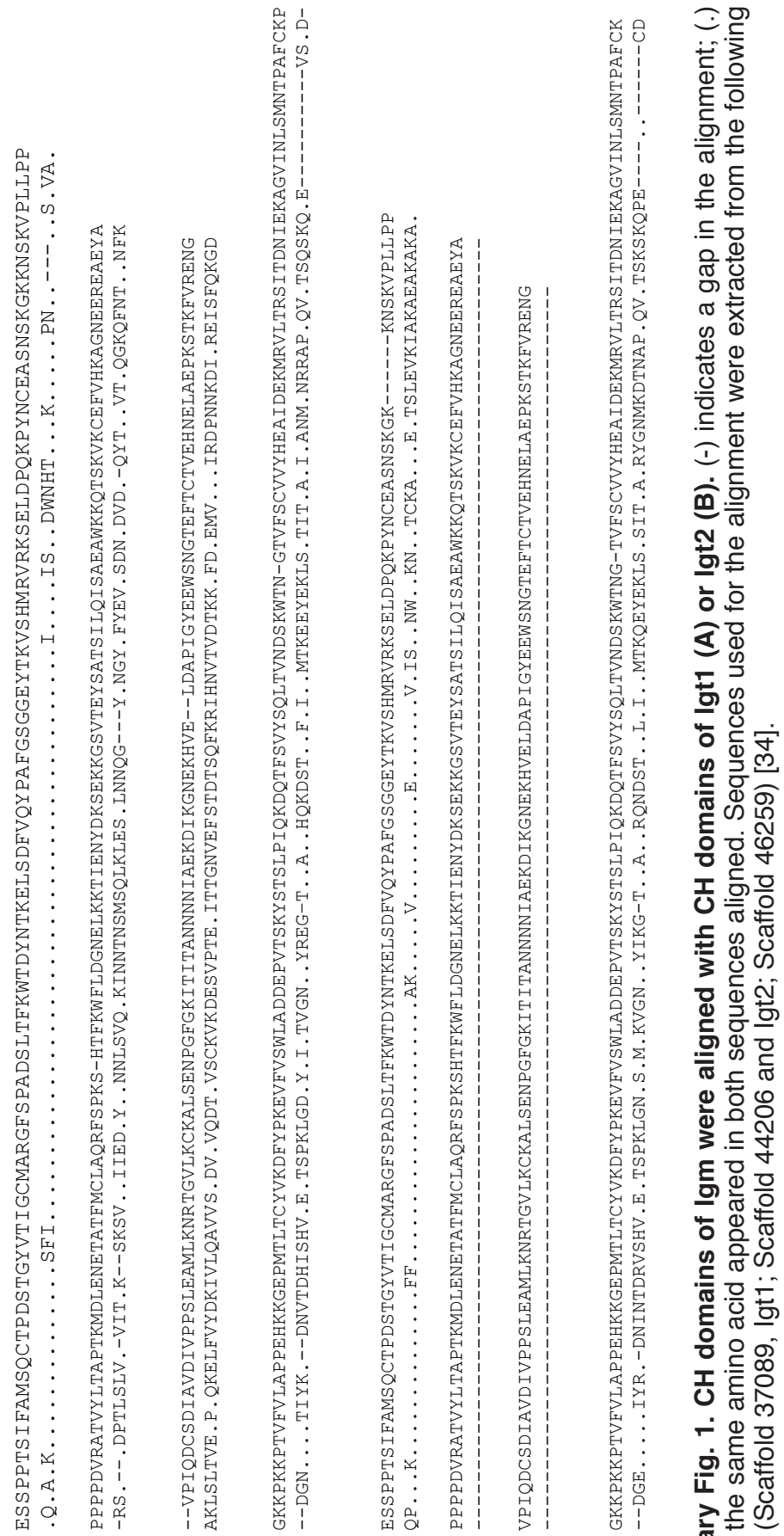

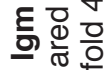

응

क $\frac{0}{\circ} \omega$

흐음

응

던

$\dot{1} \stackrel{0}{0}$

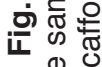

$\geq \stackrel{0}{\oplus}$

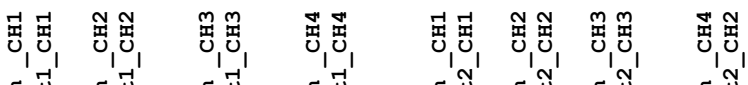

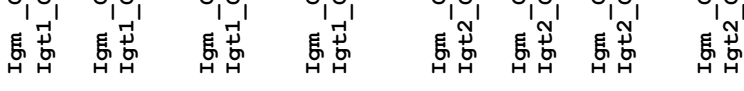
要索

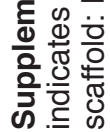



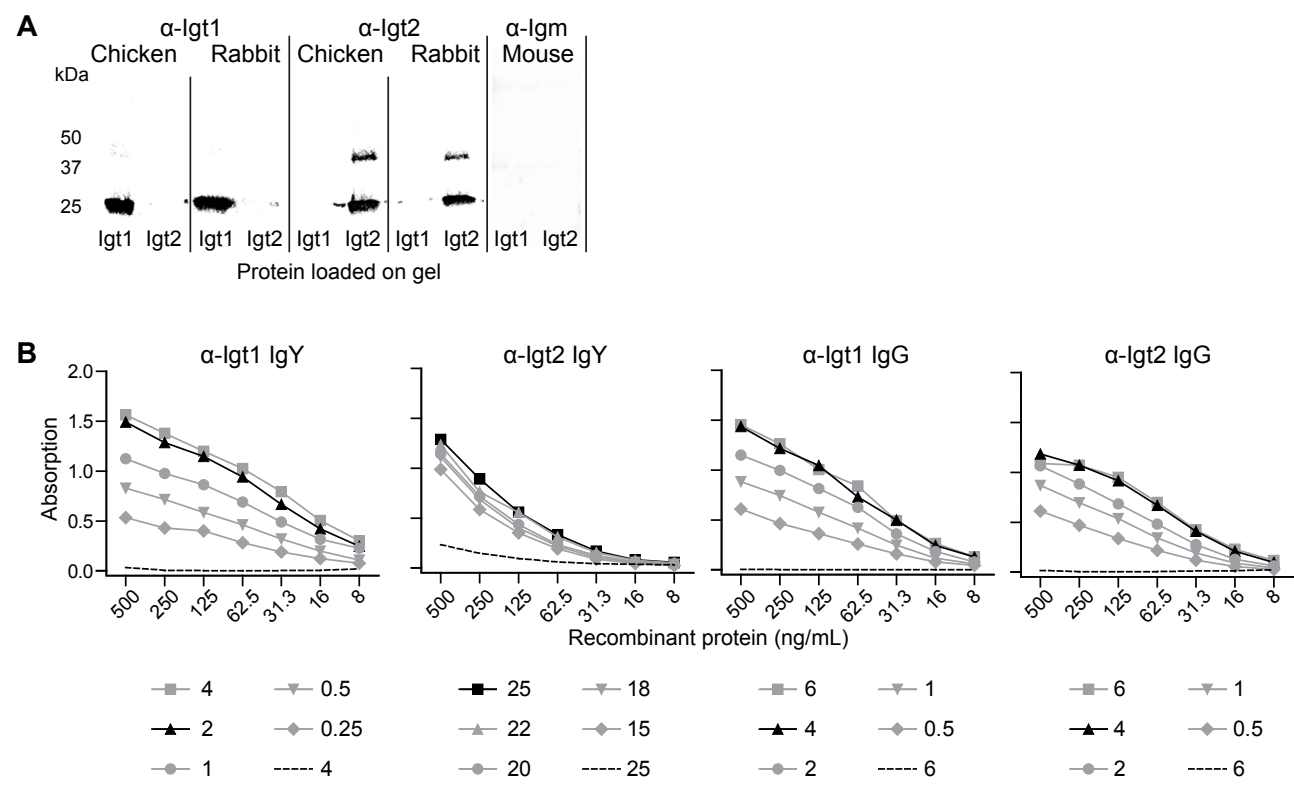

Recombinant protein $(\mathrm{ng} / \mathrm{mL})$

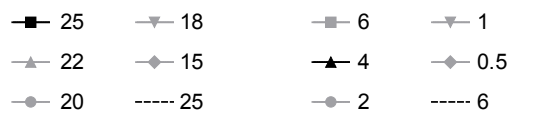

$$
\begin{aligned}
& \text { Antibody concentrations (total } \lg Y \text { or } \operatorname{lgG} \text { ) in } \mu \mathrm{g} / \mathrm{mL}
\end{aligned}
$$

Fig. 1. Validation of antibody specificity to recombinant Igt1 or Igt2. A) Recombinant proteins (100 ng/lane) were loaded on 4-20\% SDS-PAGE under reducing conditions. Western blot analysis was performed using total $\lg G$ from rabbits and total $\lg Y$ from chicken (all 5 $\mu \mathrm{g} / \mathrm{mL}$ ), or a 1:100 dilution of the anti-Igm monoclonal antibody (WCl12). B) Plates were coated with the indicated concentrations of recombinant proteins. Various concentrations of antibodies were used to detect the autologous recombinant proteins (full lines) or the heterologous recombinant protein (dotted lines) coated at the same concentration. Black lines indicate the concentration that was selected as optimal for detection of the recombinants. Absorption was measured at $O D_{405 \mathrm{~nm}}$.

\section{Polyclonal antibodies recognize native carp Igt1 and lgt2}

After confirming that the antibodies raised in chicken and rabbit specifically recognize the recombinant Igt1 and Igt2, we set out to validate their reactivity to native Igt1 and Igt2. Prior to ELISA analysis, rabbit total IgG were further affinity purified against the respective proteins whereas total IgY were used as polyclonal pool. Using serum of naïve carp, no signal was obtained using the chicken anti-Igt1, anti-Igt2, or the rabbit anti-Igt1, whereas a strong reactivity was obtained when using the rabbit-antiIgt2 (Fig. 2A). The absorption levels however, completely matched those of the antiIgm antibody (clone WCI12), hinting at the possibility that the rabbit-anti Igt2 might cross-react with Igm. In a subsequent sandwich ELISA, where we coated with the anti-Igm antibody (WCI12) and then used the chicken or rabbit anti-Igt antibodies to detect WCI12-captured immunoglobulins, we confirmed that the affinity purified rabbit anti-Igt2, but not the other antibodies, cross-react with carp Igm (Fig. 2B). 
The specificity of the anti-Igt1 and anti-Igt2 polyclonal antibodies was further investigated by Western blot (Fig. 2C). A specific reactivity of rabbit and chicken antiIgt1 antibodies to protein of the expected size for carp Igt1 (61 kDa) was observed in naïve serum (Fig. 2C, lanes 1-2). In naïve serum, chicken anti-Igt2 antibodies did not show any reactivity (lane 3 ), whereas the rabbit anti-Igt2 showed reactivity to a high molecular weight protein corresponding to carp Igm (lanes 4 and 5). When using sera from parasite-infected fish, chicken anti-Igt2 reacted to two high molecular weight (75 and $100 \mathrm{kDa}$ ) proteins (lane 6); based on the intensity of the signal however, the $75 \mathrm{kDa}$ protein does not correspond to the Igm molecule in the same serum (lane 8). Using the same parasite-infected serum, rabbit anti-Igt 2 recognized a high (75 $\mathrm{kDa}$ ) as well as low molecular weight protein (39 kDa) (Fig. 2C, lane 7). The high molecular weight protein, based on the intensity of the reaction, corresponded to carp Igm (lane 8). The low molecular weight protein was of the expected size of carp Igt2 $(39 \mathrm{kDa})$ and was induced during infection.

Altogether the data from the western blot analysis indicate that both rabbit and chicken anti-Igt1 specifically recognize the native Igt1 protein in carp serum; rabbit anti-Igt2 recognize the native protein in carp serum, but they also show crossreactivity to carp Igm. The cross-reactivity is most likely due to the similarity of the $\mathrm{CH} 1 \tau_{2}$ domain to the $\mathrm{CH} 1 \mu$ of Igm (supplementary Fig. 1B). Chicken anti-Igt2 did not show cross-reactivity to the native Igt2 protein but revealed the presence of additional high molecular weight proteins that require further investigation. For this reason, from now on, the affinity purified rabbit anti-Igt1 was used to further characterize the presence of soluble Igt1 and $\operatorname{Igt}^{+} \mathrm{B}$ cell distribution, whereas the affinity purified rabbit anti-Igt 2 was preferentially used for western blot analysis and for preliminary immunohistochemical analysis, aware that the staining would reflect a mixed $\operatorname{Igm}^{+} / \operatorname{Igt} 2^{+}$population.

\section{Characterization of B cell populations of common carp by flow cytometry}

Next, the antibodies were used to characterize the distribution of $\operatorname{Igm}^{+}$and $\operatorname{Igt1}^{+} \mathrm{B}$ cell populations in common carp by flow cytometric analysis. Preliminary analysis in peripheral blood leukocytes (PBLs) revealed that while no reactivity was observed using freshly isolated non-fixed cells, an additional fixation step with 4\% PFA allowed us to visualize $\operatorname{Igt} 1^{+} \mathrm{B}$ cells This suggests that an epitope demasking step is required for the antibodies to recognize their respective Igt1 epitopes. 
In agreement with the ELISA and western blot analysis, rabbit and chicken anti-Igt1 specifically recognized a distinct population of Igt $1^{+} \mathrm{B}$ cells, and no cross-reactivity with $\mathrm{Igm}^{+} \mathrm{B}$ cells was observed (Fig 2D). However, while the chicken anti-Igt2 reacted to lymphoid-like cells that were Igm; the rabbit-anti-Igt2 antibody crossreacted with $\mathrm{Igm}^{+}$cells as expected (Fig. 2E). Interestingly, also Igm-Igt2 ${ }^{+}$cells were observed (Fig. 2E, white arrowheads).

We next characterized the percentages of Igt $1^{+}$and Igm $^{+} \mathrm{B}$ cells in various organs (Fig. 2F). Although Igm ${ }^{+}$B cells were the most abundant B cell subtype in all tested organs, Igt $1^{+}$cells were present at lower percentages in all investigated organs. PBLs and spleen contained the highest amount of Igt $1^{+}$cells and in spleen and gills.

Altogether, we generated polyclonal antibodies against carp Igt1 and Igt2. The rabbit and chicken antibodies against carp Igt 1 specifically recognize Igt1 in western blot and flow cytometric analysis, and do not show any cross reactivity to Igm. The rabbit anti-Igt2 however showed cross reactivity to Igt2 and Igm in ELISA, Western blot and flow cytometric analysis.

\section{Characterization of Igm and Igt at mucosal sites}

After having characterized the distribution of $\operatorname{Igm}^{+}$and Igt $1^{+} \mathrm{B}$ cell subsets in various organs, we next investigated to presence of soluble Igm and Igt1/2 in skin, intestine and gill mucus and bacteria, as well as the distribution of $\mathrm{Igm}^{+}$and $\mathrm{Igt}^{+}$cells in the intestine. Western blot analysis revealed (Fig. 3A) that there seems to be site-specific preferences with regards to the coating of mucosal bacteria; while skin bacteria are mainly coated with Igm and Igt2, intestinal bacteria are preferentially coated with Igt1 and to a lesser extent with Igt2. Gill bacteria, on the other hand, show the highest coating with Igt 2 and to a lesser extent with Igm and Igt1. Similar trends were observed in the mucus, although in the intestinal mucus only weak signal was detected, possibly due to a quick protein breakdown.

Next, we visualized the presence of the different B cell subtypes in the intestine by immunohistochemistry. Given that the chicken antibodies did not gave any signal in immunohistochemistry, the rabbit-anti-Igt2 was used, despite the previously assessed cross-reactivity with Igm. Igm ${ }^{+} \mathrm{B}$ cells were most abundant in the lamina propria, whereas Igt $1^{+}$cells could be observed at the basal side of the intestinal epithelium (Fig 3B, upper panel). Double labelling between Igm and Igt1 further confirmed the spatial segregation of $\operatorname{Igm}^{+}$and Igt1 ${ }^{+}$B cells in the intestine. Strikingly, while Igm ${ }^{+}$ 
A

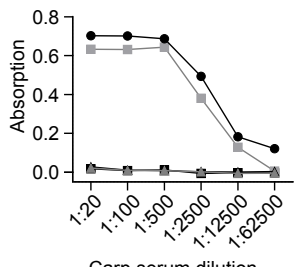

Carp serum dilution

$\rightarrow-\alpha-\operatorname{lgt} 1 \lg G \quad \leftarrow a-\lg 11 \lg Y$

$-\alpha-\operatorname{lgt} 2 \lg G \rightarrow \alpha-\lg 2 \lg Y$

$\rightarrow-\alpha-\operatorname{lgm}$
B

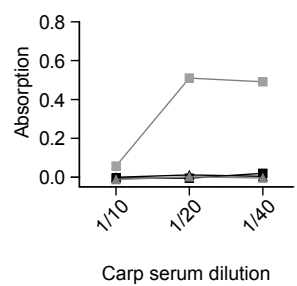

$-\alpha-\operatorname{lgt} 1 \lg G \quad \leftarrow \alpha-\lg t 1 \lg Y$

- - $-\operatorname{lgt} 2 \lg G \rightarrow \alpha-\operatorname{lgt} 2 \lg Y$
C

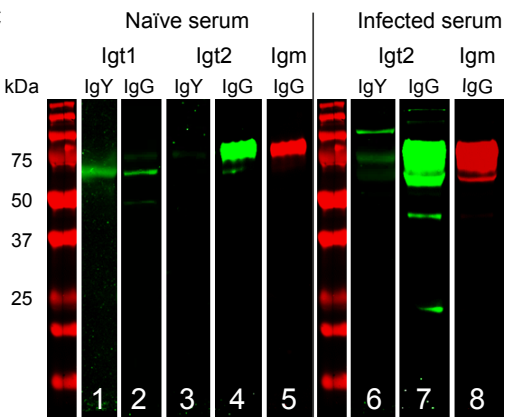

D

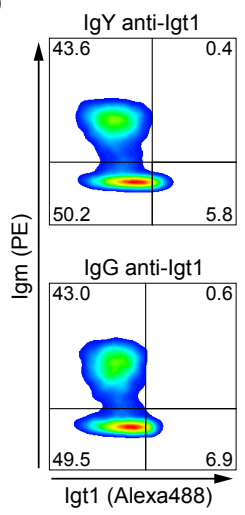

IgG anti-lgt1

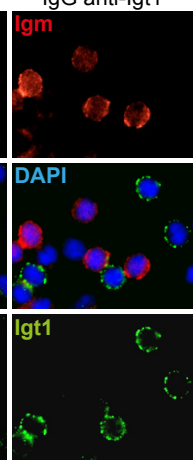

E

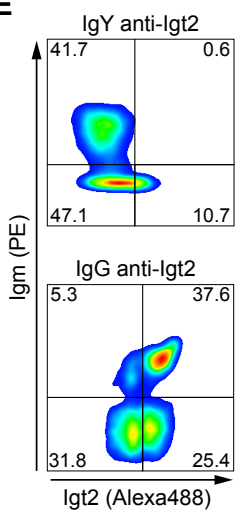

$\lg Y$ anti-lgt2

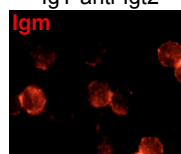

IgG anti-lgt2

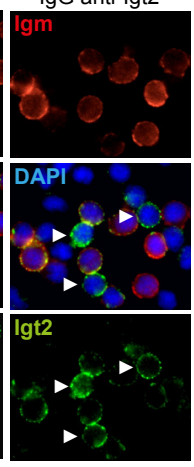

$\mathbf{F}$

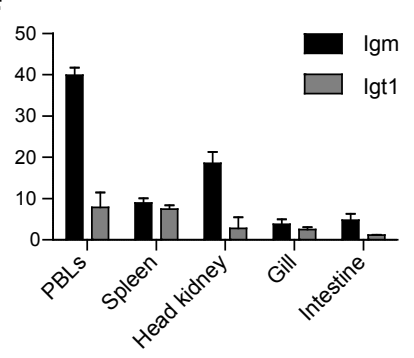

Fig. 2. Reactivity and specificity of anti-lgt1 and anti-lgt2 antibodies against native Igt1 and Igt2. A) rabbit IgG and chicken IgY were used in an indirect ELISA to detect Igt1 and Igt2 in naïve carp serum. Purified mouse anti-Igm (WCl12) was used as positive control. B) AntiIgm antibody (WCl12) was used as capture antibody, followed by incubation with naïve carp serum diluted in PBS. The indicated anti-lgt1 and anti-lgt2 were used as detection antibodies using concentrations as in (A). C) Western blot analysis of the reactivity of rabbit and chicken affinity purified IgG or IgY to naïve Igt1 or Igt2. Serum from naïve (8 $\mu \mathrm{g} / \mathrm{lane}$ ) or parasite infected carp ( $1 \mu \mathrm{g} / \mathrm{lane})$ was resolved on an SDS-PAGE gel under reducing condition as indicated; affinity purified rabbit anti-lgt1 or anti-lgt2, total chicken IgY anti-lgt1 or anti-lgt2, or mouse-anti-lgm (WCl12) were used, followed by incubation with donkey anti-mouseIRDye-680 (red signal), donkey anti-rabbit-IRDye-800 (green signal), or donkey anti-chickenIRDye-800 (green signal). Fluorescence signal was analyzed with an Odyssey scanner. D,E) PFA-fixed PBLs were labelled with the chicken or rabbit anti-lgt1 (FITC, left panel) or anti-lgt2 (FITC, right panel) in combination with anti-lgm (WCI12; PE) and analysed using a FACS Canto A (BD Biosciences). After labelling, a small sample was centrifuged on a polysine slide, stained with DAPI to visualize the cell nuclei and imaged using a Leica DM6 microscope. (F) 
Total leukocytes from the indicated organs ( $n=3$, except for PBLs, $n=6$ ) were labelled and analysed as in (D). Bars indicate the average $+S D$ of the percentage of $\operatorname{lgm}{ }^{+}, \lg 11^{+}$and $\lg 2^{+}$ cells in the total leukocyte population.

and Igt $2^{+}$cells were found to be located strictly in the lamina propria of the intestine (Fig. 3B, black arrows), Igt $1^{+}$cells could not be detected in the lamina propria but instead were detected in the epithelial layer. Even more, some $\operatorname{Igt} 1^{+}$cells at the border of the epithelial layer seemed to be actively secreting immunoglobulins into the intestinal lumen (Fig. 3B, green arrow). The presence of $\operatorname{Igt} 1^{+}$, but not $\operatorname{Igt} 2^{+} \mathrm{B}$ cells, at the luminal site might relate to the higher amount of Igt1 coating intestinal bacteria. Overall, we show that Igm, Igt1 and Igt 2 in mucus and on bacteria show differential distribution and that $\operatorname{Igm}^{+}, \operatorname{Igt}^{+}$and $\operatorname{Igt} 2^{+} \mathrm{B}$ cells are spatially segregated in the intestine. If they also serve different roles requires further investigation.

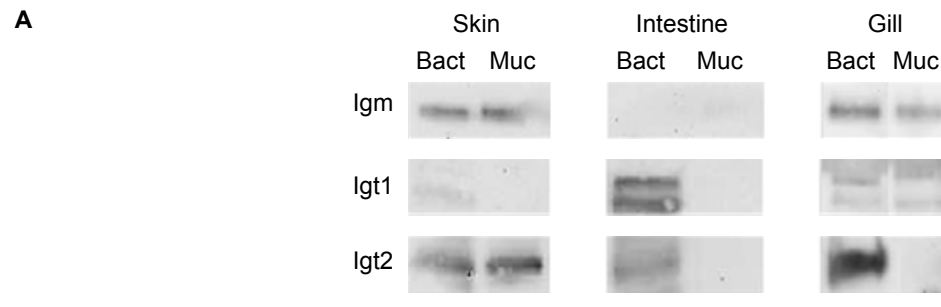

B
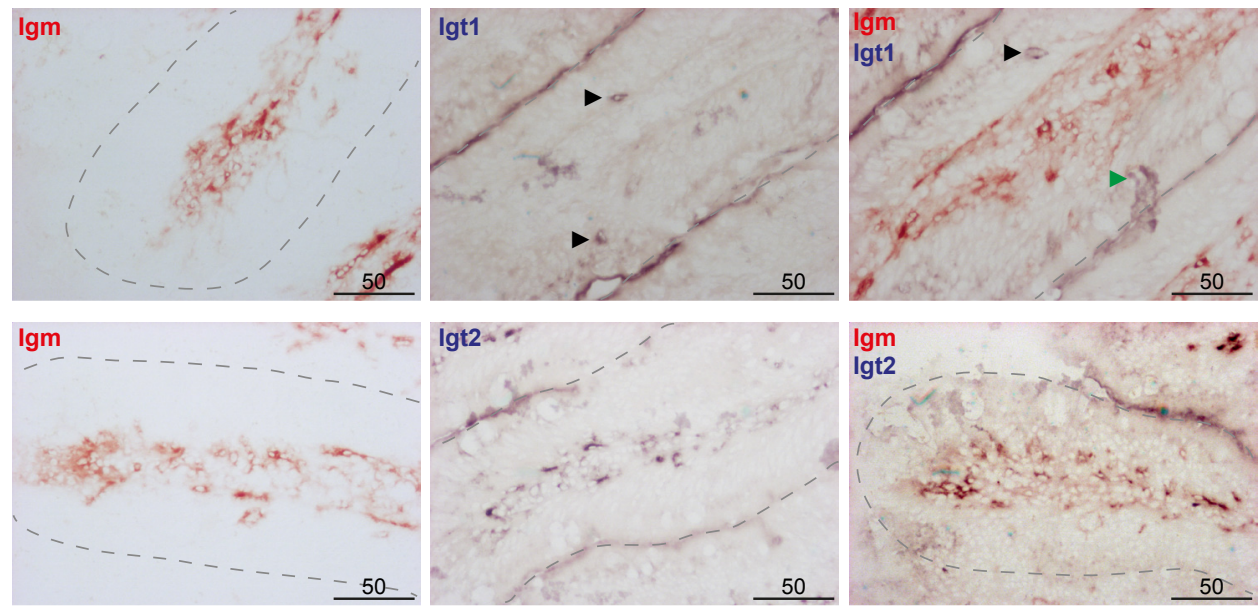

Fig. 3. Distribution of different Ig types in bacteria and mucus of skin, intestine and gills, and localisation in the intestine. A) Bacteria (equivalent to $100 \mu \mathrm{L}$ mucus) and mucus (5 $\mu \mathrm{g} / \mathrm{lane}$ ) from skin, intestine and gills were loaded onto SDS-PAGE gels and the presence of different Igs (Igm, Igt1 and Igt2) was analysed by Western blot using the anti-lgm antibody (WCl12), rabbit-anti Igt1 and rabbit anti-lgt2. B) Consecutive cryosections $(5 \mu \mathrm{m})$ from the second intestinal segment were labelled with rabbit-anti-Igt1 or anti-lgt2 (middle panel), mouse anti-lgm (clone WCl12) (left panel), or a were double labelled using the indicated anti-Igt1/2 antibodies and anti-lgm (right panel), followed by goat-anti-mouse HRP and goat- 
anti-rabbit AP. Slides were imaged using a Nikon Microphot-FXA microscope with an Olympus DP50 camera. Black arrows indicate Igt1 ${ }^{+}$cells, the green arrow indicates an Igt $1^{+}$cell that possibly secretes Igt1 into the lumen. Scalebars indicate $\mu \mathrm{m}$.

\section{Common carp Igm and Igt1 cells are phagocytic and form phagolysosomes}

It was previously reported that common carp $\operatorname{Igm}^{+} \mathrm{B}$ cells are able to phagocytose [51] and now, using our newly developed antibodies, we were able to examine whether also common carp Igt $1^{+}$cells have this ability. We examined this in two ways: with bright fluorescent beads that allow for flow cytometric analysis, and pHrodo particles that are $\mathrm{pH}$ sensitive and allow for evaluation of the formation of phagolysosomes after ingestion of particles. For both assays, freshly isolated PBLs were incubated for 2 hours with either the fluorescent beads or the pHrodo particles. Using the fluorescent beads, we show that $\operatorname{Igm}^{+}$and $\operatorname{Igt}^{+}$cells are both able to phagocytose fluorescent beads of $0.5,1$ and $2 \mu \mathrm{m}$ in vitro (Fig. 4A, B). Using the $\mathrm{pH}$-sensitive pHrodo particles we show that both $\operatorname{Igm}^{+}$and $\operatorname{Igt}^{+}$cells form phagolysosomes after ingestion of particles (Fig. 4C, D), suggesting that they both might be able of intracellular killing and clearance of microbes. Some of the cells that ingested pHrodo particles showed an enlargement of their cytoplasm and deformations of their outer membrane, most likely caused by the ingestion of particles. Combining the results from both assays we show that $\mathrm{Igm}^{+}$and $\operatorname{Igt}^{+} \mathrm{B}$ cell subsets are able to phagocytose fluorescent beads in vitro and that the uptake of $\mathrm{pH}$-sensitive particles results in the formation of phagolysosomes. The relevance of this phagocytic ability in vivo requires further investigation.

\section{B cell responses to the gill parasite $S$. molnari}

In parallel to characterizing the distribution of Igm and Igt in gill mucus and on gill bacteria (Fig. 5A), we also examined the response of $\operatorname{Igm}^{+}$and $\operatorname{Igt}^{+}$cells in the gills, using a model infection with the parasite Sphaerospora molnari (S. molnari). Due to the lack of reactivity of the chicken anti-Igt2 in histology and due to the cross-reactivity of the rabbit anti-Igt2, we decided to exclude Igt 2 from the analysis. Although this parasite has a blood-stage, it predominantly manifests in the gills of infected fish $[45,46]$, making it a good model to study immune responses in gills. Gills were isolated at $42 \mathrm{~d}$ post-infection and processed for immunohistochemical and gene expression analysis. Paraffin slides were labelled with mouse-anti-Igm

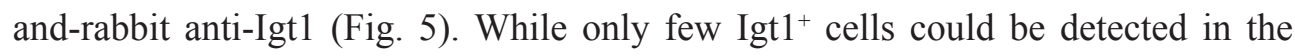


gills of healthy control carp (Fig. 5B, arrowheads), a higher number of $\operatorname{Igt} 1^{+}$cells was recruited to the gills upon infection (Fig. 5A, arrowheads), especially to the gill filaments, where parasites normally manifests. Due to the strong background obtained upon AP staining, no clear $\operatorname{Igm}^{+}$cells could be identified in both control and infected fish. In parallel, while no upregulation of Igm was observed at this time point after infection, a strong increase of both igtl and igt 2 transcripts was observed (Fig. 5C). This shows that at least at this time-point, $\operatorname{Igt} 1^{+}$and $\operatorname{Igt} 2^{+} \mathrm{B}$ cells might play a role in the gills response to $S$. molnari infection.
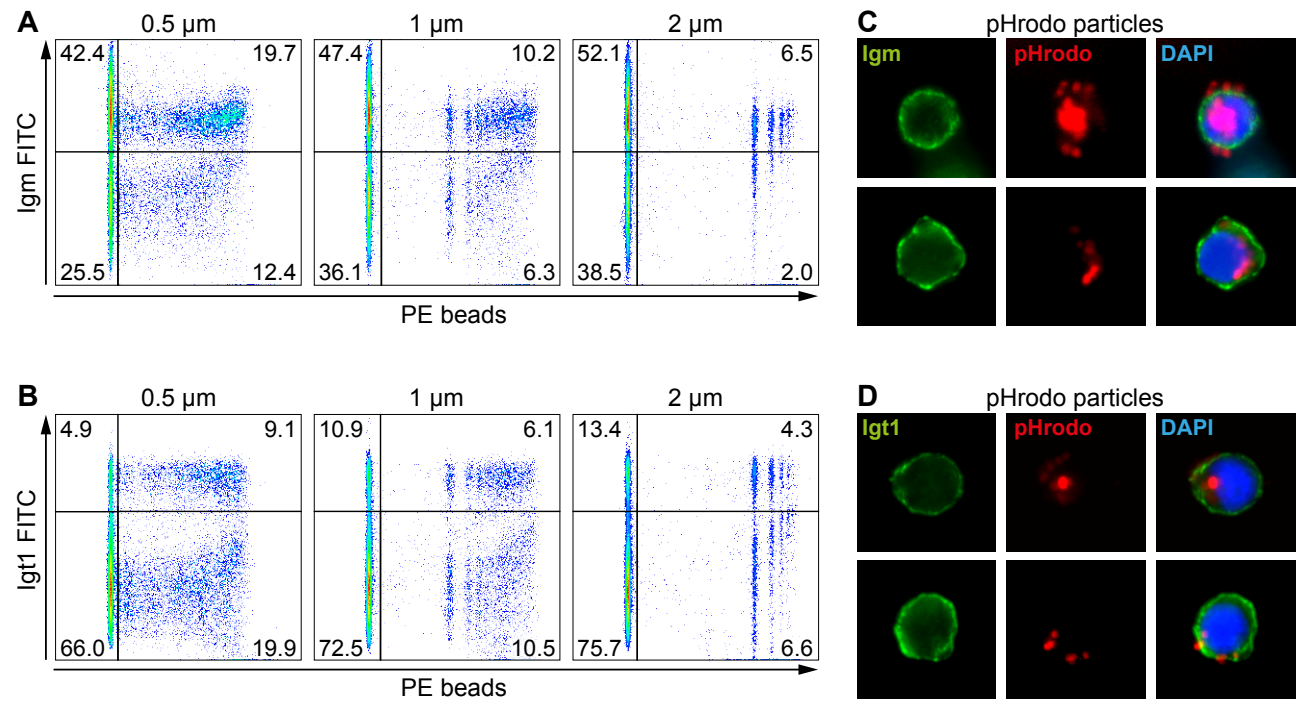

Fig. 4. Phagocytic ability of common carp $\operatorname{lgm}^{+}$and $\operatorname{lgt} 1^{+} B$ cells. A,B) PBLs were incubated for $2 \mathrm{~h}$ at $27^{\circ} \mathrm{C}\left(1 \times 10^{6}\right.$ cells/well of a 96-well plate) with PE fluorescent beads of different sized at a cell:bead ratio of $1: 10$, or with $40 \mu \mathrm{g}$ of pHrodo particles. After separation of non-ingested beads by density gradient centrifugation, cells were PFA-fixed and labelled with mouse-anti-Igm (WCI12) orrabbit-anti-Igt1. Cells incubated with fluorescent beads were analysed using a BD CantoA flow cytometer (BD Biosciences) $(\mathbf{A}, \mathbf{B})$ and cells incubated with pHrodo particles were centrifuged on a polysine glass slide, counterstained with DAPI to visualize the cell nuclei and imaged using a DM6 microscope (Leica) $(\mathbf{C}, \mathbf{D})$.

\section{B cell responses during infection with the blood-borne parasite T. borreli}

Previously it was reported that igt1, but not igt2, was strongly upregulated upon infection with the blood-borne parasite Trypanoplasma borreli [15]. However, since this observation was based on gene expression in spleen at one time point only, we used our newly developed antibodies to further characterize the role of the different used our newly developed antibodies to further characterize the role of the different $\mathrm{B}$ cell subsets during this infection. To demonstrate that both Igm and Igt are able to 
bind $T$. borreli and that parasite-specific antibodies are generated during infection, parasites were incubated with serum from naïve and infected carp and subsequently processed for either Western blot analysis or immunofluorescent labelling. Western blot analysis revealed that in serum from naïve fish low levels of (natural) Igm, and to a lesser extent Igt1 were present and would bind to the parasites (Fig. 6A lane 2-4). In serum from 3 weeks-infected fish, a strong increase in the binding was observed for all immunoglobulin types, indicating an increase in parasite-specific antibodies (Fig.6A lane 5-7). Based on the intensity of the signal, higher amount of Igm and Igt1 than Igt 2 were present in serum of infected fish. The lack of signal for Igt1 in lane 5 of Fig. 6A is due to overexposure of the film. No Igs were detected in lysates of parasites that were not incubated with serum (Fig. 6A, lane 1). In agreement with the Western blot analysis, immunofluorescence staining clearly reveals the presence of Igm and Igt1 on the parasite surface (Fig. 6B, Igt2 was not tested in this assay). Using the optimized sandwich ELISA, we were able to quantify the serum levels of total Igt1 and Igt2. As expected, Igm titers significantly increased upon infection (Fig. 6C). Currently, due to the lack of purified or recombinant Igm, the protein concentration could not be quantified. In contrast to Igm, we show that hardly any serum Igt can be detected in naïve carp. However, a strong increase was observed during infection where higher concentrations were detected for Igt1 $(77 \mathrm{ng} / \mathrm{mL})$ than for Igt2 (65 ng/mL ) (Fig. 6D).

Besides serum Ig, the presence of the different B cell subsets was analyzed in spleen by immunohistochemical analysis. During infection we observed an increase in the number of $\mathrm{Igm}^{+}$and $\mathrm{Igt1}^{+} \mathrm{B}$ cells, of which the latter was rarely present in healthy tissue (Fig. 6E). The same can be seen for Igt2, but given the cross-reactivity of the rabbit polyclonal with carp Igm, it is not clear what proportion of cells are actually Igt2 single positive B cells.

When analyzing the kinetics of igm, igt1 and igt2 gene expression in spleen during infection, we observe an increase in igt 2 transcription already 10 days post-infection (dpi), which gradually decreases overtime (Fig. 6F). Igt1 was also increased at 10 dpi, but reached its highest expression at 3 weeks post-infection (wpi), concomitantly with the peak of parasitaemia, and remained high up until 6 wpi when parasite levels significantly declined. Finally, igm transcripts were increased at 3 and 4 wpi, but were back almost to control levels at 6 wpi. These data indicate that not only igm transcription but also igt1 and igt 2 increase during $T$. borreli infection, and that they 
differ in their kinetics of expression. In conclusion, we show that all tested Ig subsets (Igm, Igt1 and Igt2) respond to an infection with the blood-borne parasite T. borreli and that depending on the time point or organ analysed, differences in reactivity of the different Ig subsets can be founds. These results show for the first time that common carp Igt1 and Igt2 respond strongly also to a systemic infection.

Finally, in this paper were able to study Igm, Igt1 and Igt 2 of European common carp at the protein level, using (newly developed) antibodies against Igt 1 and Igt2. While we found the highest number of Igt $1^{+}$cells in blood and spleen, both soluble Igt1 and Igt2 were shown to be present in mucus from different sites, and to differentially bind to local bacteria. Finally, the in vivo infection studies show that Igt1 and Igt2, like Igm, respond to both systemic and mucosal infections.

A

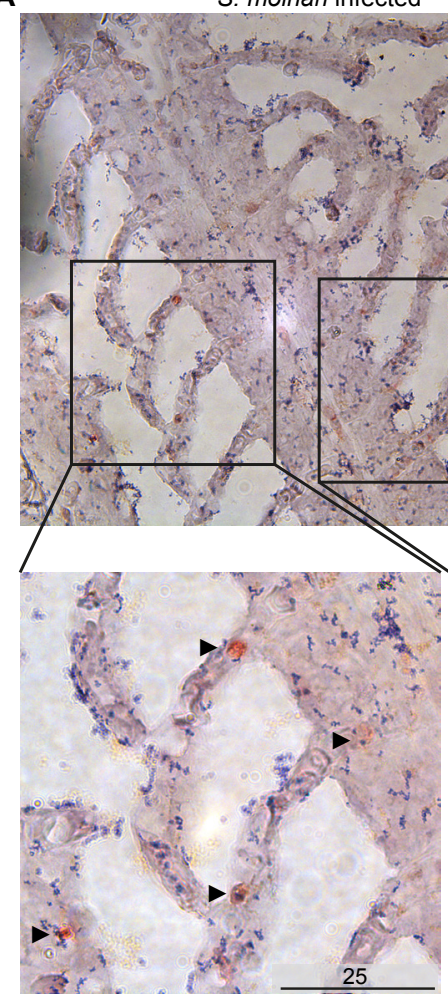

B

Healthy control

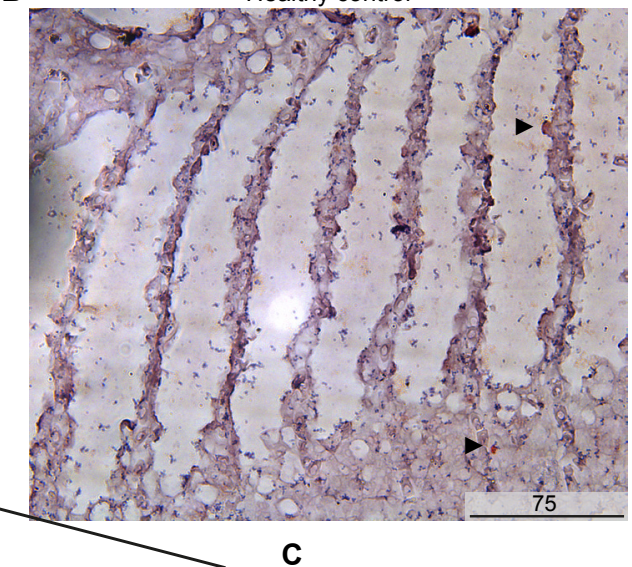

C

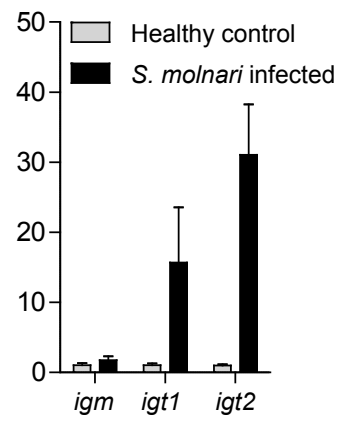

Fig. 5. B cell responses to the gill parasite $S$. molnari. Gills were isolated from $S$. molnariinfected carp at $42 \mathrm{~d}$ post-infection (A) or non-infected healthy controls (B). (A and B) Slides were labelled with rabbit-anti-lgt1 and mouse-anti-lgm followed by HRP-conjugated goat-antirabbit and AP-conjugated goat-anti-mouse. Slides were imaged using a Leica DM6 microscope. Black arrowheads indicate examples of Igt $1^{+}$B cells. (C) RT-qPCR gene expression analysis of igm, igt1 and igt2 in gill tissue. Expression ratios were calculated relative to the healthy control at the same time point. Data represents mean+SD for $n=2$ control, and $n=4$ infected gills. 


\section{Discussion}

In this study we described the different subtypes of European common carp Igt and by using antibodies raised in both rabbit and chicken we characterized their distribution in multiple organs and described their response during systemic and mucosal infection. We observed spatial segregation of Igm, Igt1 and Igt2 in the intestine, as well as in mucus and bacteria from different mucosal sites. However, for the first time in carp, we show that all tested Igs (Igm, Igt1, Igt2) responded to mucosal and systemic challenges.

Teleost B cells can be divided into $\operatorname{Igm}^{+}, \operatorname{Igt}^{+}, \operatorname{Igd}^{+}$single positive populations as well as $\operatorname{Igm}^{+} \operatorname{Igd}^{+}$double positive populations. However, the characterization of these different B cell subtypes in teleost species has suffered greatly from the lack of tools. Moreover, since complete genome data are only available in a limited number of fish species, many igt genes are yet to be discovered.

Of the three Ig types, Igt was the most recently discovered [3]. Despite the identification of igt genes in many teleost species [13,24,26,27,30,52,53], only in rainbow trout, Igt function and distribution has been characterized in greater detail owing to the availability of a monoclonal antibody [10,19-21,54]. While the studies performed in rainbow trout were the first to reveal a role for Igt in mucosal responses,studies in other teleost species suggest that Igt responses are not restricted to mucosal sites and that Igt is also involved in systemic immune responses [15,28-31]. Regretfully, most of these observations are largely based on gene expression or repertoire analysis that do not reflect the relative percentage of B cells in the organs or of immunoglobulin (Ig) protein levels in e.g. serum and mucus. Furthermore, recent studies in gilthead seabream (Sparus auratus) have highlighted the importance of using primers that discriminate between soluble and transmembrane Ig, showing that while soluble igm transcripts can be higher than soluble igt, transmembrane igt and igm transcripts can be comparable [23].

In this study, we reported the preliminary functional characterization of European common carp Igt1 and Ig2. We produced specific antibodies against Igt1 and Igt2 in rabbits and chickens using recombinant $\tau 2$ and $\tau 3 \mathrm{CH}$ domains of Igt 1 and $\tau 1$ and $\tau 2$ $\mathrm{CH}$ domains of Igt2. For Igt1, both chicken and rabbit antibodies showed a specific reactivity to a high molecular weight band of the expected size of Igt1. For Igt2, rabbit but not chicken antibodies, showed reactivity to a low molecular weight 
A

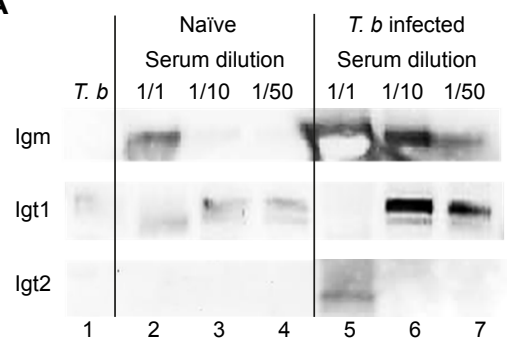

C

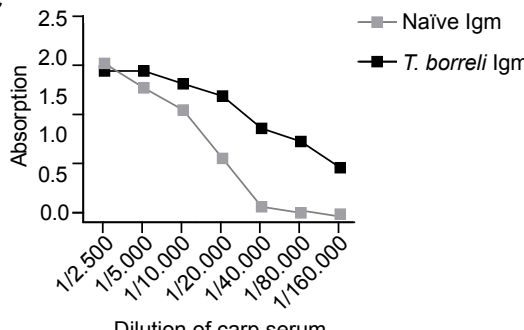

B

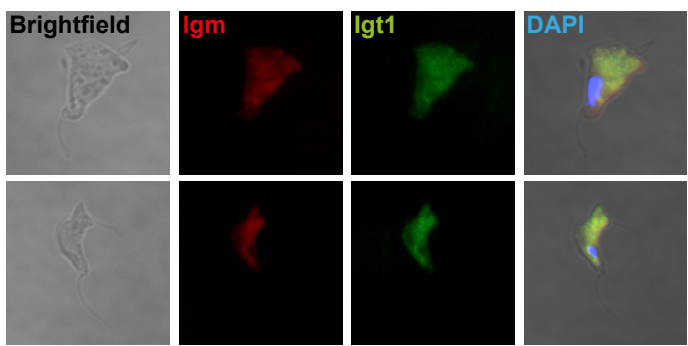

D

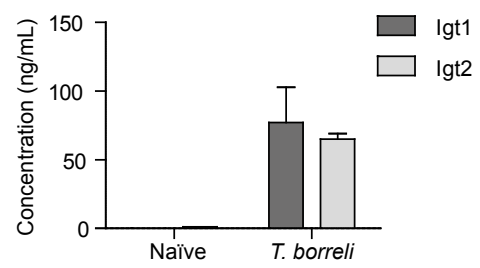

E
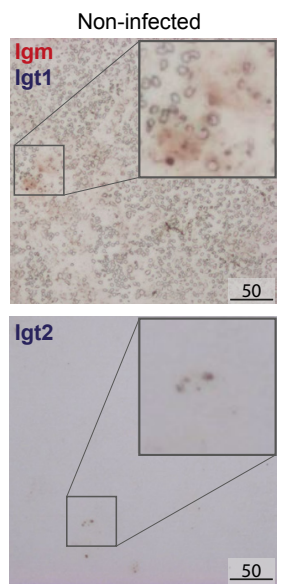

T. borreli infected
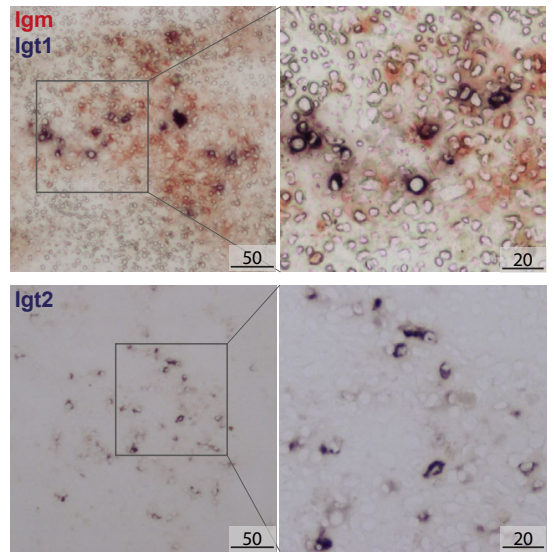

$\mathbf{F}$

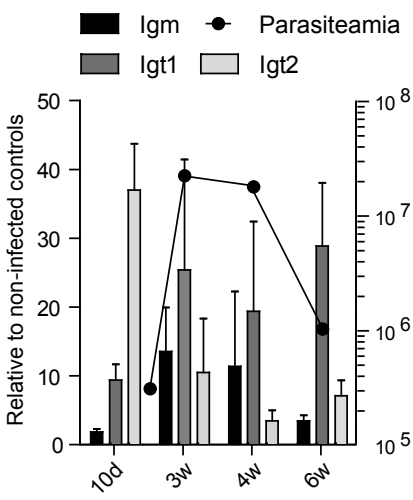

Fig. 6. B cell responses during an infection with the blood-borne parasite Trypanoplasma borreli. T. borreli parasites were incubated with the indicated dilutions of serum from naïve carp or from carp 3-weeks infected with $T$. borreli (T. b). Samples, including a control sample of parasites that were not incubated with carp serum (lane 1, T.b.) were either lysed for Western blot detection of bound Igs using the mouse-anti-Igm antibody (WCl12), rabbit-anti-lgt1 or rabbit-anti-lgt2 (A) or centrifuged on polysine slides. Slides were stained with the mouseanti-Igm and rabbit-anti-Igt1, followed by goat-anti-mouse PE and goat-anti-rabbit-Alexa488. Finally, slides were counterstained with DAPI and imaged using a Leica DM6 microscope. (C) Igm, Igt1 and lgt2 was detected in sera of naïve carp and serum of carp 3-weeks infected with T. borreli using the optimized sandwich ELISA. Y-axes indicate absorption at 405nm. (D) Igt1 and lgt2 concentrations in the sera described in $\mathrm{C}$, were quantified based on a standard curve of recombinant Igt1 and Igt2 and the optimized sandwich ELISA. (E) Spleen was isolated from healthy carp or carp that were 3-weeks infected with T. borreli. cryosections were labelled with antibodies against Igm, rabbit- anti-lgt1, and rabbit-anti-lgt2 and with goat-anti-mouse HRP and goat-anti-rabbit AEC. Slides were imaged using a Nikon Microphot-FXA microscope with an Olympus DP50 camera. Scale bars indicate $\mu \mathrm{m}$. (F) Spleens were isolated from carp 
at different time points post-infection and was processed for gene expression analysis by RT-qPCR. Y-axis indicates time post-infection in days (d) or weeks (w). Left axis represents the gene expression data in fold-change, right $y$-axis represents the parasitaemia in $\log ^{10}$ parasites $/ \mathrm{ml}$. Expression was calculated relative to the healthy controls at time point $\mathrm{Oh}$ and bars represent mean $+\mathrm{SD}$ of $n=5$ for each group.

protein of the expected size of Igt2. As expected, however, due to the similarity between the $\mathrm{CH} 1 \tau 2$ and the $\mathrm{CH} 1 \mu$ domains, cross-reactivity to Igm was also observed. Thus, the rabbit anti-Igt2 antibodies were preferably used for Western blot analysis, where it was possible to identify the Igt2-specific signal. Given the abundance of $\mathrm{Igm}^{+}$cells in several tissues, the rabbit anti-Igt 2 were not reliable by FACS analysis, and in immunohistochemistry they reflected an $\operatorname{Igm}^{+} / \operatorname{Igt} 2^{+}$mixed population. Nevertheless, they provided indication about the possible location of $\operatorname{Igt} 2^{+}$cells.

Using flow cytometry, we characterized the percentages of $\operatorname{Igm}^{+}$and $\operatorname{Igt1}^{+} \mathrm{B}$ cells in different tissues and we found the highest percentages of Igt $^{+}$cells in PBLs and spleen, which is in agreement with the gene expression data in Japanese common carp [15], as well as with the findings in rainbow trout [10,20].

Next we investigated if Igt $^{+}$cells have phagocytic abilities, as was previously reported for common carp Igm ${ }^{+}$cells [51] and for rainbow trout $\mathrm{Igm}^{+}$and $\mathrm{Igt}^{+} \mathrm{B}$ cells [10]. Indeed, both $\operatorname{Igm}^{+}$and $\operatorname{Igt}^{+} \mathrm{B}$ cells of common carp were able to phagocytose fluorescent beads of $0.5,1$ and $2 \mu \mathrm{m}$ and formed phagolysosomes after ingestion of particles, as assessed using $\mathrm{pH}$-sensitive $E$. coli particles. While this suggests a role for $\mathrm{Igt}^{+}$cells in the killing and elimination of microbes, analysis using in vivo phagocytosis or intracellular killing assays awaits further investigation.

Besides characterizing the presence of $\operatorname{Igt}^{+} \mathrm{B}$ cells and soluble Igt in PBLs and serum, we had a closer look at the presence of soluble Igt in mucus. By isolating bacteria and mucus from skin, intestine and gills, we found that bacteria in the intestine were predominantly coated with Igt and to a lesser extent with Igm, which was also reported for rainbow trout [10,20]. Furthermore, we found that while Igt1 mainly coated intestinal bacteria, Igm and Igt 2 were the main Ig types coating skin and gill bacteria. In gills, coating by Igt 2 was more predominant than by Igm. When looking at the mucus, we detected Igm and Igt 2 in the skin, and Igm and Igt1 in the gills. While we found a strong coating by Igt 2 of gill bacteria, we did not observe a Igt2 signal in the mucus. Similar to our findings, also in rainbow trout, Igm was more abundant than Igt in gill mucus [20]. Regretfully, the detection of Igt in mucus has 
not been successful so far and moreover, the isolation of the intestinal mucus require refinement since we were not able to obtain an apparent signal for any Ig on Western blot, possibly caused by high levels of protein degradation [55].

Through immunohistochemistry we identified $\operatorname{Igm}^{+}$and Igt $1^{+} \mathrm{B}$ cells and preliminary assessed the presence of $\operatorname{Igm}^{+} / \operatorname{Igt}^{+}$cells in the intestine. We show that while $\operatorname{Igm}^{+} /$ Igt $2^{+}$cells were located strictly in the lamina propria, Igt $1^{+}$cells were found in the epithelial layer. At this location, potential Ig-secreting $\operatorname{Igt} 1^{+}$cells were also identified. The observation that only Igt $1^{+}$cells were found at this location might explain why the majority of intestinal bacteria were coated with Igt1. If this spatial segregation of B cell subsets in the intestine is present in other teleosts awaits further investigation, since thus far B cell distribution in the intestine has been reported only in rainbow trout but with contrasting results. In a first study, $\operatorname{Igt}^{+} \mathrm{B}$ cells were detected exclusively in the lamina propria in naïve fish, whereas Igt $^{+} \mathrm{B}$ cells in fish that recovered from an infection with the intestinal parasite Ceratomyxa shasta were found close to the lumen [10]. In contrast, in a second study the presence of high numbers of intra-epithelial Igt $^{+}$cells was observed already in naïve fish throughout the intestinal tract. However, the number of intra-epithelial $\mathrm{Igm}^{+}$and $\mathrm{Igt}^{+} \mathrm{B}$ cells increased in the pyloric caeca upon oral vaccination against IPNV [56].

In parallel to the characterization of B cell populations in the intestine of naïve carp, we used the Sphaerospora molinari ( $S$. molnari) parasite model to investigate the differential response of B cell subtypes. This parasite infects the gill epithelium and multiplies in the interlamellar epithelium as well as epithelium filaments, making it a perfect model to study immune responses in gills [46,57]. Using this model, we observed recruitment of Igt $1^{+}$cells to the gills and more specifically to the filaments. Although the anti-Igm staining was not successful and the anti-Igt2 staining was not performed, gene expression analysis confirmed the increase in igt 1 expression and revealed an even higher increase in igt 2 transcripts, while igm expression remained unchanged. While these results point at a mucosal role of Igt, investigation of systemic responses (e.g. in serum) to S. molinari should be considered, especially given that the parasite also has a blood-stage form. Infections with the gill parasite Ich (Ichthyophtirius multifillis) have been used to characterize Igt responses in gills of rainbow trout but so far, results are contrasting. One study, focusing on the early stages after Ich infection, showed that in non-immunized fish only Igm bound to Ich, whereas in immunized fish both Igm and Igt bound to the parasite [27]. In contrast 
to this, another study showed that at 25 days after infection the Ich parasites were predominantly coated with Igt and not Igm [20]. However, in different monoclonal antibodies against trout Igm and Igt were used in these studies.

To further study the relation between Igm and Igt responses during a systemic infection we used the model of the blood-borne parasite $T$. borreli. Using this model, we showed that Igm, Igt1 and Igt2 bind to T. borreli, and that parasite-specific Igm, Igt1 and Igt2 levels increased in serum during infection. Even though total Igt1 and Igt 2 concentrations during infection increased greatly when compared to naïve carp, the calculated concentrations were still very low $(<100 \mathrm{ng} / \mathrm{mL})$, especially when compared to titers reported in rainbow trout $(3.7 \mu \mathrm{g} / \mathrm{mL})$ [10]. As we observed in flow cytometry and immunohistochemistry, epitope demasking might be required to allow for optimal antigen recognition; thus, the calculated titers by ELISA might represent an underestimation of the real Igt concentrations. Gene expression analysis in spleen of $T$. borreli-infected fish indicated an early igt 2 response, followed by an igm and igt1 response. However, while igm transcription decreased in the late phase of the infection, igtl transcripts remained high even after parasite clearance. This indicates that Igts can be strongly upregulated upon infection with a systemic parasite, and that each of the different Igs might serve different roles. This difference in kinetics between igm and igt and more specifically, an earlier increase of igt, was also reported in rohu (Labeo rohita) infected with the skin parasite Argulus siamensis [30], in mandarin fish (Siniperca chuatsi) infected with Flavobacterium columnare [31] and flounder Paralichthys olivaceus vaccinated against Edwardsiella tarda [26]. The sequences of igt 1 and igt 2 were previously cloned and characterized in Japanese carp [15]. In this study it was observed that igt 1 was highly expressed in mucosal organs and was upregulated during infection with the skin parasite Lernea while in contrast, igt 2 was highly expressed in systemic organs and was more upregulated than igt1 3 weeks after infection with the blood-borne parasite Trypanoplasma borreli [15]. Based on these results, Igt1 was proposed to play role in mucosal responses whereas Igt 2 in systemic responses. However, due to a lack of specific antibodies, no further characterization was performed. In agreement with this previous study, our data confirm a higher igt 1 response in spleen of infected fish at 3 weeks post infection, but also shows that igt 2 expression was higher than igtl at $10 \mathrm{~d}$ after infection.

Altogether, there does not seem to be a strict functional segregation in "mucosal" or "systemic" Igt in carp, given that no large differences were observed between 
Igt ${ }^{+}$and Igt $2^{+} \mathrm{B}$ cell populations in systemic and mucosal organs, and that both Igt 1 and Igt 2 responded to mucosal as well as systemic infections. Of course, the spatial differences observed in the intestine, as well as the different kinetics of expression during $T$. borreli infection, indicate that Igt 1 and Igt 2 might serve different roles in the immune response, and this requires further investigation.

While the first reports on Igt described a role for Igt mostly in mucosal immune responses, new insights from other species, using various approaches and infection models, indicate that Igt certainly plays a role also in systemic immunity. Nevertheless, since Igt concentrations in serum and mucus are much lower than Igm, and pathogen-specific Igts can be detected only when the samples are hardly diluted $[19,20]$, the true function and relevance of Igt is yet to be unraveled. To illustrate; while binding of Igts to various parasites has been shown, the effect of this binding is not shown, and while Igt $^{+} \mathrm{B}$ cells were recruited to the muscle after i.m. DNA vaccination, neutralizing capacity of Igt has never been shown.

All above shows that the measured response, and therefore the compartmentalization of the total Ig response, is multifactorial. Outcomes greatly depend on the organ or sample used, (specificity of the) technique, and in the case of a challenge/vaccination, also on the route and time of sampling. While most often mucosal vaccination or challenge models are used to characterize the role of Igt, one should always compare the response to Igm and furthermore, one should not underestimate the role of Igt also in systemic immune responses. The production of specific antibodies, especially for species that have more than one Igt, will be of utmost importance to characterize the true importance of Igt. While we show the possibilities of studying Igt1 using our newly developed antibodies, we are still working on optimizing our antibodies and assays for Igt2. Furthermore, the establishment of sensitive ELISAs to be able to detect Igt1/2 in mucus will be one of our targets, especially since they will be highly valuable for analyzing responses after mucosal vaccination. Ultimately, combined approaches including MasSpec and sorting of Igt $1^{+}$and Igt $2^{+}$cells will provide the definitive answer on the reactivity of the antibodies and will allow for a deeper characterization of $\operatorname{Igt}^{+} \mathrm{B}$ cell phenotypes. 


\section{Acknowledgements}

We thank Dr. Susana Magadan (INRA, Jouy-en-Josas) for her great effort in data mining for the different igt genes within the common carp genome. We also thank Dr. Pierre Boudinot for fruitful discussions on igt sequences and annotation. We also wish to thank Dr. Alf Dalum (Norwegian University of Life Sciences) for his discussion on immunohistochemistry and for his help in optimizing the protocols for Igt staining on paraffin slides. Lastly, we wish to thank Marloes Grobben (Wageningen University, Wageningen) and Maarten Bezemer (Wageningen University, Wageningen) for their technical support in various of the presented techniques.

This work was supported by the European Commission under the Seventh Framework Programme for Research and Technological Development (FP7) of the European Union (Grant Agreement 311993 TARGETFISH).

\section{References}

1. Bradshaw CM, Richard AS, Sigel MM. IgM Antibodies in Fish Mucus. Exp Biol Med (1971) 136:1122-1124. doi:10.3181/00379727-136-35443

2. Wilson M, Bengtén E, Miller N., Clem L., Du Pasquier L, Warr G. A novel chimeric Ig heavy chain from a teleost fish shares similarities to IgD. Proc Natl Acad Sci U S A (1997) 94:4593-4597. doi:10.1073/pnas.94.9.4593

3. Hansen JD, Landis ED, Phillips RB. Discovery of a unique Ig heavy-chain isotype (IgT) in rainbow trout: Implications for a distinctive B cell developmental pathway in teleost fish. Proc Natl Acad Sci U S A (2005) 102:6919-24. doi:10.1073/pnas.0500027102

4. Danilova N, Bussmann J, Jekosch K, Steiner LA. The immunoglobulin heavy-chain locus in zebrafish: Identification and expression of a previously unknown isotype, immunoglobulin Z. Nat Immunol (2005) 6:295-302. doi:10.1038/ni1166

5. Ramirez-Gomez F, Greene W, Rego K, Hansen JD, Costa G, Kataria P, Bromage ES. Discovery and Characterization of Secretory IgD in Rainbow Trout: Secretory IgD Is Produced through a Novel Splicing Mechanism. J Immunol (2012) 188:1341-1349. doi:10.4049/jimmunol.1101938

6. Castro R, Bromage E, Abos B, Pignatelli J, Gonzalez Granja A, Luque A, Tafalla C. CCR7 Is Mainly Expressed in Teleost Gills, Where It Defines an IgD+IgM- B Lymphocyte Subset. $J$ Immunol (2014) 192:1257-1266. doi:10.4049/jimmunol.1302471

7. Edholm E-S, Bengtén E, Stafford JL, Sahoo M, Taylor EB, Miller NW, Wilson M. Identification of two IgD+ B cell populations in channel catfish, Ictalurus punctatus. $J$ Immunol (2010) 185:4082-94. doi:10.4049/jimmunol.1000631

8. Sun Y, Wei Z, Hammarstrom L, Zhao Y. The immunoglobulin $\delta$ gene in jawed vertebrates: A comparative overview. Dev Comp Immunol (2011) 35:975-981. doi:10.1016/j. dci.2010.12.010

9. Edholm ES, Bengten E, Wilson M. Insights into the function of IgD. Dev Comp Immunol (2011) 35:1309-1316. doi:10.1016/j.dci.2011.03.002

10. Zhang Y-A, Salinas I, Li J, Parra D, Bjork S, Xu Z, LaPatra SE, Bartholomew J, Sunyer JO. 
IgT, a primitive immunoglobulin class specialized in mucosal immunity. Nat Immunol (2010) 11:827-35. doi:10.1038/ni.1913

11. Fillatreau S, Six A, Magadan S, Castro R, Sunyer JO, Boudinot P. The astonishing diversity of Ig classes and B cell repertoires in teleost fish. Front Immunol (2013) 4:28. doi:10.3389/ fimmu.2013.00028

12. Bao Y, Wang T, Guo Y, Zhao Z, Li N, Zhao Y. The immunoglobulin gene loci in the teleost Gasterosteus aculeatus. Fish Shellfish Immunol (2010) 28:40-48. doi:10.1016/j. fsi.2009.09.014

13. Gambón-Deza F, Sánchez-Espinel C, Magadán-Mompó S. Presence of an unique IgT on the IGH locus in three-spined stickleback fish (Gasterosteus aculeatus) and the very recent generation of a repertoire of VH genes. Dev Comp Immunol (2010) 34:114-122. doi:10.1016/j.dci.2009.08.011

14. $\mathrm{Hu}$ YL, Xiang LX, Shao JZ. Identification and characterization of a novel immunoglobulin $\mathrm{Z}$ isotype in zebrafish: Implications for a distinct B cell receptor in lower vertebrates. $\mathrm{Mol}$ Immunol (2010) 47:738-746. doi:10.1016/j.molimm.2009.10.010

15. Ryo S, Wijdeven RHM, Tyagi A, Hermsen T, Kono T, Karunasagar I, Rombout JHWM, Sakai M, Kemenade BMLV van, Savan R. Common carp have two subclasses of bonyfish specific antibody IgZ showing differential expression in response to infection. Dev Comp Immunol (2010) 34:1183-1190. doi:10.1016/j.dci.2010.06.012

16. Savan R, Aman A, Nakao M, Watanuki H, Sakai M. Discovery of a novel immunoglobulin heavy chain gene chimera from common carp (Cyprinus carpio L.). Immunogenetics (2005) 57:458-463. doi:10.1007/s00251-005-0015-z

17. Yasuike M, de Boer J, von Schalburg KR, Cooper GA, McKinnel L, Messmer A, So S, Davidson WS, Koop BF. Evolution of duplicated IgH loci in Atlantic salmon, Salmo salar. BMC Genomics (2010) 11: doi:10.1186/1471-2164-11-486

18. Zhang N, Zhang X-J, Chen D-D, Oriol Sunyer J, Zhang Y-A. Molecular characterization and expression analysis of three subclasses of IgT in rainbow trout (Oncorhynchus mykiss). Dev Comp Immunol (2017) 70:94-105. doi:10.1016/j.dci.2017.01.001

19. Xu Z, Parra D, Gómez D, Salinas I, Zhang Y-A, von Gersdorff Jørgensen L, Heinecke RD, Buchmann K, LaPatra S, Sunyer JO. Teleost skin, an ancient mucosal surface that elicits gut-like immune responses. Proc Natl Acad Sci U S A (2013) 110:13097-102. doi:10.1073/ pnas. 1304319110

20. Xu Z, Takizawa F, Parra D, Gómez D, von Gersdorff Jørgensen L, LaPatra SE, Sunyer JO. Mucosal immunoglobulins at respiratory surfaces mark an ancient association that predates the emergence of tetrapods. Nat Commun (2016) 7:10728. doi:10.1038/ncomms 10728

21. Tacchi L, Musharrafieh R, Larragoite ET, Crossey K, Erhardt EB, Martin S a. M, LaPatra SE, Salinas I. Nasal immunity is an ancient arm of the mucosal immune system of vertebrates. Nat Commun (2014) 5:5205. doi:10.1038/ncomms6205

22. Picchietti S, Nuñez-Ortiz N, Stocchi V, Randelli E, Buonocore F, Guerra L, Scapigliati G. Evolution of lymphocytes. Immunoglobulin T of the teleost sea bass (Dicentrarchus labrax): Quantitation of gene expressing and immunoreactive cells. Fish Shellfish Immunol (2017) 63:40-52. doi:10.1016/j.fsi.2017.02.002

23. Piazzon MC, Galindo-Villegas J, Pereiro P, Estensoro I, Calduch-Giner JA, Gómez-Casado E, Novoa B, Mulero V, Sitjà-Bobadilla A, Pérez-Sánchez J. Differential modulation of IgT and IgM upon parasitic, bacterial, viral, and dietary challenges in a perciform fish. Front Immunol (2016) 7: doi:10.3389/fimmu.2016.00637

24. Buonocore F, Stocchi V, Nunez-Ortiz N, Randelli E, Gerdol M, Pallavicini A, Facchiano A, Bernini C, Guerra L, Scapigliati G, et al. Immunoglobulin T from sea bass (Dicentrarchus labrax L.): Molecular characterization, tissue localization and expression after nodavirus infection. BMC Mol Biol (2017) 18:1-14. doi:10.1186/s12867-017-0085-0

25. Chen L, Klaric G, Wadsworth S, Jayasinghe S, Kuo T-Y, Evensen Ø, Mutoloki S, Evensen O, Mutoloki S, Evensen Ø, et al. Augmentation of the Antibody Response of Atlantic Salmon by 
Oral Administration of Alginate-Encapsulated IPNV Antigens. PLoS One (2014) 9:e109337. doi:10.1371/journal.pone.0109337

26. Du Y, Tang X, Zhan W, Xing J, Sheng X. Immunoglobulin tau heavy chain $(\operatorname{IgT})$ in flounder, Paralichthys olivaceus: Molecular cloning, characterization, and expression analyses. Int $J$ Mol Sci (2016) 17:1-21. doi:10.3390/ijms17091571

27. Von Gersdorff Jørgensen L, Heinecke RD, Skjødt K, Rasmussen KJ, Buchmann K. Experimental evidence for direct in situ binding of $\operatorname{IgM}$ and $\operatorname{IgT}$ to early trophonts of Ichthyophthirius multifiliis (Fouquet) in the gills of rainbow trout, Oncorhynchus mykiss (Walbaum). J Fish Dis (2011) 34:749-755. doi:10.1111/j.1365-2761.2011.01291.x

28. Kato G, Takano T, Sakai T, Matsuyama T, Sano N, Nakayasu C. Cloning and expression analyses of a unique IgT in ayu Plecoglossus altivelis. Fish Sci (2014) 81:29-36. doi:10.1007/s12562-014-0820-0

29. Castro R, Martínez-Alonso S, Fischer U, Haro NÁ De, Soto-Lampe V, Wang T, Secombes CJ, Lorenzen N, Lorenzen E, Tafalla C. DNA vaccination against a fish rhabdovirus promotes an early chemokine-related recruitment of B cells to the muscle. Vaccine (2014) 32:1160-1168. doi:10.1016/j.vaccine.2013.11.062

30. Kar B, Mohapatra A, Mohanty J, Sahoo PK. Transcriptional changes in three immunoglobulin isotypes of rohu, Labeo rohita in response to Argulus siamensis infection. Fish Shellfish Immunol (2015) 47:28-33. doi:10.1016/j.fsi.2015.08.023

31. Tian J, Sun B, Luo Y, Zhang Y, Nie P. Distribution of IgM, IgD and IgZ in mandarin fish, Siniperca chuatsi lymphoid tissues and their transcriptional changes after Flavobacterium columnare stimulation. Aquaculture (2009) 288:14-21. doi:10.1016/j. aquaculture.2008.11.023

32. Castro R, Jouneau L, Pham H-P, Bouchez O, Giudicelli V, Lefranc M-P, Quillet E, Benmansour A, Cazals F, Six A, et al. Teleost fish mount complex clonal IgM and IgT responses in spleen upon systemic viral infection. PLoS Pathog (2013) 9:e1003098. doi:10.1371/journal.ppat.1003098

33. Zwollo P, Hennessey E, Moore C, Marancik DP, Wiens GD, Epp L. A BCWD-resistant line of rainbow trout exhibits higher abundance of $\operatorname{IgT}+\mathrm{B}$ cells and heavy chain tau transcripts compared to a susceptible line following challenge with Flavobacterium psychrophilum. Dev Comp Immunol (2017) 74:190-199. doi:10.1016/j.dci.2017.04.019

34. Henkel C V., Dirks RP, Jansen HJ, Forlenza M, Wiegertjes GF, Howe K, van den Thillart GEEJM, Spaink HP. Comparison of the Exomes of Common Carp (Cyprinus carpio) and Zebrafish (Danio rerio). Zebrafish (2012) 9:59-67. doi:10.1089/zeb.2012.0773

35. Piazzon MC, Savelkoul HSJ, Pietretti D, Wiegertjes GF, Forlenza M. Carp I110 Has AntiInflammatory Activities on Phagocytes, Promotes Proliferation of Memory T Cells, and Regulates B Cell Differentiation and Antibody Secretion. J Immunol (2015) 194:187-99. doi:10.4049/jimmunol.1402093

36. Morar D, Tijhaar E, Negrea A, Hendriks J, van Haarlem D, Godfroid J, Michel AL, Rutten VPMG. Cloning, sequencing and expression of white rhinoceros (Ceratotherium simum) interferon-gamma (IFN- $\gamma$ ) and the production of rhinoceros IFN- $\gamma$ specific antibodies. Vet Immunol Immunopathol (2007) 115:146-154. doi:10.1016/j.vetimm.2006.10.016

37. Secombes CJ, van Groningen JJ, Egberts E. Separation of lymphocyte subpopulations in carp Cyprinus carpio L. by monoclonal antibodies: immunohistochemical studies. Immunology (1983) 48:165-175.

38. Forlenza M, Scharsack JP, Kachamakova NM, Taverne-Thiele AJ, Rombout JHWM, Wiegertjes GF. Differential contribution of neutrophilic granulocytes and macrophages to nitrosative stress in a host-parasite animal model. Mol Immunol (2008) 45:3178-3189. doi:10.1016/j.molimm.2008.02.025

39. Koumans-van Diepen JE, van de Lisdonk MHM, Taverne-Thiele AJ, Verburg-van Kemenade BML, Rombout JHWM. Characterisation of immunoglobulin-binding leucocytes in carp (Cyprinus carpio L.). Dev Comp Immunol (1994) 18:45-56. doi:10.1016/0145- 
305X(94)90251-8

40. Nakayasu C, Omori M, Hasegawa S, Kurata O, Okamoto N. Production of a monoclonal antibody for carp (Cyprinus carpio L.) phagocytic cells and separation of the cells. Fish Shellfish Immunol (1998) 8:91-100. Available at: http://ac.els-cdn.com/ S105046489790125X/1-s2.0-S105046489790125X-main.pdf?_tid=f2ecacd2-4167-11e7b339-00000aab0f01\&acdnat=1495730217_68caa61b6a51021c584816f36ce0e663 [Accessed May 25, 2017]

41. Weyts FAA, Rombout JHWM, Flik G, L Verburg-van Kemenade BM, Weyts FAA, Rombout JHWM, Flik G, Verburg-van Kemenade BML. A common carp (Cyprinus carpio L.) leucocyte cell line shares morphological and functional characteristics with macrophages. Fish Shellfish Immunol (1997) 7:123-133. doi:10.1006/fsim.1996.0069

42. Toda H, Saito Y, Koike T, Takizawa F, Araki K, Yabu T, Somamoto T, Suetake H, Suzuki Y, Ototake M, et al. Conservation of characteristics and functions of CD4 positive lymphocytes in a teleost fish. Dev Comp Immunol (2011) 35:650-660. doi:10.1016/j.dci.2011.01.013

43. Rombout JHWM, Joosten PHM, Engelsma MY, Vos AP, Taverne N, Taverne-Thiele JJ. Indications for a distinct putative $\mathrm{T}$ cell population in mucosal tissue of carp (Cyprinus carpio L.). Dev Comp Immunol (1998) 22:63-77. doi:10.1016/S0145-305X(97)00048-7

44. Li J, Barreda DR, Zhang Y-A, Boshra H, Gelman AE, LaPatra S, Tort L, Sunyer JO. B lymphocytes from early vertebrates have potent phagocytic and microbicidal abilities. Nat Immunol (2006) 7:1116-1124. doi:10.1038/ni1389

45. Holzer AS, Hartigan A, Patra S, Pecková H, Eszterbauer E. Molecular fingerprinting of the myxozoan community in common carp suffering Swim Bladder Inflammation (SBI) identifies multiple etiological agents. Parasites and Vectors (2014) 7:1-9. doi:10.1186/17563305-7-398

46. Eszterbauer E, Sipos D, Forró B, Bartošová P, Holzer AS. Molecular characterization of Sphaerospora molnari (Myxozoa), the agent of gill sphaerosporosis in common carp Cyprinus carpio carpio. Dis Aquat Organ (2013) 104:59-67. doi:10.3354/dao02584

47. Embregts CWE, Rigaudeau D, Veselý T, Pokorová D, Lorenzen N, Petit J, Houel A, Dauber M, Schütze H, Boudinot P, et al. Intramuscular DNA Vaccination of Juvenile Carp against Spring Viremia of Carp Virus Induces Full Protection and Establishes a Virus-Specific B and T Cell Response. Front Immunol (2017) 8:1340. doi:10.3389/fimmu.2017.01340

48. Forlenza M, Kaiser T, Savelkoul HFJ, Wiegertjes GF. The use of real-time quantitative PCR for the analysis of cytokine mRNA levels. Methods Mol Biol (2012) 820:7-23. doi:10.1007/978-1-61779-439-1

49. Pfaffl MW. A new mathematical model for relative quantification in real-time RT-PCR. Nucleic Acids Res (2001) 29:e45-e45. doi:10.1093/nar/29.9.e45

50. Steinhagen D, Kruse P, Kdrting W. The Parasitemia of Cloned Trypanoplasma borreli Laveran and Mesnil, 1901, in Laboratory- Infected Common Carp ( Cyprinus carpio L .). (1989) 75:685-689.

51. Nagasawa T, Nakayasu C, Rieger AM, Barreda DR, Somamoto T, Nakao M. Phagocytosis by thrombocytes is a conserved innate immune mechanism in lower vertebrates. Front Immunol (2014) 5:36-39. doi:10.3389/fimmu.2014.00445

52. Tadiso TM, Lie KK, Hordvik I. Molecular cloning of IgT from Atlantic salmon, and analysis of the relative expression of tau, mu and delta in different tissues. Vet Immunol Immunopathol (2011) 139:17-26. doi:10.1016/j.vetimm.2010.07.024

53. Savan R, Aman A, Sato K, Yamaguchi R, Sakai M. Discovery of a new class of immunoglobulin heavy chain from fugu. Eur J Immunol (2005) 35:3320-3331. doi:10.1002/ eji.200535248

54. Salinas I, Zhang Y-A, Sunyer JO. Mucosal immunoglobulins and B cells of teleost fish. Dev Comp Immunol (2011) 35:1346-1365. doi:10.1016/j.dci.2011.11.009

55. Hatten F, Fredriksen Å, Hordvik I, Endresen C. Presence of IgM in cutaneous mucus, but not in gut mucus of Atlantic salmon, Salmo salar. Serum IgM is rapidly degraded when added to 
gut mucus. Fish Shellfish Immunol (2001) 11:257-268. doi:10.1006/fsim.2000.0313

56. Ballesteros NA, Castro R, Abos B, Rodríguez Saint-Jean SS, Pérez-Prieto SI, Tafalla C. The Pyloric Caeca Area Is a Major Site for IgM+ and IgT+ B Cell Recruitment in Response to Oral Vaccination in Rainbow Trout. PLoS One (2013) 8:e66118. doi:10.1371/journal. pone. 0066118

57. Molnár K, Eszterbauer E. "Specificity of infection sites in vertebrate hosts," in Myxozoan Evolution, Ecology and Development (Springer), 295-313. doi:10.1007/978-3-319-14753-6 


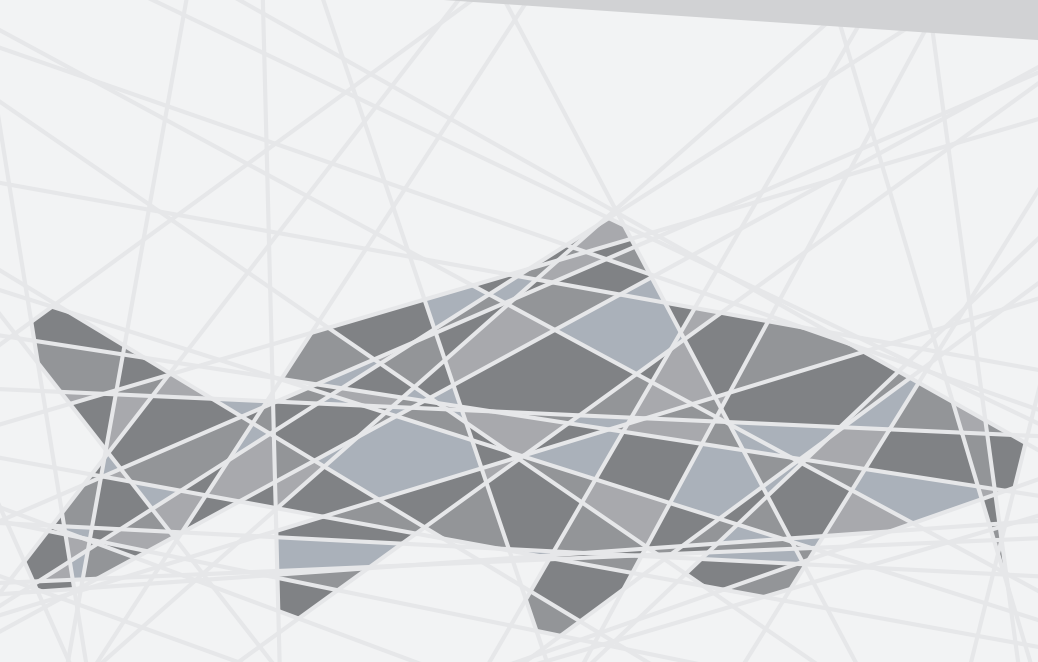




\section{General discussion}

8 
Despite the strong intensification of the aquaculture sector, as well as the sharp increase in outbreaks of aquatic infectious diseases, the amount of (commercially) available effective vaccines for aquaculture is still very limited. Given that traditional injection vaccines induce high levels of stress and can lead to severe local side-effects, mucosal vaccination would serve a good alternative. For this reason, strategies for oral vaccination of fish were extensively reviewed in this thesis (Chapter 2, [1]). After developing experimental i.m. injection vaccines against SVCV (Chapter 3, [2]) and KHV (Chapter 5) and characterizing the induced immune response after vaccination, we tested whether these vaccines could be successful when administered orally (Chapter 4; SVCV and chapter 5; KHV). Besides the knowledge required on the pathogen to select the most immunogenic proteins for vaccine use, one needs thorough knowledge on the fish immune system to identify the required protective immune mechanisms triggered by vaccination. To this end, an array of tools was developed and applied to functionally characterize common carp T cells (Chapter 6) and B cells (Chapter 7). In this chapter, chapter 8, I will discuss the obtained knowledge in the framework of the latest literature and I will once more focus on the following questions:

$1 \quad$ What would be the best vaccination approach against SVCV and KHV with regard to vaccine type, antigen and delivery route?

2 What is necessary to thoroughly study adaptive immune responses in common carp and how can this knowledge help us with vaccine design?

By considering the strengths and weaknesses of our work, I will propose a framework for future studies that could help addressing these questions. Finally, I will zoom out to put my results into a broader perspective and I will discuss the relevance of the obtained results.

\section{Vaccine approaches: every pathogen requires a tailor-made vaccine}

Making use of new molecular techniques and of the availability of pathogen's genomes, vaccine development has made a shift from using whole pathogens (inactivated or attenuated) to specific pathogen sites. This shift has given rise to 
the development of several alternative vaccine types, including DNA vaccines, recombinant attenuated vaccines, subunit vaccines and virus-like particles (VLPs). If we reflect on the knowledge obtained in this study, what would be the optimal vaccine type(s) for SVCV and KHV?

\section{DNA vaccines are safe, but are they also universally applicable?}

DNA vaccines have many benefits, which include the production process (relatively easy to produce, long shelf life) and the induced immune responses (intrinsic adjuvant properties, induce both humoral and cellular immune responses). Immune responses after i.m. DNA vaccination have been studied widely in fish and indeed, both humoral and cellular responses were found to be induced (Chapter $\mathbf{3}$ and $[2-6,8,9])$. Despite the many benefits of DNA vaccines and their success under experimental conditions, only the Apex-IHN ${ }^{\circledR}$ vaccine (Aqua Health Ltd., Novartis, now Elanco) against IHNV has been licensed in Canada after the first report on successful DNA vaccination (rainbow trout Oncorhynchus mykiss against VHSV [9]). Only recently, the CLYNAV vaccine (Aqua Health Ltd., Novartis, now Elanco) against Salmonid Alphavirus 3 (SAV3) was the first DNA vaccine that was given a positive recommendation for marketing authorization by the European Union [10]. During the difficult and long road to licensure of a DNA vaccine, extensive research was performed for both the Apex-IHN ${ }^{\circledR}$ vaccine and the CLYNAV vaccine $[11,12]$. The major concerns about DNA vaccines include the possibility of genomic integration of the vaccine DNA in reproductive cells, leading to germline transfer of the modified DNA, possible anti-DNA antibodies causing auto-immunity, or the induction of antibiotic resistance due to the resistance gene(s) being incorporated in the plasmid backbone [13]. Prior to the positive recommendation of the CLYNAV vaccine, it was extensively shown that the actual rate of genomic incorporation is negligible, as assessed close to the injection site and in the gonads [12]. Genomic incorporation at the injection site is the most likely to occur, but in fish (as in other animal species) it was found to occur at negligible levels, at magnitudes below the spontaneous rate of mutation [14-17]. Since these extensive studies all point at a safe use of DNA vaccines, there is a bright future ahead.

Having stated that DNA vaccines are safe, can we use them also for SVCV?

$\mathrm{SVCV}$ is a rhabdovirus and in general, DNA vaccines encoding the G protein were found to be extremely effective against other fish rhabdoviruses when administered 
intramuscularly $[5,9,18-22]$. The SVCV G protein is located on the virion surface and is a known target for neutralizing antibodies [23-25]. Despite that SVCV-Gbased DNA vaccines were used in multiple studies, so far only limited protection was reported $[8,26]$ In chapter 3 we show that, after elaborate optimization of multiple factors (age and temperature at vaccination, route and temperature of challenge) we obtained full protection against SVCV. The vaccine is effective at a dose of only $0.1 \mu \mathrm{g}$ DNA per gram of fish. [2]. This ground-breaking result would argue for the commercialization of the SVCV-G DNA vaccine in its current state. However, we are aware that individual vaccination of carp is currently not cost-effective, given that it is a relatively low-value species.

In chapter 4 we therefore addressed the possibility to convert our successful injectable DNA vaccine into an oral DNA vaccine that could potentially be used for mass delivery. Despite the lack of protection observed in our studies, we still obtained valuable information on vaccine dose, vaccination regime and vaccine type, that can now be used to refine future strategies for oral vaccine development in carp and were extensively discussed in chapter 2 and chapter 4.

When reflecting on our work, what can be improved to increase efficacy of our SVCV-G DNA vaccine when administered orally, and which alternative vaccine types should be explored next?

\section{Alternative vaccine types for oral vaccination of carp against SVCV}

Besides i.m. DNA vaccination, within the TargetFish project we focused on additional vaccination approaches for SVCV, which are still in the developmental stage and will be summarized below. In addition to the baculovirus-based approach described in chapter 4, we aimed at producing a recombinant Lactococcus lactis (L. lactis) and a recombinant yeast, Pichia pastoris (P. pastoris) expressing the SVCV G protein. To date, the only oral vaccine that showed a moderate protection against SVCV was based on the commensal Lactococcus plantarum expressing the G protein [27]. Furthermore, multiple Lactococcus ssp. species were shown to have probiotic effects in fish as well [28-31]. Given this, the use of lactic acid bacteria including L. lactis was of great interest. While the expression of the SVCV-G on the surface of L. lactis was unsuccessful, we were able to use L. lactis as a vehicle to carry a SVCV-G DNA vaccine in combination with a listeriolysin-O (LLO) gene. LLO is a pore-forming toxin that facilitates escape of the bacterium from phagolysosomes and through a 
mechanism that is still not understood, it facilitates the release of plasmid DNA into the cytoplasm, which will then be taken up by the nucleus [32,33].

Using 6-day cultured carp macrophages [34] it was shown that LLO indeed facilitated escape of the bacteria from endosomes/phagolysosomes, as was shown by a longer survival time of L. lactis after being phagocytosed by carp macrophages. Next, we show that L. lactis carrying both the SVCV-G and LLO plasmid was able to mediate SVCV-G expression in the fathead minnow epithelial cells line EPC, while the $L$. lactis not expressing LLO did not result in any SVCV-G expression. Although this vaccine awaits in vivo testing, the results are very promising given both the beneficial effects of $L$. lactis and the previously reported partial protection using L. plantarum expressing SVCV-G.

In parallel, we aimed at producing recombinant yeast Pichia pastoris (P. pastoris), since the immunostimulatory and even adjuvant effects of $\beta$-glucans present on the yeast cell wall have been widely described in fish [35-38], and like L. lactis it would not require further protection against degradation in the anterior part of the intestine. While the use of $\beta$-glucan particles as a vaccine vehicle have been described $[39,40]$, whole $P$. pastoris was used to express the iridovirus capsid protein in a promising oral vaccine candidate for rock bream (Oplegnatus fasciatus) [41]. For the expression of SVCV-G on the surface of $P$. pastoris we used multiple approaches, including the signal peptide of either SVCV-G or P. pastoris, and either the transmembrane region of SVCV-G or a yeast-optimized glycophosphatidylinosito (GPI)-anchor. While these approaches did not result in expression of the SVCV G protein on the surface of $P$. pastoris, when SVCV-G was expressed as a soluble construct without any transmembrane anchor, we were we able to detect the G protein in the culture supernatant. While no in vivo trials on the immunogenicity have been performed using these samples, it is questionable whether this would afford protection since 1) the SVCV G is expressed on the virion surface as a trimer [23] and 2) when delivered orally, the soluble proteins would require additional protection.

To conclude, while oral DNA vaccination still holds promise, given that the i.m. delivered vaccine conferred full protection, further optimization is needed to make it successful through oral delivery.

\section{Virus-like-particles as vaccine for KHV}

In contrast to SVCV, which has only five proteins, the large number of (surface) 
proteins encoded by KHV greatly complicates the process of selection of the best antigen(s) as vaccine targets. In chapter 5 we show that we were not able to afford protection after i.m. or oral vaccination using a DNA vaccine encoding ORF25, one of the major surface proteins of KHV previously shown to be a target of neutralizing antibodies [42-45]. To date, a large number of the KHV ORFs remains to be characterized. While 13 ORFs were previously identified as glycosylated type I integral membrane proteins, some of which were potentially immunogenic [44], not much is known about their function. After thoroughly discussing our vaccine design in chapter 5 we have come to one major conclusion: one antigen might not be sufficient to confer full protection against KHV. While there are a few studies showing successful vaccination against KHV with only one antigen [27,46,47], we were not able to replicate the results of a previous study using an ORF25-based DNA vaccine [46]. As discussed in chapter 5, differences in protection level might have been due to differences in the method used to assess vaccine efficacy. In our studies, we used a challenge model that closely resembled the natural route of infection (bath challenge), whereas other studies challenged the fish via i.p. injection. Taking all this into consideration, studies that show protection against KHV upon bath challenge are those using live (recombinant) attenuated or whole inactivated vaccines [45,4855]. In agreement, the only commercially available vaccine against $\mathrm{KHV}$ is the live attenuated KV3 vaccine (KoVAX). However, due to safety concerns, it is allowed for use in Israel only. In fact, despite their proven efficacy, live (recombinant) attenuated vaccines still raise safety and legislative concerns and are not yet allowed for worldwide use in fish.

For all these reasons, we propose a system that is safe and can more closely mimics the virus by carrying more viral antigens. To this end, virus-like particles (VLPs) would serve our goal best since they cannot replicate in the host, cannot revert to their virulent form, and accordingly, are safer than live (recombinant) attenuated viral vaccines. VLPs are based on the principle that structural proteins of most viruses self-assemble into particles that resemble the native virus structure in both, size and morphology even in the absence of viral genome. VLPs are widely applicable and are mostly used for the expression of capsid or envelope proteins. While most often they only express one viral protein, successful reports on non-enveloped multiplecapsid VLPs include the ones produced against bluetongue virus [56], infectious bursal disease [57], rotavirus [58] and enterovirus 71 [59]. The use of VLPs in 
experimental vaccines has already been exploited, and VLPs were produced using the capsid protein of IPNV [60-62], and of Nervous Necrosis Virus (NNV) [63-65]. With respect to the generation of VLPs for complex viruses such as herpesviruses, promising examples come from studies in humans against human papillomavirus [66], hepatitis B virus [67,68] and more recently Epstein-Barr virus [69,70]. They are all generally based on the expression of one or more surface glycoproteins or capsid proteins that were shown to be the target of neutralizing antibodies or to induce $\mathrm{T}$ cell-mediated immunity. For KHV, several surface proteins have been identified as immunogenic targets of neutralizing antibodies. These include the capsid proteins ORF92 and ORF72 and the surface glycoproteins ORF25, ORF65, ORF148, ORF149 and ORF81 [42-47,71,72]. Which one of these antigens, or combinations thereof, is necessary and sufficient to confer protection awaits further investigation. Nevertheless, the availability of several eukaryotic expression systems and recombinant vaccine platforms will certainly speed up the development of the first prototype KHV-VLPs.

\section{Oral vaccination - is this really the preferred route for every pathogen?}

Given that we would be able to develop an efficient vaccine against KHV, how are we going to deliver it? Throughout this thesis we discussed oral vaccination strategies in general (Chapter 2) and we tested oral vaccination against SVCV (Chapter 4) and KHV (Chapter 5). While we discussed many factors that might have caused the lack of protection we observed, we also proposed that for a cytopathic virus targeting systemic organs such as SVCV, oral vaccination might just not trigger the required response, whereas oral or mucosal vaccination for a mucosal pathogen such as KHV would hypothetically trigger the appropriate response at the right location. In fact, a few papers showed that oral vaccination against KHV using liposomeencapsulated inactivated KHV or L. lactis expressing ORF81 [27,73,74] is possible but confers only limited protection. Considering the nature of the pathogen and the aforementioned VLP approach, I would propose that besides oral vaccination, immersion vaccination should be tested in parallel. The current commercial vaccine (KV3, KoVAX, Israel) and the various experimental live attenuated vaccines $[48,52]$ have all proven their efficacy through immersion vaccination. Therefore, combining KHV-VLPs with immersion vaccination should provide an alternative to oral vaccination that addresses not only efficacy but also vaccine safety. 


\section{Correlates of protection - (how) can we use them in the development of vaccines for fish?}

Without a way to validate a newly developed vaccine, the risk of over- or underestimating vaccine efficacy is tremendous. While for this reason challenge models that resemble natural infection routes are necessary, alternatives to laborious in vivo challenges are also being investigated. In our case, the immune responses that were characterized after the successful i.m. DNA vaccination against SVCV (Chapter 3) can be used to identify correlates of protection. Even more, given that we did not succeed in inducing protection against SVCV through oral vaccination (Chapter 4), it can help us improve suboptimal vaccines more rapidly by performing analysis of the identified correlates of protection first, instead of performing elaborate in vivo vaccination trials. For mammalian viruses, correlates of protection are mainly based on thresholds of neutralizing antibodies, and have been determined for different pathogens [75-78], However, clear threshold units are not (yet) available for most vaccines against fish virus, mostly because the mechanisms behind vaccine efficacy are still not understood [79,80]. For example, in many instances neutralizing titres do not always correlate with protection, and neither does the presence of antigenspecific antibodies. However, an extensive study on Atlantic salmon assessed longterm protection and antibody titres after vaccination against Piscirickettsia salmonis. A full protection was observed when specific Igm titres in serum were above 2000 $\mathrm{ng} / \mathrm{mL}$, whereas lower titres created an window of infection [81]. Another study about vaccination of salmon against IPNV showed a strong vaccine-dose effect on virus replication, antibody titres and survival. However, since for IPNV circulating antibodies often do not correlate with protection, other correlates of protection and biomarkers for disease progression were assessed as well [80]. Given that minimal protective titres, or other correlates of protection including complement or cytokines, allow for small-scale testing of new vaccine formulations, it should be a worthy investment to identify them for vaccination against SVCV and KHV. Depending on the route of vaccination, the correlates of protection should be identified in samples other than serum. For example, after immersion vaccination, changes in humoral parameters in skin mucus might be considered as well. While correlates of protection will be extremely valuable in vaccine testing and validation, is obvious that this approach requires substantial research efforts, as immune responses might differ per fish species, pathogen and vaccine delivery route. However, given the 
new developments in available molecular tools to study immune responses in fish, techniques including transcriptome analysis will reveal valuable candidates. Many studies characterized local and systemic gene expression profiles after vaccination and an overview of potential correlates of protection in fish was published recently [82]. This review showed that $m x$ and several Interferon Stimulated Genes (ISGS) were upregulated for all tested vaccines, indicating their potential to serve as correlates of protection. One study of interest included gene expression analysis in spleen after i.m. and oral DNA vaccination against IHNV using various doses of the oral DNA vaccine. Indeed, although protection as well as specific antibody titres in the oral vaccinated groups were not as high as in the i.m. vaccinated group, gene expression levels in the oral group showing the highest protection (receiving the highest dose) closely matched those of the i.m. vaccinated group [83]. Related to our results obtained in chapter 3, 4 and 5, I would propose to study the potential of $m x 1$, isg15.2, ifn $\varphi 1$ and ifn $\varphi 2$ as correlates of protection for SVCV since these genes showed a strong increase after successful i.m. DNA vaccination [2], but not after unsuccessful oral DNA vaccination. If we aim at identifying correlates of protection based on gene expression, we need to extend our analysis to systemic organs as well in order to compare different vaccination methods. However, the response is expected to be much lower than the local response, as was indeed observed after oral DNA vaccination (chapter 4).

In my opinion, correlates of protection can be a valuable way of assessing protection but still require elaborate optimization before they can be applied for screening of fish vaccines. Each pathogen will require the identification of specific immune parameters, and the timing and location where they are analysed need to be standardized. Given the rising interest in mucosal and especially oral vaccination, the optimization of sensitive ELISAs to detect Igt in mucus will also be of much value, however, the true role of Igt in protection first needs to be confirmed.

\section{Studying adaptive immune responses after vaccination and during infection}

In the first section of the discussion I focused on vaccine development and already there, I mentioned the importance of studying protective mechanisms in detail. In the 
second section I will therefore focus on how we can best study immune responses in common carp, which will also reflect back on the importance of correlates of protection as described earlier in the discussion. First, I will elaborate on some discussion points raised in chapter 6 and 7, where we presented the preliminary characterization of $\mathrm{T}$ and $\mathrm{B}$ cells, and will combine the discussion points with recent developments that might aid in the further characterization of adaptive immune responses in common carp.

Even though fish immunology lags behind human and murine immunology, many important and interesting discoveries have been made in the last 10-15 years, leading to a whole new view on cell functions in teleosts. The most striking discoveries include those of phagocytic B cells and thrombocytes $[84,85]$, the presence of a Cd4$1^{+}$macrophage subset in rainbow trout [86] and possibly in flounder [87] and the fact that red blood cells might have immune-relevant functions [88]. These data indicate that one needs multiple approaches to truly characterize cell populations and their functions in fish, and that 'like books, you cannot judge a cell by its cover'.

\section{Potentially uncharacterized $\mathrm{Cd} 8 \beta \beta \mathrm{T}$ cell populations}

During the course of this study there were some interesting findings which made us wonder whether Cd8$\beta \beta$ T cells would exist in common carp. Until now, no reports have shown the presence of a $\operatorname{Cd} 8 \beta \beta \mathrm{T}$ cell subset in any fish species, and human, but not mice, CD8 T cells can express $\beta \beta$ on their surface. However, unlike the $\alpha \alpha$ homodimer or $\alpha \beta$ heterodimer, they are not able to efficiently bind to MHC class I or signal due to the lack of an lck signalling motif [89]. In contrast, it has been shown that common carp cd8 8 has an unconventional lck signalling motif $(\mathrm{CXH})$ and therefor it was hypothesised that a functional $\operatorname{Cd} 8 \beta \beta \mathrm{T}$ cell might exist in common carp [90]. First, as was shown in chapter 7, $c d 8 \beta 1$ is the only $c d 8$ gene that was not fully depleted after sorting with the anti-Cd8 $\alpha 1$ antibody, indicating that a possible small $\mathrm{Cd} 8 \beta 1 \beta 1$ population might exist in the intestine. The existence of $\mathrm{Cd} 8 \beta 1 \beta 1$ cells in carp was also suggested based on the observation that during infection with KHV $c d 8 \beta 1$, but not $c d 8 \alpha 1$, $c d 8 \alpha 2$ or $c d 8 \beta 2$, was upregulated [91]. Second, while $c d 8 \alpha 2$ and $c d 8 \beta 1$ showed enrichment in the transcriptome dataset of the WCL38 ${ }^{+}$ sorted cells, the $c d 8 \beta 2$ showed no enrichment and was highest expressed in the WCL38- fraction. This would argue that besides Cd8 $\beta 1 \beta 1 \mathrm{~T}$ cells, Cd8$\beta 2 \beta 2 \mathrm{~T}$ cells might also exist. However, the $c d 8 \alpha 1$ was not incorporated in this dataset since it was 
wrongly annotated in the carp genome, resulting in an incomplete picture regarding the $c d 8$ genes.

In order to better characterize $\mathrm{Cd} 8$ subsets of common carp, we attempted to make a monoclonal antibody against the extracellular domain of Cd8 $\beta 1$ by immunizing rats with Normal Rat Kidney (NRK) cells expressing the carp Cd8 $\beta 1$. While one clone was found to react with Cd8ß1-expressing Vero cells (Fig. 1A) and to also reacted against carp thymocytes (Fig. 1B), regrettably, the subcloning failed and the hybridoma lost its reactivity. However, we were able to perform an initial staining on multiple cell types using a double labelling with an antibody against Zap70. While of course this needs further validation, it looks like two Cd8 $\beta 1$ populations can be identified; a dim and a bright, especially in the intestine (Fig. 1C). In mammals, $\mathrm{CD} 8 \beta 1^{\mathrm{dim}}$ and $\mathrm{CD} 8 \beta 1^{\text {bright }} \mathrm{T}$ cells populations were described as well $[92,93]$. In humans it was found that $\mathrm{CD} 8 \beta 1^{\text {bright }} \mathrm{CD} 28^{+}$cells could differentiate into either $\mathrm{CD} 8 \beta 1^{\mathrm{dim}} \mathrm{CD} 28^{+}$or $\mathrm{CD} 8 \beta 1^{\mathrm{dim}} \mathrm{CD} 28^{-}$cells upon TCR stimulation [93]. Based on the levels of expression of multiple intracellular cytokines, effector molecules and surface $C D 8 \beta^{\text {bright }} T$ cells were classified as naïve and $C D 8 \beta^{\text {dim }}$ as activates/effector or memory $\mathrm{T}$ cell. After the observation of the $\mathrm{Cd} 8 \beta 1^{\mathrm{dim}}$ and $\mathrm{Cd} 8 \beta 1^{\text {bright }}$ populations in the intestine, we examined the proportion $\mathrm{dim} / \mathrm{bright}$ in various organs and found that besides the thymus, the intestine had the highest proportion of $\mathrm{Cd} 8 \beta 1^{\text {bright }}$ cells, indicating that $\sim 15 \%$ of the $\mathrm{Cd} 8 \beta 1^{+}$cells in the intestine might be of the "naïve" type. On the other hand, $\mathrm{Cd} 8 \beta^{\text {bright }}$ might possibly reflect a population of $\mathrm{Cd} 8 \beta \beta$ cells, which is also suggested based on the RT-qPCR and transcriptome data of $\mathrm{Cd} 8 \alpha 1$ and WCL38 sorted cell populations. While we show the evidence of the potential Cd8 $\beta \beta$ $\mathrm{T}$ cells, their function and $\mathrm{Cd} 8 \beta$ signalling needs to be revealed. Unfortunately, we no longer have an antibody against $\mathrm{Cd} 8 \beta 1$ nor against $\mathrm{Cd} 8 \beta 2$. Therefore, other techniques including in situ hybridization or single-cell sorting are required to better characterize $\mathrm{Cd} 8 \beta^{+}$cells as well as the different dimerization states of the $\mathrm{Cd} 8 \alpha$ and $\mathrm{Cd} 8 \beta$ proteins of common carp. To add to this, recently developed techniques that combine in situ hybridization and flow cytometry (FISH-Flow $[94,95]$ or the commercially available PrimeFlow (Invitrogen, eBiosceinces), would allow for the quantification of cells as well. This technique is widely applicable and would also be of great value to study the presence of the different Cd4 subsets (Cd4-1-Cd4-2+, Cd4$1^{+} \mathrm{Cd} 42^{-}$and $\mathrm{Cd} 4-1^{+} \mathrm{Cd} 4-2^{+}$), especially since we observed (albeit low) expression of $c d 4-2 a$ and $c d 4-1 a$ in the Cd4-1- fraction after sorting with the Cd4-1 antibody. 
However, since this technique relies on the hybridization of 20-40 gene-specific probes, it depends on the sequence similarity whether an adequate number of probes can be designed to study different isoforms of genes (like the $c d 4$ genes) as well.

A
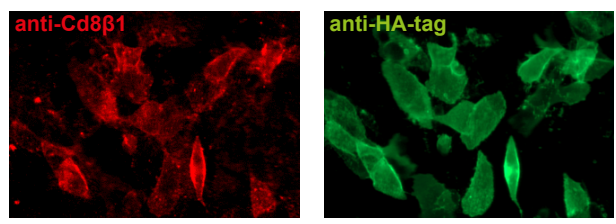

C

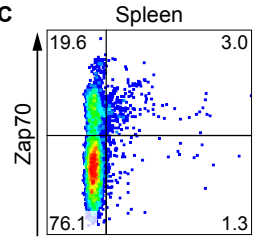

Gill

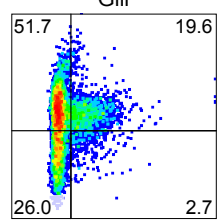

Intestine

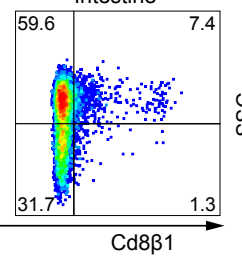

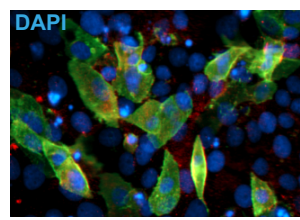

Intestine

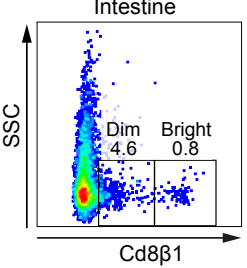

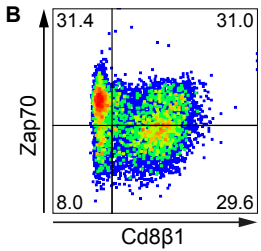

D

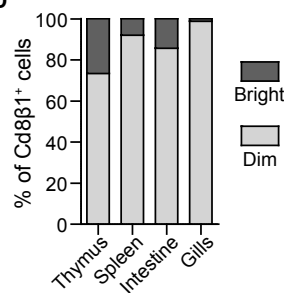

Fig. 1. Initial tests of a rat-anti-Cd8 $\beta 1$ antibody using Cd8 $\beta 1$-expressing Vero cells and flow cytometry. A) Rat-anti-Cd8 $\beta 1$ hybridoma supernatant (1:4, followed by rabbit-anti-rat $\mathrm{PE}$ ) and anti-HA-Tag antibody (1:1000, followed by goat-anti-mouse FITC) were used to label Vero cells transfected with pDisplay-Cd8 $\beta 1$, encoding the cytoplasmic domain of Cd8 $\beta 1$. A staining with DAPI was included to stain the cell nuclei and images were acquired using an EVOS fl LED microscope (Advanced Microscopy group). B) Freshly isolated thymocytes were labelled with rat-anti-Cd8 $\beta 1$ hybridoma supernatant $(1: 4)$ and an antibody against Zap70 (1:100) followed by goat-anti-rat PE and goat-anti-rabbit FITC. Cells were analysed using a CantoA flow cytometer (BD Biosciences). C) Freshly isolated leukocytes from spleen, gills and intestine were labelled as in C. D) Cd8 $\beta 1^{\mathrm{dim}}$ and $\mathrm{Cd} 8 \beta 1^{\text {bright }}$ populations are expressed relative to the total population of $\mathrm{Cd} 8 \beta 1^{+}$cells in the given organ.

\section{WCL38 and Cd8 populations in the intestine}

As was shown in chapter 6, we used multiple approaches to characterize different $\mathrm{T}$ cell subsets in common carp and we attempted to characterize the exact cell type marked by the WCL38 antibody [96]. Based on the facts that high numbers of WCL38 ${ }^{+}$, small, lymphoid-like cells were found in gills and intestine but not in systemic organs, and no reactivity was found to B cells, macrophages or non-specific cytotoxic cells, it was proposed that this antibody most likely recognizes a distinct population of putative mucosal T cells [96]. Furthermore, it was shown that in gill and skin, but not in intestine, large granular lymphoid cells were also recognized. This indicates that 1) WCL38 might recognize multiple cell types and 2) WCL38+ populations might differ between organs. However, using the available tools and datasets, we were not able to draw definite conclusions on the exact cell type. Since we observed three main populations upon double labelling with WCL38 and anti- 
$\mathrm{Cd} 8 \alpha 1\left(\mathrm{Cd} 8 \alpha 1^{+} \mathrm{WCL} 38^{-}, \mathrm{CD} 8 \alpha 1^{-} \mathrm{WCL} 38^{+}\right.$and CD $\left.8 \alpha 1^{+} \mathrm{WCL} 8^{+}\right)$, we are currently sorting all of these three populations separately in order to 1) verify the different cell populations recognized by WCL38 and 2) identify differentially expressed markers between "mucosal/WCL38+" Cd8 $\alpha 1^{+}$cells and "systemic/WCL38-“ $\mathrm{CD} 8 \alpha 1^{+}$cells. Interestingly, similar to the labelling patterns observed when using the $\operatorname{Cd} 8 \beta 1$ antibody, we could also discriminate between a $\mathrm{Cd} 8 \alpha 1^{\mathrm{dim}}$ and a $\mathrm{Cd} 8 \alpha 1^{\text {bright }}$ population when using the $\mathrm{Cd} 8 \alpha 1$ antibody (Fig. 2A). Highest ratios of $\mathrm{Cd} 8 \alpha 1^{\text {bright }}$ were found in intestine and head kidney, while lowest ratios were found in PBLs and spleen (Fig. 2B). A double labelling in the intestine with WCL38 rules out the possibility that $\mathrm{WCL}_{3} 8^{+}$cells would be either the $\mathrm{Cd} 8 \alpha 1^{\mathrm{dim}}$ or $\mathrm{Cd} 8 \alpha 1^{\text {bright }}$ population, since both $\mathrm{Cd} 8 \alpha 1$ populations were found to have equal proportions of WCL38+ cells (Fig $2 \mathrm{C}$ ). One possible hypothesis is that the $\mathrm{Cd} 8 \alpha 1^{\mathrm{dim}}$ would reflect $\mathrm{Cd} 8 \alpha \beta$ cells while $\mathrm{Cd} 8 \alpha 1^{\text {bright }}$ cells reflect $\mathrm{Cd} 8 \alpha \alpha$ cells, however, at least in humans, it was found that $\alpha \beta$ heterodimers and $\alpha \alpha$ homodimers can both be expressed on a single cell [97]. The characterization of different $\mathrm{Cd} 8 \alpha$ and $\mathrm{Cd} 8 \beta$ dimerization profiles is not straightforward, since we cannot rule out that the antibody against $\mathrm{Cd} 8 \alpha 1$ might also react to $\mathrm{Cd} 8 \alpha 2$ (and thereby potential $\mathrm{Cd} 8 \alpha 2 \alpha 2$ cells). An anti-Cd8 82 antibody would allow for investigation of the presence of the $\mathrm{Cd} 8 \alpha 1 \alpha 1, \mathrm{Cd} 8 \alpha 1 \alpha 2$ and $\mathrm{Cd} 8 \alpha 2 \alpha 2$ populations, it would be even more important in combination with antibodies against $\mathrm{Cd} 8 \beta 1$ and $\mathrm{Cd} 8 \beta 2$, since for example $c d 8 \alpha 2$ and $c d 8 \beta 1$, but not $c d 8 \alpha 1$ and $c d 8 \beta 2$, were upregulated at 4 days post-infection with SVCV, possible indicating the specific increase of a $\mathrm{Cd} 8 \alpha 2^{+} \mathrm{Cd} 8 \beta 1^{+}$population [90]. Furthermore, besides studying the potential dimerization states of $\mathrm{Cd} 8 \alpha \alpha$ and $\operatorname{Cd} 8 \alpha \beta$ (and potentially $\mathrm{Cd} 8 \beta \beta$ ), the characterization of their signalling efficiency might reflect possible differences in functional roles.

Besides the description of the Cd8 $\alpha 1^{+} \mathrm{WCL} 38^{-}, \mathrm{CD} 8 \alpha 1^{-} \mathrm{WCL} 8^{+}$and CD $8 \alpha 1^{+} \mathrm{WCL} 38^{+}$ populations in the carp intestine, we have evidence that a small subpopulation of Cd4-2a ${ }^{+}$WCL $38^{+}$cells exist, given that moderate counts (417-665) of $c d 4-2 a$ were found in the database of WCL38 $8^{+}$sorted cells, while almost no counts $(<200)$ were observed for the two $c d 4-1$ genes as well as $c d 4-2 b$. While the existence of this population needs confirmation, it is an observation that we should take into account when further characterizing ( $\mathrm{T}$ cell) subpopulations recognized by the WCL38 antibody. 

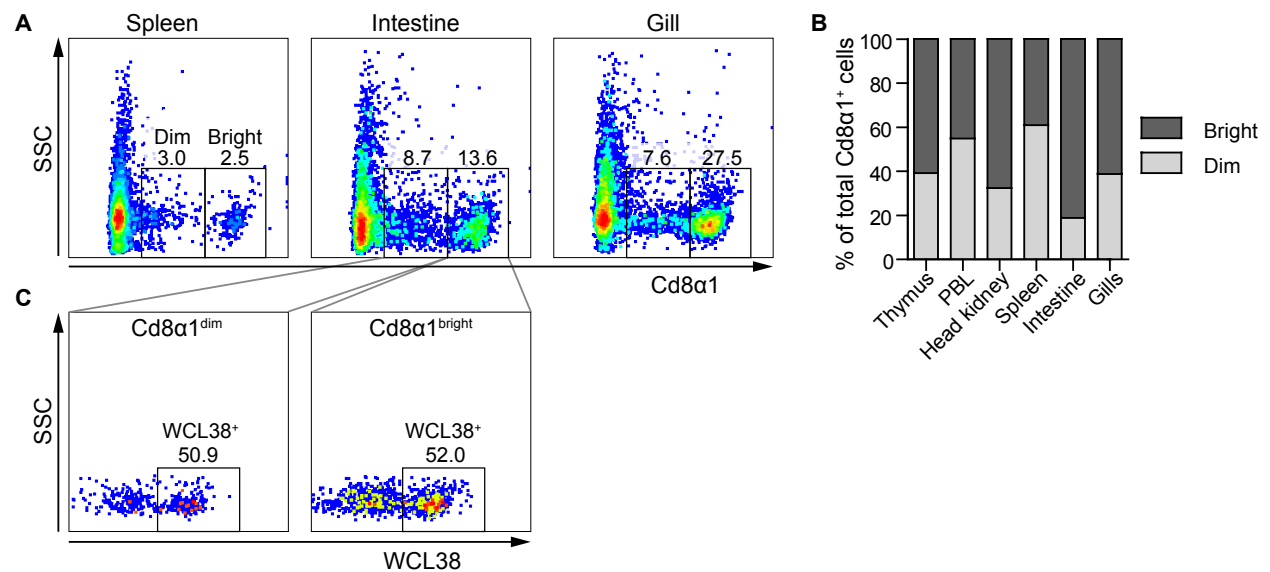

Fig. 2. Characterization of $\mathrm{Cd} 8 \mathrm{a} 1^{\mathrm{dim}}$ and $\mathrm{Cd} 8 \mathrm{a} 1^{\text {bright }}$ populations in various organs. Leukocytes isolated from various organs were stained with the anti-Cd8a1 antibody alone (A), after which proportions of Cd8a1 $1^{\text {dim }}$ and Cd8a $1^{\text {bright }}$ were quantified relative to the total population of Cd8a1 cells (B). Alternatively, double labelling was performed with the WCL38 antibody and cells were first gated based on their Cd8a1 expression, and secondly for their staining with WCL38 (C).

\section{WCL38 and T cell populations in gills}

As mentioned before, the initial characterization of the WCL38 antibody showed differences in cells recognized by the antibody. Besides the small lymphoid cells that were detected in gills and intestine, large granular lymphoid cells were found to be positive in the gills only [96]. Given this difference, it would be necessary to sort both small lymphoid and larger granular lymphoid WCL38 ${ }^{+}$cells, and perform RNA sequencing on these populations separately. While the intestine is seen as a strictly mucosal organ, the gills can be considered both a mucosal and a systemic organ, since they have a true mucosal layer but are also highly vascularized and in close contact with the environment [98]. Given this contact with the environment, a strong local immune defence is required. While immune cells and immune factors can be found scattered throughout the whole gill (Reviewed in [99]), a defined T cell-rich immune structure has been identified, referred to as the interbranchial lymphoid tissue (ILT) [100-104]. Given its T cell richness and its systemic as well as mucosal nature, the gills would serve as a perfect tool to study the reactivity of the WCL38 antibody. By collaborating with the group that performed the initial characterization of the ILT, we were able to study WCL38 ${ }^{+}$and WCL38- $\mathrm{T}$ cell populations in the ILT of common carp. Given that regrettably, the rat-anti-Cd4-1 and rat-anti-Cd8 $\alpha 1$ do not work in immunohistochemistry, we combined the WCL38 antibody with 
an antibody against the pan-(NK)T-cell marker Zap70. Using this combination of antibodies we revealed that while Zap $70^{+}$cells are homogeneously distributed in the whole proximal ILT except the epithelial layer (Fig. 3A,C), WCL38 ${ }^{+}$cells are only found at the intermediate proximal ILT, which is immediately underneath the epithelial layer and therefore close to the environment (Fig. 3B,D). While we see that in the intermediate proximal ILT Zap $70^{+}$and WCL38 ${ }^{+}$cells co-localize (Fig. $3 \mathrm{E}, \mathrm{H}$ ), it is difficult to distinguish which proportion is single positive (either Zap70 or WCL $38^{+}$) or double positive. What we also observed is that besides the small lymphoid cells, larger cells with less-dense nuclei are recognized by the WCL38 antibody (Fig. $3 \mathrm{G}, \mathrm{H}$, white arrowheads). This result shows that the WCL38 antibody

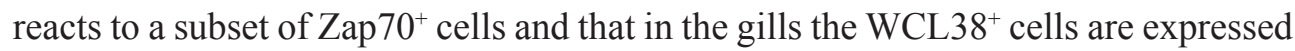
in a strict location. In agreement to previous observations, we observed that in gills WCL38 does not only recognize small lymphoid cells, but also larger cells with lessdense nuclei.

This observation led to the hypothesis that even within the ILT, a division can be made between the "mucosal" and the "systemic" part. Using laser-dissection of the two regions of the ILT followed by RNA Sequencing, we are currently investigating whether a true mucosal vs. systemic profile can be identified. While the gills have a much higher population of $\mathrm{Cd} 4^{+}$cells than the intestine, it needs to be examined whether the WCL $38^{+}$in the gills also is only exclusively co-expressed with $\mathrm{Cd} 8$ or not. If the WCL $38^{+} \mathrm{Cd} 8 \alpha 1^{+}$cells play such a crucial role in the immune defence in the gills, it would make sense for the $\mathrm{Cd} 4^{+}$cells to be more abundantly present in the basal proximal ILT, since there is still a large Zap $70^{+}$population there. To this end, techniques like in situ hybridization can be used to further study the spatial distribution of various T cell populations in the ILT. However, since we observed an additional population of WCL $38^{+}$in the gills that we did not observe in the intestine, these cells should be identified separately. FACS sorting and RNA sequencing will reveal important information about this larger lymphoid WCL $38^{+}$cells, and when these populations are sorted and analysed separately, it will certainly aid to determine the different cell types recognized by the WCL38 antibody.

\section{From tools to study WCL38 to WCL38 as a study tool}

While we now put considerable effort in characterizing which cell populations are recognized by the WCL38 antibody, how could we use it to our best once we have confirmed exactly which epitope it recognizes? One main conclusion we can already 
draw is that WCL38 requires additional antibodies in order to draw conclusions. For example, even with the $\mathrm{Cd} 8 \alpha 1$ antibody we detected multiple populations. However, if we in the end reveal the epitope of WCL38, we can potentially establish an antibody panel that would allow us to study mucosal (Cd8) T cells and compare them to nonmucosal T cells. Especially when looking at the increased focus on and interest in the development of mucosal vaccines, the availability of such panel will be of great value and would allow us to study in greater detail compartmentalization of the gill and intestine, as well as to further characterize immune responses at mucosal surfaces during infection and after (mucosal) vaccination.
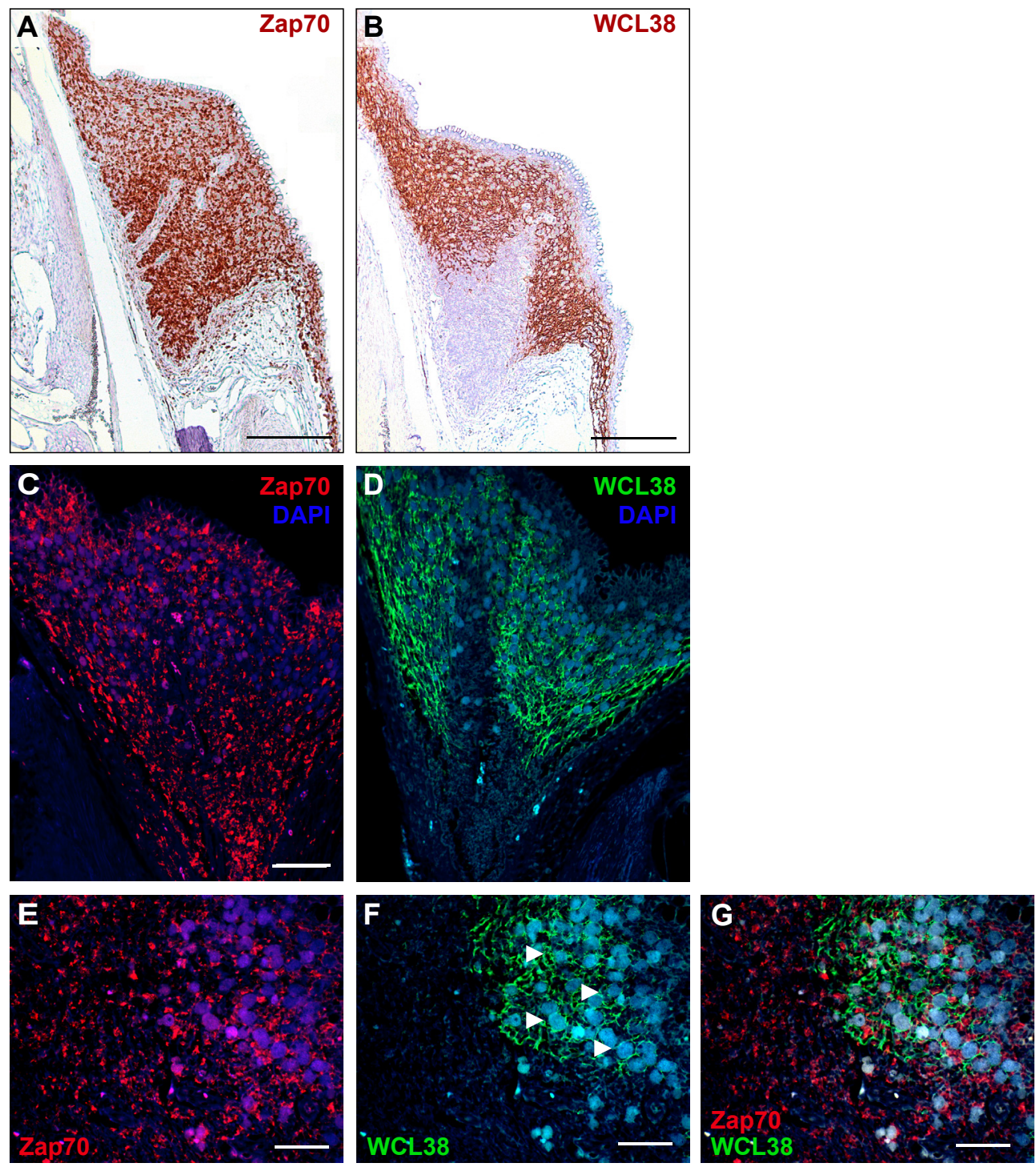
Fig. 3. WCL38 and Zap70 staining of the proximal ILT of common carp. Paraffin sections $(4 \mu \mathrm{m})$ of formalin-fixed gills of naîve carp were processed and stained as described before [101] using the anti-Zap-70 or/and the WCL38 antibody, followed by goat-anti-rabbit-HRP and goat-anti-mouse-HRP and development using 3,30-diaminobenzidine (DAB) and a counterstaining with haematoxylin (A,B), or followed by goat-anti-mouse Alexa488 (green) and goat-anti-rabbit-Alexa594 (red) and a staining with DAPI to visualize the cell nuclei (C-H). White arrowheads in $(\mathrm{H})$ indicate the staining of WCL38+ large granular cells with less-dense nuclei. Scale bars represent $400 \mu \mathrm{m}(A-D)$ or $100 \mu \mathrm{m}(E-G)$.

\section{Characterizing B cells: may the force B with you.}

Similar to the challenges in developing antibodies against specific $\mathrm{T}$ cell subsets, not many antibodies are yet available against the different $\mathrm{B}$ cell subsets of teleost fish. While we show the initial characterization of Igt $1^{+} \mathrm{B}$ cells in carp in chapter 7, we are currently still optimizing the available tools to also obtain full insight into Igt2 responses in common carp. While there is no doubt about the abundance of Igm in serum and in mucus, as well as the presence of pathogen-specific Igm, the true function for Igt still needs to be unravelled. When looking at the few studies where antibodies were available against Igt, pathogen-specific Igt titres could only be detected in samples that were minimally diluted [105-107] or in samples in which Ig enrichment had to be performed before Igt could be detected [108]. Also in this thesis, we show that by sandwich ELISA Igt1 and Igt2 could be detected only in serum of fish that survived an infection with the blood-borne parasite Trypanoplasma borreli. While for detection of Igt1 the serum could only be diluted 20 times, Igm in serum was detected when the serum was diluted up to 160.000 times. This shows that in serum, Igts are present at much lower concentrations than Igm. While the first papers describing Igt in teleost species highlighted the mucosal role of Igt and hereby focused on mucosal infections $[106,107,109]$ we, amongst others, show that Igts are not strictly mucosal and might also be involved in systemic responses, despite their relatively low abundance.

Nevertheless, two questions remain; 1) what is the function of Igt and 2) how relevant is the function of Igt?

Although Igt responses were described after vaccination against and during infection with various viruses [2,110-113] whether Igts are able to neutralize viruses and/or fix complement is still not known. If not, what then is the role of Igt in the response to viruses? Since neutralizing antibody tests are always performed with total serum, the relative contribution of the different Igs is not easy to ascertain. Furthermore, given the high amounts of Igm in serum, Igts are easily overshadowed and depletion or 
pre-absorption of the Igm in the serum would be required to quantify the neutralizing capacities of Igt. Similar to serum samples, mucus samples could be used to test neutralizing abilities, or in the case of parasites, motility assays. However, the presence of many factors in the mucus that might interact with the pathogen, together with the low amounts of Igt present, will increase the difficulty to point out the exact contribution of Igts to the observed effect. In this case, depletion of Igt from the samples might provide indirect evidence of their involvement.

Since our newly developed antibody against Igt1 allowed for the visualization of parasite-bound Igt1, we used a similar approach to preliminary visualize virus-specific Igts. Here, we used heat-inactivated serum from fish that were DNA vaccinated against SVCV ( $1 \mu \mathrm{g} / \mathrm{g}$ of fish by i.m. injection, 3 months after vaccination) to stain SVCV-infected Common Carp Brain (CCB) cells and afterwards stained with antiIgm or anti-Igt1 antibodies. As can be seen in Fig. 4, we were able to detect both SVCV-specific Igm and Igt1, while the serum only showed low background against non-infected cells. However, this should be compared to serum from naïve fish or fish receiving the control DNA vaccine. Furthermore, while this shows binding of the Igs, it does not necessarily indicate neutralization. Given the general difficulties with characterizing the function of the different B cell subsets, we should maybe aim for completely different approaches. For example, now that antibodies specific for Igt1 and Igm are available, we can use them to specifically deplete either Igm or Igt1 from serum, and use the depleted serum to assess (residual) passive transfer of immunity. Using the same antibodies, cells can be sorted, (re-)exposed in vitro to antigens and afterwards, the cell culture supernatant can be used to assess the presence of antigen-specific Ig and their neutralizing ability. Furthermore, we can make use of the zebrafish model. While Igt transgenic fish would allow for visualization of (trafficking of) the cells in vivo, the use of transgenic lines bearing the gene for the Escherichia coli nitroreductase enzyme (nfsB) would allow us to specifically ablate cell populations [114,115] (Igm, Igt1, Igt2), allowing to study their function using various infection models.

\section{Genomes and transcriptomes: is more data better?}

Rapidly, an increasing amount of genome information is becoming available for many teleost species mostly driven by a decrease in costs for next-generation sequencing (NGS) approaches. This development has also been ongoing for common carp, with 

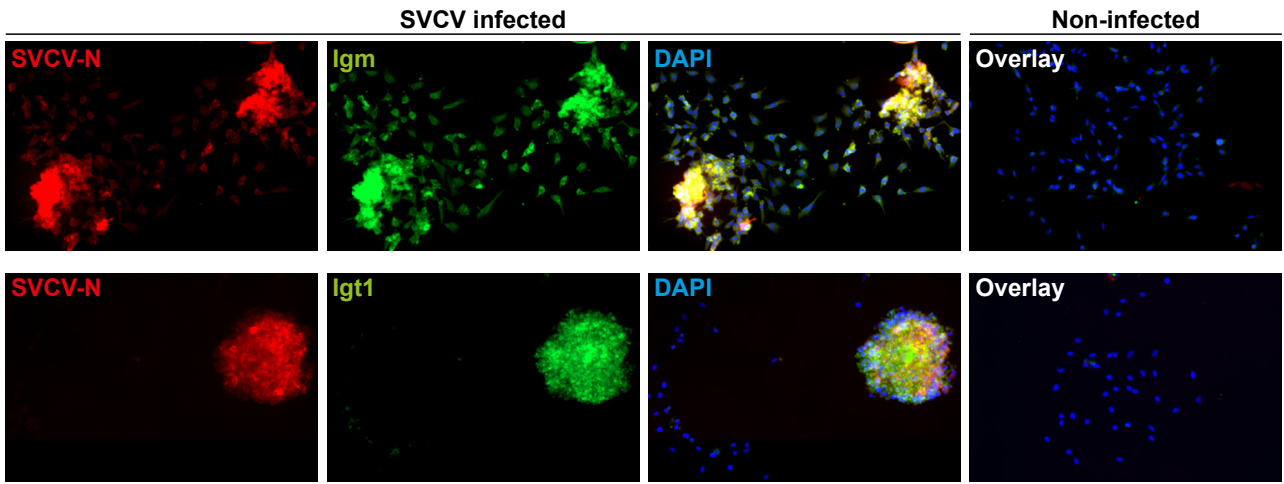

Fig. 4. Binding of common carp Igm and Igt1 to SVCV-infected CCB cells. CCBs were seeded on glass cover slides in 12-well plates (175.000/well). The next day, cells were infected for 2 hours with SVCV at an MOI of 1 . At 36 hours post-infection, cells were fixed, permeabilized with methanol, blocked with $5 \%$ normal goat serum in PBS and incubated with serum from carp that were i.m. DNA vaccinated (1 $\mu \mathrm{g}$ DNA/g of fish pcDNA3-SVCV-G). Serum was collected 3 months after vaccination. Bound lgs were detected with FITC-labelled mouse-anti-lgm (1:200, WCl12), or rabbit-anti-lgt1 $(5 \mu \mathrm{g} / \mathrm{mL})$ followed by goat-anti-rabbitAlexa488 (1:2000). SVCV infection was visualized using a mouse-anti-SVCV-N (1:500, Bio311) and goat-anti-mouse PE (1:200). A final staining with DAPI was included to visualize the cell nuclei and afterwards, cover slips were mounted on polysine slides and imaged using a DM6 microscope (Leica). Non-infected CCBs were included to detect the presence of nonspecific binding of the carp Igs to CCBs.

the first partial de novo assembly of the carp genome published relatively recently in 2011 [116], but quickly followed by a more complete genome and transcriptome assemblies in 2012 [117] and 2016 [118]. Indeed, RNA sequencing allows for studying complete transcriptome profiles of given samples, an approach rapidly outcompeting RT-qPCR analysis which typically addresses limited sets of target genes. Where RNA sequencing provides a broad range of unbiased information on large numbers of genes, and may thus lead to renewed biological insights, there are also shortcomings associated with the analysis of these large datasets. Apart from their somewhat overwhelming nature naturally associated with the large amount of information, an important limitation is that often RNA sequences are mapped on fish genomes that suffer from incomplete annotation, like we encountered when analysing the carp transcriptome datasets described in chapter 6. For the carp genome, many genes have not (yet) been placed on final linkage maps and many genes remain unidentified. Often, sequences ranking as highly expressed or highly regulated in large transcriptome datasets appear as 'unknown gene sequence', which only rarely can be repaired by manual blasts against the most recent information in the NCBI database. It is important to realize, especially in the case of tetraploid common carp which underwent several genome duplication events, that current annotation 
tools have not been optimized to handle subtle differences in nucleotide sequence between gene duplicates [119]. This can potentially lead to misinterpretations when duplicated genes exert different biological functions. In the near future, these errors can probably be prevented by the introduction of nanopore and PacBio long read sequencing techniques that are inherently less sensitive to these types of errors than short read sequencing techniques. This opens up the route for routine analysis of complete transcriptome profiles of samples including post-vaccination and/or postchallenge analyses in immune-relevant organs. These experiments can then include time-course studies, but also single cell sequencing approaches of B or T cell subpopulations sorted to purity with the antibodies described in this thesis.

\section{Integration of the results into common practise; societal relevance and scientific impact}

Common carp culture is threatened by many different pathogens, of which SVCV and KHV are the most relevant, and no effective vaccines are yet available for worldwide use. Furthermore, the lack of tools to study (adaptive) immune responses severely hampers the understanding of protective mechanisms induced by (experimental) vaccination. This knowledge is urgently needed to identify the aforementioned correlates of protection. In this thesis, we have developed experimental vaccines for SVCV and KHV and tested them using challenge models that resemble natural routes of infections. Besides studying their afforded protection, we characterized local and systemic immune responses that were induced through vaccination.

For SVCV we show that while i.m. vaccination using a DNA vaccine encoding the SVCV-G protein induces full protection even when administered at $0.1 \mu \mathrm{g} / \mathrm{g}$ of fish (chapter 3), we were not able to obtain sufficient protection when the same vaccine was administered orally (chapter 4). However, the insights on the immune response after successful DNA vaccination will be very valuable when improving the non-optimal subunit vaccines and the oral DNA vaccine reported in chapter 4. Furthermore, in chapter 2 we discussed oral vaccination for fish using a wider perspective, also including veterinary and human insights, which will be valuable for oral vaccine development for all fish species. The insights on protective mechanisms will also be of much use for the optimization of the DNA vaccine against KHV (chapter 5), however, we also discuss and propose alternative vaccine strategies. In order to better adaptive study immune responses of common carp, we developed tools to characterize $\mathrm{T}$ cells (chapter 6) and B cells (chapter 7), which will be 
widely applicable and of much use to characterize immune responses at a basal level, during infection as well as after vaccination.

On the longer term, the results from this thesis could also contribute to improving the aquaculture industry. The development of targeted vaccines for fish aquaculture not only increases animal welfare by decreasing disease incidence, but also contributes to more sustainable aquaculture by decreasing the need for antibiotics. As demonstrated in this thesis, DNA vaccines are a promising technique for targeted vaccination. Considering the recent first legal approval of a DNA vaccine, widespread application of these vaccines may be possible in the near future.

Over the course of this thesis, the overarching TargetFish project directly and immediately involved stakeholders in society. The results of this thesis, and other research within the project, were reported to industrial stakeholders during several so-called 'Industrial Forum' meetings. At these meetings, representatives of the major fish vaccine companies were invited to attend presentations reporting on the most recent updates in fish vaccine development within the project. Through monthly newsletters, the TargetFish project updated fish farmers on our progress, and invited them to 'demonstration' sessions, where scientists would demonstrate the use of novel experimental vaccines.

In conclusion, this thesis contributed to the protection of common carp against viral diseases in common carp culture by 1) the development of an effective i.m. DNA vaccine against SVCV, 2) reporting on suboptimal subunit vaccines for SVCV as well as DNA vaccines against KHV and proposing how they can be improved and 3) greatly expanding the toolbox available to study adaptive immune responses in common carp that will prove instrumental to evaluate host-pathogen interaction and future vaccination strategies. Lastly, outcomes presented in this thesis might have a broader sustained impact on the fish immunology society as well as to the aquaculture sector, given that the outcomes have been presented at many international meetings as well as to stakeholders, through the Industrial Platform of TargetFish. 


\section{References}

1. Embregts CWE, Forlenza M. Oral vaccination of fish: Lessons from humans and veterinary species. Dev Comp Immunol (2016) 64:118-137. doi:10.1016/j.dci.2016.03.024

2. Embregts CWE, Rigaudeau D, Veselý T, Pokorová D, Lorenzen N, Petit J, Houel A, Dauber M, Schütze H, Boudinot P, et al. Intramuscular DNA Vaccination of Juvenile Carp against Spring Viremia of Carp Virus Induces Full Protection and Establishes a Virus-Specific B and T Cell Response. Front Immunol (2017) 8:1340. doi:10.3389/fimmu.2017.01340

3. Utke K, Kock H, Schuetze H, Bergmann SM, Lorenzen N, Einer-Jensen K, Köllner B, Dalmo RA, Vesely T, Ototake M, et al. Cell-mediated immune responses in rainbow trout after DNA immunization against the viral hemorrhagic septicemia virus. Dev Comp Immunol (2008) 32:239-252. doi:10.1016/j.dci.2007.05.010

4. Boudinot P, Bernard D, Boubekeur S, Thoulouze MI, Bremont M, Benmansour A. The glycoprotein of a fish rhabdovirus profiles the virus-specific T-cell repertoire in rainbow trout. J Gen Virol (2004) 85:3099-3108. doi:10.1099/vir.0.80135-0

5. Takano T, Iwahori A, Hirono I, Aoki T. Development of a DNA vaccine against hirame rhabdovirus and analysis of the expression of immune-related genes after vaccination. Fish Shellfish Immunol (2004) 17:367-374. doi:10.1016/j.fsi.2004.04.012

6. Cuesta A, Tafalla C. Transcription of immune genes upon challenge with viral hemorrhagic septicemia virus (VHSV) in DNA vaccinated rainbow trout (Oncorhynchus mykiss). Vaccine (2009) 27:280-9. doi:10.1016/j.vaccine.2008.10.029

7. Lorenzen N, Lorenzen E, Einer-Jensen K, Heppell J, Davis HL. Genetic vaccination of rainbow trout against viral haemorrhagic septicaemia virus: small amounts of plasmid DNA protect against a heterologous serotype. Virus Res (1999) 63:19-25. Available at: www. elsevier.com/locate/virusres [Accessed October 30, 2017]

8. Kanellos T, Sylvester ID, D'Mello F, Howard CR, Mackie A, Dixon PF, Chang KC, Ramstad A, Midtlyng PJ, Russell PH. DNA vaccination can protect Cyprinus Carpio against spring viraemia of carp virus. Vaccine (2006) 24:4927-4933. doi:10.1016/j.vaccine.2006.03.062

9. Lorenzen N, Lorenzen E, Einer-Jensen K, Heppell J, Wu T, Davis H. Protective immunity to VHS in rainbow trout (Oncorhynchus mykiss, Walbaum) following DNA vaccination. Fish Shellfish Immunol (1998) 8:261-270.

10. EMA. First DNA vaccine in the EU recommended for use in salmon. 2016-04-22 (2016)1. Available at: http://www.ema.europa.eu/ema/index.jsp?curl=pages/news_and_events/ news/2016/04/news_detail_002516.jsp\&mid=WC0b01ac058004d5c1 [Accessed September $18,2017]$

11. Salonius K, Simard N, Harland R, Ulmer JB. The road to licensure of a DNA vaccine. Curr Opin Investig Drugs (2007) 8:635-641.

12. Houston R, Moxon S, Nogué F, Papadopoulou N, Ramon M, Waigmann E. Assessment of the potential integration of the DNA plasmid vaccine CLYNAV into the salmon genome. EFSA J (2017) 15:1-15. doi:10.2903/j.efsa.2017.4689

13. Kutzler MA, Weiner DB. DNA vaccines: Ready for prime time? Nat Rev Genet (2008) 9:776-788. doi:10.1038/nrg2432

14. Ledwith BJ, Manam S, Troilo PJ, Barnum AB, Pauley CJ, Griffiths TG, Harper LB, Schock HB, Zhang H, Faris JE, et al. Plasmid DNA vaccines: assay for integration into host genomic DNA. Dev Biol (Basel) (2000) 104:33 - 43. Available at: http://europepmc.org/abstract/ MED/11713822

15. Pal R, Yu Q, Wang S, Kalyanaraman VS, Nair BC, Hudacik L, Whitney S, Keen T, Hung CL, Hocker L, et al. Definitive toxicology and biodistribution study of a polyvalent DNA prime/ protein boost human immunodeficiency virus type 1 (HIV-1) vaccine in rabbits. Vaccine (2006) 24:1225-1234. doi:10.1016/j.vaccine.2005.07.112

16. Manam S, Ledwith B, Barnum AB, Troilo PJ, Pauley CJ, Harper LB, Griffiths TG, Niu ZT, Denisova L, Follmer TT, et al. Plasmid DNA vaccines: Tissue distribution and effects of 
DNA sequence, adjuvants and delivery method on integration into host DNA. Intervirology (2000) 43:273-281. doi:10.1159/000053994

17. Sheets RL, Stein J, Manetz TS, Andrews C, Bailer R, Rathmann J, Gomez PL. Toxicological safety evaluation of DNA plasmid vaccines against HIV-1, Ebola, Severe Acute Respiratory Syndrome, or West Nile virus is similar despite differing plasmid backbones or gene-inserts. Toxicol Sci (2006) 91:620-630. doi:10.1093/toxsci/kfj170

18. Byon JY, Ohira T, Hirono I, Aoki T. Comparative immune responses in Japanese flounder, Paralichthys olivaceus after vaccination with viral hemorrhagic septicemia virus (VHSV) recombinant glycoprotein and DNA vaccine using a microarray analysis. Vaccine (2006) 24:921-30. doi:10.1016/j.vaccine.2005.08.087

19. Pereiro P, Martinez-Lopez A, Falco A, Dios S, Figueras A, Coll JM, Novoa B, Estepa A. Protection and antibody response induced by intramuscular DNA vaccine encoding for viral haemorrhagic septicaemia virus (VHSV) G glycoprotein in turbot (Scophthalmus maximus). Fish Shellfish Immunol (2012) 32:1088-1094. doi:10.1016/j.fsi.2012.03.004

20. Hart LM, Lorenzen N, Lapatra SE, Grady CA, Roon SE, O’Reilly J, Gregg JL, Hershberger PK. Efficacy of a glycoprotein DNA vaccine against viral haemorrhagic septicaemia (VHS) in pacific herring, clupea pallasii valenciennes. J Fish Dis (2012) 35:775-779. doi:10.1111/ j.1365-2761.2012.01364.x

21. Garver KA, LaPatra SE, Kurath G. Efficacy of an infectious hematopoietic necrosis (IHN) virus DNA vaccine in Chinook Oncorhynchus tshawytscha and sockeye O. nerka salmon. Dis Aquat Organ (2005) 64:13-22. doi:10.3354/dao064013

22. Kurath G, Garver KA, Corbeil S, Elliott DG, Anderson ED, LaPatra SE. Protective immunity and lack of histopathological damage two years after DNA vaccination against infectious hematopoietic necrosis virus in trout. Vaccine (2006) 24:345-354. doi:10.1016/j. vaccine. 2005.07.068

23. Ahne W, Bjorklund HV, Essbauer S, Fijan N, Kurath G, Winton JR. Spring viremia of carp (SVC). Dis Aquat Organ (2002) 52:261-272. doi:10.3354/dao052261

24. Dauber M, Schütze H, Fichtner D. Development and characterization of monoclonal antibodies raised against a viral haemorrhagic septicaemia virus (VHSV) isolate which failed to be identified by a commercial kit. Bull Eur Assoc Fish Pathol (2001) 21:170-177.

25. Teng Y, Liu H, Lv JQ, Fan WH, Zhang QY, Qin QW. Characterization of complete genome sequence of the spring viremia of carp virus isolated from common carp (Cyprinus carpio) in China. Arch Virol (2007) 152:1457-1465. doi:10.1007/s00705-007-0971-8

26. Emmenegger EJ, Kurath G. DNA vaccine protects ornamental koi (Cyprinus carpio koi) against North American spring viremia of carp virus. Vaccine (2008) 26:6415-6421. doi:10.1016/j.vaccine.2008.08.071

27. Cui L-C, Guan X-TX-T, Liu Z-MZ-M, Tian C-YC-Y, Xu YGY-G. Recombinant lactobacillus expressing $\mathrm{G}$ protein of spring viremia of carp virus (SVCV) combined with ORF81 protein of koi herpesvirus (KHV): A promising way to induce protective immunity against SVCV and KHV infection in cyprinid fish via oral vaccination. Vaccine (2015) 33: doi:10.1016/j. vaccine. 2015.05.002

28. Akhter N, Wu B, Memon AM, Mohsin M. Probiotics and prebiotics associated with aquaculture: A review. Fish Shellfish Immunol (2015) 45:733-741. doi:10.1016/j. fsi.2015.05.038

29. Balcázar JL, Vendrell D, de Blas I, Ruiz-Zarzuela I, Gironés O, Muzquiz JL. Immune modulation by probiotic strains: Quantification of phagocytosis of Aeromonas salmonicida by leukocytes isolated from gut of rainbow trout (Oncorhynchus mykiss) using a radiolabelling assay. Comp Immunol Microbiol Infect Dis (2006) 29:335-343. doi:10.1016/j. cimid.2006.09.004

30. Mohapatra S, Chakraborty T, Prusty AK, Kumar K, Pani Prasad K, Mohanta KN. Fenvalerate induced stress mitigation by dietary supplementation of multispecies probiotic mixture in a tropical freshwater fish, Labeo rohita (Hamilton). Pestic Biochem Physiol (2012) 104:28-37. 
doi:10.1016/j.pestbp.2012.06.006

31. Nayak SK. Probiotics and immunity: A fish perspective. Fish Shellfish Immunol (2010) 29:214. doi:10.1016/j.fsi.2010.02.017

32. Wells J. Mucosal Vaccination and Therapy with Genetically Modified Lactic Acid Bacteria. Annu Rev Food Sci Technol (2011) 2:423-445. doi:10.1146/annurev-food-022510-133640

33. de Azevedo MSP, Santos Rocha C, Pereira VB, de Oliveira Junior AF, de Sousa CS, Azevedo V, LeBlanc JG, Chatel JM, Miyoshi A. Prospective uses of recombinant Lactococcus lactis expressing both listeriolysin $\mathrm{O}$ and mutated internalin A from Listeria monocytogenes as a tool for DNA vaccination. Genet Mol Res (2015) 14:18485-18493. doi:10.4238/2015. December.23.36

34. Joerink M, Ribeiro CMS, Stet RJM, Hermsen T, Savelkoul HFJ, Wiegertjes GF. Head Kidney-Derived Macrophages of Common Carp (Cyprinus carpio L.) Show Plasticity and Functional Polarization upon Differential Stimulation. J Immunol (2006) 177:61-69. doi:10.4049/jimmunol.177.1.61

35. Petit J, Wiegertjes GF. Long-lived effects of administering $\beta$-glucans: Indications for trained immunity in fish. Dev Comp Immunol (2016) 64:93-102. doi:10.1016/j.dci.2016.03.003

36. Ogier de Baulny M, Quentel C, Fournier V, Lamour F, Le Gouvello R. Effect of long-term oral administration of beta-glucan as an immunostimulant or an adjuvant on some nonspecific parameters of the immune response of turbot Scophthalmus maximus. Dis Aquat Organ (1996) 26:139-147. doi:10.3354/dao026139

37. Bonaldo a, Bonaldo a, Thompson KD, Thompson KD, Manfrin a, Manfrin a, Adams a, Adams a, Murano E, Murano E, et al. The influence of dietary beta-glucans on the adaptive and innate immune responses of European sea bass (Dicentrarchus labrax) vaccinated against vibriosis. Ital J Anim Sci (2007) 6:151-164.

38. Selvaraj V, Sampath K, Sekar V. Adjuvant and immunostimulatory effects of $\beta$-glucan administration in combination with lipopolysaccharide enhances survival and some immune parameters in carp challenged with Aeromonas hydrophila. Vet Immunol Immunopathol (2006) 114:15-24. doi:10.1016/j.vetimm.2006.06.011

39. De Smet R, Demoor T, Verschuere S, Dullaers M, Ostroff GR, Leclercq G, Allais L, Pilette $\mathrm{C}$, Dierendonck M, De Geest BG, et al. B-Glucan microparticles are good candidates for mucosal antigen delivery in oral vaccination. J Control Release (2013) 172:671-678. doi:10.1016/j.jconrel.2013.09.007

40. Huang H, Ostroff GR, Lee CK. Robust Stimulation of Humoral and Cellular Immune Responses following Vaccination with Antigen-Loaded $\beta$-Glucan Particles Robust Stimulation of Humoral and Cellular Immune Responses following Vaccination with Antigen-Loaded $\square$-Glucan Particles. MBio (2010)1-7. doi:10.1128/mBio.00164-10.Editor

41. Seo JY, Chung HJ, Kim TJ. Codon-optimized expression of fish iridovirus capsid protein in yeast and its application as an oral vaccine candidate. J Fish Dis (2013) 36:763-768. doi:10.1111/jfd.12037

42. Fuchs W, Granzow H, Dauber M, Fichtner D, Mettenleiter TC. Identification of structural proteins of koi herpesvirus. Arch Virol (2014) 159:3257-3268. doi:10.1007/s00705-0142190-4

43. Han JE, Kim JH, Renault T, Casiano C, Shin SP, Jun JW, Park SC. Identifying the viral genes encoding envelope glycoproteins for differentiation of Cyprinid herpesvirus 3 isolates. Viruses (2013) 5:568-576. doi:10.3390/v5020568

44. Michel B, Leroy B, Raj VS, Lieffrig F, Mast J, Wattiez R, Vanderplasschen AF, Costes B. The genome of cyprinid herpesvirus 3 encodes 40 proteins incorporated in mature virions. $J$ Gen Virol (2010) 91:452-462. doi:10.1099/vir.0.015198-0

45. Vancsok C, Peñaranda MMD, Raj VS, Leroy B, Jazowiecka-Rakus J, Boutier M, Gao Y, Wilkie GS, Suárez NM, Wattiez R, et al. Proteomic and Functional Analyses of the Virion Transmembrane Proteome of Cyprinid Herpesvirus 3. J Virol (2017) 91:JVI.01209-17. doi:10.1128/JVI.01209-17 
46. Zhou J-X, Wang H, Li X-W, Zhu X, Lu W-L, Zhang D-M. Construction of KHV-CJ ORF25 DNA vaccine and immune challenge test. J Fish Dis (2014) 37:319-325. doi:10.1111/ jfd.12105

47. Zhou J-X, Xue J, Wang Q, Zhu X, Li X, Lv. W, Zhang D, Lu. W, D. Z. Vaccination of plasmid DNA encoding ORF81 gene of CJ strains of KHV provides protection to immunized carp. Vitr Cell Dev Biol - Anim (2014) 50:489-495. doi:10.1007/s11626-014-9737-2

48. Boutier M, Ronsmans M, Ouyang P, Fournier G, Reschner A, Rakus K, Wilkie GS, Farnir FF, Bayrou C, Lieffrig FF, et al. Rational Development of an Attenuated Recombinant Cyprinid Herpesvirus 3 Vaccine Using Prokaryotic Mutagenesis and In Vivo Bioluminescent Imaging. PLoS Pathog (2015) 11: doi:10.1371/journal.ppat.1004690

49. Boutier M, Gao Y, Vancsok C, Suárez NM, Davison AJ, Vanderplasschen A. Identification of an essential virulence gene of cyprinid herpesvirus 3. Antiviral Res (2017) 145:60-69. doi:10.1016/j.antiviral.2017.07.002

50. Costes B, Fournier G, Michel B, Delforge C, Raj VS, Dewals B, Gillet L, Drion P, Body A, Schynts F, et al. Cloning of the koi herpesvirus genome as an infectious bacterial artificial chromosome demonstrates that disruption of the thymidine kinase locus induces partial attenuation in Cyprinus carpio koi. J Virol (2008) 82:4955-64. doi:10.1128/JVI.00211-08

51. Fuchs W, Fichtner D, Bergmann SM, Mettenleiter TC. Generation and characterization of koi herpesvirus recombinants lacking viral enzymes of nucleotide metabolism. Arch Virol (2011) 156:1059-1063. doi:10.1007/s00705-011-0953-8

52. Perelberg A, Ronen A, Hutoran M, Smith Y, Kotler M. Protection of cultured Cyprinus carpio against a lethal viral disease by an attenuated virus vaccine. Vaccine (2005) 23:33963403. doi:10.1016/j.vaccine.2005.01.096

53. Ronen A, Perelberg A, Abramowitz J, Hutoran M, Tinman S, Bejerano I, Steinitz M, Kotler M. Efficient vaccine against the virus causing a lethal disease in cultured Cyprinus carpio. Vaccine (2003) 21:4677-4684. doi:10.1016/S0264-410X(03)00523-1

54. Schmid T, Gaede L, Böttcher K, Bräuer G, Fichtner D, Beckmann R, Speck S, Becker F, Truyen U. Efficacy assessment of three inactivated koi herpes virus antigen preparations against experimental challenge virus infection in common carp. J Fish Dis (2016) 39:10071013. doi:10.1111/jfd. 12428

55. Yasumoto S, Yoshimura T, Miyazaki T. Oral immunization of common carp with a liposome vaccine containing Aeromonas hydrophila antigen. Fish Pathol (2006) 41:45-49. doi: $10.3147 /$ jsfp. 41.45

56. French TJ, Roy P. Synthesis of bluetongue virus (BTV) corelike particles by a recombinant baculovirus expressing the two major structural core proteins of BTV. J Virol (1990) 64:1530-1536.

57. Kibenge FSB, Qian B, Nagy É, Cleghorn JR, Wadowska D. Formation of virus-like particles when the polyprotein gene (segment A) of infectious bursal disease virus is expressed in insect cells. Can J Vet Res (1999) 63:49-55.

58. Vieira HLA, Estêvão C, Roldão A, Peixoto CC, Sousa MFQ, Cruz PE, Carrondo MJT, Alves PM. Triple layered rotavirus VLP production: Kinetics of vector replication, mRNA stability and recombinant protein production. J Biotechnol (2005) 120:72-82. doi:10.1016/j. jbiotec.2005.03.026

59. Chung Y, Huang J, Lai C, Sheng H, Shih S, Ho M, Hu Y. Expression , purification and characterization of enterovirus-71 virus-like particles. (2006) 12:921-927.

60. Allnutt FCTT, Bowers RM, Rowe CG, Vakharia VN, LaPatra SE, Dhar AK. Antigenicity of infectious pancreatic necrosis virus VP2 subviral particles expressed in yeast. Vaccine (2007) 25:4880-4888. doi:10.1016/j.vaccine.2007.04.068

61. Dhar AK, Bowers RM, Rowe CG, Allnutt FCT. Expression of a foreign epitope on infectious pancreatic necrosis virus VP2 capsid protein subviral particle (SVP) and immunogenicity in rainbow trout. Antiviral Res (2010) 85:525-531. doi:10.1016/j.antiviral.2009.12.009

62. Martinez-Alonso S, Vakharia VN, Saint-Jean SR, Pérez-Prieto S, Tafalla C. Immune 
responses elicited in rainbow trout through the administration of infectious pancreatic necrosis virus-like particles. Dev Comp Immunol (2012) 36:378-384. doi:10.1016/j. dci.2011.07.010

63. Thiery R, Cozien J, Cabon J, Lamour F, Baud M, Schneemann A. Induction of a Protective Immune Response against Viral Nervous Necrosis in the European Sea Bass Dicentrarchus labrax by Using Betanodavirus Virus-Like Particles. J Virol (2006) 80:10201-10207. doi:10.1128/JVI.01098-06

64. Lin CS, Lu MW, Tang L, Liu W, Chao C Ben, Lin CJ, Krishna NK, Johnson JE, Schneemann A. Characterization of virus-like particles assembled in a recombinant baculovirus system expressing the capsid protein of a fish nodavirus. Virology (2001) 290:50-58. doi:10.1006/ viro.2001.1157

65. Wi GR, Hwang JY, Kwon M-G, Kim HJ, Kang HA, Kim H-J. Protective immunity against nervous necrosis virus in convict grouper Epinephelus septemfasciatus following vaccination with virus-like particles produced in yeast Saccharomyces cerevisiae. Vet Microbiol (2015) 177:214-218. doi:10.1016/j.vetmic.2015.02.021

66. Wang JW, Roden RBS. Virus-like particles for the prevention of human papillomavirusassociated malignancies. Expert Rev Vaccines (2013) 12:129-141. doi:10.1586/erv.12.151

67. Mohsen MO, Zha L, Cabral-Miranda G, Bachmann MF. Major findings and recent advances in virus-like particle (VLP)-based vaccines. Semin Immunol (2017) 34:123-132. doi:10.1016/j.smim.2017.08.014

68. Valenzuela P, Medina A, Rutter WJ, Ammerer G, Hall BD. Synthesis and assembly of hepatitis B virus surface antigen particles in yeast. Nature (1982) 298:347-50. doi:10.1038/298347a0

69. Perez EM, Foley J, Tison T, Silva R, Ogembo JG, Perez EM, Foley J, Tison T, Silva R, Ogembo JG. Novel Epstein-Barr virus-like particles incorporating gH/gL-EBNA1 or gB-LMP2 induce high neutralizing antibody titers and EBV-specific T-cell responses in immunized mice. Oncotarget (2016) 5:19255-19273. doi:10.18632/oncotarget.13770

70. Ruiss R, Jochum S, Wanner G, Reisbach G, Hammerschmidt W, Zeidler R. A Virus-Like Particle-Based Epstein-Barr Virus Vaccine. J Virol (2011) 85:13105-13113. doi:10.1128/ JVI.05598-11

71. Torrent F, Villena A, Lee PA, Fuchs W, Bergmann SM, Coll JM. The amino-terminal domain of ORF149 of koi herpesvirus is preferentially targeted by IgM from carp populations surviving infection. (2016) 161:2653-2665. doi:10.1007/s00705-016-2934-4

72. Tu C, Lu YP, Hsieh CY, Huang SM, Chang SK, Chen MM. Production of monoclonal antibody against ORF72 of koi herpesvirus isolated in Taiwan. Folia Microbiol (Praha) (2014) 59:159-165. doi:10.1007/s12223-013-0261-7

73. Miyazaki T, Yasumoto S, Kuzuya Y, Yoshimura T. "A primary study on oral vaccination with liposomes entrapping Koi Herpesvirus ( KHV) antigens against KHV infection in carp," in Diseases in Asian Aquaculture, ed. R. P. Bondad-Reantaso, M.G., Mohan, C.V., Crumlish, M. and Subasinghe (Manila: Asian Fisheries Society), 99-184.

74. Yasumoto S, Kuzuya Y, Yasuda M, Yoshimura T, Miyazaki T. Oral immunization of common carp with a liposome vaccine fusing Koi Herpesvirus antigen. Fish Pathol (2006) 41:141145. doi:10.3147/jsfp. 41.141

75. Zaaijer HL, Leentvaar-Kuijpers A, Rotman H, Lelie PN. Hepatitis A antibody titres after infection and immunization: implications for passive and active immunization. J Med Virol (1993) 40:22-27.

76. Elia G, Cavalli A, Cirone F, Lorusso E, Camero M, Buonavoglia D, Tempesta M. Antibody levels and protection to canine parvovirus type 2. J Vet Med Ser B Infect Dis Vet Public Heal (2005) 52:320-322. doi:10.1111/j.1439-0450.2005.00870.x

77. Huang Y, Anderson SA, Forshee RA, Yang H. A modified dose-response model that describes the relationship between haemagglutination inhibition titre and protection against influenza infection. J Appl Microbiol (2018) 124:294-301. doi:10.1111/jam.13628 
78. Plotkin SA. Vaccines: Correlates of Vaccine $\square$ Induced Immunity. Clin Infect Dis (2008) 47:401-409. doi:10.1086/589862

79. Skjold P, Sommerset I, Frost P, Villoing S. Vaccination against pancreas disease in Atlantic salmon, Salmo salar L., reduces shedding of salmonid alphavirus. Vet Res (2016) 47:10-15. doi:10.1186/s13567-016-0362-9

80. Munang'Andu HM, Fredriksen BN, Mutoloki S, Dalmo RA, Evensen Ø. Antigen dose and humoral immune response correspond with protection for inactivated infectious pancreatic necrosis virus vaccines in Atlantic salmon (Salmo salar L). Vet Res (2013) 44:1-16. doi:10.1186/1297-9716-44-7

81. Tobar I, Arancibia S, Torres C, Vera V, Soto P, Carrasco C, Alvarado M, Neira E, Arcos S, Tobar J a. Successive Oral Immunizations Against Piscirickettsia Salmonis and Infectious Salmon Anemia Virus are Required to Maintain a Long-Term Protection in Farmed Salmonids. Front Immunol (2015) 6:1-7. doi:10.3389/fimmu.2015.00244

82. Dalmo RA. DNA vaccines for fish: Review and perspectives on correlates of protection. $J$ Fish Dis (2018) 41:1-9. doi:10.1111/jfd.12727

83. Ballesteros NA, Alonso M, Rodríguez Saint-Jean S, Perez-Prieto SI. An oral DNA vaccine against infectious haematopoietic necrosis virus (IHNV) encapsulated in alginate microspheres induces dose-dependent immune responses and significant protection in rainbow trout (Oncorrhynchus mykiss). Fish Shellfish Immunol (2015) 45:877-888. doi:10.1016/j.fsi.2015.05.045

84. Nagasawa T, Nakayasu C, Rieger AM, Barreda DR, Somamoto T, Nakao M. Phagocytosis by thrombocytes is a conserved innate immune mechanism in lower vertebrates. Front Immunol (2014) 5:36-39. doi:10.3389/fimmu.2014.00445

85. Li J, Barreda DR, Zhang Y-A, Boshra H, Gelman AE, LaPatra S, Tort L, Sunyer JO. B lymphocytes from early vertebrates have potent phagocytic and microbicidal abilities. Nat Immunol (2006) 7:1116-1124. doi:10.1038/ni1389

86. Takizawa F, Magadan S, Parra D, Xu Z, Korytář T, Boudinot P, Sunyer JO. Novel Teleost CD4-Bearing Cell Populations Provide Insights into the Evolutionary Origins and Primordial Roles of CD4 ${ }^{+}$Lymphocytes and CD4 ${ }^{+}$Macrophages. J Immunol (2016) 196:4522-4535. doi:10.4049/jimmunol.1600222

87. Kato G, Goto K, Akune I, Aoka S, Kondo H, Hirono I. CD4 and CD8 homologues in Japanese flounder, Paralichthys olivaceus: Differences in the expressions and localizations of CD4-1, CD4-2, CD8 $\alpha$ and CD8ß. Dev Comp Immunol (2013) 39:293-301. doi:10.1016/j. dci.2012.09.004

88. Morera D, MacKenzie SA. Is there a direct role for erythrocytes in the immune response? Vet Res (2011) 42:89. doi:10.1186/1297-9716-42-89

89. Devine L, Kieffer LJ, Aitken V, Kavathas PB. Human CD8 beta, but not mouse CD8 beta, can be expressed in the absence of CD8 alpha as a beta beta homodimer. J Immunol (2000) 164:833-838. doi:10.4049/jimmunol.164.2.833

90. Forlenza M, de Carvalho Dias JDA, Veselý T, Pokorová D, Savelkoul HFJ, Wiegertjes GF. Transcription of signal-3 cytokines, IL-12 and IFN alpha beta, coincides with the timing of CD8 alpha beta up-regulation during viral infection of common carp (Cyprinus carpio L). Mol Immunol (2008) 45:1531-47. doi:10.1016/j.molimm.2007.10.010

91. Rakus KL, Irnazarow I, Adamek M, Palmeira L, Kawana Y, Hirono I, Kondo H, Matras M, Steinhagen D, Flasz B, et al. Gene expression analysis of common carp (Cyprinus carpio L.) lines during Cyprinid herpesvirus 3 infection yields insights into differential immune responses. Dev Comp Immunol (2012) 37:65-76. doi:10.1016/j.dci.2011.12.006

92. Kronsteiner B, Bassaganya-Riera J, Philipson C, Viladomiu M, Carbo A, Pedragosa M, Vento S, Hontecillas R. Helicobacter pylori infection in a pig model is dominated by Th1 and cytotoxic CD8+ T cell responses. Infect Immun (2013) 81:3803-3813. doi:10.1128/ IAI.00660-13

93. Werwitzke S, Tiede A, Drescher BE, Schmidt RE, Witte T. CD8beta/CD28 expression 
defines functionally distinct populations of peripheral blood T lymphocytes. Clin Exp Immunol (2003) 133:334-343. doi:2226 [pii]

94. Arrigucci R, Bushkin Y, Radford F, Lakehal K, Vir P, Pine R, Martin D, Sugarman J, Zhao Y, Yap GS, et al. FISH-Flow, a protocol for the concurrent detection of mRNA and protein in single cells using fluorescence in situ hybridization and flow cytometry HHS Public Access. Nat Protoc (2017) 12:1245-1260. doi:10.1038/nprot.2017.039

95. Zahedipour F, Ranjbaran R, Behbahani AB, Afshari KT, Okhovat MA, Tamadon G, Sharifzadeh S. Development of flow cytometry-fluorescent in situ hybridization (Flow-FISH) method for detection of PML/RARa chromosomal translocation in acute promyelocytic leukemia cell line. Avicenna J Med Biotechnol (2017) 9:104-108.

96. Rombout JHWM, Joosten PHM, Engelsma MY, Vos AP, Taverne N, Taverne-Thiele JJ. Indications for a distinct putative T cell population in mucosal tissue of carp (Cyprinus carpio L.). Dev Comp Immunol (1998) 22:63-77. doi:10.1016/S0145-305X(97)00048-7

97. Moebius U, Kober G, Griscelli AL, Hercend T, Meuer SC. Expression of different CD8 isoforms on distinct human lymphocyte subpopulations. Eur J Immunol (1991) 21:17931800. doi:10.1002/eji.1830210803

98. Olson KR. Vascular anatomy of the fish gill. J Exp Zool (2002) 293:214-231. doi:10.1002/ jez.10131

99. Salinas I. The Mucosal Immune System of Teleost Fish. Biology (Basel) (2015) 4:525-39. doi:10.3390/biology4030525

100. Haugarvoll E, Bjerkås I, Nowak BF, Hordvik I, Koppang EO. Identification and characterization of a novel intraepithelial lymphoid tissue in the gills of Atlantic salmon. $J$ Anat (2008) 213:202-209. doi:10.1111/j.1469-7580.2008.00943.x

101. Dalum AS, Austbø L, Bjørgen H, Skjødt K, Hordvik I, Hansen T, Fjelldal PG, Press CM, Griffiths DJ, Koppang EO. The interbranchial lymphoid tissue of Atlantic Salmon (Salmo salar L) extends as a diffuse mucosal lymphoid tissue throughout the trailing edge of the gill filament. J Morphol (2015) 276:1075-1088. doi:10.1002/jmor.20403

102. Austbø L, Aas IB, König M, Weli SC, Syed M, Falk K, Koppang EO. Transcriptional response of immune genes in gills and the interbranchial lymphoid tissue of Atlantic salmon challenged with infectious salmon anaemia virus. Dev Comp Immunol (2014) 45:107-114. doi:10.1016/j.dci.2014.02.007

103. Koppang EO, Fischer U, Moore L, Tranulis MA, Dijkstra JM, Köllner B, Aune L, Jirillo E, Hordvik I. Salmonid T cells assemble in the thymus, spleen and in novel interbranchial lymphoid tissue. J Anat (2010) 217:728-739. doi:10.1111/j.1469-7580.2010.01305.x

104. Aas IB, Austbø L, König M, Syed M, Falk K, Hordvik I, Koppang EO. Transcriptional Characterization of the T Cell Population within the Salmonid Interbranchial Lymphoid Tissue. J Immunol (2014) 193:3463-3469. doi:10.4049/jimmunol.1400797

105. Piazzon MC, Galindo-Villegas J, Pereiro P, Estensoro I, Calduch-Giner JA, Gómez-Casado E, Novoa B, Mulero V, Sitjà-Bobadilla A, Pérez-Sánchez J. Differential modulation of IgT and IgM upon parasitic, bacterial, viral, and dietary challenges in a perciform fish. Front Immunol (2016) 7: doi:10.3389/fimmu.2016.00637

106. Xu Z, Takizawa F, Parra D, Gómez D, von Gersdorff Jørgensen L, LaPatra SE, Sunyer JO. Mucosal immunoglobulins at respiratory surfaces mark an ancient association that predates the emergence of tetrapods. Nat Commun (2016) 7:10728. doi:10.1038/ncomms 10728

107. Xu Z, Parra D, Gómez D, Salinas I, Zhang Y-A, von Gersdorff Jørgensen L, Heinecke RD, Buchmann K, LaPatra S, Sunyer JO. Teleost skin, an ancient mucosal surface that elicits gut-like immune responses. Proc Natl Acad Sci U S A (2013) 110:13097-102. doi:10.1073/ pnas. 1304319110

108. Picchietti S, Nuñez-Ortiz N, Stocchi V, Randelli E, Buonocore F, Guerra L, Scapigliati G. Evolution of lymphocytes. Immunoglobulin T of the teleost sea bass (Dicentrarchus labrax): Quantitation of gene expressing and immunoreactive cells. Fish Shellfish Immunol (2017) 63:40-52. doi:10.1016/j.fsi.2017.02.002 
109. Zhang Y-A, Salinas I, Li J, Parra D, Bjork S, Xu Z, LaPatra SE, Bartholomew J, Sunyer JO. IgT, a primitive immunoglobulin class specialized in mucosal immunity. Nat Immunol (2010) 11:827-35. doi:10.1038/ni.1913

110. Castro R, Martínez-Alonso S, Fischer U, Haro NÁ De, Soto-Lampe V, Wang T, Secombes CJ, Lorenzen N, Lorenzen E, Tafalla C. DNA vaccination against a fish rhabdovirus promotes an early chemokine-related recruitment of B cells to the muscle. Vaccine (2014) 32:1160-1168. doi:10.1016/j.vaccine.2013.11.062

111. Castro R, Jouneau L, Pham H-P, Bouchez O, Giudicelli V, Lefranc M-P, Quillet E, Benmansour A, Cazals F, Six A, et al. Teleost fish mount complex clonal IgM and IgT responses in spleen upon systemic viral infection. PLoS Pathog (2013) 9:e1003098. doi:10.1371/journal.ppat.1003098

112. Ballesteros NA, Castro R, Abos B, Rodríguez Saint-Jean SS, Pérez-Prieto SI, Tafalla C. The Pyloric Caeca Area Is a Major Site for IgM+ and IgT+ B Cell Recruitment in Response to Oral Vaccination in Rainbow Trout. PLoS One (2013) 8:e66118. doi:10.1371/journal. pone. 0066118

113. Buonocore F, Stocchi V, Nunez-Ortiz N, Randelli E, Gerdol M, Pallavicini A, Facchiano A, Bernini C, Guerra L, Scapigliati G, et al. Immunoglobulin T from sea bass (Dicentrarchus labrax L.): Molecular characterization, tissue localization and expression after nodavirus infection. BMC Mol Biol (2017) 18:1-14. doi:10.1186/s12867-017-0085-0

114. Pisharath H, Parsons MJ. "Nitroreductase-Mediated Cell Ablation in Transgenic Zebrafish Embryos," in Zebrafish: Methods and Protocols, eds. G. J. Lieschke, A. C. Oates, K. Kawakami (Totowa, NJ: Humana Press), 133-143. doi:10.1007/978-1-60327-977-2_9

115. Gray C, Loynes CA, Whyte MKB, Crossman DC, Renshaw SA, Chico TJA. Simultaneous intravital imaging of macrophage and neutrophil behaviour during inflammation using a novel transgenic zebrafish. Thromb Haemost (2011) 105:811-819. doi:10.1160/TH10-080525

116. Zhang Y, Stupka E, Henkel C V, Jansen HJ, Spaink HP, Verbeek FJ. Identification of common carp innate immune genes with whole-genome sequencing and RNA-Seq data. $J$ Integr Bioinform (2011) 8:169. doi:10.2390/biecoll-jib-2011-169

117. Henkel C V., Dirks RP, Jansen HJ, Forlenza M, Wiegertjes GF, Howe K, van den Thillart GEEJM, Spaink HP. Comparison of the Exomes of Common Carp (Cyprinus carpio) and Zebrafish (Danio rerio). Zebrafish (2012) 9:59-67. doi:10.1089/zeb.2012.0773

118. Kolder ICRM, van der Plas-Duivesteijn SJ, Tan G, Wiegertjes GF, Forlenza M, Guler AT, Travin DY, Nakao M, Moritomo T, Irnazarow I, et al. A full-body transcriptome and proteome resource for the European common carp. BMC Genomics (2016) 17:701. doi:10.1186/s12864-016-3038-y

119. Petit J, David L, Dirks R, Wiegertjes GF. Genomic and transcriptomic approaches to study immunology in cyprinids: What is next? Dev Comp Immunol (2017) 75:48-62. doi:10.1016/j.dci.2017.02.022 


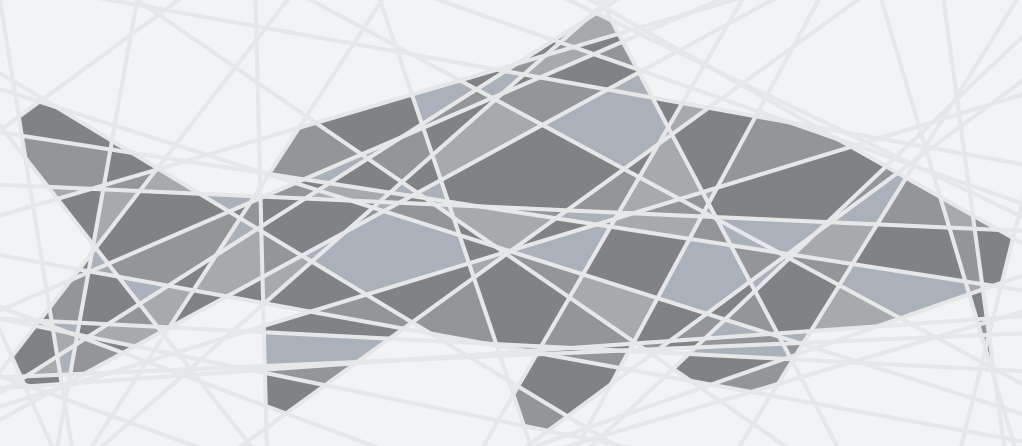




\section{Summaries}

Summary (English)

Samenvatting (Nederlands) 


\section{Summary}

To meet the ever-growing demand for animal protein sources related to the increasing world population, the field of aquaculture has undergone a significant intensification over the last decades. While this has increased production tremendously, it also led to an increase in outbreaks of known and emerging infectious diseases. For most of the aquatic diseases, no effective commercial vaccines are available. For example, for carp - the target species of this thesis - no vaccines are available against Spring Viremia of Carp Virus (SVCV) and Koi Herpes Virus (KHV), the two viruses affecting carp production the most. In general, if effective vaccines are available for aquaculture species, they are most often administered through intraperitoneal (i.p.) injection. With respect to animal welfare, labor and costs for mass vaccination, alternative routes of vaccination would be preferred. These include the oral and immersion vaccination route, which are not only suitable for mass vaccination, but also reduce the stress of the animal. Finally: extensive knowledge on the fish immune response is not only required to characterize the protective mechanisms triggered by effective vaccination, but also to further improve existing vaccines by identifying correlates of protection.

With the above mentioned in mind, the two major aims of this thesis are 1) to design experimental (injection and/or oral) vaccines against SVCV and KHV and to assess their efficacy, and 2) to characterize protective immune responses of carp after infection and vaccination, with a focus on adaptive immune responses.

In chapter 1, I provide a framework for this thesis by introducing the rising problem of disease outbreaks in aquaculture. First, I introduce our target viruses: SVCV and KHV. Next, I compare (novel) vaccine types and give a comprehensive overview of the different delivery routes. Since we evaluate the efficacy of DNA vaccines against both SVCV and KHV in this thesis, I also introduce the mechanism of DNA vaccination in detail. Finally, I explain the importance of studying adaptive immune responses in order to understand the protective mechanisms induced by vaccination, and give an overview on how $\mathrm{B}$ and $\mathrm{T}$ cell responses can be examined.

Considering that oral vaccination would be the most practical route of vaccination, in chapter 2 we review the current approaches to oral vaccination of fish, taking examples from humans and veterinary species. We discuss the major factors to be considered in oral vaccine design, including identification of protective antigens, 
vaccine encapsulation, prevention of tolerance, and vaccination regime. Besides reviewing existing literature, we propose additional approaches for fish oral vaccine development and discuss how to improve suboptimal vaccines by using novel adjuvants.

For the development of a vaccine against SVCV, we started by identifying the protective antigen. For this, we used an i.m. DNA vaccination approach which was previously shown to be effective against other fish rhabdoviruses. In chapter 3 we show that i.m. vaccination using a DNA vaccine encoding the SVCV G protein confers $90-100 \%$ protection when administered at a dose of only $0.1 \mu$ g DNA per gram of fish. This protection was observed in juvenile carp by infecting them through a newly established bath challenge model, which resembles the natural route of infection. After this breakthrough in the identification of the protective antigen and in the optimization of vaccination conditions, we investigated rapid local as well as long-term systemic immune responses. We observed an early immune response to the vaccine, characterized by changes in gene expression of various pro-inflammatory cytokines and antiviral genes. Most importantly, we show that virus-neutralizing antibodies are present in the serum of DNA vaccinated fish, and that i.m. DNA vaccination also leads to the generation of virus-specific (memory) $T$ cells. Together, these immune parameters represent correlates to protection that might help the evaluation of novel experimental vaccines.

After the success reported in chapter 3, in chapter 4 we examined the possibilities to use our DNA vaccine via an oral route against SVCV. For this, we used alginates as an encapsulation vehicle to protect the DNA plasmid prior to oral administration to carp. Regrettably, oral administration of low or high doses (up to $100 \mu \mathrm{g} / \mathrm{fish}$ ) of alginate-encapsulated DNA plasmid did not induce protection, despite testing various vaccination regimes and adding the mucosal adjuvant lymphotoxin-B (LTB). Immune response analysis by gene expression and flow cytometry revealed that the orally delivered vaccine did not trigger a strong local or systemic response, at least not when compared to the response triggered by the injected DNA vaccine. In the same study, we also designed a subunit vaccine based on the baculovirus-mediated recombinant expression of the SVCV-G protein. Unfortunately, despite using multiple vaccination routes (i.m., i.p. and oral) and vaccine doses, no protection could be induced. Finally, we discuss the reason for this observed lack of protection and propose multiple strategies for improvement of oral vaccines against SVCV. 
Given the success of the i.m. DNA vaccine against SVCV, in chapter 5 we developed a DNA vaccine against KHV. We chose the Open Reading Frame (ORF) 25 as the vaccine antigen, since it is one of the major structural membrane proteins and was previously shown to induce neutralizing antibodies against KHV. We expressed the ORF25 as a soluble or transmembrane protein. Despite the use of multiple vaccine doses for both i.m. and oral delivery, no protection was observed when fish were challenged by cohabitation. Although we did observe expression of the ORF25 locally and in the spleen after i.m. injection, no apparent changes in local gene expression of multiple chemokines, cytokines and antiviral genes was found. Based on these results, we propose future vaccination strategies based on the use of multiple antigens, which might be required to confer protection against a complex virus such as KHV.

As mentioned before, studying innate and adaptive immune responses after vaccination will provide insights into the protective mechanism triggered by effective vaccination. For this reason, the last two chapters of this thesis focus on the molecular and cellular characterization of B and T cells in common carp. In chapter 6, we report the presence of two Cd4 genes ( $c d 4-1$ and $c d 4-2$ ), each present in two isoforms ( $a$ and $b$ ). Using cross-reactive antibodies against $\mathrm{Cd} 4-1$ and $\mathrm{Cd} 8 \alpha 1$ we characterized $\mathrm{T}$ cell populations in common carp. Interestingly, different from what was reported for rainbow trout and zebrafish, Cd4-1 expression seems to be restricted to T cells and is not present in monocytes/macrophages. RNA sequencing transcriptome-analysis of FACS-sorted Cd4-1+ $1^{+}$and $\mathrm{Cd} 8 \alpha 1^{+}$cells was used to confirm the specificity of the antibodies. This approach was also used to gain insight into the cells recognized by the WCL38 antibodies, which were previously shown to recognize putative mucosal $\mathrm{T}$ cells. Transcriptome and FACS analysis confirmed that at mucosal sites, including the intestine, WCL38 recognizes at least two populations of cells: WCL $38^{+} / \mathrm{Cd} 8 \alpha 1^{+} /$ Cd4 ${ }^{-} \mathrm{T}$ cells as well as a $\mathrm{WCL} 38^{+} / \mathrm{Cd} 8 \alpha 1^{-} / \mathrm{Cd} 4^{-}$population. We propose that the latter cells might include NKT cells and $\mathrm{Cd} 8 \alpha 1^{-} / \gamma \delta$-T cells. Further analysis of the $\mathrm{WCL} 38^{+} / \mathrm{Cd} 8 \alpha 1^{+} / \mathrm{Cd} 4-$ population and identification of the target antigen/epitope is needed to confirm this hypothesis.

In chapter 7, we characterized B cell subsets in common carp. Using newly developed antibodies against Igt1 and Igt2 we show that they are found at different locations in the intestines: $\operatorname{Igt} 1^{+}$cells in the epithelial layer and $\operatorname{Igm}^{+} / \operatorname{Igt} 2^{+}$cells in the lamina propria. In the gills we observed that most bacteria were coated with 
Igt2. These data indicate spatial differences between Igt1 and Igt 2 and suggest a prominent role for Igt1 in the intestine and for Igt 2 in the gills. Furthermore, with both a mucosal infection model (Sphaerospora molnari) and a systemic infection model (Trypanoplasma borreli) we demonstrated for the first time that both Igt1 and Igt2 are involved in the immune response to both types of infection. However, since the antibody against Igt 2 reacted not only to Igt2, but also to Igm, more research is needed to further characterize the function of Igt1 and Igt 2 in carp.

Lastly, in chapter 8 I reflect on the results obtained in this thesis and focus on two major aspects: 1) future approaches to mucosal vaccination against SVCV and KHV, considering the successes and challenges encountered in this thesis, 2) tools for analyzing the immune response of $\mathrm{B}$ and $\mathrm{T}$ cells. In the first part, I focus on alternative methods for oral vaccination against SVCV, describing preliminary work on using the yeast Pichia pastoris or the gram-positive bacterium Lactococcus lactis for oral delivery of respectively subunit vaccines or DNA plasmids. For KHV I suggest that not only oral, but also immersion vaccination should be considered, given the nature of the pathogen. In particular, I discuss the possibility to use several KHV antigens in the form of VLPs. In the second part, based on preliminary data, I propose a novel $\mathrm{T}$ cell subset in carp, i.e. a $\mathrm{Cd} 8 \beta \beta^{+} \mathrm{T}$ cell population. I show that in the gills, the WCL38 antibody recognizes a population of larger granular lymphoid-like cells distinct from conventional T cells. Furthermore, I suggest how the WCL38 antibody, in combination with antibodies against Cd4-1, Cd8 $\alpha 1$, and Zap70 can help us characterize cell types other than $T$ cells.

In conclusion, in this thesis we describe the optimization of the first successful i.m. DNA vaccine against SVCV in carp. Furthermore, we generated several additional experimental vaccines for injection as well as oral vaccination against SVCV and KHV. Although these vaccines did not provide sufficient protection upon pathogen challenge, the knowledge that has been generated on vaccine doses, regime and immune responses, will be instrumental for the optimization of future vaccination studies. Finally, the new set of tools that were developed will be widely applicable when characterizing the response to other infections, or to novel vaccines. 


\section{Samenvatting}

Om aan de stijgende vraag voor dierlijke eiwitten (gerelateerd aan de groeiende wereldpopulatie) te kunnen voldoen, heeft de aquacultuur sector de laatste tientallen jaren een significante intensivering doorgemaakt. Deze ontwikkeling heeft geleid tot een forse groei in productie, maar ook tot een vermeerdering in het uitbreken van bekende en onbekende infectieziektes. Voor de meeste visziekten zijn nog geen effectieve vaccins beschikbaar. Zo zijn er geen vaccins beschikbaar voor Spring Viraemia of Carp Virus (SVCV) en Koi Herpes Virus (KHV), de twee virussen die de meeste schade toebrengen aan de kweek van karpers. De vaccins die op dit moment beschikbaar zijn voor visziekten worden meestal toegediend door middel van een intra-peritoneale (i.p.) injectie. Als men kijkt naar dierenwelzijn, arbeid en kosten voor massavaccinatie hebben alternatieve routes de voorkeur. Voorbeelden van alternatieve vaccinatieroutes zijn orale vaccinatie en badvaccinatie, welke niet alleen toepasbaar zijn voor vaccinatie van grote groepen vis tegelijk, maar ook minder stressvol zijn dan injectie-vaccinatie. Een goed begrip van de immuunreactie is hier van groot belang. Niet alleen om de immuunreactie na een succesvolle vaccinatie te begrijpen, maar ook om bestaande vaccins te verbeteren door het identificeren van voorspellende immuunmarkers.

Met het bovenstaande in gedachten zijn de twee hoofddoelen van dit proefschrift om: 1) experimentele vaccins (injectie en oraal) voor SVCV en KHV te ontwikkelen en te testen in vivo en 2) om immuunreacties na vaccinatie te karakteriseren met een focus op adaptieve immuunreacties.

In hoofdstuk 1 verschaf ik een kader voor dit proefschrift door het introduceren van het rijzende probleem van ziekte-uitbraken binnen de viskweeksector. Als eerste introduceer ik de twee virussen waartegen we later in dit proefschrift vaccines gaan produceren en testen: SVCV en KHV. Hierna vergelijk ik (vernieuwende) vaccintypes en geef een overzicht van de verschillende toegepaste vaccinatieroutes. Omdat we in dit proefschrift DNA-vaccins testen voor zowel SVCV als KHV leg ik ook het mechanisme van DNA-vaccinatie uit. Als laatste leg ik het belang van het bestuderen van adaptieve immuunreacties uit om de mechanismen achter succesvolle vaccinatie te begrijpen en geef ik een overzicht van hoe B- en T- cel reacties kunnen worden bestudeerd.

Omdat orale vaccinatie de meest praktische vaccinatieroute is vatten we in hoofd- 
stuk 2 de huidige benaderingen van orale vaccinatie van vis samen, en we gebruiken hiervoor humane en veterinaire voorbeelden. We bespreken de belangrijkste factoren waarmee rekening moet worden gehouden bij de ontwikkeling van orale vaccins, inclusief de identificatie van beschermende antigenen, vaccin-encapsulatie, voorkoming van tolerantie, en vaccinatieregime. Naast het samenvatten van bestaande literatuur stellen we extra mogelijkheden voor om visvaccins te ontwikkelen en we zetten uiteen hoe bestaande vaccins kunnen worden verbeterd bij vernieuwende adjuvantia.

Voor het ontwikkelen van een vaccin tegen SVCV zijn we begonnen met het identificeren van het beschermende antigen. Hiervoor hebben we gebruik gemaakt van een intramusculaire (i.m.) DNA-techniek die eerder succesvol was bij het vaccineren tegen andere rhabdovirussen in vis. In hoofdstuk 3 laten we zie dat i.m. vaccinatie van een DNA-vaccin dat codeert voor het SVCV glycoproteïne zorgt voor een bescherming van $90-100 \%$, zelfs als er slechts $0.1 \mu$ g DA per gram vis wordt geïnjecteerd. Deze bescherming werd getest in een nieuw geoptimaliseerd bad-infectiemodel, dat de natuurlijke infectieroute weerspiegelt. $\mathrm{Na}$ deze doorbraak in de identificatie van het beschermende antigen en de optimalisatie van vaccinatie hebben we de snelle (lokale) en geheugen (systemische) immuunreacties gekarakteriseerd. Hier zagen we een snelle immuunreactie tegen het vaccin dat gekenmerkt werd door veranderingen in genexpressie van verschillende ontstekingsbevorderende cytokines en antivirale genen. De belangrijkste observaties waren d circulatie van virus-neutraliserende antilichamen in het serum van gevaccineerde vissen, en dat i.m. DNA-vaccinatie ook zorgt voor de voortbrenging van virus-specifieke (geheugen) cellen.

$\mathrm{Na}$ het in hoofdstuk 3 gerapporteerde succes, gaan we in hoofdstuk 4 in op de mogelijkheden om ons DNA-vaccin tegen SVCV te gebruiken als een oraal vaccin. Om dit mogelijk te maken hebben we alginaat (een polymeer afkomstig van algen) gebruikt om een kapsel om ons DNA-vaccin te maken alvorens het vaccin oraal toe te dienen aan karpers. Helaas gaf orale toediening van dit vaccin geen bescherming tegen SVCV, ook niet nadat verschillende lage en hoge doseringen (tot 100 $\mu \mathrm{g}$ per vis) en vaccinatiechema's werden getest, en ook niet na toevoeging van het mucosale adjuvans lymphotoxine-B (LTB). We bestudeerden de immuunreactie na vaccinatie met flow-cytometrie en genexpressie en vonden dat orale toediening van het DNA-vaccin geen sterke lokale en/of systemische immuunreactie induceert, als we de resultaten vergelijken met de immuunreactie na i.m. injectie van hetzelfde 
DNA-vaccin tegen SVCV. In dezelfde studie hebben we ook een subunit-vaccin tegen SVCV geproduceerd, gebaseerd op een baculovirus-gemedieerde recombinante expressie van het SVCV-G eiwit. Helaas constateerden we dat dit vaccin geen bescherming induceerde tegen SVCV, al hebben we meerdere vaccinatieroutes (i.m., i.p. en oraal) en verschillende doseringen getest. Uiteindelijk beargumenteren we de redenen voor het gebrek aan bescherming en stellen we verschillende strategieën voor om orale vaccins voor SVCV te verbeteren.

Omdat het i.m. DNA-vaccin tegen SVCV zo goed werkt hebben we in hoofdstuk 5 een DNA-vaccin tegen KHV ontwikkeld. We hebben het eiwit ORF25 gekozen als antigen omdat dit eiwit een belangrijk structureel eiwit van KHV is en omdat het eerder werd aangetoond dat ORF25 neutraliserende antilichamen tegen KHV induceert. We hebben ORF25 tot expressie gebracht als een oplosbaar en als een transmembraan eiwit. Ondanks het testen van meerdere doseringen voor i.m. en orale toediening van het vaccin zagen we geen bescherming als de vissen werden geïnfecteerd door middel van cohabitatie. Ondanks dat we ORF25-expressie zagen in de spier en in de milt na i.m. injectie, zagen we geen sterke veranderingen in de genexpressie van verschillende cytokines en antivirale genen. Gebaseerd op deze resultaten stellen we toekomstige vaccinatiestrategieën voor gebaseerd op het gebruik van meerdere antigenen en we bediscussiëren dat meerdere antigenen nodig zijn om een volledige bescherming tegen het complexe virus KHV te induceren.

Zoals al eerder genoemd zal het bestuderen van aangeboren en adaptieve immuunreacties na vaccinatie belangrijke inzichten geven in de mechanismen die geactiveerd worden na succesvolle vaccinatie. Om deze reden gaan de laatste twee hoofdstukken van mijn proefschrift over de moleculaire en cellulaire karakterisering van B- en Tcellen. In hoofdstuk 6 rapporteren we de aanwezigheid van twee Cd4 genen (cd4-1 en cd4-2) met ieder twee isovormen ( $a$ en b). Met het gebruik van kruisreagerende antilichamen tegen $\mathrm{Cd} 4-1$ en $\mathrm{Cd} 8 \alpha 1$ hebben we T-cel populaties van de karper gekarakteriseerd. We constateerden dat bij karpers, anders dan bij forel en zebravis, Cd4-1 expressie beperkt is tot T-cellen en is niet aanwezig is op monocyten en macrofagen. RNA sequencing transcriptoomanalyse van FACS-gesorteerde Cd4-1+ en $\mathrm{Cd} 8 \alpha 1+$ cellen werd verder gebruikt om de specificiteit van de antilichamen aan te tonen. Deze benadering werd ook gebruikt om de cellen te karakteriseren die worden herkend door het WCL38 antilichaam, eerder beschreven als een antilichaam dat vermeende mucosale T-cellen herkent. Transcriptoom en FACS analyse bevestigde dat 
in mucosale gebieden zoals de darm, het antilichaam WCL38 meerdere populaties herkent: WCL38+/Cd8 $\alpha 1+/$ Cd4-1- T cellen en een tot nu toe onbekende WCL38+/ $\mathrm{Cd} 8 \alpha 1-/ \mathrm{Cd} 4-1-$ en we stellen voor dat deze tweede populatie NKT cellen kan bevatten en ook Cd8 $\alpha 1-1-\gamma \delta$-T cellen. Verdere analyse van deze WCL38+/Cd8 $11-/ C d 4-$ 1- populatie is nodig om deze hypothese te bevestigen, alsmede de identificatie van het herkende antigen/epitoop.

In hoofdstuk 7 hebben we B-cel populaties in de karper gekarakteriseerd. We hebben antilichamen geproduceerd tegen Igt 1 en Igt2 en met gebruik van deze antilichamen hebben we ontdekt dat Igt1 en Igt2 op verschillende locaties in de darm zitten: Igt1 ${ }^{+}$ cellen in de epitheellaag en Igt $2^{+} / \operatorname{Igm}^{+}$cellen in de lamina propria. In de kieuwen constateerden we dat de meeste bacteriën waren gecoat met Igt2, hetgeen laat zien dat Igt 1 wellicht een grotere rol speelt in de darmen, en Igt 2 in de kieuwen. Door het in kaart brengen van de immuunreactie tijdens een mucosale infectie (Sphaerospora molnari) en een systemische infectie (Trypanoplasma borreli) laten we, voor de eerste keer, zien dat Igt1 en Igt2 beiden een rol spelen tijdens de verschillende infecties. Omdat we observeerden dat het antilichaam tegen Igt2 ook reageerde met Igm is er meer ondezoek nodig om de functie van Igt1 en Igt2 in karper te beschrijven.

Als laatste reflecteer ik in hoofdstuk 8 op de resultaten behaald in dit proefschrift en ik focus op de volgende twee aspecten: 1) toekomstperspectieven voor mucosale vaccinatie tegen SVCV en KHV met een focus op de behaalde successen en ook de uitdagingen die we tegen zijn gekomen binnen dit project, en 2) tools om Ben T- cellen beter te kunnen bestuderen. In het eerste deel focus ik op alternatieve methoden voor orale vaccinatie tegen SVCV en beschrijf het voorlopige werk met het gist Pichia pastoris en de grampositieve bacterie Lactococcus lactis voor de toediening van respectievelijk subunit vaccins en DNA plasmiden. Voor KHV stel ik dat naast orale vaccinatie, immersie-vaccinatie getest zou moeten worden gezien de natuur van het virus. Daarnaast beargumenteer ik dat meerdere KHV antigenen samen gebruikt kunnen worden in VLPs. In het tweede deel laat ik voorlopige data zien en bespreek de mogelijke aanwezigheid van $\mathrm{Cd} 8 \beta \beta^{+} \mathrm{T}$ cellen. Ik laat zien dat het WCL38 antilichaam in de kieuwen naast de T-cellen zoals eerder beschreven, een extra populatie van grotere granulaire lymfocyt-achtige cellen herkent. Verder geef ik suggesties over hoe het WCL38 antilichaam, in combinatie met antilichamen tegen Cd4-1, Cd8 1 en Zap70 ons kan helpen om naast T-cellen ook andere celtypes te karakteriseren. 
Om samen te vatten, in dit proefschrift beschrijven we de optimalisatie van een succesvol i.m. DNA-vaccin tegen SVCV in karper. Verder hebben we verscheidene experimentele injectie-en orale vaccins voor SVCV en KHV ontwikkeld en getest. Hoewel deze vaccins niet voldoende bescherming gaven tijdens een virale infectie hebben we veel kennis opgedaan over vaccindosering, regime en immuunreacties en deze kennis is van groot belang bij verdere vaccinontwikkeling voor vissen. Tot slot, de nieuwe set van antilichamen en technieken/assays is breed inzetbaar bij het karakteriseren van immuunreacties tijdens infectie en na vaccinatie. 


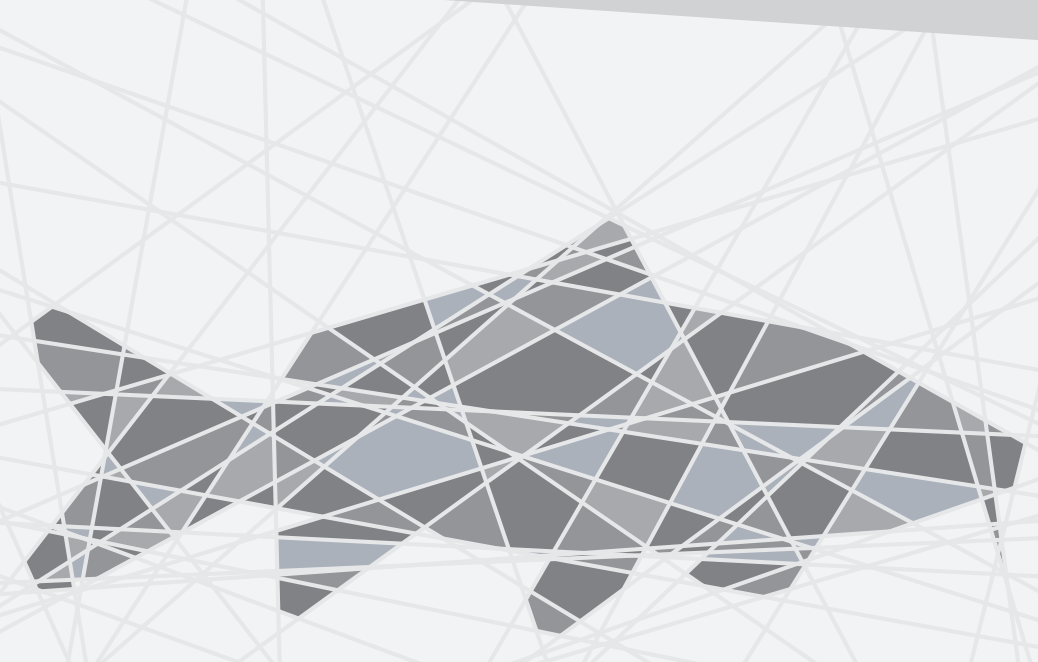




\section{About the author}

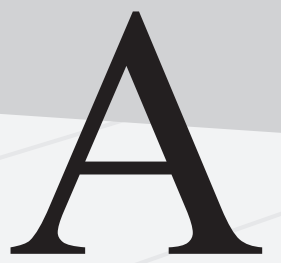

Curriculum Vitae

List of publications

Overview of completed training activities Acknowledgements 


\section{Curriculum vitae}

Carmen Wilhelmina Elisabeth was born in Maastricht on the 31rd of March, 1989. From her early days she spent lots of time around animals and took care of many orphaned birds or animals that fell prey to one of her cats. This was never a problem, since the house was already filled with a colorful variety of animals including cats and dogs, multiple types of rodents, birds, and even reptiles. Given this all, it is no surprise that Carmen went to study Animal Sciences in Wageningen after obtaining her high school degree in Maastricht.

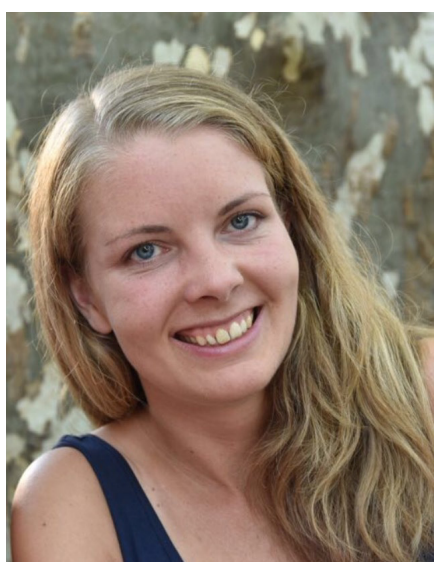

During her BSc and MSc Carmen investigated the effects of feed restriction on productivity and offspring performance in broiler breeder hens (BSc thesis) and the role of the 14-3-3 protein in baculovirus-induced behavioral changes in caterpillars (MSc minor thesis at the Laboratory of Virology). After this, she turned to the "fishy side" and she devoted 9 months at Insel Riems (Greifswald, Germany), where she worked on the production of monoclonal antibodies against common carp $\mathrm{T}$ cell markers (MSc major thesis and post-graduate research assistant). Given that 9 months were not enough to achieve this goal, she was happy to continue with this challenge in 2013 when she started as a PhD candidate at the Cell Biology and Immunology Group of Wageningen University. Her PhD was part of the European Union FP7 Framework Project TARGETFISH and during the period 2013-2017 Carmen produced and tested multiple vaccines for common carp against Spring Vireamia of Carp Virus and Koi Herpes Virus. Next to this she studied immune responses during infection and after vaccination, with a focus on adaptive immune mechanisms. The results of her $\mathrm{PhD}$ can be found in this thesis.

From April 2018 Carmen works as a post-doc at the Comparative Pathology and Pathogenesis group at the Viroscience department of the Erasmus Medical Centre (Rotterdam, the Netherlands). In this function she is investigating rabies virus-induced immunosuppression. 


\section{List of publications}

Embregts, C.W.E., Rigaudeau, D., Tacchi, L., Pijlman, G.P., Kampers, L., Veselý, T., Pokorová, D., Boudinot, P., Wiegertjes, G.F. and Forlenza, M., 2018. Vaccination of carp against SVCV with an oral DNA vaccine or an insect cells-based subunit vaccine. Fish \& Shellfish Immunology (accepted for publication). doi: 10.1016/j. fsi.2018.03.028

Embregts, C.W.E, Tadmor-Levi, R., Veselý, T., Pokorová, D., David, L., Wiegertjes, G.F. and Forlenza, M., 2018. Intra-muscular and oral vaccination using a Koi Herpesvirus ORF25 DNA vaccine does not confer protection in common carp (Cyprinus carpio L.). Fish \& Shellfish Immunology (accepted for publication).

doi: 10.1016/j.fsi.2018.03.037

Embregts, C.W.E., Reyes-Lopez, F., Pall, A.C., Stratmann, A., Tort, L., Lorenzen, N., Engell-Sorensen, K., Wiegertjes, G.F., Forlenza, M., Sunyer, O., Parra, D., 2018, The potential use of the yeast Pichia pastroris as a vehicle for oral vaccination in larval and adult teleosts. Submitted for publication.

Embregts, C.W.E., Rigaedeau, D., Veselý, T., Pokorova, D., Lorenzen, N., Petit, J., Houel, A., Dauber, M., Schütze, H., Boudinot, P., Wiegertjes, G.F., Forlenza, M. "Intra-muscular DNA vaccination against SVCV induces full protection in juvenile carp and establishes a virus-specific B- and T-cell memory". Frontiers in Immunology 8 (2017): article 1340. doi:10.3389/fimmu.2017.01340

Embregts, C.W.E., Forlenza, M., "Oral vaccination of fish: Lessons from humans and veterinary species." Developmental \& Comparative Immunology 64 (2016): 118-137. doi: 10.1016/j.dci.2016.03.024 


\section{Overview of completed training activities}

The basic package

WIAS introduction course

Ethics and philosophy in science

Scientific exposure

\section{International conferences}

Young Scientists Symposium, Insel Vilm, Germany

International Fish and Shellfish Immunology Conference,

Vigo, Spain

ISDCI Immunology conference, Murcia, Spain

International Fish and Shellfish Immunology Conference,

Portland (Maine), US

International Conference on Diseases of Fish and Shellfish,

Belfast, Ireland

\section{Seminars and workshops}

WIAS Science Day, Wageningen, the Netherlands

Wageningen PhD symposium, Wageningen, the Netherlands

Dutch Society for Immunology (NVVI),

Lunteren, the Netherlands

\section{Poster presentations}

WIAS Science Day, Wageningen, the Netherlands

Fish Immunology Workshop, Wageningen, the Netherlands

\section{Oral presentations}

Young Scientists Symposium, Insel Vilm, Germany

2012

ISDCI Immunology conference, Murcia, Spain 2015

Wageningen PhD Symposium, Wageningen, the Netherlands 2015

International Fish and Shellfish Immunology Conference,

Portland US

WIAS Science Day, Wageningen, the Netherlands

Fish Immunology Workshop, Wageningen, the Netherlands 2017

International Conference on Diseases of Fish and Shellfish, 2017

Belfast, Ireland, $2 x$ oral 
In-depth studies

Design of experiments

Advanced Immunology Course, Utrecht, the Netherlands

Fish Immunology and Vaccinology Workshop, Wageningen

Summerschool Vaccinology, Antwerp, Belgium

\section{Professional skills support courses}

Course Supervising MSc thesis work

PhD Competence assessment or Job assessment

Project and time management

Data management

Efficient writing strategies

Scientific writing

The final touch: Writing the introduction and discussion

Research skills training

Preparing own PhD research proposal

\section{ECTS}

2013

2014

2014

2014

\section{ECTS}

2013

2014

2014

2014

2015

2016

2017

\section{ECTS}

2013

Didactic skills training

35 ECTS

Lecturing

Cell biology I

2013, 2014, 2015, 2016

Supervising practicals and excursions

Cell Biology I

2013, 2014, 2015, 2016

Fish Immunology Workshop

2014, 2015, 2016, 2017

\section{Supervising theses}

Supervising four MSc theses

2013-2016

Supervising one BSC thesis

\section{Education and training total}

\section{ECTS}

Completion of the training activities is in fulfilment of the requirements for the education certificate of the Graduate School of the Wageningen Institute of Animal Sciences (WIAS). One ECTS equals a study load of 28 hours. 


\section{Acknowledgements}

Five years, many hours in the lab, many

succeses and also many "challenges", where to start to thank you all! I know you will all read (only) this pages so in order to avoid the embarassing situation where I (most likely) forget to mention some of you, you can pick the statements below that fit you best. You all know I am really thankful to you all and I hope I mentioned this already often enough throughout the years. I am looking back at a great and exciting time and I hope to see you all soon again, easy!
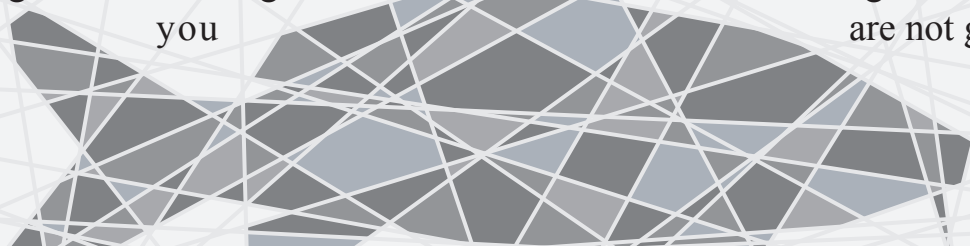

Giving enroll in 21 this

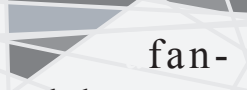
last-minute orders of laboratory tastic project - Arranging vaccination or sampling trials - All the elaborate discussions and feedback on my work (sorry for writing such a large thesis..!) - Coming to work during the Christmas holiday to de-block my account - Taking care of me when I cut my hand, and again, and again..! Cheering me up with making Elephant Toothpaste - All the therapeutic "Tol-Tripjes" and our shared mini-plant addiction - All the amazing \#fun we had at the PhD weekends, WE-Days, coffee and lunch breaks - All the (international) collaborators within the TARGETFISH project - Making the fish immunology field a warm family to work in - Delivering meals during the most stressful writing phase - Given me the opportunity to put my own creativity into my project - Tolerating my "not-so-clean" desk policy - Teaching me all techniques in the lab - Helping me out when I got stuck with the microscope/ FACS/ ... - Helping me when the flow cytometer decided to become a fountain - Taking care of my fish and setting up beautiful vaccination tanks - Making me a better/ stronger/ more confident person - Giving me the opportunity to present at multiple (international) conferences -Supporting me with my new challenge/ job - Making sure my desert-stomach was never empty - All the nice evenings we had with the " fish-unit" - Making the E-Wing the best wing of the Zodiac and the nicest place to work - Always supporting me in my decisions, even if this meant that I spent too little time with friends and family - Standing next to me in the Aula - Let me borrow money (twice..) when I lost or forgot my wallet during a conference - Staying friends even after leaving the CBI gang - Getting me coffee without even asking for it - 
Helping me out with experiments even when I am gone already - Taking over the legacy of the carp B and T cell saga - Always making some time (in the evening) for elaborate brainstorm sessions - Making the really stunning figures for my introduction - Staying positive on turning the IgTerrible into IgTeriffic - Raising strange discussions during coffee breaks to reset the brain - Being proud of what I do even when it is hard to grasp - Always trying to see positive things in negative (vaccination..) outcomes - Always purring loud and happy when I come home, even when it is far after cat dinner time - Being there for me when I need(ed) it - Tolerating all the jokes and office make-overs we performed throughout the years - All the help in the lab I got from colleagues, students, collaborators - Always appreciating the friemels I put in your desk Helping us CBIs out with computer problems - Teaching me how to make alginates, or to perform phagocytosis assays, IH stainings,... - Thank you all for all the support!!

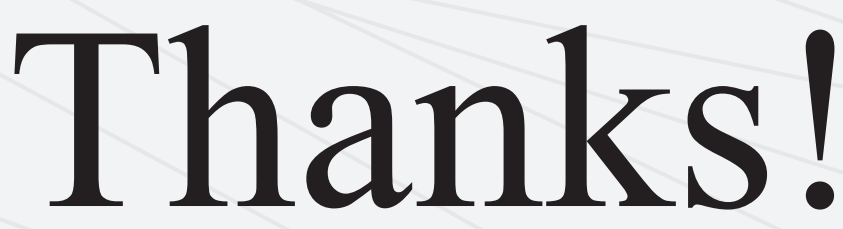


This research described in this thesis was financially supported by the European Commission under the 7th Framework Programme for Research and Technological Development (FP7) of the European Union (Grant Agreement 311993 TARGETFISH).

Cover design by Cees Voesenek and Carmen Embregts Layout by Carmen Embregts 